\title{
Basic data on fertility in the provinces of China, 1940-82
}

Ansley J. Coale and Chen Sheng Li

\begin{tabular}{|c||}
\hline PAPERS \\
OF THE \\
EAST-WEST \\
POPULATION \\
INSTITUTE \\
\hline NUMBER \\
104 \\
JANUARY \\
1987 \\
\hline F. \\
EAST-WEST \\
CENTER \\
HONOLULU, \\
HAWAII \\
\hline
\end{tabular}


PAPERS OF THE EAST-WEST POPULATION INSTITUTE, published about eight times a year, facilitate early dissemination of research findings and policyrelevant reports on the populations of Asia, the Pacific, and the United States. Cost per copy, $\$ 3$.

NOTE TO CONTRIBUTORS: The Population Institute considers unsolicited as well as commissioned manuscripts for the Paper Series. Appropriate topics are demographic trends and estimation, fertility and family structure, economic development and human resources, urbanization and migration, and population policies and programs. All manuscripts are reviewed. In selecting manuscripts for publication, the Institute considers quality of scholarship and usefulness to public officials and other professionals in the field of population; it also seeks contributions reflecting diverse cultural and disciplinary perspectives on population. The series can accommodate articles not necessarily suited for journals because of unusual length or treatment of subject. All copy must be typed double-spaced. For additional information on manuscript preparation, write to Editor, Paper Series.

\section{OTHER SERIAL PUBLICATIONS OF THE EAST-WEST POPULATION INSTITUTE}

Working Papers are circulated for comment and to inform interested colleagues about work in progress at the East-West Population Institute. They are intended to complement evidence of completed work as reflected in Papers of the EastWest Population Institute and the Reprint Series. \$1 per copy.

Reprint Series brings selected articles originating from Institute research but published elsewhere to the attention of population specialists who might not otherwise see them. Single copies available upon request.

Asian and Pacific Population Forum brings articles of potential value in policy formulation, program administration, and research to the notice of policymakers, professionals, and scholars concerned with population matters in the AsiaPacific region. Published quarterly in November, February, May, and August. Annual subscription rate, $\$ 12$ (free to individuals and organizations involved in population-related work).

Serial publications except Working Papers are available without charge to libraries serving population specialists and to professionals and scholars in the field of population. Requests describing the nature of the research or program and the intended use of the publications should be addressed to: Publication Sales and Distribution, East-West Center.

East-West Population Institute

East-West Center

1777 East-West Road

Director Lee-Jay Cho

Honolulu, Hawaii 96848

Senior Editor Sandra E. Ward

Editor David Ellis 


\title{
Basic data on fertility in the provinces of China, 1940-82
}

\author{
Ansley J. Coale \\ and Chen Sheng $\mathrm{Li}$
}

Number $104 \cdot$ January 1987 
ANSLEY J. COALE is William Church Osborne Professor Emeritus of Public Affairs and Associate Director (retired) of the Office of Population Research at Princeton University. CHEN SHENG LI is Head of Family Planning Statistics, Jilin Provincial Family Planning Commission, People's Republic of China.

Library of Congress Cataloging-in-Publication Data

Coale, Ansley J.

Basic data on fertility in the provinces of China, 1940-82.

(Papers of the East-West Population Institute, ISSN 0732-0531; no. 104)

"January 1987."

1. Fertility, Human-China-Provinces-History. I. Chen Sheng $\mathrm{Li}, 1945-\quad$. II. Title. III. Series. HB1064.A3C63 $1987 \quad 304.6^{\prime} 32^{\prime} 0951 \quad 86-32765$ ISBN 0-86638-088-4 


\section{ERRATA}

Basic Data on Fertility in the Provinces of China, 1940-82 by Ansley J. Coale and Chen Sheng $\mathrm{Li}$

Papers of the East-West Population Institute, No. 104, January 1987

1. Page 1, line 3 of the Abstract: The phrase "within each province for total urban, and rural populations" should be "within each province for total, urban, and rural populations."

2. Page 6, Table 1: This table contains several errors. A corrected table follows.

Basic Data on Fertility in the Provinces of China, 1940-82

Table 1. Number of respondents and number of sample units in each province: One per Thousand Sample Fertility Survey, 1982

\begin{tabular}{|c|c|c|c|c|c|}
\hline \multirow{2}{*}{\multicolumn{2}{|c|}{ Province }} & \multicolumn{3}{|c|}{ Number of respondents } & \multirow{3}{*}{$\begin{array}{c}\begin{array}{c}\text { Number of } \\
\text { sample units }\end{array} \\
7\end{array}$} \\
\hline & & \multirow{2}{*}{$\frac{\text { Total }}{3,103}$} & \multirow{2}{*}{$\frac{\text { Urban }}{1,941}$} & \multirow{2}{*}{$\frac{\text { Rural }}{1,162}$} & \\
\hline 1 & Beijing & & & & \\
\hline 2 & Tianjin & 3,044 & 1,719 & 1,325 & 6 \\
\hline 3 & Hebei & 17,030 & 1,575 & 15,455 & 53 \\
\hline 4 & Shanxi & 7,681 & 955 & 6,726 & 30 \\
\hline 5 & Inner Mongolia & 6,257 & 1,843 & 4,414 & 16 \\
\hline 6 & Liaoning & 12,513 & 4,836 & 7,677 & 22 \\
\hline 7 & Jilin & 6,744 & 1,892 & 4,852 & 14 \\
\hline 8 & Heilongjiang & 9,599 & 3,133 & 6,466 & 23 \\
\hline 9 & Shanghai & 4,416 & 2,888 & 1,528 & 6 \\
\hline 10 & Jiangsu & 21,019 & 3,447 & 17,572 & 41 \\
\hline 11 & Zhejiang & 11,705 & 1,440 & 10,265 & 44 \\
\hline 12 & Anhui & 14,357 & 1,662 & 12,695 & 32 \\
\hline 13 & Fujian & 7,414 & 826 & 6,588 & 17 \\
\hline 14 & Jiangxi & 9,509 & 1,094 & 8,415 & 24 \\
\hline 15 & Shandong & 23,409 & 1,559 & 21,850 & 88 \\
\hline 16 & Henan & 21,717 & 1,992 & 19,725 & 50 \\
\hline 17 & Hubei & 15,638 & 2,419 & 13,219 & 38 \\
\hline 18 & Hunan & 16,727 & 1,939 & 14,788 & 50 \\
\hline 19 & Guangdong' & 17,692 & 3,020 & 14,672 & 32 \\
\hline 20 & Guangxi & 10,512 & 973 & 9,539 & 17 \\
\hline 21 & Sichuan & 30,114 & 4,385 & 25,729 & 85 \\
\hline 22 & Guizhou & 7,841 & 1,108 & 6,733 & 27 \\
\hline 23 & Yunnan & 10,088 & 1,254 & 8,834 & 16 \\
\hline 24 & Shaanxi & 8,956 & 1,545 & 7,411 & 35 \\
\hline 25 & Gansu & 5,732 & 1,057 & 4,675 & 19 \\
\hline 26 & Qinghai & 1,489 & 283 & 1,206 & 6 \\
\hline 27 & Ningxia & 1,290 & 387 & 903 & 4 \\
\hline 28 & Xinjiang & 4,418 & 1,241 & 3,177 & 13 \\
\hline Tot & & 310,014 & 52,413 & 257,601 & 815 \\
\hline
\end{tabular}




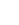
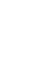


\section{CONTENTS}

Acknowledgments $\quad x v i i$

Abstract 1

Part I. Changes in fertility, 1940-82 1

Age-specific and duration-specific fertility rates for twenty-eight provinces 2

Total fertility rates by age and duration since first marriage 2

Truncation of fertility records for early years (before 1964) 4

Variability of rates attributable to the limited sample sizes

in individual provinces 5

Features of China's fertility rates 7

Fertility differences and fertility trends in the provinces 12

How age-specific and duration-specific rates were calculated 17

Truncation of estimates because of upper age limit of respondents 18

Estimation of TDFR and TAFR, 1945-63 18

Estimation of TAFR from duration-specific rates, and total firstmarriage rates of preceding years 20

Part II. Basic Tables: Fertility rates by age of woman, and fertility rates of ever-married women by duration since first marrriage, 1940-82, for Mainland China and twenty-eight provinces: total, urban, and rural 23

Appendix A. Comments on the total age-specific fertility rate, total age-specific married fertility rate, and total duration-specific fertility rate 356

Hypothetical example of duration-specific and age-specific fertility when age at marriage changes 358

Conversion of TDFR into TAFR in China 360

TAFR and TDFR: Summary 362

Appendix B. Proofs of two propositions about the relation of TDFR and TAFR (by German Rodriguez and Ansley J. Coale) 363 


\section{TABLES}

\section{Text Tables}

1. Number of respondents and number of sample units in each province: One per Thousand Sample Fertility Survey, $1982 \quad 6$

2. Median marital fertility rates at duration 5-9 years in selected periods, for the provinces of Mainland China 15

3. Ratios of fertility rates at different durations employed in the calculation of TDFR for 1940-63 20

4. Calculation of TDFR , and estimated TAFR, for the rural population of China: $1980 \quad 21$

5. Calculation of TDFR*, and estimated TAFR, for the rural population of China: 195022

A.1. Total duration-specific fertility, total age-specific fertility, and adjusted total duration-specific fertility, for rural Liaoning Province, 1960-82, and for China and selected provinces, 1979361

Basic Tables

1.A. Total China: age-specific fertility rates, 1940-82 24

B. Urban China: age-specific fertility rates, 1940-82 26

C. Rural China: age-specific fertility rates, 1940-82 28

2.A. Total Beijing Province (1): age-specific fertility rates, 1940-82 30

B. Urban Beijing Province (1): age-specific fertility rates, 1940-82 32

C. Rural Beijing Province (1): age-specific fertility rates, 1940-82 34

3.A. Total Tianjin Province (2): age-specific fertility rates, 1940-82 36

B. Urban Tianjin Province (2): age-specific fertility rates, 1940-82 38

C. Rural Tianjin Province (2): age-specific fertility rates, 1940-82 40

4.A. Total Hebei Province (3): age-specific fertility rates, 1940-82 42

B. Urban Hebei Province (3): age-specific fertility rates, 1940-82 44

C. Rural Hebei Province (3): age-specific fertility rates, 1940-82 46

5.A. Total Shanxi Province (4): age-specific fertility rates, 1940-82 48 
5.B. Urban Shanxi Province (4): age-specific fertility rates, 1940-82 50

C. Rural Shanxi Province (4): age-specific fertility rates, 1940-82 52

6. A. Total Inner Mongolia Province (5): age-specific fertility rates, 1940-82 54

B. Urban Inner Mongolia Province (5): age-specific fertility rates, 1940-82 56

C. Rural Inner Mongolia Province (5): age-specific fertility rates, 1940-82 58

7.A. Total Liaoning Province (6): age-specific fertility rates, 1940-82 60

B. Urban Liaoning Province (6): age-specific fertility rates, 1940-82 62

C. Rural Liaoning Province (6): age-specific fertility rates, 1940-82 64

8.A. Total Jilin Province (7): age-specific fertility rates, 1940-82 66

B. Urban Jilin Province (7): age-specific fertility rates, 1940-82 68

C. Rural Jilin Province (7): age-specific fertility rates, 1940-82 70

9.A. Total Heilongjiang Province (8): age-specific fertility rates, 1940-82 72

B. Urban Heilongiiang Province (8): age-specific fertility rates, 1940-82 74

C. Rural Heilongjiang Province (8): age-specific fertility rates, 1940-82 76

10.A. Total Shanghai Province (9): age-specific fertility rates, 1940-82 78

B. Urban Shanghai Province (9): age-specific fertility rates, 1940-82 80

C. Rural Shanghai Province (9): age-specific fertility rates, 1940-82. 82

11.A. Total Jiangsu Province (10): age-specific fertility rates, 1940-82 84

B. Urban Jiangsu Province (10): age-specific fertility rates, 1940-82 86

C. Rural Jiangsu Province (10): age-specific fertility rates, 1940-82 88

12.A. Total Zhejiang Province (11): age-specific fertility rates, 1940-82 90

B. Urban Zhejiang Province (11): age-specific fertility rates, 1940-82 92 
12.C. Rural Zhejiang Province (11): age-specific fertility rates, 1940-82 94

13.A. Total Anhui Province (12): age-specific fertility rates, 1940-82 96

B. Urban Anhui Province (12): age-specific fertility rates, 1940-82 98

C. Rural Anhui Province (12): age-specific fertility rates, 1940-82 100

14.A. Total Fujian Province (13): age-specific fertility rates, 1940-82 102

B. Urban Fujian Province (13): age-specific fertility rates, 1940-82 104

C. Rural Fujian Province (13): age-specific fertility rates, 1940-82 106

15.A. Total Jiangxi Province (14): age-specific fertility rates, 1940-82 108

B. Urban Jiangxi Province (14): age-specific fertility rates, 1940-82 110

C. Rural Jiangxi Province (14): age-specific fertility rates, 1940-82 112

16.A. Total Shandong Province (15): age-specific fertility rates, 1940-82 114

B. Urban Shandong Province (15): age-specific fertility rates, 1940-82 116

C. Rural Shandong Province (15): age-specific fertility rates, 1940-82 118

17.A. Total Henan Province (16): age-specific fertility rates, 1940-82 120

B. Urban Henan Province (16): age-specific fertility rates, 1940-82 122

C. Rural Henan Province (16): age-specific fertility rates, 1940-82 124

18.A. Total Hubei Province (17): age-specific fertility rates, 1940-82 126

B. Urban Hubei Province (17): age-specific fertility rates, 1940-82 128

C. Rural Hubei Province (17): age-specific fertility rates, 1940-82 130

19.A. Total Hunan Province (18): age-specific fertility rates, 1940-82 132 
19.B. Urban Hunan Province (18): age-specific fertility rates, 1940-82 134

C. Rural Hunan Province (18): age-specific fertility rates, 1940-82 136

20.A. Total Guangdong Province (19): age-specific fertility rates, 1940-82 138

B. Urban Guangdong Province (19): age-specific fertility rates, 1940-82 140

C. Rural Guangdong Province (19): age-specific fertility rates, 1940-82 142

21.A. Total Guangxi Province (20): age-specific fertility rates, 1940-82 144

B. Urban Guangxi Province (20): age-specific fertility rates, 1940-82 146

C. Rural Guangxi Province (20): age-specific fertility rates, 1940-82 148

22.A. Total Sichuan Province (21): age-specific fertility rates, 1940-82 150

B. Urban Sichuan Province (21): age-specific fertility rates, 1940-82 152

C. Rural Sichuan Province (21): age-specific fertility rates, 1940-82 154

23.A. Total Guizhou Province (22): age-specific fertility rates, 1940-82 156

B. Urban Guizhou Province (22): age-specific fertility rates, 1940-82 158

C. Rural Guizhou Province (22): age-specific fertility rates, 1940-82 160

24.A. Total Yunnan Province (23): age-specific fertility rates, 1940-82 162

B. Urban Yunnan Province (23): age-specific fertility rates, 1940-82 164

C. Rural Yunnan Province (23): age-specific fertility rates, 1940-82 166

25.A. Total Shaanxi Province (25): age-specific fertility rates, 1940-82 168

B. Urban Shaanxi Province (25): age-specific fertility rates, 1940-82 170

C. Rural Shaanxi Province (25): age-specific fertility rates, 1940-82 172 
26.A. Total Gansu Province (26): age-specific fertility rates, 1940-82 174

B. Urban Gansu Province (26): age-specific fertility rates, 1940-82 176

C. Rural Gansu Province (26): age-specific fertility rates, 1940-82 178

27. Total Qinghai Province (27): age-specific fertility rates, 1940-82 180

28. Total Ningxia Province (28): age-specific fertility rates, 1940-82 182

29.A. Total Xinjian Province (29): age-specific fertility rates, 1940-82 184

B. Urban Xinjian Province (29): age-specific fertility rates, 1940-82 186

C. Rural Xinjian Province (29): age-specific fertility rates, 1940-82 188

30.A. Total China: duration-specific fertility rates, 1940-82 190

B. Urban China: duration-specific fertility rates, 1940-82 192

C. Rural China: duration-specific fertility rates, 1940-82 194

31.A. Total Beijing Province (1): duration-specific fertility rates, 1940-82 196

B. Urban Beijing Province (1): duration-specific fertility rates, 1940-82 198

C. Rural Beijing Province (1): duration-specific fertility rates, 1940-82 200

32.A. Total Tianjin Province (2): duration-specific fertility rates, 1940-82 202

B. Urban Tianjin Province (2): duration-specific fertility rates, 1940-82 204

C. Rural Tianjin Province (2): duration-specific fertility rates, 1940-82 206

33.A. Total Hebei Province (3): duration-specific fertility rates, 1940-82 208

B. Urban Hebei Province (3): duration-specific fertility rates, 1940-82 210

C. Rural Hebei Province (3): duration-specific fertility rates, 1940-82 212

34.A. Total Shanxi Province (4): duration-specific fertility rates, 1940-82 214 
34.B. Urban Shanxi Province (4): duration-specific fertility rates, 1940-82 216

C. Rural Shanxi Province (4): duration-specific fertility rates, 1940-82 218

35.A. Total Inner Mongolia Province (5): duration-specific fertility rates, 1940-82 220

B. Urban Inner Mongolia Province (5): duration-specific fertility rates, 1940-82 222

C. Rural Inner Mongolia Province (5): duration-specific fertility rates, 1940-82 224

36.A. Total Liaoning Province (6): duration-specific fertility rates, 1940-82 226

B. Urban Liaoning Province (6): duration-specific fertility rates, 1940-82 228

C. Rural Liaoning Province (6): duration-specific fertility rates, 1940-82 230

37.A. Total Jilin Province (7): duration-specific fertility rates, 1940-82 232

B. Urban Jilin Province (7): duration-specific fertility rates, 1940-82 234

C. Rural Jilin Province (7): duration-specific fertility rates, 1940-82 236

38.A. Total Heilongjiang Province (8): duration-specific fertility rates, 1940-82 238

B. Urban Heilongjiang Province (8): duration-specific fertility rates, 1940-82 240

C. Rural Heilongjiang Province (8): duration-specific fertility rates, 1940-82 242

39.A. Total Shanghai Province (9): duration-specific fertility rates, 1940-82 244

B. Urban Shanghai Province (9): duration-specific fertility rates, 1940-82 246

C. Rural Shanghai Province (9): duration-specific fertility rates, 1940-82 248

40.A. Total Jiangsu Province (10): duration-specific fertility rates, 1940-82 250

B. Urban Jiangsu Province (10): duration-specific fertility rates, 1940-82 252

C. Rural Jiangsu Province (10): duration-specific fertility rates, 1940-82 254 
41.A. Total Zhejiang Province (11): duration-specific fertility rates, 1940-82 256

B. Urban Zhejiang Province (11): duration-specific fertility rates, 1940-82 258

C. Rural Zhejiang Province (11): duration-specific fertility rates, 1940-82 260

42.A. Total Anhui Province (12): duration-specific fertility rates, 1940-82 262

B. Urban Anhui Province (12): duration-specific fertility rates, 1940-82 264

C. Rural Anhui Province (12): duration-specific fertility rates, 1940-82 266

43.A. Total Fujian Province (13): duration-specific fertility rates, 1940-82 268

B. Urban Fujian Province (13): duration-specific fertility rates, 1940-82 270

C. Rural Fujian Province (13): duration-specific fertility rates, 1940-82 272

44.A. Total Jiangxi Province (14): duration-specific fertility rates, 1940-82 274

B. Urban Jiangxi Province (14): duration-specific fertility rates, 1940-82 276

C. Rural Jiangxi Province (14): duration-specific fertility rates, 1940-82 278

45.A. Total Shandong Province (15): duration-specific fertility rates, 1940-82 280

B. Urban Shandong Province (15): duration-specific fertility rates, 1940-82 282

C. Rural Shandong Province (15): duration-specific fertility rates, 1940-82 284

46.A. Total Henan Province (16): duration-specific fertility rates, 1940-82 286

B. Urban Henan Province (16): duration-specific fertility rates, 1940-82 288

C. Rural Henan Province (16): duration-specific fertility rates, 1940-82 290

47.A. Total Hubei Province (17): duration-specific fertility rates, 1940-82 292

B. Urban Hubei Province (17): duration-specific fertility rates, 1940-82 294 
47.C. Rural Hubei Province (17): duration-specific fertility rates, 1940-82 296

48.A. Total Hunan Province (18): duration-specific fertility rates, 1940-82 298

B. Urban Hunan Province (18): duration-specific fertility rates, 1940-82 300

C. Rural Hunan Province (18): duration-specific fertility rates, 1940-82 302

49.A. Total Guangdong Province (19): duration-specific fertility rates, 1940-82 304

B. Urban Guangdong Province (19): duration-specific fertility rates, 1940-82 306

C. Rural Guangdong Province (19): duration-specific fertility rates, 1940-82 308

50.A. Total Guangxi Province (20): duration-specific fertility rates, 1940-82 310

B. Urban Guangxi Province (20): duration-specific fertility rates, 1940-82 312

C. Rural Guangxi Province (20): duration-specific fertility rates, 1940-82 314

51.A. Total Sichuan Province (21): duration-specific fertility rates, 1940-82 316

B. Urban Sichuan Province (21): duration-specific fertility rates, 1940-82 318

C. Rural Sichuan Province (21): duration-specific fertility rates, 1940-82 320

52.A. Total Guizhou Province (22): duration-specific fertility rates, 1940-82 322

B. Urban Guizhou Province (22): duration-specific fertility rates, 1940-82 324

C. Rural Guizhou Province (22): duration-specific fertility rates, 1940-82 326

53.A. Total Yunnan Province (23): duration-specific fertility rates, 1940-82 328

B. Urban Yunnan Province (23): duration-specific fertility rates, 1940-82 330

C. Rural Yunnan Province (23): duration-specific fertility rates, 1940-82 332

54.A. Total Shaanxi Province (25): duration-specific fertility rates, 1940-82 334 
54.B. Urban Shaanxi Province (25): duration-specific fertility rates, 1940-82 336

C. Rural Shaanxi Province (25): duration-specific fertility rates, 1940-82 338

55.A. Total Gansu Province (26): duration-specific fertility rates, 1940-82 340

B. Urban Gansu Province (26): duration-specific fertility rates, 1940-82 342

C. Rural Gansu Province (26): duration-specific fertility rates, 1940-82 344

56. Total Qinghai Province (27): duration-specific fertility rates, 1940-82 346

57. Total Ningxia Province (28): duration-specific fertility rates, 1940-82 348

58.A. Total Xinjiang Province (29): duration-specific fertility rates, 1940-82 350

B. Urban Xinjiang Province (29): duration-specific fertility rates, 1940-82 352

C. Rural Xinjiang Province (29): duration-specific fertility rates, 1940-82 354 



\section{FIGURES}

1. Marital fertility rates, marriage duration of 0-4 years: Rural Beijing Province, $1940-80$, as recorded, and five-term moving average 8

2. Fertility rates of ever-married women by five-year intervals of duration since first marriage: Rural China, 1940-82 9

3. Fertility rates of ever-married women by five-year intervals of duration since first marriage: Urban China, 1940-82 11

4. Fertility rates by five-year age intervals: Rural China, 1940-82 13

5. Fertility rates by five-year age intervals: Urban China, 1940-82 14

6. Marital fertility rates at marriage duration of 5-9 years: Provinces of China, different periods 16 



\section{ACKNOWLEDGMENTS}

Thanks are due to the State Family Planning Commission, China, for their support of this project. Victoria Ho of the East-West Population Institute provided the computations for the fertility tables. Financial support for the institute's research on China's demography has come from the Hewlett Foundation, the Rockefeller Foundation, and the United Nations Fund for Population Activities. 

ABSTRACT This paper presents age-specific and marriage duration-specific fertility rates for Mainland China and each of twenty-eight provinces, and within each province for total urban, and rural populations, during the period 1940-82. The rates were tabulated from data obtained by China's One per Thousand Sample Fertility Survey, conducted by the State Family Planning Commission in 1982 . The basic tables supplement the history of fertility change in China as a whole already revealed in the report of the survey published by the Chinese government in 1983. The additional information on marital fertility illustrates the substantial effect of changes in nuptiality on the course of total fertility in China. The provincial data show the geographical diversity of fertility from the earliest years to 1982 . This fundamental information about China's population will be useful to students of the Chinese society, both within China and elsewhere.

\section{PART I. CHANGES IN FERTILITY, 1940-82}

Because China's population is the world's largest, and because fertility and mortality have changed dramatically since the People's Republic was founded, there is special interest in demographic information about China. Much detailed and accurate information has been published in reports on the census taken in 1982 and on the censuses of 1953 and 1964 belatedly released in 1983. An invaluable additional source of information is the very large, One per Thousand Sample Fertility Survey conducted in 1982 by the State Family Planning Commission. Published reports on the survey provide a remarkably complete and detailed record of fertility in China since the 1940s.

In the sample fertility survey, all women 15 to 67 years of age in sampled households were interviewed. The households contained a total population of more than one million persons. The survey questionnaire asked for the birth date of the respondent, the date of her first marriage, the date of every birth she had experienced, the sex of each child born, whether the child had survived to the time of the survey, the education and occupation of the respondent, and her current contraceptive practice. The survey is exceptional because of its very large sample, the care with which it was conducted, and the accuracy of the dates of births and marriages reported by the respondents. Results of extensive analysis of the survey for China as a whole were published in 1983 in a special issue of the journal Population and Economy, and in 1984 in English translation (Analysis on China's National One-per-Thousand Population Fertility Sampling Survey) by the China Population Information Centre. The English version of the report is 182 pages long and contains many detailed tables and charts. Among other details, it lists first-marriage rates and rates of childbearing, by single years of age for the rural, urban, and total populations of China for each year 
from 1950 to 1981, with supplementary data and estimates for the 1940s.

During the summer of 1985, tapes containing the complete original data from this survey for each of twenty-eight provinces (all of the provinces in Mainland China except Tibet, Province 24) were analyzed on the computer at the East-West Population Institute in Honolulu in a collaborative enterprise involving experts from the State Family Planning Commission and staff and consultants at the Population Institute.

The data on these tapes are an extraordinary resource. Because the sample is so large, many forms of detailed analysis are feasible. An example, presented here, is the calculation of fertility rates since 1940 for each province. Because there are 311,000 respondents, the average number per province-over 11,000-exceeds the number in most national surveys of fertility.

\section{Age-specific and duration-specific fertility rates for twenty-eight provinces}

Part II of this paper contains 166 tables, six for each of twenty-six provinces, two each for two provinces in which the urban sample is exceptionally small, and six for China as a whole. The tables present fertility rates for women at each five-year age interval from 15-19 to 45-49 years of age for each year from 1940 to 1982. Fertility rates for ever-married women at each five-year interval of duration since first marriage from 0-4 to 30-34 years are also presented for the period from 1940 to 1982 . For each province (except Qinghai and Ningxia, where the urban samples are so small that the annual rates are extremely erratic), separate tables have been prepared for the urban, rural, and total populations.

\section{Total fertility rates by age and duration since first marriage}

Each table of age-specific or duration-specific fertility rates also contains a column of total fertility rates: total age-specific rates (TAFRs) and total duration-specific rates (TDFRs). These totals, which are the sum of the single-year (of age or duration) rates, or five times the sum of the rates by five-year intervals, show how many children would be born per woman in a hypothetical population subject to the rates that occurred during the year in question. The TAFR shows the average number of lifetime births for all women subject to that year's fertility rates by age; the TDFR shows the average number of lifetime births for ever-married women subject to that year's marital fertility rates at each duration since first marriage. The total age-specific fertility rate is a measure of the total fertility of all women, whereas the total duration-specific fertility rate is a measure of the total fertility of ever-married women; the two rates are linked by the recent history of total first-marriage rates. 
In China in a cohort of women (i.e., women born in the same year, for example those who were 50 years old in 1982), TAFR and TDFR are almost exactly equal because the proportion ever married by age 35 is greater than 99 percent, negligibly different from 1.0 . On the assumption that all women marry, the number of children they will have borne by age $50(5.62$ for women in China who reached age 50 in 1982) is the total of the agespecific rates for that cohort, and also the sum of the duration-specific rates to which those women have been subject since first marriage. The two totals are the same because each represents the average number of children ever born to the same women.

Mean age at first marriage in China rose from a little more than 18 years in the 1940s to about 23 years in the late 1970s. The increase in age at first marriage occurred without any change in the universality of marriage for women in China. The proportion remaining single among respondents in the survey was less than 1.0 percent at every age above 30 .

The total first-marriage rate (TMR) is analogous to the total fertility rate. It equals the proportion ever married at the highest age of first marriage that would be produced in a hypothetical cohort experiencing the rate of first marriage at each age in a given year. The total first-marriage rate for birth cohorts in China is very near 1.0, but the TMR for individual calendar years differs, sometimes by a large margin, from 1.0. A crisis can cause many potential marriages to be postponed, and postponed marriages can be crowded into the year after the crisis, so that the TMR falls well below 1.0, and then rises well above 1.0. A second factor causing TMRs different from 1.0 in China is a change in the mean age at marriage. Even when all women in every birth cohort ultimately marry, an increase in age at marriage reduces the annual TMR below 1.0. To see how such a reduction in TMR is brought about, consider a hypothetical population in which all marriages occur (hypothetically mandated by law) at exact age 22. Suppose a new law increases the required exact age at marriage to exact age 23 . All women initially marry at 22; under the new regime all marry at 23 . In the year of the new edict, there are no marriages. The women who would have married at $22 \mathrm{do}$ not; those who attain their twenty-second birthday wait one year until reaching 23 before marrying. Cohort TMR remains at 1.0, but the TMR for one year drops to zero. A real increase by one year in the mean age at first marriage has an effect related to this unrealistic example, since a real increase also causes the loss of one year's normal number of marriages, but over a span of several years, rather than abruptly as in the example.

Mean age at first marriage rose from 18.21 in 1940 to 23.12 in 1979. The result was an average TMR of only 0.882 during 1940-79, despite the continued universality of first marriage. According to a formula suggested by Norman Ryder, TMR for a period of years approximately equals cohort TMR 
multiplied by 1.0 minus the annual rate of change in the mean age at first marriage. The recorded increase in mean age at first marriage is 4.91 years in thirty-nine years, or an average increase of 0.126 years per year. The average TMR during the period 1940-79 should be, by this approximation, 0.874 , quite close to the actual 0.882 .

The reduction in the number of marriages caused by increasing age at marriage has no immediate effect on marital fertility at each duration of marriage. Women married at 23 instead of 22 bear about the same number of children in the early years after marriage. A rise in age at marriage does reduce the number of married women at early durations, however, and does reduce age-specific fertility rates at the ages at which married women in early durations of marriage are found.

It can be proven that, if duration-specific fertility rates are adjusted by multiplying the duration-specific rate at each duration $d$ by the total firstmarriage rate $d$ years earlier, the sum of the adjusted duration-specific rates equals (within a margin of 2 or 3 percent) the sum of the age-specific rates. If age at marriage had been constant in China, the total first-marriage rate would have been close to 1.0 and total duration-specific fertility would have been approximately equal to total age-specific fertility.

In China the degree to which TAFR falls short of TDFR (or, more precisely, falls short of 0.98 TDFR) is a measure of the extent to which TAFR has been lowered by changes in nuptiality. In the 1960s, TAFR was 5 to 7 percent lower than it would have been had age at marriage been constant; but in the 1970s, TAFR was as much as 22 percent lower than it would have been with the same marital fertility by duration of marriage and a history of constant instead of rising age at marriage. By 1981 the contribution of nuptiality change to lower fertility had been reduced by one-half of its contribution during 1977-79 as a result of the marriage boom in 1980 and 1981. (TMR averaged about 0.75 in the 1970 s but rose to an average above 1.20 in 1980-81. See Appendix A for further discussion of TAFR and TDFR.)

\section{Truncation of fertility records for early years (before 1964)}

The survey design called for interviews with all women in the sample households who were 15 to 67 years of age. The upper age limit of 67 means that the marriage and childbearing histories contained no information about events experienced by women above age 25 in 1940, 30 in 1945, 35 in 1950, 40 in 1955, and 45 in 1960. Complete data for women of ages up to 49, which is considered the end of the reproductive age span, are available only for the years since 1964. The basic tables that are the major part of this paper show age-specific fertility rates up to only age 25 in 1940, 30 in 1945, etc., with corresponding truncation of rates tabulated by duration of marriage. In the years for which the data are incomplete, total fertility rates by age 
and duration since first marriage have been estimated. The estimates are based on an assumption of a constant relation between fertility rates at different durations since first marriage, and on the proven relation of TAFR to the sum of duration-specific rates, after multiplication by TMR (displaced an appropriate number of years earlier). The estimates begin in 1945 because the severely truncated data for the earliest years provide an insufficient basis for calculation.

\section{Variability of rates attributable to the limited sample sizes in individual provinces}

A very large sample was interviewed in the fertility survey in order to yield at the national level reliable single-year-of-age fertility rates for single years of time in the period just before the survey. When allowance is made for the greater variance resulting from cluster sampling, the estimated sampling variance at the age of peak fertility $(0.301$ at age 25 in 1981) is $43 \times$ $10^{-6}$, implying a sampling standard deviation of this rate of 0.0066 . Thus the 95 percent confidence limits for the true value of this fertility rate are $0.301 \pm 0.0132$, or 0.288 to 0.314 . The confidence limits for the highest fertility rate in a five-year age interval in China as a whole are about $0.216 \pm$ 0.0059 , or 0.210 to 0.222 . The stochastic variability of the total fertility rates is still less, except in the early years when the estimated rates are based on duration-specific fertility for only two or three duration intervals.

The sample size for most individual provinces is quite respectable in comparison with many national fertility surveys. Nevertheless, stochastic variation is much greater in the fertility rates calculated for individual provinces than for all China. The number of respondents and the number of sample points in each province are listed in Table 1 . In four provinces (Beijing, Tianjin, Qinghai, and Ningxia) the number of respondents is less than 4,000; in two of these (Qinghai and Ningxia) the number is less than 1,500. Roughly speaking, sampling variability increases in inverse proportion to the square root of the number of respondents. Since the number of respondents in Beijing and Tianjin is about 1.0 percent of the national number, the standard deviation of a given fertility rate there is about ten times the corresponding national figure. The data for urban populations are even more affected by small numbers. There are five provinces with fewer than 1,000 urban respondents and only seven with more than 2,000.

The effect of the small number of respondents (exacerbated by clustering into a small number of sampling units in some provinces) is to produce fertility rates that may in some years differ by a wide margin from true rates and that vary erratically from year to year. In rural Beijing (1,162 respondents), for example, there were only about 60 respondents who had been married five years or less during the early 1940s, and they reported an aver- 
Table 1. Number of respondents and number of sample units in each province: One per Thousand Sample Fertility Survey, 1982

\begin{tabular}{|c|c|c|c|c|c|}
\hline & & Num & of resp & ents & Number of \\
\hline Pro & vince & Total & Urban & Rural & sample units \\
\hline 1 & Beijing & 3,103 & 1,941 & 1,162 & 7 \\
\hline 2 & Tianjin & 3,044 & 1,719 & 1,325 & 6 \\
\hline 3 & Hebei & 17,030 & 1,575 & 15,455 & 53 \\
\hline 4 & Shanxi & 7,681 & 955 & 6,726 & 30 \\
\hline 5 & Inner Mongolia & 6,257 & 1,843 & 4,414 & 16 \\
\hline 6 & Liaoning & 12,513 & 4,836 & 7,677 & 22 \\
\hline 7 & Jilin & 6,744 & 1,892 & 4,852 & 14 \\
\hline 8 & Heilongjiang & 9,599 & 3,133 & 6,466 & 23 \\
\hline 9 & Shanghai & 4,416 & 2,888 & 1,528 & 6 \\
\hline 10 & Jiangsu & 21,019 & 3,447 & 17,572 & 41 \\
\hline 11 & Zhejiang & 11,705 & 1,440 & 10,265 & 44 \\
\hline 12 & Anhui & 14,357 & 1,662 & 12,695 & 32 \\
\hline 13 & Fujian & 7,414 & 826 & 6,588 & 17 \\
\hline 14 & Jiangxi & 9,505 & 1,094 & 8,415 & 24 \\
\hline 15 & Shandong & 23,409 & 1,559 & 21,850 & 88 \\
\hline 16 & Henan & 21,717 & 1,992 & 19,725 & 50 \\
\hline 17 & Hubei & 15,638 & 2,419 & 13,219 & 38 \\
\hline 18 & Hunan & 16,727 & 1,939 & 14,788 & 50 \\
\hline 19 & Guangdong & 17,692 & 3,020 & 14,672 & 32 \\
\hline 20 & Guangxi & 10,512 & 973 & 9,539 & 17 \\
\hline 21 & Sichuan & 30,114 & 4,385 & 26,729 & 85 \\
\hline 22 & Guizhou & 7,841 & 1,108 & 6,733 & 27 \\
\hline 23 & Yunnan & 10,688 & 1,254 & 8,834 & 16 \\
\hline 24 & Shaanxi & 8,956 & 1,545 & 7,411 & 35 \\
\hline 25 & Gansu & 5,732 & 1,057 & 4,675 & 19 \\
\hline 26 & Qinghai & 1,489 & 283 & 1,206 & 6 \\
\hline 27 & Ningxia & 1,290 & 387 & 903 & 4 \\
\hline & Xinjiang & 4,418 & 1,241 & 2,517 & 13 \\
\hline Tot & & 310,610 & 52,421 & & 815 \\
\hline
\end{tabular}

age of only about twelve births each year. That the fertility rate at duration $0-4$ in 1943 is more than twice as high as in 1940 could be an artifact of the small sample; for all of rural Beijing the rates in the two years might well have been nearly equal. The erratic rates from year to year at early durations in rural Beijing in the 1940s (resulting from the small number of events reported) lead to erratic estimates of the total duration-specific 
rate (and the total age-specific rate) derived, as they are, from data limited to the early years of marriage of older respondents.

Variability in annual data resulting from small numbers of observations can be reduced by combining data from a sequence of several calendar years, or by other forms of smoothing. In Figure 1, fertility rates at duration 0-4 for rural Beijing Province are shown in a five-year running average (e.g., the average of rates for the period 1940-44 is shown at the middle year, 1942). The averaged rates are much less variable, but of course genuine fluctuations are smoothed out along with stochastic variation. Users of the data for any province are cautioned to bear in mind the sample size when interpreting the annual sequences listed in the basic tables. If a provincial sample is small, some form of smoothing is advisable; the possibility of explaining dips and peaks in any annual sequence is limited to the national population, to larger provinces, and to regional data obtained by combining figures from adjacent provinces.

\section{Features of China's fertility rates}

Fertility rates at different durations since first marriage in rural China (Figure 2) varied in a highly synchronized way until 1969, after which fertility at duration less than five years remained high and fertility at higher durations underwent a large sustained decline. The synchronism of the variation until the late 1960s suggests that the variations from year to year had a quasi-biological source, since voluntary control by means of contraception or induced abortion normally affects fertility at higher durations more than at lower durations, as is evident in the downward trend after 1969. Possible quasi-biological factors were disruptions of married life caused by a change in living arrangements due to collectivization and disruptions of married life or even reduced fecundability caused by an economic crisis, including widespread famine.

Fertility at duration 0-4 was lower than at duration 5-9 in rural China in the 1940s, rose to equality in the early 1950s, and fell slightly below during the big dip in the agricultural crisis years beginning in 1958. The two rates were virtually identical from 1963 until 1969, when fertility at duration 5-9 (and at higher durations) began a steep decline. The almost perfect synchronism of the first two duration-specific rates (and fertility at other durations as well) until the late 1960s signals the absence of fertility control related to parity until after 1969. Lower fertility at duration 0-4 than at 5-9 in the 1940 s was the result of very early marriage before 1950 . When mean age at first marriage is only 18.3 years (its average in the 1940s), many women in their first five years of marriage have lower fecundability than slightly older women. As the frequency of very early marriage diminishes, the rate of childbearing at early duration of marriage rises. (In rural China from 


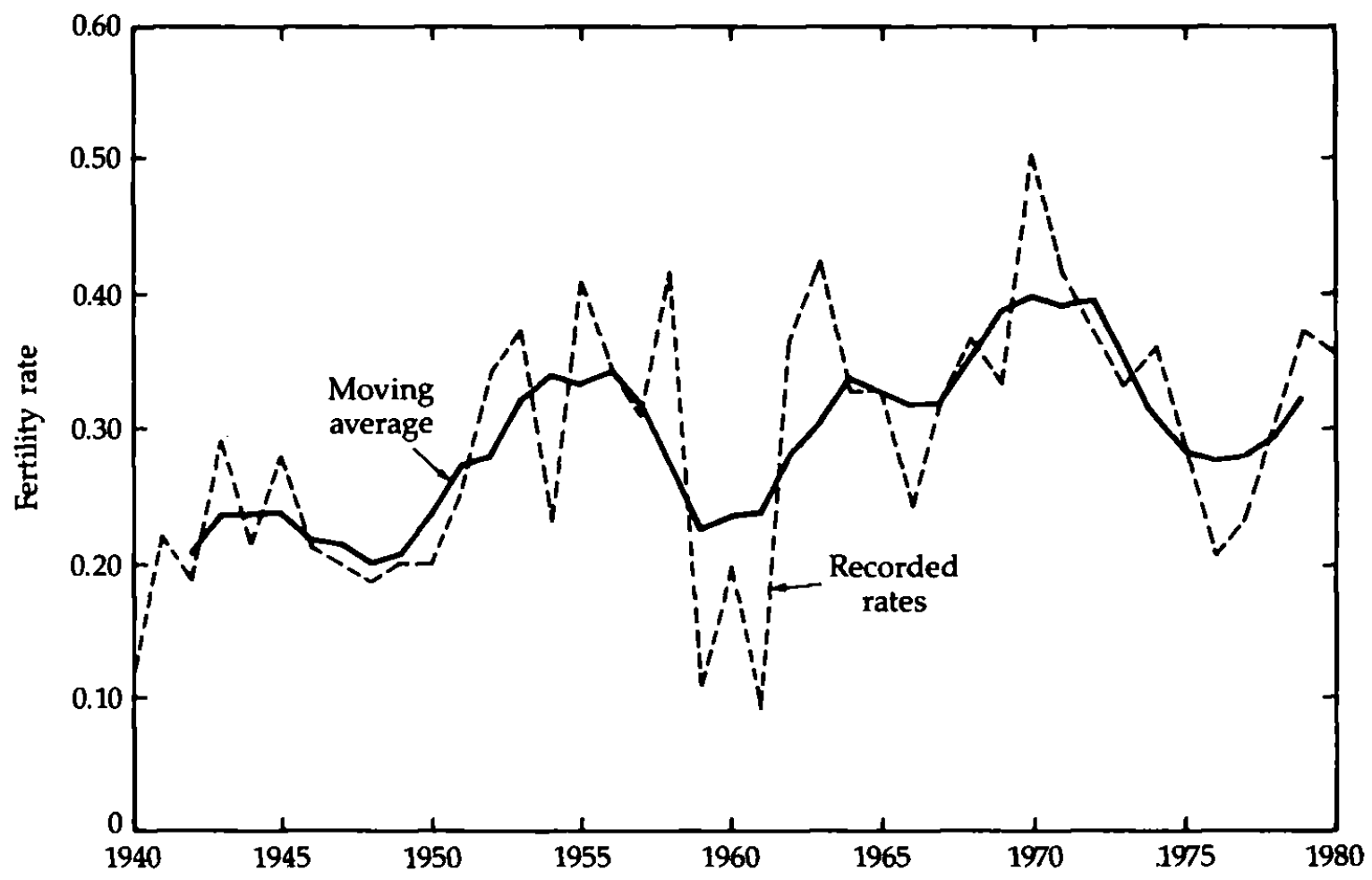

Figure 1. Marital fertility rates, marriage duration of 0-4 years: Rural Beijing Province, $1940-80$, as recorded, and five-term moving average 


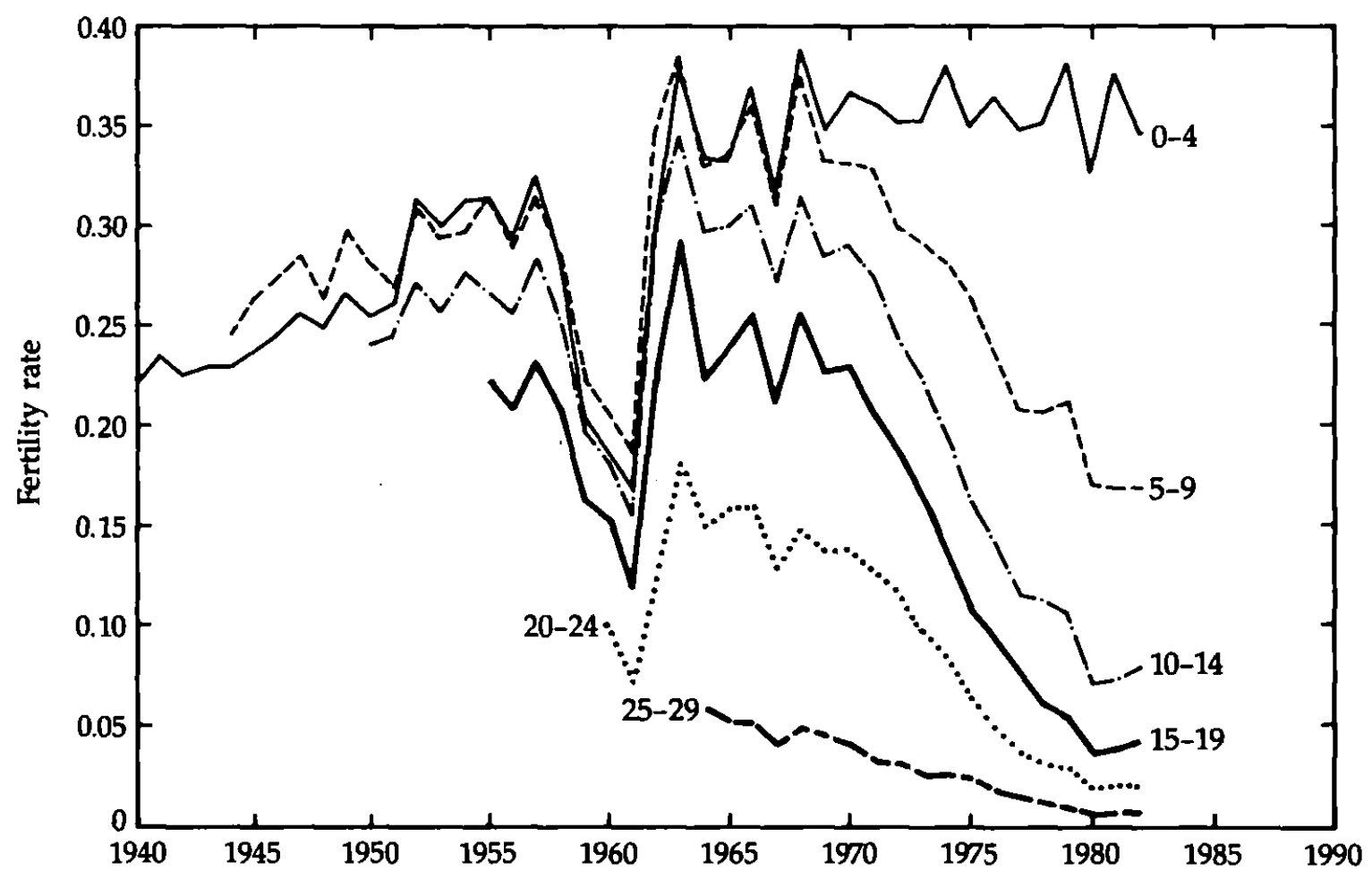

Figure 2. Fertility rates of ever-married women by five-year intervals of duration since first marriage: Rural China, 1940-82 
1945-49 to 1950-54, the proportion of first marriages occurring before age 16 fell from 16.9 to 11.6 percent, before age 17 from 33.0 to 27.6 percent, and before age 18 from 50.1 to 47.2 percent.) The decline in very early marriages has the greatest effect on fertility at duration 0-4 years.

In urban China, duration-specific fertility was higher at duration 0-4 years than at 5-9, even in the 1940s (Figure 3). The proportion of marriages at very early ages in the urban population was less in 1945-49 than five years later in the rural population: $\mathbf{3 8 . 9}$ percent of urban marriages took place before age 18 in 1945-49, compared with 47.2 percent of rural marriages in 1950-54.

There are surprising time trends in duration-specific fertility, and conspicuous differences between urban and rural trends. In the rural population, fertility at the earliest durations rose between the 1940s and the 1950s partly because of fewer very early marriages. Rural marital fertility also increased between the late 1940s and the 1950s at duration 5-9 (the median rate for $1945-49$ is 0.273 ; for $1952-56,0.296$ ). Fertility at all durations fell dramatically beginning in 1958, reaching a low in 1961 of 42 to 48 percent below the fertility level in 1957. After reaching a postcrisis peak in 1963, fertility at durations $0-4,5-9$, and 10-14 returned to a near plateau above the corresponding rates in the 1950s. (Median fertility in 1964-68 was 5.7, 12.8 , and 12.4 percent higher than in 1952-56 at durations 0-4, 5-9, and $10-14$ respectively.)

In the 1970 s marital fertility at duration 0-4 varied moderately from year to year around a median level about 5 percent higher than in 1964-68; at greater durations marital fertility fell dramatically. The family planning policy led rural women married more than five years to a strong restriction of fertility, but it did not affect childbearing in the first five years after marriage.

Two well-known episodes in the history of the People's Republic have visibly distorted the sequence of rural duration-specific fertility rates. One was the economic crisis of the Great Leap Forward, which caused a major decline in fertility at all durations from 1958 to 1961 and was followed by a dramatic recovery to a record peak in 1963. The second was the Cultural Revolution, initiated in 1966, which probably caused the dip in 1967 at every duration of marriage.

In China's urban population, duration-specific marital fertility at durations 0-4 and 5-9 rose fairly steadily through the 1940 s and 1950 s to a peak in 1957, which was followed by a steep decline until 1961 and recovery to a record peak in 1963, as a consequence of the economic crisis (Figure 3). From 1964 onward, the course of urban marital fertility was quite different from that of rural marital fertility. Urban fertility declined at every duration to a minimum in 1966 or 1967 well below its level in the 1950s, perhaps 


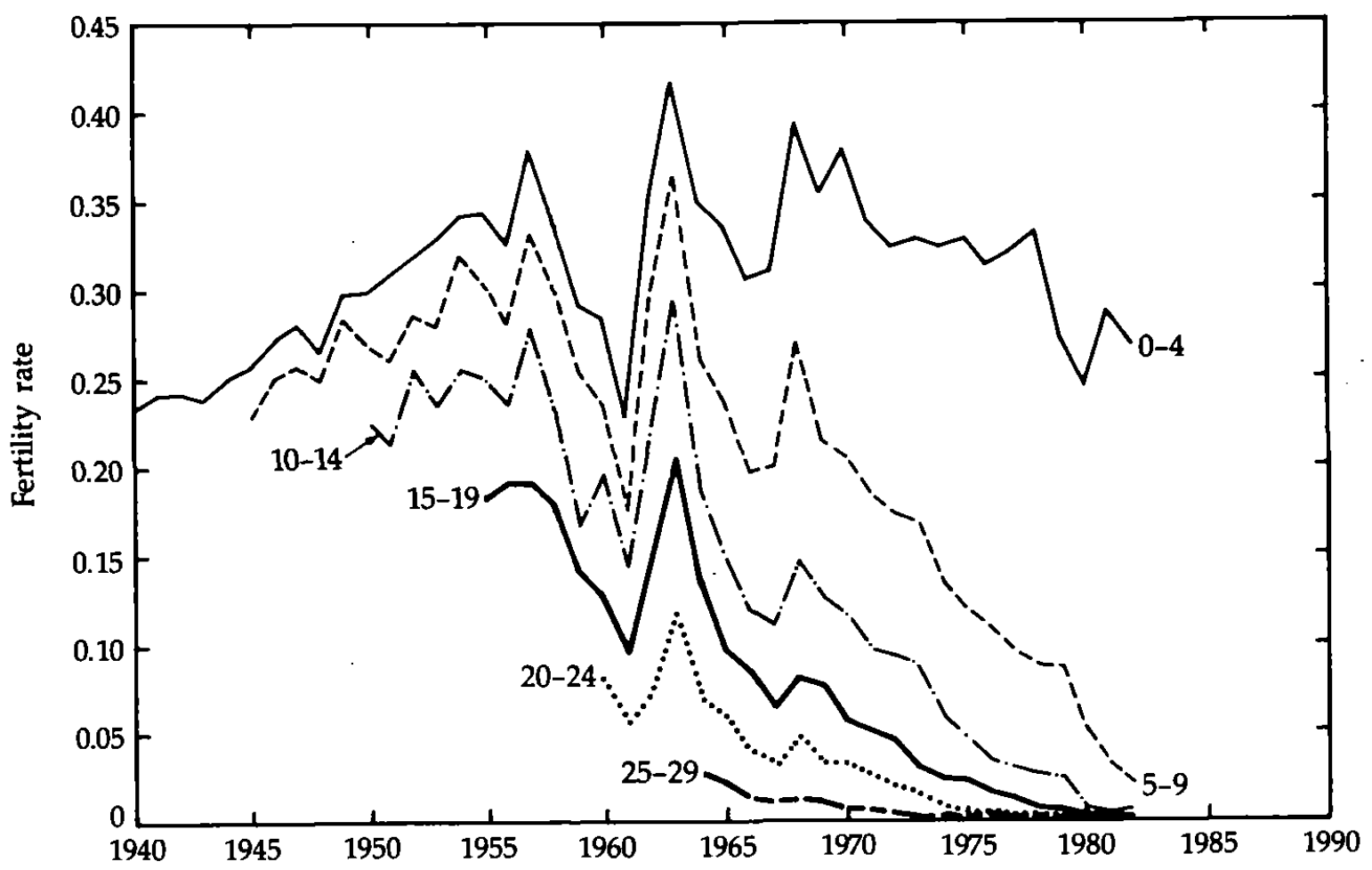

Figure 3. Fertility rates of ever-married women by five-year intervals of duration since first marriage: Urban China, 1940-82 
in response to the family planning policy introduced in the urban areas during the 1960s, and sharply increased in 1968, after the policy had been disrupted by the Cultural Revolution. The decline after 1970 was steeper than in the rural population and occurred at duration $0-4$ as well as at later durations. A sharp drop at duration 0-4 in 1979 coincided with the initiation of the one-child policy. (Note the similar sharp break one year later at durations 5-9 through 20-24 in rural China.)

The sequence of age-specific fertility rates tells a slightly different story. In rural China, fertility rates at ages 20-24 and 25-29 were virtually identical from 1945 to 1957 because the proportion married was very high even at ages 20-24, there was little difference in fecundity among women 20 to 30 years old, and essentially there was no contraceptive practice (Figure 4). In the late 1950s, fertility at 20-24 began to fall below fertility at 25-29 as later marriage reduced the proportion married among women in their early 20s. The proportion ever married at 20-24 declined from 87.4 percent in 1956 to 75.2 percent in 1969, 59.4 percent in 1974, and 43.6 percent in 1979.

Many features of the time pattern of duration-specific fertility in rural China are repeated in the age-specific rates: the big deficit in 1958-61, the peak in 1963, and the dip in 1967. Moreover, fertility rose at all observed age intervals from the 1940s to the 1950s and from the 1950s to the 1960s. The decline in the 1970s at ages 25-29 and higher was the result of the decline in marital fertility already noted; the decline at 20-24 was the result of later marriages.

In urban China, fertility at ages 20-24 was lower than at 25-29 even during the 1940s because urban marriage occurred at a later age than rural marriage. By the 1960s, fertility at ages 20-24 was lower even than at ages 30-34 because of extensive increases in age at marriage (Figure 5).

\section{Fertility differences and fertility trends in the provinces}

Both time trends and interprovincial differences in fertility in particular years must be interpreted with caution because of the lange stochastic element in the tabulated fertility rates in provinces where the respondent samples are especially small. Despite the small numbers, certain common features in fertility trends can be discerned in most, if not all, of the twenty-eight provinces. For example, the total age-specific fertility rate based on reported births in the rural area of every province was at least 33 percent lower in 1961 than in 1957 (the decline for rural China as a whole was 48 percent); the largest reductions were more than 60 percent of the 1957 fertility rate.

The fertility rate of ever-married women at duration 5-9 provides a convenient means for comparing changes in fertility in the different provinces. The total fertility rate, which is another convenient form of comparison, incorporates a large element of estimation in the early years, whereas from 


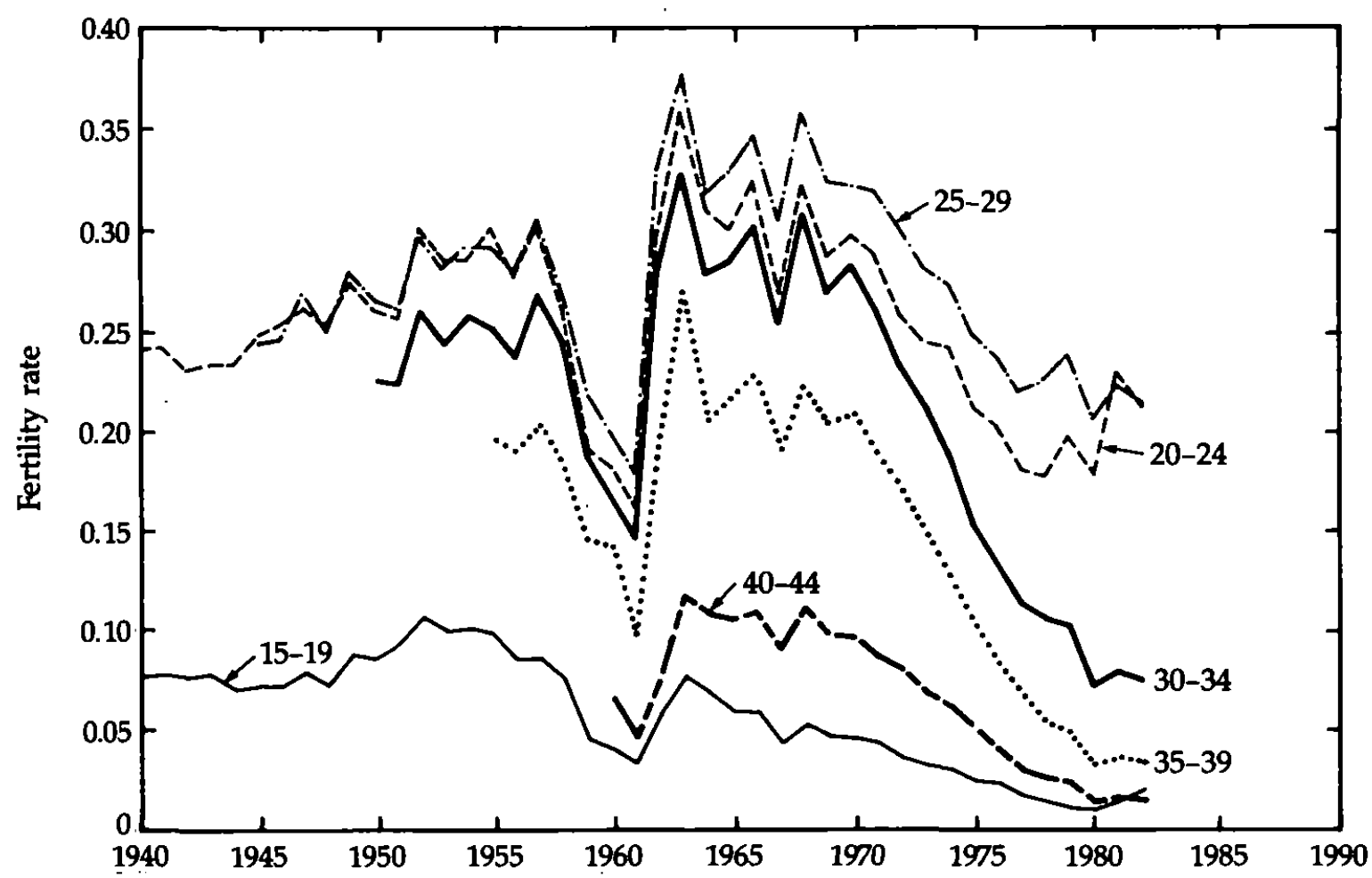

Figure 4. Fertility rates by five-year age intervals: Rural China, 1940-82 


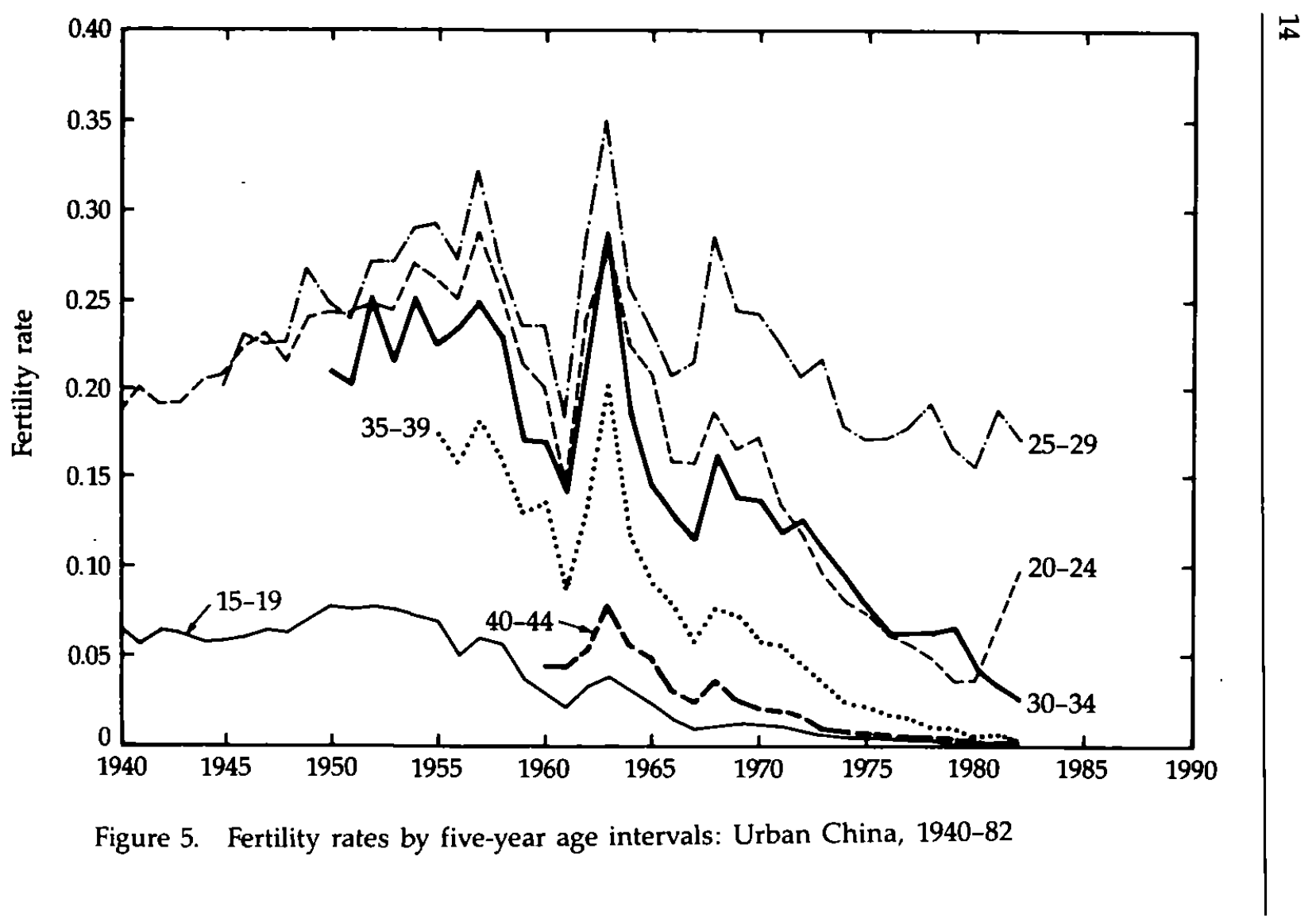


1945 onward marital fertility at duration 5-9 is derived directly from the respondents' reports. The median annual value of fertility at duration 5-9 years since first marriage in four five-year time periods in each province is presented in Table 2. The change from one period to the next is shown in Figure 6. The rise in fertility in rural China at duration 5-9 between 1945-49 and 1952-56, and the further rise in 1964-68, were noted earlier. It was also noted that the synchronism of changes in fertility at various durations indicates little influence of contraception on marital fertility dur-

Table 2. Median marital fertility rates at duration 5-9 years in selected periods, for the provinces of Mainland China

\begin{tabular}{|c|c|c|c|c|c|c|}
\hline \multicolumn{2}{|c|}{ Province } & \multirow{2}{*}{$\frac{1945-49}{.240}$} & \multirow{2}{*}{$\frac{1952-56}{.307}$} & \multirow{2}{*}{$\begin{array}{c}1964-68 \\
.238\end{array}$} & \multirow{2}{*}{$\begin{array}{c}1971-75 \\
.179\end{array}$} & \multirow{2}{*}{$\frac{1977-81}{.089}$} \\
\hline 1 & Beijing & & & & & \\
\hline 2 & Tianjin & .288 & .316 & .241 & .182 & .083 \\
\hline 3 & Hebei & .249 & .278 & .323 & .307 & .221 \\
\hline 4 & Shanxi & .225 & .279 & .292 & .275 & .163 \\
\hline 5 & Inner Mongolia & .262 & .312 & .313 & .260 & .193 \\
\hline 6 & Liaoning & .276 & .357 & .305 & .220 & .134 \\
\hline 7 & Jilin & .290 & .353 & .340 & .278 & .140 \\
\hline 8 & Heilongjiang & .275 & .334 & .317 & .293 & .165 \\
\hline 9 & Shanghai & .284 & .298 & .160 & .082 & .063 \\
\hline 10 & Jiangsu & .258 & .277 & .302 & .179 & .108 \\
\hline 11 & Zhejiang & .284 & .300 & .333 & .243 & .192 \\
\hline 12 & Anhui & .241 & .268 & .322 & .286 & .223 \\
\hline 13 & Fujian & .275 & .296 & .334 & .311 & .210 \\
\hline 14 & Jiangxi & .286 & .286 & .365 & .337 & .238 \\
\hline 15 & Shandong & .263 & .300 & .334 & .249 & .153 \\
\hline 16 & Henan & .262 & .295 & .308 & .247 & .176 \\
\hline 17 & Hubei & .284 & .305 & .362 & .261 & .167 \\
\hline 18 & Hunan & .315 & .321 & .356 & .289 & .181 \\
\hline 19 & Guangdong & .239 & .260 & .321 & .294 & .235 \\
\hline 20 & Guangxi & .260 & .279 & .342 & .317 & .266 \\
\hline 21 & Sichuan & .299 & .323 & .333 & .302 & .129 \\
\hline 22 & Guizhou & .290 & .296 & .336 & .350 & .268 \\
\hline 23 & Yunnan & .300 & .344 & .337 & .303 & .232 \\
\hline 24 & Shaanxi & .275 & .273 & .315 & .292 & .190 \\
\hline 25 & Gansu & .291 & .321 & .355 & .309 & .213 \\
\hline 26 & Qinghai & .221 & .233 & .308 & .291 & .243 \\
\hline 27 & Ningxia & .335 & .320 & .351 & .306 & .261 \\
\hline 28 & Xinjiang & .216 & .242 & .327 & .292 & .225 \\
\hline
\end{tabular}



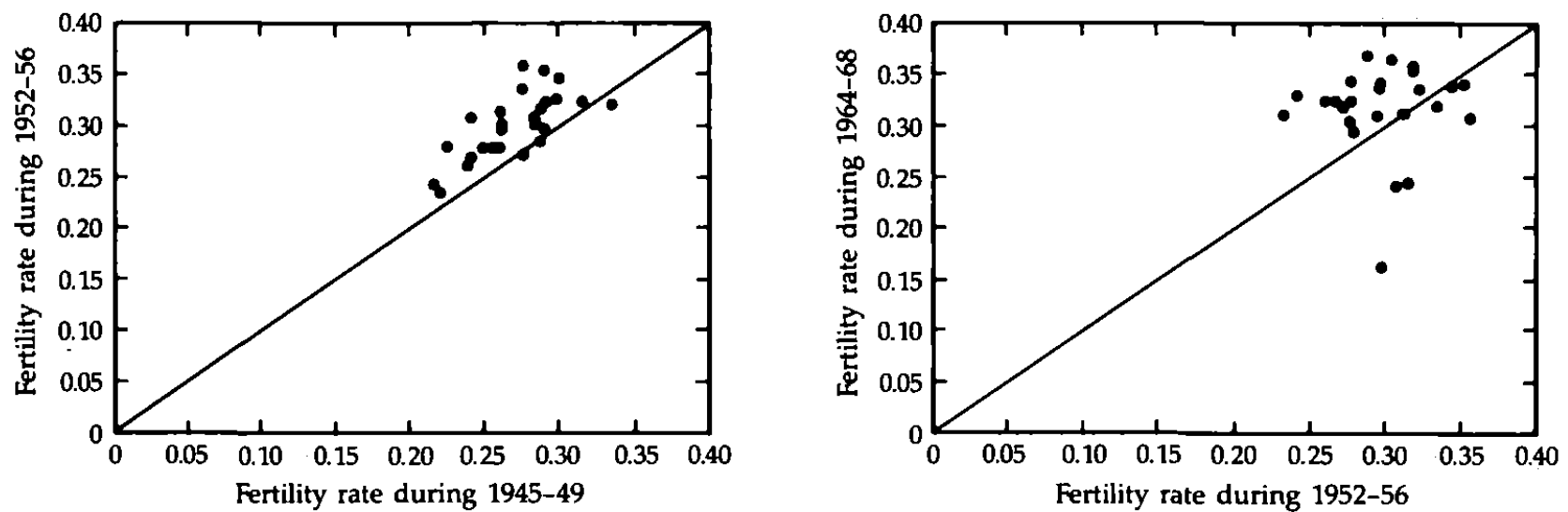

Figure 6. Marital fertility rates at marriage duration of 5-9 years: Provinces of China, different periods 
ing those early years; the major decline at durations 5-9 years and above in rural China after 1970, on the other hand, is clearly due to the birth control program.

Median fertility at duration 5-9 was higher (or within 1.0 percent of the same value) in 1952-1956 than in 1945-49 in every province but Ningxia, where fewer than 1,500 respondents were interviewed. From 1952-1956 to $1964-1968$, an increase in marital fertility at duration 5-9 was again shared by most provinces-all but seven, to be precise. In six of the seven provinces wherein fertility at duration 5-9 was lower in 1964-1968, more than 25 percent of the respondents were in urban sample units. (In the only other such province, Inner Mongolia, there was no change in the rate at duration 5-9). In other words, the data indicate a virtually universal increase in marital fertility from the 1940s to the 1960s (the years affected by the economic crisis are omitted from consideration), with the exception of provinces in which the urban population had a major influence. In such provinces marital fertility increased from the 1940s to the 1950s but fell between 1952-56 and 1964-68. In the most urbanized provinces of Beijing, Tianjin, and Shanghai, the decline in marital fertility at durations 5-9 years was 25 percent or more.

From 1964-68 to 1971-75, fertility in the second five years of marriage fell in every province except Qinghai (where, because the number of respondents was so small, the exception is highly questionable). From the early to the later years of the 1970s, the fall in marital fertility was universal. The declines in the later years are readily understood; the quasi-universal increases from the 1940 s to the 1950 s and from the 1950 s to the 1960 s can be explained only after further research.

\section{How age-specific and duration-specific rates were calculated}

Fertility rates were derived from individual marriage histories and birth histories provided by the survey respondents. The rates were calculated as the ratio of the number of events in a specified age (or duration) interval during a given year to the number of person-years lived by the respondents in the interval. For example, the fertility rate at ages 15-19 in 1940 was calculated as the number of births that occurred to women who were between exact ages 15 and 20 in 1940, divided by the number of person-years lived in 1940 in this age interval by the respondents. Person-years lived at each age are approximated by the midyear population at that age. Since the fertility rates calculated are the rates of childbearing in the past experienced by the respondents, the number of person-years lived by the respondents between exact ages 18 and 20 in 1940 is equal to the number of personyears lived between ages 57 ( 15 plus 42 ) and 62 ( 20 plus 42), forty-two years 
later, in 1982, and thus to the number of respondents who were between exact ages 57 and 62 at the time of the survey.

Duration-specific rates were derived by an analogous procedure. (It should be noted that the duration-specific rates presented here are based on births to ever-married women classified by duration since first marriage. Only the date of first marriage was recorded for each woman. No information was collected on dates of marital dissolution or remarriage.) From knowledge of date of first marriage for a given respondent, a file of personyears lived in each duration interval can be constructed. A respondent who married on 1 July 1953 contributed half of one person-year to duration 0-4 in 1953, and one person-year in 1954, 1955, 1956, and 1957. In 1958 she contributed half of one person-year to duration $0-4$, and half of one personyear to duration 5-9. A birth to her before 1 July 1958 would contribute to the duration-specific fertility rate at $0-4$; if it occurred after 1 July 1958, it would contribute to the rate at duration 5-9.

This sytem of storing the data makes it possible to calculate fertility rates by single years of age and duration of marriage. Such rates have been calculated for some provinces but are too strongly affected by sampling fluctuations to be reproduced for general use. The basic tables and the figures present rates for five-year intervals of age and marriage duration.

Truncation of estimates because of upper age limit of respondents

The detailed questionnaire that obtained marriage and fertility histories in 1982 from respondents in every sample household was administered to all women 15 to 67 years of age in the household. The upper age limit of 67 means that marriage and fertility data pertain to women who were not older than 25 in 1980, 30 in 1985, or 49 in 1964. As a result, age-specific rates can be calculated from actual responses for ages 15-19 and 20-24 from 1940 to 1944, for the three five-year age intervals less than 30 from 1945 to 1949, for age intervals less than 45 from 1960 to 1964, and for all age intervals up to 50 after 1964.

Duration-specific rates are equally affected by truncation, but in a less clearcut way. Almost all women who were age 25 in 1940 (i.e., those 67 in 1982) had at that date completed five years of marriage, since the mean age at marriage was about 18 years. For 1940 to 1944 , the fertility at duration 0-4 is presented; for 1945 to 1949 , the rates of duration $0-4$ and 5-9; etc.

\section{Estimation of TDFR and TAFR, 1945-63}

The restriction of directly calculated age-specific fertility rates for early years to younger ages, and of directly calculated duration-specific rates to early durations of marriage, leads to incomplete listing of rates until 1964 and 
later. Such partial records of fertility are valuable in themselves. Nevertheless, it seemed worthwhile to complete the sequences of total fertility rates by age (TAFRs) and total marital fertility rates by duration (TDFRs) by estimating these totals for years 1945-63, even though only partial information is available.

Estimates of TDFR and TAFR have been constructed on the basis of two relations among fertility rates that can be assumed to prevail in the early years. One relation is the observed tendency for duration-specific fertility rates to fluctuate in unison from year to year, preserving more or less constant ratios between rates at different durations. The second relation is expressed by the equation TAFR $=\int_{0}^{35} f(d, t) \operatorname{TMR}(t-d) d d$, where $f(d, t)$ is the fertility of ever-married women at duration $d$ at time $t$, and TMR $(t-d)$ is total first marriage rate at time $t-d$. On the basis of the first relation, unrecorded duration-specific rates for early years are estimated from recorded rates in the same year. On the basis of the second relation, TAFR for each year is calculated from the recorded and estimated duration-specific rates of that year and the total first marriage rate in the years preceding the year in question.

The approximate constancy of the ratio of duration-specific fertility at different durations is evident in Figures 2 and 3, showing duration-specific rates for rural and urban China. Rates at different durations move up and down synchronously until the mid-1960s in the urban population and the late $1960 \mathrm{~s}$ in the rural population (with the exceptions noted earlier).

The total duration-specific fertility rate (TDFR) for the years in which the data are truncated is estimated by using the ratios of duration-specific rates at later duration intervals to the rates at earlier intervals. The ratios are from the rural and urban populations of all Mainland China, during the first years in which truncation permits their calculation. Specifically, when $D_{1}$ is the marital fertility rate at duration $0-4$ years, $D_{2}$ at duration 5-9 years, and $D_{7}$ at duration 30-34 years, we make use of the median value of $D_{3} / D_{2}$ for $1950-54, D_{4} / D_{3}$ for $1955-59, D_{5} / D_{4}$ for for $1960-63$, and $D_{6} / D_{5}$ and $D_{7} / D_{6}$ for 1964-68. We assume the ratios to be fixed during the years in which truncation requires estimation, and to be the same in every province.

The ratios employed in estimation are shown in Table 3 . Estimates were not attempted for 1940-44, where only $D_{1}$ is available. The ratio of $D_{i}$ to $D_{1}$ was not utilized because $D_{1}$ was rising in relation to other durationspecific ratios in the early years.

$D_{3}, D_{4}, D_{5}, D_{6}$, and $D_{7}$ are estimated for 1945-49 from $D_{2}$. In later years, separate estimates at later durations are based on $D_{2}$ and $D_{3}$ (1950-54), $D_{2}$, $D_{3}$, and $D_{4}$ (1955-59), and $D_{2}, D_{3}, D_{4}$, and $D_{5}(1960-63)$; the separate estimates are averaged. After duration-specific fertility rates were estimated 
Table 3. Ratios of fertility rates at different durations employed in the calculation of TDFR for 1940-63

\begin{tabular}{lll}
\hline Ratio & Rural & Urban \\
\hline Ratios at adjacent five-year intervals & & \\
$D_{3} / D_{2}$ & .874 & .841 \\
$D_{4} / D_{3}$ & .830 & .764 \\
$D_{5} / D_{4}$ & .620 & .596 \\
$D_{6} / D_{5}$ & .324 & .325 \\
$D_{7} / D_{6}$ & .155 & .111 \\
& & \\
Ratios to earlier intervals obtained by chaining & & \\
$D_{4} / D_{2}$ & .725 & .695 \\
$D_{5} / D_{2}$ & .450 & .384 \\
$D_{6} / D_{2}$ & .146 & .125 \\
$D_{7} / D_{2}$ & .023 & .014 \\
$D_{5} / D_{3}$ & .515 & .457 \\
$D_{6} / D_{3}$ & .167 & .149 \\
$D_{7} / D_{3}$ & .026 & .017 \\
$D_{6} / D_{4}$ & .201 & .144 \\
$D_{7} / D_{4}$ & .031 & .022 \\
$D_{7} / D_{5}$ & .050 & .036 \\
\hline
\end{tabular}

at the later duration intervals, omitted because of truncation, TDFRs for the urban and rural populations were computed as $5\left(D_{1}+D_{2}+D_{3}+D_{4}+D_{5}\right.$ $+D_{6}+D_{7}$ ), combining recorded and estimated rates. TDFR for the total population of each province was calculated as the weighted average of rural and urban TDFRs; the weights were the number of person-years lived by evermarried women in each sector.

Estimation of TAFR from duration-specific rates, and total first-marriage rates of preceding years

The total age-specific fertility rate for the years affected by truncation could have been estimated by combining the age-specific rates recorded up to the age that 67-year-olds had attained in a given year with estimated rates above that age. Estimates so obtained are as valid as those presented here, but they are often inconsistent with the relation derived earlier, namely that TAFR $\equiv .98$ TDFR $^{\star}$, where TDFR ${ }^{*}=\int_{0}^{35} f(d, t) \operatorname{TMR}(t-d) d d ; f(d, t)$ is the fertility rate at duration $t$ and time $d$; and $\operatorname{TMR}(t-d)$ is the total marriage rate $d$ years before $t$. To preserve the correct relation between the two total rates, and thereby to illustrate correctly the effect of changing nuptiality on total 
fertility, TAFR for 1945-63 was estimated from duration-specific fertility rates (recorded and estimated) and total first-marriage rates, according to the above formula.

The calculation of TDFR $\left(\right.$ TAFR $\cong .98$ TDFR $^{\star}$ ) was based on durationspecific rates tabulated in five-year duration intervals, and on mean values of the total first-marriage rate, over five-year intervals. Specifically, the formula modified for use with five-year intervals is TDFR ${ }^{*}=5 \sum_{1}^{7}\left(D_{i}\right)\left(\mathrm{TMR}_{i}\right)$. $D_{1}$ is fertility at duration $0-4$ years, $D_{2}$ at duration 5-9 years, etc., and $\mathrm{TMR}_{i}$ is the total first-marriage rate in the time interval from $t-5_{i}$ to $t-5_{i}+5$, where $t$ is the midpoint of the year in which the fertility rates are experienced.

The value of TMR for a five-year interval is taken as the average of TMRs for the individual years. The five-year TMRs required for these calculations are in time intervals bounded by the midpoints of calendar years five years apart. To calculate TMR for an interval (such as 1975.5 to 1980.5) that begins and ends in the middle of a calendar year, TMR for the years 1975 through 1979 is averaged with TMR for 1976 through 1980.

Table 4 illustrates the conversion of recorded duration-specific fertility rates for 1980 and recorded first-marriage rates from 1945 to 1980 into an estimate of TAFR for rural China in 1980. For the years from 1945 to 1963, when truncation requires that TAFR be estimated, duration-specific fertility rates are incompletely listed; in addition, first-marriage rates are unrecorded for women over age 23 in 1938, 24 in 1939, 25 in 1940, etc. The required values of $\mathrm{TMR}_{i}$ for early time intervals (realistically, for intervals before 1938-42) are not on record. Table 5 illustrates the calculation of TDFR for 1950. Values of duration-specific fertility at durations 15-19, 20-24, 25-29,

Table 4. Calculation of TDFR*, and estimated TAFR, for the rural population of China: 1980

\begin{tabular}{lcccc}
\hline Duration interval & $D_{i}$ & $\mathrm{TMR}_{i}$ & $D_{i} \cdot \mathrm{TMR}_{1}$ \\
\hline 1 & $0-4$ & .324 & .843 & .2731 \\
2 & $5-9$ & .170 & .710 & .1207 \\
3 & $10-14$ & .070 & .864 & .0605 \\
4 & $15-19$ & .035 & .972 & .0340 \\
5 & $20-24$ & .018 & .865 & .0156 \\
6 & $25-29$ & .005 & .919 & .0046 \\
7 & $30-34$ & .001 & 1.037 & .0010 \\
& & & Sum of $D_{i} \cdot \mathrm{TMR}_{i}=.5095$ \\
\hline
\end{tabular}

Note: TAFR $=0.98 \mathrm{TDFR}^{*}=(0.98)(5)(.5095)=2.497$. (TAFR, directly calculated, is $2.502 ;$ TDFR is 3.081.) 
Table 5. Calculation of TDFR*, and estimated TAFR, for the rural population of China: 1950

\begin{tabular}{|c|c|c|c|c|}
\hline Duration interval & $D_{i}$ & $\mathrm{TMR}_{i}$ & $\begin{array}{c}\text { Years of } \\
\text { first marriages }\end{array}$ & $D_{i} \cdot \mathrm{TMR}_{1}$ \\
\hline $1 \quad(0-4)$ & .254 & 1.037 & $1945.5-1950.5$ & .263 \\
\hline $2 \quad(5-9)$ & .281 & .943 & $1940.5-1945.5$ & .265 \\
\hline $3(10-14)$ & .240 & .946 & $1938-42$ & .227 \\
\hline $4(15-19)$ & $.201^{\star}$ & .946 & $1938-42$ & .190 \\
\hline $5(20-24)$ & $.125^{\star}$ & .946 & $1938-42$ & .118 \\
\hline $6 \quad(25-29)$ & $.041^{\star}$ & .946 & $1938-42$ & .039 \\
\hline \multirow[t]{2}{*}{$7(30-34)$} & $.006^{*}$ & .946 & $1938-42$ & .006 \\
\hline & & & Sum of $D_{i} \cdot \mathrm{TMR}_{i}=$ & $=1.108$ \\
\hline
\end{tabular}

Note: $D_{4}^{*}=[(.240)(.830)+(.281)(.725)] / 2 ;$

$D_{5}^{*}=[(.240)(.515)+(.281)(.450)] / 2$

$D_{6}^{*}=[(.240)(.167)+(.281)(.146)] / 2 ;$

$D_{7}^{*}=[(.240)(.026)+(.281)(.023)] / 2 ;$

$0.98 \mathrm{TDFR}^{*}=(0.98)(5)(1.108)=5.431$.

and 30-34 ( $D_{4}$ to $\left.D_{7}\right)$ must be estimated. Only TMR 1 and TMR (for years 1945.5 to 1950.5 and 1940.5 to 1945.5 ) can be derived from recorded data. $\mathrm{TMR}_{3}$ to $\mathrm{TMR}_{7}$ are assumed equal to the mean TMR from 1938 to 1942. The estimated values of $D_{4}$ to $D_{7}$ (based on recorded $D_{2}$ and $D_{3}$ plus the ratios listed in Table 3 ) are those incorporated in the estimate of TDFR for 1950 (5.72). The estimate of TAFR for 1950 (5.42) thus differs from the estimated TDFR (5.72) precisely because the total marriage rates before 1950 are calculated or estimated as below 1.00 for all periods except 1945.5 to 1950.5 . 


\section{PART II.}

BASIC TABLES: FERTILITY RATES BY AGE OF WOMAN, AND FERTILITY RATES OF EVER-MARRIED WOMEN BY DURATION SINCE FIRST MARRIAGE, 1940-82, FOR MAINLAND CHINA AND TWENTY-EIGHT PROVINCES: TOTAL, URBAN, AND RURAL 
Basic Table 1.A. Total China: age-specific fertility rates, 1940-82

\begin{tabular}{|c|c|c|c|c|}
\hline $\begin{array}{l}\text { Birth year } \\
\text { of child }\end{array}$ & $15-19$ & $20-24$ & $25-29$ & $30-34$ \\
\hline 1940 & 0.074 & - & - & • \\
\hline 1941 & 0.075 & - & - & - \\
\hline 1942 & 0.074 & - & - & - \\
\hline 1943 & 0.076 & - & - & - \\
\hline 1944 & 0.068 & - & - & - \\
\hline 1945 & 0.069 & 0.241 & - & - \\
\hline 1946 & 0.070 & 0.249 & - & . \\
\hline 1947 & 0.076 & 0.257 & - & - \\
\hline 1948 & 0.072 & 0.246 & - & - \\
\hline 1949 & 0.084 & 0.269 & - & - \\
\hline 1950 & 0.083 & 0.258 & 0.263 & - \\
\hline 1951 & 0.090 & 0.254 & 0.257 & - \\
\hline 1952 & 0.101 & 0.292 & 0.292 & - \\
\hline 1953 & 0.094 & 0.278 & 0.280 & - \\
\hline 1954 & 0.095 & 0.283 & 0.292 & - \\
\hline 1955 & 0.092 & 0.294 & 0.293 & 0.248 \\
\hline 1956 & 0.078 & 0.272 & 0.278 & 0.236 \\
\hline 1957 & 0.081 & 0.299 & 0.308 & 0.266 \\
\hline 1958 & 0.072 & 0.260 & 0.269 & 0.241 \\
\hline 1959 & 0.044 & 0.196 & 0.222 & 0.184 \\
\hline 1960 & 0.038 & 0.186 & 0.205 & 0.167 \\
\hline 1961 & 0.031 & 0.157 & 0.179 & 0.143 \\
\hline 1962 & 0.054 & 0.284 & 0.319 & 0.266 \\
\hline 1963 & 0.072 & 0.345 & 0.371 & 0.321 \\
\hline 1904 & 0.063 & 0.294 & 0.305 & 0.259 \\
\hline 1905 & 0.053 & 0.284 & 0.309 & 0.257 \\
\hline 1966 & 0.052 & 0.296 & 0.317 & 0.266 \\
\hline 1967 & 0.038 & 0.250 & 0.285 & 0.224 \\
\hline 1968 & 0.046 & 0.301 & 0.344 & 0.277 \\
\hline 1969 & 0.041 & 0.267 & 0.309 & 0.241 \\
\hline 1970 & 0.039 & 0.278 & 0.308 & 0.252 \\
\hline 1971 & 0.038 & 0.263 & 0.303 & 0.231 \\
\hline 1972 & 0.031 & 0.235 & 0.285 & 0.211 \\
\hline 1973 & 0.027 & 0.220 & 0.270 & 0.193 \\
\hline 1974 & 0.026 & 0.215 & 0.257 & 0.171 \\
\hline 1975 & 0.020 & 0.188 & 0.235 & 0.139 \\
\hline 1976 & 0.019 & 0.177 & 0.226 & 0.121 \\
\hline 1977 & 0.014 & 0.159 & 0.212 & 0.104 \\
\hline 1978 & 0.011 & 0.153 & 0.218 & 0.098 \\
\hline 1979 & 0.009 & 0.167 & 0.225 & 0.096 \\
\hline 1980 & 0.008 & 0.151 & 0.197 & 0.066 \\
\hline 1981 & 0.013 & 0.197 & 0.216 & 0.071 \\
\hline 1982 & 0.018 & 0.190 & 0.206 & 0.066 \\
\hline
\end{tabular}

Note: In this and the following Basic Tables, a value of 0.000 represents a rate of fewer than cohort means there were no births in a given year and age group. Total fertility rates 


\begin{tabular}{|c|c|c|c|}
\hline $35-39$ & $40-44$ & $45-49$ & Total \\
\hline - & - & • & - \\
\hline - & $\bullet$ & • & - \\
\hline - & - & - & - \\
\hline - & - & - & - \\
\hline - & - & • & - \\
\hline - & - & - & 4.766 \\
\hline - & - & - & 4.999 \\
\hline - & - & $\bullet$ & 5.199 \\
\hline - & - & - & 4.906 \\
\hline - & - & - & 5.537 \\
\hline - & - & - & 5.292 \\
\hline - & - & - & 5.262 \\
\hline - & . & - & 5.973 \\
\hline . & . & - & 5.669 \\
\hline - & - & - & 5.955 \\
\hline - & - & • & 5.978 \\
\hline - & - & - & 5.605 \\
\hline - & - & - & 6.205 \\
\hline - & - & - & 5.496 \\
\hline - & - & - & 4.233 \\
\hline 0.140 & - & - & 3.986 \\
\hline 0.096 & - & - & 3.280 \\
\hline 0.182 & - & - & 5.969 \\
\hline 0.253 & - & - & 7.413 \\
\hline 0.190 & 0.099 & 0.013 & 6.120 \\
\hline 0.194 & 0.096 & 0.012 & 6.022 \\
\hline 0.201 & 0.096 & 0.013 & 6.211 \\
\hline 0.106 & 0.078 & 0.009 & 5.252 \\
\hline 0.196 & 0.098 & 0.012 & 6.370 \\
\hline 0.179 & 0.085 & 0.013 & 5.670 \\
\hline 0.179 & 0.083 & 0.010 & 5.746 \\
\hline 0.162 & 0.074 & 0.008 & 5.396 \\
\hline 0.146 & 0.069 & 0.003 & 4.920 \\
\hline 0.126 & 0.057 & 0.008 & 4.506 \\
\hline 0.105 & 0.051 & 0.006 & 4.150 \\
\hline 0.086 & 0.041 & 0.000 & 3.570 \\
\hline 0.070 & 0.033 & 0.005 & 3.254 \\
\hline 0.056 & 0.024 & 0.004 & $2.860^{\circ}$ \\
\hline 0.044 & 0.021 & 0.005 & 2.749 \\
\hline 0.041 & 0.019 & 0.003 & 2.798 \\
\hline 0.028 & 0.011 & 0.003 & 2.316 \\
\hline 0.031 & 0.013 & 0.002 & 2.713 \\
\hline 0.029 & 0.012 & 0.003 & 2.513 \\
\hline
\end{tabular}

5 births per 10,000 women in a given year and age group. A blank cell in a complete age were not calculated for $\mathbf{1 9 4 0 - 4 4}$ because of insufficient data. 
Basic Table 1.B. Urban China: age-specific fertility rates, 1940-82

\begin{tabular}{|c|c|c|c|c|}
\hline $\begin{array}{l}\text { Birth year } \\
\text { of child }\end{array}$ & $15-19$ & $20-24$ & $25-29$ & $30-34$ \\
\hline 1940 & 0.066 & • & - & - \\
\hline 1941 & 0.057 & - & - & - \\
\hline 1942 & 0.064 & - & - & - \\
\hline 1943 & 0.062 & - & - & $\bullet$ \\
\hline 1944 & 0.058 & - & - & - \\
\hline 1945 & 0.059 & 0.208 & - & $\bullet$ \\
\hline 1946 & 0.061 & 0.224 & - & - \\
\hline 1947 & 0.065 & 0.232 & • & $\bullet$ \\
\hline 1948 & 0.063 & 0.215 & • & $\bullet$ \\
\hline 1949 & 0.071 & 0.241 & - & - \\
\hline 1950 & 0.078 & 0.244 & 0.250 & - \\
\hline 1951 & 0.076 & 0.243 & 0.240 & - \\
\hline 1952 & 0.078 & 0.248 & 0.272 & • \\
\hline 1953 & 0.076 & 0.244 & 0.273 & $\bullet$ \\
\hline 1954 & 0.073 & 0.271 & 0.290 & - \\
\hline 1955 & 0.069 & 0.262 & 0.293 & 0.223 \\
\hline 1956 & 0.050 & 0.251 & 0.273 & 0.235 \\
\hline 1957 & 0.061 & 0.289 & 0.323 & 0.249 \\
\hline 1958 & 0.057 & 0.255 & 0.271 & 0.230 \\
\hline 1959 & 0.038 & 0.214 & 0.235 & 0.171 \\
\hline 1960 & 0.030 & 0.201 & 0.236 & 0.170 \\
\hline 1961 & 0.021 & 0.141 & 0.183 & 0.140 \\
\hline 1962 & 0.033 & 0.238 & 0.281 & 0.213 \\
\hline 1963 & 0.039 & 0.283 & 0.349 & 0.286 \\
\hline 1964 & 0.031 & 0.225 & 0.258 & 0.186 \\
\hline 1965 & 0.023 & 0.209 & 0.234 & 0.145 \\
\hline 1906 & 0.014 & 0.158 & 0.206 & 0.127 \\
\hline 1967 & 0.009 & 0.157 & 0.214 & 0.114 \\
\hline 1908 & 0.010 & 0.187 & 0.285 & 0.162 \\
\hline 1969 & 0.012 & 0.165 & 0.244 & 0.138 \\
\hline 1970 & 0.011 & 0.172 & 0.242 & 0.136 \\
\hline 1971 & 0.010 & 0.135 & 0.225 & 0.118 \\
\hline 1972 & 0.007 & 0.118 & 0.206 & 0.126 \\
\hline 1973 & 0.005 & 0.096 & 0.216 & 0.109 \\
\hline 1974 & 0.004 & 0.081 & 0.178 & 0.094 \\
\hline 1975 & 0.004 & 0.073 & 0.170 & 0.077 \\
\hline 1976 & 0.003 & 0.061 & 0.171 & 0.062 \\
\hline 1977 & 0.002 & 0.056 & 0.177 & 0.062 \\
\hline 1978 & 0.002 & 0.048 & 0.190 & 0.063 \\
\hline 1979 & 0.001 & 0.036 & 0.165 & 0.066 \\
\hline 1980 & 0.001 & 0.037 & 0.154 & 0.043 \\
\hline 1981 & 0.002 & 0.067 & 0.186 & 0.033 \\
\hline 1982 & 0.002 & 0.097 & 0.172 & 0.025 \\
\hline
\end{tabular}




\begin{tabular}{|c|c|c|c|}
\hline $35-39$ & $40-44$ & $45-49$ & Total \\
\hline - & - & - & - \\
\hline - & - & - & - \\
\hline - & - & - & - \\
\hline$\bullet$ & $\bullet$ & - & - \\
\hline - & $\bullet$ & - & - \\
\hline - & - & - & 4.394 \\
\hline - & - & - & 4.786 \\
\hline - & - & - & 4.952 \\
\hline - & - & - & 4.801 \\
\hline • & - & - & 5.512 \\
\hline - & - & - & 5.300 \\
\hline - & - & - & $5 \cdot 136$ \\
\hline - & - & - & 5.683 \\
\hline - & - & - & 5.510 \\
\hline - & • & - & 6.041 \\
\hline - & - & - & 5.670 \\
\hline - & - & - & 5.403 \\
\hline - & - & - & 6.165 \\
\hline • & - & - & 5.452 \\
\hline - & - & - & 4.360 \\
\hline 0.135 & - & - & 4.195 \\
\hline 0.086 & - & - & 3.130 \\
\hline 0.133 & - & - & 4.911 \\
\hline 0.202 & - & . & 6.341 \\
\hline 0.118 & 0.056 & 0.009 & 4.416 \\
\hline 0.091 & 0.048 & 0.006 & 3.779 \\
\hline 0.078 & 0.030 & 0.007 & 3.101 \\
\hline 0.058 & 0.023 & 0.006 & 2.899 \\
\hline 0.077 & 0.037 & 0.002 & 3.797 \\
\hline 0.072 & 0.025 & 0.004 & 3.301 \\
\hline 0.059 & 0.020 & 0.002 & 3.216 \\
\hline 0.057 & 0.019 & 0.003 & 2.831 \\
\hline 0.045 & 0.016 & 0.001 & 2.589 \\
\hline 0.035 & 0.009 & 0.001 & 2.362 \\
\hline 0.024 & 0.007 & 0.001 & 1.944 \\
\hline 0.021 & 0.006 & 0.001 & 1.759 \\
\hline 0.017 & 0.005 & 0.001 & 1.604 \\
\hline 0.014 & 0.003 & 0.000 & 1.571 \\
\hline 0.009 & 0.003 & 0.001 & 1.575 \\
\hline 0.009 & 0.002 & 0.000 & 1.401 \\
\hline 0.004 & 0.001 & $\bullet$ & 1.196 \\
\hline 0.005 & 0.000 & 0.001 & 1.474 \\
\hline 0.003 & 0.000 & - & 1.499 \\
\hline
\end{tabular}


Basic Table 1.C. Rural China: age-specific fertility rates, 1940-82

\begin{tabular}{|c|c|c|c|c|}
\hline $\begin{array}{l}\text { Birth year } \\
\text { of child }\end{array}$ & $15-19$ & $20-24$ & $25-29$ & $30-34$ \\
\hline 1940 & 0.076 & - & - & - \\
\hline 1941 & 0.078 & - & - & - \\
\hline 1942 & 0.076 & - & - & - \\
\hline 1943 & 0.078 & - & - & - \\
\hline 1944 & 0.070 & - & - & - \\
\hline 1945 & 0.072 & 0.248 & - & - \\
\hline 1946 & 0.072 & 0.254 & - & $\bullet$ \\
\hline 1947 & 0.079 & 0.262 & - & - \\
\hline 1948 & 0.074 & 0.252 & $\bullet$ & - \\
\hline 1949 & 0.088 & 0.275 & - & - \\
\hline 1950 & 0.085 & 0.261 & 0.266 & - \\
\hline 1951 & 0.093 & 0.256 & 0.261 & - \\
\hline 1952 & 0.107 & 0.302 & 0.297 & • \\
\hline 1953 & 0.099 & 0.285 & 0.281 & - \\
\hline 1954 & 0.101 & 0.286 & 0.292 & $\bullet$ \\
\hline 1955 & 0.098 & 0.301 & 0.292 & 0.252 \\
\hline 1956 & 0.085 & 0.277 & 0.279 & 0.236 \\
\hline 1957 & 0.086 & 0.302 & 0.305 & 0.269 \\
\hline 1958 & 0.076 & 0.262 & 0.268 & 0.244 \\
\hline 1959 & 0.045 & 0.191 & 0.219 & 0.187 \\
\hline 1960 & 0.040 & 0.182 & 0.197 & 0.166 \\
\hline 1961 & 0.033 & 0.162 & 0.177 & 0.144 \\
\hline 1962 & 0.058 & 0.295 & 0.330 & 0.278 \\
\hline 1963 & 0.077 & 0.359 & 0.377 & 0.329 \\
\hline 1964 & 0.069 & 0.309 & 0.317 & 0.277 \\
\hline 1963 & 0.059 & 0.299 & 0.328 & 0.284 \\
\hline 1960 & 0.059 & 0.323 & 0.346 & 0.302 \\
\hline 1967 & 0.043 & 0.268 & 0.303 & 0.253 \\
\hline 1968 & 0.053 & 0.322 & 0.358 & 0.308 \\
\hline 1969 & 0.046 & 0.286 & 0.323 & 0.268 \\
\hline 1970 & 0.045 & 0.297 & 0.321 & 0.282 \\
\hline 1971 & 0.043 & 0.287 & 0.318 & 0.260 \\
\hline 1972 & 0.036 & 0.257 & 0.300 & 0.232 \\
\hline 1973 & 0.032 & 0.243 & 0.280 & 0.212 \\
\hline 1974 & 0.030 & 0.240 & 0.272 & 0.187 \\
\hline 1975 & 0.024 & 0.211 & 0.247 & 0.152 \\
\hline 1976 & 0.023 & 0.201 & 0.236 & 0.132 \\
\hline 1977 & 0.016 & 0.180 & 0.219 & 0.112 \\
\hline 1978 & 0.013 & 0.176 & .0 .224 & 0.104 \\
\hline 1979 & 0.010 & 0.197 & 0.237 & 0.101 \\
\hline 1980 & 0.009 & 0.178 & 0.205 & 0.071 \\
\hline 1981 & 0.014 & 0.229 & 0.222 & 0.079 \\
\hline 1982 & 0.020 & 0.212 & 0.213 & 0.074 \\
\hline
\end{tabular}




\begin{tabular}{|c|c|c|c|}
\hline $35-39$ & $40-44$ & $45-49$ & Total \\
\hline - & - & - & - \\
\hline - & • & - & $\bullet$ \\
\hline - & $\bullet$ & - & - \\
\hline$\bullet$ & - & • & - \\
\hline$\bullet$ & • & • & 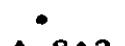 \\
\hline$\bullet$ & - & - & 4.842 \\
\hline • & • & - & 5.043 \\
\hline - & • & - & 5.250 \\
\hline - & - & - & $4.92 B$ \\
\hline - & - & - & 5.543 \\
\hline - & - & - & 5.290 \\
\hline - & - & - & 5.289 \\
\hline$\bullet$ & - & - & 6.038 \\
\hline - & - & - & 5.705 \\
\hline - & - & - & $5 \cdot 935$ \\
\hline - & - & - & 6.048 \\
\hline - & - & - & 5.651 \\
\hline - & - & - & 6.213 \\
\hline - & • & - & 5.506 \\
\hline - & - & - & 4.204 \\
\hline 0.141 & - & - & 3.940 \\
\hline 0.098 & - & - & 3.313 \\
\hline 0.192 & - & - & 6.200 \\
\hline 0.269 & - & - & 7.645 \\
\hline 0.205 & 0.108 & 0.014 & 6.496 \\
\hline 0.217 & 0.105 & 0.014 & 6.526 \\
\hline 0.229 & 0.109 & 0.014 & 6.905 \\
\hline 0.190 & 0.090 & 0.009 & 5.776 \\
\hline 0.223 & 0.111 & 0.014 & 6.938 \\
\hline 0.204 & 0.097 & 0.014 & 6.196 \\
\hline 0.209 & 0.096 & 0.012 & 6.313 \\
\hline 0.189 & 0.087 & 0.010 & 5.963 \\
\hline 0.172 & 0.080 & 0.009 & 5.430 \\
\hline 0.150 & 0.068 & 0.009 & 4.975 \\
\hline 0.126 & 0.061 & 0.008 & 4.623 \\
\hline 0.103 & 0.050 & 0.007 & 3.966 \\
\hline 0.083 & 0.040 & 0.006 & 3.605 \\
\hline 0.067 & 0.029 & 0.005 & 3.143 \\
\hline 0.053 & 0.025 & 0.005 & 3.001 \\
\hline 0.048 & 0.023 & 0.004 & 3.100 \\
\hline 0.033 & 0.013 & 0.003 & 2.563 \\
\hline 0.036 & 0.016 & 0.003 & 2.988 \\
\hline 0.034 & 0.015 & 0.003 & 2.857 \\
\hline
\end{tabular}


Basic Table 2.A. Total Beijing Province (1): age-specific fertility rates,

\begin{tabular}{|c|c|c|c|c|}
\hline $\begin{array}{l}\text { Birth year } \\
\text { of child }\end{array}$ & $15-19$ & $20-24$ & $25-29$ & $30-34$ \\
\hline 1940 & 0.069 & • & - & - \\
\hline 1941 & 0.068 & $\bullet$ & - & - \\
\hline 1942 & 0.070 & - & • & - \\
\hline 1943 & 0.087 & - & - & - \\
\hline 1944 & 0.073 & - & - & - \\
\hline 1945 & 0.084 & 0.238 & - & - \\
\hline 1946 & 0.052 & 0.204 & • & - \\
\hline 1947 & 0.071 & 0.269 & • & - \\
\hline 1948 & 0.116 & 0.200 & - & - \\
\hline 1949 & 0.072 & 0.287 & - & - \\
\hline 1950 & 0.050 & 0.258 & 0.307 & $\bullet$ \\
\hline 1951 & 0.104 & 0.235 & 0.258 & - \\
\hline 1952 & 0.102 & 0.290 & 0.263 & - \\
\hline 1953 & 0.078 & 0.232 & 0.260 & - \\
\hline 1954 & 0.089 & 0.248 & 0.266 & - \\
\hline 1955 & 0.072 & 0.281 & 0.319 & 0.245 \\
\hline 1956 & 0.047 & 0.256 & 0.252 & 0.177 \\
\hline 1957 & 0.050 & 0.286 & 0.316 & 0.240 \\
\hline 1958 & 0.088 & 0.235 & 0.236 & 0.211 \\
\hline 1959 & 0.035 & 0.182 & 0.264 & 0.172 \\
\hline 1960 & 0.030 & 0.204 & 0.196 & 0.164 \\
\hline 1961 & 0.014 & 0.132 & 0.160 & 0.131 \\
\hline 1962 & 0.025 & 0.214 & 0.279 & 0.245 \\
\hline 1963 & 0.046 & 0.301 & 0.361 & 0.318 \\
\hline 1904 & 0.011 & 0.193 & 0.239 & 0.212 \\
\hline 1965 & 0.020 & 0.154 & 0.257 & 0.139 \\
\hline 1966 & 0.025 & 0.131 & 0.188 & 0.137 \\
\hline 1967 & 0.006 & 0.126 & 0.205 & 0.113 \\
\hline 1968 & 0.017 & 0.172 & 0.269 & 0.123 \\
\hline 1969 & 0.016 & 0.179 & 0.277 & 0.132 \\
\hline 1970 & 0.008 & 0.198 & 0.268 & 0.132 \\
\hline 1971 & 0.002 & 0.128 & 0.252 & 0.136 \\
\hline 1972 & 0.010 & 0.101 & 0.235 & 0.118 \\
\hline 1973 & 0.008 & 0.096 & 0.247 & 0.144 \\
\hline 1974 & - & 0.086 & 0.168 & 0.049 \\
\hline 1975 & - & 0.066 & 0.128 & 0.055 \\
\hline 1976 & - & 0.057 & 0.166 & 0.054 \\
\hline 1977 & - & 0.043 & 0.174 & 0.071 \\
\hline 1978 & - & 0.038 & 0.151 & 0.092 \\
\hline 1979 & - & 0.041 & 0.174 & 0.061 \\
\hline 1980 & 0.002 & 0.045 & 0.207 & 0.047 \\
\hline 1981 & 0.002 & 0.068 & 0.209 & 0.031 \\
\hline 1982 & $\bullet$ & 0.079 & 0.172 & 0.033 \\
\hline
\end{tabular}




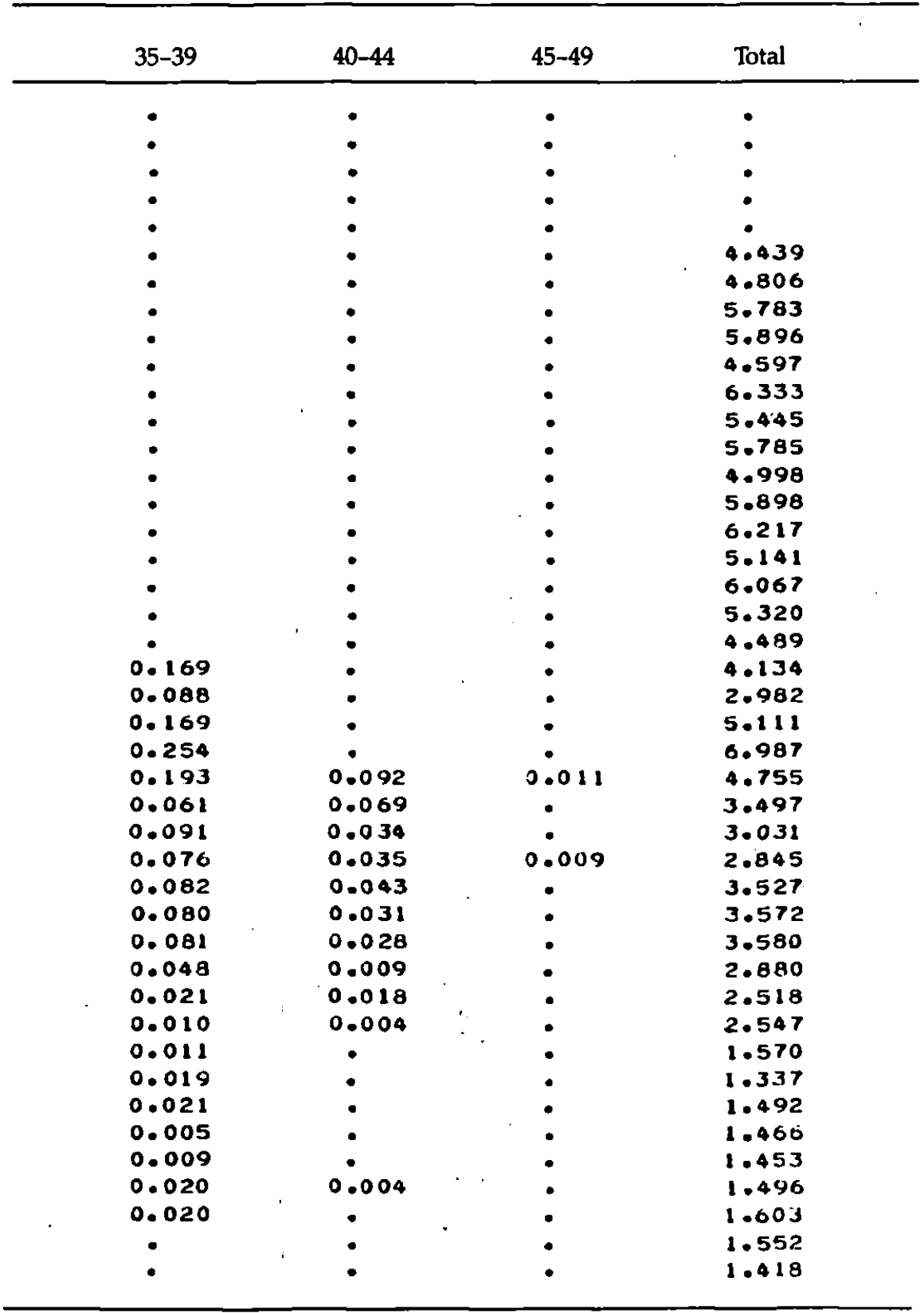


Basic Table 2.B. Urban Beijing Province (1): age-specific fertility rates,

\begin{tabular}{|c|c|c|c|c|}
\hline $\begin{array}{l}\text { Birth year } \\
\text { of child }\end{array}$ & $15-19$ & $20-24$ & $25-29$ & $30-34$ \\
\hline 1940 & 0.090 & - & $\bullet$ & • \\
\hline 1941 & 0.050 & - & - & - \\
\hline 1942 & 0.063 & • & - & - \\
\hline 1943 & 0.057 & - & - & - \\
\hline 1944 & 0.081 & - & - & - \\
\hline 1945 & 0.061 & 0.119 & - & - \\
\hline 1946 & 0.043 & 0.188 & - & $\bullet$ \\
\hline 1947 & 0.035 & 0.229 & - & - \\
\hline 1948 & 0.100 & 0.227 & - & - \\
\hline 1949 & 0.068 & 0.270 & - & - \\
\hline 1950 & 0.057 & 0.228 & 0.373 & - \\
\hline 1951 & 0.067 & 0.229 & 0.226 & - \\
\hline 1952 & 0.081 & 0.240 & 0.250 & - \\
\hline 1953 & 0.045 & 0.234 & 0.284 & - \\
\hline 1954 & 0.093 & 0.278 & 0.288 & - \\
\hline 1955 & 0.065 & 0.269 & 0.266 & 0.269 \\
\hline 1950 & 0.029 & 0.243 & 0.300 & 0.138 \\
\hline 1957 & 0.046 & 0.291 & 0.289 & 0.260 \\
\hline 1958 & 0.095 & 0.198 & 0.247 & 0.217 \\
\hline 1959 & 0.047 & 0.199 & 0.247 & 0.207 \\
\hline 1960 & 0.025 & 0.210 & 0.206 & 0.129 \\
\hline 1961 & 0.016 & 0.138 & 0.155 & 0.143 \\
\hline 1962 & 0.015 & 0.183 & 0.277 & 0.183 \\
\hline 1963 & 0.042 & 0.269 & 0.346 & 0.341 \\
\hline 1964 & 0.006 & 0.142 & 0.227 & 0.1 ล5 \\
\hline 1965 & 0.011 & 0.126 & 0.230 & 0.109 \\
\hline 1966 & 0.021 & 0.111 & 0.189 & 0.103 \\
\hline 1967 & - & 0.067 & 0.183 & 0.115 \\
\hline 1968 & 0.024 & 0.155 & 0.255 & 0.099 \\
\hline 1969 & 0.004 & 0.181 & 0.253 & 0.102 \\
\hline 1970 & 0.003 & 0.128 & 0.269 & 0.115 \\
\hline 1971 & • & 0.078 & 0.190 & 0.109 \\
\hline 1972 & $\bullet$ & 0.064 & 0.186 & 0.085 \\
\hline 1973 & 0.003 & 0.081 & 0.184 & 0.116 \\
\hline 1974 & $\bullet$ & $0.02 \mathrm{~s}$ & 0.133 & 0.024 \\
\hline 1975 & - & 0.047 & 0.111 & 0.042 \\
\hline 1976 & - & 0.050 & 0.197 & 0.071 \\
\hline 1977 & - & 0.046 & 0.183 & 0.074 \\
\hline 1978 & - & 0.024 & 0.138 & 0.078 \\
\hline 1979 & - & 0.009 & 0.177 & 0.073 \\
\hline 1980 & - & 0.015 & 0.194 & 0.050 \\
\hline 1981 & - & 0.034 & 0.179 & 0.031 \\
\hline 1982 & - & 0.079 & 0.139 & 0.018 \\
\hline
\end{tabular}


Total

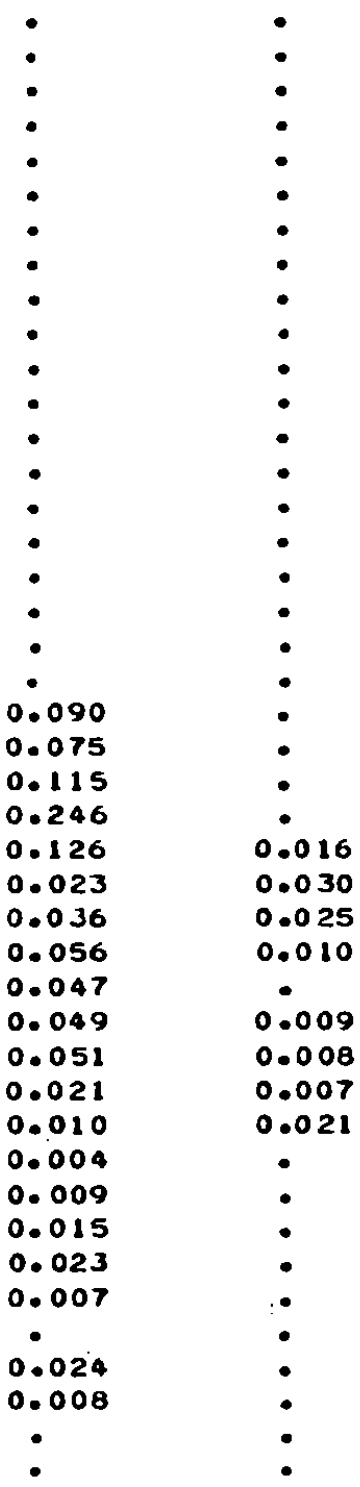

$\bullet$

$\bullet$

3.169

6.057

5.530

6.227

4.117

7.250

5.077

5.403

5.092

6.538

6.025

5.041

6.129

5. 350

5.002

3.820

3.199

4.277

6.870

3.509

2.642

2.425

2.154

2.900

2.989

2.872

2.024

1.827

1.943

0.977

1.075

1.709

1.550

1.196

1.409

1. 336

1. 225

1.179 
Basic Table 2.C. Rural Beijing Province (1): age-specific fertility rates,

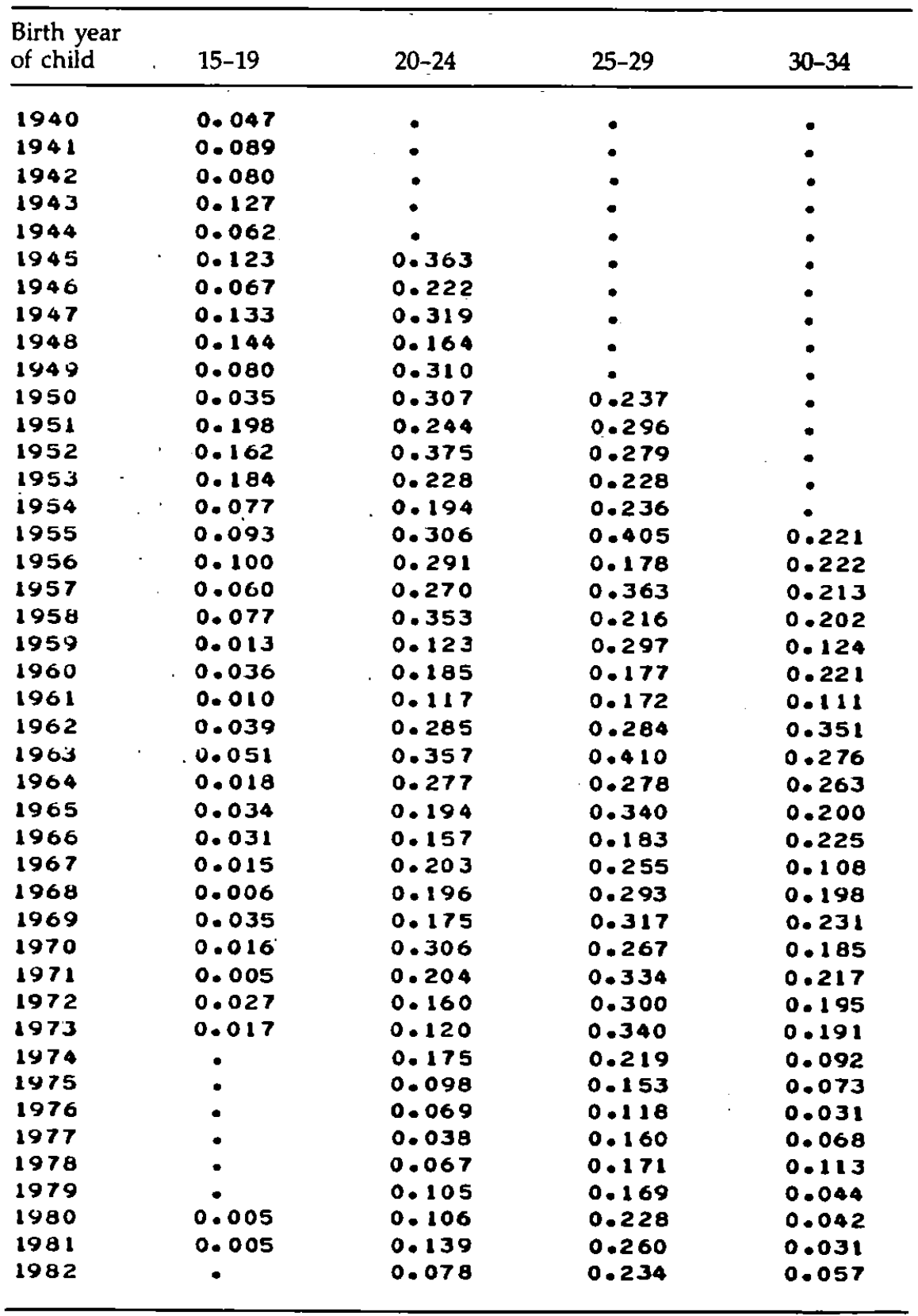




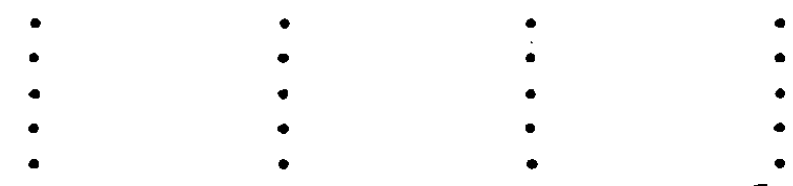

5.996

3.240

6.113

5.449

5.262

4.983

6.026

6.422

4.834

4.772

6.556

5.322

5.956

5.268

3.593

0.252

0.104

0.239

0.266

0.286

0.123

0.178

0.109

0.144

0.137

0.141

0.119

0.054

0.028

0.015

0.031

0.017

.

0.026

0.013

0.036

0.177

4.670

2.615

6.508

7.185

6.594

0.110

0.044

0.067

0.101

0.062

0.061

5.006

4.092

3.867

4.691

4.789

4.885

0.011

4.455

0.012

3.742

0.012

3.541

2.508

1.772

1.174

1.329

1.881

1.730

2.091 
Basic Table 3.A. Total Tianjin Province (2): age-specific fertility rates,

\begin{tabular}{|c|c|c|c|c|}
\hline $\begin{array}{l}\text { Birth year } \\
\text { of child }\end{array}$ & $15-19$ & $20-24$ & $25-29$ & $30-34$ \\
\hline 1940 & 0.098 & - & - & - \\
\hline 1941 & 0.059 & - & - & $\bullet$ \\
\hline 1942 & 0.115 & - & - & - \\
\hline 1943 & 0.100 & - & - & - \\
\hline 1944 & 0.063 & - & - & • \\
\hline 1945 & 0.078 & 0.209 & - & - \\
\hline 1940 & 0.096 & 0.253 & - & - \\
\hline 1947 & 0.100 & 0.262 & $\bullet$ & - \\
\hline 1948 & 0.093 & 0.196 & - & - \\
\hline 1949 & 0.046 & 0.249 & - & - \\
\hline 1950 & 0.102 & 0.271 & 0.216 & - \\
\hline 1951 & 0.099 & 0.268 & 0.306 & $\bullet$ \\
\hline 1952 & 0.113 & 0.282 & 0.294 & $\bullet$ \\
\hline 1953 & 0.093 & 0.237 & 0.291 & - \\
\hline 1954 & 0.118 & 0.370 & 0.268 & - \\
\hline 1955 & 0.114 & 0.312 & 0.363 & 0.294 \\
\hline 1956 & 0.084 & 0.282 & 0.323 & 0.277 \\
\hline 1957 & 0.098 & 0.356 & 0.327 & 0.316 \\
\hline 1958 & 0.065 & 0.333 & 0.313 & 0.291 \\
\hline 1959 & 0.052 & 0.236 & 0.249 & 0.161 \\
\hline 1960 & 0.054 & 0.273 & 0.316 & 0.175 \\
\hline 1961 & 0.014 & 0.147 & 0.167 & 0.237 \\
\hline 1962 & 0.021 & 0.278 & 0.305 & 0.250 \\
\hline 1963 & 0.029 & 0.316 & 0.405 & 0.300 \\
\hline 1964 & 0.023 & 0.235 & 0.208 & 0.204 \\
\hline 1965 & 0.036 & 0.209 & 0.282 & 0.131 \\
\hline 1966 & 0.003 & 0.142 & 0.194 & 0.091 \\
\hline 1967 & 0.003 & 0.124 & 0.242 & 0.109 \\
\hline 1968 & 0.006 & 0.161 & 0.236 & 0.135 \\
\hline 1969 & 0.003 & 0.163 & 0.266 & 0.150 \\
\hline 1970 & - & 0.180 & 0.248 & 0.134 \\
\hline 1971 & - & 0.120 & 0.276 & 0.115 \\
\hline 1972 & - & 0.105 & 0.265 & 0.113 \\
\hline 1973 & - & 0.046 & 0.228 & 0.135 \\
\hline 1974 & 0.002 & 0.047 & 0.205 & 0.115 \\
\hline 1975 & - & 0.036 & 0.234 & 0.102 \\
\hline 1976 & 0.002 & 0.032 & 0.236 & 0.110 \\
\hline 1977 & - & 0.015 & 0.207 & 0.073 \\
\hline 1978 & • & 0.039 & 0.197 & 0.079 \\
\hline 1979 & - & 0.013 & 0.207 & 0.072 \\
\hline 1980 & - & 0.017 & 0.195 & 0.065 \\
\hline 1981 & 0.002 & 0.039 & 0.194 & 0.038 \\
\hline 1982 & - & 0.051 & 0.169 & 0.039 \\
\hline
\end{tabular}


1940-82

\begin{tabular}{|c|c|c|c|}
\hline 35-39 & $40-44$ & $45-49$ & Total \\
\hline$\bullet$ & - & - & - \\
\hline$\bullet$ & $\bullet$ & $\bullet$ & - \\
\hline - & $\bullet$ & $\bullet$ & - \\
\hline - & - & • & - \\
\hline - & - & • & $\bullet$ \\
\hline - & - & - & 4.284 \\
\hline - & - & - & 6.296 \\
\hline - & - & • & 6.633 \\
\hline - & - & - & 4.596 \\
\hline - & - & $\bullet$ & 6.556 \\
\hline - & - & - & 6.202 \\
\hline - & - & - & 6.346 \\
\hline - & - & - & 6.968 \\
\hline - & - & - & 6.062 \\
\hline - & - & - & 7.072 \\
\hline - & - & - & 7.915 \\
\hline - & - & - & 7.184 \\
\hline - & - & - & 7.714 \\
\hline - & - & - & 6.851 \\
\hline - & - & - & 4.706 \\
\hline 0.222 & - & - & 5.864 \\
\hline 0.130 & - & $\bullet$ & 3.963 \\
\hline 0.164 & - & - & 5.975 \\
\hline 0.259 & - & - & 7.426 \\
\hline 0.185 & 0.068 & 0.010 & 4.664 \\
\hline 0.138 & 0.072 & 0.009 & 4.385 \\
\hline 0.086 & 0.059 & - & 2.883 \\
\hline 0.086 & 0.016 & - & 2.905 \\
\hline 0.080 & 0.042 & 0.007 & 3.338 \\
\hline 0.062 & 0.034 & - & 3.389 \\
\hline 0.066 & 0.037 & 0.007 & 3.355 \\
\hline 0.064 & 0.027 & 0.006 & 3.037 \\
\hline 0.031 & 0.032 & - & 2.732 \\
\hline 0.046 & 0.004 & - & 2.301 \\
\hline 0.027 & $\bullet$ & - & 1.977 \\
\hline 0.015 & 0.008 & - & 1.977 \\
\hline 0.021 & • & - & 2.006 \\
\hline 0.036 & - & 0.005 & 1.670 \\
\hline 0.015 & • & 0.004 & 1.669 \\
\hline 0.005 & 0.005 & - & 1.509 \\
\hline$\bullet$ & - & • & 1.387 \\
\hline 0.009 & - & - & 1.408 \\
\hline$\bullet$ & 0.010 & - & 1.350 \\
\hline
\end{tabular}


Basic Table 3.B. Urban Tianjin Province (2): age-specific fertility rates,

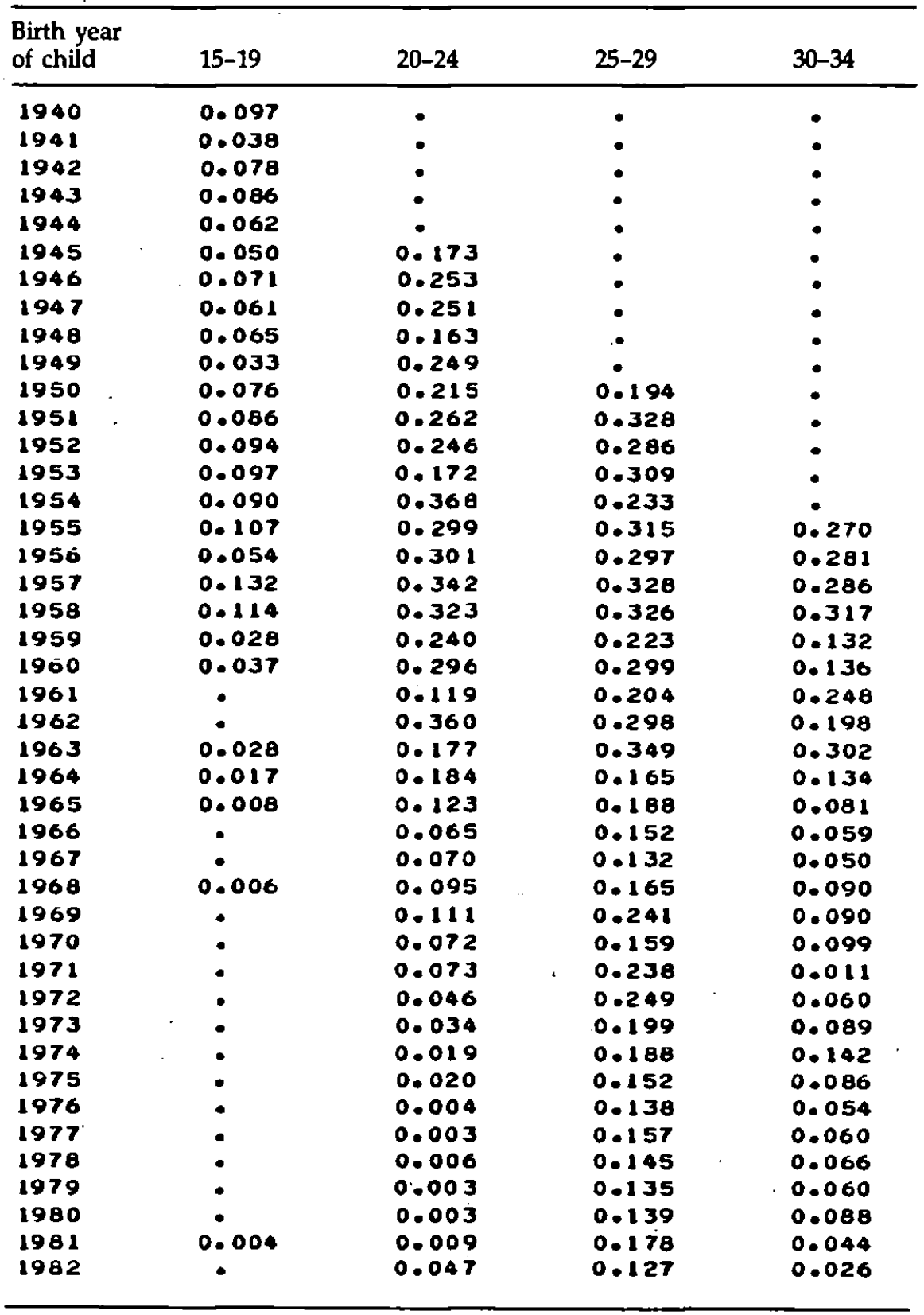


1940-82

\begin{tabular}{|c|c|c|c|}
\hline $35-39$ & $40-44$ & $45-49$ & Total \\
\hline - & - & $\bullet$ & - \\
\hline - & • & - & • \\
\hline - & - & - & $\bullet$ \\
\hline - & - & • & - \\
\hline - & - & - & - \\
\hline - & - & - & 4.173 \\
\hline - & - & - & 6.376 \\
\hline - & - & - & 6.049 \\
\hline - & • & - & 3.930 \\
\hline - & - & • & 6.244 \\
\hline - & - & - & 5.856 \\
\hline - & • & - & 6.617 \\
\hline - & $\bullet$ & - & 6.466 \\
\hline • & - & - & 5.932 \\
\hline - & - & - & 6.324 \\
\hline - & - & • & 7.401 \\
\hline - & - & - & 7.278 \\
\hline - & - & - & 7.603 \\
\hline - & - & - & $7 \cdot 234$ \\
\hline - & - & $\bullet$ & 4.039 \\
\hline 0.205 & $\bullet$ & - & 5.596 \\
\hline 0.141 & $\bullet$ & - & 4.434 \\
\hline 0.139 & $\bullet$ & - & 5.472 \\
\hline 0.257 & - & - & 6.296 \\
\hline 0.148 & 0.033 & 0.016 & 3.484 \\
\hline 0.072 & 0.054 & - & 2.629 \\
\hline 0.028 & 0.009 & - & 1.567 \\
\hline 0.041 & $\bullet$ & - & 1.463 \\
\hline 0.036 & 0.017 & - & 2.041 \\
\hline 0.045 & 0.023 & - & 2.550 \\
\hline 0.027 & 0.014 & - & 1.858 \\
\hline 0.038 & 0.007 & - & 1.828 \\
\hline - & 0.007 & - & 1.810 \\
\hline 0.019 & $\bullet$ & - & 1.704 \\
\hline 0.007 & - & - & 1.783 \\
\hline 0.009 & 0.005 & - & 1.365 \\
\hline$\bullet$ & $\bullet$ & - & 0.977 \\
\hline 0.012 & - & - & 1.159 \\
\hline 0.013 & - & 0.000 & 1.181 \\
\hline$\bullet$ & - & • & 0.989 \\
\hline$\bullet$ & - & • & 1.150 \\
\hline 0.022 & $\bullet$ & • & 1.253 \\
\hline$\bullet$ & • & • & 0.998 \\
\hline
\end{tabular}


Basic Table 3.C. Rural Tianjin Province (2): age-specific fertility rates,

\begin{tabular}{|c|c|c|c|c|}
\hline $\begin{array}{l}\text { Birth year } \\
\text { of child }\end{array}$ & $15-19$ & $20-24$ & $25-29$ & $30-34$ \\
\hline 1940 & 0.099 & - & • & - \\
\hline 1941 & 0.095 & $\bullet$ & • & - \\
\hline 1942 & 0.176 & - & - & - \\
\hline 1943 & 0.124 & - & - & $\bullet$ \\
\hline 1944 & 0.065 & $\bullet$ & $\bullet$ & - \\
\hline 1945 & 0.128 & 0.265 & $\bullet$ & 6 \\
\hline 1946 & 0.140 & 0.253 & - & - \\
\hline 1947 & 0.177 & 0.279 & - & - \\
\hline 1948 & 0.162 & 0.248 & - & - \\
\hline 1949 & 0.082 & 0.249 & - & - \\
\hline 1950 & 0.184 & 0.372 & 0.248 & - \\
\hline 1951 & 0.136 & 0.281 & 0.269 & $\bullet$ \\
\hline 1952 & 0.161 & 0.354 & 0.309 & - \\
\hline 1953 & 0.085 & 0.399 & 0.262 & - \\
\hline 1954 & 0.160 & 0.376 & 0.327 & - \\
\hline 1955 & 0.122 & 0.351 & 0.449 & 0.331 \\
\hline 1956 & 0.112 & 0.227 & 0.370 & 0.269 \\
\hline 1957 & 0.072 & 0.389 & 0.327 & 0.368 \\
\hline 1958 & 0.033 & 0.354 & 0.281 & 0.248 \\
\hline 1959 & 0.066 & 0.229 & 0.327 & 0.209 \\
\hline 1960 & 0.064 & 0.244 & 0.368 & 0.244 \\
\hline 1961 & 0.024 & 0.173 & 0.061 & 0.217 \\
\hline 1962 & 0.037 & 0.216 & 0.322 & 0.354 \\
\hline 1963 & 0.029 & 0.407 & 0.512 & 0.295 \\
\hline 1964 & 0.027 & 0.264 & 0.275 & 0.408 \\
\hline 1965 & 0.059 & 0.266 & 0.399 & 0.284 \\
\hline 1966 & 0.006 & 0.200 & 0.233 & 0.182 \\
\hline 1967 & 0.007 & 0.164 & 0.324 & 0.255 \\
\hline 1968 & 0.006 & 0.213 & 0.282 & 0.220 \\
\hline 1909 & 0.006 & 0.205 & 0.280 & 0.241 \\
\hline 1970 & - & 0.269 & 0.306 & 0.177 \\
\hline 1971 & - & 0.162 & 0.304 & 0.213 \\
\hline 1972 & - & 0.166 & 0.276 & 0.153 \\
\hline 1973 & - & 0.060 & 0.249 & 0.166 \\
\hline 1974 & 0.005 & 0.078 & 0.219 & 0.099 \\
\hline 1975 & - & 0.055 & 0.302 & 0.113 \\
\hline 1976 & 0.006 & 0.069 & 0.323 & 0.152 \\
\hline 1977 & - & 0.032 & 0.259 & 0.082 \\
\hline 1978 & - & 0.093 & 0.253 & 0.088 \\
\hline 1979 & - & 0.030 & 0.290 & 0.082 \\
\hline 1980 & - & 0.043 & 0.264 & 0.046 \\
\hline 1981 & - & 0.094 & 0.215 & 0.032 \\
\hline 1982 & - & 0.058 & 0.231 & 0.053 \\
\hline
\end{tabular}


1940-82

\begin{tabular}{|c|c|c|c|}
\hline $35-39$ & $40-44$ & $45-49$ & Total \\
\hline - & - & - & - \\
\hline$\bullet$ & - & - & - \\
\hline - & - & - & - \\
\hline • & • & $\bullet$ & - \\
\hline - & $\bullet$ & - & $\bullet$ \\
\hline - & - & - & 4.468 \\
\hline - & - & - & 6.161 \\
\hline • & - & - & 7.660 \\
\hline - & - & - & 5.857 \\
\hline - & - & - & 7.172 \\
\hline - & - & - & 6.891 \\
\hline$\bullet$ & - & - & 5.815 \\
\hline$\bullet$ & - & - & 7.938 \\
\hline - & - & - & 6.308 \\
\hline - & - & - & 8.459 \\
\hline - & - & - & 8.838 \\
\hline • & - & - & 7.027 \\
\hline$\bullet$ & - & - & 7.893 \\
\hline - & - & - & 6.260 \\
\hline - & - & - & 5.710 \\
\hline 0.248 & - & - & 6.264 \\
\hline 0.111 & - & - & 3.281 \\
\hline 0.206 & - & - & 6.674 \\
\hline 0.262 & - & - & 8.955 \\
\hline 0.249 & $0 . i 26$ & - & 6.744 \\
\hline 0.257 & 0.099 & 0.022 & 6.929 \\
\hline 0.191 & 0.142 & - & 4.778 \\
\hline 0.177 & 0.044 & - & 4.853 \\
\hline 0.192 & 0.083 & 0.020 & 5.078 \\
\hline 0.114 & 0.052 & $\bullet$ & 4.491 \\
\hline 0.184 & 0.077 & 0.017 & 5.149 \\
\hline 0.136 & 0.064 & 0.016 & 4.475 \\
\hline 0.107 & 0.082 & - & 3.920 \\
\hline 0.098 & 0.015 & - & 2.941 \\
\hline 0.057 & $\bullet \quad$ & • & $2 \cdot 291$ \\
\hline 0.022 & 0.017 & - & 2.540 \\
\hline 0.041 & $\bullet$ & - & 2.952 \\
\hline 0.054 & - & 0.014 & 2.201 \\
\hline 0.017 & - & - & 2.251 \\
\hline 0.008 & 0.011 & - & 2.110 \\
\hline • & $\bullet$ & - & 1.764 \\
\hline - & - & - & 1.706 \\
\hline - & 0.018 & - & 1.802 \\
\hline
\end{tabular}


Basic Table 4.A. Total Hebei Province (3): age-specific fertility rates,

\begin{tabular}{|c|c|c|c|c|c|}
\hline $\begin{array}{l}\text { Birth year } \\
\text { of child }\end{array}$ & $15-19$ & $20-24$ & $25-29$ & & $30-34$ \\
\hline $19+0$ & 0.056 & - & - & & $\bullet$ \\
\hline 1941 & 0.065 & - & - & & - \\
\hline 1942 & 0.085 & - & - & & - \\
\hline 1943 & 0.063 & - & - & & - \\
\hline 1944 & 0.049 & - & - & & - \\
\hline 1945 & 0.078 & 0.248 & - & & - \\
\hline 1940 & 0.077 & 0.194 & - & & - \\
\hline 1947 & 0.073 & $0.2 \in 1$ & - & & - \\
\hline 1948 & 0.066 & 0.199 & • & & - \\
\hline 1949 & 0.102 & 0.262 & - & & - \\
\hline 1950 & 0.075 & 0.251 & 0.247 & & - \\
\hline 1951 & 0.092 & 0.213 & 0.244 & & - \\
\hline 1952 & 0.112 & 0.307 & 0.274 & & - \\
\hline 1953 & 0.093 & 0.265 & 0.240 & & - \\
\hline 1954 & 0.098 & 0.243 & 0.276 & & - \\
\hline 1955 & 0.101 & 0.292 & 0.283 & & 0.242 \\
\hline 1956 & 0.081 & 0.291 & 0.258 & & 0.217 \\
\hline 1957 & 0.083 & 0.270 & 0.278 & & 0.250 \\
\hline 1958 & 0.070 & 0.248 & 0.260 & & 0.229 \\
\hline 1959 & 0.057 & 0.200 & 0.203 & & 0.162 \\
\hline 1960 & 0.042 & 0.234 & 0.224 & & 0.174 \\
\hline 1961 & 0.029 & 0.114 & 0.133 & & 0.096 \\
\hline 1962 & 0.040 & 0.240 & 0.285 & & 0.213 \\
\hline 1963 & 0.058 & 0.336 & 0.361 & & 0.325 \\
\hline 1964 & 0.053 & 0.261 & 0.276 & . & 0.268 \\
\hline 1965 & 0.051 & 0.266 & 0.305 & & 0.267 \\
\hline 1966 & 0.034 & 0.241 & 0.312 & & 0.221 \\
\hline 1967 & 0.024 & 0.225 & 0.274 & & 0.225 \\
\hline 1968 & 0.028 & 0.239 & 0.304 & & 0.263 \\
\hline 1969 & 0.023 & 0.205 & 0.284 & . & 0.218 \\
\hline 1970 & 0.019 & 0.261 & 0.307 & . & 0.247 \\
\hline 1971 & 0.021 & 0.223 & 0.295 & & 0.230 \\
\hline 1972 & 0.017 & 0.189 & 0.279 & & 0.190 \\
\hline 1973 & 0.010 & 0.182 & 0.257 & & 0.167 \\
\hline 1974 & 0.010 & 0.156 & 0.217 & & 0.111 \\
\hline 1975 & 0.007 & 0.119 & 0.216 & & 0.094 \\
\hline 1976 & 0.006 & 0.127 & 0.223 & & 0.100 \\
\hline 1977 & 0.009 & 0.103 & 0.202 & . & 0.096 \\
\hline 1978 & 0.005 & 0.115 & 0.233 & & 0.083 \\
\hline 1979 & 0.004 & 0.119 & 0.224 & & 0.084 \\
\hline 1980 & 0.002 & 0.129 & 0.232 & & 0.081 \\
\hline 1981 & 0.010 & 0.178 & 0.277 & & 0.108 \\
\hline 1982 & 0.009 & 0.151 & 0.225 & & 0.101 \\
\hline
\end{tabular}


Total

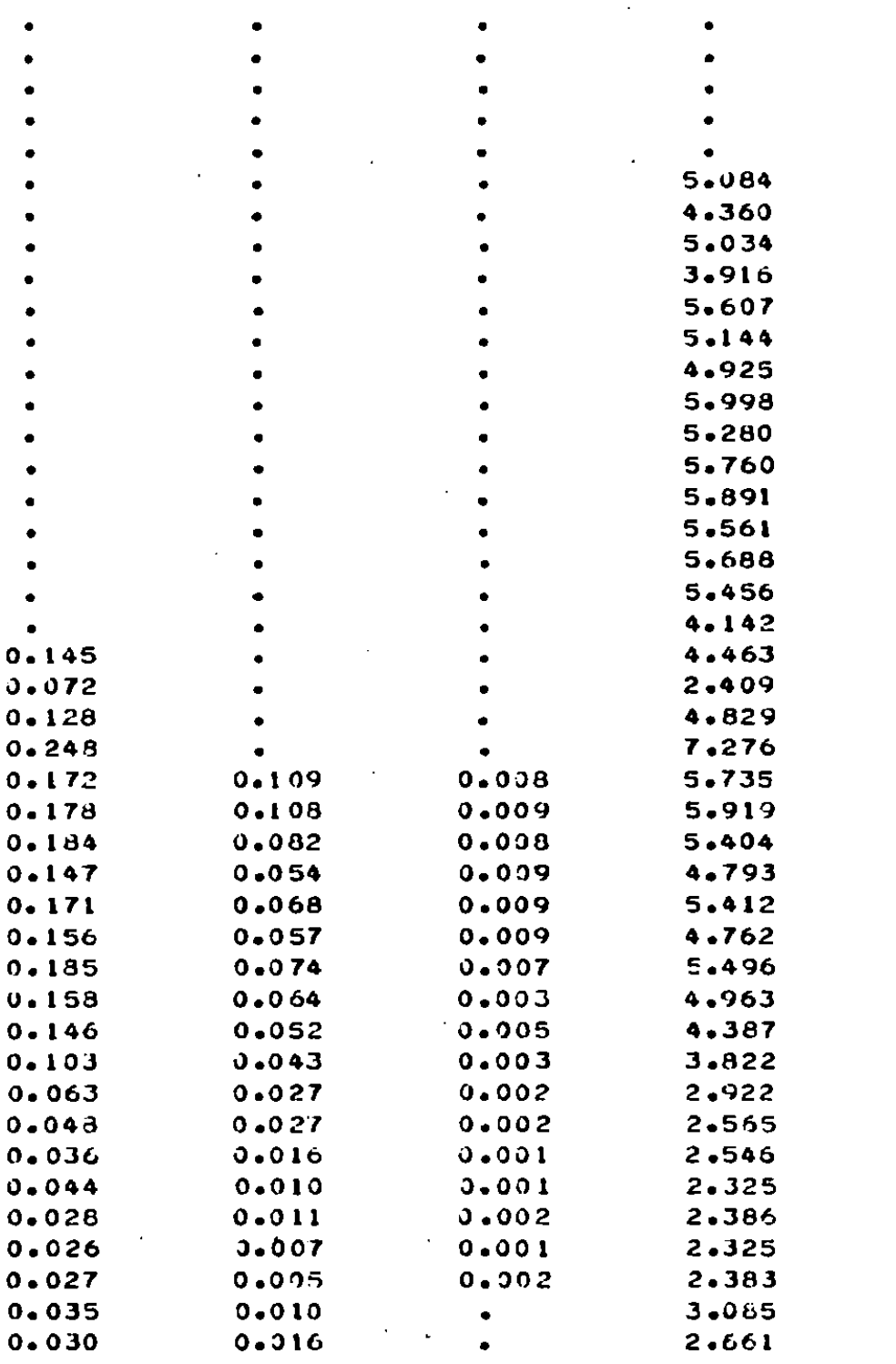


Basic Table 4.B. Urban Hebei Province (3): age-specific fertility rates,

\begin{tabular}{|c|c|c|c|c|}
\hline $\begin{array}{l}\text { Birth year } \\
\text { of child }\end{array}$ & $15-19$ & $20-24$ & $25-29$ & $30-34$ \\
\hline 1940 & 0.026 & - & - & • \\
\hline 1941 & 0.039 & - & - & - \\
\hline 1942 & 0.087 & - & - & - \\
\hline 1943 & 0.038 & - & - & - \\
\hline 1944 & 0.050 & - & - & - \\
\hline 1945 & 0.100 & 0.232 & - & - \\
\hline 1940 & 0.058 & 0.195 & - & $\bullet$ \\
\hline 1947 & 0.036 & 0.198 & - & - \\
\hline 1948 & 0.049 & 0.152 & - & - \\
\hline 1949 & 0.079 & 0.237 & - & - \\
\hline 1950 & 0.098 & 0.249 & 0.168 & . \\
\hline 1951 & 0.109 & 0.202 & 0.352 & - \\
\hline 1952 & 0.136 & 0.218 & 0.161 & • \\
\hline 1953 & 0.073 & 0.213 & 0.241 & - \\
\hline 1954 & 0.034 & 0.357 & 0.250 & - \\
\hline 1955 & 0.062 & 0.227 & 0.361 & 0.220 \\
\hline 1950 & 0.027 & 0.301 & 0.231 & 0.209 \\
\hline 1957 & 0.042 & 0.273 & 0.291 & 0.273 \\
\hline 1958 & 0.057 & 0.212 & 0.278 & 0.241 \\
\hline 1959 & 0.025 & 0.162 & 0.222 & 0.187 \\
\hline 1960 & 0.024 & 0.221 & 0.242 & 0.187 \\
\hline 1961 & 0.016 & 0.087 & 0.100 & 0.086 \\
\hline 1962 & 0.016 & 0.167 & 0.321 & 0.209 \\
\hline 1963 & 0.015 & 0.307 & 0.365 & 0.262 \\
\hline 1964 & 0.015 & 0.212 & 0.236 & 0.191 \\
\hline 1965 & 0.028 & 0.163 & 0.207 & 0.113 \\
\hline 1966 & - & 0.130 & 0.255 & 0.125 \\
\hline 1907 & - & 0.172 & 0.209 & 0.144 \\
\hline 1968 & 0.015 & 0.137 & 0.339 & 0.139 \\
\hline 1969 & 0.009 & 0.099 & 0.237 & $0.1 \in 2$ \\
\hline 1970 & . & 0.162 & 0.285 & 0.152 \\
\hline 1971 & 0.004 & 0.112 & 0.268 & 0.161 \\
\hline 1972 & - & 0.088 & 0.242 & 0.097 \\
\hline 1973 & - & 0.067 & 0.198 & 0.105 \\
\hline 1974 & - & 0.050 & 0.152 & 0.068 \\
\hline 1975 & - & 0.037 & 0.211 & 0.057 \\
\hline 1976 & - & 0.026 & 0.151 & 0.057 \\
\hline 1977 & - & 0.054 & 0.159 & 0.078 \\
\hline 1978 & - & 0.019 & 0.200 & 0.092 \\
\hline 1979 & 0.004 & 0.043 & 0.153 & 0.076 \\
\hline 1980 & - & 0.032 & 0.212 & 0.049 \\
\hline 1981 & - & 0.142 & 0.347 & 0.066 \\
\hline 1982 & - & 0.133 & 0.100 & 0.057 \\
\hline
\end{tabular}


1940-82

\begin{tabular}{|c|c|c|c|c|}
\hline $35-39$ & $40-44$ & $45-49$ & Total & - \\
\hline - & - & - & - & \\
\hline - & - & - & - & \\
\hline - & - & - & - & \\
\hline - & - & - & - & \\
\hline - & - & - & - & \\
\hline$\bullet$ & - & - & 6.500 & \\
\hline - & - & - & 3.039 & \\
\hline - & - & - & 4.963 & \\
\hline - & - & - & 4.200 & \\
\hline - & - & - & 4.533 & \\
\hline - & - & . & 4.931 & \\
\hline - & - & - & 6.050 & \\
\hline$\bullet$ & - & - & 4.936 & \\
\hline - & - & - & 5.945 & \\
\hline - & - & - & 7.253 & \\
\hline - & - & - & 5.641 & \\
\hline - & - & - & 4.957 & \\
\hline - & - & - & 6.374 & \\
\hline - & - & - & 6.028 & \\
\hline • & - & - & 4.273 & \\
\hline 0.142 & - & - & 4.619 & \\
\hline 0.091 & - & - & 2.173 & \\
\hline 0.124 & - & - & 4.486 & \\
\hline 0.330 & - & - & 7.204 & \\
\hline 0.112 & 0.064 & . & 4.146 & \\
\hline 0.100 & 0.065 & . & 3.376 & \\
\hline 0.077 & 0.052 & 0.014 & 3.263 & \\
\hline 0.073 & $\bullet$ & 0.013 & 3.055 & \\
\hline 0.164 & 0.101 & - & 4.481 & \\
\hline 0.056 & 0.012 & - & 2.872 & \\
\hline 0.098 & 0.050 & - & 3.732 & \\
\hline 0.067 & 0.029 & - & 3.201 & \\
\hline 0.064 & 0.036 & - & 2.642 & \\
\hline 0.015 & 0.008 & - & 1.966 & \\
\hline 0.027 & - & - & 1.481 & \\
\hline 0.014 & - & - & 1.597 & \\
\hline 0.020 & 0.008 & - & 1.316 & \\
\hline 0.007 & - & - & 1.488 & \\
\hline 0.024 & - & • & 1.678 & \\
\hline 0.034 & - & - & 1.549 & \\
\hline$\bullet$ & • & - & 1.470 & \\
\hline - & • & - & 2.778 & \\
\hline - & • & - & 1.850 & \\
\hline
\end{tabular}


Basic Table 4.C. Rural Hebei Province (3): age-specific fertility rates,

\begin{tabular}{|c|c|c|c|c|}
\hline $\begin{array}{l}\text { Birth year } \\
\text { of child }\end{array}$ & 15-19 & $20-24$ & 25-29 & $30-34$ \\
\hline 1940 & 0.059 & - & - & - \\
\hline 1941 & 0.067 & $\bullet$ & - & - \\
\hline 1942 & 0.085 & - & - & - \\
\hline 1943 & 0.065 & - & - & - \\
\hline 1944 & 0.049 & • & - & - \\
\hline 1945 & 0.076 & 0.250 & - & - \\
\hline 1946 & 0.079 & 0.193 & - & - \\
\hline 1947 & 0.077 & 0.267 & - & - \\
\hline 1948 & 0.068 & 0.203 & - & - \\
\hline 1949 & 0.104 & 0.264 & - & - \\
\hline 1950 & 0.072 & 0.251 & 0.255 & • \\
\hline 1951 & 0.090 & 0.214 & 0.234 & - \\
\hline 1952 & 0.109 & 0.317 & 0.284 & - \\
\hline 1953 & 0.096 & 0.271 & 0.240 & • \\
\hline 1954 & 0.107 & 0.229 & 0.279 & • \\
\hline 1955 & 0.107 & 0.300 & 0.276 & 0.244 \\
\hline 1956 & 0.089 & 0.290 & 0.261 & 0.217 \\
\hline 1957 & 0.089 & 0.270 & 0.277 & 0.255 \\
\hline 1958 & 0.071 & 0.253 & 0.257 & 0.228 \\
\hline 1959 & 0.061 & 0.205 & 0.201 & 0.160 \\
\hline 1960 & 0.045 & 0.236 & 0.222 & 0.173 \\
\hline 1961 & 0.031 & 0.118 & 0.136 & 0.097 \\
\hline 1962 & 0.042 & 0.250 & 0.281 & 0.214 \\
\hline 1963 & 0.062 & 0.340 & 0.360 & 0.333 \\
\hline 1964 & 0.056 & 0.268 & 0.282 & 0.277 \\
\hline 1965 & 0.053 & 0.279 & 0.319 & 0.286 \\
\hline 1966 & 0.036 & 0.253 & 0.321 & 0.232 \\
\hline 1967 & 0.027 & 0.230 & 0.284 & 0.234 \\
\hline 1968 & 0.029 & 0.248 & 0.300 & 0.278 \\
\hline 1969 & 0.025 & 0.214 & 0.290 & 0.226 \\
\hline 1970 & $0.02 i$ & 0.270 & 0.310 & 0.260 \\
\hline 1971 & 0.022 & 0.232 & 0.298 & 0.240 \\
\hline 1972 & 0.019 & 0.198 & 0.282 & 0.203 \\
\hline 1973 & 0.011 & 0.192 & 0.263 & 0.175 \\
\hline 1974 & 0.011 & 0.167 & 0.222 & 0.116 \\
\hline $197 \mathrm{~g}$ & 0.008 & 0.127 & 0.217 & 0.098 \\
\hline 1976 & 0.007 & 0.138 & 0.229 & 0.105 \\
\hline 1977 & .0 .010 & 0.109 & 0.206 & 0.097 \\
\hline 1978 & 0.006 & 0.125 & 0.236 & 0.083 \\
\hline 1979 & 0.004 & 0.128 & 0.231 & 0.084 \\
\hline 1980 & 0.002 & 0.140 & 0.234 & 0.083 \\
\hline 1981 & 0.011 & 0.182 & 0.269 & 0.111 \\
\hline 1982 & 0.009 & 0.153 & 0.230 & 0.105 \\
\hline
\end{tabular}


1940-82

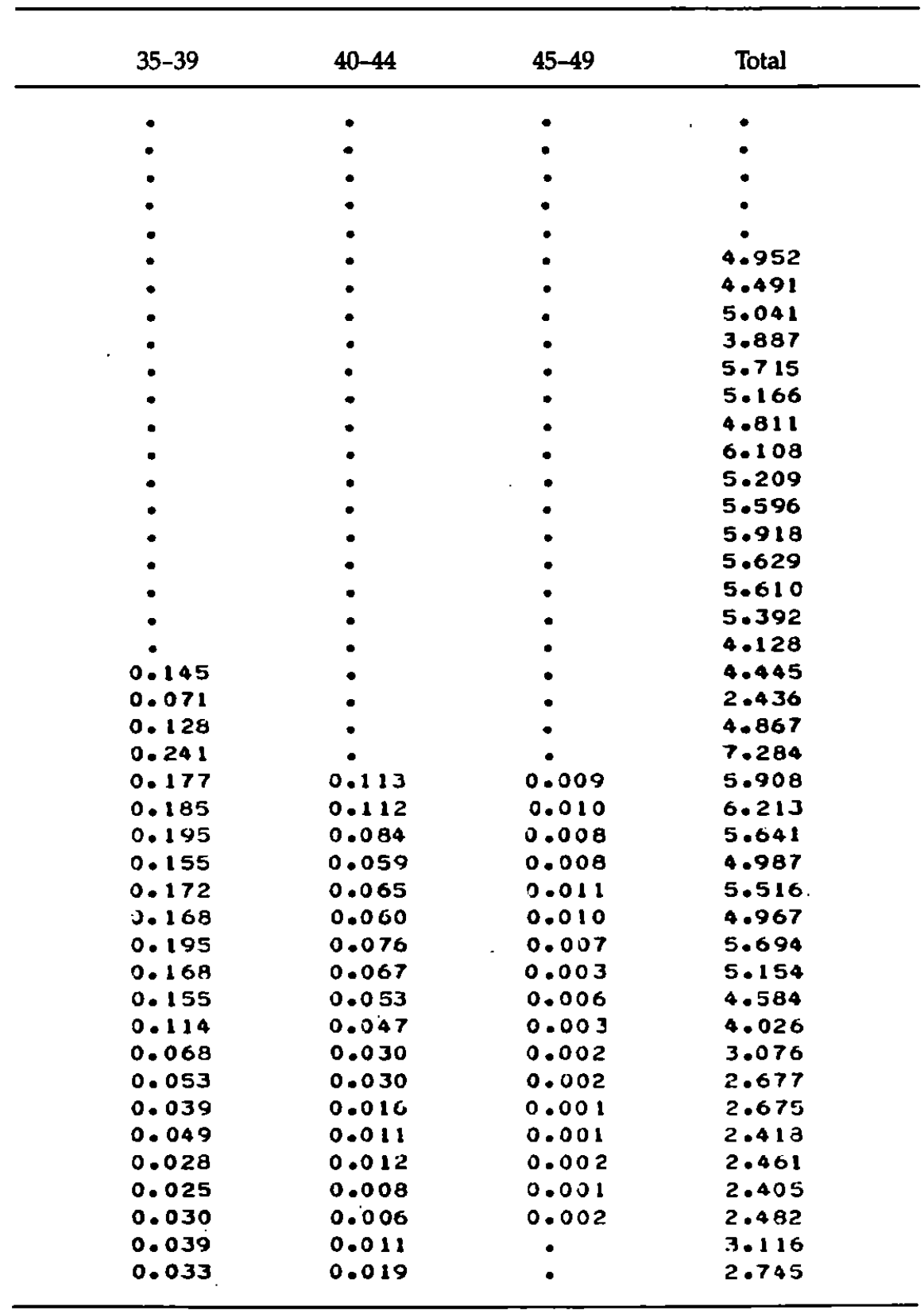


Basic Table 5.A. Total Shanxi Province (4): age-specific fertility rates,

\begin{tabular}{|c|c|c|c|c|}
\hline $\begin{array}{l}\text { Birth year } \\
\text { of child }\end{array}$ & $15-19$ & $20-24$ & $25-29$ & $30-34$ \\
\hline 1940 & 0.124 & - & - & - \\
\hline 1941 & 0.111 & - & - & - \\
\hline 1942 & 0.128 & - & - & - \\
\hline 1943 & 0.112 & - & - & - \\
\hline 1944 & 0.110 & - & - & - \\
\hline 1945 & 0.105 & 0.199 & - & - \\
\hline 1946 & 0.114 & 0.294 & - & - \\
\hline 1947 & 0.110 & 0.209 & - & - \\
\hline 1948 & 0.108 & 0.175 & - & $\bullet$ \\
\hline 1949 & 0.111 & 0.206 & - & $\bullet$ \\
\hline 1950 & 0.115 & 0.262 & 0.197 & $\bullet$ \\
\hline 1951 & 0.149 & 0.258 & 0.224 & - \\
\hline 1952 & 0.131 & 0.254 & 0.245 & - \\
\hline 1953 & 0.158 & 0.271 & 0.218 & - \\
\hline 1954 & 0.151 & 0.259 & 0.231 & $\bullet$ \\
\hline 1950 & 0.142 & 0.292 & 0.230 & 0.236 \\
\hline $\begin{array}{l}1956 \\
1957\end{array}$ & $\begin{array}{l}0.106 \\
0.154\end{array}$ & $\begin{array}{l}0.274 \\
0.305\end{array}$ & $\begin{array}{l}0.255 \\
0.257\end{array}$ & $\begin{array}{l}0.171 \\
0.231\end{array}$ \\
\hline 1958 & 0.093 & 0.289 & 0.215 & 0.175 \\
\hline 1959 & 0.079 & 0.235 & 0.227 & 0.220 \\
\hline 1960 & 0.094 & 0.259 & 0.216 & 0.144 \\
\hline 1901 & 0.082 & 0.204 & 0.200 & 0.174 \\
\hline 1962 & 0.119 & 0.321 & 0.322 & 0.225 \\
\hline 1963 & 0.125 & 0.344 & 0.348 & 0.254 \\
\hline 1964 & 0.140 & 0.294 & 0.270 & 0.245 \\
\hline 1965 & 0.107 & 0.282 & 0.313 & 0.216 \\
\hline 1960 & 0.088 & 0.287 & 0.233 & 0.195 \\
\hline 1967 & 0.064 & 0.287 & 0.339 & 0.246 \\
\hline 1968 & 0.067 & 0.304 & 0.315 & 0.282 \\
\hline 1969 & 0.077 & 0.252 & 0.323 & 0.233 \\
\hline 1970 & 0.076 & 0.322 & 0.269 & 0.214 \\
\hline 1971 & 0.086 & 0.313 & 0.274 & 0.236 \\
\hline 1972 & 0.060 & 0.285 & 0.278 & 0.198 \\
\hline 1973 & 0.060 & 0.264 & 0.251 & 0.170 \\
\hline 1974 & 0.050 & 0.261 & 0.258 & 0.133 \\
\hline 1975 & 0.025 & 0.221 & 0.195 & 0.096 \\
\hline 1976 & 0.034 & 0.191 & 0.205 & 0.129 \\
\hline 1977 & 0.023 & 0.173 & 0.179 & 0.091 \\
\hline 1978 & 0.012 & 0.158 & 0.165 & 0.077 \\
\hline 1979 & 0.013 & 0.157 & 0.163 & 0.074 \\
\hline 1980 & 0.017 & 0.173 & 0.188 & 0.052 \\
\hline 1981 & 0.021 & 0.187 & 0.146 & 0.050 \\
\hline 1982 & 0.026 & 0.224 & 0.210 & 0.089 \\
\hline
\end{tabular}


1940-82

\begin{tabular}{|c|c|c|c|}
\hline $35-39$ & $40-44$ & $45-49$ & Total \\
\hline - & $\bullet$ & - & • \\
\hline - & • & $\bullet$ & - \\
\hline - & - & - & - \\
\hline - & $\bullet$ & - & - \\
\hline$\bullet$ & - & - & - \\
\hline$\bullet$ & - & - & 4.101 \\
\hline$\bullet$ & $\bullet$ & - & 5.097 \\
\hline$\bullet$ & - & - & 4.350 \\
\hline - & $\bullet$ & - & 3.774 \\
\hline - & - & - & 4.649 \\
\hline$\bullet$ & - & - & 4.631 \\
\hline - & - & - & 4.899 \\
\hline - & - & - & 5.110 \\
\hline - & - & - & 5.112 \\
\hline - & - & - & 5.375 \\
\hline - & - & - & 5.667 \\
\hline - & - & - & 5.003 \\
\hline - & - & - & 5.835 \\
\hline - & - & - & 4.873 \\
\hline - & - & - & 4.893 \\
\hline 0.153 & $\bullet$ & - & 4.601 \\
\hline 0.082 & - & - & 4.008 \\
\hline 0.188 & - & - & 6.349 \\
\hline 0.175 & - & - & 6.685 \\
\hline 0.157 & 0.091 & 0.008 & 6.021 \\
\hline 0.174 & 0.073 & 0.016 & 5.911 \\
\hline 0.170 & 0.072 & 0.003 & 5.236 \\
\hline 0.123 & 0.069 & 0.006 & 5.690 \\
\hline 0.185 & 0.077 & 0.013 & 6.210 \\
\hline 0.153 & 0.061 & 0.010 & 5.546 \\
\hline 0.178 & 0.073 & 0.017 & 5.751 \\
\hline 0.147 & 0.079 & 0.010 & 5.726 \\
\hline 0.133 & 0.062 & 0.002 & 5.094 \\
\hline 0.127 & 0.060 & 0.005 & 4.687 \\
\hline 0.080 & 0.044 & 0.002 & 4.140 \\
\hline 0.088 & 0.046 & 0.009 & 3.401 \\
\hline 0.067 & 0.029 & 0.004 & 3.299 \\
\hline 0.053 & 0.022 & 0.004 & 2.750 \\
\hline 0.029 & 0.013 & 0.007 & $2 \cdot 300$ \\
\hline 0.027 & 0.013 & 0.002 & 2.235 \\
\hline 0.016 & - & 0.005 & 2.254 \\
\hline 0.024 & 0.002 & - & 2.159 \\
\hline 0.010 & 0.004 & $\bullet$ & 2.917 \\
\hline
\end{tabular}


Basic Table 5.B. Urban Shanxi Province (4): age-specific fertility rates,

\begin{tabular}{|c|c|c|c|c|}
\hline $\begin{array}{l}\text { Birth year } \\
\text { of child }\end{array}$ & $15-19$ & $20-24$ & $25-29$ & $30-34$ \\
\hline 1940 & 0.111 & - & - & - \\
\hline 1941 & 0.066 & - & - & - \\
\hline 1942 & 0.059 & - & - & - \\
\hline 1943 & 0.137 & - & • & . \\
\hline 1944 & 0.051 & - & - & . \\
\hline 1945 & 0.203 & 0.200 & - & - \\
\hline 1946 & 0.142 & 0.309 & - & - \\
\hline 1947 & 0.097 & 0.255 & - & - \\
\hline 1948 & 0.109 & 0.215 & - & - \\
\hline 1949 & 0.058 & 0.051 & - & - \\
\hline 1950 & 0.117 & 0.328 & 0.177 & - \\
\hline 1951 & 0.122 & 0.199 & 0.243 & - \\
\hline 1952 & 0.115 & 0.262 & 0.295 & - \\
\hline 1953 & 0.150 & 0.266 & 0.234 & - \\
\hline 1954 & 0.095 & 0.278 & 0.254 & - \\
\hline 1955 & 0.130 & 0.308 & 0.235 & 0.244 \\
\hline 1936 & 0.044 & 0.265 & 0.241 & 0.177 \\
\hline 1957 & 0.122 & 0.303 & 0.290 & 0.275 \\
\hline 1958 & 0.031 & 0.265 & 0.302 & 0.332 \\
\hline 1959 & 0.075 & 0.248 & 0.186 & 0.186 \\
\hline 1960 & 0.124 & 0.208 & 0.180 & 0.250 \\
\hline 1961 & 0.034 & 0.192 & 0.204 & 0.128 \\
\hline 1962 & 0.035 & 0.260 & 0.303 & 0.235 \\
\hline 1963 & 0.067 & 0.294 & 0.358 & 0.229 \\
\hline 1964 & 0.155 & 0.225 & 0.295 & 0.186 \\
\hline 1965 & 0.108 & 0.194 & 0.299 & 0.149 \\
\hline 1966 & 0.065 & 0.222 & 0.163 & 0.143 \\
\hline 1967 & 0.033 & 0.138 & 0.413 & 0.157 \\
\hline 1968 & - & 0.270 & 0.186 & 0.277 \\
\hline 1969 & 0.047 & 0.140 & 0.356 & 0.165 \\
\hline 1970 & 0.022 & 0.154 & 0.229 & 0.156 \\
\hline 1971 & 0.041 & 0.220 & 0.188 & 0.089 \\
\hline 1972 & 0.025 & 0.178 & 0.242 & 0.168 \\
\hline 1973 & 0.025 & 0.128 & 0.253 & 0.108 \\
\hline 1974 & 0.024 & 0.101 & 0.171 & 0.112 \\
\hline 1975 & 0.018 & 0.093 & 0.170 & 0.106 \\
\hline 1976 & 0.026 & 0.118 & 0.194 & 0.085 \\
\hline 1977 & - & 0.114 & 0.190 & 0.052 \\
\hline 1978 & 0.007 & 0.055 & 0.247 & 0.067 \\
\hline 1979 & 0.007 & 0.060 & 0.109 & 0.124 \\
\hline 1980 & • & 0.084 & 0.208 & 0.062 \\
\hline 1981 & 0.006 & 0.078 & 0.131 & 0.052 \\
\hline 1982 & $\bullet$ & 0.113 & 0.240 & 0.022 \\
\hline
\end{tabular}




\begin{tabular}{|c|c|c|c|}
\hline $35-39$ & $40-44$ & $45-49$ & Total \\
\hline - & - & - & - \\
\hline - & - & - & - \\
\hline - & $\bullet$ & - & - \\
\hline - & - & - & - \\
\hline • & - & - & - \\
\hline - & - & - & 4.549 \\
\hline - & - & - & 5.845 \\
\hline - & - & - & 3.928 \\
\hline - & - & • & 4.640 \\
\hline - & - & - & 3.221 \\
\hline - & - & - & 5.024 \\
\hline - & - & - & 4.946 \\
\hline - & - & - & $5 \cdot 986$ \\
\hline - & - & - & 5.141 \\
\hline$\bullet$ & - & - & 6.302 \\
\hline - & 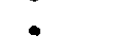 & - & 5.778 \\
\hline - & . & - & 5.114 \\
\hline - & - & - & 5.889 \\
\hline - & . & - & 6.105 \\
\hline$\bullet$ & . & . & 4.576 \\
\hline 0.044 & - & . & 4.418 \\
\hline 0.066 & - & - & 3.508 \\
\hline 0.255 & - & - & 5.951 \\
\hline 0.195 & - & . & 6.163 \\
\hline 0.169 & 0.060 & - & 5.452 \\
\hline 0.109 & 0.022 & . & 4.404 \\
\hline 0.128 & 0.022 & 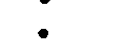 & 3.709 \\
\hline 0.110 & 0.059 & 0.023 & 4.673 \\
\hline 0.157 & 0.020 & - & 4.546 \\
\hline 0.070 & 0.034 & - & 4.057 \\
\hline 0.106 & 0.047 & - & 3.570 \\
\hline 0.102 & 0.071 & - & 3.553 \\
\hline 0.073 & 0.028 & - & 3.575 \\
\hline 0.046 & 0.024 & - & 2.922 \\
\hline - & 0.035 & - & 2.217 \\
\hline 0.039 & 0.011 & - & 2.182 \\
\hline 0.044 & 0.010 & - & 2.387 \\
\hline 0.031 & $\bullet$ & - & 1.931 \\
\hline - & - & - & 1.884 \\
\hline - & - & - & 1.500 \\
\hline • & - & - & 1.769 \\
\hline 0.017 & - & - & 1.421 \\
\hline - & - & - & 1.873 \\
\hline
\end{tabular}


Basic Table 5.C. Rural Shanxi Province (4): age-specific fertility rates,

\begin{tabular}{|c|c|c|c|c|}
\hline $\begin{array}{l}\text { Birth year } \\
\text { of child }\end{array}$ & $15-19$ & $20-24$ & 25-29 & $30-34$ \\
\hline 1940 & 0.126 & - & - & - \\
\hline 1941 & 0.116 & - & - & $\bullet$ \\
\hline 1942 & 0.138 & - & - & - \\
\hline 1943 & 0.109 & $\bullet$ & - & - \\
\hline 1944 & 0.119 & - & - & - \\
\hline 1945 & 0.090 & 0.199 & - & - \\
\hline 1946 & 0.109 & 0.292 & $\bullet$ & - \\
\hline 1947 & 0.113 & 0.203 & - & $\bullet$ \\
\hline 1448 & 0.108 & 0.169 & - & - \\
\hline 1949 & $0 \cdot 120$ & 0.230 & - & - \\
\hline 1950 & 0.115 & 0.252 & 0.199 & - \\
\hline 1951 & 0.154 & 0.268 & 0.221 & $\bullet$ \\
\hline 1952 & 0.134 & 0.252 & 0.238 & - \\
\hline 1953 & 0.159 & 0.272 & 0.215 & - \\
\hline 1954 & 0.162 & 0.255 & 0.228 & • \\
\hline 1955 & 0.144 & 0.288 & 0.229 & 0.235 \\
\hline 1956 & 0.115 & 0.275 & 0.258 & 0.170 \\
\hline 1957 & 0.158 & 0.305 & 0.252 & 0.225 \\
\hline 1958 & 0.102 & 0.293 & 0.200 & 0.153 \\
\hline 1959 & 0.079 & 0.233 & 0.235 & 0.225 \\
\hline 1960 & 0.091 & 0.268 & 0.223 & 0.127 \\
\hline 1961 & 0.088 & 0.205 & 0.199 & 0.182 \\
\hline 1962 & 0.128 & 0.330 & 0.326 & 0.223 \\
\hline 1963 & 0.131 & 0.351 & 0.346 & 0.258 \\
\hline 1964 & 0.138 & 0.302 & 0.265 & 0.255 \\
\hline 1965 & 0.107 & 0.292 & 0.316 & 0.229 \\
\hline 1960 & 0.090 & 0.294 & 0.243 & 0.205 \\
\hline 1967 & 0.068 & 0.303 & 0.328 & 0.264 \\
\hline 1968 & 0.076 & 0.307 & 0.334 & 0.283 \\
\hline 1969 & 0.081 & 0.264 & 0.319 & 0.246 \\
\hline 1970 & 0.084 & 0.334 & 0.274 & 0.224 \\
\hline 1971 & 0.092 & 0.324 & 0.284 & 0.258 \\
\hline 1972 & 0.066 & 0.297 & 0.282 & 0.203 \\
\hline 1973 & 0.065 & 0.282 & 0.251 & 0.179 \\
\hline 1974 & 0.054 & 0.285 & 0.267 & 0.135 \\
\hline 1975 & 0.027 & 0.240 & 0.198 & 0.095 \\
\hline 1976 & 0.035 & 0.202 & 0.206 & 0.134 \\
\hline 1977 & 0.026 & 0.182 & 0.178 & 0.095 \\
\hline 1978 & 0.013 & 0.175 & 0.154 & 0.078 \\
\hline 1979 & 0.013 & 0.173 & 0.171 & 0.068 \\
\hline 1980 & 0.019 & 0.188 & 0.185 & 0.051 \\
\hline 1981 & 0.023 & 0.205 & 0.149 & 0.050 \\
\hline 1982 & 0.030 & 0.241 & 0.206 & 0.097 \\
\hline
\end{tabular}


1940-82

35-39

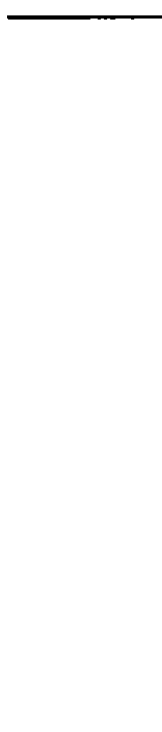

0.166

0.084

0.179

0.172

0.155

0.185

0.178

0.131

0.189

0.168

0.192

0.156

0.144

0.142

0.095

0.096

0.071

0.062

0.033

0.030

0.018

0.025

0.011
40-44

45-49

Total

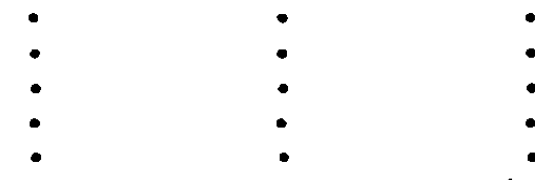

4.041

4.993

4.408

3.649

4.862

4.571

4.891

4.974

5.108

5.229

5.649

4.986

5.826

4.685

4.940

4.628

4.082

6.406

6.759

6.099

6.130

5.459

5.832

6.442

5.773

6.051

6.029

5.314

4.958

4.417

3.593

3.431

2.874

2.375

2.360

2.335

2.273

2.943 
Basic Table 6.A. Total Inner Mongolia Province (5): age-specific fertility

\begin{tabular}{|c|c|c|c|c|}
\hline $\begin{array}{l}\text { Birth year } \\
\text { of child }\end{array}$ & $15-19$ & $20-24$ & 25-29 & $30-34$ \\
\hline 1940 & 0.164 & - & - & - \\
\hline 1941 & 0.169 & - & - & - \\
\hline 1942 & 0.086 & - & - & - \\
\hline 1943 & 0.103 & - & - & - \\
\hline 1944 & 0.071 & - & - & - \\
\hline 1945 & 0.103 & 0.272 & - & - \\
\hline 1946 & 0.090 & 0.257 & - & - \\
\hline 1947 & 0.092 & 0.302 & - & - \\
\hline 1948 & 0.134 & 0.218 & - & - \\
\hline 1949 & 0.101 & 0.251 & - & - \\
\hline 1950 & 0.109 & 0.274 & 0.263 & - \\
\hline 1951 & 0.105 & 0.305 & 0.245 & - \\
\hline 1932 & 0.116 & 0.287 & 0.302 & - \\
\hline 1953 & 0.105 & 0.300 & 0.302 & - \\
\hline 1954 & 0.128 & 0.254 & 0.279 & - \\
\hline 1955 & 0.101 & 0.314 & 0.335 & 0.289 \\
\hline 1956 & 0.147 & 0.294 & 0.302 & 0.241 \\
\hline 1957 & 0.092 & 0.280 & 0.337 & 0.313 \\
\hline 1958 & 0.097 & 0.265 & 0.305 & 0.230 \\
\hline 1959 & 0.067 & 0.263 & 0.273 & 0.198 \\
\hline 1900 & 0.103 & 0.235 & 0.288 & 0.298 \\
\hline 1961 & 0.042 & 0.164 & 0.219 & 0.178 \\
\hline 1962 & 0.115 & 0.281 & 0.320 & 0.257 \\
\hline 1963 & 0.137 & 0.303 & 0.343 & 0.342 \\
\hline 1964 & 0.101 & 0.311 & 0.314 & 0.254 \\
\hline 1965 & 0.096 & 0.280 & 0.280 & 0.257 \\
\hline 1906 & 0.075 & 0.267 & 0.268 & 0.254 \\
\hline 1967 & 0.047 & 0.310 & 0.232 & 0.217 \\
\hline 1968 & 0.060 & 0.358 & 0.357 & 0.267 \\
\hline 1969 & 0.060 & 0.292 & 0.311 & 0.207 \\
\hline 1970 & $0.060^{\circ}$ & 0.303 & 0.363 & 0.245 \\
\hline 1971 & 0.059 & 0.307 & 0.257 & 0.218 \\
\hline 1972 & 0.034 & 0.270 & 0.274 & 0.238 \\
\hline 1973 & 0.034 & 0.236 & 0.232 & 0.178 \\
\hline 1974 & 0.045 & 0.260 & 0.279 & 0.135 \\
\hline 1475 & 0.019 & 0.208 & 0.228 & 0.113 \\
\hline 1976 & 0.020 & 0.178 & 0.225 & 0.085 \\
\hline 1977 & 0.020 & 0.153 & 0.218 & 0.083 \\
\hline 1978 & 0.008 & 0.137 & 0.229 & 0.104 \\
\hline 1979 & 0.005 & 0.159 & 0.202 & 0.080 \\
\hline 1980 & 0.014 & 0.155 & 0.210 & 0.092 \\
\hline 1981 & 0.012 & 0.192 & 0.198 & 0.070 \\
\hline 1982 & 0.022 & 0.197 & 0.273 & 0.121 \\
\hline
\end{tabular}


rates, $1940-82$

\begin{tabular}{|c|c|c|c|}
\hline $35-39$ & $40-44$ & $45-49$ & Total \\
\hline - & - & • & - \\
\hline - & • & • & • \\
\hline$\bullet$ & $\bullet$ & - & - \\
\hline - & - & $\bullet$ & - \\
\hline$\bullet$ & • & $\bullet$ & • \\
\hline • & $\bullet$ & - & 4.567 \\
\hline - & - & - & 4.362 \\
\hline - & - & • & 5.394 \\
\hline .. & - & • & 4.685 \\
\hline$\bullet$ & $\bullet$ & • & 4.621 \\
\hline - & - & - & 4.912 \\
\hline - & - & - & 5.144 \\
\hline - & - & - & 5.968 \\
\hline$\bullet$ & - & - & 5.690 \\
\hline - & - & • & 5.844 \\
\hline - & • & - & 6.180 \\
\hline$\bullet$ & - & • & 6.202 \\
\hline - & • & • & 6.695 \\
\hline$\bullet$ & - & $\bullet$ & 6.049 \\
\hline - & - & - & 5.017 \\
\hline 0.225 & - & - & 6.149 \\
\hline 0.088 & $\bullet$ & $\bullet$ & $3 \cdot 936$ \\
\hline 0.235 & - & - & 6.518 \\
\hline 0.237 & $\therefore$ & • & 7.480 \\
\hline 0.191 & 0.133 & 0.041 & 6.726 \\
\hline 0.209 & 0.112 & 0.043 & 6.378 \\
\hline 0.165 & 0.084 & 0.017 & 5.661 \\
\hline 0.136 & 0.090 & 0.016 & 5.240 \\
\hline 0.181 & 0.073 & 0.005 & 6.505 \\
\hline 0.177 & 0.109 & 0.009 & 5.827 \\
\hline 0.163 & 0.068 & 0.013 & 0.075 \\
\hline 0.145 & 0.072 & 0.008 & 5.328 \\
\hline 0.120 & 0.062 & 0.004 & 5.011 \\
\hline 0.121 & 0.037 & 0.004 & 4.201 \\
\hline 0.077 & 0.042 & 0.004 & 4.211 \\
\hline 0.058 & 0.050 & 0.007 & 3.415 \\
\hline 0.041 & 0.027 & - & 2.881 \\
\hline 0.032 & 0.023 & • & 2.646 \\
\hline 0.028 & 0.009 & 0.003 & 2.586 \\
\hline 0.025 & 0.024 & 0.002 & 2.499 \\
\hline 0.026 & 0.004 & - & 2.505 \\
\hline 0.032 & 0.004 & 0.004 & 2.555 \\
\hline 0.023 & 0.004 & - & 3.202 \\
\hline
\end{tabular}


Basic Table 6.B. Urban Inner Mongolia Province (5): age-specific

\begin{tabular}{|c|c|c|c|c|}
\hline $\begin{array}{l}\text { Birth year } \\
\text { of child }\end{array}$ & $15-19$ & $20-24$ & $25-29$ & $30-34$ \\
\hline 1940 & 0.081 & - & $\bullet$ & - \\
\hline 1941 & 0.071 & - & - & - \\
\hline 1942 & 0.044 & - & - & - \\
\hline 1943 & 0.065 & - & - & - \\
\hline 1944 & 0.044 & - & - & - \\
\hline 1945 & 0.062 & 0.264 & - & - \\
\hline 1946 & 0.026 & 0.178 & - & - \\
\hline 1947 & 0.016 & 0.205 & • & - \\
\hline 1948 & 0.037 & 0.194 & . & . \\
\hline 1949 & 0.041 & 0.211 & - & . \\
\hline 1950 & 0.084 & 0.228 & 0.102 & . \\
\hline 1951 & 0.045 & 0.251 & 0.284 & $\bullet$ \\
\hline 1952 & 0.039 & 0.236 & 0.293 & . \\
\hline 1953 & 0.048 & 0.200 & 0.272 & - \\
\hline 1954 & 0.034 & 0.218 & 0.256 & - \\
\hline 1955 & 0.029 & 0.215 & 0.353 & 0.163 \\
\hline 1956 & 0.035 & 0.193 & 0.277 & 0.284 \\
\hline 1957 & 0.028 & 0.166 & 0.318 & 0.249 \\
\hline 1958 & 0.021 & 0.200 & 0.311 & 0.168 \\
\hline 1959 & 0.027 & 0.184 & 0.245 & 0.100 \\
\hline 1960 & 0.025 & 0.167 & 0.268 & 0.290 \\
\hline 1961 & 0.012 & 0.083 & 0.210 & 0.113 \\
\hline 1962 & 0.059 & 0.169 & 0.264 & 0.253 \\
\hline 1963 & 0.070 & 0.155 & 0.331 & 0.311 \\
\hline 1964 & 0.027 & 0.221 & 0.248 & 0.143 \\
\hline 1965 & 0.018 & 0.162 & 0.171 & 0.155 \\
\hline 1966 & 0.008 & 0.210 & 0.213 & 0.147 \\
\hline 1967 & 0.014 & 0.198 & 0.212 & 0.161 \\
\hline 1968 & 0.006 & 0.237 & 0.331 & 0.196 \\
\hline 1969 & $\bullet$ & 0.225 & 0.257 & 0.167 \\
\hline 1970 & 0.018 & 0.162 & 0.270 & 0.167 \\
\hline 1971 & 0.016 & 0.173 & 0.181 & 0.130 \\
\hline 1972 & - & 0.188 & 0.220 & 0.197 \\
\hline 1973 & - & 0.074 & 0.219 & 0.146 \\
\hline 1974 & $\bullet$ & 0.100 & 0.144 & 0.077 \\
\hline 1975 & - & 0.066 & 0.226 & 0.069 \\
\hline 1976 & - & 0.035 & 0.173 & 0.058 \\
\hline 1977 & 0.003 & 0.025 & 0.145 & 0.059 \\
\hline 1978 & - & 0.036 & 0.277 & 0.105 \\
\hline 1979 & - & 0.013 & 0.120 & 0.054 \\
\hline 1980 & - & 0.032 & 0.175 & 0.099 \\
\hline 1981 & - & 0.036 & 0.220 & 0.025 \\
\hline 1982 & - & 0.102 & 0.330 & 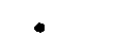 \\
\hline
\end{tabular}


fertility rates, $1940-82$

\begin{tabular}{|c|c|c|c|}
\hline $35-39$ & $40-44$ & $45-49$ & Total \\
\hline - & - & - & - \\
\hline - & - & - & • \\
\hline - & • & - & - \\
\hline - & - & - & - \\
\hline - & - & - & $\bullet$ \\
\hline - & - & - & 3.881 \\
\hline - & - & - & 2.667 \\
\hline • & • & - & 3.693 \\
\hline • & - & - & $3 \cdot 287$ \\
\hline - & - & - & 4.698 \\
\hline - & - & - & 3.691 \\
\hline - & - & - & 4.850 \\
\hline - & $\bullet$ & - & 5.774 \\
\hline - & - & - & 4.440 \\
\hline - & - & - & 5.694 \\
\hline - & - & - & 4.867 \\
\hline - & - & - & 5.113 \\
\hline - & - & - & 5.627 \\
\hline - & - & - & 5.500 \\
\hline - & - & - & 3.824 \\
\hline 0.264 & - & - & 5.443 \\
\hline 0.089 & - & - & 3.256 \\
\hline 0.088 & - & - & 4.545 \\
\hline 0.220 & - & - & $6 \cdot 292$ \\
\hline 0.067 & 0.068 & - & 3.367 \\
\hline 0.093 & 0.041 & 0.031 & $3 \cdot 360$ \\
\hline 0.095 & • & • & 3.371 \\
\hline 0.073 & 0.044 & $\bullet$ & 3.517 \\
\hline 0.089 & 0.026 & 0.028 & 4.559 \\
\hline 0.095 & 0.011 & $\bullet$ & 3.774 \\
\hline 0.048 & 0.021 & - & 3.429 \\
\hline 0.085 & 0.026 & - & 3.055 \\
\hline 0.059 & 0.024 & - & 3.438 \\
\hline 0.065 & - & - & 2.524 \\
\hline 0.009 & - & 0.011 & 1.701 \\
\hline 0.029 & 0.018 & - & 2.035 \\
\hline 0.020 & - & - & 1.432 \\
\hline 0.004 & - & - & 1.177 \\
\hline 0.008 & - & - & 2.132 \\
\hline 0.005 & - & - & 0.958 \\
\hline 0.015 & - & - & 1.607 \\
\hline 0.006 & $\cdot$ & - & 1.434 \\
\hline$\bullet$ & 0.008 & - & 2.200 \\
\hline
\end{tabular}


Basic Table 6.C. Rural Inner Mongolia Province (5): age-specific

\begin{tabular}{|c|c|c|c|c|}
\hline $\begin{array}{l}\text { Birth year } \\
\text { of child }\end{array}$ & $15-19$ & $20-24$ & $25-29$ & $30-34$ \\
\hline 1940 & 0.186 & - & $\bullet$ & - \\
\hline 1941 & 0.197 & - & - & • \\
\hline 1942 & 0.102 & - & - & • \\
\hline 1943 & 0.120 & - & - & - \\
\hline 1944 & 0.083 & - & - & • \\
\hline 1945 & 0.123 & 0.274 & - & - \\
\hline 1946 & 0.127 & 0.280 & - & - \\
\hline 1947 & 0.135 & 0.337 & - & - \\
\hline 1948 & 0.188 & $0.22 \mathrm{e}$ & • & - \\
\hline 1949 & 0.133 & 0.269 & - & - \\
\hline 1950 & 0.124 & 0.296 & 0.307 & - \\
\hline 1951 & 0.140 & 0.336 & 0.233 & - \\
\hline 1952 & 0.165 & 0.316 & 0.305 & • \\
\hline 1953 & 0.145 & 0.355 & 0.315 & - \\
\hline 1954 & 0.198 & 0.274 & 0.290 & • \\
\hline 1955 & 0.155 & 0.371 & 0.327 & 0.324 \\
\hline 1956 & 0.239 & 0.354 & 0.317 & 0.228 \\
\hline 1957 & 0.146 & 0.352 & 0.348 & 0.337 \\
\hline 1958 & 0.159 & 0.311 & 0.302 & 0.256 \\
\hline 1959 & 0.097 & 0.323 & 0.289 & 0.243 \\
\hline 1900 & 0.159 & 0.286 & 0.299 & 0.301 \\
\hline 1901 & 0.060 & 0.232 & 0.224 & 0.214 \\
\hline 1962 & 0.139 & 0.375 & 0.355 & 0.260 \\
\hline 1963 & 0.161 & 0.419 & 0.351 & 0.359 \\
\hline 1964 & 0.122 & 0.380 & 0.364 & 0.314 \\
\hline 1965 & 0.117 & 0.362 & 0.362 & 0.316 \\
\hline 1966 & 0.094 & 0.300 & 0.314 & 0.317 \\
\hline 1967 & 0.055 & 0.360 & 0.249 & 0.253 \\
\hline 1968 & 0.076 & 0.400 & 0.379 & 0.317 \\
\hline 1969 & 0.081 & 0.311 & 0.353 & 0.237 \\
\hline 1970 & 0.074 & 0.340 & 0.428 & 0.305 \\
\hline 1971 & 0.074 & 0.344 & 0.300 & 0.291 \\
\hline 1972 & 0.047 & 0.293 & 0.298 & 0.272 \\
\hline 1973 & 0.047 & $0 \cdot 283$ & 0.236 & 0.203 \\
\hline 1974 & 0.063 & 0.314 & 0.319 & 0.180 \\
\hline 1975 & 0.028 & 0.257 & 0.228 & 0.145 \\
\hline 1976 & 0.030 & 0.227 & 0.239 & 0.100 \\
\hline 1977 & $0.02 B$ & 0.201 & 0.237 & 0.094 \\
\hline 1978 & 0.011 & 0.179 & 0.214 & 0.103 \\
\hline 1979 & 0.006 & 0.217 & 0.230 & 0.088 \\
\hline 1980 & 0.019 & 0.209 & 0.222 & 0.090 \\
\hline 1981 & 0.015 & 0.263 & 0.190 & 0.083 \\
\hline 1982 & 0.029 & 0.238 & 0.251 & 0.155 \\
\hline
\end{tabular}


fertility rates, $1940-82$

\begin{tabular}{|c|c|c|c|}
\hline $35-39$ & $40-44$ & $45-49$ & Total \\
\hline - & - & - & - \\
\hline$\bullet$ & $\bullet$ & - & - \\
\hline - & - & • & - \\
\hline$\bullet$ & - & - & $\bullet$ \\
\hline$\bullet$ & - & - & • \\
\hline - & - & - & 4.801 \\
\hline$\bullet$ & - & - & 4.981 \\
\hline - & - & - & 6.039 \\
\hline - & - & - & 5.236 \\
\hline - & - & - & 4.590 \\
\hline - & - & • & 5.429 \\
\hline - & - & - & 5.274 \\
\hline - & - & - & 6.059 \\
\hline$\bullet$ & - & - & 6.306 \\
\hline - & - & - & 5.919 \\
\hline - & - & - & 6.858 \\
\hline - & - & - & 6.791 \\
\hline - & - & - & 7.290 \\
\hline - & - & - & $6 \cdot 359$ \\
\hline - & - & - & 5.684 \\
\hline 0.214 & - & - & 6.540 \\
\hline 0.088 & - & - & 4.308 \\
\hline 0.289 & - & - & 7.574 \\
\hline 0.245 & $\bullet$ & - & 8.098 \\
\hline 0.248 & 0.150 & 0.050 & 0.144 \\
\hline 0.266 & 0.132 & 0.045 & 7.993 \\
\hline 0.205 & 0.109 & 0.021 & 6.796 \\
\hline 0.172 & 0.107 & 0.019 & 6.071 \\
\hline 0.232 & 0.092 & - & 7.480 \\
\hline 0.220 & 0.155 & 0.012 & 6.842 \\
\hline 0.230 & 0.092 & 0.016 & 7.427 \\
\hline 0.180 & 0.097 & 0.010 & 6.482 \\
\hline 0.159 & 0.084 & 0.005 & 5.791 \\
\hline 0.160 & 0.057 & 0.005 & 4.959 \\
\hline 0.128 & 0.065 & - & 5.345 \\
\hline 0.079 & 0.069 & 0.010 & 4.080 \\
\hline 0.059 & 0.043 & - & 3.490 \\
\hline 0.050 & 0.037 & • & 3.271 \\
\hline 0.044 & 0.015 & 0.004 & 2.854 \\
\hline 0.042 & 0.042 & 0.004 & 3.141 \\
\hline 0.034 & 0.006 & $\bullet$ & 2.900 \\
\hline 0.047 & 0.007 & 0.007 & 3.053 \\
\hline 0.032 & - & - & 3.528 \\
\hline
\end{tabular}


Basic Table 7.A. Total Liaoning Province (6): age-specific fertility rates,

\begin{tabular}{|c|c|c|c|c|}
\hline $\begin{array}{l}\text { Birth year } \\
\text { of child }\end{array}$ & $15-19$ & $20-24$ & $25-29$ & $30-34$ \\
\hline 1940 & 0.087 & - & - & $\bullet$ \\
\hline 1941 & 0.062 & - & - & • \\
\hline 1942 & 0.058 & - & - & - \\
\hline 1943 & 0.082 & - & • & - \\
\hline 1944 & 0.065 & • & - & - \\
\hline 1945 & 0.071 & 0.222 & - & - \\
\hline 1946 & 0.068 & 0.256 & - & - \\
\hline 1947 & 0.086 & 0.276 & • & - \\
\hline 1948 & 0.076 & 0.216 & - & - \\
\hline 1449 & 0.103 & 0.301 & • & - \\
\hline 1950 & 0.114 & 0.239 & 0.257 & - \\
\hline $\begin{array}{l}1951 \\
1952\end{array}$ & $\begin{array}{l}0.087 \\
0.089\end{array}$ & $\begin{array}{l}0.256 \\
0.317\end{array}$ & $\begin{array}{l}0.302 \\
0.329\end{array}$ & : \\
\hline 1953 & 0.093 & 0.312 & 0.298 & - \\
\hline 1954 & 0.096 & 0.306 & 0.335 & • \\
\hline 1950 & 0.099 & 0.336 & 0.373 & 0.312 \\
\hline 1956 & 0.068 & 0.294 & 0.285 & 0.253 \\
\hline 1957 & 0.083 & 0.323 & 0.358 & 0.329 \\
\hline 1958 & 0.069 & 0.284 & 0.297 & 0.258 \\
\hline 1959 & 0.039 & 0.230 & 0.262 & 0.239 \\
\hline 1960 & 0.046 & 0.263 & 0.295 & 0.245 \\
\hline 1961 & 0.019 & 0.115 & 0.147 & 0.130 \\
\hline 1962 & 0.043 & 0.265 & 0.300 & 0.225 \\
\hline 1963 & 0.071 & 0.358 & 0.398 & 0.377 \\
\hline 1964 & 0.061 & 0.305 & 0.313 & 0.249 \\
\hline 1905 & 0.029 & 0.262 & 0.296 & 0.249 \\
\hline 1966 & 0.033 & 0.264 & 0.309 & 0.213 \\
\hline 1967 & 0.021 & 0.208 & 0.273 & 0.151 \\
\hline 1968 & 0.022 & 0.240 & 0.330 & 0.231 \\
\hline 1969 & 0.026 & 0.234 & 0.255 & 0.204 \\
\hline 1970 & $0.019^{\circ}$ & 0.227 & 0.288 & 0.161 \\
\hline 1971 & 0.014 & 0.227 & 0.241 & 0.184 \\
\hline 1972 & 0.013 & 0.160 & 0.246 & 0.168 \\
\hline 1973 & 0.011 & 0.171 & 0.239 & 0.152 \\
\hline 1974 & 0.011 & 0.125 & 0.244 & 0.115 \\
\hline 1975 & 0.007 & 0.110 & 0.185 & 0.077 \\
\hline 1976 & 0.010 & 0.082 & 0.198 & 0.071 \\
\hline 1977 & 0.003 & 0.094 & 0.186 & 0.062 \\
\hline 1978 & 0.004 & 0.120 & 0.226 & 0.074 \\
\hline 1979 & 0.003 & 0.112 & 0.223 & 0.067 \\
\hline 1980 & 0.006 & 0.102 & 0.196 & 0.040 \\
\hline 1981 & 0.003 & 0.143 & 0.191 & 0.035 \\
\hline 1982 & 0.007 & 0.172 & 0.201 & 0.017 \\
\hline
\end{tabular}


1940-82

\begin{tabular}{|c|c|c|c|}
\hline $35-39$ & $40-44$ & $45-49$ & Total \\
\hline - & - & - & - \\
\hline$\bullet$ & $\bullet$ & $\bullet$ & - \\
\hline - & - & - & - \\
\hline - & - & - & - \\
\hline - & - & - & - \\
\hline - & - & - & 4.514 \\
\hline - & - & - & 5.314 \\
\hline - & - & - & 5.220 \\
\hline - & - & - & 4.476 \\
\hline - & • & - & 6.278 \\
\hline • & - & - & 5.470 \\
\hline - & - & - & 5.719 \\
\hline - & - & - & 6.117 \\
\hline • & - & - & 6.263 \\
\hline - & - & - & 6.713 \\
\hline - & - & - & 7.342 \\
\hline - & • & - & 5.874 \\
\hline • & - & - & 7.292 \\
\hline - & - & - & 5.821 \\
\hline - & - & - & $4.97 B$ \\
\hline 0.217 & - & • & 5.781 \\
\hline 0.103 & - & - & 2.809 \\
\hline 0.190 & - & - & 5.547 \\
\hline 0.288 & - & - & 8.199 \\
\hline 0.188 & 0.144 & 0.019 & 6.397 \\
\hline 0.188 & 0.084 & 0.002 & 5.542 \\
\hline 0.166 & 0.094 & 0.011 & 5.451 \\
\hline 0.143 & 0.077 & 0.006 & 4.393 \\
\hline 0.154 & 0.078 & 0.006 & 5.305 \\
\hline 0.130 & 0.053 & 0.011 & 4.563 \\
\hline 0.106 & 0.041 & 0.003 & 4.226 \\
\hline 0.091 & 0.031 & 0.005 & 3.963 \\
\hline 0.068 & 0.024 & 0.001 & 3.397 \\
\hline 0.059 & 0.036 & 0.001 & 3.351 \\
\hline 0.050 & 0.018 & 0.001 & 2.816 \\
\hline 0.035 & 0.010 & 0.004 & 2.134 \\
\hline 0.023 & 0.006 & 0.003 & 1.960 \\
\hline 0.025 & 0.007 & - & 1.882 \\
\hline 0.015 & 0.014 & - & 2.266 \\
\hline 0.020 & 0.002 & 0.001 & 2.151 \\
\hline 0.007 & $\bullet$ & $\bullet$ & 1.756 \\
\hline 0.011 & 0.003 & 0.001 & 1.942 \\
\hline 0.008 & 0.005 & • & 2.052 \\
\hline
\end{tabular}


Basic Table 7.B. Urban Liaoning Province (6): age-specific fertility

\begin{tabular}{|c|c|c|c|c|}
\hline $\begin{array}{l}\text { Birth year } \\
\text { of child }\end{array}$ & $15-19$ & $20-24$ & $25-29$ & $30-34$ \\
\hline 1940 & 0.063 & - & - & - \\
\hline 1941 & 0.050 & - & - & - \\
\hline 1942 & 0.059 & - & - & $\bullet$ \\
\hline 1943 & 0.067 & - & • & - \\
\hline 1944 & 0.056 & - & - & - \\
\hline 1945 & 0.061 & 0.229 & - & - \\
\hline 1946 & 0.066 & 0.241 & - & $\bullet$ \\
\hline 1947 & 0.067 & 0.214 & - & - \\
\hline 1948 & 0.053 & 0.197 & - & - \\
\hline 1949 & 0.108 & 0.221 & - & - \\
\hline 1950 & 0.078 & 0.245 & 0.241 & $\bullet$ \\
\hline 1951 & 0.081 & 0.251 & 0.273 & - \\
\hline 1952 & 0.078 & 0.264 & 0.324 & - \\
\hline 1953 & 0.088 & 0.302 & 0.317 & - \\
\hline 1954 & 0.065 & 0.286 & 0.311 & - \\
\hline 1955 & 0.099 & 0.303 & 0.377 & 0.301 \\
\hline 1956 & 0.058 & 0.280 & 0.266 & 0.259 \\
\hline 1957 & 0.057 & 0.299 & 0.371 & 0.258 \\
\hline 1958 & 0.056 & 0.272 & 0.279 & 0.247 \\
\hline 1959 & 0.023 & 0.201 & 0.242 & 0.218 \\
\hline 1960 & 0.037 & 0.235 & 0.246 & 0.199 \\
\hline 1901 & 0.007 & 0.096 & 0.158 & 0.113 \\
\hline 1962 & 0.016 & 0.218 & 0.262 & 0.197 \\
\hline 1963 & 0.033 & 0.307 & 0.372 & 0.374 \\
\hline 1964 & 0.045 & 0.268 & 0.276 & 0.178 \\
\hline 1965 & 0.008 & 0.189 & 0.257 & 0.184 \\
\hline 1966 & 0.009 & 0.153 & 0.212 & 0.081 \\
\hline 1967 & 0.008 & 0.108 & 0.210 & 0.083 \\
\hline 1968 & 0.003 & 0.162 & 0.319 & 0.181 \\
\hline 1969 & 0.006 & 0.131 & 0.200 & 0.112 \\
\hline 1970 & 0.004 & 0.149 & 0.203 & 0.102 \\
\hline 1971 & 0.004 & 0.142 & 0.193 & 0.127 \\
\hline 1972 & 0.001 & 0.077 & 0.190 & 0.124 \\
\hline 1973 & 0.004 & 0.061 & 0.227 & 0.093 \\
\hline 1974 & - & 0.034 & 0.201 & 0.081 \\
\hline 1975 & - & 0.022 & 0.124 & 0.064 \\
\hline 1976 & - & 0.018 & 0.151 & 0.052 \\
\hline 1977 & 0.001 & 0.030 & 0.199 & 0.044 \\
\hline 1978 & 0.001 & 0.051 & 0.262 & 0.072 \\
\hline 1979 & • & 0.018 & 0.194 & 0.064 \\
\hline 1980 & 0.003 & 0.028 & 0.171 & 0.033 \\
\hline 1981 & 0.005 & 0.076 & 0.202 & 0.021 \\
\hline 1982 & 0.003 & 0.136 & 0.234 & - \\
\hline
\end{tabular}


rates, $1940-82$

\begin{tabular}{|c|c|c|c|}
\hline $35-39$ & $40-44$ & $45-49$ & Total \\
\hline - & - & - & - \\
\hline - & • & - & - \\
\hline - & - & - & - \\
\hline - & - & • & - \\
\hline - & - & - & - \\
\hline - & - & - & 4.383 \\
\hline - & - & - & 5.571 \\
\hline - & - & - & 4.985 \\
\hline - & - & . & 4.129 \\
\hline - & • & • & 6.175 \\
\hline - & - & - & 5.516 \\
\hline - & - & - & 5.674 \\
\hline - & - & - & 5.951 \\
\hline - & - & - & 6.719 \\
\hline - & - & - & 6.431 \\
\hline - & - & • & $7 \cdot 326$ \\
\hline • & - & • & 5.786 \\
\hline - & - & - & 6.680 \\
\hline - & - & - & 5.720 \\
\hline$\bullet$ & - & - & 4.625 \\
\hline 0.174 & - & - & 4.688 \\
\hline 0.086 & - & • & 2.473 \\
\hline 0.152 & - & - & 4.517 \\
\hline 0.244 & - & . & 7.423 \\
\hline 0.096 & 0.097 & - & 4.800 \\
\hline 0.107 & 0.043 & - & 3.938 \\
\hline 0.072 & 0.050 & 0.005 & 2.917 \\
\hline 0.070 & 0.021 & 0.005 & 2.516 \\
\hline 0.071 & 0.033 & - & 3.849 \\
\hline 0.075 & 0.028 & 0.004 & 2.785 \\
\hline 0.041 & 0.009 & - & 2.841 \\
\hline 0.053 & 0.009 & - & 2.038 \\
\hline 0.044 & 0.006 & - & 2.209 \\
\hline 0.022 & 0.012 & - & 2.099 \\
\hline 0.023 & - & - & 1.695 \\
\hline 0.011 & - & - & 1.101 \\
\hline 0.006 & 0.005 & . & 1.162 \\
\hline 0.014 & - & - & 1.445 \\
\hline 0.003 & 0.010 & - & 1.998 \\
\hline 0.013 & - & - & 1.441 \\
\hline 0.007 & - & • & 1.205 \\
\hline 0.003 & - & - & 1.533 \\
\hline 0.006 & - & - & 1.897 \\
\hline
\end{tabular}


Basic Table 7.C. Rural Liaoning Province (6): age-specific fertility rates,

\begin{tabular}{|c|c|c|c|c|}
\hline $\begin{array}{l}\text { Birth year } \\
\text { of child }\end{array}$ & $15-19$ & $20-24$ & $25-29$ & $30-34$ \\
\hline 1940 & 0.104 & - & - & - \\
\hline 1941 & 0.070 & - & - & - \\
\hline 1942 & 0.058 & - & - & - \\
\hline 1943 & 0.093 & - & - & - \\
\hline 1944 & 0.072 & - & - & - \\
\hline 1945 & 0.079 & 0.217 & • & - \\
\hline 1946 & 0.070 & 0.267 & - & - \\
\hline 1947 & 0.101 & 0.321 & $\bullet$ & - \\
\hline 1940 & 0.095 & 0.229 & - & - \\
\hline 1949 & 0.099 & 0.360 & - & - \\
\hline 1950 & 0.146 & 0.235 & 0.269 & - \\
\hline 1951 & 0.092 & 0.261 & 0.323 & - \\
\hline 1952 & 0.100 & 0.359 & 0.334 & - \\
\hline 1953 & 0.097 & 0.318 & 0.284 & - \\
\hline 1954 & 0.122 & 0.323 & 0.353 & - \\
\hline 1955 & 0.099 & 0.365 & 0.369 & 0.321 \\
\hline 1956 & 0.075 & 0.307 & 0.298 & 0.248 \\
\hline 1957 & 0.100 & 0.346 & 0.347 & 0.382 \\
\hline 1958 & 0.076 & 0.295 & 0.312 & 0.265 \\
\hline 1959 & 0.047 & 0.256 & 0.279 & 0.254 \\
\hline 1960 & 0.051 & 0.286 & 0.340 & 0.279 \\
\hline 1901 & 0.025 & 0.130 & 0.138 & 0.143 \\
\hline 1962 & 0.056 & 0.295 & 0.335 & 0.246 \\
\hline 1963 & 0.089 & 0.358 & 0.421 & 0.380 \\
\hline 1964 & 0.069 & 0.326 & 0.345 & 0.312 \\
\hline 1965 & 0.038 & 0.299 & 0.327 & 0.307 \\
\hline 1966 & 0.045 & 0.320 & 0.381 & 0.329 \\
\hline 1967 & 0.027 & 0.257 & 0.313 & 0.215 \\
\hline 1968 & 0.032 & 0.278 & 0.337 & 0.279 \\
\hline 1969 & 0.038 & 0.282 & 0.286 & 0.283 \\
\hline 1970 & 0.028 & 0.265 & 0.300 & 0.209 \\
\hline 1971 & 0.021 & 0.268 & 0.265 & 0.227 \\
\hline 1972 & 0.021 & 0.202 & 0.273 & 0.197 \\
\hline 1973 & 0.016 & 0.232 & 0.244 & 0.186 \\
\hline 1974 & 0.019 & 0.179 & 0.264 & 0.133 \\
\hline 1975 & 0.012 & 0.166 & 0.214 & 0.034 \\
\hline 1976 & 0.017 & 0.125 & 0.220 & 0.080 \\
\hline 1977 & 0.005 & 0.139 & 0.179 & 0.070 \\
\hline 1978 & 0.006 & 0.170 & 0.206 & 0.075 \\
\hline 1979 & 0.006 & 0.184 & 0.241 & 0.069 \\
\hline 1980 & 0.008 & 0.159 & 0.213 & 0.043 \\
\hline 1981 & 0.002 & 0.194 & 0.184 & 0.042 \\
\hline 1982 & 0.010 & 0.197 & 0.178 & 0.027 \\
\hline
\end{tabular}


40-44

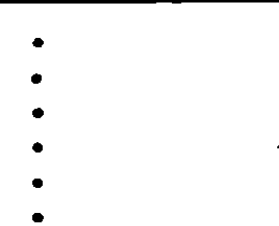

-

:

$\bullet$

•

.

•

•

-

•

$\bullet$

•

$\bullet$

.

0.249

0.116

0.217

0.320

0.256

0.249

0.237

0.199

0.222

0.177

0.164

0.124

0.090

0.094

0.073

0.054

0.036

0.031

0.021

0.025

0.007

0.015

0.009
45-49

Total

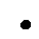

-

-

-

-

4.612

5.120

5. 397

4.739

6.358

5.433

5.755

6.251

5.895

6.940

7.356

5.942

7.759

5.896

5.240

6.575

3.050

6.269

0.732

7.594

6.684

7.266

5.690

6.332

5.765

5.183

4.802

4.115

4.157

3.516

2.778

2.451

2.190

2.485

2.652

2.147

2.225

2.137 
Basic Table 8.A. Total Jilin Province (7): age-specific fertility rates,

\begin{tabular}{|c|c|c|c|c|}
\hline $\begin{array}{l}\text { Birth year } \\
\text { of child }\end{array}$ & 15-19 & $20-24$ & $25-29$ & $30-34$ \\
\hline 1940 & 0.105 & - & $\bullet$ & - \\
\hline 1941 & 0.099 & • & - & - \\
\hline 1942 & 0.117 & - & - & - \\
\hline 1943 & 0.101 & $\bullet$ & - & - \\
\hline 1944 & 0.081 & - & - & - \\
\hline 1945 & 0.077 & 0.249 & - & - \\
\hline 1940 & 0.102 & 0.269 & - & - \\
\hline 1947 & 0.097 & 0.294 & - & - \\
\hline 1948 & 0.107 & 0.296 & - & $\bullet$ \\
\hline 1949 & 0.143 & 0.261 & - & - \\
\hline 1950 & 0.125 & 0.327 & 0.319 & - \\
\hline 1951 & 0.112 & 0.257 & 0.258 & - \\
\hline 1952 & 0.149 & 0.302 & 0.294 & • \\
\hline 1953 & 0.104 & 0.311 & 0.353 & - \\
\hline 1954 & 0.126 & 0.317 & 0.358 & - \\
\hline 1955 & 0.132 & 0.350 & 0.294 & 0.249 \\
\hline 1956 & 0.073 & 0.328 & 0.296 & 0.307 \\
\hline 1957 & 0.088 & 0.289 & 0.342 & 0.252 \\
\hline 1958 & 0.101 & 0.345 & 0.325 & 0.256 \\
\hline 1959 & 0.090 & 0.244 & 0.292 & 0.238 \\
\hline 1960 & 0.058 & 0.302 & 0.364 & 0.244 \\
\hline 1961 & 0.061 & 0.198 & 0.237 & 0.171 \\
\hline 1962 & 0.061 & 0.290 & 0.344 & 0.291 \\
\hline 1963 & 0.083 & 0.315 & 0.364 & 0.377 \\
\hline 1964 & 0.083 & 0.283 & 0.349 & 0.271 \\
\hline 1965 & 0.067 & 0.264 & 0.332 & 0.303 \\
\hline 1966 & 0.053 & 0.261 & 0.280 & 0.274 \\
\hline 1967 & 0.044 & 0.259 & 0.304 & 0.223 \\
\hline 1968 & 0.066 & 0.288 & 0.333 & 0.292 \\
\hline 1969 & 0.061 & 0.226 & 0.341 & 0.246 \\
\hline 1970 & 0.059 & 0.336 & 0.308 & 0.244 \\
\hline 1971 & 0.049 & 0.242 & 0.316 & 0.265 \\
\hline 1972 & 0.033 & 0.248 & 0.337 & 0.201 \\
\hline 1973 & 0.040 & 0.225 & 0.261 & 0.146 \\
\hline 1974 & 0.038 & 0.222 & 0.214 & 0.119 \\
\hline 1975 & 0.016 & 0.143 & 0.204 & 0.087 \\
\hline 1976 & 0.016 & 0.182 & 0.189 & 0.067 \\
\hline 1977 & 0.010 & 0.141 & 0.190 & 0.069 \\
\hline 1978 & 0.020 & 0.170 & 0.222 & 0.061 \\
\hline 1979 & 0.010 & 0.196 & 0.231 & 0.076 \\
\hline 1980 & 0.008 & 0.135 & 0.169 & 0.036 \\
\hline 1981 & 0.005 & 0.187 & 0.182 & 0.027 \\
\hline 1982 & 0.008 & 0.186 & 0.162 & 0.022 \\
\hline
\end{tabular}




\begin{tabular}{|c|c|c|c|}
\hline $35-39$ & $40-44$ & $45-49$ & Total \\
\hline - & - & - & $\bullet$ \\
\hline - & - & $\bullet$ & - \\
\hline - & - & - & $\bullet$ \\
\hline - & • & - & $\bullet$ \\
\hline - & $\bullet$ & $\bullet$ & $\bullet$ \\
\hline$\bullet$ & - & $\bullet$ & 5.694 \\
\hline$\bullet$ & - & - & 6.139 \\
\hline • & - & - & 6.357 \\
\hline - & - & - & 6.033 \\
\hline - & - & - & 6.242 \\
\hline - & - & - & 7.098 \\
\hline - & - & - & 6.300 \\
\hline - & - & - & 6.677 \\
\hline - & - & - & 7.178 \\
\hline - & - & - & 7.409 \\
\hline - & - & - & 6.883 \\
\hline - & - & $\bullet$ & 6.958 \\
\hline - & - & - & 6.979 \\
\hline - & - & - & 6.955 \\
\hline - & • & - & 5.699 \\
\hline 0.221 & - & - & 6.669 \\
\hline 0.167 & - & - & 4.843 \\
\hline 0.191 & - & $\bullet$ & 6.804 \\
\hline 0.306 & - & - & 8.447 \\
\hline 0.179 & 0.118 & 0.031 & 6.570 \\
\hline 0.211 & 0.113 & 0.026 & 6.580 \\
\hline 0.207 & 0.121 & 0.023 & 6.095 \\
\hline 0.191 & 0.110 & 0.020 & 5.754 \\
\hline 0.183 & 0.118 & 0.009 & 6.447 \\
\hline 0.179 & 0.104 & 0.004 & 5.806 \\
\hline 0.181 & 0.110 & 0.004 & 6.208 \\
\hline 0.144 & 0.053 & 0.008 & 5.378 \\
\hline 0.151 & 0.074 & 0.007 & 5.258 \\
\hline 0.106 & 0.049 & 0.007 & $4 \cdot 165$ \\
\hline 0.046 & 0.030 & - & 3.377 \\
\hline 0.032 & 0.024 & - & 2.528 \\
\hline 0.025 & 0.023 & 0.008 & 2.548 \\
\hline 0.042 & 0.009 & 0.006 & $2 \cdot 329$ \\
\hline 0.012 & 0.008 & 0.005 & 2.492 \\
\hline 0.021 & 0.004 & 0.005 & 2.718 \\
\hline 0.010 & 0.008 & - & 1.836 \\
\hline 0.006 & 0.004 & - & 2.054 \\
\hline 0.007 & 0.004 & - & 1.950 \\
\hline
\end{tabular}


Basic Table 8.B. Urban Jilin Province (7): age-specific fertility rates,

\begin{tabular}{|c|c|c|c|c|}
\hline $\begin{array}{l}\text { Birth year } \\
\text { of child }\end{array}$ & $15-19$ & $20-24$ & $25-29$ & $30-34$ \\
\hline 1940 & 0.085 & - & • & - \\
\hline 1941 & 0.096 & - & - & - \\
\hline 1942 & 0.179 & - & - & - \\
\hline 1943 & 0.094 & - & - & - \\
\hline 1944 & 0.077 & - & - & - \\
\hline 1945 & 0.055 & 0.238 & - & - \\
\hline 1946 & 0.053 & 0.305 & - & - \\
\hline 1947 & 0.108 & 0.228 & - & - \\
\hline 1948 & 0.087 & 0.204 & - & - \\
\hline 1949 & 0.133 & 0.248 & - & . \\
\hline 1950 & 0.081 & 0.234 & 0.221 & - \\
\hline 1951 & 0.066 & 0.278 & 0.241 & - \\
\hline $\begin{array}{l}1952 \\
1953\end{array}$ & $\begin{array}{l}0.042 \\
0.046\end{array}$ & $\begin{array}{l}0.297 \\
0.309\end{array}$ & 0.310 & - \\
\hline 1954 & 0.118 & 0.311 & 0.434 & . \\
\hline 1955 & 0.074 & 0.334 & 0.221 & 0.187 \\
\hline 1956 & 0.056 & 0.304 & 0.304 & 0.257 \\
\hline 1957 & 0.043 & 0.257 & 0.351 & 0.244 \\
\hline 1958 & 0.060 & 0.325 & 0.309 & 0.283 \\
\hline 1959 & 0.025 & 0.189 & 0.233 & 0.201 \\
\hline 1960 & 0.014 & 0.228 & 0.324 & 0.248 \\
\hline 1961 & 0.014 & 0.096 & 0.222 & 0.132 \\
\hline 1962 & 0.035 & 0.240 & 0.362 & 0.297 \\
\hline 1963 & 0.026 & 0.223 & 0.319 & 0.408 \\
\hline 1964 & 0.039 & 0.178 & 0.360 & 0.245 \\
\hline 1965 & 0.032 & 0.187 & 0.273 & 0.212 \\
\hline 1960 & 0.010 & 0.167 & 0.186 & 0.164 \\
\hline 1967 & 0.010 & 0.151 & 0.224 & 0.139 \\
\hline 1968 & 0.013 & 0.173 & 0.303 & 0.202 \\
\hline 1969 & 0.021 & 0.167 & 0.301 & 0.165 \\
\hline 1970 & 0.024 & 0.211 & 0.308 & 0.176 \\
\hline 1971 & 0.015 & 0.117 & 0.305 & 0.242 \\
\hline 1972 & 0.008 & 0.134 & $0.28 B$ & 0.182 \\
\hline 1973 & 0.004 & 0.156 & 0.247 & 0.099 \\
\hline 1974 & - & 0.121 & 0.167 & 0.104 \\
\hline 1975 & 0.004 & 0.073 & 0.197 & 0.077 \\
\hline 1976 & 0.004 & 0.080 & 0.227 & 0.076 \\
\hline 1977 & - & 0.075 & 0.258 & 0.080 \\
\hline 1978 & - & 0.073 & 0.264 & 0.069 \\
\hline 1979 & - & 0.042 & 0.193 & 0.058 \\
\hline 1980 & - & 0.041 & 0.132 & 0.039 \\
\hline 1981 & $\bullet$ & 0.086 & 0.232 & 0.020 \\
\hline 1982 & - & 0.153 & 0.210 & 0.013 \\
\hline
\end{tabular}




\begin{tabular}{|c|c|c|c|}
\hline $35-39$ & $40-44$ & $45-49$ & Total \\
\hline - & - & - & - \\
\hline - & - & - & - \\
\hline - & - & - & - \\
\hline - & - & - & - \\
\hline - & - & - & - \\
\hline - & - & - & 9.542 \\
\hline • & - & - & 8.850 \\
\hline - & - & - & 6.678 \\
\hline - & - & - & 7.146 \\
\hline • & • & - & 6.700 \\
\hline - & - & - & 6.936 \\
\hline - & - & - & 6.681 \\
\hline - & - & - & 7.017 \\
\hline - & - & - & 7.127 \\
\hline - & - & - & 9.732 \\
\hline • & - & - & 6.259 \\
\hline - & - & - & 7.158 \\
\hline - & - & - & 6.523 \\
\hline - & - & - & 7.538 \\
\hline - & - & . & 4.572 \\
\hline 0.170 & - & - & 5.736 \\
\hline 0.160 & - & - & 3.975 \\
\hline 0.147 & - & - & 6.341 \\
\hline 0.251 & - & - & 7.469 \\
\hline 0.108 & 0.133 & 0.036 & 5.492 \\
\hline 0.179 & 0.102 & • & 4.932 \\
\hline 0.066 & 0.032 & 0.029 & 3.269 \\
\hline 0.054 & 0.081 & 0.023 & 3.412 \\
\hline 0.074 & 0.063 & • & 4.139 \\
\hline 0.111 & $\bullet$ & - & 3.826 \\
\hline 0.051 & 0.014 & - & 3.918 \\
\hline 0.057 & 0.040 & - & 3.879 \\
\hline 0.063 & 0.013 & • & 3.435 \\
\hline 0.039 & - & - & 2.728 \\
\hline 0.012 & - & - & 2.017 \\
\hline 0.023 & - & - & 1.868 \\
\hline 0.023 & 0.016 & 0.013 & 2.199 \\
\hline 0.027 & 0.007 & $\bullet$ & 2.235 \\
\hline$\bullet$ & $\bullet$ & $\bullet$ & 2.030 \\
\hline 0.005 & - & - & 1.494 \\
\hline$\bullet$ & 0.006 & - & 1.092 \\
\hline - & • & - & 1.090 \\
\hline - & - & - & 1.879 \\
\hline
\end{tabular}


Basic Table 8.C. Rural Jilin Province (7): age-specific fertility rates,

\begin{tabular}{|c|c|c|c|c|}
\hline $\begin{array}{l}\text { Birth year } \\
\text { of child }\end{array}$ & $15-19$ & $20-24$ & $25-29$ & $30-34$ \\
\hline 1940 & 0.111 & $\bullet$ & - & - \\
\hline 1941 & 0.099 & - & - & - \\
\hline 1942 & 0.100 & - & - & - \\
\hline 1943 & 0.103 & - & - & - \\
\hline 1944 & 0.082 & - & - & • \\
\hline 1945 & 0.083 & 0.252 & - & - \\
\hline 1946 & 0.115 & 0.258 & - & - \\
\hline 1947 & 0.094 & 0.312 & - & - \\
\hline 1948 & 0.112 & 0.321 & - & - \\
\hline 1949 & 0.146 & 0.264 & - & - \\
\hline 1950 & 0.139 & 0.352 & 0.348 & - \\
\hline 1951 & 0.129 & 0.252 & 0.263 & • \\
\hline 1952 & 0.196 & 0.304 & 0.290 & - \\
\hline 1953 & 0.132 & 0.312 & 0.364 & - \\
\hline 1954 & 0.131 & 0.319 & 0.338 & - \\
\hline 1955 & 0.167 & 0.355 & 0.314 & 0.267 \\
\hline 1956 & 0.083 & 0.338 & 0.294 & 0.323 \\
\hline 1957 & 0.116 & 0.303 & 0.340 & 0.254 \\
\hline 1958 & 0.128 & 0.354 & 0.329 & 0.248 \\
\hline 1959 & 0.132 & 0.275 & 0.309 & 0.247 \\
\hline 1960 & 0.088 & 0.346 & 0.377 & 0.242 \\
\hline 1961 & 0.093 & 0.259 & 0.243 & 0.182 \\
\hline 1962 & 0.080 & 0.319 & 0.337 & 0.289 \\
\hline 1963 & 0.120 & 0.376 & 0.385 & 0.368 \\
\hline 1964 & 0.112 & 0.351 & 0.343 & 0.279 \\
\hline 1965 & 0.089 & 0.315 & 0.366 & 0.332 \\
\hline 1966 & 0.078 & 0.324 & 0.335 & 0.316 \\
\hline 1907 & 0.061 & 0.337 & 0.352 & 0.260 \\
\hline 1908 & 0.092 & 0.362 & 0.353 & 0.335 \\
\hline 1969 & 0.078 & 0.263 & 0.367 & 0.291 \\
\hline 1970 & 0.073 & 0.411 & 0.309 & 0.283 \\
\hline 1971 & 0.061 & 0.312 & 0.324 & 0.279 \\
\hline 1972 & 0.041 & 0.306 & 0.372 & 0.213 \\
\hline 1973 & 0.052 & 0.257 & 0.270 & 0.177 \\
\hline 1974 & 0.051 & 0.265 & 0.243 & 0.129 \\
\hline 1975 & 0.020 & 0.170 & 0.208 & 0.094 \\
\hline 1976 & 0.020 & 0.218 & 0.167 & 0.061 \\
\hline 1977 & 0.013 & 0.163 & 0.156 & 0.060 \\
\hline 1978 & 0.026 & 0.201 & 0.203 & 0.056 \\
\hline 1979 & 0.014 & 0.245 & 0.246 & 0.087 \\
\hline 1980 & 0.011 & 0.164 & 0.183 & 0.035 \\
\hline 1981 & 0.007 & 0.218 & 0.164 & 0.030 \\
\hline 1982 & 0.011 & 0.197 & 0.147 & $0.020^{\circ}$ \\
\hline
\end{tabular}




\begin{tabular}{|c|c|c|c|}
\hline $35-39$ & $40-44$ & $45-49$ & Total \\
\hline - & - & $\bullet$ & - \\
\hline - & - & - & - \\
\hline$\bullet$ & $\bullet$ & - & - \\
\hline - & - & $\bullet$ & - \\
\hline - & $\bullet$ & - & $\bullet$ \\
\hline - & - & • & 4.914 \\
\hline - & $\bullet$ & - & 5.398 \\
\hline - & - & - & 6.270 \\
\hline - & - & • & 5.727 \\
\hline - & - & - & 6.115 \\
\hline - & - & - & 7.145 \\
\hline - & - & • & 6.183 \\
\hline - & - & - & 6.566 \\
\hline • & • & • & $7 \cdot 195$ \\
\hline$\bullet$ & - & - & 6.593 \\
\hline - & - & - & 7.109 \\
\hline - & - & - & 6.883 \\
\hline • & - & - & 7.156 \\
\hline - & - & - & 6.718 \\
\hline - & - & - & 6.167 \\
\hline 0.237 & - & - & 7.066 \\
\hline 0.169 & - & - & 5.219 \\
\hline 0.204 & - & - & 7.014 \\
\hline 0.321 & - & - & 8.892 \\
\hline 0.198 & 0.114 & 0.029 & 7.130 \\
\hline 0.220 & 0.116 & 0.033 & 7.354 \\
\hline 0.245 & 0.149 & 0.021 & 7.338 \\
\hline 0.228 & 0.118 & 0.019 & 6.874 \\
\hline 0.214 & 0.133 & 0.012 & 7.502 \\
\hline 0.199 & 0.132 & 0.005 & 6.677 \\
\hline 0.222 & 0.136 & 0.005 & 7.197 \\
\hline 0.177 & 0.056 & 0.010 & 6.093 \\
\hline 0.190 & 0.090 & 0.009 & $6 \cdot 109$ \\
\hline 0.138 & 0.063 & 0.009 & 4.824 \\
\hline 0.065 & 0.047 & - & 3.997 \\
\hline 0.037 & 0.032 & - & 2.802 \\
\hline 0.027 & 0.025 & 0.007 & 2.619 \\
\hline 0.052 & 0.009 & 0.007 & 2.299 \\
\hline 0.020 & 0.013 & 0.007 & 2.621 \\
\hline 0.032 & 0.007 & 0.007 & $3.1 \mathrm{es}$ \\
\hline 0.016 & 0.010 & • & 2.090 \\
\hline 0.010 & 0.007 & - & 2.179 \\
\hline 0.013 & 0.000 & - & 2.000 \\
\hline
\end{tabular}


Basic Table 9.A. Total Heilongjiang Province (8): age-specific fertility

\begin{tabular}{|c|c|c|c|c|}
\hline $\begin{array}{l}\text { Birth year } \\
\text { of child }\end{array}$ & $15-19$ & $20-24$ & $25-29$ & $30-34$ \\
\hline 1940 & 0.075 & - & - & - \\
\hline 1941 & 0.069 & - & - & - \\
\hline 1942 & 0.077 & $\bullet$ & - & $\bullet$ \\
\hline 1943 & 0.093 & - & - & - \\
\hline 1944 & 0.068 & - & - & - \\
\hline 1945 & 0.099 & 0.285 & - & - \\
\hline 1946 & 0.104 & 0.219 & - & - \\
\hline 1947 & 0.092 & 0.339 & - & - \\
\hline 1948 & 0.108 & 0.277 & $\bullet$ & $\bullet$ \\
\hline 1949 & 0.134 & 0.311 & - & - \\
\hline 1950 & 0.154 & 0.285 & 0.306 & - \\
\hline 1951 & 0.100 & 0.286 & 0.265 & • \\
\hline 1952 & 0.109 & 0.321 & 0.339 & - \\
\hline 1953 & 0.110 & 0.280 & 0.243 & - \\
\hline 1954 & 0.102 & 0.288 & 0.302 & - \\
\hline 1955 & 0.088 & 0.331 & 0.283 & 0.311 \\
\hline 1956 & 0.096 & 0.297 & 0.324 & 0.243 \\
\hline 1957 & 0.102 & 0.345 & 0.297 & 0.318 \\
\hline 1958 & 0.100 & 0.327 & 0.304 & 0.255 \\
\hline 1959 & 0.107 & 0.275 & 0.255 & 0.214 \\
\hline 1960 & 0.091 & 0.289 & 0.326 & 0.251 \\
\hline 1961 & 0.063 & 0.227 & 0.236 & 0.198 \\
\hline 1962 & 0.071 & 0.283 & 0.327 & 0.272 \\
\hline 1963 & 0.086 & 0.344 & 0.358 & 0.286 \\
\hline 1964 & 0.077 & 0.312 & 0.286 & 0.239 \\
\hline 1965 & 0.056 & 0.302 & 0.286 & 0.241 \\
\hline 1966 & 0.073 & 0.239 & 0.308 & 0.267 \\
\hline 1967 & 0.064 & 0.275 & 0.286 & 0.220 \\
\hline 1968 & 0.073 & 0.315 & 0.364 & 0.242 \\
\hline 1969 & 0.058 & 0.282 & 0.299 & 0.211 \\
\hline 1970 & 0.070 & 0.312 & 0.286 & 0.201 \\
\hline 2971 & 0.053 & 0.274 & 0.299 & 0.184 \\
\hline 1972 & 0.042 & 0.289 & 0.284 & 0.231 \\
\hline 1973 & 0.039 & 0.258 & 0.317 & 0.204 \\
\hline 1974 & 0.030 & 0.264 & 0.258 & 0.160 \\
\hline 1975 & 0.031 & 0.234 & 0.212 & 0.101 \\
\hline 1976 & 0.026 & 0.186 & 0.193 & 0.075 \\
\hline 1977 & 0.016 & 0.178 & 0.177 & 0.078 \\
\hline 1978 & 0.015 & 0.185 & 0.187 & 0.085 \\
\hline 1979 & 0.014 & 0.193 & 0.232 & 0.093 \\
\hline 1980 & 0.016 & 0.196 & 0.174 & 0.041 \\
\hline 1981 & 0.021 & 0.205 & 0.167 & 0.043 \\
\hline 1982 & 0.026 & 0.238 & 0.203 & 0.052 \\
\hline
\end{tabular}


rates, $1940-82$

\begin{tabular}{|c|c|c|c|}
\hline $35-39$ & $40-44$ & $45-49$ & Total \\
\hline • & - & - & - \\
\hline - & - & - & - \\
\hline - & - & - & - \\
\hline - & - & - & - \\
\hline - & - & - & - \\
\hline - & - & - & 4.923 \\
\hline - & - & - & 4.954 \\
\hline - & - & - & $5 \cdot 735$ \\
\hline - & - & - & 5.509 \\
\hline - & - & - & 0.370 \\
\hline - & - & - & 6.670 \\
\hline - & - & - & 5.960 \\
\hline - & - & - & 6.820 \\
\hline - & - & - & 5.756 \\
\hline - & - & - & 6.784 \\
\hline - & - & - & 6.451 \\
\hline - & - & - & 6.727 \\
\hline$\bullet$ & - & - & 7.126 \\
\hline$\bullet$ & - & - & 6.427 \\
\hline - & - & - & 5.824 \\
\hline 0.283 & - & - & 6.918 \\
\hline 0.133 & - & - & 4.789 \\
\hline 0.234 & - & - & 6.734 \\
\hline 0.261 & - & - & 7.404 \\
\hline 0.203 & 0.130 & 0.018 & 6.321 \\
\hline 0.229 & 0.090 & 0.015 & 6.096 \\
\hline 0.190 & 0.102 & 0.021 & 5.999 \\
\hline 0.153 & 0.098 & 0.013 & 5.539 \\
\hline 0.202 & 0.098 & 0.018 & 6.562 \\
\hline 0.137 & 0.086 & 0.011 & 5.414 \\
\hline 0.166 & 0.076 & 0.013 & 5.619 \\
\hline 0.133 & 0.067 & 0.012 & 5.145 \\
\hline 0.154 & 0.071 & 0.005 & 5.377 \\
\hline 0.111 & 0.059 & 0.007 & 4.971 \\
\hline 0.068 & 0.049 & 0.005 & 4.165 \\
\hline 0.058 & 0.023 & 0.004 & 3.317 \\
\hline 0.032 & 0.0 .25 & 0.002 & 2.699 \\
\hline 0.038 & 0.011 & 0.002 & 2.499 \\
\hline 0.029 & 0.016 & 0.002 & 2.591 \\
\hline 0.016 & 0.015 & 0.007 & 2.849 \\
\hline 0.012 & 0.004 & 0.002 & 2.220 \\
\hline 0.012 & 0.007 & - & 2.273 \\
\hline 0.005 & 0.009 & - & 2.669 \\
\hline
\end{tabular}


Basic Table 9.B. Urban Heilongjiang Province (8): age-specific fertility

\begin{tabular}{|c|c|c|c|c|}
\hline $\begin{array}{l}\text { Birth year } \\
\text { of child }\end{array}$ & $15-19$ & $20-24$ & $25-29$ & $30-34$ \\
\hline 1940 & 0.053 & - & $\bullet$ & - \\
\hline 1941 & 0.032 & - & - & - \\
\hline 1942 & 0.038 & - & - & - \\
\hline 1943 & 0.080 & - & - & - \\
\hline 1944 & 0.046 & - & $\bullet$ & $\bullet$ \\
\hline 1945 & 0.086 & 0.258 & - & - \\
\hline 1946 & 0.063 & 0.219 & - & - \\
\hline 1947 & 0.080 & 0.299 & . & - \\
\hline 1948 & 0.110 & 0.278 & • & - \\
\hline 1949 & 0.081 & 0.283 & - & - \\
\hline 1950 & 0.102 & 0.271 & 0.365 & - \\
\hline 1951 & 0.061 & 0.270 & 0.235 & - \\
\hline 1952 & 0.058 & 0.273 & 0.291 & - \\
\hline 1953 & 0.106 & 0.244 & 0.278 & - \\
\hline 1954 & 0.069 & 0.238 & 0.310 & - \\
\hline 1955 & 0.053 & 0.292 & 0.302 & 0.347 \\
\hline 1956 & 0.043 & 0.280 & 0.304 & 0.325 \\
\hline 1957 & 0.071 & 0.295 & 0.278 & 0.322 \\
\hline 1950 & 0.057 & 0.328 & 0.311 & 0.285 \\
\hline $\begin{array}{l}1959 \\
1960\end{array}$ & $\begin{array}{l}0.054 \\
0.038\end{array}$ & $\begin{array}{l}0.230 \\
0.280\end{array}$ & $\begin{array}{l}0.242 \\
0.271\end{array}$ & $\begin{array}{l}0.178 \\
0.246\end{array}$ \\
\hline 1961 & 0.052 & 0.172 & 0.253 & 0.161 \\
\hline 1962 & 0.038 & $0.220^{\circ}$ & 0.313 & 0.283 \\
\hline 1963 & 0.071 & 0.330 & 0.396 & 0.287 \\
\hline 1964 & 0.026 & 0.258 & 0.278 & 0.211 \\
\hline 1965 & 0.023 & 0.260 & 0.249 & 0.191 \\
\hline 1966 & 0.027 & 0.149 & 0.251 & 0.149 \\
\hline 1907 & 0.022 & 0.236 & 0.212 & 0.158 \\
\hline 1968 & 0.015 & 0.190 & 0.327 & 0.212 \\
\hline 1969 & 0.014 & 0.158 & 0.251 & 0.158 \\
\hline 1970 & 0.018 & 0.248 & 0.279 & 0.161 \\
\hline 1971 & 0.014 & 0.155 & 0.253 & 0.133 \\
\hline 1972 & 0.004 & 0.204 & 0.215 & 0.152 \\
\hline 1973 & 0.010 & 0.187 & 0.309 & 0.167 \\
\hline 1974 & 0.006 & 0.167 & 0.209 & 0.136 \\
\hline 1975 & 0.004 & 0.104 & 0.184 & 0.092 \\
\hline 1976 & 0.004 & 0.102 & 0.176 & 0.052 \\
\hline 1977 & 0.007 & 0.097 & 0.194 & 0.072 \\
\hline 1978 & 0.002 & 0.073 & 0.216 & 0.071 \\
\hline 1979 & 0.002 & 0.069 & 0.220 & 0.077 \\
\hline 1980 & 0.003 & 0.095 & 0.172 & 0.038 \\
\hline 1981 & 0.007 & 0.116 & 0.159 & 0.027 \\
\hline 1982 & 0.012 & 0.169 & 0.189 & 0.055 \\
\hline
\end{tabular}


rates, $1940-82$

\begin{tabular}{|c|c|c|c|c|}
\hline $35-39$ & $40-44$ & & $45-49$ & Total \\
\hline$\bullet$ & $\bullet$ & & - & - \\
\hline • & - & & • & - \\
\hline - & - & & $\bullet$ & $\bullet$ \\
\hline$\bullet$ & • & & - & - \\
\hline - & $\bullet$ & & $\bullet$ & - \\
\hline$\bullet$ & $\bullet$ & & - & $5 \cdot 368$ \\
\hline • & - & & - & 4.809 \\
\hline$\bullet$ & • & & • & 4.442 \\
\hline - & $\bullet$ & & $\bullet$ & 6.880 \\
\hline - & - & & • & 5.140 \\
\hline$\bullet$ & - & & $\bullet$ & 7.684 \\
\hline - & - & & - & 5.774 \\
\hline - & $\bullet$ & & - & 6.374 \\
\hline • & $\bullet$ & $\therefore$ & • & 6.257 \\
\hline - & - & & - & $7 \cdot 163$ \\
\hline - & - & & - & 6.469 \\
\hline - & - & & - & 7.215 \\
\hline - & - & & • & 6.875 \\
\hline - & - & & - & 6.906 \\
\hline - & . & & - & 4.965 \\
\hline 0.222 & . & & - & 6.307 \\
\hline 0.138 & - & & - & 4.495 \\
\hline 0.222 & • & & - & 6.285 \\
\hline 0.241 & • & & - & 7.280 \\
\hline 0.132 & 0.080 & & 0.032 & 5.081 \\
\hline 0.166 & 0.089 & & 0.026 & 5.014 \\
\hline 0.115 & 0.049 & & 0.013 & 3.766 \\
\hline 0.102 & 0.054 & & 0.036 & 4.093 \\
\hline 0.115 & 0.058 & & • & 4.583 \\
\hline 0.076 & 0.026 & - & 0.010 & 3.463 \\
\hline 0.089 & 0.037 & & - & 4.161 \\
\hline 0.084 & 0.034 & & - & 3.371 \\
\hline 0.086 & 0.037 & & - & 3.492 \\
\hline $0.0<8$ & $0.010^{\circ}$ & & - & 3.755 \\
\hline 0.041 & 0.027 & & - & 2.929 \\
\hline 0.025 & 0.004 & & - & 2.064 \\
\hline 0.032 & 0.015 & & - & 1.907 \\
\hline 0.025 & 0.004 & & $\bullet$ & 1.993 \\
\hline 0.007 & 0.007 & & • & 1.873 \\
\hline 0.014 & $\bullet$ & & - & 1.905 \\
\hline 0.004 & • & & - & 1.553 \\
\hline 0.008 & 0.004 & & - & 1.599 \\
\hline - & - & & - & 2.125 \\
\hline
\end{tabular}


Basic Table 9.C. Rural Heilongjiang Province (8): age-specific fertility

\begin{tabular}{|c|c|c|c|c|}
\hline $\begin{array}{l}\text { Birth year } \\
\text { of child }\end{array}$ & $15-19$ & $20-24$ & $25-29$ & $30-34$ \\
\hline 1940 & 0.083 & - & • & • \\
\hline 1941 & 0.084 & - & - & - \\
\hline 1942 & 0.094 & - & - & - \\
\hline 1943 & 0.099 & - & - & - \\
\hline 1944 & 0.079 & $\bullet$ & - & • \\
\hline 1945 & 0.107 & 0.296 & - & . \\
\hline 1946 & 0.127 & 0.219 & - & - \\
\hline 1947 & 0.100 & 0.357 & - & - \\
\hline 1948 & 0.106 & 0.277 & $\bullet$ & • \\
\hline 1949 & 0.168 & 0.325 & : & • \\
\hline 1950 & 0.190 & 0.293 & 0.282 & - \\
\hline 1951 & 0.130 & 0.295 & 0.277 & - \\
\hline 1952 & 0.151 & 0.349 & 0.361 & - \\
\hline 1953 & 0.113 & 0.303 & 0.228 & - \\
\hline $\begin{array}{l}1954 \\
1955\end{array}$ & $\begin{array}{l}0.128 \\
0.115\end{array}$ & $\begin{array}{l}0.321 \\
0.359\end{array}$ & $\begin{array}{l}0.297 \\
0.273\end{array}$ & 0.296 \\
\hline 1956 & 0.133 & 0.311 & 0.336 & 0.209 \\
\hline 1957 & 0.125 & 0.385 & 0.308 & 0.317 \\
\hline 1958 & 0.129 & 0.326 & 0.300 & 0.241 \\
\hline 1959 & 0.143 & 0.312 & 0.263 & 0.232 \\
\hline 1960 & 0.123 & 0.296 & 0.365 & 0.253 \\
\hline 1961 & 0.070 & 0.265 & 0.223 & 0.219 \\
\hline 1962 & 0.088 & 0.323 & 0.339 & 0.265 \\
\hline 1963 & 0.092 & 0.353 & 0.326 & 0.285 \\
\hline 1964 & 0.096 & 0.347 & 0.293 & 0.257 \\
\hline 1905 & 0.069 & 0.328 & 0.314 & 0.276 \\
\hline 1966 & 0.089 & 0.290 & 0.348 & 0.359 \\
\hline 1967 & 0.078 & 0.293 & 0.338 & 0.269 \\
\hline 1908 & 0.093 & 0.367 & 0.390 & 0.268 \\
\hline 1969 & 0.073 & 0.329 & 0.331 & 0.254 \\
\hline 1970 & 0.089 & $0 \cdot 336$ & 0.291 & 0.231 \\
\hline 1971 & 0.068 & 0.318 & 0.325 & 0.220 \\
\hline 1972 & 0.057 & 0.319 & 0.318 & 0.288 \\
\hline 1973 & 0.051 & 0.283 & 0.320 & 0.229 \\
\hline 1974 & 0.042 & 0.298 & 0.276 & 0.176 \\
\hline 1975 & 0.044 & 0.280 & 0.223 & 0.107 \\
\hline 1976 & 0.037 & 0.218 & 0.200 & 0.088 \\
\hline 1977 & 0.021 & 0.210 & 0.170 & 0.081 \\
\hline 1978 & 0.022 & 0.234 & 0.177 & 0.090 \\
\hline 1979 & 0.021 & 0.251 & 0.237 & 0.099 \\
\hline 1980 & 0.023 & 0.247 & 0.175 & 0.042 \\
\hline 1981 & 0.028 & 0.250 & 0.170 & 0.048 \\
\hline 1982 & 0.033 & 0.277 & 0.209 & 0.051 \\
\hline
\end{tabular}


rates, $1940-82$

\begin{tabular}{|c|c|c|c|}
\hline $35-39$ & $40-44$ & $45-49$ & Total \\
\hline - & - & - & $\bullet$ \\
\hline - & - & - & - \\
\hline - & - & - & - \\
\hline - & - & • & - \\
\hline$\bullet$ & $\bullet$ & - & - \\
\hline - & - & - & 4.720 \\
\hline$\bullet$ & $\bullet$ & - & 5.020 \\
\hline$\bullet$ & - & - & 6.347 \\
\hline - & - & - & 4.838 \\
\hline - & - & - & 6.995 \\
\hline - & - & - & $6 \cdot 133$ \\
\hline • & - & - & 6.063 \\
\hline - & - & - & 7.071 \\
\hline - & $\bullet$ & - & 5.465 \\
\hline - & - & - & 6.562 \\
\hline - & $\bullet$ & $\bullet$ & 6.441 \\
\hline - & - & - & 6.442 \\
\hline - & - & - & 7.277 \\
\hline - & - & - & 6.138 \\
\hline - & - & - & 6.341 \\
\hline 0.307 & - & - & 7.279 \\
\hline 0.132 & - & - & 4.959 \\
\hline 0.239 & - & • & 6.991 \\
\hline 0.271 & - & • & 7.473 \\
\hline 0.239 & 0.149 & 0.013 & 6.971 \\
\hline 0.263 & 0.090 & 0.010 & 6.754 \\
\hline 0.231 & 0.125 & 0.024 & 7.328 \\
\hline 0.184 & 0.118 & 0.004 & 6.424 \\
\hline 0.257 & 0.115 & 0.025 & 7.577 \\
\hline 0.176 & 0.116 & 0.011 & 6.451 \\
\hline 0.220 & 0.097 & 0.018 & 6.403 \\
\hline 0.181 & 0.086 & 0.017 & 6.076 \\
\hline 0.208 & 0.090 & 0.007 & 6.434 \\
\hline 0.147 & 0.091 & 0.010 & 5.654 \\
\hline 0.089 & 0.064 & 0.007 & 4.756 \\
\hline 0.084 & 0.036 & 0.007 & 3.900 \\
\hline 0.033 & 0.033 & 0.003 & 3.058 \\
\hline 0.048 & 0.017 & 0.003 & 2.756 \\
\hline 0.044 & 0.023 & 0.003 & 2.966 \\
\hline 0.017 & 0.023 & 0.012 & 3.316 \\
\hline 0.016 & 0.008 & 0.003 & $2 \cdot 568$ \\
\hline 0.013 & 0.010 & • & 2.604 \\
\hline 0.008 & 0.015 & - & 2.959 \\
\hline
\end{tabular}


Basic Table 10.A. Total Shanghai Province (9): age-specific fertility

\begin{tabular}{|c|c|c|c|c|}
\hline $\begin{array}{l}\text { Birth year } \\
\text { of child }\end{array}$ & $15-19$ & $20-24$ & $25-29$ & $30-34$ \\
\hline 1940 & 0.059 & - & • & - \\
\hline 1941 & 0.055 & • & • & - \\
\hline 1942 & 0.062 & - & - & - \\
\hline 1943 & 0.049 & - & - & - \\
\hline 1944 & 0.066 & - & - & - \\
\hline 1945 & 0.050 & 0.200 & - & - \\
\hline 1946 & 0.034 & 0.267 & - & - \\
\hline 1947 & 0.078 & 0.251 & - & - \\
\hline 1948 & 0.057 & 0.259 & $\bullet$ & $\bullet$ \\
\hline 1949 & 0.059 & 0.240 & - & - \\
\hline 1950 & 0.078 & 0.249 & 0.296 & - \\
\hline 1951 & 0.054 & 0.194 & 0.241 & - \\
\hline 1952 & 0.067 & 0.243 & 0.319 & - \\
\hline $\begin{array}{l}1953 \\
1954\end{array}$ & $\begin{array}{l}0.051 \\
0.074\end{array}$ & $\begin{array}{l}0.235 \\
0.275\end{array}$ & $\begin{array}{l}0.283 \\
0.303\end{array}$ & - \\
\hline 1955 & 0.042 & 0.230 & 0.314 & 0.240 \\
\hline 1956 & 0.073 & 0.210 & 0.247 & 0.177 \\
\hline $\begin{array}{l}1957 \\
1958\end{array}$ & $\begin{array}{l}0.051 \\
0.033\end{array}$ & $\begin{array}{l}0.269 \\
0.206\end{array}$ & $\begin{array}{l}0.333 \\
0.258\end{array}$ & $\begin{array}{l}0.273 \\
0.189\end{array}$ \\
\hline 1959 & 0.021 & 0.205 & 0.192 & 0.120 \\
\hline 1960 & 0.033 & 0.129 & 0.209 & 0.100 \\
\hline 1901 & 0.018 & 0.177 & 0.185 & 0.132 \\
\hline 1962 & 0.016 & 0.199 & 0.210 & 0.159 \\
\hline 1963 & 0.020 & 0.197 & 0.252 & 0.178 \\
\hline 1964 & 0.020 & 0.157 & 0.186 & $0: 088$ \\
\hline 1965 & 0.012 & 0.142 & 0.178 & 0.099 \\
\hline 1966 & 0.004 & 0.122 & 0.223 & 0.126 \\
\hline 1967 & 0.002 & 0.096 & 0.164 & 0.062 \\
\hline 1968 & 0.005 & 0.162 & 0.223 & 0.122 \\
\hline 1969 & 0.003 & 0.120 & 0.225 & 0.087 \\
\hline 1970 & 0.003 & 0.130 & 0.218 & 0.080 \\
\hline 1971 & 0.003 & 0.130 & 0.169 & 0.005 \\
\hline 1972 & 0.001 & 0.061 & 0.151 & 0.051 \\
\hline 1973 & 0.001 & 0.078 & 0.152 & 0.059 \\
\hline 1974 & - & 0.051 & 0.133 & 0.068 \\
\hline 1975 & - & 0.050 & 0.116 & 0.046 \\
\hline 1976 & 0.003 & 0.046 & 0.141 & 0.053 \\
\hline 1977 & 0.002 & 0.046 & 0.129 & 0.035 \\
\hline 1978 & - & 0.045 & 0.135 & 0.052 \\
\hline 1979 & - & 0.050 & 0.122 & 0.063 \\
\hline 1980 & - & 0.033 & 0.113 & 0.018 \\
\hline 1981 & & 0.065 & 0.140 & 0.032 \\
\hline 1982 & 0.005 & 0.071 & 0.142 & 0.039 \\
\hline
\end{tabular}


rates, $1940-82$

\begin{tabular}{|c|c|c|c|}
\hline $35-39$ & $40-44$ & $45-49$ & Total \\
\hline • & - & - & - \\
\hline - & • & - & $\bullet$ \\
\hline$\bullet$ & $\bullet$ & - & - \\
\hline - & - & - & - \\
\hline$\bullet$ & - & - & - \\
\hline - & $\bullet$ & $\bullet$ & 4.637 \\
\hline - & - & - & 5.108 \\
\hline - & $\bullet$ & - & 5.748 \\
\hline - & $\bullet$ & - & 5.528 \\
\hline - & - & • & 5.868 \\
\hline$\bullet$ & - & - & 5.604 \\
\hline - & - & • & 5.098 \\
\hline - & - & - & $5.84 A$ \\
\hline - & - & - & 5.391 \\
\hline - & - & - & $6 \cdot 297$ \\
\hline - & - & - & 5.590 \\
\hline - & - & - & 4.466 \\
\hline - & • & - & 6.317 \\
\hline - & - & - & 4.426 \\
\hline$\bullet$ & - & - & $3 \cdot 380$ \\
\hline 0.079 & - & - & 2.901 \\
\hline 0.064 & - & - & 2.904 \\
\hline 0.065 & - & - & 3.452 \\
\hline 0.131 & • & - & 4.173 \\
\hline 0.039 & 0.020 & - & 2.555 \\
\hline 0.056 & 0.023 & - & 2.552 \\
\hline 0.048 & 0.023 & - & 2.724 \\
\hline 0.038 & 0.006 & - & 1.837 \\
\hline 0.045 & 0.015 & - & 2.860 \\
\hline 0.035 & 0.003 & - & 2.359 \\
\hline 0.021 & 0.003 & - & 2.280 \\
\hline 0.013 & 0.006 & : & 1.930 \\
\hline 0.019 & 0.006 & - & 1.446 \\
\hline 0.014 & 0.006 & - & 1.556 \\
\hline 0.003 & - & - & 1.275 \\
\hline 0.003 & 0.008 & - & 1.122 \\
\hline 0.008 & - & $\bullet$ & 1.257 \\
\hline 0.023 & - & - & 1.174 \\
\hline 0.007 & - & 0.003 & 1.211 \\
\hline 0.007 & 0.003 & • & 1.227 \\
\hline 0.007 & 0.003 & - & 0.867 \\
\hline 0.003 & $\bullet$ & $\bullet$ & 1.201 \\
\hline 0.010 & - & - & $1 \cdot 336$ \\
\hline
\end{tabular}


Basic Table 10.B. Urban Shanghai Province (9): age-specific fertility

\begin{tabular}{|c|c|c|c|c|}
\hline $\begin{array}{l}\text { Birth year } \\
\text { of child }\end{array}$ & $15-19$ & $20-24$ & $25-29$ & $30-34$ \\
\hline 1940 & 0.070 & - & - & - \\
\hline 1941 & 0.066 & - & - & - \\
\hline 1942 & 0.068 & - & - & • \\
\hline 1943 & 0.045 & $\bullet$ & - & - \\
\hline 1944 & 0.057 & - & - & - \\
\hline 1945 & 0.034 & 0.207 & - & - \\
\hline 1946 & 0.031 & 0.232 & - & - \\
\hline 1947 & 0.063 & 0.241 & • & - \\
\hline 1948 & 0.055 & 0.268 & - & - \\
\hline 1949 & 0.064 & 0.214 & - & - \\
\hline 1950 & 0.078 & 0.248 & 0.272 & - \\
\hline 2951 & 0.048 & 0.230 & 0.213 & - \\
\hline 1952 & 0.052 & 0.209 & 0.323 & - \\
\hline 1953 & 0.055 & 0.223 & 0.304 & - \\
\hline 1954 & 0.067 & 0.265 & 0.284 & $\bullet$ \\
\hline 1955 & 0.040 & 0.241 & 0.315 & 0.235 \\
\hline 1956 & 0.075 & 0.215 & 0.234 & 0.189 \\
\hline 1957 & 0.031 & 0.228 & 0.311 & 0.237 \\
\hline 1958 & 0.029 & 0.199 & 0.235 & 0.170 \\
\hline 1959 & 0.022 & 0.191 & 0.187 & 0.087 \\
\hline 1960 & 0.021 & 0.131 & 0.215 & 0.113 \\
\hline 1961 & 0.024 & 0.145 & 0.197 & 0.138 \\
\hline 1962 & 0.004 & 0.189 & 0.179 & 0.110 \\
\hline 1963 & 0.008 & 0.117 & 0.202 & 0.121 \\
\hline 1964 & 0.004 & 0.095 & 0.142 & 0.067 \\
\hline 1965 & 0.006 & 0.064 & 0.136 & 0.048 \\
\hline 1966 & - & 0.053 & 0.127 & 0.070 \\
\hline 1967 & • & 0.069 & 0.147 & 0.049 \\
\hline 1963 & - & 0.117 & 0.211 & 0.078 \\
\hline 1969 & 0.002 & 0.066 & 0.212 & 0.062 \\
\hline 1970 & 0.002 & 0.068 & 0.196 & 0.066 \\
\hline 1971 & 0.002 & 0.076 & 0.149 & 0.064 \\
\hline 1972 & $\bullet$ & 0.034 & 0.100 & 0.049 \\
\hline 1973 & 0.002 & 0.031 & 0.121 & 0.076 \\
\hline 1974 & - & 0.019 & 0.117 & 0.084 \\
\hline 1975 & - & 0.022 & 0.094 & 0.048 \\
\hline 1976 & . & 0.008 & 0.103 & 0.058 \\
\hline 1977 & $\bullet$ & 0.012 & 0.092 & 0.039 \\
\hline 1978 & • & 0.004 & 0.114 & 0.061 \\
\hline 1979 & - & 0.002 & 0.065 & 0.073 \\
\hline 1980 & - & 0.007 & 0.080 & 0.029 \\
\hline 1981 & - & 0.010 & 0.118 & 0.049 \\
\hline 1982 & $\bullet$ & 0.022 & 0.148 & 0.055 \\
\hline
\end{tabular}


rates, $1940-82$

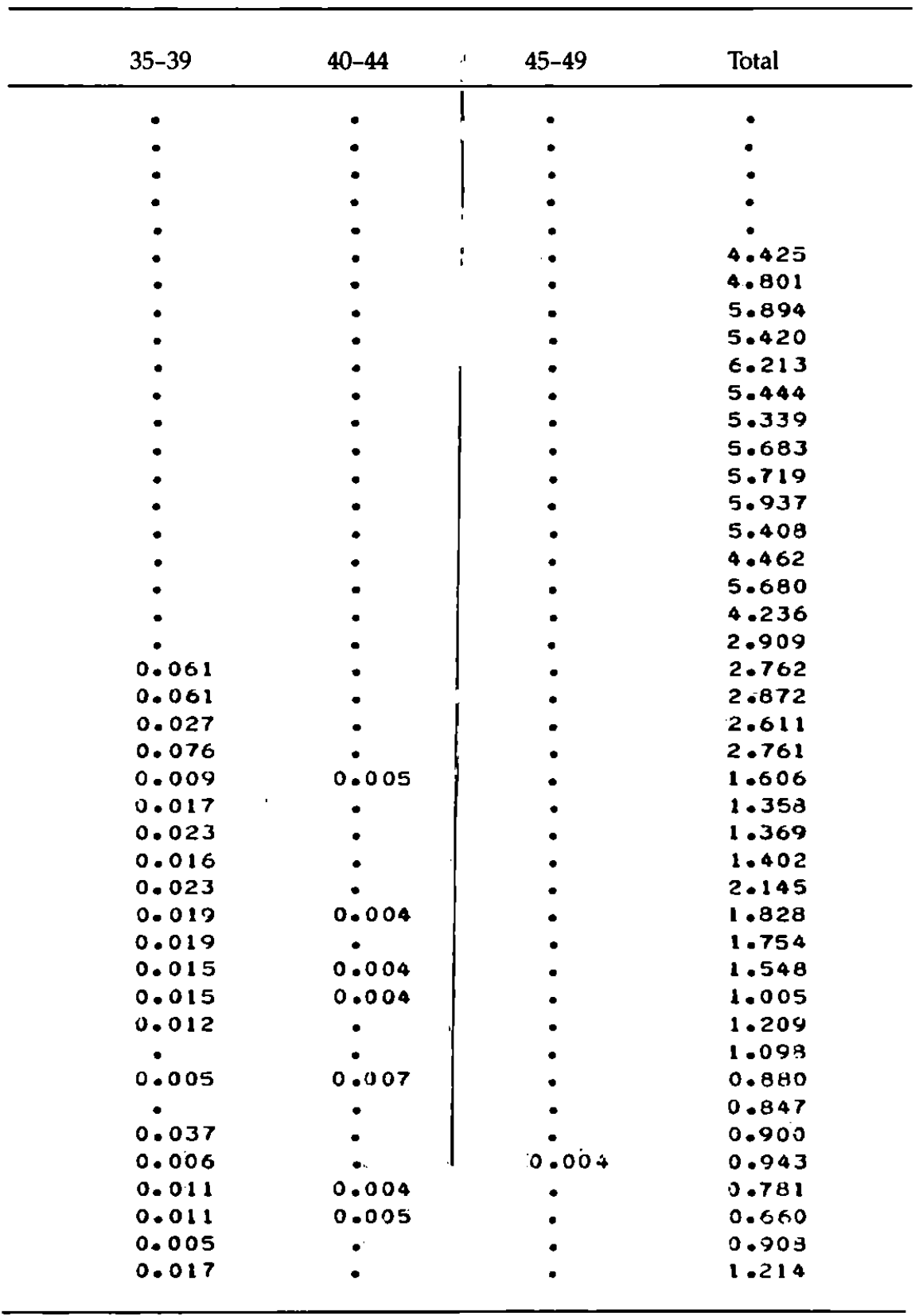


Basic Table 10.C. Rural Shanghai Province (9): age-specific fertility

\begin{tabular}{|c|c|c|c|c|}
\hline $\begin{array}{l}\text { Birth year } \\
\text { of child }\end{array}$ & $15-19$ & $20-24$ & $25-29$ & $30-34$ \\
\hline 1940 & 0.033 & - & - & • \\
\hline 1941 & 0.030 & - & - & - \\
\hline 1942 & 0.049 & - & - & • \\
\hline 1943 & 0.057 & - & - & - \\
\hline 1944 & 0.087 & - & - & - \\
\hline 1945 & 0.087 & 0.186 & - & - \\
\hline 1940 & 0.042 & 0.342 & • & - \\
\hline 1947 & 0.120 & 0.273 & - & - \\
\hline 1948 & 0.062 & 0.239 & - & - \\
\hline 1949 & 0.047 & 0.299 & - & - \\
\hline 1950 & 0.077 & 0.252 & 0.350 & • \\
\hline 1951 & 0.068 & 0.094 & 0.302 & - \\
\hline 1952 & 0.105 & 0.338 & 0.312 & - \\
\hline 1953 & 0.041 & 0.267 & 0.239 & - \\
\hline 1954 & 0.093 & 0.299 & 0.347 & - \\
\hline 1955 & 0.046 & 0.203 & 0.311 & 0.252 \\
\hline $1950^{\circ}$ & 0.068 & 0.195 & 0.282 & 0.151 \\
\hline 1957 & 0.087 & 0.374 & 0.393 & 0.351 \\
\hline 1958 & 0.041 & 0.227 & 0.308 & 0.229 \\
\hline 1959 & 0.020 & 0.244 & 0.206 & 0.212 \\
\hline 1960 & 0.052 & 0.125 & 0.194 & 0.068 \\
\hline 1961 & 0.008 & 0.239 & $0.150^{\circ}$ & 0.115 \\
\hline 1962 & 0.034 & 0.217 & 0.288 & 0.294 \\
\hline 1963 & 0.039 & 0.336 & 0.382 & 0.328 \\
\hline 1964 & 0.047 & 0.267 & 0.302 & 0.140 \\
\hline 1965 & 0.022 & 0.270 & 0.273 & 0.232 \\
\hline 1906 & 0.010 & 0.233 & 0.410 & 0.273 \\
\hline 1967 & 0.005 & 0.137 & 0.195 & 0.096 \\
\hline 1968 & 0.014 & 0.235 & 0.244 & 0.237 \\
\hline 1964 & 0.005 & 0.207 & 0.247 & 0.151 \\
\hline 1970 & 0.004 & 0.236 & 0.253 & 0.114 \\
\hline 1971 & 0.004 & 0.219 & 0.202 & 0.068 \\
\hline 1972 & 0.004 & 0.110 & 0.233 & 0.054 \\
\hline 1973 & - & 0.163 & 0.203 & 0.031 \\
\hline 1974 & - & 0.113 & 0.159 & 0.039 \\
\hline 1975 & - & 0.107 & 0.154 & 0.044 \\
\hline 1976 & 0.010 & 0.124 & 0.204 & 0.047 \\
\hline 1977 & 0.005 & 0.115 & 0.195 & 0.027 \\
\hline 1978 & - & 0.129 & 0.172 & 0.039 \\
\hline 1979 & - & 0.148 & 0.230 & 0.047 \\
\hline 1980 & - & 0.087 & 0.176 & - \\
\hline 1981 & - & 0.179 & 0.183 & 0.005 \\
\hline 1982 & 0.008 & 0.166 & 0.131 & 0.010 \\
\hline
\end{tabular}


rates, $1940-82$

\begin{tabular}{|c|c|c|c|}
\hline $35-39$ & $40-44$ & $45-49$ & Total \\
\hline - & - & - & - \\
\hline - & - & $\bullet$ & - \\
\hline - & - & $\bullet$ & - \\
\hline - & - & - & - \\
\hline - & - & - & - \\
\hline$\bullet$ & - & - & 5.219 \\
\hline - & - & - & 5.976 \\
\hline$\bullet$ & $\bullet$ & - & $5 \cdot 333$ \\
\hline - & - & - & 5.825 \\
\hline - & - & $\bullet$ & 4.950 \\
\hline - & - & • & 6.038 \\
\hline - & - & - & 4.431 \\
\hline - & - & - & 6.304 \\
\hline - & - & - & 4.502 \\
\hline - & - & - & 7.254 \\
\hline - & - & - & 6.067 \\
\hline - & - & - & 4.478 \\
\hline - & - & - & 7.953 \\
\hline - & - & - & 4.907 \\
\hline • & - & • & 4.550 \\
\hline 0.120 & - & - & 3.236 \\
\hline 0.070 & - & • & 2.981 \\
\hline 0.146 & - & - & 5.420 \\
\hline 0.248 & - & - & 7.441 \\
\hline 0.106 & 0.060 & - & 4.608 \\
\hline 0.146 & 0.077 & - & 5.100 \\
\hline 0.115 & 0.070 & - & 5.558 \\
\hline 0.098 & 0.019 & • & $2 \cdot 756$ \\
\hline 0.103 & 0.048 & • & 4.406 \\
\hline 0.075 & - & - & 3.417 \\
\hline 0.029 & 0.010 & - & 3.228 \\
\hline 0.010 & 0.010 & $\bullet$ & 2.571 \\
\hline 0.029 & 0.011 & - & 2.206 \\
\hline 0.021 & 0.021 & - & 2.186 \\
\hline 0.012 & • & - & 1.616 \\
\hline$\bullet$ & 0.010 & - & 1.571 \\
\hline 0.023 & • & - & 2.034 \\
\hline - & - & - & 1.715 \\
\hline 0.010 & - & - & 1.754 \\
\hline - & - & - & $2 \cdot 120$ \\
\hline - & - & - & 1.313 \\
\hline - & - & - & 1.835 \\
\hline - & - & - & 1.574 \\
\hline
\end{tabular}


Basic Table 11.A. Total Jiangsu Province (10): age-specific fertility rates,

\begin{tabular}{|c|c|c|c|c|}
\hline $\begin{array}{l}\text { Birth year } \\
\text { of child }\end{array}$ & $15-19$ & $20-24$ & $25-29$ & $30-34$ \\
\hline 1940 & 0.075 & - & - & - \\
\hline 1941 & 0.073 & - & - & - \\
\hline 1942 & 0.069 & - & - & - \\
\hline 1943 & .0 .091 & - & - & - \\
\hline 1944 & 0.071 & • & - & - \\
\hline 1945 & 0.062 & 0.266 & - & - \\
\hline 1946 & 0.056 & 0.268 & - & $\bullet$ \\
\hline 1947 & 0.081 & 0.267 & - & . \\
\hline 1940 & 0.058 & 0.249 & - & - \\
\hline 1949 & 0.072 & 0.258 & - & - \\
\hline 1950 & 0.073 & 0.231 & 0.255 & - \\
\hline 1951 & 0.061 & 0.243 & 0.252 & - \\
\hline 1952 & 0.071 & 0.292 & 0.270 & - \\
\hline 1953 & 0.074 & 0.254 & 0.282 & - \\
\hline 1954 & 0.064 & 0.297 & 0.288 & - \\
\hline 1955 & 0.074 & 0.309 & 0.283 & 0.218 \\
\hline 1956 & 0.057 & 0.260 & 0.260 & 0.225 \\
\hline 1957 & 0.002 & 0.290 & 0.313 & 0.251 \\
\hline 1958 & 0.059 & 0.217 & 0.246 & 0.231 \\
\hline 1959 & 0.035 & 0.176 & 0.209 & 0.186 \\
\hline 1960 & 0.027 & 0.168 & 0.204 & 0.159 \\
\hline 1961 & 0.023 & 0.148 & 0.176 & 0.151 \\
\hline 1962 & 0.025 & 0.256 & 0.303 & 0.249 \\
\hline 1963 & 0.039 & 0.305 & 0.343 & 0.306 \\
\hline 1964 & 0.043 & 0.244 & 0.292 & 0.231 \\
\hline 1965 & 0.026 & 0.279 & 0.305 & 0.221 \\
\hline 1966 & 0.024 & 0.283 & 0.302 & 0.247 \\
\hline 1967 & 0.022 & 0.226 & 0.256 & 0.180 \\
\hline 1968 & 0.022 & 0.262 & 0.321 & 0.217 \\
\hline 1969 & 0.023 & 0.233 & 0.274 & 0.188 \\
\hline 1970 & 0.021 & 0.254 & 0.267 & 0.182 \\
\hline 1971 & 0.022 & 0.221 & 0.263 & 0.145 \\
\hline 1972 & 0.012 & 0.194 & 0.213 & $0.10 \epsilon$ \\
\hline 1973 & 0.005 & 0.161 & 0.206 & 0.092 \\
\hline 1974 & 0.008 & 0.148 & 0.203 & 0.079 \\
\hline 1975 & 0.005 & 0.138 & 0.177 & 0.072 \\
\hline 1976 & 0.005 & 0.144 & 0.185 & 0.058 \\
\hline 1977 & 0.005 & 0.125 & 0.174 & 0.062 \\
\hline 1978 & 0.003 & 0.121 & 0.180 & 0.058 \\
\hline 1979 & 0.002 & 0.127 & 0.181 & 0.055 \\
\hline 1930 & 0.001 & 0.105 & 0.154 & 0.024 \\
\hline 1981 & 0.004 & 0.161 & 0.193 & 0.026 \\
\hline 1982 & 0.010 & 0.158 & 0.166 & 0.031 \\
\hline
\end{tabular}




\begin{tabular}{|c|c|c|c|}
\hline $35-39$ & $40-44$ & $45-49$ & Total \\
\hline - & - & - & • \\
\hline - & - & - & - \\
\hline - & - & - & - \\
\hline - & - & - & - \\
\hline - & - & - & - \\
\hline - & - & - & $5 \cdot 252$ \\
\hline - & - & - & 5.249 \\
\hline - & - & • & 5.916 \\
\hline - & - & - & 5.190 \\
\hline - & - & - & 5.013 \\
\hline - & - & - & 5.076 \\
\hline - & - & - & 5.309 \\
\hline - & - & - & 6.049 \\
\hline - & - & - & 5.498 \\
\hline - & - & • & 6.083 \\
\hline - & - & - & 5.913 \\
\hline - & - & - & 5.440 \\
\hline - & $\bullet$ & - & 6.088 \\
\hline - & - & - & 5.024 \\
\hline - & - & - & 3.981 \\
\hline 0.122 & - & - & 3.675 \\
\hline 0.094 & - & - & $3 \cdot 2.71$ \\
\hline $0.173:$ & - & - & 5.535 \\
\hline 0.232 & - & - & 6.666 \\
\hline 0.160 & 0.076 & 0.007 & 5.264 \\
\hline 0.160 & 0.077 & 0.010 & $5 \cdot 388$ \\
\hline 0.180 & 0.063 & 0.007 & $5 \cdot 534$ \\
\hline 0.113 & 0.059 & 0.006 & 4.307 \\
\hline 0.133 & 0.063 & $0.00 ?$ & 5.132 \\
\hline 0.124 & 0.0 .59 & 0.007 & 4.538 \\
\hline 0.132 & 0.047 & 0.003 & 4.535 \\
\hline 0.097 & 0.054 & 0.005 & 4.040 \\
\hline 0.066 & 0.039 & 0.002 & 3.160 \\
\hline 0.0 .51 & 0.017 & 0.002 & 2.671 \\
\hline 0.0 .35 & 0.022 & 0.002 & 2.491 \\
\hline 0.039 & 0.009 & 0.002 & 2.210 \\
\hline 0.026 & 0.009 & 0.004 & 2.157 \\
\hline 0.020 & 0.010 & 0.001 & 1.988 \\
\hline 0.019 & $0.00 E$ & - & 1.931 \\
\hline 0.017 & 0.004 & • & 1.932 \\
\hline 0.006 & 0.003 & 0.001 & 1.462 \\
\hline 0.006 & 0.003 & 0.001 & $1.96,7$ \\
\hline 0.000 & U. $00 \mathrm{O}$ & 0.003 & 1.379 \\
\hline
\end{tabular}


Basic Table 11.B. Urban Jiangsu Province (10): age-specific fertility

\begin{tabular}{|c|c|c|c|c|}
\hline $\begin{array}{l}\text { Birth year } \\
\text { of child }\end{array}$ & $15-19$ & $20-24$ & $25-29$ & $30-34$ \\
\hline 1940 & 0.081 & - & - & - \\
\hline 1941 & 0.084 & - & - & - \\
\hline 1942 & 0.083 & - & - & . \\
\hline 1943 & 0.074 & - & . & 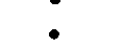 \\
\hline 1944 & 0.058 & - & - & - \\
\hline 1945 & 0.068 & 0.205 & - & - \\
\hline 1946 & 0.042. & 0.240 & - & - \\
\hline 1947 & 0.084 & 0.260 & - & - \\
\hline 1948 & 0.053 & 0.216 & - & - \\
\hline 1949 & 0.081 & 0.259 & - & - \\
\hline 1950 & 0.096 & 0.222 & 0.253 & - \\
\hline 1951 & 0.044 & 0.240 & 0.246 & - \\
\hline 1952 & 0.055 & 0.292 & 0.203 & - \\
\hline 1953 & 0.042 & 0.193 & 0.231 & - \\
\hline 1954 & 0.070 & 0.294 & 0.300 & - \\
\hline 1955 & 0.062 & 0.210 & 0.291 & 0.151 \\
\hline 1956 & 0.033 & 0.230 & 0.244 & 0.229 \\
\hline 1957 & 0.035 & 0.258 & 0.331 & 0.192 \\
\hline 1958 & 0.072 & 0.176 & 0.259 & 0.206 \\
\hline 1959 & 0.043 & 0.201 & 0.308 & 0.174 \\
\hline 1900 & 0.024 & 0.174 & 0.259 & 0.184 \\
\hline 1961 & 0.014 & 0.172 & 0.193 & 0.215 \\
\hline 1962 & 0.021 & 0.238 & 0.258 & 0.177 \\
\hline 1963 & 0.019 & 0.263 & 0.359 & 0.276 \\
\hline 1964 & 0.036 & 0.163 & 0.254 & 0.145 \\
\hline 1905 & 0.026 & 0.182 & 0.200 & 0.136 \\
\hline 1966 & 0.021 & 0.161 & 0.195 & 0.125 \\
\hline 1967 & 0.005 & 0.150 & 0.200 & 0.089 \\
\hline 1968 & 0.012 & 0.184 & 0.223 & 0.106 \\
\hline 1909 & 0.009 & 0.152 & 0.169 & 0.117 \\
\hline 1970 & 0.013 & 0.191 & 0.213 & 0.128 \\
\hline 1971 & 0.022 & 0.135 & 0.229 & 0.078 \\
\hline 1972 & 0.004 & 0.110 & 0.179 & 0.068 \\
\hline 1973 & 0.004 & 0.100 & 0.204 & 0.059 \\
\hline 1974 & - & 0.068 & 0.161 & 0.066 \\
\hline 1975 & 0.002 & 0.110 & 0.159 & 0.074 \\
\hline 1976 & - & 0.089 & 0.135 & 0.057 \\
\hline 1977 & 0.004 & 0.083 & 0.140 & 0.047 \\
\hline 1978 & 0.004 & 0.037 & 0.150 & 0.048 \\
\hline 1979 & - & 0.049 & 0.134 & 0.084 \\
\hline 1980 & - & 0.034 & 0.121 & 0.023 \\
\hline 1981 & - & 0.101 & 0.233 & 0.029 \\
\hline 1982 & - & 0.116 & 0.136 & 0.015 \\
\hline
\end{tabular}


rates, $1940-82$

\begin{tabular}{|c|c|c|c|}
\hline $35-39$ & $40-44$ & $45-49$ & Total \\
\hline - & - & $\bullet$ & • \\
\hline • & - & - & • \\
\hline - & • & $\bullet$ & $\bullet$ \\
\hline - & $\bullet$ & - & - \\
\hline$\bullet$ & $\bullet$ & $\bullet$ & - \\
\hline$\bullet$ & - & $\bullet$ & $4 \cdot 591$ \\
\hline - & - & - & 4.797 \\
\hline - & $\bullet$ & - & $5 \cdot 724$ \\
\hline • & • & - & 4.950 \\
\hline$\bullet$ & $\bullet$ & - & 6.504 \\
\hline - & - & $\bullet$ & $5 \cdot 224$ \\
\hline - & - & - & 4.895 \\
\hline - & - & - & 5.675 \\
\hline - & - & - & 4.392 \\
\hline - & - & - & 6.522 \\
\hline • & - & $\bullet$ & 4.930 \\
\hline - & $\bullet$ & - & 4.932 \\
\hline - & - & - & 5.618 \\
\hline - & - & - & 4.773 \\
\hline - & - & $\bullet$ & 4.711 \\
\hline 0.097 & $\bullet$ & - & 4.033 \\
\hline 0.117 & $\bullet$ & - & 3.968 \\
\hline 0.109 & - & - & 4.320 \\
\hline 0.182 & - & - & 5.810 \\
\hline 0.103 & 0.039 & 0.007 & 3.726 \\
\hline 0.043 & 0.049 & • & 3.178 \\
\hline 0.046 & 0.039 & - & 2.437 \\
\hline 0.027 & 0.010 & - & 2.403 \\
\hline 0.039 & 0.015 & $\bullet$ & 2.896 \\
\hline 0.027 & 0.004 & - & $2 \cdot 396$ \\
\hline 0.026 & 0.009 & - & 2.897 \\
\hline 0.036 & 0.013 & 0.006 & 2.592 \\
\hline 0.022 & 0.004 & • & 1.934 \\
\hline 0.021 & - . & - & 1.938 \\
\hline 0.017 & - & - & 1.563 \\
\hline 0.010 & - & - & 1.813 \\
\hline 0.007 & - & • & 1.442 \\
\hline 0.006 & - & • & 1.398 \\
\hline 0.003 & 0.004 & $\bullet$ & 1.234 \\
\hline 0.007 & 0.003 & - & 1.382 \\
\hline$\bullet$ & - & - & 0.890 \\
\hline 0.004 & $\bullet$ & 0.004 & 1.052 \\
\hline • & - & $\bullet$ & 1.334 \\
\hline
\end{tabular}


Basic Table 11.C. Rural Jiangsu Province (10): age-specific fertility

\begin{tabular}{|c|c|c|c|c|}
\hline $\begin{array}{l}\text { Birth year } \\
\text { of child }\end{array}$ & $15-19$ & $20-24$ & $25-29$ & $30-34$ \\
\hline 1940 & 0.073 & - & - & - \\
\hline 1941 & 0.071 & - & - & - \\
\hline 1942 & 0.066 & - & - & - \\
\hline 1943 & 0.095 & - & - & - \\
\hline 1944 & 0.074 & - & - & • \\
\hline 1945 & 0.061 & 0.277 & - & - \\
\hline 1946 & 0.059 & 0.273 & - & - \\
\hline 1947 & 0.080 & 0.268 & - & - \\
\hline 1948 & 0.059 & 0.256 & $\bullet$ & - \\
\hline 1949 & 0.070 & 0.257 & - & - \\
\hline 1950 & 0.068 & 0.232 & 0.256 & - \\
\hline 1951 & 0.065 & 0.244 & $0 \cdot 253$ & - \\
\hline 1952 & 0.074 & 0.291 & 0.283 & - \\
\hline 1953 & 0.081 & 0.266 & 0.292 & - \\
\hline 1954 & 0.063 & 0.298 & 0.286 & - \\
\hline 1955 & 0.077 & 0.329 & 0.281 & 0.230 \\
\hline 1950 & 0.062 & 0.266 & 0.263 & 0.224 \\
\hline 1957 & 0.068 & 0.298 & 0.309 & 0.263 \\
\hline 1958 & 0.056 & 0.227 & 0.244 & 0.236 \\
\hline 1959 & 0.033 & 0.170 & 0.191 & 0.188 \\
\hline 1960 & 0.028 & 0.167 & 0.193 & 0.154 \\
\hline 1901 & 0.024 & 0.142 & 0.172 & 0.138 \\
\hline 1962 & 0.025 & 0.260 & 0.313 & 0.264 \\
\hline 1963 & 0.043 & 0.315 & 0.340 & 0.312 \\
\hline 1964 & 0.044 & 0.262 & 0.301 & 0.248 \\
\hline 1965 & 0.026 & 0.298 & 0.331 & 0.238 \\
\hline 1906 & 0.025 & 0.306 & 0.327 & 0.273 \\
\hline 1967 & 0.025 & 0.239 & 0.269 & 0.201 \\
\hline 1960 & 0.024 & 0.276 & 0.343 & 0.243 \\
\hline 1969 & 0.026 & 0.248 & 0.296 & 0.205 \\
\hline 1970 & 0.022 & 0.266 & 0.278 & 0.195 \\
\hline 1971 & 0.023 & 0.238 & 0.269 & 0.162 \\
\hline 1972 & 0.013 & 0.211 & 0.219 & 0.115 \\
\hline 1973 & 0.006 & 0.173 & 0.207 & 0.099 \\
\hline 1974 & 0.009 & 0.164 & 0.211 & 0.081 \\
\hline 1973 & 0.006 & 0.143 & 0.181 & 0.072 \\
\hline 1976 & 0.006 & 0.155 & 0.195 & 0.058 \\
\hline 1977 & 0.005 & 0.133 & 0.1 คО & 0.064 \\
\hline 1978 & 0.002 & 0.138 & 0.185 & 0.060 \\
\hline 1979 & 0.002 & 0.143 & 0.191 & 0.050 \\
\hline 1980 & 0.001 & 0.121 & 0.160 & 0.024 \\
\hline 1981 & 0.004 & 0.175 & 0.186 & 0.025 \\
\hline 1982 & 0.011 & 0.168 & 0.172 & 0.034 \\
\hline
\end{tabular}


rates, $1940-82$

\begin{tabular}{|c|c|c|c|}
\hline $35-39$ & $40-44$ & $45-49$ & Total \\
\hline - & - & - & $\bullet$ \\
\hline - & $\bullet$ & - & - \\
\hline - & $\bullet$ & $\bullet$ & - \\
\hline • & - & - & - \\
\hline - & - & - & - \\
\hline - & - & - & $5 \cdot 387$ \\
\hline - & - & - & $5 \cdot 339$ \\
\hline - & - & - & $5 \cdot 953$ \\
\hline - & - & - & 5.245 \\
\hline - & - & - & 5.438 \\
\hline - & - & - & 5.046 \\
\hline • & - & - & 5.393 \\
\hline - & - & - & 6.126 \\
\hline - & - & - & 5.726 \\
\hline - & - & - & 5.997 \\
\hline - & - & - & 6.120 \\
\hline - & - & - & 5.547 \\
\hline • & • & - & 6.187 \\
\hline - & - & - & 5.077 \\
\hline - & - & - & 3.829 \\
\hline $0.12 \epsilon$ & - & - & 3.601 \\
\hline 0.090 & $\cdots$ & - & 3.128 \\
\hline 0.185 & - & - & 5.781 \\
\hline 0.242 & - & - & 6.840 \\
\hline 0.172 & 0.084 & 0.006 & 5.579 \\
\hline 0.184 & 0.083 & 0.012 & 5.855 \\
\hline 0.200 & 0.068 & 0.009 & 6.072 \\
\hline 0.131 & 0.067 & 0.007 & 4.695 \\
\hline 0.151 & 0.072 & 0.011 & 5.597 \\
\hline 0.142 & 0.070 & 0.008 & 4.978 \\
\hline 0.153 & 0.055 & 0.004 & 4.870 \\
\hline 0.110 & 0.062 & 0.00 .5 & $4 \cdot 3+2$ \\
\hline 0.076 & 0.045 & 0.003 & 3.411 \\
\hline 0.058 & 0.021 & 0.003 & 2.823 \\
\hline 0.040 & 0.027 & 0.003 & 2.675 \\
\hline 0.044 & 0.011 & 0.003 & 2.300 \\
\hline 0.030 & 0.011 & 0.005 & 2.302 \\
\hline 0.024 & 0.012 & 0.002 & 2.106 \\
\hline 0.022 & 0.007 & - & 2.071 \\
\hline 0.019 & 0.004 & - & 2.047 \\
\hline 0.007 & 0.003 & 0.001 & 1.594 \\
\hline 0.007 & 0.003 & - & 2.000 \\
\hline 0.008 & 0.001 & 0.003 & 1.991 \\
\hline
\end{tabular}


Basic Table 12.A. Total Zhejiang Province (11): age-specific fertility

\begin{tabular}{|c|c|c|c|c|}
\hline $\begin{array}{l}\text { Birth year } \\
\text { of child }\end{array}$ & $15-19$ & $20-24$ & 25-29 & $30-34$ \\
\hline 1940 & 0.091 & • & - & - \\
\hline 1941 & 0.115 & - & $\bullet$ & $\bullet$ \\
\hline 1942 & 0.105 & - & - & - \\
\hline 1943 & 0.082 & $\bullet$ & - & - \\
\hline 1944 & 0.088 & - & - & • \\
\hline 1945 & 0.086 & 0.284 & - & - \\
\hline 1946 & 0.095 & 0.256 & • & - \\
\hline 1947 & 0.093 & 0.276 & • & • \\
\hline 1948 & 0.107 & 0.284 & - & - \\
\hline 1949 & 0.096 & 0.314 & - & - \\
\hline 1950 & 0.099 & 0.293 & 0.275 & • \\
\hline 1951 & 0.122 & 0.244 & 0.267 & • \\
\hline 1952 & 0.106 & 0.312 & 0.293 & $\bullet$ \\
\hline 1953 & 0.105 & 0.289 & 0.295 & - \\
\hline 1954 & 0.146 & 0.301 & 0.311 & $\bullet$ \\
\hline 1955 & 0.118 & 0.300 & 0.290 & 0.245 \\
\hline 1956 & 0.106 & 0.278 & 0.308 & 0.227 \\
\hline 1957 & 0.114 & 0.359 & 0.340 & 0.287 \\
\hline 1958 & 0.105 & 0.239 & 0.293 & 0.237 \\
\hline 1959 & 0.069 & 0.226 & 0.248 & 0.164 \\
\hline 1960 & 0.061 & 0.230 & 0.238 & 0.192 \\
\hline 1961 & 0.032 & 0.190 & 0.182 & 0.158 \\
\hline 1962 & 0.090 & 0.343 & 0.332 & 0.329 \\
\hline 1963 & 0.096 & 0.388 & 0.390 & 0.331 \\
\hline 1964 & 0.088 & 0.357 & 0.303 & 0.261 \\
\hline 1905 & 0.100 & 0.327 & 0.299 & 0.233 \\
\hline 1906 & 0.103 & 0.372 & 0.302 & 0.219 \\
\hline 1907 & 0.076 & 0.300 & 0.259 & 0.186 \\
\hline 1968 & 0.084 & 0.333 & 0.299 & 0.191 \\
\hline 1969 & 0.080 & 0.313 & 0.289 & 0.172 \\
\hline 1970 & 0.070 & 0.295 & 0.227 & 0.154 \\
\hline 1971 & 0.070 & 0.308 & 0.223 & 0.132 \\
\hline 1972 & 0.048 & 0.259 & 0.198 & 0.107 \\
\hline 1973 & 0.045 & 0.243 & 0.175 & 0.091 \\
\hline 1974 & 0.039 & 0.226 & 0.171 & 0.082 \\
\hline 1975 & 0.027 & 0.198 & 0.156 & 0.090 \\
\hline 1976 & 0.023 & 0.222 & 0.152 & 0.067 \\
\hline 1977 & 0.021 & 0.192 & 0.147 & 0.065 \\
\hline 1978 & 0.010 & 0.183 & 0.171 & 0.049 \\
\hline 1979 & 0.017 & 0.203 & 0.174 & 0.047 \\
\hline 1980 & 0.012 & 0.165 & 0.140 & 0.033 \\
\hline 1981 & 0.017 & 0.232 & 0.153 & 0.030 \\
\hline 1982 & 0.017 & 0.220 & 0.159 & 0.031 \\
\hline
\end{tabular}


rates, $1940-82$

\begin{tabular}{|c|c|c|c|}
\hline $35-39$ & $40-44$ & $45-49$ & Total \\
\hline - & - & - & $\bullet$ \\
\hline - & - & $\bullet$ & - \\
\hline - & - & - & $\bullet$ \\
\hline - & • & $\bullet$ & • \\
\hline - & - & $\bullet$ & - \\
\hline - & - & - & 5.151 \\
\hline - & - & - & $5 \cdot 338$ \\
\hline$\bullet$ & - & - & 5.690 \\
\hline - & - & - & 5.547 \\
\hline - & - & - & 5.946 \\
\hline - & - & - & 5.567 \\
\hline - & - & - & 5.470 \\
\hline - & - & - & 6.096 \\
\hline - & - & - & 5.879 \\
\hline - & - & - & 6.002 \\
\hline - & - & - & 5.971 \\
\hline - & - & - & 5.963 \\
\hline - & - & - & 6.993 \\
\hline - & - & - & 5.442 \\
\hline • & - & - & 4.539 \\
\hline 0.149 & - & - & 4.709 \\
\hline 0.102 & - & • & 3.491 \\
\hline 0.190 & - & - & 6.854 \\
\hline 0.240 & - & - & 7.617 \\
\hline 0.221 & 0.070 & 0.007 & 6.534 \\
\hline 0.199 & 0.082 & 0.006 & 6.227 \\
\hline 0.182 & 0.069 & 0.002 & $6 \cdot 239$ \\
\hline 0.134 & 0.066 & 0.004 & 5.122 \\
\hline 0.153 & 0.072 & - & 5.667 \\
\hline 0.130 & 0.052 & 0.004 & 5.200 \\
\hline 0.097 & 0.035 & 0.005 & 4.418 \\
\hline 0.090 & 0.049 & 0.003 & 4.380 \\
\hline 0.061 & 0.030 & 0.003 & 3.532 \\
\hline 0.058 & 0.030 & 0.007 & 3.244 \\
\hline 0.040 & 0.018 & 0.001 & 2.883 \\
\hline 0.037 & 0.026 & 0.001 & 2.678 \\
\hline 0.037 & 0.019 & 0.001 & 2.602 \\
\hline 0.021 & 0.019 & 0.001 & 2.333 \\
\hline 0.013 & 0.009 & 0.003 & 2.195 \\
\hline 0.014 & 0.008 & - & 2.315 \\
\hline 0.013 & 0.003 & 0.002 & 1.839 \\
\hline 0.010 & 0.004 & 0.001 & 2.229 \\
\hline 0.015 & 0.003 & 0.005 & 2.246 \\
\hline
\end{tabular}


Basic Table 12.B. Urban Zhejiang Province (11): age-specific fertility

\begin{tabular}{|c|c|c|c|c|}
\hline $\begin{array}{l}\text { Birth year } \\
\text { of child }\end{array}$ & $15-19$ & $20-24$ & $25-29$ & $30-34$ \\
\hline 1940 & 0.013 & - & - & - \\
\hline 1941 & 0.060 & - & . & - \\
\hline 1942 & 0.065 & - & - & - \\
\hline 1943 & 0.074 & - & - & - \\
\hline 1944 & 0.070 & - & $\bullet$ & . \\
\hline 1945 & 0.079 & 0.240 & - & - \\
\hline 1946 & 0.069 & 0.229 & - & - \\
\hline 1947 & 0.142 & 0.338 & • & - \\
\hline 1948 & 0.094 & 0.223 & - & - \\
\hline 1949 & 0.067 & 0.342 & - & - \\
\hline 1950 & 0.069 & 0.356 & 0.359 & - \\
\hline 1951 & 0.103 & 0.188 & 0.265 & . \\
\hline 1952 & 0.054 & 0.315 & 0.327 & - \\
\hline 1953 & 0.044 & 0.222 & 0.244 & - \\
\hline 1954 & 0.090 & 0.275 & 0.292 & - \\
\hline 1955 & 0.026 & 0.240 & 0.277 & 0.173 \\
\hline 1956 & 0.069 & 0.191 & 0.257 & 0.241 \\
\hline 1957 & 0.051 & 0.282 & 0.457 & 0.262 \\
\hline 1958 & 0.043 & 0.197 & 0.265 & 0.202 \\
\hline 1959 & 0.042 & 0.222 & 0.238 & 0.181 \\
\hline 1960 & 0.025 & 0.244 & 0.281 & 0.208 \\
\hline 1961 & 0.015 & 0.171 & 0.205 & 0.129 \\
\hline 1962 & 0.037 & 0.194 & 0.249 & 0.244 \\
\hline 1963 & 0.064 & 0.312 & 0.343 & 0.273 \\
\hline 1964 & 0.027 & 0.236 & 0.205 & 0.156 \\
\hline 1965 & 0.038 & 0.215 & 0.106 & 0.117 \\
\hline 1966 & 0.020 & 0.161 & 0.103 & 0.109 \\
\hline 1967 & 0.006 & 0.180 & 0.144 & 0.087 \\
\hline 1908 & 0.012 & 0.184 & 0.182 & 0.073 \\
\hline 1969 & 0.018 & 0.115 & 0.203 & 0.082 \\
\hline 1970 & 0.033 & 0.166 & 0.124 & 0.105 \\
\hline 1971 & 0.025 & 0.146 & 0.177 & 0.060 \\
\hline 1972 & 0.009 & 0.115 & 0.180 & 0.076 \\
\hline 1973 & 0.004 & 0.087 & 0.170 & 0.078 \\
\hline 1974 & - & 0.112 & 0.183 & 0.051 \\
\hline 1975 & - & 0.044 & 0.128 & 0.075 \\
\hline 1976 & - & 0.071 & 0.160 & $0.03 \theta$ \\
\hline 1977 & 0.005 & 0.042 & 0.140 & 0.052 \\
\hline 1978 & - & 0.040 & 0.137 & 0.043 \\
\hline 1979 & - & 0.027 & 0.176 & 0.075 \\
\hline 1980 & 0.005 & 0.018 & 0.094 & 0.026 \\
\hline 1981 & - & 0.037 & 0.172 & 0.027 \\
\hline 1982 & - & 0.038 & 0.120 & 0.013 \\
\hline
\end{tabular}


rates, $1940-82$

\begin{tabular}{|c|c|c|c|}
\hline 35-39 & $40-44$ & $45-49$ & Total \\
\hline - & - & • & - \\
\hline - & • & - & - \\
\hline - & - & - & - \\
\hline • & • & • & $\bullet$ \\
\hline$\bullet$ & $\bullet$ & • & - \\
\hline - & - & • & 3.985 \\
\hline - & - & - & 4.114 \\
\hline - & - & - & 6.845 \\
\hline - & - & - & 5.286 \\
\hline - & - & • & 5.603 \\
\hline - & - & • & 6.408 \\
\hline - & - & • & 4.426 \\
\hline - & - & - & 5.733 \\
\hline • & - & • & 4.624 \\
\hline - & - & • & 5.674 \\
\hline - & - & - & 4.535 \\
\hline - & • & - & 5.004 \\
\hline • & $\bullet$ & - & 6.542 \\
\hline$\bullet$ & - & - & 4.794 \\
\hline$\bullet$ & - & • & 4.260 \\
\hline 0.253 & • & • & 5.395 \\
\hline 0.121 & - & • & 3.385 \\
\hline 0.131 & - & - & 4.618 \\
\hline 0.159 & - & - & 6.044 \\
\hline 0.111 & 0.029 & - & 3.822 \\
\hline 0.040 & 0.027 & - & 3.012 \\
\hline 0.030 & 0.012 & - & 2.177 \\
\hline 0.071 & 0.011 & - & 2.495 \\
\hline 0.034 & - & - & 2.428 \\
\hline 0.030 & 0.040 & $\bullet$ & 2.438 \\
\hline 0.041 & - & - & 2.348 \\
\hline 0.007 & . & - & 2.077 \\
\hline 0.013 & - & - & 1.965 \\
\hline 0.022 & - & - & 1.807 \\
\hline 0.016 & 0.007 & - & 1.846 \\
\hline 0.017 & - & $\bullet$ & 1.321 \\
\hline 0.009 & 0.007 & - & 1.422 \\
\hline 0.008 & - & - & 1.237 \\
\hline$\bullet$ & - & - & 1.099 \\
\hline 0.008 & - & - & 1.429 \\
\hline - & - & • & 0.713 \\
\hline 0.008 & - & - & 1.219 \\
\hline • & • & $\bullet$ & 0.855 \\
\hline
\end{tabular}


Basic Table 12.C. Rural Zhejiang Province (11): age-specific fertility

\begin{tabular}{|c|c|c|c|c|}
\hline $\begin{array}{l}\text { Birth year } \\
\text { of child }\end{array}$ & $15-19$ & $20-24$ & $25-29$ & $30-34$ \\
\hline 1940 & 0.102 & - & - & • \\
\hline 1941 & 0.123 & - & - & ? \\
\hline 1942 & 0.112 & - & . & - \\
\hline 1943 & 0.084 & - & - & - \\
\hline 1944 & 0.090 & . & . & 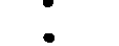 \\
\hline 1945 & 0.087 & 0.290 & - & - \\
\hline 1946 & 0.099 & 0.260 & - & - \\
\hline 1947 & 0.086 & 0.266 & - & - \\
\hline 1948 & 0.109 & 0.293 & • & - \\
\hline 1949 & 0.101 & 0.309 & - & - \\
\hline 1950 & 0.105 & 0.283 & 0.263 & - \\
\hline 1951 & 0.126 & 0.252 & 0.267 & • \\
\hline 1952 & 0.117 & 0.312 & 0.288 & - \\
\hline 1953 & 0.118 & 0.300 & 0.303 & - \\
\hline 1954 & 0.156 & 0.305 & 0.314 & • \\
\hline 1955 & 0.134 & 0.312 & 0.292 & 0.255 \\
\hline 1956 & 0.113 & 0.296 & 0.316 & 0.225 \\
\hline 1957 & 0.125 & 0.376 & 0.324 & 0.291 \\
\hline 1958 & 0.116 & 0.247 & 0.297 & 0.243 \\
\hline 1959 & 0.074 & 0.227 & 0.250 & 0.162 \\
\hline 1960 & 0.067 & 0.228 & 0.230 & 0.190 \\
\hline 1961. & 0.035 & 0.193 & 0.178 & 0.158 \\
\hline 1962 & 0.099 & 0.370 & 0.350 & 0.341 \\
\hline 1963 & 0.101 & 0.402 & 0.400 & 0.340 \\
\hline 1964 & 0.097 & 0.380 & 0.321 & 0.280 \\
\hline 1965 & 0.109 & 0.345 & 0.323 & 0.256 \\
\hline 1966 & 0.114 & 0.408 & 0.339 & 0.241 \\
\hline 1967 & 0.085 & 0.320 & 0.280 & 0.207 \\
\hline 1968 & 0.093 & 0.356 & 0.321 & 0.216 \\
\hline 1909 & 0.088 & 0.342 & 0.305 & 0.189 \\
\hline 1970 & 0.075 & 0.314 & 0.245 & 0.162 \\
\hline 1971 & 0.076 & 0.329 & 0.231 & 0.146 \\
\hline 1972 & 0.054 & 0.278 & 0.201 & 0.113 \\
\hline 1973 & 0.050 & 0.263 & 0.175 & 0.094 \\
\hline 1974 & 0.044 & 0.241 & 0.169 & 0.088 \\
\hline 1975 & 0.031 & 0.218 & 0.160 & 0.092 \\
\hline 1976 & 0.027 & 0.242 & 0.151 & 0.072 \\
\hline 1977 & 0.024 & 0.213 & 0.148 & 0.067 \\
\hline 1978 & 0.012 & 0.203 & 0.175 & 0.051 \\
\hline 1979 & 0.019 & 0.229 & 0.174 & 0.043 \\
\hline 1980 & 0.012 & 0.186 & 0.146 & 0.034 \\
\hline 1981 & 0.018 & 0.263 & 0.150 & 0.030 \\
\hline 1962 & 0.018 & 0.249 & 0.165 & 0.033 \\
\hline
\end{tabular}


rates, $1940-82$

\begin{tabular}{|c|c|c|c|}
\hline 35-39 & $40-44$ & $45-49$ & Total \\
\hline - & - & - & - \\
\hline - & - & - & - \\
\hline - & - & - & - \\
\hline - & - & - & - \\
\hline - & - & - & - \\
\hline - & - & • & $5 \cdot 3.39$ \\
\hline - & - & $\bullet$ & 5.534 \\
\hline - & - & - & 5.507 \\
\hline - & - & - & 5.590 \\
\hline - & - & - & 6.004 \\
\hline - & - & • & 5.422 \\
\hline - & - & - & 5.051 \\
\hline - & - & - & 6.158 \\
\hline • & - & - & 6.097 \\
\hline - & - & - & 6.058 \\
\hline - & - & - & 6.221 \\
\hline - & - & - & 6.131 \\
\hline • & $\bullet$ & $\bullet$ & 7.072 \\
\hline • & - & - & 5.556 \\
\hline$\bullet$ & • & - & 4.588 \\
\hline 0.133 & - & - & 4.590 \\
\hline 0.099 & - & • & 3.509 \\
\hline 0.200 & - & - & 7.240 \\
\hline 0.253 & - & - & 7.884 \\
\hline 0.238 & 0.076 & 0.009 & 6.996 \\
\hline 0.223 & 0.090 & 0.007 & 6.769 \\
\hline 0.204 & 0.077 & 0.002 & 6.928 \\
\hline 0.142 & 0.076 & 0.004 & 5.568 \\
\hline 0.172 & 0.084 & - & 6.205 \\
\hline 0.148 & 0.054 & 0.004 & 5.649 \\
\hline 0.108 & 0.041 & 0.006 & 4.753 \\
\hline 0.107 & 0.056 & 0.004 & 4.748 \\
\hline 0.071 & 0.034 & 0.004 & 3.772 \\
\hline 0.065 & 0.035 & 0.008 & 3.455 \\
\hline 0.044 & 0.020 & 0.002 & 3.043 \\
\hline 0.041 & 0.031 & 0.002 & 2.878 \\
\hline 0.043 & 0.020 & 0.001 & 2.780 \\
\hline 0.023 & 0.023 & 0.001 & 2.499 \\
\hline 0.016 & 0.011 & 0.004 & $2 \cdot 355$ \\
\hline 0.015 & 0.009 & - & 2.450 \\
\hline 0.015 & 0.003 & 0.003 & 2.005 \\
\hline 0.011 & 0.005 & 0.001 & 2.388 \\
\hline 0.017 & 0.003 & 0.006 & 2.455 \\
\hline
\end{tabular}


Basic Table 13.A. Total Anhui Province (12): age-specific fertility rates,

\begin{tabular}{|c|c|c|c|c|}
\hline $\begin{array}{l}\text { Birth year } \\
\text { of child }\end{array}$ & $15-19$ & $20-24$ & $25-29$ & $30-34$ \\
\hline 1940 & 0.083 & - & - & - \\
\hline 1941 & 0.084 & - & - & - \\
\hline 1942 & 0.066 & - & - & - \\
\hline $194 \mathrm{~J}$ & 0.076 & - & - & - \\
\hline 1944 & 0.062 & - & - & - \\
\hline 1945 & 0.069 & 0.249 & - & - \\
\hline $1940^{\circ}$ & 0.057 & 0.242 & - & - \\
\hline 1947 & 0.080 & 0.291 & - & - \\
\hline 1948 & 0.066 & 0.250 & - & - \\
\hline 1945 & 0.061 & 0.256 & - & - \\
\hline 1950 & 0.061 & 0.226 & 0.278 & - \\
\hline 1951 & 0.053 & 0.263 & 0.242 & • \\
\hline 1952 & 0.104 & 0.310 & 0.288 & - \\
\hline 1953 & 0.102 & 0.294 & 0.270 & - \\
\hline 1954 & 0.108 & 0.275 & 0.286 & • \\
\hline 1955 & 0.081 & 0.256 & 0.242 & 0.231 \\
\hline 195 & 0.068 & 0.236 & 0.232 & 0.233 \\
\hline 1957 & 0.078 & 0.267 & 0.275 & 0.231 \\
\hline 1958 & 0.054 & 0.228 & 0.197 & 0.179 \\
\hline 1959 & 0.029 & 0.109 & 0.141 & 0.120 \\
\hline 1900 & 0.018 & 0.074 & 0.107 & 0.097 \\
\hline 1901 & 0.017 & 0.098 & 0.144 & 0.095 \\
\hline 1902 & 0.042 & 0.296 & 0.390 & 0.317 \\
\hline 1963 & 0.066 & 0.393 & 0.371 & 0.315 \\
\hline 1964 & 0.053 & 0.287 & 0.289 & 0.253 \\
\hline 1965 & 0.048 & $0.32 \varepsilon$ & 0.311 & 0.286 \\
\hline 1900 & 0.052 & 0.358 & 0.339 & 0.298 \\
\hline 1967 & 0.048 & 0.278 & 0.302 & 0.245 \\
\hline 1900 & 0.051 & 0.343 & 0.364 & 0.327 \\
\hline 1909 & 0.038 & 0.312 & 0.339 & 0.270 \\
\hline 1970 & 0.039 & 0.317 & 0.323 & 0.294 \\
\hline $\begin{array}{l}1971 \\
1972\end{array}$ & $\begin{array}{l}0.038 \\
0.035\end{array}$ & $\begin{array}{l}0.289 \\
0.293\end{array}$ & $\begin{array}{l}0.337 \\
0.293\end{array}$ & $\begin{array}{l}0.264 \\
0.227\end{array}$ \\
\hline 1973 & 0.026 & 0.243 & 0.283 & 0.203 \\
\hline 1974 & 0.025 & 0.233 & 0.241 & 0.165 \\
\hline 1975 & 0.024 & 0.188 & 0.261 & 0.130 \\
\hline 1976 & 0.016 & 0.195 & 0.230 & 0.129 \\
\hline 1977 & 0.010 & 0.184 & 0.225 & 0.098 \\
\hline 1978 & 0.012 & 0.196 & 0.269 & 0.108 \\
\hline 1979 & 0.005 & 0.225 & 0.272 & 0.126 \\
\hline 1980 & 0.010 & 0.195 & 0.218 & 0.094 \\
\hline 1981 & 0.019 & 0.229 & 0.243 & 0.065 \\
\hline 1982 & 0.031 & 0.137 & 0.208 & 0.067 \\
\hline
\end{tabular}




\begin{tabular}{|c|c|c|c|}
\hline $35-39$ & $40-44$ & $45-49$ & Total \\
\hline • & $\bullet$ & $\bullet$ & - \\
\hline$\bullet$ & • & - & - \\
\hline$\bullet$ & $\bullet$ & $\bullet$ & $\bullet$ \\
\hline$\bullet$ & $\bullet$ & $\bullet$ & $\bullet$ \\
\hline$\bullet$ & $\bullet$ & $\bullet$ & $\bullet$ \\
\hline • & $\bullet$ & • & 4.507 \\
\hline • & • & $\bullet$ & 4.216 \\
\hline • & $\bullet$ & $\bullet$ & 5.866 \\
\hline$\bullet$ & - & - & 4.419 \\
\hline$\bullet$ & • & • & 5.106 \\
\hline$\bullet$ & $\bullet$ & - & 4.946 \\
\hline$\bullet$ & $\bullet$ & • & 4.795 \\
\hline - & • & • & 5.966 \\
\hline • & • & - & 5.469 \\
\hline$\bullet$ & - & - & 5.810 \\
\hline • & $\bullet$ & • & 5.094 \\
\hline$\bullet$ & $\bullet$ & $\bullet$ & 5.268 \\
\hline • & • & $\bullet$ & 5.408 \\
\hline • & • & • & 4.380 \\
\hline • & • & • & 2.583 \\
\hline 0.059 & • & • & 1.907 \\
\hline 0.072 & $\bullet$ & • & 2.271 \\
\hline 0.217 & - & - & 6.847 \\
\hline 0.256 & • & • & 7.572 \\
\hline 0.163 & 0.091 & 0.011 & 5.740 \\
\hline 0.222 & 0.091 & 0.011 & 6.474 \\
\hline 0.209 & 0.126 & 0.026 & 7.037 \\
\hline 0.211 & 0.077 & 0.004 & 5.821 \\
\hline 0.237 & 0.098 & 0.015 & 7.178 \\
\hline 0.220 & 0.098 & 0.016 & 6.463 \\
\hline 0.205 & 0.101 & 0.008 & 6.435 \\
\hline 0.208 & 0.088 & 0.009 & 0.165 \\
\hline 0.174 & 0.070 & 0.015 & 5.530 \\
\hline 0.143 & 0.067 & 0.007 & 4.873 \\
\hline 0.089 & 0.048 & 0.001 & 4.012 \\
\hline 0.080 & 0.041 & 0.009 & 3.666 \\
\hline 0.077 & 0.032 & 0.003 & 3.432 \\
\hline 0.062 & 0.031 & • & 3.045 \\
\hline 0.058 & 0.028 & 0.003 & 3.397 \\
\hline 0.047 & 0.023 & 0.005 & 3.519 \\
\hline 0.03 .3 & 0.012 & 0.004 & 2.826 \\
\hline 0.026 & 0.009 & • & 2.980 \\
\hline 0.016 & 0.015 & 0.002 & 2.379 \\
\hline
\end{tabular}


Basic Table 13.B. Urban Anhui Province (12): age-specific fertility

\begin{tabular}{|c|c|c|c|c|}
\hline $\begin{array}{l}\text { Birth year } \\
\text { of child }\end{array}$ & $15-19$ & $20-24$ & $25-29$ & $30-34$ \\
\hline 1940 & 0.047 & - & - & - \\
\hline 1941 & 0.110 & - & - & - \\
\hline 1942 & 0.038 & - & - & - \\
\hline 1943 & 0.085 & - & - & - \\
\hline 1944 & 0.025 & - & - & - \\
\hline 1945 & 0.023 & 0.154 & - & - \\
\hline 1946 & 0.043 & 0.135 & - & - \\
\hline 1947 & 0.020 & $0.23 \mathrm{e}$ & - & - \\
\hline 1948 & 0.053 & 0.109 & - & - \\
\hline 1949 & 0.008 & 0.267 & - & - \\
\hline 1950 & 0.030 & 0.128 & 0.236 & - \\
\hline 1951 & 0.050 & 0.258 & 0.258 & - \\
\hline 1952 & 0.094 & 0.235 & 0.225 & - \\
\hline 1953 & 0.058 & 0.272 & 0.170 & - \\
\hline 1954 & 0.091 & 0.295 & 0.343 & - \\
\hline 1955 & 0.063 & 0.290 & 0.233 & 0.225 \\
\hline 1956 & 0.067 & 0.262 & 0.280 & 0.172 \\
\hline 1957 & 0.080 & 0.307 & 0.304 & 0.250 \\
\hline 1958 & 0.065 & 0.283 & 0.193 & 0.230 \\
\hline 1959 & 0.043 & 0.202 & 0.295 & 0.203 \\
\hline 1960 & 0.054 & 0.139 & 0.201 & 0.140 \\
\hline 1961 & 0.027 & 0.113 & 0.113 & 0.086 \\
\hline 1962 & 0.063 & 0.281 & 0.321 & 0.343 \\
\hline 1903 & 0.109 & 0.394 & 0.356 & 0.324 \\
\hline 1964 & 0.041 & 0.334 & 0.258 & 0.191 \\
\hline 1965 & 0.046 & 0.248 & 0.292 & 0.231 \\
\hline 1966 & 0.016 & 0.198 & 0.253 & 0.099 \\
\hline 1967 & 0.014 & 0.283 & 0.214 & 0.167 \\
\hline 1908 & 0.006 & 0.230 & 0.239 & 0.189 \\
\hline 1969 & 0.024 & 0.143 & 0.300 & 0.167 \\
\hline 1970 & 0.004 & 0.194 & 0.209 & 0.188 \\
\hline 1971 & 0.011 & 0.130 & 0.225 & 0.113 \\
\hline 1972 & 0.007 & 0.129 & 0.210 & 0.121 \\
\hline 1973 & 0.007 & 0.067 & 0.153 & 0.097 \\
\hline 1974 & 0.003 & 0.097 & 0.174 & 0.092 \\
\hline 1975 & 0.019 & 0.073 & 0.176 & 0.047 \\
\hline 1976 & 0.012 & 0.088 & 0.163 & 0.063 \\
\hline 1977 & - & 0.071 & 0.123 & 0.042 \\
\hline 1978 & 0.008 & 0.079 & 0.223 & 0.077 \\
\hline 1979 & 0.004 & 0.057 & 0.276 & 0.071 \\
\hline 1980 & - & 0.070 & 0.191 & 0.074 \\
\hline 1981 & 0.010 & 0.085 & 0.169 & 0.041 \\
\hline 1982 & - & 0.071 & 0.156 & 0.013 \\
\hline
\end{tabular}


rates, $1940-82$

\begin{tabular}{|c|c|c|c|}
\hline $35-39$ & $40-44$ & $45-49$ & Total \\
\hline - & • & • & • \\
\hline - & - & $\bullet$ & • \\
\hline - & - & - & - \\
\hline$\bullet$ & - & - & - \\
\hline - & - & - & - \\
\hline - & - & - & $2 \cdot 643$ \\
\hline - & - & - & 2.764 \\
\hline - & - & - & 5.308 \\
\hline - & - & - & 3.915 \\
\hline - & - & - & 4.688 \\
\hline - & - & - & 4.260 \\
\hline - & - & - & 5.237 \\
\hline - & - & - & 5.962 \\
\hline - & - & • & 4.323 \\
\hline - & - & - & 6.426 \\
\hline - & - & - & 5.422 \\
\hline - & - & - & 5.550 \\
\hline - & - & - & 6.289 \\
\hline • & - & - & 5.573 \\
\hline - & - & - & 5.054 \\
\hline 0.106 & - & - & 3.540 \\
\hline 0.037 & - & - & 2.142 \\
\hline 0.150 & - & - & 6.730 \\
\hline 0.145 & - & - & 7.314 \\
\hline 0.114 & 0.094 & 0.020 & $5 \cdot 261$ \\
\hline 0.151 & 0.035 & 0.017 & 5.105 \\
\hline 0.086 & - & 0.016 & 3.342 \\
\hline 0.038 & 0.013 & - & 3.895 \\
\hline 0.132 & 0.049 & 0.013 & 4.278 \\
\hline 0.120 & 0.025 & - & 3.924 \\
\hline 0.060 & 0.023 & - & 3.393 \\
\hline 0.085 & • & 0.012 & 2.884 \\
\hline 0.040 & 0.010 & - & 2.580 \\
\hline 0.051 & - & - & 1.872 \\
\hline 0.014 & - & - & 1.901 \\
\hline 0.021 & 0.015 & 0.023 & 1.966 \\
\hline 0.027 & 0.007 & • & 1.801 \\
\hline 0.007 & 0.013 & - & 1.581 \\
\hline 0.006 & 0.007 & - & 2.006 \\
\hline$\bullet$ & 0.007 & - & 2.074 \\
\hline$\bullet$ & • & - & 1.677 \\
\hline 0.009 & - & - & 1.569 \\
\hline - & - & - & 1.198 \\
\hline
\end{tabular}


Basic Table 13.C. Rural Anhui Province (12): age-specific fertility rates,

\begin{tabular}{|c|c|c|c|c|}
\hline $\begin{array}{l}\text { Birth year } \\
\text { of child }\end{array}$ & $15-19$ & $20-24$ & $25-29$ & $30-34$ \\
\hline 1940 & 0.087 & - & - & - \\
\hline 1941 & 0.081 & - & $\bullet$ & - \\
\hline 1942 & 0.069 & - & - & - \\
\hline 1943 & 0.075 & - & - & - \\
\hline 1944 & 0.065 & - & - & • \\
\hline 1945 & 0.074 & 0.260 & - & - \\
\hline 1940 & 0.059 & 0.253 & - & - \\
\hline 1947 & 0.088 & 0.297 & - & • \\
\hline 1940 & 0.068 & 0.264 & - & - \\
\hline 1949 & 0.070 & 0.255 & - & - \\
\hline 1950 & 0.066 & 0.236 & 0.283 & • \\
\hline 1951 & 0.053 & 0.264 & 0.240 & - \\
\hline 1952 & 0.106 & 0.320 & 0.294 & - \\
\hline 1953 & 0.108 & 0.297 & 0.281 & - \\
\hline 1954 & 0.111 & 0.272 & 0.280 & .. \\
\hline 1955 & 0.084 & 0.251 & 0.243 & 0.231 \\
\hline 1956 & 0.068 & 0.290 & 0.226 & 0.240 \\
\hline 1957 & 0.077 & 0.260 & 0.271 & 0.229 \\
\hline 1958 & 0.053 & 0.220 & 0.198 & 0.174 \\
\hline 1959 & 0.027 & 0.094 & 0.118 & 0.111 \\
\hline 1960 & 0.014 & 0.063 & 0.093 & 0.093 \\
\hline 1901 & 0.016 & 0.096 & 0.149 & 0.096 \\
\hline 1902 & 0.041 & 0.299 & 0.400 & 0.314 \\
\hline 1963 & 0.062 & 0.393 & 0.374 & 0.314 \\
\hline 1964 & 0.054 & 0.231 & 0.294 & 0.263 \\
\hline 1965 & 0.048 & 0.337 & 0.314 & 0.294 \\
\hline 1966 & 0.055 & 0.374 & 0.351 & 0.330 \\
\hline 1907 & 0.051 & 0.277 & 0.316 & 0.257 \\
\hline 1968 & 0.056 & 0.351 & 0.384 & 0.347 \\
\hline 1909 & 0.040 & 0.325 & 0.343 & 0.280 \\
\hline 1970 & 0.044 & 0.326 & 0.336 & 0.311 \\
\hline 1971 & 0.042 & 0.303 & 0.348 & 0.288 \\
\hline 1972 & 0.039 & 0.309 & 0.299 & 0.244 \\
\hline 1973 & 0.030 & 0.262 & 0.293 & 0.220 \\
\hline 1974 & 0.029 & 0.250 & 0.246 & 0.175 \\
\hline 1975 & 0.026 & 0.204 & 0.268 & 0.140 \\
\hline 1976 & 0.017 & 0.211 & 0.235 & 0.135 \\
\hline 1977 & 0.012 & 0.202 & 0.229 & 0.103 \\
\hline 1978 & 0.013 & 0.217 & 0.274 & 0.110 \\
\hline 1979 & 0.005 & 0.260 & 0.272 & 0.131 \\
\hline 1980 & 0.011 & 0.224 & 0.222 & 0.095 \\
\hline 1981 & 0.020 & 0.267 & 0.254 & 0.071 \\
\hline 1982 & 0.034 & 0.151 & 0.217 & 0.073 \\
\hline
\end{tabular}




\begin{tabular}{|c|c|c|c|}
\hline - & - & - & - \\
\hline - & - & - & - \\
\hline - & - & - & - \\
\hline$\bullet$ & - & - & - \\
\hline - & - & - & - \\
\hline • & - & - & 4.709 \\
\hline - & - & - & 4.376 \\
\hline - & - & - & 5.929 \\
\hline - & - & - & 4.479 \\
\hline - & - & - & 5.156 \\
\hline - & - & - & 5.029 \\
\hline - & - & - & 4.740 \\
\hline - & - & - & $5 \cdot 966$ \\
\hline - & - & - & 5.613 \\
\hline - & - & - & 5.730 \\
\hline - & - & - & 5.051 \\
\hline - & - & - & 5.231 \\
\hline - & - & - & 5.292 \\
\hline - & - & - & 4.222 \\
\hline • & - & - & 2.261 \\
\hline 0.054 & - & - & 1.698 \\
\hline 0.076 & - & - & 2.237 \\
\hline 0.224 & - & - & 6.862 \\
\hline 0.268 & - & - & 7.603 \\
\hline 0.168 & 0.091 & 0.010 & 5.803 \\
\hline 0.230 & 0.097 & 0.010 & 6.645 \\
\hline 0.224 & 0.139 & 0.027 & 7.504 \\
\hline 0.228 & 0.03 .3 & 0.004 & 6.087 \\
\hline 0.253 & 0.103 & 0.015 & 7.549 \\
\hline 0.235 & 0.105 & 0.018 & 6.761 \\
\hline 0.228 & 0.109 & 0.009 & 6.820 \\
\hline 0.227 & 0.098 & 0.009 & 6.580 \\
\hline 0.195 & 0.078 & 0.017 & 5.904 \\
\hline 0.157 & 0.077 & 0.010 & 5.240 \\
\hline 0.101 & 0.055 & 0.001 & 4.288 \\
\hline 0.090 & 0.045 & 0.008 & 3.896 \\
\hline 0.085 & 0.0 .36 & 0.009 & 3.643 \\
\hline 0.071 & 0.033 & • & 3.247 \\
\hline 0.066 & 0.032 & 0.009 & 3.604 \\
\hline 0.053 & 0.026 & 0.006 & 3.762 \\
\hline 0.036 & 0.014 & 0.005 & 3.035 \\
\hline 0.028 & 0.011 & - & 3.257 \\
\hline 0.018 & 0.017 & 0.002 & 2.550 \\
\hline
\end{tabular}


Basic Table 14.A. Total Fujian Province (13): age-specific fertility rates,

\begin{tabular}{|c|c|c|c|c|}
\hline $\begin{array}{l}\text { Birth year } \\
\text { of child }\end{array}$ & $15-19$ & $20-24$ & $25-29$ & $30-34$ \\
\hline $\begin{array}{l}1940 \\
1941\end{array}$ & $\begin{array}{l}0.075 \\
0.080\end{array}$ & $\dot{\bullet}$ & - & • \\
\hline 1942 & 0.072 & 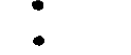 & $\bullet$ & 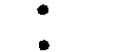 \\
\hline 1943 & 0.064 & - & - & - \\
\hline 1944 & 0.085 & - & - & - \\
\hline 1945 & 0.098 & 0.251 & - & - \\
\hline 1946 & 0.080 & 0.253 & - & - \\
\hline 1947 & 0.061 & 0.289 & - & - \\
\hline 1948 & 0.093 & 0.238 & - & - \\
\hline 1949 & 0.066 & 0.303 & - & - \\
\hline 1950 & 0.075 & 0.286 & 0.270 & - \\
\hline 1951 & 0.092 & 0.253 & 0.261 & - \\
\hline 1952 & 0.092 & 0.335 & 0.327 & - \\
\hline 1953 & 0.115 & 0.316 & 0.295 & • \\
\hline 1954 & 0.085 & 0.342 & 0.264 & $\bullet$ \\
\hline 1955 & 0.097 & 0.296 & 0.308 & 0.299 \\
\hline 1956 & 0.078 & 0.257 & 0.314 & 0.208 \\
\hline 1957 & 0.096 & 0.340 & 0.329 & 0.289 \\
\hline 1958 & 0.087 & 0.315 & 0.248 & 0.253 \\
\hline 1959 & 0.070 & 0.242 & 0.298 & 0.231 \\
\hline 1960 & 0.033 & 0.233 & 0.255 & 0.208 \\
\hline 1961 & 0.037 & 0.143 & 0.157 & 0.128 \\
\hline 1962 & 0.082 & 0.346 & 0.342 & 0.261 \\
\hline 1963 & 0.091 & 0.360 & 0.371 & 0.356 \\
\hline 1964 & 0.086 & 0.374 & 0.303 & 0.272 \\
\hline 1965 & 0.068 & 0.268 & 0.334 & 0.294 \\
\hline 1966 & 0.071 & 0.338 & 0.345 & 0.290 \\
\hline 1967 & 0.060 & 0.276 & 0.290 & 0.242 \\
\hline 1968 & 0.068 & 0.355 & 0.349 & 0.297 \\
\hline 1969 & 0.075 & 0.298 & 0.346 & 0.261 \\
\hline 1970 & 0.076 & 0.266 & 0.341 & 0.268 \\
\hline 1971 & 0.072 & 0.298 & 0.332 & 0.255 \\
\hline 1972 & 0.056 & 0.265 & 0.317 & 0.237 \\
\hline 1973 & 0.050 & 0.295 & 0.300 & 0.201 \\
\hline 1974 & 0.048 & 0.289 & 0.224 & 0.200 \\
\hline 1975 & 0.045 & 0.284 & 0.258 & 0.126 \\
\hline 1976 & 0.046 & 0.278 & 0.273 & 0.174 \\
\hline 1977 & 0.030 & 0.257 & 0.253 & 0.128 \\
\hline 1978 & 0.026 & 0.270 & 0.229 & 0.125 \\
\hline 1979 & 0.023 & 0.216 & 0.195 & 0.069 \\
\hline 1980 & 0.015 & 0.170 & 0.168 & 0.034 \\
\hline 1981 & 0.023 & 0.232 & 0.190 & 0.054 \\
\hline 1982 & 0.023 & 0.237 & 0.178 & 0.044 \\
\hline
\end{tabular}




\begin{tabular}{|c|c|c|c|}
\hline $35-39$ & $40-44$ & $45-49$ & Total \\
\hline - & - & - & - \\
\hline$\bullet$ & - & - & - \\
\hline - & - & - & - \\
\hline - & - & - & $\bullet$ \\
\hline - & - & - & - \\
\hline • & - & - & 4.207 \\
\hline - & - & - & 4.996 \\
\hline$\bullet$ & - & - & 5.041 \\
\hline - & - & - & 4.879 \\
\hline - & - & - & 5.756 \\
\hline - & - & - & 5.268 \\
\hline - & - & - & 5.039 \\
\hline - & - & - & 6.518 \\
\hline - & - & - & 5.944 \\
\hline - & - & - & 5.984 \\
\hline - & - & - & 6.226 \\
\hline - & - & - & 5.635 \\
\hline - & - & - & 6.728 \\
\hline$\bullet$ & - & - & 5.888 \\
\hline$\bullet$ & - & - & 5.524 \\
\hline 0.182 & - & - & 5.041 \\
\hline 0.070 & $\bullet$ & - & 2.896 \\
\hline 0.194 & - & - & 6.581 \\
\hline 0.298 & - & - & 8.054 \\
\hline 0.166 & 0.109 & 0.004 & 6.571 \\
\hline 0.201 & 0.112 & 0.014 & 6.455 \\
\hline 0.229 & 0.113 & 0.006 & 6.964 \\
\hline 0.159 & 0.065 & 0.009 & 5.510 \\
\hline 0.186 & 0.115 & - & 6.854 \\
\hline 0.193 & 0.068 & 0.014 & 6.264 \\
\hline 0.169 & 0.087 & 0.011 & 6.092 \\
\hline 0.194 & 0.089 & 0.008 & 6.245 \\
\hline 0.165 & 0.039 & 0.007 & $5 \cdot 439$ \\
\hline 0.132 & 0.077 & 0.007 & 5.304 \\
\hline 0.099 & 0.042 & 0.009 & 4.549 \\
\hline 0.087 & 0.039 & 0.004 & 4.216 \\
\hline 0.090 & 0.027 & 0.006 & 4.472 \\
\hline 0.107 & 0.030 & 0.002 & 4.028 \\
\hline 0.022 & 0.027 & 0.002 & 3.503 \\
\hline 0.039 & 0.021 & 0.004 & 2.838 \\
\hline 0.017 & 0.004 & 0.002 & 2.048 \\
\hline 0.043 & 0.016 & - & 2.788 \\
\hline 0.028 & 0.018 & - & 2.636 \\
\hline
\end{tabular}


Basic Table 14.B. Urban Fujian Province (13): age-specific fertility rates,

\begin{tabular}{|c|c|c|c|c|}
\hline $\begin{array}{l}\text { Birth year } \\
\text { of child }\end{array}$ & $15-19$ & $20-24$ & $25-29$ & $30-34$ \\
\hline 1940 & 0.065 & - & - & • \\
\hline 1941 & 0.041 & • & • & - \\
\hline 1942 & - & - & - & . \\
\hline 1943 & 0.087 & - & - & - \\
\hline 1944 & 0.017 & - & - & - \\
\hline 1945 & 0.140 & 0.194 & - & - \\
\hline 1946 & 0.070 & 0.224 & . & - \\
\hline 1947 & 0.058 & 0.192 & $\bullet$ & - \\
\hline 1948 & 0.099 & 0.226 & - & • \\
\hline 1949 & 0.119 & 0.201 & - & $\bullet$ \\
\hline 1950 & 0.018 & 0.350 & 0.301 & - \\
\hline 1951 & 0.157 & 0.262 & 0.245 & - \\
\hline 1952 & 0.108 & 0.251 & 0.173 & $\bullet$ \\
\hline 1953 & 0.094 & 0.355 & 0.313 & - \\
\hline 1954 & 0.164 & 0.357 & 0.234 & - \\
\hline 1955 & 0.112 & 0.277 & 0.298 & 0.237 \\
\hline 1956 & 0.079 & 0.255 & 0.314 & 0.265 \\
\hline 1957 & 0.039 & 0.355 & 0.405 & 0.230 \\
\hline 1958 & 0.060 & 0.376 & 0.276 & 0.174 \\
\hline 1959 & 0.039 & 0.345 & 0.317 & 0.234 \\
\hline 1960 & 0.061 & 0.351 & 0.370 & 0.175 \\
\hline 1961 & 0.039 & 0.126 & 0.216 & 0.210 \\
\hline 1962 & 0.125 & 0.393 & 0.308 & 0.212 \\
\hline 1963 & 0.046 & 0.282 & 0.376 & 0.138 \\
\hline 1964 & 0.028 & 0.197 & 0.329 & 0.158 \\
\hline 1965 & 0.050 & 0.284 & 0.240 & 0.166 \\
\hline 1960 & 0.012 & 0.137 & 0.110 & 0.118 \\
\hline 1967 & 0.022 & 0.142 & 0.137 & 0.093 \\
\hline 1968 & 0.011 & 0.168 & 0.261 & 0.141 \\
\hline 1969 & 0.021 & 0.236 & 0.217 & 0.082 \\
\hline 1970 & 0.037 & 0.088 & 0.142 & 0.080 \\
\hline 1971 & 0.016 & 0.185 & 0.216 & 0.047 \\
\hline 1972 & 0.043 & 0.132 & 0.036 & 0.079 \\
\hline 1973 & 0.026 & 0.185 & 0.198 & 0.080 \\
\hline 1974 & 0.019 & 0.103 & 0.083 & 0.039 \\
\hline 1975 & 0.026 & 0.158 & 0.139 & 0.041 \\
\hline 1976 & 0.014 & 0.073 & 0.231 & 0.059 \\
\hline 1977 & - & 0.072 & 0.099 & 0.036 \\
\hline 1978 & - & 0.053 & 0.131 & 0.015 \\
\hline 1979 & 0.007 & 0.019 & 0.145 & 0.042 \\
\hline 1980 & - & 0.013 & 0.111 & 0.050 \\
\hline 1981 & - & 0.048 & 0.131 & 0.023 \\
\hline 1982 & - & 0.084 & 0.125 & 0.044 \\
\hline
\end{tabular}


1940-82

\begin{tabular}{|c|c|c|c|}
\hline $35-39$ & $40-44$ & $45-49$ & Total \\
\hline - & • & - & - \\
\hline - & - & • & $\bullet$ \\
\hline - & - & - & - \\
\hline - & - & - & - \\
\hline$\bullet$ & - & • & - \\
\hline - & - & - & 3.682 \\
\hline - & - & - & 5.281 \\
\hline - & - & • & 3.607 \\
\hline - & - & - & 5.188 \\
\hline • & • & - & 4.980 \\
\hline - & - & - & 6.126 \\
\hline$\bullet$ & - & - & 5.797 \\
\hline - & - & - & 5.528 \\
\hline - & - & - & 6.692 \\
\hline - & - & - & 6.502 \\
\hline - & - & - & $6 \cdot 162$ \\
\hline - & - & - & 6.648 \\
\hline$\bullet$ & - & - & 6.880 \\
\hline - & - & - & 6.013 \\
\hline • & - & - & 6.226 \\
\hline 0.108 & - & - & 6.070 \\
\hline 0.020 & - & - & 3.409 \\
\hline 0.115 & - & - & 6.275 \\
\hline 0.157 & $\bullet$ & - & 5.792 \\
\hline 0.067 & 0.066 & - & 4.224 \\
\hline 0.053 & 0.022 & $\bullet$ & 4.073 \\
\hline 0.017 & 0.020 & - & 2.074 \\
\hline & 0.019 & $\bullet$ & 2.068 \\
\hline 0.020 & 0.070 & $\bullet$ & 3.351 \\
\hline 0.040 & - & - & 2.977 \\
\hline 0.037 & - & - & 1.921 \\
\hline 0.039 & 0.017 & - & 2.605 \\
\hline - & 0.019 & - & 1.545 \\
\hline - & • & - & 2.451 \\
\hline$\bullet$ & - & • & 1.226 \\
\hline 0.016 & - & - & 1.891 \\
\hline 0.032 & - & - & 2.042 \\
\hline - & - & • & 1.034 \\
\hline - & - & - & 0.995 \\
\hline$\bullet$ & - & - & 1.062 \\
\hline 0.020 & - & • & 0.973 \\
\hline - & - & - & 1.006 \\
\hline - & - & - & 1.269 \\
\hline
\end{tabular}


Basic Table 14.C. Rural Fujian Province (13): age-specific fertility rates,

\begin{tabular}{|c|c|c|c|c|}
\hline $\begin{array}{l}\text { Birth year } \\
\text { of child }\end{array}$ & $15-19$ & $20-24$ & $25-29$ & $30-34$ \\
\hline 1940 & 0.076 & - & - & • \\
\hline 1941 & 0.086 & - & - & - \\
\hline 1942 & 0.082 & - & - & - \\
\hline 1943 & 0.061 & - & - & - \\
\hline 1944 & 0.095 & - & - & $\bullet$ \\
\hline 1945 & 0.092 & 0.259 & - & - \\
\hline 1946 & 0.081 & 0.257 & - & - \\
\hline 1947 & 0.061 & 0.303 & - & - \\
\hline 1948 & 0.092 & 0.239 & - & - \\
\hline 1949 & 0.059 & 0.318 & - & - \\
\hline 1950 & 0.082 & 0.277 & 0.265 & - \\
\hline 1951 & 0.084 & 0.252 & $0.2 \in 3$ & - \\
\hline 1952 & 0.090 & 0.346 & 0.348 & - \\
\hline 1953 & 0.118 & 0.311 & 0.293 & - \\
\hline 1954 & 0.074 & 0.340 & 0.268 & - \\
\hline 1955 & 0.095 & 0.298 & 0.310 & 0.308 \\
\hline 1956 & 0.077 & 0.257 & 0.313 & 0.200 \\
\hline 1957 & 0.103 & 0.338 & 0.313 & 0.297 \\
\hline 1958 & 0.090 & 0.306 & 0.244 & 0.265 \\
\hline 1959 & 0.073 & 0.228 & 0.296 & 0.231 \\
\hline 1900 & 0.030 & 0.217 & 0.240 & 0.212 \\
\hline 1961 & 0.036 & 0.146 & 0.150 & 0.116 \\
\hline 1962 & 0.077 & 0.341 & 0.347 & 0.267 \\
\hline 1963 & 0.097 & 0.369 & 0.370 & 0.384 \\
\hline 1904 & 0.094 & 0.396 & 0.300 & 0.286 \\
\hline 1965 & 0.070 & 0.267 & 0.347 & 0.311 \\
\hline 1960 & 0.078 & 0.360 & 0.380 & 0.311 \\
\hline 1907 & 0.065 & 0.291 & 0.309 & 0.264 \\
\hline 1908 & 0.075 & 0.379 & 0.359 & 0.319 \\
\hline 1909 & 0.081 & 0.305 & 0.362 & 0.285 \\
\hline 1970 & 0.080 & 0.290 & 0.363 & 0.294 \\
\hline 1971 & 0.079 & 0.314 & 0.345 & 0.286 \\
\hline 1472 & 0.058 & 0.282 & 0.349 & 0.257 \\
\hline 1973 & 0.053 & 0.307 & 0.312 & 0.215 \\
\hline 1974 & 0.053 & 0.309 & 0.242 & 0.220 \\
\hline 1975 & 0.048 & 0.299 & 0.273 & 0.136 \\
\hline 1970 & 0.051 & 0.304 & 0.278 & 0.187 \\
\hline 1977 & 0.034 & 0.283 & 0.272 & 0.139 \\
\hline 1978 & 0.029 & 0.303 & 0.240 & 0.138 \\
\hline 1979 & 0.026 & 0.247 & 0.200 & 0.072 \\
\hline 1980 & 0.017 & 0.196 & 0.174 & 0.032 \\
\hline 1981 & 0.026 & 0.261 & 0.197 & 0.058 \\
\hline 1982 & 0.025 & 0.261 & 0.186 & 0.043 \\
\hline
\end{tabular}




\begin{tabular}{|c|c|c|c|}
\hline $35-39$ & $40-44$ & $45-49$ & Total \\
\hline - & • & • & • \\
\hline$\bullet$ & - & - & - \\
\hline - & - & - & - \\
\hline - & - & - & - \\
\hline - & - & - & - \\
\hline - & - & • & 4.286 \\
\hline - & - & - & 4.953 \\
\hline - & - & - & $5 \cdot 247$ \\
\hline - & - & - & 4.835 \\
\hline - & - & - & 5.868 \\
\hline$\dot{0}$ & - & - & 5.144 \\
\hline - & • & - & 4.933 \\
\hline - & - & - & 6.661 \\
\hline - & - & - & 5.836 \\
\hline - & - & - & 5.911 \\
\hline - & - & - & 6.235 \\
\hline - & - & - & 5.491 \\
\hline - & - & - & 6.706 \\
\hline - & $\bullet$ & - & 5.870 \\
\hline • & - & - & 5.427 \\
\hline 0.192 & - & - & 4.900 \\
\hline 0.077 & $\bullet$ & • & 2.827 \\
\hline 0.205 & - & - & 6.623 \\
\hline 0.318 & - & - & 8.363 \\
\hline 0.180 & 0.115 & 0.005 & 6.380 \\
\hline 0.222 & 0.125 & 0.016 & $6.7 A 8$ \\
\hline 0.259 & 0.126 & 0.007 & 7.609 \\
\hline 0.179 & 0.072 & 0.011 & 5.953 \\
\hline 0.207 & 0.122 & -. & 7.305 \\
\hline 0.212 & 0.078 & 0.016 & 6.687 \\
\hline 0.137 & 0.100 & 0.012 & 6.632 \\
\hline 0.213 & 0.099 & 0.009 & 6.723 \\
\hline 0.189 & 0.042 & 0.009 & 5.920 \\
\hline 0.151 & 0.087 & 0.008 & 5.668 \\
\hline 0.112 & 0.047 & 0.010 & 4.962 \\
\hline 0.097 & 0.044 & 0.005 & 4.511 \\
\hline 0.099 & 0.030 & 0.007 & 4.783 \\
\hline 0.120 & 0.034 & 0.002 & 4.419 \\
\hline 0.024 & 0.031 & 0.002 & 3.844 \\
\hline 0.044 & 0.024 & 0.005 & 3.091 \\
\hline 0.016 & 0.004 & 0.002 & 2.210 \\
\hline 0.047 & 0.019 & • & 3.040 \\
\hline 0.031 & 0.020 & - & 2.030 \\
\hline
\end{tabular}


Basic Table 15.A. Total Jiangxi Province (14): age-specific fertility rates,

\begin{tabular}{|c|c|c|c|c|}
\hline $\begin{array}{l}\text { Birth year } \\
\text { of child }\end{array}$ & 15-19 & $20-24$ & $25-29$ & $30-34$ \\
\hline 1940 & 0.095 & - & - & • \\
\hline 1941 & 0.088 & - & - & - \\
\hline 1942 & 0.084 & • & • & - \\
\hline 1943 & 0.091 & - & - & - \\
\hline 1944 & 0.095 & - & - & - \\
\hline 1945 & 0.087 & 0.231 & - & - \\
\hline 1946 & 0.097 & 0.285 & - & - \\
\hline 1947 & 0.104 & 0.277 & - & - \\
\hline 1948 & 0.091 & 0.284 & - & - \\
\hline 1949 & 0.125 & 0.309 & • & - \\
\hline 1950 & 0.122 & 0.329 & 0.283 & - \\
\hline 1951 & 0.142 & 0.277 & 0.282 & . \\
\hline 1952 & 0.145 & 0.270 & 0.275 & - \\
\hline 1953 & 0.124 & 0.302 & 0.282 & - \\
\hline 1954 & 0.122 & 0.288 & 0.277 & - \\
\hline 1955 & 0.140 & 0.265 & 0.274 & 0.200 \\
\hline 1956 & 0.114 & 0.236 & 0.275 & 0.242 \\
\hline 1957 & 0.112 & 0.319 & 0.284 & 0.271 \\
\hline 1958 & 0.098 & 0.326 & 0.282 & 0.279 \\
\hline 1959 & 0.062 & 0.241 & 0.236 & 0.191 \\
\hline 1960 & 0.058 & $\begin{array}{l}0.205 \\
0.233\end{array}$ & 0.251 & $\begin{array}{l}0.182 \\
0.160\end{array}$ \\
\hline 1462 & 0.087 & 0.295 & 0.321 & 0.245 \\
\hline 1963 & 0.106 & 0.354 & 0.344 & 0.306 \\
\hline 1964 & 0.089 & 0.337 & 0.332 & 0.259 \\
\hline 1905 & 0.096 & 0.336 & 0.277 & 0.261 \\
\hline 1966 & 0.099 & 0.330 & 0.369 & 0.356 \\
\hline 1967 & 0.079 & 0.309 & 0.340 & 0.294 \\
\hline 1908 & 0.107 & 0.358 & 0.373 & 0.303 \\
\hline 1969 & 0.082 & 0.305 & 0.350 & 0.256 \\
\hline 1970 & 0.079 & 0.327 & 0.327 & 0.331 \\
\hline 1971 & 0.091 & 0.308 & 0.310 & 0.246 \\
\hline 1972 & 0.070 & 0.303 & 0.334 & 0.258 \\
\hline 1973 & 0.070 & 0.316 & 0.332 & 0.271 \\
\hline 1974 & 0.064 & 0.334 & 0.317 & 0.271 \\
\hline 1975 & 0.045 & 0.313 & 0.324 & 0.228 \\
\hline 1970 & 0.079 & 0.291 & 0.304 & 0.219 \\
\hline 1977 & 0.054 & 0.291 & 0.275 & 0.220 \\
\hline 1978 & 0.043 & 0.278 & 0.273 & 0.176 \\
\hline 1979 & 0.033 & 0.278 & 0.239 & 0.125 \\
\hline 1980 & 0.032 & 0.269 & 0.185 & 0.070 \\
\hline 1981 & 0.034 & 0.292 & 0.184 & 0.097 \\
\hline 1982 & 0.040 & 0.229 & 0.177 & 0.000 \\
\hline
\end{tabular}


35-39

\begin{tabular}{|c|c|c|c|}
\hline - & - & - & - \\
\hline - & - & - & • \\
\hline - & - & - & - \\
\hline - & - & - & - \\
\hline - & - & - & • \\
\hline - & - & - & 4.269 \\
\hline - & - & - & 5.143 \\
\hline - & - & - & 5.131 \\
\hline - & - & - & 5.230 \\
\hline - & - & • & 5.349 \\
\hline - & - & - & 5.895 \\
\hline - & - & - & 5.520 \\
\hline - & - & - & 5.497 \\
\hline - & - & - & 5.907 \\
\hline - & - & - & 5.595 \\
\hline - & - & - & 5.523 \\
\hline - & - & - & 5.719 \\
\hline - & - & - & 6.077 \\
\hline - & - & - & 0.203 \\
\hline$\bullet$ & - & - & 4.700 \\
\hline 0.133 & • & - & 4.527 \\
\hline 0.139 & - & - & 4.162 \\
\hline 0.189 & - & - & 6.075 \\
\hline 0.270 & - & - & 7.276 \\
\hline 0.230 & 0.095 & 0.020 & 6.810 \\
\hline 0.223 & 0.104 & 0.011 & $\epsilon .536$ \\
\hline 0.244 & 0.119 & 0.012 & 7.645 \\
\hline 0.224 & 0.095 & 0.002 & 6.710 \\
\hline 0.248 & 0.114 & 0.012 & 7.578 \\
\hline 0.234 & 0.103 & 0.011 & 6.709 \\
\hline 0.218 & 0.104 & 0.009 & 6.970 \\
\hline 0.200 & 0.092 & 0.005 & 6.261 \\
\hline 0.217 & 0.031 & C. 009 & 0.390 \\
\hline 0.241 & 0.102 & 0.019 & 0.756 \\
\hline 0.201 & 0.090 & - & 0.383 \\
\hline 0.165 & 0.094 & 0.005 & 5.823 \\
\hline 0.198 & 0.088 & 0.007 & 5.925 \\
\hline 0.149 & 0.055 & 0.003 & 5.240 \\
\hline 0.105 & 0.055 & 0.010 & 4.746 \\
\hline 0.088 & 0.037 & 0.002 & 4.010 \\
\hline 0.031 & 0.013 & $0.0 O B$ & 3.032 \\
\hline 0.035 & 0.022 & 0.005 & 3.339 \\
\hline 0.050 & 0.010 & 0.003 & 2.872 \\
\hline
\end{tabular}

Total 
Basic Table 15.B. Urban Jiangxi Province (14): age-specific fertility

\begin{tabular}{|c|c|c|c|c|}
\hline $\begin{array}{l}\text { Birth year } \\
\text { of child }\end{array}$ & $15-19$ & $20-24$ & $25-29$ & $30-34$ \\
\hline 1940 & 0.062 & $\bullet$ & - & - \\
\hline 1941 & 0.022 & - & • & - \\
\hline 1942 & 0.082 & - & - & - \\
\hline 1943 & 0.078 & $\bullet$ & • & - \\
\hline 1944 & 0.154 & • & - & - \\
\hline 1945 & 0.126 & 0.165 & - & - \\
\hline 1940 & 0.116 & 0.305 & - & - \\
\hline 1947 & 0.114 & 0.185 & - & $\bullet$ \\
\hline $\begin{array}{l}1948 \\
1949\end{array}$ & $\begin{array}{l}0.054 \\
0.136\end{array}$ & $\begin{array}{l}0.388 \\
0.212\end{array}$ & : & - \\
\hline 1950 & 0.112 & 0.379 & 0.412 & - \\
\hline 1951 & 0.166 & 0.193 & 0.283 & • \\
\hline 1952 & 0.110 & 0.246 & 0.226 & - \\
\hline 1953 & 0.102 & 0.306 & 0.407 & - \\
\hline 1954 & 0.119 & 0.341 & 0.269 & $\bullet$ \\
\hline 1955 & 0.175 & 0.160 & 0.253 & 0.165 \\
\hline 1956 & 0.149 & 0.361 & 0.309 & 0.327 \\
\hline 1957 & 0.100 & 0.316 & 0.246 & 0.309 \\
\hline 1958 & 0.111 & 0.379 & 0.432 & 0.291 \\
\hline 1959 & 0.050 & 0.356 & 0.085 & 0.269 \\
\hline 1960 & 0.058 & 0.320 & 0.384 & 0.253 \\
\hline 1961 & 0.044 & 0.193 & 0.125 & 0.116 \\
\hline 1962 & 0.029 & 0.258 & 0.289 & 0.265 \\
\hline 1963 & 0.034 & 0.319 & 0.262 & 0.234 \\
\hline 1964 & 0.023 & 0.225 & 0.311 & 0.256 \\
\hline 1965 & 0.061 & 0.256 & 0.160 & 0.176 \\
\hline 1906 & 0.024 & 0.178 & 0.208 & 0.264 \\
\hline 1907 & 0.018 & 0.163 & 0.258 & 0.138 \\
\hline 1968 & 0.035 & 0.252 & 0.375 & 0.146 \\
\hline 1969 & 0.022 & 0.249 & 0.250 & 0.148 \\
\hline 1970 & 0.017 & 0.312 & 0.280 & 0.175 \\
\hline 1971 & 0.023 & 0.175 & 0.267 & 0.119 \\
\hline 1972 & 0.017 & 0.248 & 0.230 & 0.172 \\
\hline 1973 & 0.006 & 0.179 & 0.244 & 0.069 \\
\hline 1974 & 0.023 & 0.145 & 0.233 & 0.113 \\
\hline 1975 & 0.018 & 0.189 & 0.278 & 0.163 \\
\hline 1976 & 0.0 .33 & 0.087 & 0.247 & 0.167 \\
\hline 1977 & $\bullet$ & 0.188 & 0.242 & 0.115 \\
\hline 1978 & 0.022 & 0.168 & 0.162 & 0.084 \\
\hline 1979 & 0.015 & 0.086 & 0.173 & 0.031 \\
\hline 1980 & 0.007 & 0.085 & 0.117 & 0.054 \\
\hline 1981 & $\bullet$ & 0.093 & 0.121 & 0.018 \\
\hline 1982 & - & 0.135 & 0.123 & 0.037 \\
\hline
\end{tabular}


rates, $1940-82$

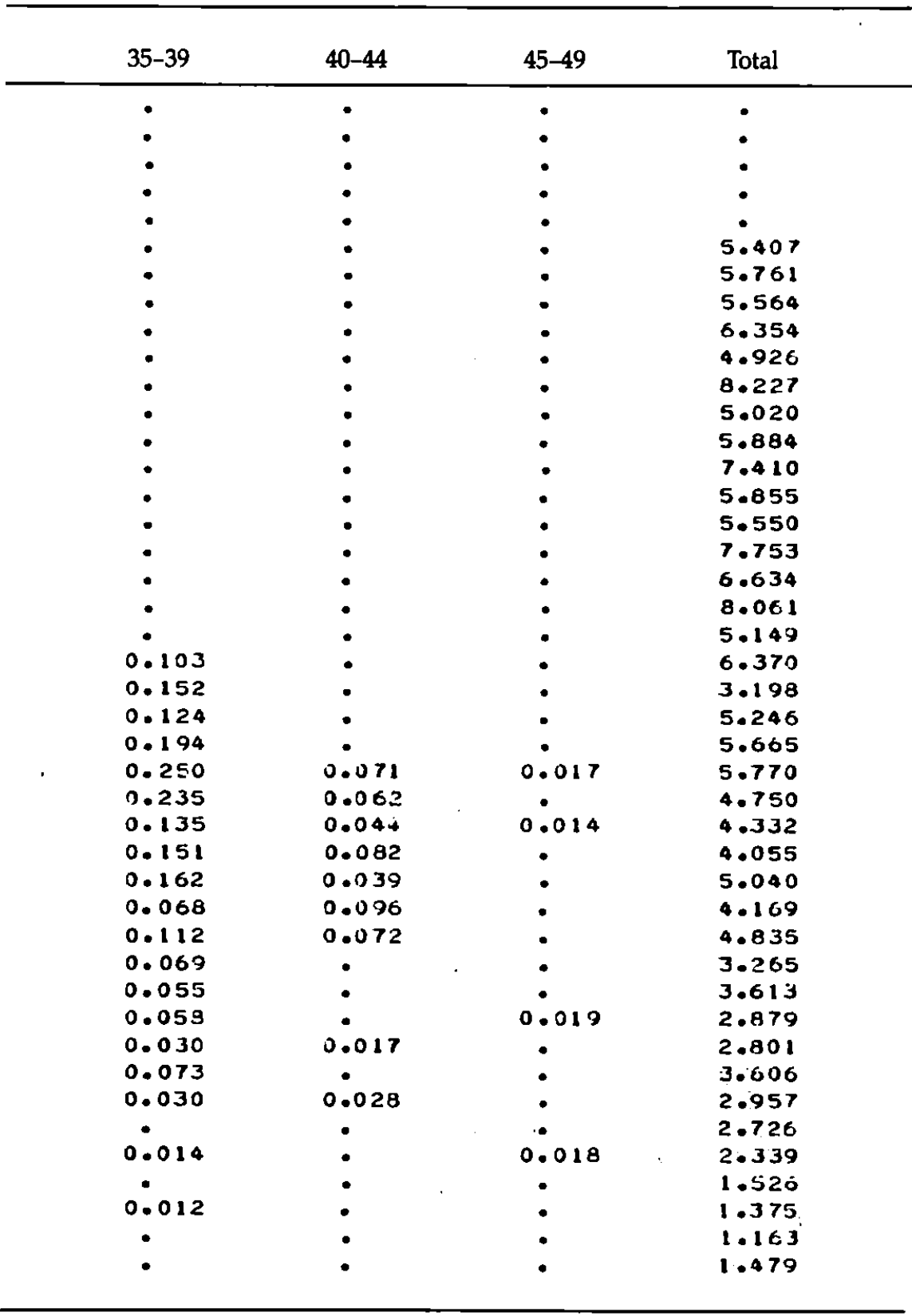


Basic Table 15.C. Rural Jiangxi Province (14): age-specific fertility rates,

\begin{tabular}{|c|c|c|c|c|}
\hline $\begin{array}{l}\text { Birth year } \\
\text { of child }\end{array}$ & 15-19 & $20-24$ & $25-29$ & $30-34$ \\
\hline 1940 & 0.099 & - & • & - \\
\hline 1941 & 0.095 & - & - & - \\
\hline 1942 & 0.085 & - & - & - \\
\hline 1943 & 0.093 & - & - & - \\
\hline 1984 & 0.089 & - & - & - \\
\hline 1945 & 0.083 & 0.237 & - & - \\
\hline 1946 & 0.096 & 0.284 & - & - \\
\hline 1947 & 0.103 & 0.285 & - & - \\
\hline 1948 & 0.094 & 0.274 & - & - \\
\hline 1949 & 0.123 & 0.318 & - & - \\
\hline 1950 & 0.124 & 0.324 & 0.270 & - \\
\hline 1951 & 0.139 & 0.285 & 0.282 & $\bullet$ \\
\hline 1952 & 0.150 & 0.272 & 0.279 & - \\
\hline 1953 & 0.127 & 0.308 & 0.270 & - \\
\hline 1954 & 0.122 & 0.283 & 0.278 & $\bullet$ \\
\hline 1955 & 0.136 & 0.277 & 0.276 & 0.203 \\
\hline 1956 & 0.110 & 0.277 & 0.272 & 0.234 \\
\hline 1957 & 0.114 & 0.320 & 0.288 & 0.268 \\
\hline 1958 & 0.096 & 0.320 & 0.267 & 0.278 \\
\hline 1959 & 0.064 & 0.228 & 0.252 & 0.183 \\
\hline 1960 & 0.058 & 0.191 & 0.236 & 0.175 \\
\hline 1901 & 0.058 & 0.238 & 0.204 & 0.164 \\
\hline 1962 & 0.097 & 0.300 & 0.32 .5 & 0.243 \\
\hline 1963 & 0.119 & 0.359 & 0.354 & 0.314 \\
\hline 1964 & 0.101 & 0.354 & 0.335 & 0.259 \\
\hline 1965 & 0.102 & 0.348 & 0.291 & 0.270 \\
\hline 1966 & 0.113 & 0.353 & 0.389 & 0.367 \\
\hline 1967 & 0.089 & 0.334 & 0.350 & 0.313 \\
\hline 1968 & 0.119 & 0.376 & 0.373 & 0.321 \\
\hline 1969 & 0.092 & 0.314 & 0.365 & 0.269 \\
\hline 1970 & 0.085 & 0.330 & 0.334 & 0.350 \\
\hline 1971 & 0.101 & 0.332 & 0.316 & 0.262 \\
\hline 1972 & 0.085 & 0.312 & 0.351 & 0.270 \\
\hline 1973 & 0.079 & 0.338 & 0.347 & 0.300 \\
\hline 1974 & 0.070 & 0.365 & 0.332 & 0.295 \\
\hline 1975 & 0.049 & 0.333 & 0.332 & 0.238 \\
\hline 1976 & 0.085 & 0.322 & 0.314 & 0.227 \\
\hline 1977 & 0.061 & 0.306 & 0.280 & 0.238 \\
\hline 1978 & 0.046 & 0.293 & 0.291 & 0.192 \\
\hline 1979 & 0.035 & 0.307 & 0.249 & 0.141 \\
\hline 1980 & 0.035 & 0.295 & 0.196 & 0.072 \\
\hline 1981 & 0.037 & 0.319 & 0.193 & 0.111 \\
\hline 1982 & 0.050 & 0.241 & 0.185 & 0.064 \\
\hline
\end{tabular}




\begin{tabular}{|c|c|c|c|}
\hline $35-39$ & $40-44$ & $45-49$ & Total \\
\hline - & - & - & - \\
\hline$\bullet$ & $\bullet$ & - & - \\
\hline - & - & - & - \\
\hline - & - & $\bullet$ & - \\
\hline - & - & - & • \\
\hline - & - & - & 4.134 \\
\hline - & $\bullet$ & - & 3.071 \\
\hline - & - & - & 5.081 \\
\hline - & - & - & 5.099 \\
\hline - & - & - & 5.956 \\
\hline - & - & - & 5.625 \\
\hline$\bullet$ & - & - & 5.579 \\
\hline$\bullet$ & - & - & 5.452 \\
\hline - & - & - & 5.732 \\
\hline - & $\bullet$ & - & 5.565 \\
\hline - & • & - & 5.520 \\
\hline - & - & - & 5.476 \\
\hline - & - & - & 6.010 \\
\hline - & - & - & 5.977 \\
\hline - & - & - & 4.645 \\
\hline $0.13 \epsilon$ & - & • & 4.299 \\
\hline 0.138 & - & - & 4.282 \\
\hline 0.195 & - & $\bullet$ & 6.182 \\
\hline 0.278 & • & $\bullet$ & 7.488 \\
\hline 0.228 & 0.098 & 0.020 & 6.971 \\
\hline 0.222 & 0.109 & 0.012 & 6.768 \\
\hline 0.254 & 0.126 & 0.011 & 8.073 \\
\hline 0.231 & 0.096 & 0.002 & 7.075 \\
\hline 0.257 & 0.121 & 0.014 & $7 \cdot 90.3$ \\
\hline 0.252 & 0.104 & 0.012 & 7.042 \\
\hline 0.22 .9 & 0.107 & 0.010 & 7.245 \\
\hline 0.216 & 0.101 & 0.000 & 6.676 \\
\hline 0.237 & 0.089 & 0.010 & 6.767 \\
\hline 0.262 & 0.112 & 0.019 & 7.291 \\
\hline 0.221 & 0.098 & - & 6.904 \\
\hline 0.177 & 0.094 & 0.006 & 6.144 \\
\hline 0.219 & 0.096 & 0.007 & 6.350 \\
\hline 0.169 & 0.062 & 0.004 & 5.603 \\
\hline 0.118. & 0.073 & 0.009 & 5.106 \\
\hline 0.102 & 0.041 & 0.002 & 4.385 \\
\hline 0.034 & 0.014 & 0.009 & 3.272 \\
\hline 0.040 & 0.025 & 0.005 & 3.654 \\
\hline 0.059 & 0.012 & 0.003 & 3.064 \\
\hline
\end{tabular}


Basic Table 16.A. Total Shandong Province (15): age-specific fertility

\begin{tabular}{|c|c|c|c|c|}
\hline $\begin{array}{l}\text { Birth year } \\
\text { of child }\end{array}$ & $15-19$ & $20-24$ & $25-29$ & $30-34$ \\
\hline 1940 & 0.063 & • & - & - \\
\hline 1941 & 0.073 & - & - & • \\
\hline 1942 & 0.063 & - & - & • \\
\hline 1943 & 0.051 & - & - & - \\
\hline 1944 & 0.049 & - & - & - \\
\hline 1945 & 0.060 & 0.268 & - & - \\
\hline 1946 & 0.056 & 0.236 & - & - \\
\hline 1947 & 0.065 & 0.234 & - & - \\
\hline 1948 & 0.056 & 0.233 & - & - \\
\hline 1949 & 0.083 & 0.228 & - & - \\
\hline 1950 & 0.063 & 0.217 & 0.243 & - \\
\hline 1951 & 0.075 & 0.262 & 0.246 & - \\
\hline 1952 & 0.067 & 0.267 & 0.278 & - \\
\hline 1953 & 0.074 & 0.263 & 0.280 & - \\
\hline 1954 & . 0.004 & 0.202 & 0.264 & - \\
\hline 1955 & 0.078 & 0.238 & 0.326 & 0.246 \\
\hline 1956 & 0.046 & 0.255 & 0.261 & 0.229 \\
\hline 1957 & 0.059 & 0.309 & 0.340 & 0.262 \\
\hline 1958 & 0.042 & 0.221 & 0.241 & 0.231 \\
\hline 1959 & 0.023 & 0.176 & 0.212 & 0.162 \\
\hline 1960 & 0.025 & 0.179 & 0.197 & 0.147 \\
\hline 1961 & 0.021 & $0.1+9$ & 0.188 & 0.135 \\
\hline 1962 & 0.034 & 0.261 & 0.314 & 0.262 \\
\hline 1963 & 0.041 & 0.339 & 0.394 & 0.321 \\
\hline 1904 & 0.026 & 0.251 & 0.255 & 0.244 \\
\hline 1965 & 0.022 & 0.234 & 0.340 & 0.241 \\
\hline 1906 & 0.021 & 0.283 & 0.313 & 0.280 \\
\hline 1967 & 0.012 & 0.214 & 0.277 & 0.202 \\
\hline 1968 & 0.016 & 0.263 & 0.327 & 0.246 \\
\hline 1409 & 0.011 & 0.235 & 0.322 & 0.238 \\
\hline 1970 & 0.019 & 0.263 & 0.344 & 0.303 \\
\hline 1971 & 0.007 & 0.234 & 0.322 & 0.226 \\
\hline 1972 & 0.010 & 0.190 & 0.275 & 0.208 \\
\hline 1973 & 0.008 & 0.171 & 0.249 & 0.166 \\
\hline 1974 & 0.008 & 0.165 & 0.232 & 0.141 \\
\hline 1975 & 0.005 & 0.141 & 0.236 & 0.131 \\
\hline 1976 & 0.007 & 0.136 & 0.235 & 0.109 \\
\hline 1977 & 0.004 & 0.107 & 0.227 & 0.086 \\
\hline 1978 & 0.003 & 0.101 & 0.211 & 0.074 \\
\hline 1979 & 0.002 & 0.122 & 0.244 & 0.089 \\
\hline 1980 & 0.001 & 0.098 & 0.203 & 0.047 \\
\hline 1901 & 0.003 & 0.141 & 0.217 & 0.048 \\
\hline 1982 & 0.004 & 0.149 & 0.199 & 0.049 \\
\hline
\end{tabular}


rates, $1940-82$

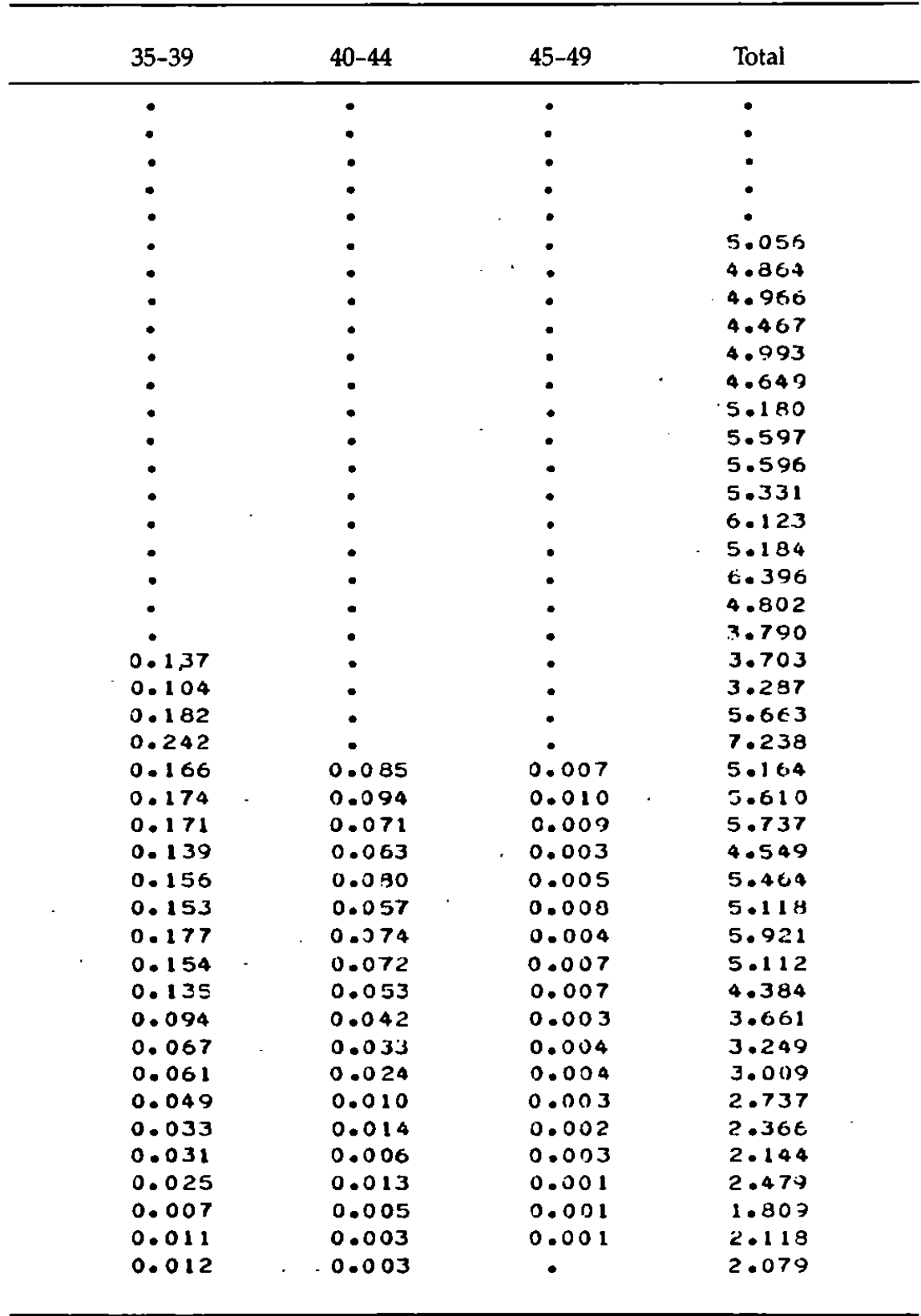


Basic Table 16.B. Urban Shandong Province (15): age-specific fertility

\begin{tabular}{|c|c|c|c|c|}
\hline $\begin{array}{l}\text { Birth year } \\
\text { of child }\end{array}$ & $15-19$ & $20-24$ & $25-29$ & $30-34$ \\
\hline 1940 & 0.059 & - & - & - \\
\hline 1941 & 0.063 & - & - & - \\
\hline 1942 & 0.063 & - & - & - \\
\hline 1943 & 0.046 & - & - & - \\
\hline 1944 & 0.044 & - & - & - \\
\hline 1945 & 0.026 & 0.138 & - & - \\
\hline 1946 & 0.035 & 0.244 & - & - \\
\hline 1947 & 0.063 & 0.260 & • & - \\
\hline 1948 & 0.035 & 0.119 & - & - \\
\hline 1949 & 0.035 & 0.221 & - & - \\
\hline 1950 & 0.053 & 0.165 & 0.295 & - \\
\hline 1951 & 0.053 & 0.300 & 0.217 & - \\
\hline 1952 & 0.067 & 0.305 & 0.251 & - \\
\hline 1953 & 0.043 & 0.191 & 0.273 & - \\
\hline 1954 & 0.042 & 0.303 & 0.301 & - \\
\hline 1955 & 0.054 & 0.213 & 0.382 & 0.197 \\
\hline 1956 & 0.030 & 0.228 & 0.265 & 0.262 \\
\hline 1957 & 0.056 & 0.300 & 0.385 & 0.269 \\
\hline 1958 & 0.012 & 0.242 & 0.313 & 0.301 \\
\hline 1959 & 0.015 & 0.203 & 0.182 & 0.248 \\
\hline 1900 & $\bullet$ & 0.206 & 0.310 & 0.156 \\
\hline 1901 & - & 0.111 & 0.158 & 0.150 \\
\hline 1962 & 0.028 & 0.169 & 0.308 & 0.242 \\
\hline 1903 & 0.036 & 0.308 & 0.397 & 0.356 \\
\hline 1964 & 0.020 & 0.180 & 0.296 & 0.165 \\
\hline 1965 & 0.009 & 0.179 & 0.340 & 0.168 \\
\hline 1966 & • & 0.070 & 0.162 & 0.132 \\
\hline 1907 & - & 0.098 & 0.270 & 0.125 \\
\hline 1968 & 0.007 & 0.108 & 0.222 & 0.164 \\
\hline 1969 & 0.006 & 0.113 & 0.314 & 0.110 \\
\hline 1970 & 0.005 & 0.214 & 0.344 & 0.143 \\
\hline 1971 & - & 0.106 & 0.252 & 0.121 \\
\hline 1972 & 0.004 & 0.083 & 0.252 & 0.101 \\
\hline 1973 & - & 0.066 & 0.193 & 0.123 \\
\hline 1974 & - & 0.057 & 0.164 & 0.075 \\
\hline 1975 & - & 0.005 & 0.233 & 0.105 \\
\hline 1970 & - & 0.031 & 0.196 & 0.042 \\
\hline 1977 & - & 0.023 & 0.165 & 0.042 \\
\hline 1978 & - & • & 0.206 & $\bullet$ \\
\hline 1979 & - & - & 0.137 & 0.051 \\
\hline 1980 & - & 0.007 & 0.165 & 0.037 \\
\hline 1981 & - & 0.014 & 0.201 & 0.016 \\
\hline 1982 & - & 0.065 & 0.212 & 0.029 \\
\hline
\end{tabular}


rates, $1940-82$

\begin{tabular}{|c|c|c|c|}
\hline $35-39$ & $40-44$ & $45-49$ & Total \\
\hline - & - & • & - \\
\hline - & - & $\bullet$ & $\bullet$ \\
\hline - & - & - & - \\
\hline - & - & - & - \\
\hline - & - & - & - \\
\hline - & - & - & 3.052 \\
\hline •. & - & - & 4.721 \\
\hline - & - & - & 4.448 \\
\hline - & - & - & 4.374 \\
\hline - & - & - & 5.777 \\
\hline - & - & - & 4.398 \\
\hline - & $\bullet$. & - & 4.933 \\
\hline - & - & - & 6.197 \\
\hline - & - & - & 5.416 \\
\hline - & - & - & $6 \cdot 388$ \\
\hline - & - & - & 5.764 \\
\hline - & - & - & 5.364 \\
\hline - & - & - & 7.110 \\
\hline - & - & - & 5.501 \\
\hline - & - & - & 4.730 \\
\hline 0.177 & • & - & 4.633 \\
\hline 0.081 & - & - & 2.715 \\
\hline 0.179 & - & - & 5.211 \\
\hline 0.237 & - & - & $7 \cdot 283$ \\
\hline 0.124 & 0.051 & - & 4.180 \\
\hline 0.052 & 0.030 & 0.014 & 3.962 \\
\hline 0.027 & 0.027 & 0.012 & 2.146 \\
\hline 0.027 & - & - & 2.599 \\
\hline 0.043 & $\bullet$ & • & 2.720 \\
\hline 0.026 & - & $\bullet$ & 2.845 \\
\hline 0.062 & 0.026 & - & 3.972 \\
\hline 0.018 & 0.009 & - & 2.530 \\
\hline 0.008 & - & - & 2.241 \\
\hline 0.009 & - & - & 1.954 \\
\hline 0.034 & 0.017 & - & 1.736 \\
\hline 0.045 & - & - & 2.237 \\
\hline 0.010 & 0.009 & • & 1.441 \\
\hline 0.011 & $\bullet$ & - & 1.200 \\
\hline • & $\bullet$ & $\bullet$ & 1.030 \\
\hline 0.015 & - & - & 1.013 \\
\hline 0.015 & - & - & 1.120 \\
\hline$\bullet$ & - & • & 1.152 \\
\hline - & - & - & 1.532 \\
\hline
\end{tabular}


Basic Table 16.C. Rural Shandong Province (15): age-specific fertility

\begin{tabular}{|c|c|c|c|c|}
\hline $\begin{array}{l}\text { Birth year } \\
\text { of child }\end{array}$ & $15-19$ & $20-24$ & $25-29$ & $30-34$ \\
\hline 1940 & 0.063 & • & - & - \\
\hline 1941 & 0.074 & - & - & • \\
\hline 1942 & 0.063 & - & - & • \\
\hline 1943 & 0.051 & - & • & - \\
\hline 1944 & 0.050 & - & - & - \\
\hline 1945 & 0.062 & 0.280 & - & - \\
\hline 1946 & 0.058 & 0.235 & - & - \\
\hline 1947 & 0.065 & 0.232 & - & - \\
\hline 1948 & 0.058 & 0.244 & - & - \\
\hline 1949 & 0.086 & 0.229 & - & - \\
\hline 1950 & .0 .004 & 0.221 & 0.239 & - \\
\hline 1951 & $=0.077$ & 0.259 & 0.249 & - \\
\hline 1952 & 0.067 & 0.264 & 0.281 & - \\
\hline 1953 & 0.076 & 0.268 & 0.280 & - \\
\hline 1954 & 0.066 & 0.260 & 0.261 & - \\
\hline 1955 & 0.080 & 0.293 & 0.322 & 0.250 \\
\hline 1956 & 0.047 & 0.257 & 0.261 & 0.226 \\
\hline 1957 & 0.059 & 0.309 & 0.337 & 0.261 \\
\hline 1958 & 0.044 & 0.219 & 0.236 & 0.224 \\
\hline 1959 & 0.023 & 0.173 & 0.214 & 0.155 \\
\hline 1960 & 0.026 & 0.177 & 0.189 & 0.146 \\
\hline 1961 & 0.022 & 0.151 & 0.190 & 0.138 \\
\hline 1962 & 0.035 & 0.267 & 0.315 & 0.264 \\
\hline 1903 & 0.041 & 0.340 & 0.394 & 0.319 \\
\hline 1964 & 0.026 & 0.254 & 0.251 & 0.250 \\
\hline 1965 & 0.023 & 0.236 & 0.347 & 0.246 \\
\hline 1960 & 0.022 & 0.291 & 0.323 & 0.290 \\
\hline 1967 & 0.013 & 0.219 & 0.277 & 0.208 \\
\hline 1968 & 0.016 & 0.270 & 0.332 & 0.252 \\
\hline 1969 & 0.012 & 0.241 & 0.322 & 0.248 \\
\hline 1970 & 0.020 & $0.2 \epsilon 6$ & 0.344 & 0.315 \\
\hline 1971 & 0.008 & 0.241 & 0.325 & 0.233 \\
\hline 1972 & 0.010 & 0.196 & 0.275 & 0.215 \\
\hline 1973 & 0.009 & 0.177 & 0.251 & 0.168 \\
\hline 1974 & 0.009 & 0.171 & 0.236 & 0.144 \\
\hline 1975 & 0.006 & 0.146 & 0.236 & 0.132 \\
\hline 1976 & 0.008 & 0.143 & 0.238 & 0.111 \\
\hline 1977 & 0.004 & 0.114 & 0.231 & 0.088 \\
\hline 1978 & 0.004 & 0.110 & 0.211 & 0.077 \\
\hline 1979 & 0.003 & 0.134 & 0.250 & 0.091 \\
\hline 1980 & 0.001 & 0.108 & 0.205 & 0.048 \\
\hline 1981 & 0.003 & 0.155 & 0.218 & 0.050 \\
\hline 1982 & 0.004 & 0.158 & 0.198 & 0.051 \\
\hline
\end{tabular}


rates, $1940-82$

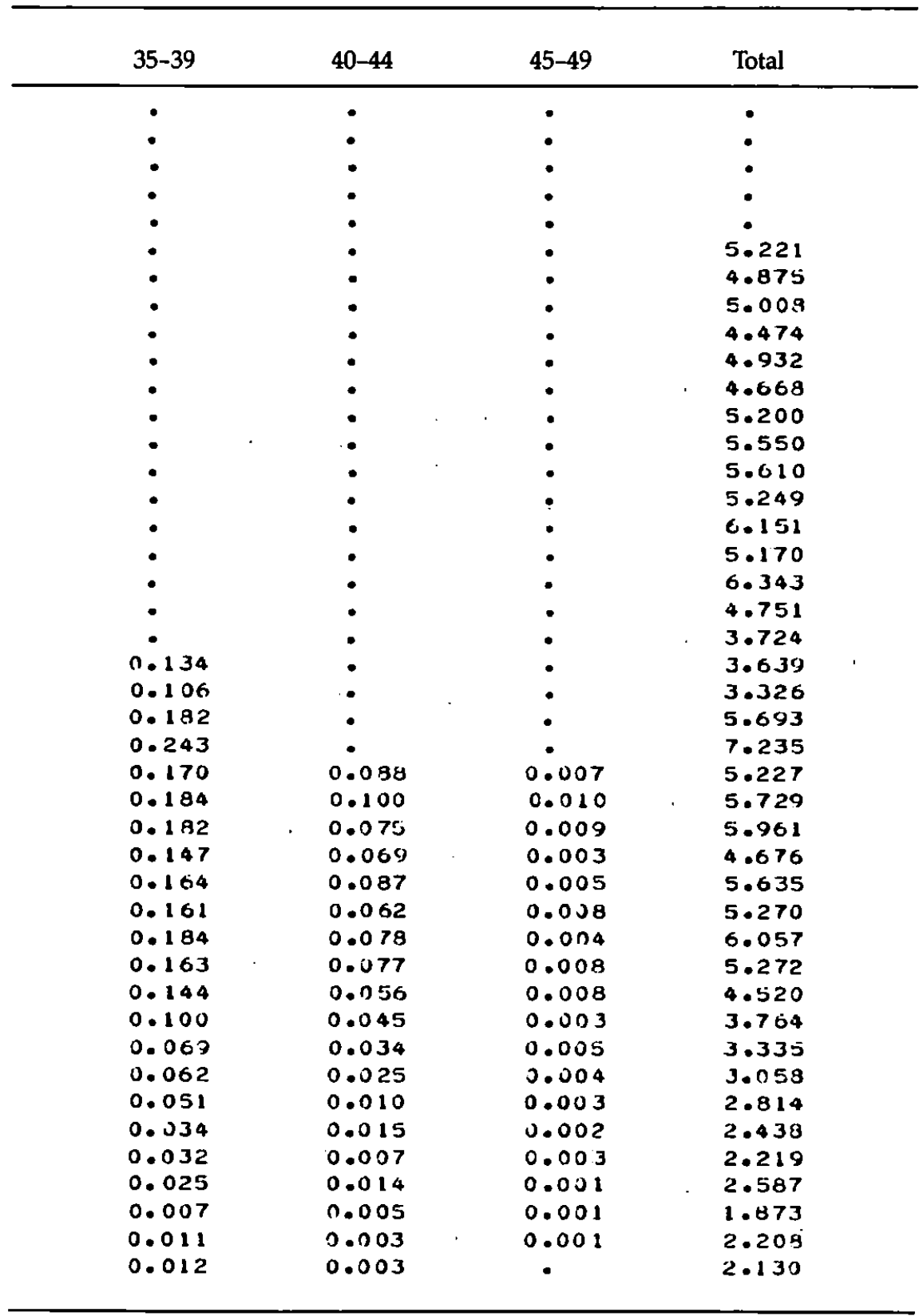


Basic Table 17.A. Total Henan Province (16): age-specific fertility rates,

\begin{tabular}{|c|c|c|c|c|}
\hline $\begin{array}{l}\text { Birth year } \\
\text { of child }\end{array}$ & $15-19$ & $20-24$ & $25-29$ & $30-34$ \\
\hline 1940 & 0.070 & - & - & $\bullet$ \\
\hline 1941 & 0.066 & - & - & - \\
\hline 1942 & 0.057 & - & - & . \\
\hline 1943 & 0.048 & - & - & - \\
\hline 1944 & 0.049 & - & - & - \\
\hline 1945 & 0.066 & 0.234 & - & - \\
\hline 1946 & 0.080 & 0.229 & . & - \\
\hline 1947 & 0.061 & 0.249 & - & - \\
\hline 1948 & 0.060 & 0.257 & - & - \\
\hline 1949 & 0.074 & 0.235 & - & - \\
\hline 1950 & 0.065 & 0.261 & 0.252 & - \\
\hline 1951 & 0.092 & 0.272 & 0.246 & - \\
\hline 1952 & 0.109 & 0.296 & 0.283 & - \\
\hline 1953 & 0.084 & 0.275 & 0.243 & - \\
\hline 1954 & 0.084 & 0.273 & 0.282 & - \\
\hline 1955 & 0.085 & 0.285 & 0.270 & 0.250 \\
\hline 1956 & 0.070 & 0.255 & 0.226 & 0.214 \\
\hline 1957 & 0.074 & 0.281 & 0.275 & 0.236 \\
\hline 1958 & 0.039 & 0.209 & 0.226 & 0.214 \\
\hline 1959 & 0.023 & 0.160 & 0.187 & 0.162 \\
\hline 1960 & 0.027 & 0.151 & 0.165 & 0.135 \\
\hline 1961 & 0.019 & 0.134 & 0.153 & 0.116 \\
\hline 1962 & 0.034 & 0.276 & 0.319 & 0.257 \\
\hline 1963 & 0.047 & 0.380 & 0.402 & 0.354 \\
\hline 1964 & 0.045 & 0.249 & 0.297 & 0.264 \\
\hline 1965 & 0.041 & 0.280 & 0.300 & 0.291 \\
\hline 1906 & 0.036 & 0.318 & 0.321 & 0.296 \\
\hline 1967 & 0.030 & 0.266 & 0.305 & 0.270 \\
\hline 1968 & 0.024 & 0.317 & 0.362 & 0.304 \\
\hline 1969 & 0.027 & 0.259 & 0.302 & 0.269 \\
\hline 1970 & 0.026 & 0.258 & 0.341 & 0.281 \\
\hline 1971 & 0.021 & 0.255 & 0.324 & 0.268 \\
\hline 1972 & 0.017 & 0.225 & 0.325 & 0.251 \\
\hline 1973 & 0.016 & 0.201 & 0.291 & 0.230 \\
\hline 1974 & 0.013 & 0.215 & 0.299 & 0.219 \\
\hline 1975 & 0.009 & 0.158 & 0.242 & 0.155 \\
\hline 1970 & 0.007 & 0.149 & 0.239 & 0.138 \\
\hline 1977 & 0.004 & 0.145 & 0.238 & 0.128 \\
\hline 1978 & 0.004 & 0.133 & 0.253 & 0.154 \\
\hline 1979 & 0.002 & 0.1 .52 & 0.273 & 0.136 \\
\hline 1980 & 0.003 & 0.134 & 0.228 & 0.081 \\
\hline 1981 & 0.005 & 0.154 & 0.259 & 0.099 \\
\hline 1982 & 0.007 & 0.157 & 0.210 & 0.072 \\
\hline
\end{tabular}




\begin{tabular}{|c|c|c|c|}
\hline $35-39$ & $40-44$ & $45-49$ & Total \\
\hline - & - & - & - \\
\hline - & - & $\bullet$ & • \\
\hline - & $\bullet$ & - & - \\
\hline - & - & $\bullet$ & - \\
\hline - & - & - & $\bullet$ \\
\hline - & - & - & 4.608 \\
\hline - & - & - & 4.524 \\
\hline - & - & - & 5.205 \\
\hline - & - & - & 4.563 \\
\hline - & - & - & 4.723 \\
\hline - & - & - & 4.997 \\
\hline - & - & - & 5.358 \\
\hline • & - & - & 5.795 \\
\hline - & - & - & 5.278 \\
\hline - & - & - & 5.626 \\
\hline - & - & - & 5.657 \\
\hline - & - & - & 5.020 \\
\hline - & - & - & 5.670 \\
\hline • & - & - & 4.510 \\
\hline$\bullet$ & - & • & 3.570 \\
\hline 0.126 & - & - & 3.281 \\
\hline 0.068 & - & - & 2.618 \\
\hline 0.200 & - & - & 5.887 \\
\hline 0.308 & . & . & 8.068 \\
\hline 0.195 & 0.095 & 0.015 & 5.803 \\
\hline 0.197 & 0.107 & 0.010 & 6.129 \\
\hline 0.257 & 0.118 & 0.012 & 6.791 \\
\hline 0.204 & 0.111 & 0.006 & 5.952 \\
\hline 0.255 & 0.135 & 0.008 & 7.025 \\
\hline 0.223 & 0.108 & 0.019 & 6.035 \\
\hline $0.24 B$ & 0.103 & 0.019 & 6.381 \\
\hline 0.207 & 0.097 & 0.009 & 5.897 \\
\hline 0.204 & 0.091 & 0.016 & 5.651 \\
\hline 0.163 & 0.080 & 0.005 & 4.926 \\
\hline 0.144 & 0.072 & 0.007 & 4.846 \\
\hline 0.093 & 0.047 & 0.004 & 3.534 \\
\hline 0.07 .3 & 0.038 & 0.001 & 3.226 \\
\hline 0.053 & 0.029 & 0.004 & 3.004 \\
\hline 0.072 & 0.022 & 0.005 & 3.208 \\
\hline 0.052 & 0.022 & 0.001 & 3.192 \\
\hline 0.030 & 0.003 & 0.002 & 2.428 \\
\hline 0.034 & 0.009 & 0.002 & 2.303 \\
\hline 0.026 & 0.007 & 0.001 & 2.400 \\
\hline
\end{tabular}


Basic Table 17.B. Urban Henan Province (16): age-specific fertility

\begin{tabular}{|c|c|c|c|c|}
\hline $\begin{array}{l}\text { Birth year } \\
\text { of child }\end{array}$ & $15-19$ & $20-24$ & $25-29$ & $30-34$ \\
\hline 1940 & 0.095 & - & - & - \\
\hline 1941 & 0.031 & - & - & - \\
\hline 1942 & 0.038 & - & - & - \\
\hline 1943 & 0.046 & - & - & - \\
\hline 1944 & 0.033 & - & - & - \\
\hline 1945 & 0.024 & 0.226 & - & $\bullet$ \\
\hline 1946 & 0.075 & 0.224 & - & - \\
\hline 1947 & 0.058 & 0.162 & $\bullet$ & - \\
\hline 1948 & 0.056 & 0.192 & - & - \\
\hline 1949 & 0.076 & 0.284 & - & - \\
\hline 1950 & 0.033 & 0.257 & 0.202 & - \\
\hline 1951 & 0.102 & 0.225 & 0.184 & - \\
\hline 1952 & 0.105 & 0.292 & 0.323 & - \\
\hline 1953 & 0.058 & 0.200 & 0.201 & - \\
\hline 1954 & 0.064 & 0.271 & 0.268 & - \\
\hline 1955 & 0.067 & 0.222 & 0.217 & 0.250 \\
\hline 1956 & 0.045 & 0.180 & 0.233 & 0.163 \\
\hline 1957 & 0.075 & 0.232 & 0.267 & 0.190 \\
\hline 1958 & 0.026 & 0.206 & 0.272 & 0.146 \\
\hline 1959 & 0.027 & 0.216 & 0.188 & 0.195 \\
\hline 1960 & 0.045 & 0.195 & 0.215 & 0.121 \\
\hline 1961 & 0.023 & 0.140 & 0.150 & 0.142 \\
\hline 1962 & 0.032 & 0.257 & 0.242 & 0.208 \\
\hline 1963 & 0.069 & 0.355 & 0.317 & 0.336 \\
\hline 1964 & 0.013 & 0.255 & 0.314 & 0.222 \\
\hline 1965 & 0.055 & 0.335 & 0.240 & 0.189 \\
\hline 1966 & 0.013 & 0.215 & 0.189 & 0.180 \\
\hline 1967 & - & 0.227 & 0.286 & 0.132 \\
\hline 1968 & 0.006 & 0.234 & 0.308 & 0.197 \\
\hline 1969 & 0.011 & 0.174 & 0.260 & 0.221 \\
\hline 1970 & 0.005 & 0.198 & 0.315 & 0.191 \\
\hline 1971 & 0.009 & 0.126 & 0.203 & 0.140 \\
\hline 1972 & $\bullet$ & 0.175 & 0.233 & 0.191 \\
\hline 1973 & 0.004 & 0.100 & 0.234 & 0.147 \\
\hline 1974 & 0.004 & 0.094 & 0.221 & 0.159 \\
\hline 1975 & 0.004 & 0.035 & 0.239 & 0.120 \\
\hline 1976 & 0.004 & $0.06 \mathrm{G}$ & 0.140 & 0.116 \\
\hline 1977 & - & 0.049 & 0.194 & 0.058 \\
\hline 1978 & $\bullet$ & 0.048 & 0.176 & 0.103 \\
\hline 1979 & - & 0.063 & 0.193 & 0.134 \\
\hline 1980 & - & 0.040 & 0.182 & 0.034 \\
\hline 1981 & 0.002 & 0.040 & 0.183 & 0.047 \\
\hline 1982 & - & 0.061 & 0.174 & 0.026 \\
\hline
\end{tabular}


rates, $1940-82$

\begin{tabular}{|c|c|c|c|}
\hline $35-39$ & $40-44$ & $45-49$ & Total \\
\hline - & • & - & - \\
\hline$\bullet$ & - & - & - \\
\hline - & - & - & - \\
\hline - & • & - & - \\
\hline - & - & - & - \\
\hline - & - & - & 4.363 \\
\hline - & - & - & 4.375 \\
\hline • & - & - & 4.552 \\
\hline - & • & - & 3.809 \\
\hline - & $\bullet$ & - & 4.544 \\
\hline • & - & - & 4.812 \\
\hline - & - & - & 4.474 \\
\hline - & - & - & 6.780 \\
\hline - & - & - & 3.852 \\
\hline - & - & - & 5.813 \\
\hline - & - & - & 4.931 \\
\hline - & - & - & 4.326 \\
\hline - & - & - & 5.141 \\
\hline - & - & - & $4 \cdot 375$ \\
\hline • & - & - & 4.413 \\
\hline 0.131 & - & - & 4.022 \\
\hline 0.071 & - & - & 2.751 \\
\hline 0.181 & - & - & 4.996 \\
\hline 0.293 & - & - & 7.278 \\
\hline 0.187 & 0.013 & 0.039 & 5.219 \\
\hline 0.088 & 0.071 & • & 4.892 \\
\hline 0.158 & 0.061 & 0.044 & 4.304 \\
\hline 0.092 & 0.057 & • & 3.905 \\
\hline 0.152 & 0.18 .3 & • & 5.397 \\
\hline 0.160 & 0.065 & 0.026 & 4.583 \\
\hline 0.085 & 0.024 & 0.024 & 4.203 \\
\hline 0.1 .32 & 0.058 & $\bullet$ & 3.342 \\
\hline 0.074 & 0.033 & 0.019 & 3.624 \\
\hline 0.072 & 0.003 & - & 2.828 \\
\hline 0.088 & 0.021 & - & 2.938 \\
\hline 0.027 & 0.026 & - & 2.254 \\
\hline 0.041 & 0.012 & • & 1.907 \\
\hline 0.033 & 0.026 & - & 1.807 \\
\hline 0.035 & 0.010 & 3.003 & 1.900 \\
\hline 0.018 & 0.010 & - & 2.093 \\
\hline & $\bullet$ & - & 1.281 \\
\hline 0.017 & - & - & 1.440 \\
\hline - & - & - & 1.305 \\
\hline
\end{tabular}


Basic Table 17.C. Rural Henan Province (16): age-specific fertility rates,

\begin{tabular}{|c|c|c|c|c|}
\hline $\begin{array}{l}\text { Birth year } \\
\text { of child }\end{array}$ & $15-19$ & $20-24$ & $25-29$ & $30-34$ \\
\hline 1940 & 0.068 & - & - & - \\
\hline 1941 & 0.069 & - & - & - \\
\hline 1942 & 0.058 & - & - & - \\
\hline 1943 & 0.048 & $\bullet$ & $\bullet$ & - \\
\hline 1944 & 0.051 & - & - & - \\
\hline 1945 & 0.070 & 0.235 & - & - \\
\hline 1946 & 0.081 & 0.230 & - & - \\
\hline 1947 & 0.061 & 0.257 & - & - \\
\hline 1948 & 0.061 & 0.262 & - & - \\
\hline 1949 & 0.074 & 0.230 & - & - \\
\hline 1950 & 0.069 & 0.262 & 0.256 & - \\
\hline 1951 & 0.091 & 0.277 & 0.251 & - \\
\hline 1952 & 0.109 & 0.296 & 0.280 & $\bullet$ \\
\hline $\begin{array}{l}1953 \\
1954\end{array}$ & $\begin{array}{l}0.088 \\
0.087\end{array}$ & $\begin{array}{l}0.283 \\
0.273\end{array}$ & $\begin{array}{l}0.247 \\
0.283\end{array}$ & • \\
\hline 1955 & 0.098 & 0.292 & 0.276 & 0.250 \\
\hline 1950 & 0.074 & 0.264 & 0.225 & 0.218 \\
\hline 1957 & 0.074 & 0.288 & 0.276 & 0.240 \\
\hline 1958 & 0.042 & 0.210 & 0.221 & 0.220 \\
\hline 1959 & 0.023 & 0.152 & 0.187 & 0.158 \\
\hline 1960 & 0.024 & 0.143 & 0.159 & 0.136 \\
\hline 1961 & 0.019 & 0.133 & 0.153 & 0.113 \\
\hline 1962 & 0.034 & 0.280 & 0.328 & 0.262 \\
\hline 1963 & 0.046 & 0.384 & 0.415 & 0.356 \\
\hline 1964 & 0.047 & 0.248 & 0.294 & 0.269 \\
\hline 1905 & 0.040 & 0.272 & 0.310 & 0.302 \\
\hline 1966 & 0.037 & 0.330 & 0.346 & 0.310 \\
\hline 1967 & 0.032 & 0.269 & 0.309 & 0.288 \\
\hline 1908 & 0.025 & 0.324 & 0.372 & 0.320 \\
\hline 1969 & 0.028 & 0.265 & 0.309 & 0.276 \\
\hline 1970 & 0.028 & 0.262 & 0.345 & 0.296 \\
\hline 1971 & 0.022 & 0.263 & 0.339 & 0.292 \\
\hline 1972 & 0.019 & 0.228 & 0.334 & 0.263 \\
\hline 1973 & 0.017 & 0.208 & 0.296 & 0.244 \\
\hline 1974 & 0.014 & 0.223 & 0.305 & 0.229 \\
\hline 1975 & 0.009 & 0.166 & 0.242 & 0.160 \\
\hline 1976 & 0.007 & 0.155 & 0.245 & 0.141 \\
\hline 1977 & 0.005 & 0.153 & 0.240 & 0.135 \\
\hline 1978 & 0.004 & 0.140 & 0.258 & 0.158 \\
\hline 1979 & 0.002 & 0.160 & 0.278 & 0.137 \\
\hline 1980 & 0.004 & 0.143 & 0.231 & 0.084 \\
\hline 1981 & 0.005 & 0.168 & 0.265 & 0.102 \\
\hline 1982 & 0.008 & 0.169 & 0.213 & 0.075 \\
\hline
\end{tabular}




\begin{tabular}{|c|c|c|c|}
\hline $35-39$ & $40-44$ & $45-49$ & Total \\
\hline - & - & - & $\bullet$ \\
\hline - & - & - & - \\
\hline - & - & - & - \\
\hline - & • & - & - \\
\hline - & - & - & $\bullet$ \\
\hline - & $\bullet$ & - & 4.628 \\
\hline$\bullet$ & - & - & 4.536 \\
\hline - & • & - & 5.259 \\
\hline - & - & - & 4.627 \\
\hline - & - & - & 4.739 \\
\hline - & - & - & 5.013 \\
\hline - & - & - & 5.439 \\
\hline - & • & • & 5.700 \\
\hline - & - & - & 5.421 \\
\hline - & - & - & 5.607 \\
\hline • & . & - & 5.736 \\
\hline - & . & . & 5.057 \\
\hline - & - & - & 5.730 \\
\hline - & - & - & 4.525 \\
\hline - & • & • & 3.473 \\
\hline 0.126 & - & - & 3.196 \\
\hline 0.067 & - & - & 2.603 \\
\hline 0.208 & - & - & 5.985 \\
\hline 0.309 & - & - & 8.154 \\
\hline 0.196 & 0.101 & 0.014 & 5.845 \\
\hline 0.208 & 0.110 & 0.011 & 6.266 \\
\hline 0.266 & 0.123 & 0.010 & 7.113 \\
\hline 0.215 & 0.115 & 0.000 & 6.169 \\
\hline 0.265 & 0.131 & 0.009 & 7.225 \\
\hline 0.230 & 0.113 & 0.019 & 6.193 \\
\hline 0.267 & 0.111 & 0.019 & 6.636 \\
\hline 0.216 & 0.100 & 0.009 & 6.206 \\
\hline 0.221 & 0.097 & 0.016 & 5.888 \\
\hline 0.176 & 0.087 & 0.005 & 5.166 \\
\hline 0.153 & 0.077 & 0.007 & 5.043 \\
\hline 0.105 & 0.049 & 0.004 & 3.676 \\
\hline 0.079 & 0.041 & 0.002 & 3.350 \\
\hline 0.057 & 0.029 & 0.004 & 3.116 \\
\hline 0.079 & 0.023 & 0.005 & 3.334 \\
\hline 0.058 & 0.024 & 0.002 & 3.300 \\
\hline 0.034 & 0.009 & 0.002 & 2.541 \\
\hline 0.036 & 0.011 & 0.002 & 2.942 \\
\hline 0.028 & 0.009 & 0.001 & 2.512 \\
\hline
\end{tabular}


Basic Table 18.A. Total Hubei Province (17): age-specific fertility rates,

\begin{tabular}{|c|c|c|c|c|}
\hline $\begin{array}{l}\text { Birth year } \\
\text { of child }\end{array}$ & $15-19$ & $20-24$ & $25-29$ & $30-34$ \\
\hline 1940 & 0.062 & $\bullet$ & - & - \\
\hline 1941 & 0.084 & - & - & - \\
\hline $\begin{array}{l}1942 \\
1943\end{array}$ & $\begin{array}{l}0.062 \\
0.108\end{array}$ & : & : & : \\
\hline 1944 & 0.083 & . & . & 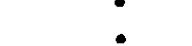 \\
\hline 1945 & 0.073 & 0.241 & - & - \\
\hline 1946 & 0.074 & 0.272 & - & - \\
\hline 1947 & 0.074 & 0.258 & - & - \\
\hline 1948 & 0.069 & 0.316 & - & - \\
\hline 1949 & 0.070 & 0.286 & - & - \\
\hline 1950 & 0.083 & 0.269 & 0.238 & - \\
\hline 1951 & 0.088 & 0.278 & 0.295 & - \\
\hline 1952 & 0.109 & 0.313 & 0.309 & - \\
\hline 1953 & 0.113 & 0.290 & 0.267 & - \\
\hline 1954 & 0.128 & 0.311 & 0.298 & - \\
\hline 1955 & 0.106 & 0.304 & 0.310 & 0.252 \\
\hline 1956 & 0.076 & 0.299 & 0.275 & 0.210 \\
\hline 1957 & 0.091 & 0.358 & 0.350 & 0.294 \\
\hline 1958 & 0.088 & 0.322 & 0.288 & 0.247 \\
\hline 1959 & 0.046 & 0.238 & 0.277 & 0.212 \\
\hline 1960 & 0.030 & 0.157 & 0.161 & 0.140 \\
\hline 1961 & 0.043 & 0.215 & 0.274 & 0.207 \\
\hline 1962 & 0.070 & 0.380 & 0.382 & 0.314 \\
\hline 1963 & 0.093 & 0.371 & 0.383 & 0.332 \\
\hline 1964 & 0.066 & 0.333 & 0.315 & 0.260 \\
\hline 1965 & 0.058 & 0.309 & 0.315 & 0.261 \\
\hline 1966 & 0.043 & 0.319 & 0.342 & 0.276 \\
\hline 1967 & 0.033 & 0.256 & 0.271 & 0.248 \\
\hline 1968 & 0.046 & 0.345 & 0.395 & 0.328 \\
\hline 1969 & 0.046 & 0.279 & 0.314 & 0.269 \\
\hline 1970 & 0.040 & 0.278 & 0.331 & 0.254 \\
\hline 1971 & 0.027 & 0.277 & 0.327 & 0.236 \\
\hline 1972 & 0.027 & 0.225 & 0.283 & 0.207 \\
\hline 1973 & 0.014 & 0.204 & 0.249 & 0.179 \\
\hline 1974 & 0.015 & 0.197 & 0.254 & 0.139 \\
\hline 1975 & 0.018 & 0.178 & 0.246 & 0.104 \\
\hline 1976 & 0.017 & 0.202 & 0.213 & 0.105 \\
\hline 1977 & 0.006 & 0.167 & 0.229 & 0.079 \\
\hline 1978 & 0.006 & 0.151 & 0.233 & 0.078 \\
\hline 1979 & 0.008 & 0.191 & 0.251 & 0.092 \\
\hline 1980 & 0.005 & 0.159 & 0.196 & 0.053 \\
\hline 1981 & 0.011 & 0.211 & 0.207 & 0.048 \\
\hline 1982 & 0.017 & 0.197 & 0.156 & 0.047 \\
\hline
\end{tabular}


1940-82

35-39

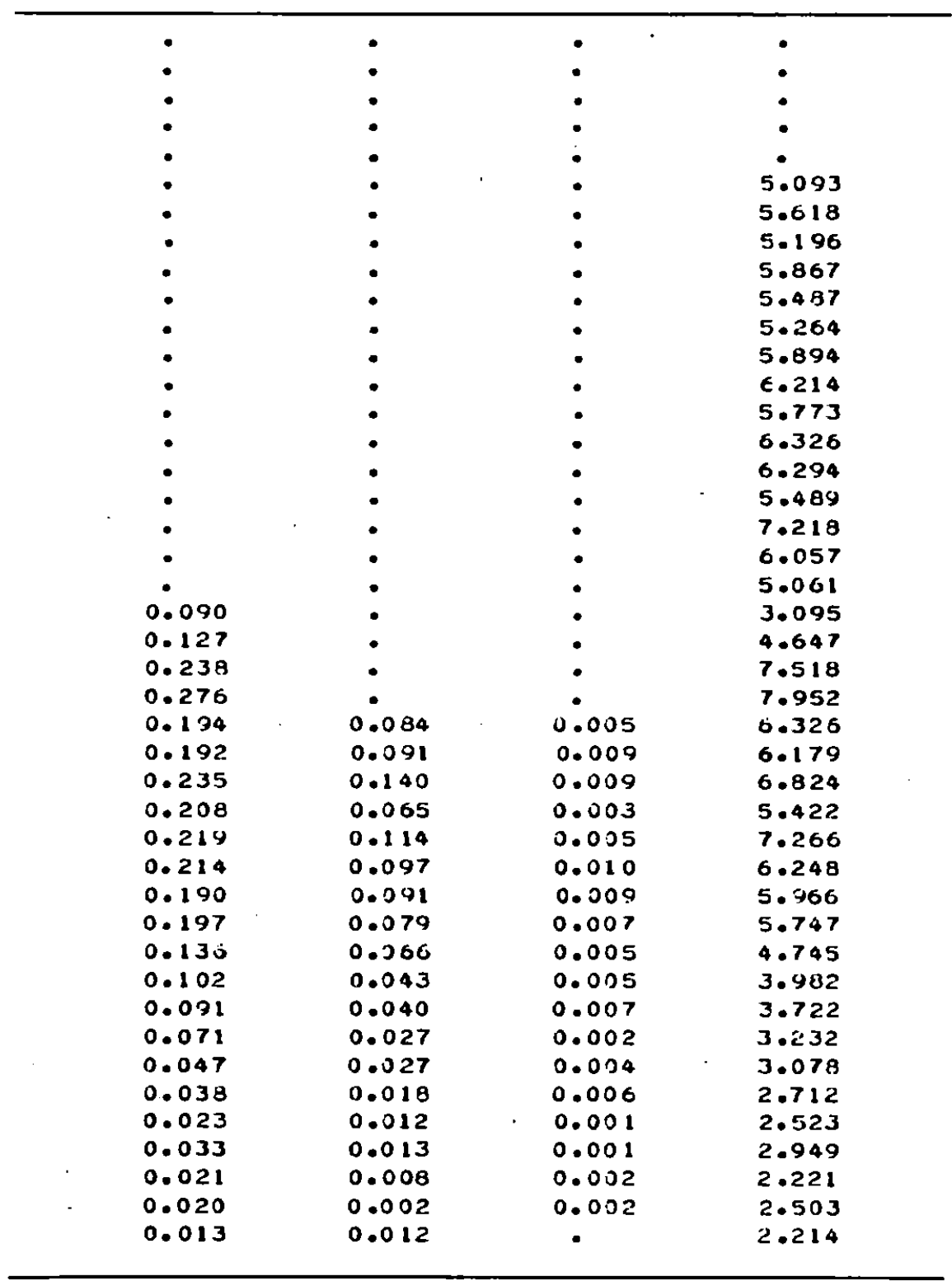


Basic Table 18.B. Urban Hubei Province (17): age-specific fertility rates,

\begin{tabular}{|c|c|c|c|c|}
\hline $\begin{array}{l}\text { Birth year } \\
\text { of child }\end{array}$ & $15-19$ & $20-24$ & $25-29$ & $30-34$ \\
\hline 1940 & 0.097 & • & $\bullet$ & - \\
\hline 1941 & 0.119 & - & - & - \\
\hline 1942 & 0.073 & - & $\bullet$ & - \\
\hline 1943 & 0.116 & - & - & - \\
\hline 1944 & 0.066 & - & - & - \\
\hline 1945 & 0.045 & 0.172 & - & - \\
\hline 1946 & 0.085 & 0.173 & - & . \\
\hline 1947 & 0.072 & 0.198 & $\bullet$ & $\bullet$ \\
\hline 1948 & 0.068 & 0.294 & - & - \\
\hline $194 \bar{y}$ & 0.025 & 0.273 & - & - \\
\hline 1950 & 0.118 & 0.318 & 0.205 & - \\
\hline 1951 & 0.090 & 0.237 & 0.227 & - \\
\hline 1952 & 0.081 & 0.200 & 0.188 & - \\
\hline 1953 & 0.126 & 0.328 & 0.189 & - \\
\hline 1954 & 0.113 & 0.240 & 0.255 & - \\
\hline 1955 & 0.074 & 0.281 & 0.294 & 0.162 \\
\hline 1956 & 0.028 & 0.295 & 0.262 & 0.184 \\
\hline 1957 & 0.058 & 0.373 & 0.328 & 0.261 \\
\hline 1958 & 0.060 & 0.298 & 0.192 & 0.137 \\
\hline 1959 & 0.035 & 0.290 & 0.339 & 0.198 \\
\hline 1900 & 0.023 & 0.251 & 0.242 & 0.200 \\
\hline 1961 & 0.020 & 0.153 & 0.280 & 0.186 \\
\hline 1962 & 0.038 & 0.333 & 0.387 & 0.200 \\
\hline 1963 & 0.057 & 0.276 & 0.365 & 0.335 \\
\hline 1964 & 0.016 & 0.229 & 0.248 & 0.197 \\
\hline 1905 & 0.011 & 0.192 & 0.181 & 0.141 \\
\hline 1966 & 0.006 & 0.118 & 0.209 & 0.105 \\
\hline 1907 & 0.008 & 0.104 & 0.173 & 0.153 \\
\hline 1968 & 0.008 & 0.177 & 0.359 & 0.155 \\
\hline 1969 & 0.008 & 0.151 & 0.234 & 0.185 \\
\hline 1970 & 0.003 & 0.168 & 0.229 & 0.119 \\
\hline 1971 & 0.005 & 0.101 & 0.270 & 0.141 \\
\hline 1972 & 0.011 & 0.065 & 0.208 & 0.115 \\
\hline 1973 & - & 0.047 & 0.208 & 0.104 \\
\hline 1974 & - & 0.046 & 0.188 & 0.078 \\
\hline 1975 & 0.003 & 0.066 & 0.216 & 0.064 \\
\hline 1976 & 0.003 & 0.049 & 0.174 & 0.064 \\
\hline 1977 & - & 0.050 & 0.249 & 0.080 \\
\hline 1978 & 0.003 & 0.059 & 0.253 & 0.080 \\
\hline 1979 & 0.003 & 0.046 & 0.204 & 0.094 \\
\hline 1980 & - & 0.022 & 0.177 & 0.048 \\
\hline 1981 & 0.003 & 0.086 & 0.211 & 0.044 \\
\hline 1982 & - & 0.117 & 0.123 & 0.016 \\
\hline
\end{tabular}


1940-82

\begin{tabular}{|c|c|c|c|}
\hline $35-39$ & $40-44$ & $45-49$ & Total \\
\hline - & - & - & - \\
\hline$\bullet$ & - & - & - \\
\hline - & - & - & - \\
\hline - & - & - & - \\
\hline - & - & - & - \\
\hline - & - & - & 5.014 \\
\hline - & - & - & 5.395 \\
\hline - & - & - & 4.705 \\
\hline - & - & - & 5.092 \\
\hline - & - & - & 5.247 \\
\hline - & • & • & 5.866 \\
\hline - & - & - & 5.520 \\
\hline - & - & - & 4.650 \\
\hline - & - & - & 5.900 \\
\hline - & - & - & 5.527 \\
\hline - & - & - & 6.104 \\
\hline - & - & - & 4.961 \\
\hline - & - & - & 7.620 \\
\hline - & - & - & 4.460 \\
\hline - & - & - & 6.017 \\
\hline 0.108 & - & - & 4.438 \\
\hline 0.097 & • & - & 3.991 \\
\hline 0.136 & - & - & 6.206 \\
\hline 0.221 & - & - & 7.018 \\
\hline 0.170 & 0.034 & - & 4.474 \\
\hline 0.082 & 0.043 & - & 3.246 \\
\hline 0.093 & $0.0 \in 5$ & - & 2.982 \\
\hline 0.050 & 0.010 & - & 2.523 \\
\hline 0.048 & 0.021 & - & 3.836 \\
\hline 0.105 & 0.035 & - & 3.597 \\
\hline 0.073 & 0.036 & - & 3.140 \\
\hline 0.080 & - & 0.032 & 3.150 \\
\hline 0.058 & 0.016 & - & $2 \cdot 365$ \\
\hline 0.034 & 0.014 & 0.011 & 2.082 \\
\hline 0.013 & 0.012 & 0.009 & 1.729 \\
\hline 0.025 & 0.006 & - & 1.892 \\
\hline 0.020 & • & - & 1.550 \\
\hline 0.012 & - & - & 1.956 \\
\hline 0.004 & - & - & 1.995 \\
\hline - & 0.004 & - & 1.756 \\
\hline 0.009 & 0.004 & - & 1.293 \\
\hline 0.005 & - & - & 1.747 \\
\hline • & - & - & 1.280 \\
\hline
\end{tabular}


Basic Table 18.C. Rural Hubei Province (17): age-specific fertility.rates,

\begin{tabular}{|c|c|c|c|c|}
\hline $\begin{array}{l}\text { Birth year } \\
\text { of child }\end{array}$ & $15-19$ & $20-24$ & $25-29$ & $30-34$ \\
\hline 1940 & 0.058 & - & - & • \\
\hline 1941 & 0.080 & - & $\bullet$ & - \\
\hline 1942 & 0.060 & - & - & - \\
\hline 1943 & 0.108 & $\bullet$ & - & - \\
\hline 1944 & 0.085 & • & - & - \\
\hline 1945 & 0.078 & 0.250 & - & - \\
\hline 1946 & 0.072 & 0.284 & • & - \\
\hline 1947 & 0.075 & 0.265 & - & - \\
\hline 1948 & 0.069 & 0.319 & - & - \\
\hline 1949 & 0.079 & $0.28 B$ & - & $\bullet$ \\
\hline 1950 & 0.076 & 0.261 & 0.242 & - \\
\hline 1951 & 0.087 & 0.285 & 0.303 & $\bullet$ \\
\hline 1952 & 0.116 & 0.332 & 0.324 & - \\
\hline 1953 & 0.110 & 0.283 & 0.277 & - \\
\hline 1954 & 0.132 & 0.325 & 0.304 & • \\
\hline 1955 & 0.115 & 0.309 & 0.312 & 0.263 \\
\hline 1956 & 0.089 & 0.300 & 0.277 & 0.213 \\
\hline 1957 & 0.100 & 0.354 & 0.354 & 0.298 \\
\hline 1958 & 0.095 & 0.329 & 0.305 & 0.261 \\
\hline 1959 & 0.048 & 0.223 & 0.264 & 0.214 \\
\hline 1960 & 0.031 & 0.132 & 0.144 & 0.131 \\
\hline 1961 & 0.048 & 0.232 & 0.272 & 0.210 \\
\hline 1962 & 0.076 & 0.392 & 0.381 & 0.332 \\
\hline 1963 & 0.099 & 0.393 & 0.388 & 0.332 \\
\hline 1964 & 0.075 & 0.356 & 0.334 & $0.2 e_{1}$ \\
\hline 1905 & 0.068 & 0.332 & 0.353 & 0.287 \\
\hline 1966 & 0.051 & 0.355 & 0.379 & 0.317 \\
\hline 1967 & 0.039 & 0.284 & 0.296 & 0.272 \\
\hline 1968 & 0.055 & 0.376 & 0.404 & 0.376 \\
\hline 1969 & 0.056 & 0.327 & 0.332 & 0.293 \\
\hline 1970 & 0.048 & 0.301 & 0.351 & 0.291 \\
\hline 1971 & 0.032 & 0.317 & 0.337 & 0.262 \\
\hline 1972 & 0.031 & 0.263 & 0.296 & 0.232 \\
\hline 1973 & 0.017 & 0.243 & 0.256 & 0.198 \\
\hline 1974 & 0.018 & 0.235 & 0.267 & 0.153 \\
\hline 1975 & 0.021 & 0.204 & 0.253 & 0.112 \\
\hline 1976 & 0.020 & 0.237 & 0.222 & 0.113 \\
\hline 1977 & 0.008 & 0.192 & 0.224 & 0.079 \\
\hline 1978 & 0.007 & 0.170 & 0.228 & 0.077 \\
\hline 1979 & 0.009 & 0.221 & 0.263 & 0.092 \\
\hline 1980 & 0.006 & 0.189 & 0.200 & 0.055 \\
\hline 1981 & 0.012 & 0.237 & 0.206 & 0.049 \\
\hline 1982 & 0.019 & 0.213 & 0.163 & 0.054 \\
\hline
\end{tabular}


40-44

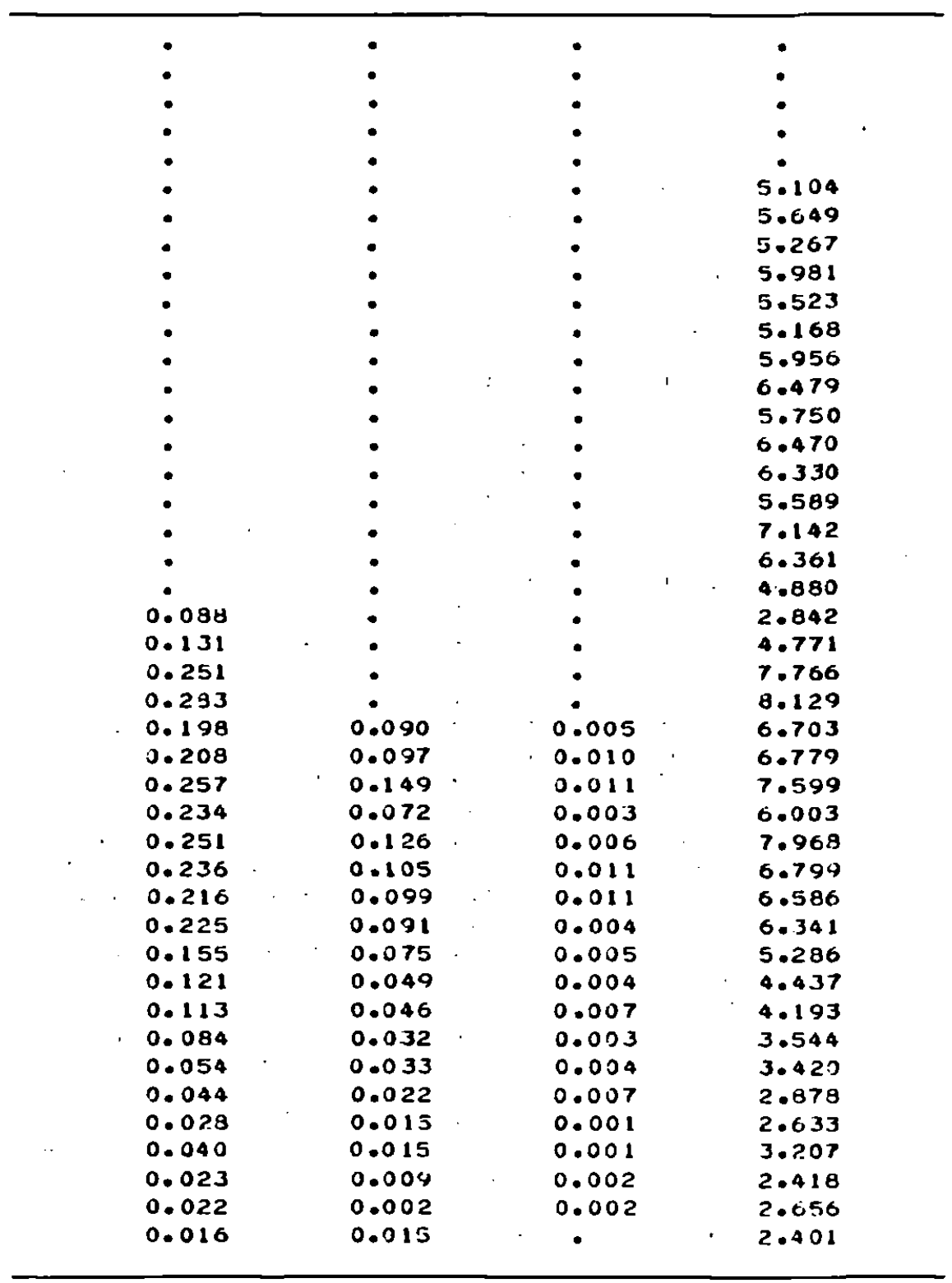


Basic Table 19.A. Total Hunan Province (18): age-specific fertility rates,

\begin{tabular}{|c|c|c|c|c|}
\hline $\begin{array}{l}\text { Birth year } \\
\text { of child }\end{array}$ & $15-19$ & $20-24$ & $25-29$ & $30-34$ \\
\hline 1940 & 0.073 & - & - & $\bullet$ \\
\hline 1941 & 0.084 & - & - & - \\
\hline 1942 & 0.084 & • & - & - \\
\hline 1943 & 0.082 & - & - & - \\
\hline 1944 & 0.091 & - & - & - \\
\hline 1945 & 0.075 & 0.236 & - & . \\
\hline 1946 & 0.067 & 0.270 & - & - \\
\hline 1947 & 0.092 & 0.267 & - & - \\
\hline 1948 & 0.085 & 0.310 & - & - \\
\hline 1949 & 0.099 & 0.320 & - & - \\
\hline 1950 & 0.089 & 0.304 & 0.296 & • \\
\hline 1951 & 0.123 & 0.298 & 0.287 & - \\
\hline 1952 & 0.111 & 0.329 & 0.313 & - \\
\hline 1953 & 0.116 & 0.326 & 0.321 & - \\
\hline 1954 & 0.101 & 0.301 & 0.318 & - \\
\hline 1955 & 0.102 & 0.280 & 0.293 & 0.240 \\
\hline 1950 & 0.099 & 0.298 & 0.325 & 0.261 \\
\hline 1957 & 0.092 & 0.323 & 0.313 & 0.263 \\
\hline 1958 & 0.071 & 0.258 & 0.267 & 0.254 \\
\hline 1959 & 0.025 & 0.200 & 0.219 & 0.183 \\
\hline $\begin{array}{l}1900 \\
1901\end{array}$ & $\begin{array}{l}0.023 \\
0.019\end{array}$ & $\begin{array}{l}0.176 \\
0.137\end{array}$ & $\begin{array}{l}0.190 \\
0.145\end{array}$ & 0.152 \\
\hline $\begin{array}{l}1901 \\
1962\end{array}$ & 0.054 & 0.331 & 0.348 & 0.331 \\
\hline 1963 & 0.091 & 0.401 & 0.397 & 0.329 \\
\hline 1964 & 0.074 & 0.335 & 0.360 & 0.296 \\
\hline 1965 & 0.060 & 0.329 & 0.339 & 0.285 \\
\hline 1906 & 0.063 & 0.344 & 0.337 & 0.293 \\
\hline 1967 & 0.043 & 0.256 & 0.284 & 0.242 \\
\hline 1968 & 0.056 & 0.395 & 0.407 & 0.324 \\
\hline 1964 & 0.051 & 0.308 & 0.285 & 0.302 \\
\hline 1970 & 0.046 & 0.308 & 0.308 & 0.272 \\
\hline 1971 & 0.041 & 0.309 & 0.326 & 0.223 \\
\hline 1972 & 0.030 & 0.303 & 0.271 & 0.227 \\
\hline 1973 & 0.030 & 0.251 & 0.281 & 0.168 \\
\hline 1974 & 0.025 & 0.254 & 0.279 & 0.170 \\
\hline 1975 & 0.016 & 0.248 & 0.257 & 0.137 \\
\hline 1976 & 0.013 & 0.214 & 0.209 & 0.108 \\
\hline 1977 & 0.008 & 0.196 & 0.203 & 0.081 \\
\hline 1978 & 0.007 & 0.173 & 0.198 & 0.070 \\
\hline 1979 & 0.008 & 0.203 & 0.202 & 0.064 \\
\hline 1980 & 0.003 & 0.200 & 0.201 & 0.049 \\
\hline 1981 & 0.013 & 0.275 & 0.222 & 0.054 \\
\hline 1982 & 0.019 & 0.258 & 0.265 & 0.060 \\
\hline
\end{tabular}




\section{5-39}

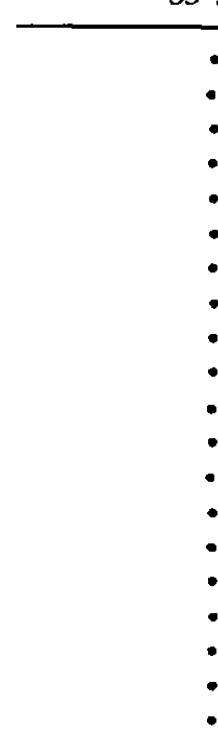

0.140

0.085

0.213

0.286

0.231

0.223

0.239

0.207

0.227

0.204

0.194

0.155

0.139

0.121

0.125

0.090

0.046

0.033

0.025

0.022

0.016

0.027

0.025
$40-44$

45-49

Total

$\bullet$

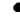

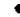

.

4. 892

5.460

5.573

5.790

6.146

5.870

5.951

6.325

6. 359

6.202

5.831

6.260

6.302

5.540

4.093

3.723

2.689

6.684

3.100

7.126

6.759

6.999

5.582

7.680

6.337

6.110

5.745

5. 260

4.545

4.561

3.971

3.142

2.729

2.459

2.576

2.379

2.995

3.241 
Basic Table 19.B. Urban Hunan Province (18): age-specific fertility

\begin{tabular}{|c|c|c|c|c|}
\hline $\begin{array}{l}\text { Birth year } \\
\text { of child }\end{array}$ & $15-19$ & $20-24$ & $25-29$ & $30-34$ \\
\hline 1940 & 0.060 & - & - & - \\
\hline 1941 & 0.080 & - & - & - \\
\hline 1942 & 0.104 & - & - & - \\
\hline 1943 & 0.060 & - & - & - \\
\hline 1944 & 0.090 & - & • & - \\
\hline 1945 & 0.072 & 0.195 & $\bullet$ & $\bullet$ \\
\hline 1946 & 0.057 & 0.161 & - & - \\
\hline 1947 & 0.104 & 0.264 & - & - \\
\hline 1948 & 0.079 & 0.238 & • & - \\
\hline 1949 & 0.067 & 0.317 & $\bullet$ & $\bullet$ \\
\hline 1950 & 0.098 & 0.228 & 0.222 & - \\
\hline 1951 & 0.110 & 0.258 & 0.230 & - \\
\hline 1952 & 0.079 & 0.227 & 0.264 & - \\
\hline 1953 & 0.128 & 0.282 & 0.192 & - \\
\hline 1954 & 0.076 & 0.202 & 0.317 & - \\
\hline 1955 & 0.093 & 0.269 & 0.254 & 0.206 \\
\hline 1956 & 0.044 & 0.258 & 0.258 & 0.201 \\
\hline 1957 & 0.091 & 0.335 & 0.325 & 0.203 \\
\hline 1958 & 0.024 & 0.313 & 0.265 & 0.225 \\
\hline 1959 & 0.040 & 0.280 & 0.208 & 0.124 \\
\hline 1960 & 0.017 & 0.207 & 0.232 & 0.117 \\
\hline 1961 & 0.017 & 0.140 & 0.180 & 0.126 \\
\hline 1962 & 0.050 & 0.280 & 0.263 & 0.202 \\
\hline 1963 & 0.024 & 0.297 & 0.306 & 0.209 \\
\hline 1964 & 0.015 & 0.298 & 0.341 & 0.174 \\
\hline 1965 & 0.013 & 0.253 & 0.236 & 0.141 \\
\hline 1966 & 0.006 & 0.084 & 0.229 & 0.129 \\
\hline 1967 & 0.006 & 0.201 & 0.205 & 0.072 \\
\hline 1960 & 0.015 & 0.206 & 0.193 & 0.121 \\
\hline 1469 & 0.018 & 0.146 & 0.217 & 0.151 \\
\hline 1970 & 0.009 & 0.121 & 0.228 & 0.114 \\
\hline 1971 & 0.020 & 0.117 & 0.168 & 0.022 \\
\hline 1972 & 0.004 & 0.117 & 0.142 & 0.114 \\
\hline 1973 & 0.004 & 0.049 & 0.103 & 0.064 \\
\hline 1974 & - & 0.071 & 0.161 & 0.072 \\
\hline 1975 & 0.004 & 0.090 & 0.148 & 0.067 \\
\hline 1976 & 0.005 & 0.073 & 0.160 & 0.025 \\
\hline 1977 & - & 0.061 & 0.200 & 0.058 \\
\hline 1978 & - & 0.061 & 0.153 & 0.032 \\
\hline 1979 & - & 0.048 & 0.138 & 0.038 \\
\hline 1980 & - & 0.035 & 0.181 & 0.067 \\
\hline 1981 & • & 0.129 & 0.121 & 0.025 \\
\hline 1982 & - & 0.062 & 0.168 & 0.033 \\
\hline
\end{tabular}


rates, $1940-82$

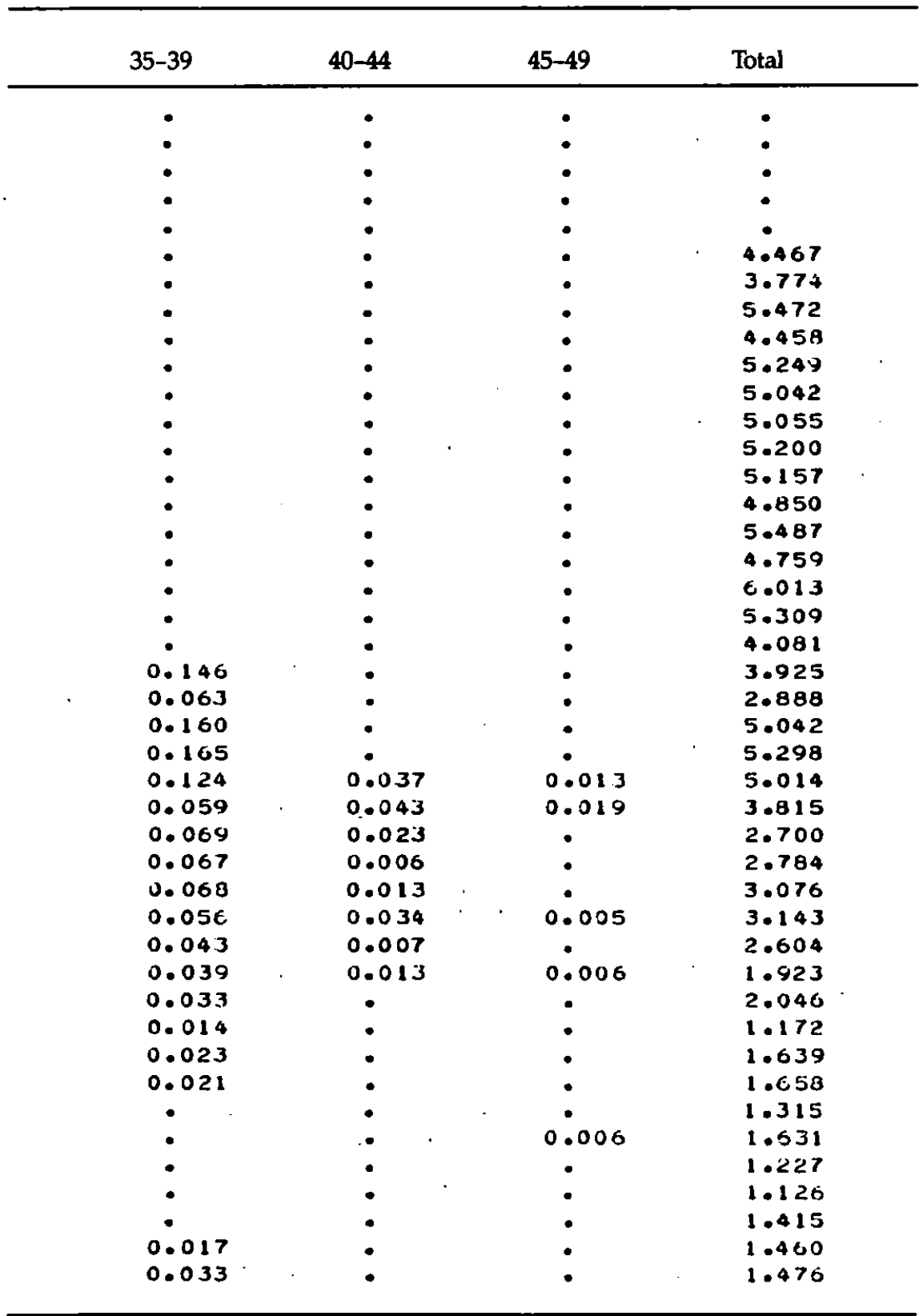


Basic Table 19.C. Rural Hunan Province (18): age-specific fertility rates,

\begin{tabular}{|c|c|c|c|c|}
\hline $\begin{array}{l}\text { Birth year } \\
\text { of child }\end{array}$ & $15-19$ & $20-24$ & 25-29 & $30-34$ \\
\hline 1940 & 0.076 & - & • & • \\
\hline 1941 & 0.085 & - & - & - \\
\hline 1942 & 0.081 & - & - & $\bullet$ \\
\hline 1943 & 0.086 & - & - & - \\
\hline 1944 & 0.091 & - & - & - \\
\hline 1945 & 0.075 & 0.245 & - & • \\
\hline 1946 & 0.069 & 0.293 & • & - \\
\hline 1947 & 0.090 & 0.268 & - & - \\
\hline 1948 & 0.086 & 0.323 & $\bullet$ & - \\
\hline 1949 & 0.104 & 0.320 & - & - \\
\hline 1950 & 0.088 & 0.317 & 0.313 & - \\
\hline 1951 & 0.125 & 0.304 & 0.299 & $\bullet$ \\
\hline 1952 & 0.115 & 0.346 & 0.322 & - \\
\hline 1953 & 0.115 & 0.334 & 0.344 & - \\
\hline $1954 \ldots$ & 0.104 & 0.318 & 0.318 & - \\
\hline 1955 & 0.103 & 0.281 & 0.300 & 0.247 \\
\hline 1956. & 0.106 & 0.304 & 0.336 & 0.274 \\
\hline $\begin{array}{l}1957 \\
1958\end{array}$ & $\begin{array}{l}0.092 \\
0.077\end{array}$ & $\begin{array}{l}0.321 \\
0.252\end{array}$ & $\begin{array}{l}0.311 \\
0.267\end{array}$ & $\begin{array}{l}0.275 \\
0.260\end{array}$ \\
\hline 1959 & 0.023 & 0.190 & 0.221 & 0.193 \\
\hline 1960 & 0.023 & 0.172 & 0.184 & 0.158 \\
\hline 1961 & 0.020 & 0.136 & 0.140 & 0.125 \\
\hline 1962 & 0.054 & 0.338 & 0.360 & 0.352 \\
\hline 1963 & 0.097 & 0.413 & 0.409 & 0.350 \\
\hline 1964 & 0.079 & 0.339 & 0.362 & 0.317 \\
\hline 1965 & 0.064 & 0.337 & 0.352 & 0.307 \\
\hline 1906 & 0.068 & 0.309 & 0.351 & 0.317 \\
\hline 1907 & 0.048 & 0.262 & 0.294 & 0.265 \\
\hline 1968 & 0.061 & 0.412 & 0.432 & 0.349 \\
\hline 1969 & 0.056 & 0.322 & 0.293 & 0.320 \\
\hline 1970 & 0.051 & 0.326 & 0.316 & 0.293 \\
\hline 1971 & 0.043 & 0.328 & 0.342 & 0.248 \\
\hline 1972 & 0.034 & 0.323 & 0.283 & 0.242 \\
\hline 1973 & 0.034 & 0.274 & 0.298 & 0.180 \\
\hline 1974 & 0.028 & 0.277 & 0.289 & 0.181 \\
\hline 1975 & 0.018 & 0.268 & 0.267 & 0.144 \\
\hline 1970 & 0.014 & 0.232 & 0.214 & 0.116 \\
\hline 1977 & 0.009 & 0.214 & 0.203 & 0.083 \\
\hline 1978 & 0.008 & 0.187 & 0.203 & 0.073 \\
\hline 1979 & 0.009 & 0.223 & 0.210 & 0.066 \\
\hline 1980 & 0.004 & 0.222 & 0.204 & 0.047 \\
\hline 1981 & 0.014 & 0.294 & 0.235 & 0.057 \\
\hline 1982 & 0.021 & 0.282 & 0.278 & 0.063 \\
\hline
\end{tabular}


1940-82

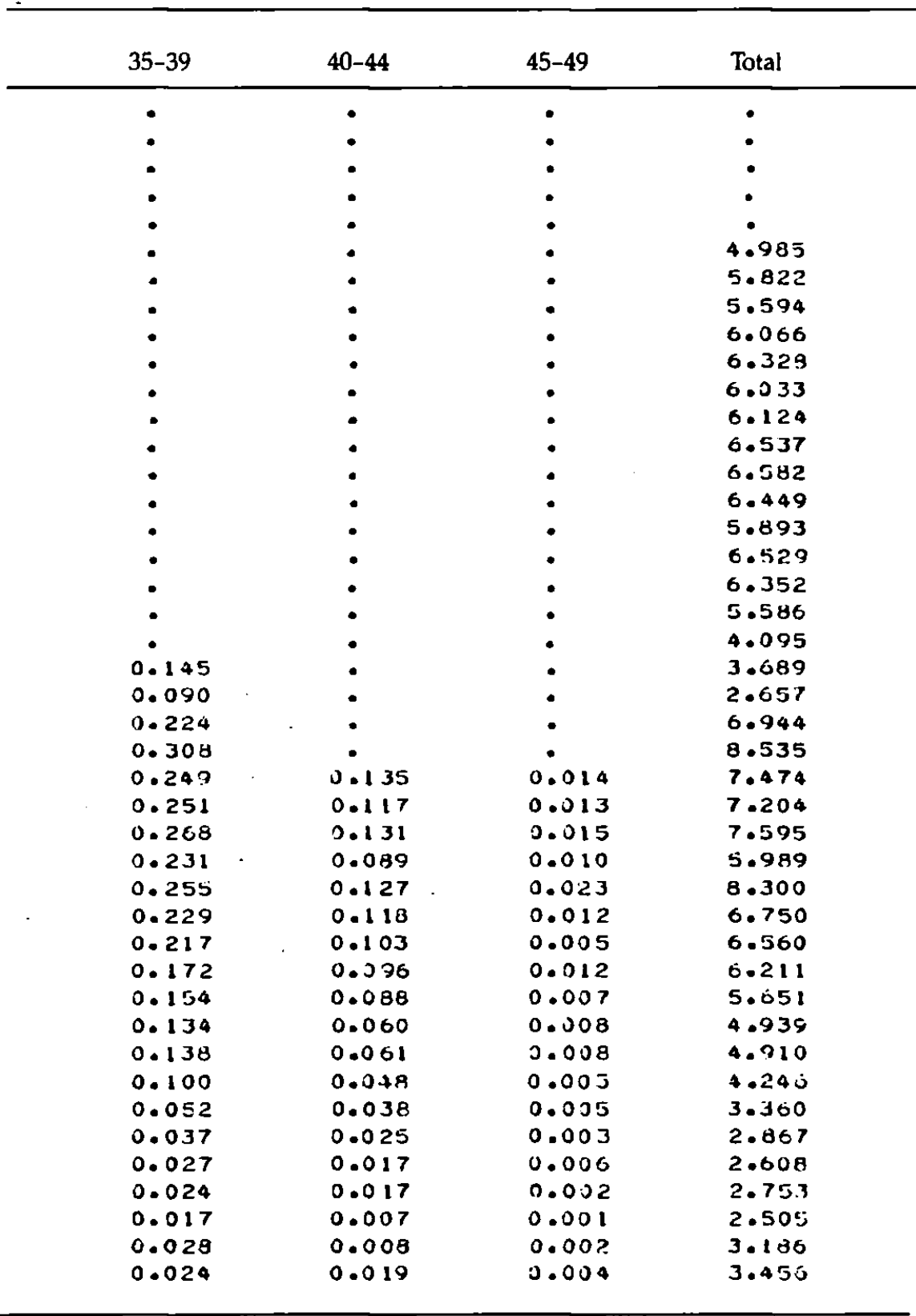


Basic Table 20.A. Total Guangdong Province (19): age-specific fertility

\begin{tabular}{|c|c|c|c|c|}
\hline $\begin{array}{l}\text { Birth year } \\
\text { of child }\end{array}$ & $15-19$ & $20-24$ & $25-29$ & $30-34$ \\
\hline 1940 & 0.040 & - & - & - \\
\hline 1941 & 0.046 & $\bullet$ & - & • \\
\hline 1942 & 0.044 & - & • & . \\
\hline 1943 & 0.057 & - & - & - \\
\hline 1944 & 0.050 & - & - & - \\
\hline 1945 & 0.044 & 0.211 & - & - \\
\hline 1946 & 0.040 & 0.178 & - & - \\
\hline 1947 & 0.030 & 0.214 & - & - \\
\hline 1948 & 0.035 & 0.203 & - & - \\
\hline 1949 & 0.041 & 0.206 & - & - \\
\hline 1950 & 0.041 & 0.222 & 0.247 & - \\
\hline 1951 & 0.042 & 0.206 & 0.265 & - \\
\hline 1952 & 0.062 & 0.212 & 0.246 & - \\
\hline 354 19 & 0.048 & 0.255 & 0.273 & - \\
\hline 1954 & 0.045 & 0.234 & 0.279 & - \\
\hline 1955 & 0.053 & 0.247 & 0.260 & 0.231 \\
\hline 1956 & 0.047 & 0.222 & 0.282 & 0.238 \\
\hline 1957 & 0.050 & 0.268 & 0.298 & 0.272 \\
\hline 190 & 0.042 & 0.235 & 0.275 & 0.237 \\
\hline 1959 & 0.028 & 0.195 & 0.242 & 0.225 \\
\hline 1960 & 0.018 & 0.160 & 0.183 & 0.170 \\
\hline 1961 & 0.024 & 0.169 & 0.223 & 0.169 \\
\hline 1962 & 0.030 & 0.266 & 0.354 & 0.300 \\
\hline 1963 & 0.041 & 0.266 & 0.337 & 0.283 \\
\hline 1964 & 0.044 & 0.262 & 0.322 & 0.256 \\
\hline 1965 & 0.048 & 0.233 & 0.285 & 0.248 \\
\hline 1966 & 0.037 & 0.244 & 0.320 & 0.269 \\
\hline 1967 & 0.027 & 0.217 & 0.315 & 0.219 \\
\hline 1968 & 0.027 & 0.245 & 0.319 & 0.306 \\
\hline 1969 & 0.024 & 0.236 & 0.311 & 0.248 \\
\hline 1970 & 0.027 & 0.229 & 0.305 & 0.260 \\
\hline 1971 & 0.022 & 0.219 & 0.314 & 0.257 \\
\hline 1972 & 0.024 & 0.211 & 0.304 & 0.253 \\
\hline 1973 & 0.019 & 0.208 & 0.289 & 0.222 \\
\hline 1974 & 0.021 & 0.194 & 0.284 & 0.208 \\
\hline 1975 & 0.011 & 0.187 & 0.242 & 0.174 \\
\hline 1976 & 0.012 & 0.168 & 0.271 & 0.153 \\
\hline 1977 & 0.015 & 0.151 & 0.245 & 0.143 \\
\hline 1978 & 0.014 & 0.154 & 0.268 & 0.154 \\
\hline 1979 & 0.011 & 0.188 & 0.276 & 0.147 \\
\hline 1980 & 0.008 & 0.161 & 0.284 & 0.134 \\
\hline 1981 & 0.015 & 0.218 & 0.271 & 0.138 \\
\hline 1982 & 0.025 & 0.174 & 0.248 & 0.096 \\
\hline
\end{tabular}


rates, $1940-82$

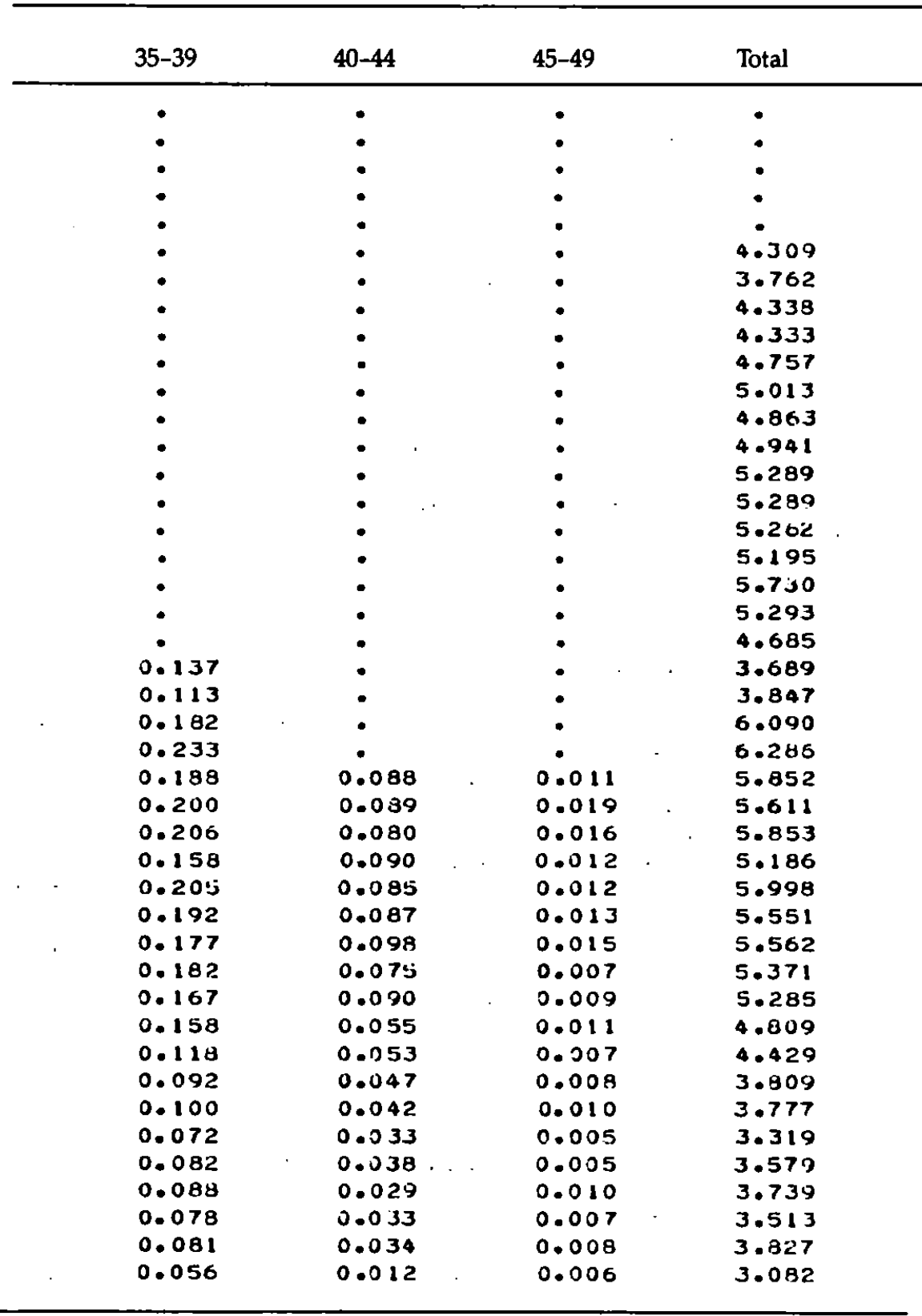


Basic Table 20.B. Urban Guangdong Province (19): age-specific fertility

\begin{tabular}{|c|c|c|c|c|}
\hline $\begin{array}{l}\text { Birth year } \\
\text { of child }\end{array}$ & $15-19$ & $20-24$ & $25-29$ & $30-34$ \\
\hline .1940 & 0.033 & - & - & - \\
\hline 1941 & 0.026 & - & - & - \\
\hline 1942 & 0.050 & - & - & - \\
\hline 1943 & 0.040 & - & - & - \\
\hline 1944 & 0.032 & - & - & - \\
\hline 1945 & 0.035 & 0.202 & - & - \\
\hline 1946 & 0.067 & 0.196 & - & - \\
\hline 1947 & 0.021 & 0.212 & • & - \\
\hline 1948 & 0.048 & 0.254 & - &. \\
\hline 1949 & 0.051 & 0.189 &. & - \\
\hline 1950 & 0.042 & 0.272 & 0.248 & - \\
\hline 1951 & 0.036 & 0.265 & 0.229 & - \\
\hline 1952 & 0.056 & 0.170 & 0.281 & - \\
\hline 1953 & 0.042 & 0.229 & 0.294 & - \\
\hline 1954 & 0.056 & 0.195 & 0.307 & - \\
\hline 1955 & 0.039 & 0.261 & 0.262 & 0.228 \\
\hline 1956 & 0.047 & 0.208 & 0.301 & 0.222 \\
\hline 1957 & 0.030 & 0.296 & 0.361 & 0.262 \\
\hline 1958 & 0.032 & 0.253 & 0.237 & 0.209 \\
\hline 1959 & 0.032 & 0.196 & 0.239 & 0.189 \\
\hline 1960 & 0.021 & 0.147 & 0.212 & 0.141 \\
\hline 1961 & 0.018 & 0.154 & 0.212 & 0.148 \\
\hline 1902 & 0.029 & 0.226 & 0.345 & 0.269 \\
\hline $1 \ngtr 63$ & 0.025 & 0.276 & 0.367 & 0.249 \\
\hline 1904 & 0.020 & 0.214 & 0.257 & 0.217 \\
\hline 1965 & 0.015 & 0.181 & 0.234 & 0.115 \\
\hline 1966 & 0.015 & 0.117 & 0.191 & 0.111 \\
\hline 1967 & 0.009 & 0.181 & 0.251 & 0.104 \\
\hline 1908 & 0.011 & 0.155 & 0.179 & 0.140 \\
\hline 1969 & 0.003 & 0.112 & 0.284 & 0.088 \\
\hline 1970 & 0.015 & 0.105 & 0.253 & 0.120 \\
\hline 1971 & - & 0.114 & 0.220 & 0.121 \\
\hline 1972 & 0.011 & 0.120 & 0.238 & 0.106 \\
\hline 1973 & 0.009 & 0.092 & 0.231 & 0.097 \\
\hline 1974 & 0.004 & 0.069 & 0.176 & 0.129 \\
\hline 1975 & - & 0.054 & 0.183 & 0.093 \\
\hline 1970 & 0.002 & 0.044 & 0.183 & 0.054 \\
\hline 1977 & 0.002 & 0.017 & 0.176 & 0.070 \\
\hline 1978 & 0.005 & 0.030 & 0.136 & 0.072 \\
\hline 1979 & 0.002 & 0.028 & 0.177 & 0.064 \\
\hline 1980 & 0.002 & 0.027 & 0.199 & 0.077 \\
\hline 1981 & - & 0.065 & 0.166 & 0.059 \\
\hline 1982 & - & 0.061 & 0.117 & 0.029 \\
\hline
\end{tabular}


rates, $1940-82$

\begin{tabular}{|c|c|c|c|c|}
\hline $35-39$ & & $40-44$ & $45-49$ & Total \\
\hline - & & - & - & - \\
\hline • & & - & - & - \\
\hline • & & - & - & - \\
\hline - & & - & - & - \\
\hline • & & • & • & - \\
\hline • & & - & - & 4.259 \\
\hline$\bullet$ & & - & - & 4.767 \\
\hline - & & - & - & 4.573 \\
\hline - & & - & - & 5.500 \\
\hline - & & - & - & 5.245 \\
\hline - & & - & - & 5.330 \\
\hline - & & - & - & 5.099 \\
\hline - & &. & - & 5.117 \\
\hline - & & - & - & 5.490 \\
\hline - & & - & - & 5.340 \\
\hline - & & - & - & 5.270 \\
\hline - & & - & - & 5.235 \\
\hline - & & - & - & 6.045 \\
\hline - & & - & - & 4.758 \\
\hline - & & - & - & 4.350 \\
\hline 0.124 & & - & - & 3.339 \\
\hline 0.059 & & - & - & 3.249 \\
\hline 0.131 & & - & - & 5.410 \\
\hline 0.203 & & - & - & 5.746 \\
\hline 0.036 & & 0.027 & 0.014 & 4.179 \\
\hline 0.081 & & 0.026 & - & 3.262 \\
\hline 0.076 & & 0.007 & - & 2.590 \\
\hline 0.037 & & 0.006 & - & 2.940 \\
\hline 0.044 & & 0.040 & 0.007 & 2.075 \\
\hline 0.066 & & 0.011 & - & 2.316 \\
\hline 0.052 & & - & - & 2.758 \\
\hline 0.050 & & 0.009 & - & 2.577 \\
\hline 0.019 & & 0.017 & - & 2.549 \\
\hline 0.03 .3 & . & 0.004 & 0.000 & 2.386 \\
\hline 0.003 & & - & - & 1.930 \\
\hline 0.013 & & 0.007 & - & 1.750 \\
\hline 0.005 & & - & - & 1.436 \\
\hline 0.005 & & - & - & 1.352 \\
\hline 0.005 & & - & - & 1.241 \\
\hline 0.005 & , & - & - & $1 \cdot 38.3$ \\
\hline$\bullet$ & & 0.004 & - & $1.5+5$ \\
\hline 0.004 & & • & 0.004 & 1.485 \\
\hline $0.00 \mathrm{~B}$ & & - & - & 1.075 \\
\hline
\end{tabular}


Basic Table 20.C. Rural Guangdong Province (19): age-specific fertility

\begin{tabular}{|c|c|c|c|c|}
\hline $\begin{array}{l}\text { Birth year } \\
\text { of child }\end{array}$ & $15-19$ & $20-24$ & $25-29$ & $30-34$ \\
\hline 1940 & 0.042 & $\bullet$ & - & $\bullet$ \\
\hline 1941 & 0.049 & - & - & - \\
\hline 1942 & 0.043 & $\bullet$ & - & $\bullet$ \\
\hline 1943 & 0.061 & - & • & - \\
\hline 1944 & 0.054 & - & - & - \\
\hline 1945 & 0.046 & 0.213 & - & - \\
\hline 1946 & 0.034 & 0.174 & - & - \\
\hline 1947 & 0.032 & 0.214 & - & $\bullet$ \\
\hline 1948 & 0.032 & 0.194 & - & - \\
\hline 1949 & 0.038 & 0.209 & - & - \\
\hline 1950 & 0.041 & 0.212 & 0.247 & • \\
\hline 1951 & 0.043 & 0.192 & 0.271 & - \\
\hline 1952 & 0.064 & 0.222 & 0.240 & - \\
\hline 1953 & 0.050 & 0.262 & 0.269 & - \\
\hline 1954 & 0.042 & 0.244 & 0.274 & - \\
\hline 1955 & 0.056 & 0.244 & 0.260 & 0.2332 \\
\hline 1956 & 0.047 & 0.225 & 0.278 & 0.241 \\
\hline 1957 & 0.054 & 0.261 & 0.283 & 0.273 \\
\hline 1958 & 0.043 & 0.231 & 0.285 & 0.242 \\
\hline 1959 & 0.027 & 0.194 & 0.243 & 0.231 \\
\hline $\begin{array}{l}1900 \\
1961\end{array}$ & $\begin{array}{l}0.018 \\
0.025\end{array}$ & $\begin{array}{l}0.162 \\
0.173\end{array}$ & $\begin{array}{l}0.176 \\
0.226\end{array}$ & 0.175 \\
\hline 1962 & 0.031 & 0.274 & 0.356 & 0.314 \\
\hline 1963 & 0.045 & 0.264 & 0.330 & 0.291 \\
\hline 1964 & 0.049 & 0.271 & 0.336 & 0.267 \\
\hline 1965 & 0.056 & 0.243 & 0.296 & 0.284 \\
\hline 1900 & 0.042 & 0.270 & 0.347 & 0.308 \\
\hline 1907 & 0.031 & 0.225 & $0.32 \mathrm{e}$ & 0.246 \\
\hline 1968 & 0.031 & 0.267 & 0.345 & 0.345 \\
\hline 1969 & 0.028 & 0.206 & 0.316 & 0.283 \\
\hline 1970 & 0.030 & 0.260 & 0.315 & 0.289 \\
\hline 1971 & 0.027 & 0.243 & 0.333 & 0.285 \\
\hline 1972 & 0.027 & 0.232 & 0.318 & 0.283 \\
\hline 1973 & 0.021 & 0.235 & 0.303 & 0.245 \\
\hline 1974 & 0.025 & 0.222 & 0.311 & 0.223 \\
\hline 1975 & 0.014 & 0.216 & 0.256 & 0.190 \\
\hline 1976 & 0.014 & 0.196 & 0.292 & 0.174 \\
\hline 1977 & 0.018 & 0.181 & 0.261 & 0.159 \\
\hline 1978 & 0.017 & 0.182 & 0.298 & 0.173 \\
\hline 1979 & 0.012 & 0.223 & 0.298 & 0.167 \\
\hline 1980 & 0.009 & 0.191 & 0.302 & 0.148 \\
\hline 1981 & 0.018 & 0.253 & 0.294 & 0.157 \\
\hline 1982 & 0.028 & 0.199 & 0.277 & 0.112 \\
\hline
\end{tabular}


rates, $1940-82$

\begin{tabular}{|c|c|c|c|}
\hline $35-39$ & $40-44$ & $45-49$ & Total \\
\hline • & - & • & - \\
\hline - & - & - & - \\
\hline - & - & - & • \\
\hline - & • & • & - \\
\hline • & - & $\bullet$ & $\bullet$ \\
\hline - & - & - & 4.319 \\
\hline - & - & $\bullet$ & 3.555 \\
\hline - & - & - & 4.237 \\
\hline - & - & - & 4.086 \\
\hline - & - & - & 4.652 \\
\hline - & - & - & 4.944 \\
\hline - & - & - & 4.812 \\
\hline - & - & - & 4.903 \\
\hline - & - & - & 5.245 \\
\hline - & - & - & 5.278 \\
\hline - & - & - & 5.260 \\
\hline - & - & - & 5.186 \\
\hline - & - & - & 5.663 \\
\hline - & - & - & 5.407 \\
\hline - & - & - & 4.757 \\
\hline 0.139 & - & - & 3.763 \\
\hline 0.122 & • & - & 3.975 \\
\hline 0.190 & • & - & 6.237 \\
\hline 0.233 & - & - & 6.404 \\
\hline 0.207 & 0.095 & 0.010 & 0.195 \\
\hline 0.223 & 0.101 & 0.023 & 6.126 \\
\hline 0.234 & 0.093 & 0.019 & 6.563 \\
\hline 0.187 & 0.104 & 0.014 & 5.673 \\
\hline 0.244 & 0.093 & 0.014 & 6.692 \\
\hline 0.225 & 0.101 & 0.015 & 6.175 \\
\hline 0.211 & 0.117 & 0.019 & 6.201 \\
\hline 0.215 & 0.089 & 0.008 & 6.000 \\
\hline 0.202 & 0.107 & 0.010 & 5.895 \\
\hline 0.186 & 0.067 & 0.012 & 5.345 \\
\hline 0.143 & 0.067 & 0.008 & 4.992 \\
\hline 0.109 & 0.057 & 0.010 & 4.264 \\
\hline 0.119 & 0.052 & 0.012 & 4.294 \\
\hline 0.085 & 0.041 & 0.000 & 3.754 \\
\hline 0.096 & 0.047 & 0.007 & 4.097 \\
\hline 0.103 & 0.035 & 0.012 & 4. . 56 \\
\hline 0.093 & 0.039 & 0.008 & 3.945 \\
\hline 0.097 & 0.041 & 0.009 & 4.343 \\
\hline 0.067 & 0.014 & 0.007 & 3.520 \\
\hline
\end{tabular}


Basic Table 21.A. Total Guangxi Province (20): age-specific fertility

\begin{tabular}{|c|c|c|c|c|}
\hline $\begin{array}{l}\text { Birth year } \\
\text { of child }\end{array}$ & $15-19$ & $20-24$ & $25-29$ & $30-34$ \\
\hline 1940 & 0.055 & - & - & - \\
\hline 1941 & 0.052 & • & - & - \\
\hline 1942 & 0.060 & - & - & . \\
\hline 1943 & 0.063 & - & - & . \\
\hline 1944 & 0.061 & - & - & - \\
\hline 1945 & 0.051 & 0.225 & - & . \\
\hline 1946 & 0.050 & 0.198 & - & - \\
\hline 1947 & 0.042 & 0.187 & - & - \\
\hline 1948 & 0.055 & 0.174 & . & - \\
\hline 1949 & 0.060 & 0.254 & - & - \\
\hline 1950 & 0.061 & 0.248 & 0.256 & - \\
\hline 1951 & 0.065 & 0.194 & 0.202 & - \\
\hline 1952 & 0.070 & 0.279 & 0.283 & - \\
\hline 1953 & 0.062 & 0.223 & 0.267 & - \\
\hline 1954 & 0.063 & 0.271 & 0.289 & - \\
\hline 1955 & 0.052 & 0.267 & 0.275 & 0.258 \\
\hline 1950 & 0.059 & 0.228 & 0.288 & 0.228 \\
\hline 1957 & 0.041 & 0.281 & 0.295 & 0.253 \\
\hline 1958 & 0.048 & 0.267 & 0.330 & 0.259 \\
\hline 1959 & 0.023 & 0.179 & 0.221 & 0.199 \\
\hline 1960 & 0.032 & 0.203 & 0.230 & 0.231 \\
\hline 1901 & 0.038 & 0.179 & 0.223 & 0.166 \\
\hline 1962 & 0.057 & 0.291 & 0.338 & 0.298 \\
\hline 1963 & 0.052 & 0.332 & 0.377 & 0.326 \\
\hline 1964 & 0.040 & 0.312 & 0.338 & 0.324 \\
\hline 1965 & 0.039 & 0.280 & 0.310 & 0.302 \\
\hline 1906 & 0.033 & 0.324 & 0.363 & 0.294 \\
\hline 1967 & 0.021 & 0.202 & 0.308 & 0.236 \\
\hline 1968 & 0.038 & 0.281 & 0.364 & 0.298 \\
\hline 1969 & 0.021 & 0.247 & 0.325 & 0.264 \\
\hline 1970 & 0.021 & 0.217 & 0.296 & 0.275 \\
\hline 1971 & 0.023 & 0.252 & 0.321 & 0.240 \\
\hline 1972 & 0.018 & 0.200 & 0.299 & 0.245 \\
\hline 1973 & 0.018 & 0.215 & 0.332 & 0.267 \\
\hline 1974 & 0.020 & 0.227 & 0.296 & 0.254 \\
\hline 1975 & 0.017 & 0.213 & 0.294 & 0.257 \\
\hline 1976 & 0.009 & 0.182 & 0.272 & 0.199 \\
\hline 1977 & 0.009 & 0.181 & 0.285 & 0.172 \\
\hline 1978 & 0.007 & 0.166 & 0.268 & 0.143 \\
\hline 1979 & 0.006 & 0.172 & 0.282 & 0.189 \\
\hline 1980 & 0.008 & 0.188 & 0.308 & 0.178 \\
\hline 1981 & 0.008 & 0.189 & 0.291 & 0.162 \\
\hline 1982 & 0.012 & 0.213 & 0.253 & 0.162 \\
\hline
\end{tabular}


rates, $1940-82$

\begin{tabular}{|c|c|c|c|}
\hline $35-39$ & $40-44$ & $45-49$ & Total \\
\hline - & • & • & - \\
\hline - & - & - & - \\
\hline$\bullet$ & $\bullet$ & - & - \\
\hline$\bullet$ & • & $\bullet$ & - \\
\hline$\cdot$ & $\bullet$ & - & $\cdot$ \\
\hline - & - & - & 5.427 \\
\hline - & - & - & 4.901 \\
\hline - & - & - & 4.457 \\
\hline - & - & - & 4.773 \\
\hline - & - & - & 5.712 \\
\hline - & - & - & 5.294 \\
\hline - & - & - & 4.407 \\
\hline - & - & - & 6.024 \\
\hline - & - & - & 5.239 \\
\hline - & - & - & 5.735 \\
\hline - & - & - & 5.793 \\
\hline - & - & - & 5.443 \\
\hline - & - & - & 6.146 \\
\hline - & - & - & 6.222 \\
\hline - & - & - & 4.391 \\
\hline 0.172 & - & - & 4.856 \\
\hline 0.127 & - & - & 4.124 \\
\hline 0.156 & - & - & 6.269 \\
\hline 0.209 & - & - & 7.545 \\
\hline 0.232 & 0.103 & 0.019 & $\epsilon .838$ \\
\hline 0.238 & 0.122 & 0.019 & 6.551 \\
\hline 0.250 & 0.151 & 0.014 & 7.145 \\
\hline 0.176 & 0.075 & 0.018 & 5.179 \\
\hline 0.249 & 0.125 & 0.022 & 6.889 \\
\hline 0.204 & 0.113 & 0.016 & 5.951 \\
\hline 0.205 & 0.115 & 0.023 & 5.760 \\
\hline 0.206 & 0.109 & 0.024 & 5.872 \\
\hline 0.157 & 0.093 & 0.027 & 5.137 \\
\hline 0.176 & 0.094 & 0.018 & 5.601 \\
\hline 0.190 & 0.096 & 0.021 & 5.520 \\
\hline 0.155 & 0.056 & 0.015 & 5.034 \\
\hline 0.126 & 0.062 & 0.015 & 4.332 \\
\hline 0.094 & 0.037 & 0.014 & 3.964 \\
\hline 0.101 & 0.029 & 0.008 & 3.612 \\
\hline 0.095 & 0.043 & 0.009 & 3.977 \\
\hline 0.079 & 0.014 & 0.007 & 3.914 \\
\hline 0.077 & 0.031 & 0.004 & 3.806 \\
\hline 0.085 & 0.020 & 0.005 & 3.753 \\
\hline
\end{tabular}


Basic Table 21.B. Urban Guangxi Province (20): age-specific fertility

\begin{tabular}{|c|c|c|c|c|}
\hline $\begin{array}{l}\text { Birth year } \\
\text { of child }\end{array}$ & $15-19$ & $20-24$ & $25-29$ & $30-34$ \\
\hline 1940 & 0.035 & - & - & - \\
\hline 1941 & 0.033 & - & - & - \\
\hline 1942 & 0.079 & • & - & . \\
\hline 1943 & 0.024 & - & - & - \\
\hline 1944 & 0.117 & - & - & - \\
\hline 1945 & •. & 0.211 & - & - \\
\hline 1946 & 0.140 & 0.298 & - & - \\
\hline 1947 & 0.096 & 0.183 & - & - \\
\hline 1948 & 0.098 & 0.072 & - & - \\
\hline 1949 & 0.082 & 0.328 & - & - \\
\hline 1950 & 0.095 & 0.274 & 0.106 & . \\
\hline 1951 & 0.138 & 0.280 & 0.265 & . \\
\hline 1952 & 0.073 & 0.307 & 0.236 & - \\
\hline 1953 & 0.155 & 0.358 & 0.289 & - \\
\hline 1954 & 0.047 & 0.275 & 0.375 & - \\
\hline 1955 & 0.059 & 0.311 & 0.211 & 0.282 \\
\hline 1950 & 0.073 & 0.263 & 0.320 & 0.159 \\
\hline 1957 & 0.060 & 0.327 & 0.288 & 0.367 \\
\hline 1958 & 0.013 & 0.227 & 0.309 & 0.265 \\
\hline 1959 & 0.028 & 0.223 & 0.206 & 0.117 \\
\hline 1900 & 0.041 & 0.130 & 0.284 & 0.190 \\
\hline 1901 & 0.013 & 0.207 & 0.188 & 0.080 \\
\hline 1962 & 0.029 & 0.288 & 0.327 & 0.192 \\
\hline 1963 & 0.014 & 0.271 & 0.263 & 0.358 \\
\hline 1964 & 0.037 & 0.202 & 0.212 & 0.261 \\
\hline 1965 & 0.023 & 0.245 & 0.248 & 0.122 \\
\hline 1966 & - & 0.148 & 0.268 & 0.075 \\
\hline 1967 & 0.009 & 0.189 & 0.216 & 0.061 \\
\hline 1908 & 0.016 & 0.154 & 0.296 & 0.167 \\
\hline 1909 & 0.015 & 0.185 & 0.221 & 0.141 \\
\hline 1970 & 0.006 & 0.135 & 0.204 & 0.106 \\
\hline 1971 & 0.011 & 0.220 & 0.255 & 0.073 \\
\hline 1972 & - & 0.082 & 0.348 & 0.216 \\
\hline 1973 & 0.010 & 0.065 & 0.210 & 0.103 \\
\hline 1974 & 0.010 & 0.088 & 0.185 & 0.138 \\
\hline 1975 & - & 0.079 & 0.124 & 0.136 \\
\hline 1976 & - & 0.063 & 0.147 & 0.121 \\
\hline 1977 & - & 0.052 & 0.201 & 0.160 \\
\hline 1970 & - & 0.072 & 0.178 & 0.042 \\
\hline 1979 & - & 0.070 & 0.234 & 0.062 \\
\hline 1980 & - & 0.051 & 0.103 & 0.056 \\
\hline 1981 & 0.007 & 0.039 & 0.168 & 0.042 \\
\hline 1982 & - & 0.106 & 0.149 & 0.107 \\
\hline
\end{tabular}


rates, $1940-82$

\begin{tabular}{|c|c|c|c|}
\hline $35-39$ & $40-44$ & $45-49$ & Total \\
\hline - & - & - & - \\
\hline - & - & - & - \\
\hline - & • & - & - \\
\hline - & - & - & - \\
\hline - & $\bullet$ & - & - \\
\hline - & $\bullet$ & - & 3.854 \\
\hline - & - & - & 8.846 \\
\hline - & - & - & 4.573 \\
\hline - & • & - & 3.824 \\
\hline - & . & - & 7.899 \\
\hline - & • & - & 5.336 \\
\hline - & - & - & 5.894 \\
\hline - & - & - & 6.692 \\
\hline - & $\bullet$ & - & 7.774 \\
\hline - & - & - & 6.850 \\
\hline - & - & - & 6.116 \\
\hline - & - & - & 6.476 \\
\hline - & - & - & 7.544 \\
\hline - & - & - & 5.689 \\
\hline - & - & - & 4.022 \\
\hline 0.141 & - & • & 4.807 \\
\hline 0.099 & - & - & 3.196 \\
\hline 0.236 & - & - & 6.197 \\
\hline 0.096 & - & - & 5.672 \\
\hline 0.117 & 0.104 & 0.036 & 5.146 \\
\hline 0.042 & 0.035 & - & 3.572 \\
\hline 0.060 & - & - & 2.758 \\
\hline 0.019 & - & - & 2.467 \\
\hline 0.081 & - & - & 3.577 \\
\hline 0.041 & 0.022 & - & 3.132 \\
\hline 0.054 & 0.042 & - & 2.739 \\
\hline 0.025 & 0.020 & - & 3.020 \\
\hline 0.073 & $\bullet$ & - & 3.597 \\
\hline 0.060 & 0.016 & - & 2.318 \\
\hline 0.035 & 0.027 & - & 2.419 \\
\hline - & 0.014 & . & 1.762 \\
\hline 0.012 & • & - & 1.714 \\
\hline 0.012 & - & - & 2.124 \\
\hline 0.026 & - & - & 1.586 \\
\hline • & 0.024 & - & 1.947 \\
\hline - & • & - & 1.053 \\
\hline 0.013 & - & - & 1.349 \\
\hline • & $\bullet$ & - & 1.809 \\
\hline
\end{tabular}


Basic Table 21.C. Rural Guangxi Province (20): age-specific fertility

\begin{tabular}{|c|c|c|c|c|}
\hline $\begin{array}{l}\text { Birth year } \\
\text { of child }\end{array}$ & $15-19$ & $20-24$ & $25-29$ & $30-34$ \\
\hline 1940 & 0.056 & - & - & - \\
\hline 1941 & 0.053 & • & - & - \\
\hline 1942 & 0.059 & - & - & - \\
\hline 1943 & 0.067 & - & - & - \\
\hline 1944 & 0.055 & . & . & - \\
\hline 1945 & 0.056 & 0.226 & - & - \\
\hline 1940 & 0.042 & 0.191 & - & - \\
\hline 1947 & 0.037 & 0.187 & - & - \\
\hline 1948 & 0.052 & 0.183 & - & - \\
\hline 1949 & 0.058 & 0.247 & - & • \\
\hline 1950 & 0.058 & 0.245 & 0.266 & - \\
\hline 1951 & 0.057 & 0.197 & 0.198 & - \\
\hline 1952 & 0.070 & 0.276 & 0.287 & - \\
\hline 1953 & 0.051 & 0.211 & 0.265 & - \\
\hline 1954 & 0.065 & 0.271 & 0.231 & - \\
\hline 1955 & 0.052 & 0.263 & 0.281 & 0.257 \\
\hline 1956 & 0.057 & 0.224 & 0.285 & 0.230 \\
\hline 1957 & 0.039 & 0.276 & 0.295 & 0.248 \\
\hline 1958 & 0.052 & 0.272 & 0.332 & 0.259 \\
\hline 1959 & 0.023 & 0.174 & 0.223 & 0.207 \\
\hline 1960 & 0.031 & 0.211 & 0.224 & 0.235 \\
\hline 1901 & 0.040 & 0.176 & 0.227 & 0.174 \\
\hline 1962 & 0.060 & 0.291 & 0.339 & 0.307 \\
\hline 1963 & 0.056 & 0.332 & 0.389 & 0.323 \\
\hline 1904 & 0.040 & 0.317 & 0.352 & 0.330 \\
\hline 1905 & 0.041 & 0.283 & 0.317 & 0.321 \\
\hline 1900 & 0.037 & 0.341 & 0.373 & 0.317 \\
\hline 1967 & 0.023 & 0.203 & 0.317 & 0.255 \\
\hline 1908 & 0.041 & 0.293 & 0.371 & 0.313 \\
\hline 1969 & 0.021 & 0.254 & 0.334 & 0.278 \\
\hline 1970 & 0.023 & 0.227 & 0.305 & 0.293 \\
\hline 1971 & 0.025 & 0.256 & 0.327 & 0.258 \\
\hline 1972 & 0.021 & 0.215 & 0.294 & 0.248 \\
\hline 1973 & 0.020 & 0.233 & 0.344 & 0.283 \\
\hline 1974 & 0.022 & 0.245 & 0.308 & 0.264 \\
\hline 1975 & 0.013 & 0.232 & 0.314 & 0.268 \\
\hline 1976 & 0.011 & 0.200 & 0.287 & 0.207 \\
\hline 1977 & 0.010 & 0.203 & 0.295 & 0.173 \\
\hline 1978 & 0.008 & 0.181 & 0.280 & 0.153 \\
\hline 1979 & 0.007 & 0.187 & 0.288 & 0.203 \\
\hline 1980 & 0.008 & 0.207 & 0.338 & 0.193 \\
\hline 1981 & 0.008 & 0.208 & 0.310 & 0.176 \\
\hline 1982 & 0.013 & 0.224 & 0.270 & 0.169 \\
\hline
\end{tabular}


rates, $1940-82$

\begin{tabular}{|c|c|c|c|}
\hline $35-39$ & $40-44$ & $45-49$ & Total \\
\hline - & $\bullet$ & - & - \\
\hline - & - & - & - \\
\hline - & $\bullet$ & $\bullet$ & $\bullet$ \\
\hline - & - & - & - \\
\hline - & - & - & - \\
\hline - & $\bullet$ & - & 5.550 \\
\hline - & $\bullet$ & - & 4.594 \\
\hline - & - & - & 4.448 \\
\hline - & - & - & 4.652 \\
\hline - & - & - & 5.526 \\
\hline - & • & - $\quad-$ & 5.291 \\
\hline - & - & - & 4.277 \\
\hline - & - & - & 5.965 \\
\hline - & - & - & 5.009 \\
\hline - & • & - & 5.633 \\
\hline - & - & - & 5.763 \\
\hline - & - & - & 5.349 \\
\hline - & - & - & 6.017 \\
\hline - & - & - & 6.271 \\
\hline - & - & - & 4.425 \\
\hline 0.174 & - & - & 4.861 \\
\hline 0.129 & $\bullet$ & - & 4.210 \\
\hline 0.149 & - & - & 6.276 \\
\hline 0.286 & - & - & 7.719 \\
\hline 0.243 & 0.103 & 0.017 & 7.011 \\
\hline 0.257 & 0.127 & 0.020 & 6.829 \\
\hline 0.267 & 0.161 & 0.015 & 7.561 \\
\hline 0.190 & 0.081 & 0.019 & 5.442 \\
\hline 0.265 & 0.136 & 0.024 & 7.213 \\
\hline 0.220 & 0.121 & 0.017 & 6.234 \\
\hline 0.220 & 0.122 & 0.025 & 6.072 \\
\hline 0.220 & 0.117 & 0.025 & 0.166 \\
\hline 0.166 & 0.102 & 0.029 & 5.375 \\
\hline 0.189 & 0.102 & 0.020 & $5.95 n$ \\
\hline 0.207 & 0.103 & 0.023 & 5.861 \\
\hline 0.172 & 0.061 & $0.01 \epsilon$ & 5.409 \\
\hline 0.138 & 0.069 & 0.016 & 4.041 \\
\hline 0.102 & 0.041 & 0.015 & 4.203 \\
\hline 0.108 & 0.032 & 0.009 & $: 3.852$ \\
\hline 0.104 & 0.045 & 0.010 & 4.216 \\
\hline 0.080 & 0.016 & 0.009 & 4.277 \\
\hline 0.083 & 0.034 & 0.004 & 4.115 \\
\hline 0.094 & 0.023 & 0.005 & 3.986 \\
\hline
\end{tabular}


Basic Table 22.A. Total Sichuan Province (21): age-specific fertility

\begin{tabular}{|c|c|c|c|c|}
\hline $\begin{array}{l}\text { Birth year } \\
\text { of child }\end{array}$ & $15-19$ & $20-24$ & $25-29$ & $30-34$ \\
\hline 1940 & 0.064 & - & • & - \\
\hline 1941 & 0.066 & - & - & - \\
\hline 1942 & 0.060 & - & - & - \\
\hline 1943 & 0.058 & - & - & - \\
\hline 1944 & 0.050 & - & - & $\bullet$ \\
\hline 1945 & 0.051 & 0.240 & - & - \\
\hline 1946 & 0.057 & 0.269 & - & - \\
\hline 1947 & 0.065 & 0.237 & $\bullet$ & - \\
\hline 1948 & 0.056 & 0.248 & - & - \\
\hline 1949 & 0.069 & 0.302 & - & - \\
\hline $\begin{array}{l}1950 \\
1951\end{array}$ & $\begin{array}{l}0.064 \\
0.089\end{array}$ & $\begin{array}{l}0.274 \\
0.277\end{array}$ & $\begin{array}{l}0.287 \\
0.272\end{array}$ & - \\
\hline 1952 & 0.118 & 0.309 & 0.325 & . \\
\hline 1953 & 0.104 & 0.300 & 0.323 & - \\
\hline 1954 & 0.096 & 0.303 & 0.288 & - \\
\hline 1955 & 0.085 & 0.318 & 0.305 & 0.261 \\
\hline 1956 & 0.066 & 0.286 & 0.286 & 0.280 \\
\hline 1957 & 0.064 & 0.302 & 0.303 & 0.266 \\
\hline 1958 & 0.074 & 0.275 & 0.308 & 0.274 \\
\hline 1959 & 0.032 & 0.161 & 0.191 & 0.169 \\
\hline 1960 & 0.019 & 0.116 & 0.139 & 0.129 \\
\hline 1961 & 0.013 & 0.116 & 0.135 & 0.118 \\
\hline 1962 & 0.030 & 0.209 & 0.248 & 0.210 \\
\hline 1963 & 0.071 & 0.379 & 0.407 & 0.357 \\
\hline 1964 & 0.062 & 0.328 & 0.313 & 0.276 \\
\hline 1965 & 0.039 & 0.301 & 0.324 & 0.260 \\
\hline 1966 & 0.045 & 0.317 & 0.331 & 0.293 \\
\hline 1967 & 0.025 & 0.266 & 0.298 & 0.256 \\
\hline 1968 & 0.039 & 0.319 & 0.357 & 0.285 \\
\hline 1969 & 0.034 & 0.288 & 0.344 & 0.278 \\
\hline 1970 & 0.033 & 0.314 & 0.321 & 0.280 \\
\hline 1971 & 0.047 & 0.282 & 0.341 & 0.287 \\
\hline 1972 & 0.035 & 0.257 & 0.322 & 0.245 \\
\hline 1973 & 0.033 & 0.256 & 0.309 & 0.241 \\
\hline 1974 & 0.022 & 0.248 & 0.292 & 0.222 \\
\hline 1975 & 0.019 & 0.240 & 0.269 & 0.181 \\
\hline 1976 & 0.019 & 0.185 & 0.227 & 0.126 \\
\hline 1977 & 0.013 & 0.152 & 0.185 & 0.085 \\
\hline 1978 & 0.004 & 0.129 & 0.158 & 0.056 \\
\hline 1979 & 0.002 & 0.142 & 0.169 & 0.059 \\
\hline 1980 & 0.003 & 0.141 & 0.126 & 0.035 \\
\hline 1981 & 0.007 & 0.243 & 0.194 & 0.048 \\
\hline 1982 & 0.012 & 0.232 & 0.193 & 0.040 \\
\hline
\end{tabular}


rates, $1940-82$

\begin{tabular}{|c|c|c|c|}
\hline $35-39$ & $40-44$ & $45-49$ & Total \\
\hline - & - & - & - \\
\hline - & - & - & $\bullet$ \\
\hline$\bullet$ & - & - & - \\
\hline - & • & - & $\bullet$ \\
\hline - & - & - & - \\
\hline - & - & - & 4.724 \\
\hline - & - & - & $5 \cdot 361$ \\
\hline - & - & - & 4.925 \\
\hline - & - & - & 5.214 \\
\hline - & - & - & 5.885 \\
\hline - & - & - & 5.422 \\
\hline - & - & - & 5.319 \\
\hline$\bullet$ & - & - & 6.531 \\
\hline - & - & - & 6.170 \\
\hline - & - & - & 6.030 \\
\hline • & $\bullet$ & - & 6.283 \\
\hline - & $\bullet$ & - & 5.862 \\
\hline - & - & • & 6.122 \\
\hline$\bullet$ & $\bullet$ & • & 6.061 \\
\hline - & - & - & 3.719 \\
\hline 0.117 & - & - & 2.870 \\
\hline 0.070 & - & - & 2.431 \\
\hline 0.134 & • & - & 4.457 \\
\hline 0.272 & - & - & 8.016 \\
\hline 0.210 & 0.126 & 0.020 & 6.679 \\
\hline 0.216 & 0.103 & 0.010 & 6.287 \\
\hline 0.224 & 0.100 & 0.016 & 6.634 \\
\hline 0.182 & 0.105 & 0.016 & 5.739 \\
\hline 0.230 & 0.117 & 0.018 & 6.829 \\
\hline 0.217 & 0.116 & 0.021 & 6.499 \\
\hline 0.215 & 0.106 & 0.015 & 6.426 \\
\hline 0.202 & 0.093 & 0.012 & 6.319 \\
\hline 0.183 & 0.095 & 0.008 & 5.728 \\
\hline 0.155 & 0.077 & 0.014 & 5.428 \\
\hline 0.149 & 0.076 & 0.013 & 5.113 \\
\hline 0.113 & 0.061 & 0.007 & 4.447 \\
\hline 0.082 & 0.046 & 0.007 & 3.454 \\
\hline 0.052 & 0.021 & 0.005 & 2.570 \\
\hline 0.031 & 0.009 & 0.002 & 1.949 \\
\hline 0.023 & 0.009 & 0.002 & 2.027 \\
\hline 0.012 & 0.005 & 0.000 & 1.609 \\
\hline 0.017 & 0.012 & 0.001 & 2.014 \\
\hline 0.017 & 0.011 & 0.001 & 2.525 \\
\hline
\end{tabular}


Basic Table 22.B." Urban Sichuan Province (21): age-specific fertility

\begin{tabular}{|c|c|c|c|c|}
\hline $\begin{array}{l}\text { Birth year } \\
\text { of child }\end{array}$ & $15-19$ & $20-24$ & $25-29$ & $30-34$ \\
\hline 1940 & 0.064 & - & • & - \\
\hline 1941 & 0.045 & - & - & - \\
\hline 1942 & 0.039 & - & • & - \\
\hline 1943 & 0.048 & - & - & - \\
\hline 1944 & 0.048 & - & - & - \\
\hline 1945 & 0.044 & 0.203 & - & - \\
\hline 1940 & 0.051 & 0.223 & - & - \\
\hline 1947 & 0.052 & 0.175 & - & - \\
\hline 1948 & 0.038 & 0.221 & - & - \\
\hline 1944 & 0.067 & 0.215 & - & - \\
\hline 1950 & 0.081 & 0.225 & 0.278 & - \\
\hline لـ 19 & 0.077 & 0.243 & 0.233 & - \\
\hline 1952 & 0.115 & 0.257 & 0.280 & - \\
\hline 1953 & 0.081 & 0.240 & 0.309 & - \\
\hline 1954 & 0.077 & 0.276 & 0.257 & - \\
\hline 1955 & 0.081 & 0.283 & 0.301 & 0.214 \\
\hline 1950 & 0.048 & 0.259 & 0.299 & 0.268 \\
\hline 1957 & 0.062 & 0.346 & 0.328 & $0.2 c 5$ \\
\hline 1958 & 0.085 & 0.249 & 0.281 & 0.231 \\
\hline 1954 & 0.045 & 0.194 & 0.195 & 0.135 \\
\hline 1960 & 0.016 & 0.141 & 0.178 & 0.139 \\
\hline 1961 & 0.019 & 0.138 & 0.133 & 0.106 \\
\hline 1902 & 0.019 & 0.199 & 0.222 & 0.171 \\
\hline 1963 & 0.026 & 0.319 & 0.386 & 0.329 \\
\hline 1904 & 0.018 & 0.244 & 0.235 & 0.207 \\
\hline 1965 & 0.014 & 0.206 & 0.253 & 0.144 \\
\hline 1960 & 0.007 & 0.168 & 0.188 & 0.138 \\
\hline 1967 & 0.002 & 0.139 & 0.250 & 0.108 \\
\hline 1963 & 0.010 & 0.225 & 0.339 & 0.153 \\
\hline 1969 & 0.019 & 0.249 & 0.253 & 0.149 \\
\hline 1970 & 0.021 & 0.213 & 0.232 & 0.124 \\
\hline 1971 & 0.014 & 0.176 & 0.207 & 0.080 \\
\hline $1 \times 72$ & 0.008 & 0.127 & 0.173 & 0.099 \\
\hline 1973 & 0.005 & 0.120 & 0.170 & 0.094 \\
\hline 1974 & 0.013 & 0.110 & 0.174 & 0.075 \\
\hline 1975 & 0.007 & 0.112 & 0.153 & 0.067 \\
\hline 1976 & 0.004 & 0.072 & 0.163 & 0.036 \\
\hline 1977 & 0.002 & 0.040 & 0.111 & 0.015 \\
\hline $197 \mathrm{~d}$ & - & 0.014 & 0.124 & 0.026 \\
\hline 1979 & - & 0.018 & 0.112 & 0.029 \\
\hline 1980 & - & 0.016 & 0.128 & 0.021 \\
\hline 1981 & 0.001 & 0.067 & 0.215 & 0.015 \\
\hline 1982 & 0.003 & 0.116 & 0.190 & 0.008 \\
\hline
\end{tabular}


rates, $1940-82$

\begin{tabular}{|c|c|c|c|}
\hline $35-39$ & $40-44$ & $45-49$ & Total \\
\hline - & - & - & - \\
\hline - & r• & - & - \\
\hline - & • & - & - \\
\hline • & $\cdot \bullet$ & - & • \\
\hline • & • & - & $\bullet$ \\
\hline - & - & - & 3.710 \\
\hline - & - & - & 4.669 \\
\hline - & - & - & $4 \cdot 350$ \\
\hline - & - & - & 4.826 \\
\hline - & - & - & 5.315 \\
\hline - & - & - & 5.058 \\
\hline - & - & - & 4.745 \\
\hline - & - & - & 5.889 \\
\hline - & - & - & 5.757 \\
\hline . & - & - & 5.545 \\
\hline$\bullet$ & - & - & 5.687 \\
\hline - & - & - & 5.477 \\
\hline - & - & - & 6.409 \\
\hline • & - & - & 5.394 \\
\hline - & - & - & 3.779 \\
\hline 0.135 & - & - & 3.276 \\
\hline 0.045 & - & • & 2.438 \\
\hline 0.081 & - & - & 3.664 \\
\hline 0.205 & - & - & 6.887 \\
\hline 0.130 & 0.093 & 0.013 & 4.698 \\
\hline $0.09 ?$ & 0.098 & - & 4.063 \\
\hline 0.124 & 0.033 & 0.005 & 3.349 \\
\hline 0.055 & 0.039 & 0.015 & 3.039 \\
\hline 0.102 & 0.046 & 0.015 & 4.448 \\
\hline 0.099 & 0.029 & 0.004 & 4.006 \\
\hline 0.071 & 0.026 & 0.008 & 3.472 \\
\hline 0.046 & 0.030 & 0.003 & 2.782 \\
\hline 0.042 & 0.021 & - & 2.353 \\
\hline 0.019 & 0.015 & 0.003 & 2.132 \\
\hline 0.024 & 0.007 & 0.003 & 2.031 \\
\hline 0.019 & 0.005 & - & 1.813 \\
\hline 0.015 & 0.0 .10 & 0.003 & 1.507 \\
\hline 0.005 & - & - & 0.368 \\
\hline 0.009 & 0.002 & - & 0.877 \\
\hline 0.006 & - & - & 0.823 \\
\hline - & - & - & 0.823 \\
\hline . & - & - & $1 .+95$ \\
\hline - & - & - & 1.586 \\
\hline
\end{tabular}


Basic Table 22.C. Rural Sichuan Province (21): age-specific fertility

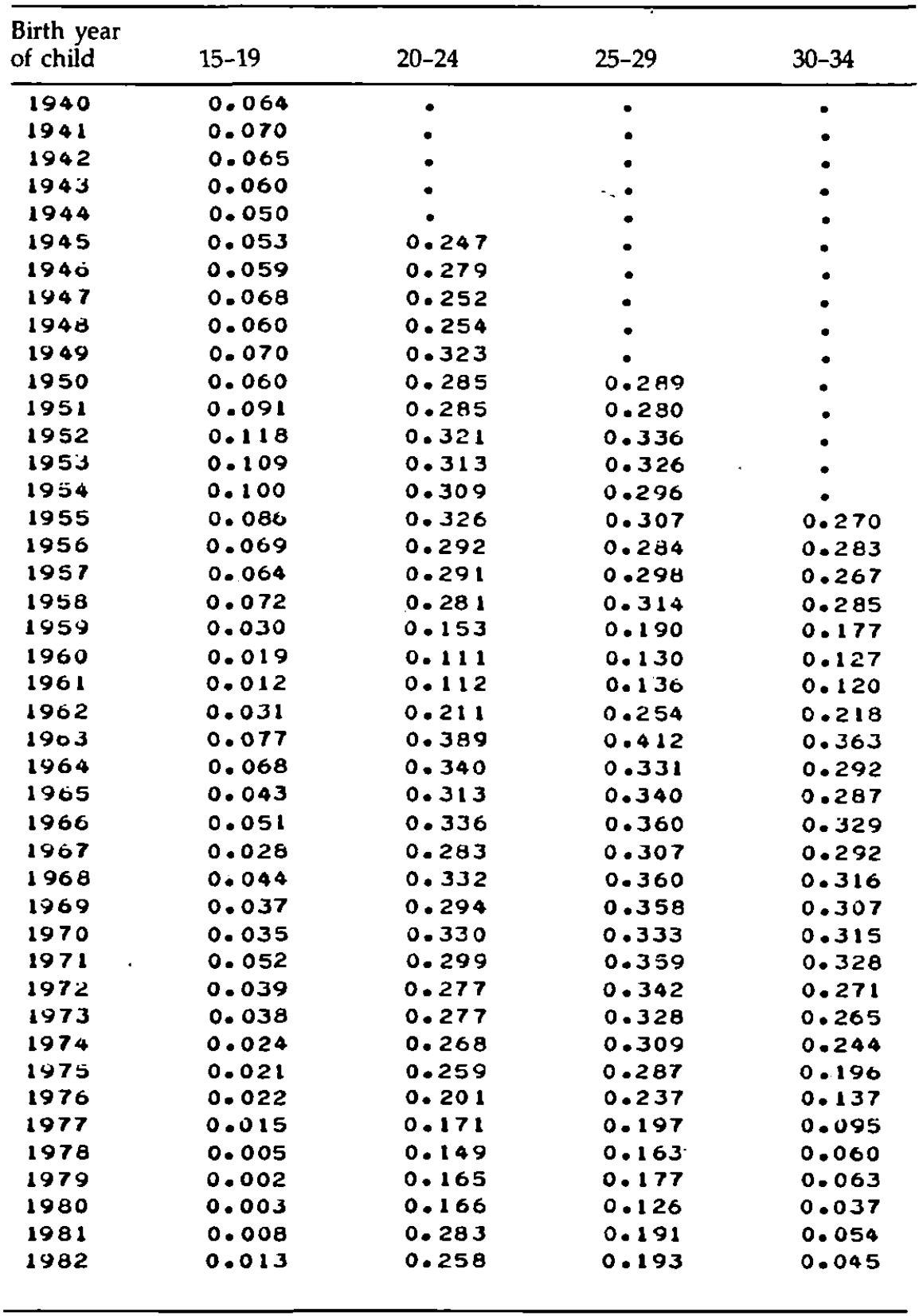


rates, $1940-82$

\begin{tabular}{|c|c|c|c|}
\hline 35-39 & $40-44$ & $45-49$ & Total \\
\hline - & - & - & - \\
\hline - & • & - & - \\
\hline - & $\bullet$ & - & - \\
\hline$\bullet$ & $\bullet$ & • & - \\
\hline - & - & - & - \\
\hline - & - & - & 4.929 \\
\hline - & - & - & 5.499 \\
\hline - & - & - & 5.041 \\
\hline - & • & - & 5.293 \\
\hline - & - & - & 6.001 \\
\hline - & - & $\bullet$ & 5.497 \\
\hline - & - & - & 5.439 \\
\hline - & - & - & 6.667 \\
\hline - & - & - & 6.257 \\
\hline - & - & - $\cdot$ & 0.132 \\
\hline - & - & - & 6.409 \\
\hline - & - & - & 5.941 \\
\hline - & - & - & 6.063 \\
\hline - & - & - & 6.194 \\
\hline - & • & - & 3.708 \\
\hline 0.113 & - & - & 2.792 \\
\hline 0.075 & • & - & 2.430 \\
\hline 0.146 & - & - & 4.606 \\
\hline 0.289 & - & - & $8 \cdot 226$ \\
\hline 0.230 & 0.132 & 0.021 & 7.073 \\
\hline 0.245 & 0.109 & 0.011 & 6.741 \\
\hline 0.249 & 0.113 & 0.018 & 7.277 \\
\hline 0.210 & 0.121 & 0.016 & 6.282 \\
\hline 0.254 & 0.135 & 0.018 & 7.319 \\
\hline 0.245 & 0.137 & 0.024 & 7.010 \\
\hline 0.249 & 0.125 & 0.017 & 7.017 \\
\hline 0.238 & 0.108 & 0.014 & 6.986 \\
\hline 0.217 & 0.112 & 0.010 & 6.340 \\
\hline 0.186 & 0.091 & 0.017 & 6.003 \\
\hline 0.178 & 0.092 & 0.015 & 5.651 \\
\hline 0.133 & 0.074 & 0.009 & 4.896 \\
\hline 0.095 & 0.055 & 0.008 & 3.777 \\
\hline 0.060 & 0.020 & 0.006 & 2.849 \\
\hline 0.034 & 0.011 & 0.003 & $2 \cdot 128$ \\
\hline 0.025 & 0.012 & 0.002 & 2.235 \\
\hline 0.013 & 0.006 & 0.001 & 1.761 \\
\hline 0.017 & 0.014 & 0.002 & 2.849 \\
\hline 0.019 & 0.012 & 0.001 & 2.700 \\
\hline
\end{tabular}


Basic Table 23.A. Total Guizhou Province (22): age-specific fertility

\begin{tabular}{|c|c|c|c|c|}
\hline $\begin{array}{l}\text { Birth year } \\
\text { of child }\end{array}$ & $15-19$ & $20-24$ & $25-29$ & $30-34$ \\
\hline 1940 & 0.059 & - & - & - \\
\hline 1941 & 0.039 & - & - & - \\
\hline 1942 & 0.058 & - & - & - \\
\hline 1943 & 0.067 & - & - & - \\
\hline 1944 & 0.054 & - & - & - \\
\hline 1945 & 0.046 & 0.197 & - & - \\
\hline 1940 & 0.047 & 0.261 & - & - \\
\hline 1947 & 0.068 & 0.223 & - & - \\
\hline 1948 & 0.051 & 0.274 & - & - \\
\hline 1949 & 0.070 & 0.289 & - & - \\
\hline 1950 & 0.069 & 0.251 & 0.270 & - \\
\hline 1951 & 0.068 & 0.210 & 0.248 & - \\
\hline 1952 & 0.073 & 0.279 & 0.322 & - \\
\hline 1953 & 0.069 & 0.279 & 0.276 & - \\
\hline 1954 & 0.074 & 0.287 & 0.325 & • \\
\hline 1955 & 0.066 & 0.312 & 0.297 & 0.270 \\
\hline 1950 & 0.093 & 0.240 & 0.342 & 0.245 \\
\hline 1957 & 0.071 & 0.273 & 0.310 & 0.299 \\
\hline 1958 & 0.061 & 0.273 & 0.301 & 0.279 \\
\hline 1959 & 0.029 & 0.216 & 0.247 & 0.215 \\
\hline 1960 & 0.029 & 0.160 & 0.183 & 0.169 \\
\hline 1901 & 0.018 & 0.141 & 0.154 & 0.142 \\
\hline 1962 & 0.044 & 0.302 & 0.297 & 0.289 \\
\hline 1963 & 0.056 & 0.310 & 0.388 & 0.371 \\
\hline 1904 & 0.049 & 0.293 & 0.330 & 0.209 \\
\hline 1965 & 0.052 & 0.302 & 0.353 & 0.334 \\
\hline 1960 & 0.062 & 0.293 & 0.346 & 0.326 \\
\hline 1907 & 0.041 & 0.250 & 0.299 & 0.265 \\
\hline 1968 & 0.061 & 0.297 & 0.414 & 0.368 \\
\hline 1909 & 0.036 & 0.275 & 0.357 & 0.298 \\
\hline 1970 & 0.045 & 0.309 & 0.339 & 0.323 \\
\hline 1971 & 0.040 & 0.300 & 0.335 & 0.317 \\
\hline 1972 & 0.035 & 0.226 & 0.352 & 0.279 \\
\hline 1973 & 0.036 & 0.264 & 0.342 & 0.337 \\
\hline 1974 & 0.041 & 0.280 & 0.362 & 0.293 \\
\hline 1975 & 0.045 & 0.257 & 0.308 & 0.264 \\
\hline 1476 & 0.034 & 0.229 & 0.292 & 0.220 \\
\hline 1977 & 0.014 & 0.225 & 0.270 & 0.206 \\
\hline 1978 & 0.012 & 0.182 & 0.278 & 0.166 \\
\hline 1979 & 0.005 & 0.223 & 0.278 & 0.163 \\
\hline 1900 & 0.000 & 0.186 & 0.264 & 0.158 \\
\hline 1981 & 0.012 & 0.263 & 0.270 & 0.177 \\
\hline 1982 & 0.028 & 0.222 & 0.293 & 0.168 \\
\hline
\end{tabular}


rates, $1940-82$

\begin{tabular}{|c|c|c|c|}
\hline $35-39$ & $40-44$ & $45-49$ & Total \\
\hline - & - & - & - \\
\hline - & - & - & - \\
\hline - & - & - & - \\
\hline - & - & - & - \\
\hline - & - & - & - \\
\hline - & - & - & 5.0 .53 \\
\hline - & - & - & 5.919 \\
\hline - & - & - & 4.937 \\
\hline - & - & - & 5.608 \\
\hline - & - & - & 6.282 \\
\hline - & - & - & 5.610 \\
\hline - & - & - & 5.074 \\
\hline - & - & - & 6.363 \\
\hline - & - & - & 5.716 \\
\hline - & - & - & 6.842 \\
\hline - & - & - & 6.552 \\
\hline - & - & - & 6.214 \\
\hline - & - & - & 6.254 \\
\hline - & - & - & 6.190 \\
\hline - & - & - & 4.726 \\
\hline 0.116 & - & - & 3.667 \\
\hline 0.083 & - & - & 2.989 \\
\hline 0.198 & - & . & 6.172 \\
\hline 0.291 & - & . & 7.843 \\
\hline 0.218 & 0.110 & 0.014 & 6.409 \\
\hline 0.236 & 0.105 & 0.018 & 7.036 \\
\hline 0.232 & 0.105 & 0.015 & 6.921 \\
\hline 0.262 & 0.112 & 0.015 & 0.222 \\
\hline 0.294 & 0.168 & 0.017 & 3.047 \\
\hline 0.222 & 0.132 & 0.027 & 6.733 \\
\hline 0.258 & 0.128 & 0.005 & 7.038 \\
\hline 0.243 & 0.096 & 0.021 & 6.784 \\
\hline 0.220 & 0.123 & 0.010 & 6.285 \\
\hline 0.240 & 0.103 & 0.017 & 6.724 \\
\hline 0.254 & 0.123 & 0.022 & 0.903 \\
\hline 0.245 & 0.102 & 0.036 & $\epsilon .283$ \\
\hline 0.141 & 0.096 & 0.012 & 5.118 \\
\hline 0.128 & 0.031 & 0.014 & 4.686 \\
\hline 0.031 & 0.049 & 0.013 & 3.909 \\
\hline 0.113 & 0.040 & 0.014 & 4.182 \\
\hline 0.094 & 0.033 & 0.005 & 3.734 \\
\hline 0.111 & 0.049 & 0.011 & 4.461 \\
\hline 0.136 & 0.044 & 0.015 & 4.532 \\
\hline
\end{tabular}


Basic Table 23.B. Urban Guizhou Province (22): age-specific fertility

\begin{tabular}{|c|c|c|c|c|}
\hline $\begin{array}{l}\text { Birth year } \\
\text { of child }\end{array}$ & $15-19$ & $20-24$ & $25-29$ & $30-34$ \\
\hline 1940 & 0.065 & - & - & - \\
\hline 1941 & 0.035 & - & - & - \\
\hline 1942 & 0.058 & - & - & - \\
\hline 1943 & 0.049 & - & - & - \\
\hline 1944 & 0.061 & - & • & - \\
\hline 1945 & 0.071 & 0.208 & - & - \\
\hline 1940 & 0.050 & 0.129 & - & - \\
\hline 1947 & 0.072 & 0.292 & - & - \\
\hline 1948 & 0.043 & 0.171 & - & - \\
\hline 1949 & 0.053 & 0.280 & - & - \\
\hline 1950 & 0.090 & 0.225 & 0.169 & - \\
\hline 1951 & 0.029 & 0.163 & $0.1 \in 4$ & - \\
\hline 1952 & 0.046 & 0.228 & 0.222 & - \\
\hline 1953 & 0.097 & 0.141 & 0.318 & - \\
\hline 1954 & 0.020 & 0.244 & 0.255 & - \\
\hline 1955 & 0.063 & 0.209 & 0.236 & 0.182 \\
\hline 1956 & 0.032 & 0.134 & 0.238 & 0.258 \\
\hline 1957 & 0.053 & 0.221 & 0.264 & 0.210 \\
\hline 1958 & 0.044 & 0.194 & 0.227 & 0.196 \\
\hline 1959 & 0.024 & 0.221 & 0.244 & 0.207 \\
\hline 1960 & - & 0.126 & 0.150 & 0.118 \\
\hline 1961 & 0.013 & 0.095 & 0.163 & 0.088 \\
\hline 1962 & 0.037 & 0.203 & 0.185 & 0.228 \\
\hline 1963 & 0.024 & 0.264 & 0.290 & 0.206 \\
\hline 1964 & 0.021 & 0.295 & 0.241 & 0.170 \\
\hline 1965 & 0.009 & 0.168 & 0.283 & 0.239 \\
\hline 1960 & 0.028 & 0.232 & 0.211 & 0.240 \\
\hline 1907 & 0.027 & 0.111 & 0.203 & 0.166 \\
\hline 1968 & - & 0.165 & 0.286 & 0.203 \\
\hline 1969 & 0.008 & 0.208 & 0.307 & 0.181 \\
\hline 1970 & 0.030 & 0.156 & 0.207 & 0.157 \\
\hline 1971 & 0.020 & 0.121 & 0.206 & 0.158 \\
\hline 1972 & - & 0.063 & 0.258 & 0.107 \\
\hline 1973 & - & 0.104 & 0.223 & 0.110 \\
\hline 1974 & 0.007 & 0.106 & 0.177 & 0.177 \\
\hline 1975 & 0.023 & 0.068 & 0.175 & 0.026 \\
\hline 1976 & 0.008 & 0.054 & 0.149 & 0.026 \\
\hline 1977 & - & 0.071 & 0.180 & 0.086 \\
\hline 1978 & - & 0.046 & 0.207 & 0.059 \\
\hline 1979 & • & 0.061 & 0.162 & 0.062 \\
\hline 1980 & 0.006 & 0.084 & 0.106 & 0.009 \\
\hline 1981 & 0.005 & 0.008 & 0.183 & 0.074 \\
\hline 1982 & - & 0.070 & 0.090 & 0.018 \\
\hline
\end{tabular}


rates, $1940-82$

\begin{tabular}{|c|c|c|c|}
\hline $35-39$ & $40-44$ & $45-49$ & Total \\
\hline$\bullet$ & & - & - \\
\hline - & - & - & • \\
\hline - & - & - & • \\
\hline • & - & $\bullet$ & • \\
\hline - & - & - & - \\
\hline - & - & - & 6.178 \\
\hline - & $\bullet$ & - & $3 \cdot 550$ \\
\hline - & - & - & 5.090 \\
\hline - & - & - & 4.010 \\
\hline - & - & - & 6.702 \\
\hline$\bullet$ & - & - & 5.023 \\
\hline - & - & - & $3 \cdot 938$ \\
\hline$\bullet$ & • & - & 5.468 \\
\hline - & - & - & 5.041 \\
\hline - & • & $\bullet$ & 5.902 \\
\hline - & - & $\bullet$ & 4.714 \\
\hline - & - & - & 4.637 \\
\hline$\bullet$ & - & - & 4.991 \\
\hline - & • & - & 5.204 \\
\hline - & - & - & 4.494 \\
\hline 0.065 & - & • & 2.705 \\
\hline 0.053 & - & - & 2.204 \\
\hline 0.094 & - & - & 4.252 \\
\hline 0.171 & - & - & 5.427 \\
\hline 0.158 & 0.080 & - & 4.822 \\
\hline 0.177 & 0.0 .39 & 0.024 & 4.697 \\
\hline 0.063 & 0.035 & 0.020 & 4.144 \\
\hline 0.060 & 0.035 & 0.018 & 3.101 \\
\hline 0.162 & 0.024 & • & 4.204 \\
\hline 0.085 & 0.061 & 0.013 & 4.311 \\
\hline 0.140 & 0.035 & • & 3.628 \\
\hline 0.077 & $\therefore$ & - & 2.912 \\
\hline 0.055 & 0.036 & - & 2.597 \\
\hline 0.048 & 0.032 & - & 2.590 \\
\hline 0.020 & 0.032 & - & 2.587 \\
\hline 0.021 & 0.010 & 0.012 & $I .67 I$ \\
\hline - & - & - & 1.186 \\
\hline - & 0.028 & - & 1.825 \\
\hline$\bullet$ & - & - & 1.563 \\
\hline 0.012 & - & 0.011 & 1.543 \\
\hline - & - & $\bullet$ & 1.023 \\
\hline - & - & 0.010 & 1.404 \\
\hline - & • & $\bullet$ & 0.895 \\
\hline
\end{tabular}


Basic Table 23.C. Rural Guizhou Province (22): age-specific fertility

\begin{tabular}{|c|c|c|c|c|}
\hline $\begin{array}{l}\text { Birth year } \\
\text { of child }\end{array}$ & $15-19$ & $20-24$ & $25-29$ & $30-34$ \\
\hline 1940 & 0.058 & - & - & - \\
\hline 1941 & 0.040 & - & - & • \\
\hline 1942 & 0.058 & - & - & - \\
\hline 1943 & 0.072 & - & - & - \\
\hline 1944 & 0.052 & - & - & - \\
\hline 1945 & 0.041 & 0.194 & - & - \\
\hline 1946 & 0.046 & 0.298 & - & - \\
\hline 1947 & 0.067 & 0.204 & - & - \\
\hline 1948 & 0.052 & 0.299 & - & - \\
\hline 1949 & 0.074 & 0.291 & - & - \\
\hline 1950 & 0.005 & 0.257 & 0.296 & - \\
\hline 1951 & 0.070 & 0.219 & 0.271 & - \\
\hline 1552 & 0.079 & 0.289 & 0.350 & - \\
\hline 1953 & $0.0 \in 4$ & 0.308 & 0.266 & - \\
\hline 1954 & 0.083 & 0.296 & 0.341 & - \\
\hline 1955 & 0.066 & 0.333 & 0.310 & 0.293 \\
\hline 1956 & 0.103 & 0.262 & 0.362 & 0.241 \\
\hline 1957 & 0.073 & 0.283 & 0.319 & 0.324 \\
\hline 1958 & 0.064 & 0.287 & 0.317 & 0.299 \\
\hline 1959 & 0.029 & 0.215 & 0.248 & 0.217 \\
\hline 1960 & 0.033 & 0.166 & 0.191 & 0.180 \\
\hline 1961 & 0.019 & 0.148 & 0.152 & 0.153 \\
\hline 1962 & 0.045 & 0.318 & 0.320 & 0.301 \\
\hline 1963 & 0.061 & 0.318 & 0.405 & 0.405 \\
\hline 1964 & 0.053 & $0.29 z$ & 0.345 & 0.289 \\
\hline 1965 & 0.059 & 0.320 & 0.364 & 0.360 \\
\hline 1906 & 0.067 & 0.301 & 0.368 & 0.344 \\
\hline 1967 & 0.043 & 0.269 & 0.315 & 0.285 \\
\hline 1968 & 0.070 & 0.315 & 0.434 & 0.397 \\
\hline 1969 & 0.040 & 0.284 & 0.364 & 0.318 \\
\hline 1970 & $0.04 a$ & 0.333 & 0.356 & 0.350 \\
\hline 1971 & 0.043 & 0.327 & 0.351 & 0.343 \\
\hline 1972 & 0.041 & 0.251 & 0.365 & 0.306 \\
\hline 1973 & 0.042 & 0.288 & 0.358 & 0.373 \\
\hline 1974 & 0.046 & 0.306 & 0.389 & 0.310 \\
\hline 1975 & 0.049 & 0.286 & 0.328 & 0.296 \\
\hline 1976 & 0.038 & 0.257 & 0.314 & 0.245 \\
\hline 1977 & 0.016 & 0.250 & 0.283 & 0.222 \\
\hline 1978 & 0.015 & 0.204 & 0.288 & 0.181 \\
\hline 1979 & 0.005 & 0.250 & 0.296 & 0.177 \\
\hline 1980 & 0.006 & 0.204 & 0.288 & 0.181 \\
\hline 1981 & 0.013 & 0.307 & 0.284 & 0.192 \\
\hline 1982 & 0.032 & 0.248 & 0.326 & 0.190 \\
\hline
\end{tabular}


rates, $1940-82$

\begin{tabular}{|c|c|c|c|}
\hline $35-39$ & $40-44$ & $45-49$ & Total \\
\hline - & - & - & - \\
\hline • & - & - & • \\
\hline - & - & - & - \\
\hline - & - & - & - \\
\hline - & - & - & - \\
\hline - & - & - & 4.816 \\
\hline - & • & - & 6.413 \\
\hline - & - & - & 4.905 \\
\hline - & - & - & 5.944 \\
\hline - & - & - & 6.195 \\
\hline - & - & - & 5.734 \\
\hline - & - & - & $5 \cdot 309$ \\
\hline - & - & - & 6.545 \\
\hline • & - & - & 5.351 \\
\hline - & - & - & 7.026 \\
\hline - & - & - & 6.911 \\
\hline • & - & - & 6.520 \\
\hline - & - & - & 6.496 \\
\hline - & - & - & 6.376 \\
\hline - & - & - & 4.769 \\
\hline 0.129 & - & • & 3.842 \\
\hline 0.090 & - & - & 3.130 \\
\hline 0.227 & - & - & 6.518 \\
\hline 0.320 & • & - & 8.272 \\
\hline 0.231 & 0.117 & 0.015 & 6.717 \\
\hline 0.249 & 0.123 & 0.019 & 7.466 \\
\hline $0.2 \in 5$ & 0.131 & 0.014 & 7.447 \\
\hline 0.301 & 0.133 & 0.014 & 6.79 .9 \\
\hline 0.310 & 0.203 & 0.021 & 8.747 \\
\hline 0.250 & 0.148 & 0.030 & 7.175 \\
\hline 0.283 & 0.147 & 0.007 & 7.616 \\
\hline 0.283 & 0.114 & 0.027 & 7.442 \\
\hline 0.255 & 0.146 & 0.013 & 6.906 \\
\hline 0.280 & 0.118 & 0.021 & 7.398 \\
\hline 0.293 & 0.148 & 0.027 & 7.601 \\
\hline 0.280 & 0.121 & 0.041 & 7.007 \\
\hline 0.163 & 0.115 & 0.015 & 5.736 \\
\hline 0.148 & 0.091 & 0.010 & 5.138 \\
\hline 0.094 & 0.057 & 0.016 & 4.274 \\
\hline 0.128 & 0.047 & 0.015 & 4.592 \\
\hline 0.107 & 0.038 & 0.006 & 4.150 \\
\hline 0.125 & 0.057 & 0.012 & 4.943 \\
\hline 0.155 & 0.051 & 0.018 & 5.100 \\
\hline
\end{tabular}


Basic Table 24.A. Total Yunnan Province (23): age-specific fertility

\begin{tabular}{|c|c|c|c|c|}
\hline $\begin{array}{l}\text { Birth year } \\
\text { of child }\end{array}$ & $15-19$ & $20-24$ & $25-29$ & $30-34$ \\
\hline 1940 & 0.069 & - & - & - \\
\hline 1941 & 0.046 & - & - & $\bullet$ \\
\hline 1942 & 0.088 & - & - & - \\
\hline 1943 & 0.064 & - & - & - \\
\hline 1944 & 0.054 & - & - & $\bullet$ \\
\hline 1945 & 0.042 & 0.258 & - & - \\
\hline 1946 & 0.077 & 0.273 & - & - \\
\hline 1947 & 0.093 & 0.267 & - & - \\
\hline 1948 & 0.084 & $0.23 \mathrm{~g}$ & - & - \\
\hline 1949 & 0.078 & 0.313 & - & - \\
\hline 1950 & 0.108 & 0.228 & 0.272 & • \\
\hline 1951 & 0.082 & 0.272 & 0.243 & - \\
\hline 1952 & 0.089 & 0.311 & 0.288 & - \\
\hline 1953 & 0.084 & 0.271 & 0.303 & $\bullet$ \\
\hline 1954 & 0.104 & 0.290 & 0.345 & - \\
\hline 1955 & 0.103 & 0.316 & 0.322 & 0.242 \\
\hline 1956 & 0.068 & 0.292 & 0.273 & 0.267 \\
\hline 1957 & 0.086 & 0.294 & 0.306 & 0.244 \\
\hline 1958 & 0.071 & 0.255 & 0.288 & 0.289 \\
\hline 1955 & 0.053 & 0.175 & 0.213 & 0.212 \\
\hline 1960 & 0.047 & 0.207 & 0.216 & 0.186 \\
\hline 1961 & 0.043 & 0.206 & 0.182 & 0.167 \\
\hline 1962 & 0.087 & 0.321 & 0.361 & 0.294 \\
\hline 1963 & 0.074 & 0.330 & 0.367 & 0.319 \\
\hline 1964 & 0.086 & 0.325 & 0.352 & 0.274 \\
\hline 1965 & 0.064 & 0.304 & 0.333 & 0.301 \\
\hline 1966 & 0.066 & 0.284 & 0.336 & 0.279 \\
\hline 1967 & 0.045 & 0.263 & 0.282 & 0.239 \\
\hline 1968 & 0.056 & 0.313 & 0.315 & 0.310 \\
\hline 1969 & 0.046 & 0.315 & 0.326 & 0.244 \\
\hline 1970 & 0.041 & 0.279 & 0.312 & 0.274 \\
\hline 1971 & 0.047 & 0.295 & 0.293 & 0.285 \\
\hline 1972 & 0.042 & 0.291 & 0.307 & 0.220 \\
\hline 1973 & 0.042 & 0.244 & 0.295 & 0.238 \\
\hline 1974 & 0.035 & 0.268 & 0.315 & 0.207 \\
\hline 1975 & 0.052 & 0.244 & 0.301 & 0.230 \\
\hline 1970 & 0.035 & 0.248 & 0.312 & 0.202 \\
\hline 1977 & 0.019 & 0.261 & 0.296 & 0.197 \\
\hline 1978 & 0.012 & 0.209 & 0.271 & 0.207 \\
\hline 1979 & 0.014 & 0.229 & 0.250 & 0.158 \\
\hline 1980 & 0.013 & 0.176 & 0.218 & 0.110 \\
\hline 1981 & 0.019 & 0.231 & 0.225 & 0.140 \\
\hline 1982 & 0.026 & 0.236 & 0.235 & 0.123 \\
\hline
\end{tabular}


rates, $1940-82$

\begin{tabular}{|c|c|c|c|}
\hline $35-39$ & $40-44$ & $45-49$ & Total \\
\hline - & - & $\bullet$ & - \\
\hline • & - & - & - \\
\hline - & $\bullet$ & - & - \\
\hline$\bullet$ & $\bullet$ & - & • \\
\hline - & - & • & - \\
\hline - & - & - & 4.706 \\
\hline - & - & - & 5.699 \\
\hline - & - & - & $5 \cdot 590$ \\
\hline - & - & - & 5.437 \\
\hline - & - & - & 6.626 \\
\hline - & - & - & 5.610 \\
\hline - & - & - & 5.429 \\
\hline - & - & - & 6.223 \\
\hline - & - & - & 5.990 \\
\hline - & - & - & 6.780 \\
\hline - & - & - & 6.497 \\
\hline - & - & - & 5.906 \\
\hline - & - & - & 6.167 \\
\hline - & - & - & 6.087 \\
\hline - & - & - & 4.363 \\
\hline 0.159 & • & - & 4.505 \\
\hline 0.108 & - & - & 3.797 \\
\hline 0.198 & - & - & 6.926 \\
\hline 0.298 & - & - & 7.787 \\
\hline 0.187 & 0.107 & 0.022 & 6.762 \\
\hline 0.219 & 0.104 & 0.017 & 6.712 \\
\hline 0.219 & 0.088 & 0.017 & 6.437 \\
\hline 0.193 & 0.069 & 0.007 & 5.494 \\
\hline 0.204 & 0.133 & 0.011 & 0.70 .9 \\
\hline 0.231 & 0.111 & 0.021 & 6.472 \\
\hline 0.179 & 0.098 & 0.014 & 5.984 \\
\hline 0.182 & 0.089 & 0.002 & 5.964 \\
\hline 0.173 & 0.079 & 0.004 & 5.584 \\
\hline 0.181 & 0.085 & 0.016 & 5.535 \\
\hline 0.155 & 0.077 & 0.012 & 5.338 \\
\hline 0.157 & 0.078 & 0.005 & 5.332 \\
\hline 0.168 & 0.074 & $0.00 E$ & 5.227 \\
\hline 0.164 & 0.053 & 0.003 & 4.983 \\
\hline 0.132 & 0.073 & 0.010 & 4.571 \\
\hline 0.082 & 0.059 & $0.00 \dot{E}$ & 3.993 \\
\hline 0.089 & 0.037 & 0.005 & 3.243 \\
\hline 0.083 & 0.044 & 0.004 & 3.727 \\
\hline 0.094 & 0.030 & 0.013 & 3.787 \\
\hline
\end{tabular}


Basic Table 24.B. Urban Yunnan Province (23): age-specific fertility

\begin{tabular}{|c|c|c|c|c|}
\hline $\begin{array}{l}\text { Birth year } \\
\text { of child }\end{array}$ & $15-19$ & $20-24$ & $25-29$ & $30-34$ \\
\hline 1940 & 0.080 & - & - & - \\
\hline 1941 & 0.014 & - & - & - \\
\hline 1942 & 0.110 & - & - & - \\
\hline 1943 & 0.042 & - & - & - \\
\hline 1944 & 0.066 & - & - & - \\
\hline 1945 & 0.017 & 0.223 & - & - \\
\hline 1946 & 0.082 & 0.317 & - & - \\
\hline 1947 & 0.097 & 0.304 & - & - \\
\hline 1940 & 0.084 & 0.168 & - & - \\
\hline 1949 & 0.084 & 0.265 & - & - \\
\hline 1950 & 0.101 & 0.222 & 0.271 & - \\
\hline 1951 & 0.139 & 0.304 & 0.144 & - \\
\hline 1952 & 0.110 & 0.266 & 0.244 & - \\
\hline 1953 & 0.128 & 0.307 & 0.262 & - \\
\hline 1954 & 0.128 & 0.245 & 0.322 & - \\
\hline 1955 & 0.133 & 0.336 & 0.264 & 0.191 \\
\hline 1956 & 0.083 & 0.253 & 0.263 & 0.159 \\
\hline 1957 & 0.129 & 0.343 & 0.275 & 0.244 \\
\hline 1958 & 0.048 & 0.323 & 0.315 & 0.273 \\
\hline 1959 & 0.076 & 0.211 & 0.217 & 0.180 \\
\hline 1960 & 0.039 & 0.233 & 0.202 & 0.136 \\
\hline 1961 & 0.040 & 0.213 & 0.209 & 0.132 \\
\hline 1902 & 0.145 & 0.257 & 0.264 & 0.170 \\
\hline 1963 & 0.095 & 0.372 & 0.424 & 0.269 \\
\hline 1964 & 0.138 & 0.247 & 0.271 & 0.231 \\
\hline 1965 & 0.040 & 0.322 & 0.291 & 0.155 \\
\hline 1966 & 0.050 & 0.231 & 0.185 & 0.153 \\
\hline 1967 & 0.011 & 0.134 & 0.148 & $0.06 \mathrm{I}$ \\
\hline 1968 & - & 0.345 & 0.315 & 0.182 \\
\hline 1969 & 0.069 & 0.226 & 0.266 & 0.136 \\
\hline 1970 & 0.015 & 0.212 & 0.234 & 0.125 \\
\hline 1971 & 0.007 & 0.225 & 0.221 & 0.120 \\
\hline 1972 & 0.021 & 0.126 & 0.145 & 0.168 \\
\hline 1973 & 0.014 & 0.148 & 0.333 & 0.095 \\
\hline 1974 & 0.014 & 0.155 & 0.126 & 0.067 \\
\hline 1975 & 0.008 & 0.141 & 0.133 & 0.045 \\
\hline 1976 & . & 0.176 & 0.213 & 0.100 \\
\hline 1977 & 0.007 & 0.152 & 0.211 & 0.078 \\
\hline 1978 & - & 0.137 & 0.197 & $0.0 \in 0$ \\
\hline 1979 & - & 0.071 & 0.121 & 0.038 \\
\hline 1980 & - & 0.070 & 0.141 & - \\
\hline 1981 & - & 0.107 & 0.081 & . \\
\hline 1962 & 0.010 & 0.145 & 0.125 & 0.021 \\
\hline
\end{tabular}


rates, $1940-82$

\begin{tabular}{|c|c|c|c|}
\hline $35-39$ & $40-44$ & $45-49$ & Total \\
\hline$\bullet$ & $\bullet$ & - & - \\
\hline - & - & - & - \\
\hline • & - & $\bullet$ & - \\
\hline - & - & - & - \\
\hline - & - & - & - \\
\hline - & - & - & 4.826 \\
\hline - & - & - & 5.652 \\
\hline - & - & - & 5.610 \\
\hline • & - & - & 4.989 \\
\hline - & - & - & $7 \cdot 434$ \\
\hline - & - & - & $5 \cdot 319$ \\
\hline - & - & - & 5.528 \\
\hline - & - & - & 5.471 \\
\hline - & - & - & 6.181 \\
\hline - & - & - & 6.502 \\
\hline - & - & - & 5.797 \\
\hline - & - & - & 5.232 \\
\hline - & - & - & 6.346 \\
\hline - & - & - & 6.148 \\
\hline - & - & - & 4.143 \\
\hline 0.127 & - & - & 4.061 \\
\hline 0.130 & - & - & 3.728 \\
\hline 0.183 & - & - & 5.387 \\
\hline 0.241 & • & - & 7.845 \\
\hline 0.151 & 0.007 & 0.017 & 5.616 \\
\hline 0.136 & 0.080 & 0.031 & 5.275 \\
\hline 0.132 & 0.029 & - & 3.923 \\
\hline 0.040 & 0.012 & - & 2.032 \\
\hline 0.001 & 0.052 & - & 4.777 \\
\hline 0.077 & 0.019 & • & 3.963 \\
\hline 0.034 & 0.020 & 0.016 & 3.309 \\
\hline 0.070 & 0.041 & - & 3.419 \\
\hline 0.055 & - & - & 2.570 \\
\hline 0.034 & 0.015 & - & 3.195 \\
\hline 0.030 & - & - & 1.959 \\
\hline 0.017 & 0.013 & - & $i .901$ \\
\hline 0.019 & 0.006 & - & 2.570 \\
\hline 0.030 & - & - & 2.390 \\
\hline 0.010 & • & - & 2.014 \\
\hline 0.019 & 0.015 & - & 1.313 \\
\hline • & $\bullet$ & - & 1.055 \\
\hline - & 0.009 & - & 0.987 \\
\hline - & - & - & 1.501 \\
\hline
\end{tabular}


Basic Table 24.C. Rural Yunnan Province (23): age-specific fertility

\begin{tabular}{|c|c|c|c|c|}
\hline $\begin{array}{l}\text { Birth year } \\
\text { of child }\end{array}$ & $15-19$ & $20-24$ & $25-29$ & $30-34$ \\
\hline 1940 & 0.068 & - & - & - \\
\hline 1941 & 0.051 & - & - & - \\
\hline 1942 & 0.084 & - & - & - \\
\hline 1943 & 0.068 & - & - & - \\
\hline 1944 & 0.051 & - & - & - \\
\hline 1945 & 0.048 & 0.263 & - & - \\
\hline 1946 & 0.076 & $0.26 \epsilon$ & - & - \\
\hline 1947 & 0.092 & 0.260 & - & - \\
\hline 1948 & 0.084 & 0.252 & $\bullet$ & - \\
\hline 1949 & 0.077 & 0.324 & - & - \\
\hline 1950 & 0.109 & 0.230 & 0.273 & - \\
\hline 1951 & 0.067 & 0.265 & 0.259 & - \\
\hline 1902 & 0.084 & 0.320 & 0.296 & - \\
\hline 1953 & 0.074 & 0.264 & 0.312 & - \\
\hline 1954 & 0.099 & 0.300 & 0.350 & - \\
\hline 1955 & 0.098 & 0.312 & 0.334 & 0.249 \\
\hline 1956 & 0.065 & 0.301 & 0.276 & 0.285 \\
\hline 1957 & 0.079 & 0.231 & 0.312 & 0.244 \\
\hline 1958 & 0.074 & 0.239 & 0.282 & 0.292 \\
\hline 1959 & 0.050 & 0.168 & 0.213 & 0.219 \\
\hline 1960 & 0.049 & 0.202 & 0.220 & 0.197 \\
\hline 1961 & 0.043 & 0.204 & 0.175 & 0.175 \\
\hline 1962 & 0.081 & 0.330 & 0.386 & 0.318 \\
\hline 1963 & 0.072 & 0.324 & 0.354 & 0.329 \\
\hline 1964 & 0.081 & 0.336 & 0.368 & 0.283 \\
\hline 1905 & 0.066 & 0.302 & 0.340 & 0.336 \\
\hline 1966 & 0.067 & 0.290 & 0.361 & 0.309 \\
\hline 1967 & 0.048 & 0.277 & 0.302 & 0.284 \\
\hline 1963 & 0.062 & 0.310 & 0.315 & 0.340 \\
\hline 1969 & 0.043 & 0.323 & 0.335 & 0.266 \\
\hline 1970 & 0.044 & 0.284 & 0.323 & 0.302 \\
\hline 1971 & 0.052 & 0.301 & 0.302 & 0.313 \\
\hline 1972 & 0.044 & 0.307 & 0.325 & 0.228 \\
\hline 1973 & 0.046 & 0.254 & 0.292 & 0.259 \\
\hline 1974 & 0.037 & 0.280 & 0.332 & 0.226 \\
\hline 1975 & 0.057 & 0.257 & 0.314 & 0.255 \\
\hline 1976 & 0.039 & 0.257 & 0.320 & 0.214 \\
\hline 1977 & 0.021 & 0.274 & 0.304 & 0.210 \\
\hline 1978 & 0.014 & 0.218 & 0.278 & 0.222 \\
\hline 1979 & 0.016 & 0.249 & 0.264 & 0.168 \\
\hline 1980 & 0.015 & 0.189 & 0.227 & 0.119 \\
\hline 1981 & 0.021 & 0.246 & 0.243 & 0.152 \\
\hline 1982 & 0.028 & 0.249 & 0.249 & 0.133 \\
\hline
\end{tabular}


rates, $1940-82$

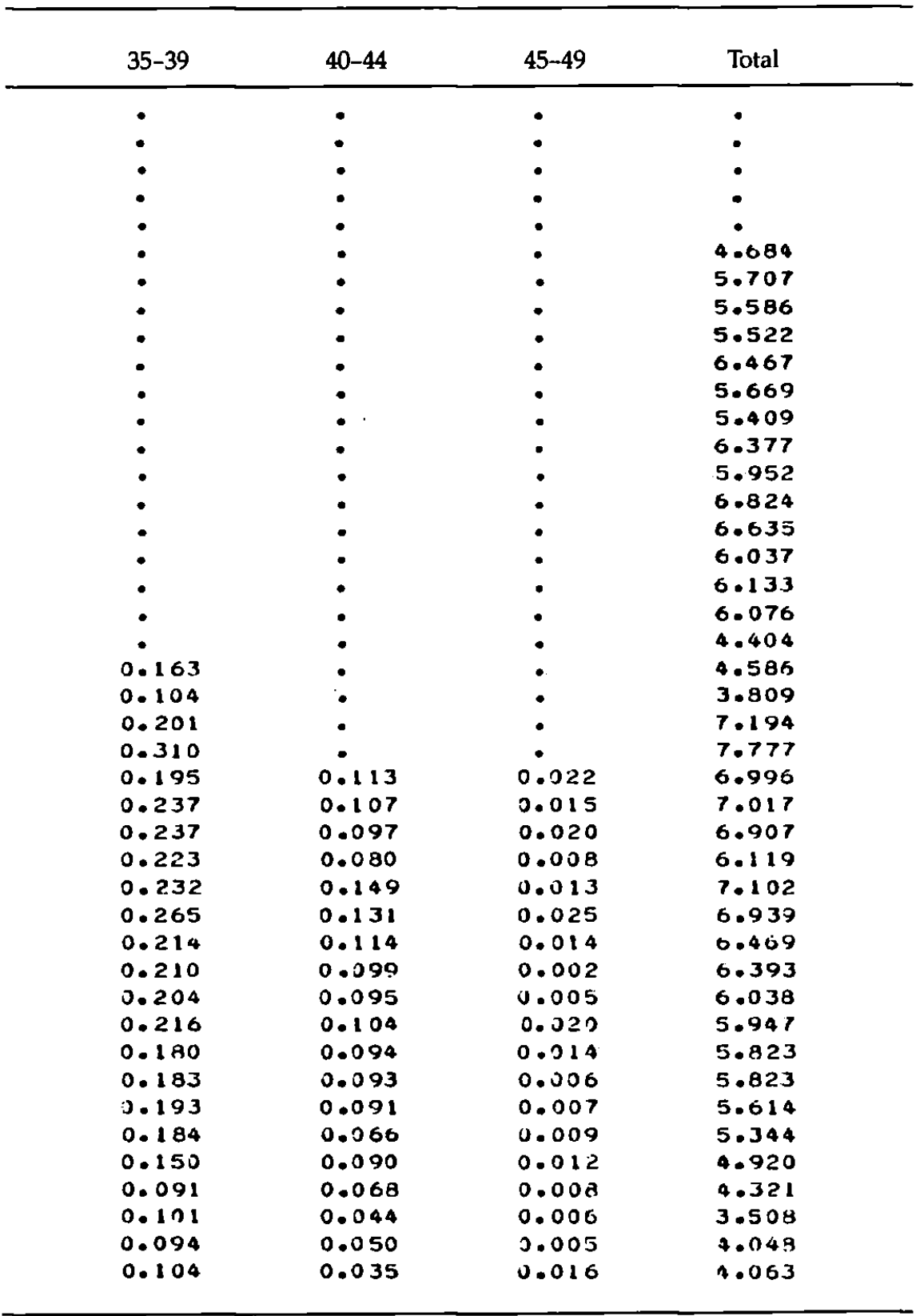


Basic Table 25.A. Total Shaanxi Province (25): age-specific fertility

\begin{tabular}{|c|c|c|c|c|}
\hline $\begin{array}{l}\text { Birth year } \\
\text { of child }\end{array}$ & 15-19 & $20-24$ & $25-29$ & $30-34$ \\
\hline 1940 & 0.095 & - & - & - \\
\hline 1941 & 0.097 & • & • & • \\
\hline 1942 & 0.119 & • & - & - \\
\hline 1943 & 0.106 & - & - & - \\
\hline 1944 & 0.128 & - & - & . \\
\hline 1945 & 0.099 & 0.240 & - & - \\
\hline 1946 & 0.097 & 0.293 & - & - \\
\hline 1947 & 0.131 & 0.280 & - & - \\
\hline 1948 & 0.086 & 0.259 & - & - \\
\hline 1949 & 0.132 & 0.260 & - & - \\
\hline 1950 & 0.147 & 0.247 & 0.227 & - \\
\hline 1951 & 0.141 & 0.270 & $0.2 \notin 4$ & - \\
\hline 1952 & 0.136 & 0.291 & 0.234 & - \\
\hline 1953 & 0.120 & 0.277 & 0.263 & - \\
\hline 1954 & 0.118 & 0.296 & 0.288 & - \\
\hline 1955 & 0.122 & 0.308 & 0.260 & 0.254 \\
\hline 1950 & 0.101 & 0.286 & 0.275 & 0.214 \\
\hline 1957 & 0.130 & 0.317 & 0.319 & 0.274 \\
\hline 1958 & 0.139 & 0.305 & 0.228 & 0.222 \\
\hline 1959 & 0.088 & 0.233 & 0.256 & 0.164 \\
\hline 1900 & 0.083 & 0.242 & 0.241 & 0.205 \\
\hline 1901 & 0.064 & 0.213 & 0.200 & 0.161 \\
\hline 1962 & 0.105 & 0.325 & 0.348 & 0.259 \\
\hline 1963 & 0.135 & 0.372 & 0.329 & 0.294 \\
\hline 1904 & 0.101 & 0.302 & 0.303 & 0.216 \\
\hline 1905 & 0.065 & 0.276 & 0.266 & 0.241 \\
\hline 1960 & 0.084 & $0.31 \mathrm{E}$ & 0.300 & 0.248 \\
\hline 1967 & 0.056 & 0.280 & 0.275 & 0.207 \\
\hline 1963 & 0.009 & 0.304 & $0.35 i$ & 0.255 \\
\hline 1969 & 0.057 & 0.303 & 0.281 & 0.205 \\
\hline 1970 & 0.057 & 0.292 & 0.270 & 0.251 \\
\hline 1971 & 0.054 & 0.306 & 0.266 & 0.205 \\
\hline 1972 & 0.044 & 0.265 & 0.310 & 0.217 \\
\hline 1973 & 0.046 & 0.251 & 0.240 & 0.186 \\
\hline 1974 & 0.043 & 0.260 & 0.246 & 0.153 \\
\hline 1973 & 0.025 & 0.194 & 0.204 & 0.130 \\
\hline 1976 & 0.019 & 0.231 & 0.193 & 0.111 \\
\hline 1977 & 0.008 & 0.176 & 0.187 & 0.101 \\
\hline 1976 & 0.008 & 0.178 & 0.210 & 0.083 \\
\hline 1979 & 0.008 & 0.188 & 0.240 & 0.072 \\
\hline 1980 & 0.004 & 0.169 & 0.182 & 0.061 \\
\hline 1981 & 0.005 & 0.188 & 0.203 & 0.060 \\
\hline 1982 & 0.016 & 0.237 & 0.186 & 0.045 \\
\hline
\end{tabular}


rates, $1940-82$

\begin{tabular}{|c|c|c|c|}
\hline $35-39$ & $40-44$ & $45-49$ & Total \\
\hline - & - & • & - \\
\hline$\bullet$ & $\bullet$ & • & • \\
\hline - & - & - & • \\
\hline - & - & - & • \\
\hline - & - & - & - \\
\hline - & - & - & 4.582 \\
\hline - & - & - & 5.682 \\
\hline - & - & - & $5 \cdot 322$ \\
\hline - & - & - & 5.082 \\
\hline - & - & - & 5.565 \\
\hline - & - & - & 5.122 \\
\hline - & - & - & 5.498 \\
\hline - & - & - & 6.040 \\
\hline - & - & - & 5.744 \\
\hline - & - & - & 6.220 \\
\hline - & - & - & 5.994 \\
\hline - & - & - & 5.657 \\
\hline - & - & - & 6.743 \\
\hline - & - & - & 5.888 \\
\hline - & - & - & 4.001 \\
\hline 0.169 & - & - & $5.10 i$ \\
\hline 0.137 & - & - & 4.208 \\
\hline 0.153 & $\cdot$ & - & 6.431 \\
\hline 0.237 & • & - & $7 \cdot 359$ \\
\hline 0.198 & 0.105 & 0.006 & 6.152 \\
\hline 0.151 & 0.056 & 0.010 & 5.332 \\
\hline 0.157 & $0.08 ?$ & 0.012 & 6.037 \\
\hline 0.158 & 0.063 & 0.007 & 5.228 \\
\hline 0.170 & 0.100 & 0.011 & 0.331 \\
\hline 0.165 & 0.068 & 0.013 & 5.461 \\
\hline 0.137 & 0.082 & 0.008 & 5.513 \\
\hline 0.126 & 0.075 & 0.008 & $5 \cdot 197$ \\
\hline 0.130 & 0.077 & 0.006 & 5.274 \\
\hline 0.106 & 0.046 & 0.012 & 4.428 \\
\hline 0.090 & 0.042 & 0.004 & 4.192 \\
\hline 0.077 & 0.026 & 0.004 & 3.293 \\
\hline 0.069 & 0.022 & 0.002 & 3.233 \\
\hline 0.043 & 0.010 & 0.005 & 2.054 \\
\hline 0.041 & 0.026 & 0.002 & 2.742 \\
\hline 0.037. & 0.019 & • & 2.925 \\
\hline 0.015 & 0.007 & 0.002 & $2 \cdot 198$ \\
\hline 0.020 & 0.006 & 0.003 & 2.424 \\
\hline 0.019 & 0.003 & 0.003 & 2.544 \\
\hline
\end{tabular}


Basic Table 25.B. Urban Shaanxi Province (25): age-specific fertility

\begin{tabular}{|c|c|c|c|c|}
\hline $\begin{array}{l}\text { Birth year } \\
\text { of child }\end{array}$ & $15-19$ & $20-24$ & $25-29$ & $30-34$ \\
\hline 1940 & 0.063 & - & - & - \\
\hline 1941 & 0.056 & - & - & • \\
\hline 1942 & 0.066 & - & - & - \\
\hline 1943 & 0.041 & - & - & - \\
\hline 1944 & 0.091 & - & - & - \\
\hline 1945 & 0.046 & 0.302 & - & • \\
\hline 1946 & 0.104 & 0.226 & - & - \\
\hline 1947 & 0.092 & 0.274 & - & - \\
\hline 1948 & 0.054 & 0.217 & - & • \\
\hline 1949 & 0.103 & 0.190 & - & - \\
\hline 1950 & 0.058 & 0.184 & 0.176 & - \\
\hline 1951 & 0.091 & 0.200 & 0.260 & - \\
\hline 1952 & 0.077 & 0.259 & 0.252 & - \\
\hline 1953 & 0.072 & 0.217 & 0.227 & - \\
\hline 1954 & 0.055 & 0.286 & 0.326 & - \\
\hline 1955 & 0.046 & 0.199 & 0.266 & 0.201 \\
\hline 1956 & 0.043 & 0.272 & 0.244 & 0.260 \\
\hline 1957 & 0.095 & 0.341 & 0.317 & 0.252 \\
\hline 1958 & 0.068 & 0.252 & 0.209 & 0.165 \\
\hline 1959 & 0.055 & 0.213 & 0.264 & 0.127 \\
\hline 1960 & 0.043 & 0.186 & 0.225 & 0.165 \\
\hline 1961 & 0.037 & 0.159 & 0.181 & 0.096 \\
\hline 1962 & 0.019 & 0.292 & 0.336 & 0.217 \\
\hline 1963 & 0.061 & 0.284 & 0.396 & 0.271 \\
\hline 1964 & 0.039 & 0.182 & 0.243 & 0.183 \\
\hline 1965 & 0.010 & 0.239 & 0.258 & 0.110 \\
\hline 1966 & - & 0.128 & 0.234 & 0.169 \\
\hline 1967 & - & 0.191 & 0.185 & 0.127 \\
\hline 1968 & - & 0.153 & 0.352 & 0.196 \\
\hline 1909 & $\bullet$ & 0.184 & 0.238 & 0.174 \\
\hline 1970 & - & 0.146 & 0.231 & 0.175 \\
\hline 1971 & 0.021 & 0.115 & 0.248 & 0.144 \\
\hline 1972 & 0.013 & 0.145 & 0.191 & 0.107 \\
\hline 1973 & 0.006 & 0.081 & 0.224 & 0.088 \\
\hline 1974 & $\bullet$ & 0.121 & 0.155 & 0.032 \\
\hline 1975 & - & 0.088 & 0.204 & 0.077 \\
\hline 1976 & - & 0.097 & 0.183 & 0.083 \\
\hline 1977 & - & 0.141 & 0.179 & 0.105 \\
\hline 1978 & - & 0.083 & 0.242 & 0.061 \\
\hline 1979 & - & 0.027 & 0.181 & 0.107 \\
\hline 1980 & - & 0.060 & 0.175 & 0.068 \\
\hline 1981 & - & 0.037 & 0.172 & 0.058 \\
\hline 1982 & - & 0.061 & 0.171 & - \\
\hline
\end{tabular}


rates, $1940-82$

\begin{tabular}{|c|c|c|c|}
\hline $35-39$ & $40-44$ & $45-49$ & Total \\
\hline • & - & - & - \\
\hline - & - & - & - \\
\hline - & $\bullet$ & - & - \\
\hline - & $\bullet$ & - & - \\
\hline - & - & - & - \\
\hline - & - & - & 5.295 \\
\hline - & - & - & 5.969 \\
\hline - & - & - & 4.503 \\
\hline - & - & - & 5.469 \\
\hline - & - & - & 5.244 \\
\hline • & - & - & $3 \cdot 345$ \\
\hline - & - & - & 5.257 \\
\hline - & - & - & 6.081 \\
\hline - & - & - & 5.049 \\
\hline - & - & - & 6.667 \\
\hline - & - & - & 4.586 \\
\hline - & - & - & 5.871 \\
\hline - & - & - & 0.587 \\
\hline - & - & - & 5.112 \\
\hline - & - & - & 4.409 \\
\hline 0.151 & - & - & 4.121 \\
\hline 0.113 & - & - & 3.393 \\
\hline 0.154 & - & - & 5.687 \\
\hline 0.186 & - & - & 6.735 \\
\hline 0.118 & 0.084 & - & 4.244 \\
\hline 0.110 & 0.025 & - & 3.787 \\
\hline 0.026 & 0.045 & 0.017 & 3.101 \\
\hline 0.067 & 0.044 & 0.015 & 3.138 \\
\hline 0.101 & 0.052 & - & 4.262 \\
\hline 0.088 & 0.018 & 0.028 & 3.648 \\
\hline 0.071 & 0.046 & - & 3.340 \\
\hline 0.060 & 0.020 & - & 3.069 \\
\hline 0.077 & 0.025 & - & 2.794 \\
\hline 0.041 & $0.0 \geq 3$ & - & 2.317 \\
\hline 0.020 & - & - & 1.038 \\
\hline 0.015 & 0.006 & - & 1.951 \\
\hline 0.032 & 0.006 & - & 1.999 \\
\hline 0.012 & - & - & 2.186 \\
\hline 0.027 & 0.010 & - & 2.115 \\
\hline 0.008 & • & - & 1.615 \\
\hline$\bullet$ & - & - & 1.515 \\
\hline 0.009 & - & - & 1.381 \\
\hline 0.020 & - & - & 1.262 \\
\hline
\end{tabular}


Basic Table 25.C. Rural Shaanxi Province (25): age-specific fertility

\begin{tabular}{|c|c|c|c|c|}
\hline $\begin{array}{l}\text { Birth year } \\
\text { of child }\end{array}$ & $15-19$ & $20-24$ & $25-29$ & $30-34$ \\
\hline 1940 & 0.101 & - & - & - \\
\hline 1941 & 0.106 & - & - & - \\
\hline 1942 & 0.131 & - & - & - \\
\hline 1943 & 0.121 & - & - & - \\
\hline 1944 & 0.138 & - & - & - \\
\hline 1945 & 0.114 & 0.227 & - & - \\
\hline 1946 & 0.095 & 0.308 & - & - \\
\hline 1947 & 0.142 & 0.281 & - & - \\
\hline 1948 & 0.095 & 0.268 & - & - \\
\hline 1949 & 0.141 & 0.278 & - & - \\
\hline 1950 & 0.179 & 0.263 & 0.237 & - \\
\hline 1951 & 0.161 & 0.289 & 0.264 & - \\
\hline 1952 & 0.161 & 0.301 & 0.290 & - \\
\hline 1953 & 0.141 & 0.294 & 0.271 & - \\
\hline 1954 & 0.145 & 0.300 & 0.278 & • \\
\hline 1955 & 0.151 & 0.347 & 0.258 & 0.264 \\
\hline $\begin{array}{l}1956 \\
1957\end{array}$ & $\begin{array}{l}0.122 \\
0.140\end{array}$ & $\begin{array}{l}0.291 \\
0.308\end{array}$ & $\begin{array}{l}0.284 \\
0.319\end{array}$ & $\begin{array}{l}0.204 \\
0.279\end{array}$ \\
\hline 1958 & 0.158 & 0.328 & 0.233 & 0.235 \\
\hline 1959 & 0.095 & 0.242 & 0.254 & 0.173 \\
\hline 1960 & 0.091 & 0.264 & 0.247 & 0.215 \\
\hline 1961 & 0.068 & 0.232 & 0.206 & 0.179 \\
\hline 1962 & 0.119 & 0.336 & 0.353 & 0.270 \\
\hline 1963 & 0.146 & 0.395 & 0.299 & 0.301 \\
\hline 1904 & 0.109 & 0.328 & 0.328 & 0.226 \\
\hline 1905 & 0.072 & 0.283 & 0.272 & 0.237 \\
\hline 1966 & 0.095 & 0.349 & 0.324 & 0.278 \\
\hline 1967 & 0.063 & 0.294 & 0.303 & 0.240 \\
\hline 1903 & 0.078 & 0.326 & 0.357 & 0.231 \\
\hline 1909 & 0.065 & 0.320 & 0.290 & 0.219 \\
\hline 1970 & 0.065 & 0.311 & 0.285 & 0.280 \\
\hline 1971 & 0.058 & 0.330 & 0.269 & 0.227 \\
\hline 1972 & 0.048 & 0.282 & 0.330 & 0.250 \\
\hline 1973 & 0.052 & 0.273 & 0.243 & 0.212 \\
\hline 1974 & 0.049 & 0.279 & 0.259 & 0.180 \\
\hline 1975 & 0.029 & 0.208 & 0.204 & 0.141 \\
\hline 1976 & 0.022 & 0.249 & 0.194 & 0.116 \\
\hline 1977 & 0.010 & 0.181 & 0.188 & 0.100 \\
\hline 1978 & 0.009 & 0.192 & $0.200^{\circ}$ & 0.087 \\
\hline 1979 & 0.010 & 0.211 & 0.248 & 0.090 \\
\hline 1980 & 0.005 & 0.185 & 0.183 & 0.060 \\
\hline 1961 & 0.006 & 0.212 & 0.207 & 0.061 \\
\hline 1982 & 0.020 & 0.271 & 0.188 & 0.051 \\
\hline
\end{tabular}


rates, $1940-82$

\begin{tabular}{|c|c|c|c|}
\hline $35-39$ & $40-44$ & $45-49$ & Total \\
\hline - & - & - & - \\
\hline - & - & - & - \\
\hline$\bullet$ & - & - & - \\
\hline - & - & - & - \\
\hline - & - & - & - \\
\hline - & - & - & 4.428 \\
\hline - & - & - & 5.619 \\
\hline - & - & - & 5.506 \\
\hline - & - & - & 4.993 \\
\hline - & - & - & 5.642 \\
\hline - & - & - & 5.574 \\
\hline - & - & - & 5.561 \\
\hline - & - & - & 6.029 \\
\hline - & - & - & 5.938 \\
\hline - & - & - & 6.093 \\
\hline - & - & - & 6.396 \\
\hline - & - & - & 5.597 \\
\hline - & - & - & 6.787 \\
\hline - & - & - & 6.102 \\
\hline - & - & - & 4.653 \\
\hline 0.173 & - & - & 5.361 \\
\hline 0.142 & - & - & 4.419 \\
\hline 0.152 & • & - & 0.619 \\
\hline 0.24 .7 & - & - & 7.513 \\
\hline 0.219 & 0.109 & 0.007 & 6.635 \\
\hline 0.162 & 0.062 & 0.012 & 5.745 \\
\hline 0.193 & 0.099 & 0.012 & 6.745 \\
\hline 0.184 & 0.067 & 0.005 & 5.782 \\
\hline 0.190 & 0.112 & 0.014 & 6.790 \\
\hline 0.189 & 0.081 & 0.010 & 5.371 \\
\hline 0.161 & 0.092 & 0.010 & 6.014 \\
\hline 0.151 & 0.088 & 0.010 & 5.667 \\
\hline 0.161 & 0.091 & 0.007 & 5.840 \\
\hline 0.134 & 0.052 & 0.014 & 4.895 \\
\hline 0.120 & 0.055 & 0.005 & 4.732 \\
\hline 0.101 & 0.033 & 0.005 & 3.598 \\
\hline 0.092 & 0.028 & 0.002 & 3.470 \\
\hline 0.053 & 0.014 & 0.007 & 2.703 \\
\hline 0.044 & 0.033 & 0.002 & 2.070 \\
\hline 0.043 & 0.027 & • & 3.149 \\
\hline 0.018 & 0.010 & 0.002 & 2.314 \\
\hline 0.022 & 0.003 & 0.005 & 2.602 \\
\hline 0.018 & 0.004 & 0.005 & 2.785 \\
\hline
\end{tabular}


Basic Table 26.A. Total Gansu Province (26): age-specific fertility rates,

\begin{tabular}{|c|c|c|c|c|}
\hline $\begin{array}{l}\text { Birth year } \\
\text { of child }\end{array}$ & $15-19$ & $20-24$ & $25-29$ & $30-34$ \\
\hline 1940 & 0.129 & $\bullet$ & - & $\bullet$ \\
\hline 1941 & 0.147 & - & $\bullet$ & - \\
\hline 1942 & 0.122 & - & - & - \\
\hline 1943 & 0.151 & - & - & - \\
\hline 1944 & 0.131 & - & - & $\bullet$ \\
\hline 1945 & 0.110 & 0.287 & - & - \\
\hline 1946 & 0.103 & 0.336 & - & - \\
\hline 1947 & 0.104 & 0.301 & - & - \\
\hline 1948 & 0.103 & 0.242 & $\bullet$ & $\bullet$ \\
\hline 1949 & 0.102 & 0.315 & $\bullet$ & - \\
\hline 1950 & 0.153 & 0.287 & 0.298 & - \\
\hline 1951 & 0.152 & 0.249 & 0.218 & - \\
\hline 1952 & 0.176 & 0.322 & 0.316 & - \\
\hline 1953 & 0.159 & 0.294 & 0.258 & - \\
\hline 1954 & 0.127 & 0.300 & 0.279 & . \\
\hline 1955 & 0.125 & 0.343 & 0.307 & 0.228 \\
\hline 1950 & 0.137 & 0.291 & 0.302 & 0.243 \\
\hline 1957 & 0.130 & 0.266 & 0.315 & 0.221 \\
\hline 1958 & 0.114 & 0.256 & 0.267 & 0.250 \\
\hline 1959 & 0.056 & 0.183 & 0.169 & 0.147 \\
\hline 1960 & 0.068 & 0.205 & 0.180 & 0.138 \\
\hline 1901 & 0.055 & 0.196 & 0.166 & 0.149 \\
\hline 1962 & 0.092 & 0.378 & 0.332 & 0.320 \\
\hline 1903 & 0.142 & 0.356 & 0.364 & 0.279 \\
\hline 1964 & 0.139 & 0.323 & 0.357 & 0.311 \\
\hline 1965 & 0.100 & 0.325 & 0.318 & 0.253 \\
\hline 1966 & 0.124 & 0.339 & 0.359 & 0.307 \\
\hline 1967 & 0.097 & 0.316 & 0.282 & 0.266 \\
\hline 1968 & 0.118 & 0.343 & 0.347 & 0.313 \\
\hline 1969 & 0.106 & 0.326 & 0.271 & 0.267 \\
\hline 1970 & 0.094 & 0.319 & 0.332 & 0.273 \\
\hline 1971 & 0.104 & 0.329 & 0.303 & 0.247 \\
\hline 1972 & 0.089 & 0.315 & 0.289 & 0.267 \\
\hline 1973 & 0.033 & 0.283 & 0.303 & 0.218 \\
\hline 1974 & 0.089 & 0.280 & 0.248 & 0.197 \\
\hline 1975 & 0.067 & 0.225 & 0.180 & 0.101 \\
\hline 1976 & 0.042 & 0.214 & 0.181 & 0.098 \\
\hline 1977 & 0.026 & 0.198 & 0.166 & 0.077 \\
\hline 1978 & 0.031 & 0.240 & 0.196 & 0.081 \\
\hline 1979 & 0.026 & 0.249 & 0.254 & 0.094 \\
\hline 1980 & 0.024 & 0.236 & 0.186 & 0.038 \\
\hline 1981 & 0.043 & 0.236 & 0.190 & 0.068 \\
\hline 1982 & 0.051 & 0.183 & 0.206 & 0.044 \\
\hline
\end{tabular}




\begin{tabular}{|c|c|c|c|}
\hline $35-39$ & $40-44$ & $45-49$ & Total \\
\hline - & $\bullet$ & $\bullet$ & - \\
\hline • & - & • & - \\
\hline$\bullet$ & - & $\bullet$ & - \\
\hline - & - & - & $\bullet$ \\
\hline • & • & - & $\bullet$ \\
\hline - & - & - & 6.030 \\
\hline - & • & - & 5.843 \\
\hline • & • & - & 5.470 \\
\hline - & • & - & 5.502 \\
\hline • & - & - & 6.696 \\
\hline - & - & - & 6.292 \\
\hline - & - & - & 5.140 \\
\hline - & - & - & 6.615 \\
\hline - & - & - & 6.073 \\
\hline - & - & - & 6.011 \\
\hline - & - & - & 6.682 \\
\hline - & - & - & 6.078 \\
\hline • & - & - & 5.754 \\
\hline - & - & • & 5.714 \\
\hline - & • & - & 3.625 \\
\hline 0.110 & - & - & 3.710 \\
\hline 0.089 & - & - & 3.454 \\
\hline 0.233 & - & - & 7.227 \\
\hline 0.179 & - & - & 6.991 \\
\hline 0.223 & 0.089 & 0.017 & 7.293 \\
\hline 0.185 & 0.136 & 0.020 & 6.681 \\
\hline 0.214 & 0.122 & 0.031 & 7.478 \\
\hline 0.176 & 0.099 & 0.016 & 0.254 \\
\hline 0.200 & 0.131 & 0.037 & 7.444 \\
\hline 0.175 & 0.088 & 0.015 & 6.233 \\
\hline 0.229 & 0.083 & 0.022 & 6.761 \\
\hline 0.180 & 0.088 & 0.014 & 6.371 \\
\hline 0.208 & 0.093 & 0.008 & 6.345 \\
\hline 0.167 & 0.094 & 0.016 & 5.820 \\
\hline 0.109 & 0.072 & 0.012 & 5.034 \\
\hline 0.060 & 0.068 & 0.008 & 3.577 \\
\hline 0.046 & 0.327 & 0.004 & 3.064 \\
\hline 0.053 & 0.026 & 0.010 & 2.779 \\
\hline 0.041 & 0.013 & 0.012 & 3.063 \\
\hline 0.056 & 0.021 & 0.003 & 3.509 \\
\hline 0.013 & 0.010 & 0.010 & 2.585 \\
\hline 0.020 & 0.006 & 0.007 & 2.855 \\
\hline 0.019 & 0.031 & 0.004 & 2.690 \\
\hline
\end{tabular}


Basic Table 26.B. Urban Gansu Province (26): age-specific fertility

\begin{tabular}{|c|c|c|c|c|}
\hline $\begin{array}{l}\text { Birth year } \\
\text { of child }\end{array}$ & $15-19$ & $20-24$ & $25-29$ & $30-34$ \\
\hline 1940 & 0.118 & • & - & - \\
\hline 1941 & 0.128 & - & - & - \\
\hline 1942 & 0.077 & - & - & - \\
\hline 1943 & 0.072 & - & - & - \\
\hline 1944 & 0.089 & - & - & - \\
\hline 1945 & 0.179 & 0.213 & - & - \\
\hline 1946 & 0.109 & 0.307 & - & - \\
\hline 1947 & 0.048 & 0.204 & - & - \\
\hline 1948 & 0.088 & 0.239 & - & - \\
\hline 1947 & 0.178 & 0.444 & - & - \\
\hline 1950 & 0.146 & 0.258 & 0.237 & - . \\
\hline 1951 & 0.074 & 0.200 & 0.230 & - \\
\hline 1952 & 0.114 & 0.288 & 0.425 & - \\
\hline 1953 & 0.117 & 0.353 & 0.192 & - \\
\hline 1954 & 0.058 & 0.260 & 0.289 & • \\
\hline 1955 & 0.060 & 0.305 & 0.333 & 0.189 \\
\hline 1950 & 0.098 & 0.295 & 0.382 & 0.281 \\
\hline 1957 & $0.0 \mathrm{BB}$ & 0.204 & 0.352 & 0.128 \\
\hline 1958 & 0.116 & 0.276 & 0.383 & 0.335 \\
\hline 1959 & 0.084 & 0.192 & 0.205 & 0.133 \\
\hline 1960 & 0.045 & 0.257 & 0.225 & 0.199 \\
\hline 1961 & 0.037 & 0.212 & 0.123 & 0.200 \\
\hline 1362 & 0.031 & 0.361 & 0.375 & 0.304 \\
\hline 1963 & 0.074 & 0.408 & 0.382 & 0.295 \\
\hline 1904 & 0.069 & 0.278 & 0.346 & 0.301 \\
\hline 1965 & 0.022 & 0.296 & 0.300 & 0.172 \\
\hline 1900 & 0.023 & 0.223 & 0.318 & 0.197 \\
\hline 1967 & 0.023 & 0.167 & 0.217 & 0.170 \\
\hline 1963 & 0.032 & 0.24 .5 & 0.299 & 0.297 \\
\hline 1969 & 0.018 & 0.195 & 0.194 & 0.096 \\
\hline 1970 & 0.009 & 0.234 & 0.215 & 0.163 \\
\hline 1971 & - & 0.150 & 0.242 & 0.147 \\
\hline 1972 & - & 0.228 & 0.262 & 0.168 \\
\hline 1973 & - & 0.140 & 0.223 & 0.116 \\
\hline 1974 & 0.008 & 0.073 & 0.218 & 0.059 \\
\hline 1975 & - & 0.053 & 0.156 & 0.054 \\
\hline 1976 & - & 0.093 & 0.161 & 0.037 \\
\hline 1977 & - & 0.036 & 0.217 & 0.084 \\
\hline 1978 & $\bullet$. & 0.079 & 0.215 & 0.053 \\
\hline 1979 & - & 0.075 & 0.238 & 0.034 \\
\hline 1980 & - & 0.069 & 0.131 & 0.022 \\
\hline 1981 & 0.005 & 0.075 & 0.224 & 0.012 \\
\hline 1982 & $\bullet$ & 0.071 & 0.186 & - \\
\hline
\end{tabular}


rates, $1940-82$

\begin{tabular}{|c|c|c|c|}
\hline $35-39$ & $40-44$ & $45-49$ & Total \\
\hline - & - & - & - \\
\hline$\bullet$ & - & - & - \\
\hline - & - & - & - \\
\hline - & - & - & - \\
\hline - & • & - & - \\
\hline - & - & - & 6.149 \\
\hline - & - & • & 5.718 \\
\hline - & - & - & 3.367 \\
\hline - & - & - & 6.303 \\
\hline - & - & - & 7.554 \\
\hline - & - & - & 4.773 \\
\hline - & - & - & 5.506 \\
\hline - & - & - & 6.511 \\
\hline - & - & - & 6.648 \\
\hline • & - & • & 5.661 \\
\hline - & - & - & 7.078 \\
\hline - & - & - & 6.381 \\
\hline - & - & - & 5.015 \\
\hline - & - & - & 7.089 \\
\hline$\bullet$ & - & - & 4.280 \\
\hline 0.118 & - & - & 4.285 \\
\hline 0.077 & - & - & 3.391 \\
\hline 0.256 & - & - & 7.102 \\
\hline 0.168 & - & $\bullet$ & 7.001 \\
\hline 0.222 & 0.043 & - & 6.294 \\
\hline 0.179 & 0.024 & 0.025 & 5.093 \\
\hline 0.132 & 0.026 & 0.043 & 5.055 \\
\hline 0.080 & 0.051 & - & 3.541 \\
\hline 0.088 & 0.072 & - & 5.167 \\
\hline 0.055 & 0.044 & 0.022 & 3.121 \\
\hline 0.093 & 0.020 & 0.024 & 3.735 \\
\hline 0.086 & 0.018 & • & 3.211 \\
\hline 0.068 & $\bullet$ & - & $3.63 ?$ \\
\hline 0.032 & 0.074 & - & 2.925 \\
\hline 0.038 & $\bullet$ & - & 1.982 \\
\hline 0.034 & $\bullet$ & • & 1.482 . \\
\hline$\bullet$ & 0.012 & 0.018 & 1.608 \\
\hline 0.008 & 0.011 & - & 1.777 \\
\hline$\bullet$ & $\bullet$ & 0.015 & 1.811 \\
\hline 0.017 & - & - & 1.823 \\
\hline$\bullet$ & - & - & 1.112 \\
\hline 0.007 & • & - & 1.626 \\
\hline$\bullet$ & - & - & 1.282 \\
\hline
\end{tabular}


Basic Table 26.C. Rural Gansu Province (26): age-specific fertility rates,

\begin{tabular}{|c|c|c|c|c|}
\hline $\begin{array}{l}\text { Birth year } \\
\text { of child }\end{array}$ & $15-19$ & $20-24$ & $25-29$ & $30-34$ \\
\hline 1940 & 0.130 & - & $\bullet$ & - \\
\hline 1941 & 0.150 & - & - & - \\
\hline 1942 & 0.130 & - & - & - \\
\hline 1943 & 0.167 & - & $\bullet$ & - \\
\hline 1944 & 0.141 & - & - & - \\
\hline 1945 & 0.093 & 0.300 & - & - \\
\hline $1940^{\circ}$ & 0.102 & 0.341 & - & - \\
\hline 1947 & 0.119 & 0.318 & - & - \\
\hline 1948 & 0.106 & 0.243 & - & - \\
\hline 1949 & 0.158 & 0.286 & - & - \\
\hline 1950 & 0.155 & 0.294 & 0.309 & • \\
\hline 1951 & 0.170 & 0.261 & 0.216 & - \\
\hline 1952 & 0.191 & 0.331 & 0.287 & - \\
\hline 1953 & 0.169 & 0.279 & 0.271 & • \\
\hline 1954 & 0.146 & 0.310 & 0.276 & - \\
\hline 1955 & 0.144 & 0.351 & 0.299 & 0.235 \\
\hline 1956 & 0.149 & 0.290 & 0.281 & 0.237 \\
\hline 1957 & 0.144 & 0.280 & 0.305 & 0.237 \\
\hline 1958 & 0.113 & 0.251 & 0.238 & 0.233 \\
\hline 1959 & 0.048 & 0.180 & 0.161 & 0.150 \\
\hline 1960 & 0.074 & 0.190 & 0.170 & 0.123 \\
\hline 1961 & 0.060 & 0.191 & 0.176 & $0.13 \epsilon$ \\
\hline 1962 & 0.105 & 0.384 & 0.322 & 0.335 \\
\hline 1963 & 0.156 & 0.341 & 0.359 & 0.275 \\
\hline 1964 & 0.152 & 0.335 & 0.360 & 0.313 \\
\hline 1965 & 0.115 & 0.332 & 0.324 & 0.271 \\
\hline 1966 & 0.143 & 0.367 & 0.372 & 0.332 \\
\hline 1967 & 0.110 & 0.348 & 0.302 & 0.288 \\
\hline 1968 & 0.133 & 0.364 & 0.361 & 0.317 \\
\hline 1969 & 0.123 & 0.351 & 0.293 & 0.313 \\
\hline 1970 & 0.111 & 0.336 & 0.362 & 0.306 \\
\hline 1971 & 0.126 & 0.362 & 0.319 & 0.278 \\
\hline 1972 & 0.108 & 0.330 & 0.295 & 0.297 \\
\hline 1973 & 0.101 & 0.309 & 0.319 & 0.248 \\
\hline 1974 & 0.108 & 0.321 & 0.254 & 0.235 \\
\hline 1975 & 0.084 & 0.260 & 0.184 & 0.113 \\
\hline 1976 & 0.054 & 0.240 & 0.185 & 0.113 \\
\hline 1977 & .0 .033 & 0.233 & 0.157 & 0.076 \\
\hline 1978 & 0.040 & 0.275 & 0.193 & 0.087 \\
\hline 1979 & 0.032 & 0.288 & 0.257 & 0.106 \\
\hline 1980 & 0.030 & 0.277 & 0.1 .97 & 0.041 \\
\hline 1981 & 0.051 & 0.280 & 0.183 & 0.079 \\
\hline 1982 & 0.061 & 0.215 & 0.211 & 0.052 \\
\hline
\end{tabular}


1940-82

\begin{tabular}{|c|c|c|c|}
\hline $35-39$ & $40-44$ & $45-49$ & Total \\
\hline - & - & - & • \\
\hline - & - & - & - \\
\hline - & - & - & - \\
\hline - & - & - & - \\
\hline$\bullet$ & - & - & - \\
\hline - & - & - & 6.004 \\
\hline - & - & - & $5 \cdot A 70$ \\
\hline - & - & - & 5.947 \\
\hline - & - & - & $5 \cdot 321$ \\
\hline - & - & - & 6.504 \\
\hline - & - & - & 6.629 \\
\hline - & - & - & 5.059 \\
\hline - & - & - & 6.639 \\
\hline - & - & - & 5.938 \\
\hline • & - & • & 6.094 \\
\hline - & - & - & 6.596 \\
\hline - & - & - & 6.004 \\
\hline - & - & - & 5.939 \\
\hline • & - & - & 5.374 \\
\hline - & - & - & 3.462 \\
\hline 0.109 & - & - & 3.568 \\
\hline 0.091 & - & - & 3.470 \\
\hline 0.228 & - & - & 7.257 \\
\hline 0.181 & - & - & 6.989 \\
\hline 0.223 & 0.098 & 0.021 & 7.519 \\
\hline 0.186 & 0.157 & 0.019 & 7.015 \\
\hline 0.223 & 0.137 & 0.028 & 8.009 \\
\hline 0.203 & 0.108 & 0.019 & 6.887 \\
\hline 0.227 & 0.143 & 0.045 & 7.951 \\
\hline 0.202 & 0.097 & 0.013 & 6.965 \\
\hline 0.259 & 0.098 & 0.022 & 7.470 \\
\hline 0.212 & 0.107 & 0.017 & 7.097 \\
\hline 0.241 & 0.119 & 0.009 & 6.997 \\
\hline 0.201 & 0.099 & 0.019 & 6.478 \\
\hline 0.128 & 0.089 & 0.015 & 5.747 \\
\hline 0.076 & 0.083 & 0.010 & 4.048 \\
\hline 0.060 & 0.031 & - & 3.412 \\
\hline 0.067 & 0.029 & 0.013 & 3.041 \\
\hline 0.053 & 0.016 & 0.011 & 3.372 \\
\hline 0.067 & 0.026 & 0.003 & 3.897 \\
\hline 0.016 & 0.013 & 0.012 & 2.932 \\
\hline 0.023 & 0.007 & 0.008 & 3.161 \\
\hline 0.023 & 0.040 & 0.005 & 3.036 \\
\hline
\end{tabular}


Basic Table 27. Total Qinghai Province (27): age-specific fertility rates,

\begin{tabular}{|c|c|c|c|c|}
\hline $\begin{array}{l}\text { Birth year } \\
\text { of child }\end{array}$ & $15-19$ & $20-24$ & $25-29$ & $30-34$ \\
\hline 1940 & 0.026 & - & • & - \\
\hline 1941 & 0.056 & - & - & - \\
\hline 1942 & 0.072 & - & - & - \\
\hline 1943 & 0.085 & - & - & - \\
\hline 1944 & 0.064 & - & - & - \\
\hline 1945 & 0.051 & 0.102 & - & - \\
\hline 1946 & 0.058 & 0.247 & - & - \\
\hline 1947 & 0.028 & 0.247 & - & - \\
\hline 1948 & 0.009 & 0.127 & - & - \\
\hline 1949 & 0.068 & 0.215 & - & - \\
\hline 1950 & 0.057 & 0.193 & 0.217 & - \\
\hline 1951 & 0.063 & 0.155 & 0.146 & - \\
\hline 1952 & 0.085 & 0.170 & 0.144 & - \\
\hline 1953 & 0.073 & 0.142 & 0.212 & - \\
\hline 1954 & 0.056 & 0.220 & 0.268 & - \\
\hline 1955 & 0.084 & 0.245 & 0.233 & 0.281 \\
\hline 1956 & 0.070 & 0.158 & 0.174 & 0.168 \\
\hline 1957 & 0.041 & 0.231 & 0.207 & 0.123 \\
\hline 1958 & 0.053 & 0.169 & 0.133 & 0.212 \\
\hline 1959 & 0.019 & 0.119 & 0.195 & 0.150 \\
\hline 1960 & 0.018 & 0.049 & 0.082 & 0.112 \\
\hline 1901 & 0.012 & 0.077 & 0.134 & 0.068 \\
\hline 1962 & 0.068 & 0.234 & 0.231 & 0.207 \\
\hline 1963 & 0.065 & 0.207 & 0.220 & 0.212 \\
\hline 1964 & 0.081 & 0.276 & 0.349 & 0.330 \\
\hline 1965 & 0.081 & .0 .202 & 0.265 & 0.204 \\
\hline 1906 & 0.098 & 0.186 & 0.323 & 0.237 \\
\hline 1967 & 0.087 & 0.242 & 0.275 & 0.192 \\
\hline 1968 & 0.138 & 0.292 & 0.287 & 0.287 \\
\hline 1969 & 0.107 & 0.270 & 0.270 & 0.210 \\
\hline 1970 & 0.086 & 0.331 & 0.257 & 0.245 \\
\hline 1971 & 0.061 & 0.216 & 0.252 & 0.267 \\
\hline 1972 & 0.063 & 0.325 & 0.335 & 0.227 \\
\hline 1973 & 0.069 & 0.238 & 0.266 & 0.234 \\
\hline 1974 & 0.101 & 0.246 & 0.337 & 0.263 \\
\hline 1975 & 0.050 & 0.247 & 0.270 & 0.141 \\
\hline 1970 & 0.114 & 0.304 & 0.222 & 0.150 \\
\hline 1977 & 0.095 & 0.219 & 0.250 & 0.186 \\
\hline 1978 & 0.084 & 0.249 & 0.238 & 0.143 \\
\hline 1979 & 0.043 & 0.271 & 0.290 & 0.155 \\
\hline 1980 & 0.014 & 0.207 & 0.210 & 0.095 \\
\hline 1981 & 0.050 & 0.324 & 0.237 & 0.203 \\
\hline 1982 & 0.046 & 0.150 & 0.230 & 0.224 \\
\hline
\end{tabular}




\begin{tabular}{|c|c|c|c|}
\hline $35-39$ & $40-44$ & $45-49$ & Total \\
\hline - & - & - & - \\
\hline - & - & $\bullet$ & - \\
\hline - & - & - & - \\
\hline - & - & - & - \\
\hline - & - & - & - \\
\hline - & - & - & 3.826 \\
\hline - & - & - & 5.212 \\
\hline - & - & - . & 4.122 \\
\hline - & $\bullet$ & - & $2 \cdot 185$ \\
\hline - & - & - & 3.856 \\
\hline - & - & • & 4.474 \\
\hline - & - & - & 3.330 \\
\hline - & - & - & 3.928 \\
\hline - & - & - & 3.969 \\
\hline • & - & - & 4.849 \\
\hline - & - & - & 5.496 \\
\hline - & - & - & 3.798 \\
\hline - & • & - & 3.966 \\
\hline - & - & - & 3.916 \\
\hline - & - & - & 2.830 \\
\hline 0.051 & - & - & 1.736 \\
\hline 0.050 & - & • & 1.904 \\
\hline 0.175 & - & - & 4.735 \\
\hline 0.127 & - & - & 4.465 \\
\hline 0.150 & 0.085 & 0.051 & 6.617 \\
\hline 0.193 & 0.153 & 0.044 & 5.716 \\
\hline 0.223 & 0.079 & 0.042 & 5.937 \\
\hline 0.132 & 0.032 & 0.021 & 5.161 \\
\hline 0.212 & 0.149 & 0.051 & 7.073 \\
\hline 0.220 & 0.086 & - & 5.813 \\
\hline 0.196 & 0.091 & 0.026 & 6.161 \\
\hline 0.190 & 0.048 & 0.022 & 5.276 \\
\hline 0.200 & 0.123 & 0.021 & 6.464 \\
\hline 0.184 & 0.142 & 0.011 & 5.708 \\
\hline 0.196 & 0.076 & 0.021 & 6.204 \\
\hline 0.133 & 0.139 & 0.020 & 4.999 \\
\hline 0.147 & 0.055 & 0.010 & 5.009 \\
\hline 0.186 & 0.046 & 0.019 & 5.006 \\
\hline 0.107 & 0.118 & 0.018 & 4.770 \\
\hline 0.135 & 0.091 & - & 4.927 \\
\hline 0.147 & 0.112 & 0.016 & 4.001 \\
\hline 0.120 & 0.077 & 0.008 & 5.121 \\
\hline 0.153 & 0.092 & $\bullet$ & 4.429 \\
\hline
\end{tabular}


Basic Table 28. Total Ningxia Province (28): age-specific fertility rates,

\begin{tabular}{|c|c|c|c|c|}
\hline $\begin{array}{l}\text { Birth year } \\
\text { of child }\end{array}$ & $15-19$ & $20-24$ & $25-29$ & $30-34$ \\
\hline 1940 & 0.068 & - & - & - \\
\hline 1941 & 0.045 & - & - & - \\
\hline 1942 & 0.063 & - & - & - \\
\hline 1943 & 0.232 & - & - & - \\
\hline 1944 & 0.116 & - & - & - \\
\hline 1945 & 0.209 & 0.250 & - & - \\
\hline 1946 & 0.142 & 0.293 & - & - \\
\hline 1947 & 0.139 & 0.399 & - & - \\
\hline 1948 & 0.146 & 0.279 & - & - \\
\hline 1949 & 0.192 & 0.232 & • & - \\
\hline 1950 & 0.172 & 0.441 & 0.363 & - \\
\hline 1951 & 0.144 & 0.159 & 0.180 & - \\
\hline 1952 & 0.168 & 0.325 & 0.315 & - \\
\hline 1953 & 0.139 & 0.278 & 0.302 & - \\
\hline 1954 & 0.210 & 0.332 & 0.372 & - \\
\hline 1955 & 0.077 & 0.382 & 0.301 & 0.159 \\
\hline 1956 & 0.144 & 0.348 & 0.407 & 0.383 \\
\hline 1957 & 0.149 & 0.277 & 0.278 & 0.315 \\
\hline 1958 & 0.178 & 0.256 & 0.336 & 0.209 \\
\hline 1959 & 0.071 & 0.158 & 0.268 & 0.209 \\
\hline 1960 & 0.030 & 0.126 & 0.185 & 0.139 \\
\hline 1961 & 0.065 & 0.144 & 0.120 & 0.124 \\
\hline 19002 & 0.205 & 0.317 & 0.361 & 0.294 \\
\hline 1963 & 0.158 & 0.367 & 0.320 & 0.336 \\
\hline 1964 & 0.122 & 0.426 & 0.263 & 0.204 \\
\hline 1965 & 0.121 & 0.314 & 0.290 & 0.246 \\
\hline 1966 & 0.090 & 0.342 & 0.356 & 0.288 \\
\hline 1967 & 0.031 & 0.351 & 0.345 & 0.241 \\
\hline 1968 & 0.087 & 0.417 & 0.367 & 0.256 \\
\hline 1969 & 0.082 & 0.349 & 0.315 & 0.242 \\
\hline 1970 & 0.074 & 0.290 & 0.264 & 0.280 \\
\hline 1971 & 0.054 & 0.275 & 0.435 & 0.202 \\
\hline 1972 & 0.040 & 0.228 & 0.359 & 0.205 \\
\hline 1973 & 0.030 & 0.350 & 0.244 & 0.277 \\
\hline 1974 & 0.047 & 0.252 & 0.304 & 0.254 \\
\hline 1975 & 0.073 & 0.305 & 0.344 & 0.213 \\
\hline 1970 & 0.030 & 0.229 & 0.281 & 0.139 \\
\hline 1977 & 0.006 & 0.271 & 0.228 & 0.154 \\
\hline 1978 & - & 0.250 & 0.292 & 0.134 \\
\hline 1979 & 0.005 & 0.228 & 0.277 & 0.213 \\
\hline 1980 & 0.013 & 0.204 & 0.310 & 0.169 \\
\hline 1931 & 0.044 & 0.342 & 0.244 & 0.089 \\
\hline 1962 & 0.016 & 0.276 & 0.246 & 0.134 \\
\hline
\end{tabular}




\begin{tabular}{|c|c|c|c|}
\hline $35-39$ & $40-44$ & $45-49$ & Total \\
\hline$\bullet$ & - & - & • \\
\hline$\bullet$ & - & - & - \\
\hline - & - & - & - \\
\hline - & - & - & - \\
\hline - & $\bullet$ & $\bullet$ & - \\
\hline$\bullet$ & - & - & 4.773 \\
\hline - & - & - & 7.157 \\
\hline - & - & - & 7.896 \\
\hline - & - & $\bullet$ & 5.837 \\
\hline - & - & - & 6.148 \\
\hline - & - & - & 8.680 \\
\hline$\bullet$ & - & - & 3.799 \\
\hline - & - & - & 6.580 \\
\hline - & - & - & 6.536 \\
\hline - & - & - & 7.391 \\
\hline - & - & - & 6.416 \\
\hline • & - & - & 8.602 \\
\hline - & $\bullet$ & - & 6.707 \\
\hline$\bullet$ & - & - & 6.105 \\
\hline • & - & - & 4.082 \\
\hline 0.091 & - & - & 3.518 \\
\hline 0.135 & • & - & 3.144 \\
\hline 0.231 & - & - & 9.164 \\
\hline 0.209 & - & - & 7.171 \\
\hline 0.163 & 0.151 & 0.053 & 6.911 \\
\hline 0.186 & 0.023 & 0.045 & 6.126 \\
\hline 0.212 & 0.158 & - & 7.262 \\
\hline 0.217 & 0.063 & - & 6.233 \\
\hline 0.219 & 0.070 & 0.086 & 7.514 \\
\hline 0.166 & 0.116 & - & 6.352 \\
\hline 0.234 & 0.023 & 0.045 & 6.051 \\
\hline 0.144 & 0.106 & - & 6.076 \\
\hline 0.168 & 0.139 & - & 5.72 .8 \\
\hline 0.160 & 0.117 & - & 5.894 \\
\hline 0.179 & 0.077 & 0.023 & 5.672 \\
\hline 0.135 & 0.148 & 0.046 & 6.321 \\
\hline 0.116 & 0.084 & 0.035 & 4.598 \\
\hline 0.056 & 0.060 & 0.046 & 4.107 \\
\hline 0.119 & 0.021 & 0.053 & 4.370 \\
\hline 0.132 & 0.074 & 0.026 & 4.772 \\
\hline 0.051 & 0.087 & 0.012 & 4.227 \\
\hline 0.037 & 0.029 & • & 3.923 \\
\hline 0.034 & 0.038 & 0.024 & 3.835 \\
\hline
\end{tabular}


Basic Table 29.A. Total Xinjian Province (29): age-specific fertility

\begin{tabular}{|c|c|c|c|c|}
\hline $\begin{array}{l}\text { Birth year } \\
\text { of child }\end{array}$ & $15-19$ & $20-24$ & $25-29$ & $30-34$ \\
\hline 1940 & 0.105 & • & - & - \\
\hline 1941 & 0.078 & - & - & - \\
\hline 1942 & 0.141 & . & . & - \\
\hline 1943 & 0.115 & - & - & - \\
\hline 1944 & 0.104 & • & - & - \\
\hline 1445 & 0.119 & 0.163 & - & - \\
\hline 1940 & 0.087 & 0.239 & - & - \\
\hline 1947 & 0.086 & 0.180 & - & - \\
\hline 1948 & 0.074 & 0.223 & - & - \\
\hline 1949 & 0.093 & 0.220 & - & - \\
\hline 1950 & 0.091 & 0.261 & 0.198 & - \\
\hline 1951 & 0.071 & 0.230 & 0.178 & - \\
\hline 1952 & 0.210 & 0.286 & 0.337 & - \\
\hline 1953 & 0.095 & 0.179 & 0.205 & - \\
\hline 1954 & 0.112 & 0.259 & 0.353 & - \\
\hline 1955 & 0.119 & 0.172 & 0.239 & 0.146 \\
\hline 1956 & 0.117 & 0.257 & 0.327 & 0.267 \\
\hline 1957 & 0.104 & 0.214 & 0.227 & 0.264 \\
\hline 1958 & 0.105 & 0.292 & 0.279 & 0.314 \\
\hline 1959 & 0.076 & 0.210 & 0.232 & 0.191 \\
\hline $\begin{array}{l}1960 \\
1961\end{array}$ & $\begin{array}{l}0.049 \\
0.080\end{array}$ & $\begin{array}{l}0.226 \\
0.185\end{array}$ & $\begin{array}{l}0.242 \\
0.208\end{array}$ & $\begin{array}{l}0.227 \\
0.179\end{array}$ \\
\hline 1962 & 0.080 & 0.285 & 0.277 & 0.290 \\
\hline 1403 & 0.083 & 0.274 & 0.321 & 0.201 \\
\hline 1964 & 0.106 & 0.242 & 0.309 & 0.294 \\
\hline 1965 & 0.117 & 0.289 & 0.289 & 0.233 \\
\hline 1960 & 0.122 & 0.332 & 0.277 & 0.242 \\
\hline 1967 & 0.098 & 0.291 & 0.288 & 0.185 \\
\hline 1968 & 0.12 .3 & 0.307 & 0.283 & 0.312 \\
\hline 1969 & 0.116 & 0.310 & 0.320 & 0.224 \\
\hline 1970 & 0.113 & 0.280 & 0.311 & 0.236 \\
\hline 1971 & 0.105 & 0.296 & 0.272 & 0.204 \\
\hline 1972 & 0.120 & 0.249 & 0.263 & 0.215 \\
\hline 1973 & 0.112 & $0: 258$ & 0.314 & $0.1: 36$ \\
\hline 1974 & 0.111 & 0.276 & 0.300 & 0.245 \\
\hline 1975 & 0.122 & 0.253 & 0.247 & 0.151 \\
\hline 1970 & 0.097 & 0.283 & 0.281 & 0.163 \\
\hline 1977 & 0.094 & 0.212 & 0.247 & 0.164 \\
\hline 1970 & 0.087 & 0.208 & 0.268 & 0.150 \\
\hline 1979 & 0.053 & 0.229 & 0.223 & 0.144 \\
\hline 1980 & 0.051 & 0.221 & 0.187 & 0.110 \\
\hline 1931 & 0.055 & 0.209 & 0.230 & 0.135 \\
\hline 1982 & 0.050 & 0.201 & 0.283 & 0.159 \\
\hline
\end{tabular}


rates, $1940-82$

\begin{tabular}{|c|c|c|c|}
\hline $35-39$ & $40-44$ & $45-49$ & Total \\
\hline - & - & - & • \\
\hline - & - & - & - \\
\hline - & - & - & - \\
\hline - & - & - & - \\
\hline - & - & - & - \\
\hline - & - & - & 4.063 \\
\hline - & - & - & 4.730 \\
\hline - & - & - & 3.795 \\
\hline$\bullet$ & - & - & 3.847 \\
\hline - & - & - & 4.950 \\
\hline - & - & - & 4.567 \\
\hline - & - & • & 4.131 \\
\hline - & - & - & 6.014 \\
\hline - & • & - & 4.132 \\
\hline - & - & - & 6.279 \\
\hline - & - & • & 4.482 \\
\hline - & - & - & 6.322 \\
\hline - & - & • & 5.120 \\
\hline - & - & - & 6.520 \\
\hline - & - & • & 4.568 \\
\hline 0.169 & • & • & 5.025 \\
\hline 0.100 & - & - & 4.463 \\
\hline 0.157 & - & - & 0.355 \\
\hline 0.175 & - & - & 5.838 \\
\hline 0.255 & 0.116 & 0.075 & 0.988 \\
\hline 0.193 & 0.151 & 0.059 & 6.655 \\
\hline 0.169 & 0.105 & 0.039 & 6.434 \\
\hline 0.141 & 0.124 & 0.004 & 5.952 \\
\hline 0.179 & 0.084 & 0.079 & 6.834 \\
\hline 0.145 & 0.104 & 0.043 & 6.318 \\
\hline 0.173 & 0.108 & 0.0 .35 & 6.285 \\
\hline 0.124 & 0.066 & 0.033 & 5.503 \\
\hline 0.102 & 0.095 & 0.011 & 5.574 \\
\hline 0.132 & 0.056 & 0.013 & 5.427 \\
\hline 0.145 & 0.076 & 0.023 & 5.877 \\
\hline 0.130 & 0.057 & 0.017 & 4.891 \\
\hline 0.107 & 0.074 & 0.026 & 5.157 \\
\hline 0.071 & 0.029 & 0.013 & 4.174 \\
\hline 0.047 & 0.032 & 0.015 & 4.02 .9 \\
\hline 0.063 & 0.025 & 0.003 & 3.703 \\
\hline 0.047 & 0.024 & 0.013 & 3.262 \\
\hline 0.062 & 0.019 & 0.006 & 3.582 \\
\hline 0.079 & 0.023 & 0.012 & 4.035 \\
\hline
\end{tabular}


Basic Table 29.B. Urban Xinjian Province (29): age-specific fertility

\begin{tabular}{|c|c|c|c|c|}
\hline $\begin{array}{l}\text { Birth year } \\
\text { of child }\end{array}$ & $15-19$ & $20-24$ & $25-29$ & $30-34$ \\
\hline 1940 & 0.125 & - & - & - \\
\hline 1941 & 0.056 & - & - & - \\
\hline 1942 & - & - & • & - \\
\hline 1943 & 0.035 & - & - & - \\
\hline 1944 & 0.051 & - & - & • \\
\hline 1945 & 0.093 & 0.376 & - & - \\
\hline 1946 & 0.040 & 0.446 & - & - \\
\hline 1947 & 0.055 & 0.086 & - & $\bullet$ \\
\hline 1948 & 0.045 & 0.244 & -. & - \\
\hline 1949 & 0.057 & 0.202 & - & - \\
\hline 1950 & 0.094 & 0.186 & 0.125 & - \\
\hline 1951 & 0.032 & 0.336 & 0.167 & - \\
\hline 1952 & 0.072 & 0.222 & 0.302 & - \\
\hline 1953 & 0.024 & 0.166 & 0.313 & - \\
\hline 1954 & 0.030 & 0.255 & 0.278 & - \\
\hline 1955 & 0.067 & 0.141 & 0.209 & 0.125 \\
\hline $1950^{\circ}$ & 0.041 & 0.346 & 0.277 & 0.335 \\
\hline 1957 & 0.059 & 0.198 & 0.222 & 0.129 \\
\hline 1958 & 0.069 & 0.253 & 0.227 & 0.488 \\
\hline $\begin{array}{l}1959 \\
1960\end{array}$ & $\begin{array}{l}0.032 \\
0.026\end{array}$ & $\begin{array}{l}0.246 \\
0.195\end{array}$ & $\begin{array}{l}0.298 \\
0.176\end{array}$ & $\begin{array}{l}0.202 \\
0.255\end{array}$ \\
\hline 1961 & 0.027 & 0.159 & 0.227 & 0.198 \\
\hline 1962 & 0.033 & 0.246 & 0.270 & 0.296 \\
\hline 1963 & 0.022 & 0.240 & 0.367 & 0.227 \\
\hline 1964 & 0.046 & 0.217 & 0.246 & 0.284 \\
\hline 1965 & 0.031 & 0.283 & 0.255 & 0.165 \\
\hline 1960 & 0.034 & 0.277 & 0.253 & 0.173 \\
\hline 1967 & 0.030 & 0.283 & 0.209 & 0.171 \\
\hline 1908 & - & 0.270 & 0.293 & 0.220 \\
\hline 1969 & 0.008 & 0.296 & 0.290 & 0.166 \\
\hline 1970 & 0.017 & 0.262 & 0.236 & 0.114 \\
\hline 1971 & 0.009 & 0.211 & 0.277 & 0.200 \\
\hline 1972 & 0.028 & 0.170 & 0.216 & 0.150 \\
\hline 1973 & 0.009 & 0.163 & 0.331 & 0.196 \\
\hline 1974 & 0.016 & 0.189 & 0.261 & 0.153 \\
\hline 1975 & - & 0.172 & 0.133 & 0.105 \\
\hline 1976 & - & 0.158 & 0.246 & 0.059 \\
\hline 1977 & 0.013 & 0.074 & 0.221 & 0.083 \\
\hline 1978 & - & 0.062 & 0.210 & 0.073 \\
\hline 1979 & - & 0.071 & 0.164 & 0.023 \\
\hline 1980 & - & 0.046 & 0.129 & 0.019 \\
\hline 1981 & - & 0.029 & 0.149 & 0.007 \\
\hline 1982 & - & 0.039 & 0.232 & 0.030 \\
\hline
\end{tabular}


rates, $1940-82$

\begin{tabular}{|c|c|c|c|}
\hline $35-39$ & $40-44$ & $45-49$ & Total \\
\hline - & - & - & - \\
\hline • & - & - & • \\
\hline$\bullet$ & - & - & • \\
\hline - & - & - & $\bullet$ \\
\hline - & - & - & - \\
\hline - & - & - & 6.706 \\
\hline - & - & - & 5.242 \\
\hline - & - & - & 5.992 \\
\hline - & - & - & 3.493 \\
\hline - & - & - & 7.598 \\
\hline - & - & - & 5.944 \\
\hline • & - & - & 5.872 \\
\hline - & - & - & 6.444 \\
\hline - & - & - & 4.977 \\
\hline - & - & - & 7.278 \\
\hline - & - & - & 4.734 \\
\hline - & - & - & 7.126 \\
\hline - & . & - & 0.919 \\
\hline - & - & - & 7.105 \\
\hline - & - & . & 5.444 \\
\hline - & - & - & 4.252 \\
\hline 0.223 & • & - & 5.128 \\
\hline 0.216 & • & • & 6.810 \\
\hline 0.070 & - & . & 5.735 \\
\hline 0.228 & - & - & 5.100 \\
\hline 0.162 & 0.125 & 0.070 & 5.458 \\
\hline 0.079 & • & - & 4.080 \\
\hline 0.074 & - & 0.076 & 4.210 \\
\hline 0.030 & 0.035 & • & 4.240 \\
\hline 0.142 & 0.076 & - & 4.391 \\
\hline 0.094 & 0.046 & - & 3.847 \\
\hline 0.076 & 0.040 & - & 4.062 \\
\hline 0.081 & 0.055 & - & 3.499 \\
\hline 0.098 & 0.015 & - & 4.015 \\
\hline 0.065 & 0.014 & - & 3.490 \\
\hline 0.054 & 0.012 & - & 2.678 \\
\hline 0.029 & - & - & 2.457 \\
\hline 0.021 & - & - & 2.066 \\
\hline 0.011 & - & - & 1.777 \\
\hline$\bullet$ & - & - & 1.294 \\
\hline 0.005 & - & - & 0.995 \\
\hline$\bullet$ & - & - & 0.923 \\
\hline - & - & - & 1.504 \\
\hline
\end{tabular}


Basic Table 29.C. Rural Xinjian Province (29): age-specific fertility

\begin{tabular}{|c|c|c|c|c|}
\hline $\begin{array}{l}\text { Birth year } \\
\text { of child }\end{array}$ & $15-19$ & $20-24$ & $25-29$ & $30-34$ \\
\hline 1940 & 0.103 & - & - & - \\
\hline 1941 & 0.080 & - & • & - \\
\hline 1942 & 0.162 & - & - & - \\
\hline 1943 & 0.131 & - & - & - \\
\hline 1944 & 0.120 & - & - & - \\
\hline 1945 & 0.128 & 0.141 & • & - \\
\hline 1946 & 0.103 & 0.216 & - & - \\
\hline 1947 & 0.096 & 0.194 & - & - \\
\hline 1948 & 0.084 & 0.219 & - & - \\
\hline 1949 & 0.105 & 0.225 & • & . \\
\hline 1950 & 0.090 & 0.286 & 0.205 & - \\
\hline 1951 & 0.087 & 0.193 & 0.179 & - \\
\hline 1952 & 0.128 & 0.306 & 0.342 & • \\
\hline 1953 & 0.133 & 0.183 & 0.182 & - \\
\hline 1954 & 0.132 & 0.261 & 0.375 & - \\
\hline 1953 & 0.154 & 0.210 & 0.248 & 0.148 \\
\hline 1956 & 0.170 & 0.222 & 0.345 & 0.259 \\
\hline 1957 & 0.139 & 0.221 & 0.228 & 0.284 \\
\hline 1958 & 0.130 & 0.314 & 0.296 & 0.277 \\
\hline 1959 & 0.106 & 0.189 & 0.210 & 0.188 \\
\hline 1.960 & 0.064 & 0.246 & 0.266 & 0.218 \\
\hline 1961 & 0.111 & 0.203 & 0.200 & 0.172 \\
\hline 1962 & 0.104 & 0.316 & 0.281 & 0.288 \\
\hline 1963 & 0.113 & 0.299 & 0.296 & 0.192 \\
\hline 1964 & 0.134 & 0.261 & 0.347 & 0.297 \\
\hline 1965 & 0.153 & 0.292 & 0.311 & 0.257 \\
\hline 1966 & 0.155 & 0.365 & 0.294 & 0.270 \\
\hline 1967 & 0.123 & 0.295 & 0.350 & 0.192 \\
\hline 1968 & 0.166 & 0.325 & 0.276 & 0.362 \\
\hline 1969 & 0.150 & 0.317 & 0.341 & 0.259 \\
\hline 1970 & 0.140 & 0.288 & 0.359 & 0.316 \\
\hline 1971 & 0.131 & 0.327 & 0.270 & 0.207 \\
\hline 1972 & 0.142 & 0.278 & 0.287 & 0.266 \\
\hline 1973 & 0.137 & 0.291 & 0.306 & 0.203 \\
\hline 1974 & 0.138 & 0.304 & 0.317 & 0.308 \\
\hline 1975 & 0.162 & 0.276 & 0.270 & 0.181 \\
\hline 1976 & 0.131 & 0.318 & 0.294 & 0.225 \\
\hline 1977 & 0.126 & 0.246 & 0.257 & 0.205 \\
\hline 1973 & 0.127 & 0.244 & 0.288 & 0.187 \\
\hline 1979 & 0.076 & 0.274 & 0.241 & 0.199 \\
\hline 1980 & 0.071 & 0.277 & 0.203 & 0.148 \\
\hline 1981 & 0.076 & 0.272 & 0.252 & 0.182 \\
\hline 1982 & 0.070 & 0.270 & 0.295 & 0.207 \\
\hline
\end{tabular}


rates, $1940-82$

\begin{tabular}{|c|c|c|c|}
\hline $35-39$ & $40-44$ & $45-49$ & Total \\
\hline - & • & - & - \\
\hline - & - & - & - \\
\hline - & - & - & - \\
\hline - & - & - & - \\
\hline - & - & - & - \\
\hline - & - & - & 3.557 \\
\hline - & - & - & 4.627 \\
\hline - & - & - & 3.350 \\
\hline - & - & - & 3.922 \\
\hline - & - & - & 4.343 \\
\hline - & - & - & 4.216 \\
\hline - & - & - & 3.662 \\
\hline - & - & - & 5.890 \\
\hline - & - & - & 3.873 \\
\hline - & - & - & 5.946 \\
\hline - & - & - & 4.391 \\
\hline - & - & - & 6.014 \\
\hline - & - & - & 5.203 \\
\hline - & - & - & 0.281 \\
\hline - & - & - & 4.197 \\
\hline 0.186 & - & - & 5.359 \\
\hline 0.086 & - & - & 4.172 \\
\hline 0.149 & - & - & 6.157 \\
\hline 0.197 & - & - & 5.882 \\
\hline 0.263 & 0.126 & 0.087 & 7.569 \\
\hline 0.203 & 0.154 & 0.057 & 7.143 \\
\hline 0.200 & 0.117 & 0.044 & $7 \cdot 224$ \\
\hline 0.162 & 0.142 & 0.063 & 6.636 \\
\hline 0.227 & 0.095 & 0.036 & 7.682 \\
\hline 0.146 & 0.113 & 0.046 & 6.867 \\
\hline 0.202 & 0.128 & 0.039 & 7.360 \\
\hline 0.144 & 0.076 & 0.037 & 5.956 \\
\hline 0.200 & 0.108 & 0.013 & 6.403 \\
\hline 0.150 & 0.069 & 0.022 & 5.888 \\
\hline 0.193 & 0.096 & 0.030 & 6.934 \\
\hline 0.180 & 0.073 & 0.023 & 5.822 \\
\hline 0.161 & 0.104 & 0.034 & 6.342 \\
\hline 0.110 & 0.043 & 0.024 & 5.049 \\
\hline 0.073 & 0.049 & 0.020 & 4.934 \\
\hline 0.106 & 0.040 & 0.005 & 4.705 \\
\hline 0.074 & 0.039 & 0.017 & 4.151 \\
\hline 0.098 & 0.033 & 0.009 & 0.015 \\
\hline 0.118 & 0.042 & 0.018 & 5.095 \\
\hline
\end{tabular}


Basic Table 30.A. Total China: duration-specific fertility rates, 1940-82

\begin{tabular}{|c|c|c|c|c|}
\hline $\begin{array}{l}\text { Birth year } \\
\text { of child }\end{array}$ & $0-4$ & $5-9$ & $10-14$ & $15-19$ \\
\hline 1940 & 0.223 & - & - & - \\
\hline 1941 & 0.235 & - & - & - \\
\hline 1942 & 0.226 & - & - & - \\
\hline 1943 & 0.230 & - & - & - \\
\hline 1944 & 0.232 & - & - & - \\
\hline 1945 & 0.240 & 0.257 & - & - \\
\hline 1946 & 0.250 & 0.270 & - & - \\
\hline 1947 & 0.200 & 0.280 & - & - \\
\hline 1948 & 0.251 & 0.261 & - & - \\
\hline 1949 & 0.271 & 0.295 & - & - \\
\hline 1950 & 0.262 & 0.279 & 0.238 & - \\
\hline 1951 & 0.269 & 0.268 & 0.240 & - \\
\hline 1952 & 0.314 & 0.305 & 0.268 & - \\
\hline 1953 & 0.304 & 0.291 & 0.253 & - \\
\hline 1954 & 0.317 & 0.300 & 0.273 & - \\
\hline 1955 & 0.319 & 0.311 & 0.263 & 0.216 \\
\hline 1950 & 0.298 & 0.287 & 0.252 & 0.205 \\
\hline 1457 & 0.335 & 0.317 & 0.282 & 0.225 \\
\hline 1958 & 0.292 & 0.288 & 0.247 & 0.203 \\
\hline 1959 & 0.220 & 0.229 & 0.192 & 0.159 \\
\hline 1960 & 0.204 & 0.211 & 0.184 & 0.148 \\
\hline 1961 & 0.179 & 0.184 & 0.152 & 0.113 \\
\hline 1902 & 0.305 & 0.334 & 0.284 & 0.214 \\
\hline 1903 & 0.384 & 0.380 & 0.335 & 0.277 \\
\hline 1964 & 0.335 & 0.314 & 0.276 & 0.207 \\
\hline 1965 & 0.331 & 0.315 & 0.271 & 0.213 \\
\hline 1960 & 0.359 & $0.32 \mathrm{e}$ & 0.273 & 0.225 \\
\hline 1907 & 0.313 & 0.290 & 0.240 & 0.185 \\
\hline 1968 & 0.388 & 0.357 & 0.281 & 0.225 \\
\hline 1909 & 0.345 & 0.313 & 0.253 & 0.198 \\
\hline 1970 & 0.366 & 0.311 & 0.256 & 0.197 \\
\hline 1971 & 0.357 & 0.307 & 0.242 & 0.177 \\
\hline 1972 & 0.346 & 0.280 & 0.218 & 0.160 \\
\hline 1973 & 0.348 & 0.274 & 0.201 & 0.138 \\
\hline 1974 & 0.371 & 0.260 & 0.175 & 0.114 \\
\hline 1975 & 0.345 & 0.242 & 0.145 & 0.091 \\
\hline 1976 & 0.356 & 0.216 & 0.125 & 0.079 \\
\hline 1977 & 0.342 & 0.193 & 0.103 & 0.065 \\
\hline 1978 & 0.347 & 0.191 & 0.100 & 0.051 \\
\hline 1979 & $0 \cdot 363$ & 0.196 & 0.094 & 0.046 \\
\hline 1980 & 0.311 & 0.155 & 0.061 & 0.030 \\
\hline 1981 & 0.359 & 0.151 & 0.064 & 0.033 \\
\hline 1982 & $0 . \overline{3} 30$ & 0.147 & 0.069 & 0.036 \\
\hline
\end{tabular}




\begin{tabular}{|c|c|c|c|}
\hline $20-24$ & $25-29$ & $30+$ & Total \\
\hline • & - & - & - \\
\hline$\bullet$ & - & • & - \\
\hline - & - & - & - \\
\hline - & - & - & - \\
\hline - & - & - & - \\
\hline - & - & - & 5.154 \\
\hline - & - & - & 5.401 \\
\hline - & - & - & 5.60 .5 \\
\hline - & - & - & 5.271 \\
\hline - & - & - & 5.903 \\
\hline - & - & - & 5.592 \\
\hline - & - & - & 5.555 \\
\hline - & - & - & 6.324 \\
\hline - & - & - & 6.021 \\
\hline - & - & - & 6.337 \\
\hline - & - & - & 6.376 \\
\hline - & - & - & 5.995 \\
\hline - & - & - & 6.656 \\
\hline - & - & - & 5.923 \\
\hline • & - & - & 4.594 \\
\hline 0.095 & - & - & 4.350 \\
\hline 0.068 & - & - & 3.592 \\
\hline 0.118 & - & - & 6.497 \\
\hline 0.172 & - & - & 8.020 \\
\hline 0.136 & 0.054 & 0.009 & 6.654 \\
\hline 0.134 & 0.046 & 0.010 & 6.605 \\
\hline 0.139 & 0.045 & 0.008 & 6.391 \\
\hline 0.111 & 0.035 & 0.006 & 5.894 \\
\hline 0.131 & 0.042 & 0.006 & 7.153 \\
\hline 0.119 & 0.039 & 0.004 & 6.351 \\
\hline 0.120 & 0.035 & 0.005 & 6.455 \\
\hline 0.109 & 0.028 & 0.003 & 0.110 \\
\hline 0.093 & 0.027 & 0.003 & 5.664 \\
\hline 0.082 & 0.021 & 0.004 & 5.337 \\
\hline 0.069 & 0.022 & 0.002 & 5.067 \\
\hline 0.052 & 0.019 & 0.002 & 4.482 \\
\hline 0.040 & 0.015 & 0.002 & 4.162 \\
\hline 0.030 & 0.011 & 0.002 & 3.726 \\
\hline 0.024 & 0.009 & 0.001 & 3.520 \\
\hline 0.023 & 0.007 & 0.001 & 3.049 \\
\hline 0.014 & 0.004 & 0.001 & 2.883 \\
\hline 0.016 & 0.005 & 0.001 & 3.149 \\
\hline 0.016 & 0.005 & 0.001 & 3.024 \\
\hline
\end{tabular}


Basic Table 30.B. Urban China: duration-specific fertility rates, 1940-82

\begin{tabular}{|c|c|c|c|c|}
\hline $\begin{array}{l}\text { Birth year } \\
\text { of child }\end{array}$ & $0-4$ & $5-9$ & $10-14$ & $15-19$ \\
\hline 1940 & 0.232 & • & - & - \\
\hline 1941 & 0.241 & - & - & - \\
\hline 1942 & 0.242 & - & - & - \\
\hline 1943 & 0.238 & - & - & - \\
\hline 1944 & 0.250 & - & • & - \\
\hline 1945 & 0.257 & 0.228 & - & - \\
\hline 1946 & 0.272 & 0.250 & - & - \\
\hline 1947 & 0.281 & 0.257 & - & - \\
\hline 1948 & 0.265 & 0.249 & $\bullet$ & - \\
\hline 1949 & 0.298 & 0.284 & - & - \\
\hline 1950 & 0.299 & 0.270 & 0.227 & - \\
\hline 1951 & 0.309 & 0.260 & 0.212 & - \\
\hline 1952 & 0.319 & 0.286 & 0.254 & • \\
\hline 1953 & 0.328 & 0.279 & 0.234 & - \\
\hline 1954 & 0.341 & 0.319 & 0.255 & - \\
\hline 1955 & 0.343 & 0.302 & 0.250 & 0.181 \\
\hline $\begin{array}{l}1956 \\
1957\end{array}$ & $\begin{array}{l}0.324 \\
0.379\end{array}$ & $\begin{array}{l}0.280 \\
0.331\end{array}$ & $\begin{array}{l}0.235 \\
0.278\end{array}$ & $\begin{array}{l}0.191 \\
0.190\end{array}$ \\
\hline 1958 & 0.337 & 0.298 & 0.232 & 0.178 \\
\hline 1959 & 0.290 & 0.254 & 0.166 & 0.141 \\
\hline 1960 & 0.283 & 0.235 & 0.195 & 0.127 \\
\hline 1961 & 0.226 & 0.175 & 0.143 & 0.054 \\
\hline 1902 & 0.349 & 0.294 & 0.223 & 0.150 \\
\hline 1963 & 0.416 & 0.362 & 0.294 & 0.205 \\
\hline 1964 & 0.348 & 0.260 & 0.186 & 0.135 \\
\hline 1965 & 0.334 & 0.236 & 0.150 & 0.096 \\
\hline 1960 & 0.304 & 0.196 & 0.119 & 0.084 \\
\hline 1967 & 0.310 & 0.200 & 0.110 & 0.063 \\
\hline 1968 & 0.393 & 0.269 & 0.147 & 0.082 \\
\hline 1969 & 0.351 & 0.213 & 0.127 & 0.077 \\
\hline 1970 & 0.377 & 0.204 & 0.116 & 0.057 \\
\hline 1971 & $0 \cdot 336$ & 0.184 & 0.097 & 0.051 \\
\hline 1972 & 0.322 & 0.173 & 0.093 & 0.046 \\
\hline 1973 & 0.327 & 0.167 & 0.086 & 0.030 \\
\hline 1974 & 0.322 & 0.136 & 0.060 & 0.023 \\
\hline 1975 & 0.327 & 0.120 & 0.047 & 0.022 \\
\hline 1976 & 0.311 & 0.109 & 0.034 & 0.016 \\
\hline 1977 & 0.319 & 0.096 & 0.030 & 0.012 \\
\hline 1978 & 0.330 & 0.088 & 0.026 & 0.007 \\
\hline 1979 & 0.271 & 0.086 & 0.024 & 0.005 \\
\hline 1980 & 0.243 & 0.053 & 0.007 & 0.001 \\
\hline 1981 & 0.286 & 0.032 & 0.004 & 0.003 \\
\hline 1982 & 0.267 & 0.021 & 0.007 & 0.001 \\
\hline
\end{tabular}




\begin{tabular}{|c|c|c|c|}
\hline $20-24$ & $25-29$ & $30+$ & Total \\
\hline - & - & - & - \\
\hline - & - & - & - \\
\hline - & - & $\bullet$ & $\bullet$ \\
\hline - & - & - & - \\
\hline - & - & - & - \\
\hline - & - & - & 4.947 \\
\hline - & - & - & $5 \cdot 379$ \\
\hline - & - & - & 5.540 \\
\hline - & - & - & 5.335 \\
\hline - & - & - & t.05s \\
\hline - & - & - & 5.762 \\
\hline - & - & - & 5.588 \\
\hline - & - & - & $6 \cdot 228$ \\
\hline - & - & - & 6.044 \\
\hline - & - & - & 6.631 \\
\hline - & - & - & 6.242 \\
\hline - & - & - & 5.987 \\
\hline • & - & - & 6.824 \\
\hline - & - & - & 6.053 \\
\hline - & - & - & 4.910 \\
\hline 0.079 & - & • & 4.760 \\
\hline 0.056 & - & - & 3.593 \\
\hline 0.073 & - & - & 5.642 \\
\hline 0.118 & - & . & 7.241 \\
\hline 0.070 & 0.027 & 0.003 & 5.151 \\
\hline 0.060 & 0.021 & - & 4.485 \\
\hline 0.040 & 0.013 & 0.002 & 3.785 \\
\hline 0.032 & c.0 10 & 0.002 & 3.635 \\
\hline 0.049 & 0.012 & 0.001 & 4.764 \\
\hline 0.033 & 0.010 & 0.002 & 4.060 \\
\hline 0.033 & 0.006 & 0.001 & 3.966 \\
\hline 0.026 & 0.006 & 0.000 & 3.500 \\
\hline 0.020 & 0.004 & 0.000 & 3.294 \\
\hline 0.015 & 0.001 & 0.001 & $3 \cdot 132$ \\
\hline 0.008 & 0.003 & 0.000 & 2.762 \\
\hline 0.004 & 0.002 & 0.001 & 2.610 \\
\hline 0.004 & 0.004 & - & 2.385 \\
\hline 0.003 & 0.000 & 0.000 & 2.301 \\
\hline 0.003 & 0.001 & - & 2.275 \\
\hline 0.002 & 0.001 & 0.001 & 1.952 \\
\hline 0.001 & 0.000 & 0.000 & 1.52 .3 \\
\hline 0.000 & 0.001 & 0.000 & 1.630 \\
\hline$\bullet$ & - & - & 1.473 \\
\hline
\end{tabular}


Basic Table 30.C. Rural China: duration-specific fertility rates, 1940-82

\begin{tabular}{|c|c|c|c|c|}
\hline $\begin{array}{l}\text { Birth year } \\
\text { of child }\end{array}$ & $0-4$ & $5-9$ & $10-14$ & $15-19$ \\
\hline 1940 & 0.222 & - & - & - \\
\hline 1941 & 0.234 & - & - & - \\
\hline 1942 & 0.224 & - & - & - \\
\hline 1943 & 0.229 & - & - & - \\
\hline 1944 & 0.229 & - & - & - \\
\hline 1945 & 0.237 & 0.262 & - & - \\
\hline 1946 & 0.245 & 0.273 & - & - \\
\hline 1947 & 0.256 & 0.284 & - & - \\
\hline 1948 & 0.248 & 0.263 & - & - \\
\hline 1949 & 0.266 & 0.297 & - & - \\
\hline 1950 & 0.254 & 0.281 & 0.240 & - \\
\hline 1951 & 0.261 & 0.269 & 0.245 & - \\
\hline 1952 & 0.313 & 0.305 & 0.271 & - \\
\hline 1953 & 0.298 & 0.293 & 0.256 & - \\
\hline 1954 & 0.312 & 0.296 & $0.27 \epsilon$ & - \\
\hline 1955 & 0.314 & 0.313 & 0.266 & 0.222 \\
\hline 1950 & 0.291 & 0.289 & 0.255 & 0.207 \\
\hline 1957 & 0.324 & 0.314 & 0.283 & 0.231 \\
\hline 1958 & 0.281 & 0.286 & 0.250 & 0.208 \\
\hline 1959 & 0.203 & 0.223 & 0.197 & 0.162 \\
\hline 1960 & 0.186 & 0.206 & 0.182 & 0.152 \\
\hline 1901 & 0.168 & 0.186 & 0.154 & 0.117 \\
\hline 1902 & 0.296 & 0.344 & 0.297 & 0.226 \\
\hline 1903 & 0.378 & 0.384 & 0.344 & 0.292 \\
\hline 1904 & 0.333 & 0.328 & 0.296 & 0.222 \\
\hline 1965 & 0.331 & 0.334 & 0.299 & 0.238 \\
\hline 1466 & 0.368 & 0.358 & 0.309 & 0.255 \\
\hline 1907 & 0.313 & 0.309 & 0.271 & 0.210 \\
\hline 1968 & 0.387 & 0.374 & 0.314 & 0.256 \\
\hline 1905 & 0.345 & 0.331 & 0.283 & 0.225 \\
\hline 1970 & 0.365 & 0.330 & 0.290 & 0.229 \\
\hline 1971 & 0.360 & 0.327 & 0.274 & 0.207 \\
\hline 1972 & 0.350 & 0.298 & 0.243 & 0.188 \\
\hline 1973 & 0.351 & 0.290 & 0.223 & 0.165 \\
\hline 1974 & 0.378 & 0.280 & 0.196 & 0.137 \\
\hline 1975 & 0.347 & 0.262 & 0.102 & 0.108 \\
\hline 1976 & 0.363 & 0.233 & 0.140 & 0.093 \\
\hline 1977 & 0.346 & 0.207 & 0.115 & 0.076 \\
\hline 1978 & 0.350 & 0.206 & 0.112 & 0.060 \\
\hline 1979 & 0.379 & 0.211 & 0.105 & 0.053 \\
\hline 1900 & 0.324 & 0.170 & 0.070 & 0.035 \\
\hline 1981 & 0.375 & 0.168 & 0.073 & 0.038 \\
\hline 1982 & 0.345 & 0.168 & 0.079 & 0.042 \\
\hline
\end{tabular}




\begin{tabular}{|c|c|c|c|}
\hline $20-24$ & $25-29$ & $30+$ & Total \\
\hline - & - & - & - \\
\hline - & • & - & • \\
\hline - & - & - & - \\
\hline$\bullet$ & - & • & - \\
\hline - & - & - & - \\
\hline - & - & - & 5.190 \\
\hline - & - & - & 5.405 \\
\hline - & - & • & 5.617 \\
\hline - & - & • & 5.259 \\
\hline - & - & - & 5.874 \\
\hline - & - & - & $5.5 t 0$ \\
\hline - & - & - & 5.549 \\
\hline - & - & - & $6 \cdot 342$ \\
\hline - & - & - & 6.016 \\
\hline - & • & - & 6.280 \\
\hline - & - & - & 6.403 \\
\hline - & - & - & 5.996 \\
\hline - & - & - & 6.623 \\
\hline - & - & - & 5.897 \\
\hline - & - & - & 4.530 \\
\hline 0.098 & - & - & 4.266 \\
\hline 0.070 & - & • & 3.591 \\
\hline 0.126 & - & • & 6.669 \\
\hline 0.181 & - & - & 8.176 \\
\hline 0.148 & 0.058 & 0.009 & 6.969 \\
\hline 0.148 & 0.051 & 0.011 & 7.057 \\
\hline 0.159 & 0.051 & 0.009 & 7.544 \\
\hline 0.127 & 0.039 & 0.006 & 6.378 \\
\hline 0.148 & $0.0+8$ & 0.006 & 7.669 \\
\hline 0.136 & 0.044 & 0.005 & 6.843 \\
\hline 0.138 & 0.040 & 0.006 & 6.989 \\
\hline 0.127 & 0.032 & 0.003 & 6.649 \\
\hline 0.116 & 0.031 & 0.003 & 6.144 \\
\hline 0.097 & 0.025 & 0.004 & 5.776 \\
\hline 0.083 & 0.026 & 0.002 & 5.506 \\
\hline 0.063 & 0.023 & 0.002 & 4.833 \\
\hline 0.048 & 0.017 & 0.002 & 4.481 \\
\hline 0.036 & 0.014 & 0.002 & 3.977 \\
\hline 0.030 & 0.011 & 0.002 & 3.849 \\
\hline 0.028 & 0.009 & 0.001 & $3 . .234$ \\
\hline 0.018 & 0.005 & 0.001 & 3.111 \\
\hline 0.020 & 0.006 & 0.001 & 3.413 \\
\hline 0.019 & 0.006 & 0.001 & 3.299 \\
\hline
\end{tabular}


Basic Table 31.A. Total Beijing Province (1): duration-specific fertility

\begin{tabular}{|c|c|c|c|c|}
\hline $\begin{array}{l}\text { Birth year } \\
\text { of child }\end{array}$ & $0-4$ & $5-9$ & $10-14$ & $15-19$ \\
\hline 1940 & 0.194 & - & - & - \\
\hline 1941 & 0.239 & - & - & - \\
\hline 1942 & 0.181 & - & - & - \\
\hline 1943 & 0.264 & - & - & - \\
\hline 1944 & 0.220 & $\bullet$ & - & - \\
\hline 1945 & 0.221 & 0.240 & - & - \\
\hline 1940 & 0.214 & 0.222 & - & - \\
\hline 1947 & 0.207 & 0.313 & . & . \\
\hline 1948 & 0.223 & 0.313 & - & - \\
\hline 1949 & 0.246 & 0.235 & - & - \\
\hline 1950 & 0.227 & 0.273 & 0.324 & - \\
\hline 1951 & 0.285 & 0.262 & 0.248 & - \\
\hline 1952 & 0.296 & 0.322 & 0.231 & - \\
\hline 1953 & 0.289 & 0.264 & $0.19 a$ & - \\
\hline 1954 & 0.303 & 0.314 & 0.264 & - \\
\hline 1955 & 0.343 & 0.307 & 0.345 & 0.194 \\
\hline 1456 & 0.311 & 0.270 & 0.179 & 0.239 \\
\hline 1957 & 0.352 & 0.319 & 0.293 & 0.206 \\
\hline 1958 & 0.314 & 0.284 & 0.239 & 0.181 \\
\hline 1959 & 0.199 & 0.287 & 0.201 & 0.191 \\
\hline 1960 & 0.234 & 0.270 & 0.165 & 0.156 \\
\hline 1901 & 0.192 & 0.131 & 0.159 & 0.113 \\
\hline 1962 & 0.326 & 0.269 & 0.216 & 0.190 \\
\hline 1903 & 0.428 & 0.395 & 0.368 & 0.249 \\
\hline 1904 & $0.270^{\circ}$ & 0.243 & 0.216 & 0.172 \\
\hline 1965 & 0.267 & 0.263 & 0.174 & 0.085 \\
\hline 1966 & 0.300 & 0.187 & 0.142 & 0.091 \\
\hline 1907 & 0.274 & 0.229 & 0.137 & 0.051 \\
\hline 1968 & 0.415 & 0.238 & 0.119 & 0.095 \\
\hline 1909 & 0.370 & 0.265 & 0.117 & 0.103 \\
\hline 1970 & 0.436 & 0.209 & 0.115 & 0.073 \\
\hline 1971 & 0.332 & 0.185 & 0.131 & 0.055 \\
\hline 1972 & 0.317 & 0.192 & 0.092 & 0.024 \\
\hline 1973 & 0.354 & 0.179 & 0.092 & 0.038 \\
\hline 1974 & 0.290 & 0.139 & 0.033 & 0.010 \\
\hline 1975 & 0.265 & 0.093 & 0.035 & 0.033 \\
\hline 1976 & 0.310 & 0.126 & 0.006 & 0.020 \\
\hline 1977 & 0.310 & 0.110 & 0.021 & 0.005 \\
\hline 1978 & .0 .264 & 0.132 & 0.032 & 0.005 \\
\hline 1975 & 0.300 & 0.089 & 0.011 & 0.006 \\
\hline 1960 & 0.290 & 0.074 & 0.024 & 0.006 \\
\hline 1981 & 0.290 & 0.038 & - & - \\
\hline 1922 & 0.219 & 0.018 & 0.025 & - \\
\hline
\end{tabular}


rates, $1940-82$

\begin{tabular}{|c|c|c|c|}
\hline $20-24$ & $25-29$ & $30+$ & Total \\
\hline - & - & - & $\bullet$ \\
\hline - & $\bullet$ & $\bullet$ & - \\
\hline$\bullet$ & - & - & - \\
\hline - & - & $\bullet$ & - \\
\hline$\bullet$ & - & $\bullet$ & - \\
\hline$\bullet$ & - & $\bullet$ & 4.838 \\
\hline$\bullet$ & - & - & 4.559 \\
\hline - & - & - & 5.976 \\
\hline • & - & - & 6.018 \\
\hline - & - & • & 4.349 \\
\hline - & - & • & 6.229 \\
\hline - & - & • & 5.623 \\
\hline - & - & - & 6.119 \\
\hline - & - & - & 5.398 \\
\hline - & - & - & 6.294 \\
\hline - & - & - & 6.933 \\
\hline - & - & - & 5.709 \\
\hline - & - & - & 6.696 \\
\hline$\bullet$ & - & - & 6.027 \\
\hline - & - & - & 5.119 \\
\hline 0.129 & - & • & 4.901 \\
\hline 0.071 & - & - & 3.423 \\
\hline 0.156 & - & - & 6.064 \\
\hline 0.165 & - & - & 8.345 \\
\hline 0.198 & 0.060 & 0.024 & 5.939 \\
\hline 0.052 & 0.037 & 0.019 & 4.486 \\
\hline 0.071 & 0.018 & • & 4.041 \\
\hline 0.040 & 0.041 & - & 3.859 \\
\hline 0.073 & 0.016 & - & 4.779 \\
\hline 0.059 & 0.022 & - & 4.677 \\
\hline 0.047 & $0.04 \epsilon$ & - & 4.626 \\
\hline 0.027 & - & - & 3.651 \\
\hline 0.023 & • & - & 3.245 \\
\hline$\bullet$ & 0.005 & - & 3.342 \\
\hline 0.005 & - & - & 2.419 \\
\hline • & - & - & $2 \cdot 1 \geq 0$ \\
\hline - & - & - & 2.311 \\
\hline - & - & - & 2.259 \\
\hline$\bullet$ & $\bullet$ & $\bullet$ & $2 \cdot 171$ \\
\hline 0.005 & - & - & 2.052 \\
\hline • & - & - & 1.968 \\
\hline - & - & - & 1.636 \\
\hline - & • & $\bullet$ & 1.307 \\
\hline
\end{tabular}


Basic Table 31.B. Urban Beijing Province (1): duration-specific fertility

\begin{tabular}{|c|c|c|c|c|}
\hline $\begin{array}{l}\text { Birth year } \\
\text { of child }\end{array}$ & $0-4$ & $5-9$ & $10-14$ & $15-19$ \\
\hline 1940 & 0.280 & - & - & • \\
\hline 1941 & 0.261 & - & - & - \\
\hline 1942 & 0.173 & - & • & - \\
\hline 1943 & 0.242 & - & • & - \\
\hline 1944 & 0.226 & - & • & - \\
\hline 1945 & 0.171 & 0.140 & • & . \\
\hline 1940 & 0.215 & 0.298 & - & - \\
\hline 1947 & 0.213 & 0.267 & - & - \\
\hline 1948 & 0.249 & 0.302 & - & - \\
\hline 1949 & 0.274 & 0.170 & - & - \\
\hline 1950 & 0.244 & 0.305 & 0.381 & - \\
\hline 1951 & 0.304 & 0.204 & 0.205 & - \\
\hline 1952 & 0.275 & 0.287 & 0.188 & - \\
\hline 1953 & 0.251 & 0.285 & 0.181 & - \\
\hline 1954 & 0.328 & 0.355 & 0.255 & - \\
\hline $\begin{array}{l}1955 \\
1956\end{array}$ & $\begin{array}{l}0.326 \\
0.302\end{array}$ & $\begin{array}{l}0.283 \\
0.304\end{array}$ & $\begin{array}{l}0.317 \\
0.158\end{array}$ & $\begin{array}{l}0.180 \\
0.168\end{array}$ \\
\hline 1957 & 0.366 & 0.309 & 0.309 & 0.157 \\
\hline 1958 & 0.280 & 0.259 & 0.276 & 0.181 \\
\hline 1959 & 0.233 & 0.300 & 0.177 & 0.240 \\
\hline 1960 & 0.248 & 0.248 & 0.157 & 0.110 \\
\hline 1961 & 0.231 & 0.126 & 0.148 & 0.102 \\
\hline 1902 & 0.308 & 0.263 & 0.186 & 0.160 \\
\hline 1963 & 0.431 & 0.382 & 0.374 & 0.223 \\
\hline 1904 & 0.247 & 0.226 & 0.175 & 0.153 \\
\hline 1905 & 0.226 & 0.254 & 0.135 & 0.031 \\
\hline 1966 & 0.341 & 0.154 & 0.112 & 0.042 \\
\hline 1907 & 0.242 & 0.203 & 0.109 & 0.041 \\
\hline 1968 & 0.445 & 0.215 & 0.083 & 0.072 \\
\hline 1964 & 0.398 & 0.238 & 0.073 & 0.063 \\
\hline 1970 & 0.385 & 0.187 & 0.085 & 0.064 \\
\hline 1971 & 0.268 & 0.176 & 0.070 & 0.021 \\
\hline 1972 & 0.270 & 0.115 & 0.005 & 0.013 \\
\hline 1973 & 0.379 & 0.103 & 0.052 & 0.019 \\
\hline 1974 & 0.232 & 0.074 & 0.009 & 0.007 \\
\hline 1975 & 0.241 & 0.086 & 0.029 & 0.026 \\
\hline 1970 & 0.385 & 0.122 & 0.011 & 0.021 \\
\hline 1977 & 0.364 & 0.100 & 0.012 & • \\
\hline 1978 & 0.248 & 0.076 & 0.010 & 0.009 \\
\hline 1979 & 0.261 & 0.097 & - & 0.005 \\
\hline 1980 & 0.253 & 0.039 & 0.017 & - \\
\hline 1981 & 0.249 & - & - & - \\
\hline 1982 & 0.206 & - & - & - \\
\hline
\end{tabular}


rates, $1940-82$

\begin{tabular}{|c|c|c|c|}
\hline $20-24$ & $25-29$ & $30+$ & Total \\
\hline - & - & - & - \\
\hline - & - & - & - \\
\hline - & - & - & - \\
\hline - & - & - & $\bullet$ \\
\hline - & • & • & - \\
\hline - & - & - & 3.111 \\
\hline - & - & - & 5.872 \\
\hline - & - & - & 5.357 \\
\hline - & - & - & 6.106 \\
\hline - & - & - & 4.102 \\
\hline - & - & - & 7.133 \\
\hline - & - & - & 5.033 \\
\hline - & - & - & 5.443 \\
\hline - & - & - & 5.243 \\
\hline - & - & - & 6.861 \\
\hline - & - & - & 6.457 \\
\hline - & - & - & 5.397 \\
\hline - & - & - & 6.609 \\
\hline - & - & • & 5.329 \\
\hline - & - & - & 5.615 \\
\hline 0.080 & - & - & 4.376 \\
\hline 0.093 & - & - & 3.640 \\
\hline 0.063 & - & - & 5.074 \\
\hline 0.166 & • & - & 0.188 \\
\hline 0.099 & - & - & 4.498 \\
\hline 0.024 & - & - & 3.354 \\
\hline 0.011 & 0.037 & - & 3.494 \\
\hline 0.011 & - & - & 3.028 \\
\hline 0.009 & - & - & 4.119 \\
\hline 0.024 & 0.014 & - & 4.053 \\
\hline 0.016 & 0.012 & - & 3.740 \\
\hline 0.007 & - & - & 2.710 \\
\hline 0.014 & - & - & 2.383 \\
\hline • & - & - & 2.766 \\
\hline 0.007 & - & - & 1.044 \\
\hline - & - & - & 1.910 \\
\hline - & - & - & 2.695 \\
\hline - & - & - & 2.377 \\
\hline - & - & - & 1.712 \\
\hline - & - & - & 1.833 \\
\hline - & - & - & 1.545 \\
\hline - & - & - & 1.243 \\
\hline - & - & - & 1.029 \\
\hline
\end{tabular}


Basic Table 31.C. Rural Beijing Province (1): duration-specific fertility

\begin{tabular}{|c|c|c|c|c|}
\hline $\begin{array}{l}\text { Birth year } \\
\text { of child }\end{array}$ & $0-4$ & $5-9$ & $10-14$ & $15-19$ \\
\hline 1940 & 0.120 & . & - & - \\
\hline 1941 & 0.220 & - & - & - \\
\hline 1942 & 0.191 & - & - & - \\
\hline 1943 & 0.290 & - & - & - \\
\hline 1944 & 0.213 & - & - & - \\
\hline 1945 & 0.279 & 0.326 & - & - \\
\hline 1946 & 0.213 & 0.152 & - & - \\
\hline 1947 & 0.200 & 0.364 & - & - \\
\hline 1948 & 0.186 & 0.326 & - & - \\
\hline 1949 & 0.201 & 0.305 & - & - \\
\hline 1950 & 0.200 & 0.237 & 0.275 & - \\
\hline 1951 & 0.253 & 0.327 & 0.287 & - \\
\hline 1952 & 0.340 & 0.362 & 0.277 & - \\
\hline 1953 & 0.373 & 0.236 & 0.218 & - \\
\hline 1954 & 0.232 & 0.251 & 0.274 & - \\
\hline 1955 & 0.410 & 0.342 & 0.376 & 0.206 \\
\hline 1956 & 0.343 & 0.228 & 0.201 & 0.304 \\
\hline 1957 & 0.309 & 0.340 & 0.274 & 0.200 \\
\hline 1958 & 0.416 & 0.341 & 0.186 & 0.181 \\
\hline 1959 & 0.100 & 0.211 & 0.239 & 0.137 \\
\hline 1900 & 0.198 & 0.355 & 0.177 & 0.209 \\
\hline 1961 & 0.090 & 0.147 & 0.177 & 0.126 \\
\hline 1902 & 0.363 & 0.289 & 0.340 & 0.224 \\
\hline 1903 & 0.423 & 0.435 & 0.357 & 0.285 \\
\hline 1964 & 0.325 & 0.289 & 0.329 & 0.201 \\
\hline 1965 & 0.327 & 0.287 & 0.328 & 0.165 \\
\hline 1900 & 0.242 & 0.271 & 0.245 & 0.177 \\
\hline 1907 & 0.321 & 0.281 & 0.227 & 0.071 \\
\hline 1908 & $0.360^{\circ}$ & 0.277 & 0.227 & 0.146 \\
\hline 1909 & 0.331 & 0.310 & 0.235 & 0.215 \\
\hline 1970 & 0.503 & 0.241 & 0.198 & 0.109 \\
\hline 1971 & 0.412 & 0.197 & 0.289 & 0.171 \\
\hline 1972 & 0.370 & 0.304 & 0.149 & 0.062 \\
\hline 1973 & 0.329 & 0.300 & 0.160 & 0.095 \\
\hline 1974 & 0.357 & 0.229 & 0.074 & 0.018 \\
\hline 1975 & 0.289 & 0.103 & 0.043 & 0.054 \\
\hline 1970 & 0.204 & 0.131 & - & 0.018 \\
\hline 1977 & 0.232 & 0.120 & 0.034 & 0.017 \\
\hline 1970 & 0.297 & 0.191 & 0.067 & - \\
\hline 1479 & 0.372 & 0.082 & 0.025 & - \\
\hline 1980 & 0.354 & 0.110 & 0.034 & 0.014 \\
\hline 1981 & 0.355 & 0.090 & $\bullet$ & - \\
\hline 1982 & 0.241 & 0.049 & 0.052 & - \\
\hline
\end{tabular}


rates, $1940-82$

\begin{tabular}{|c|c|c|c|}
\hline $20-24$ & $25-29$ & $30+$ & Total \\
\hline - & - & - & - \\
\hline - & - & - & - \\
\hline - & - & - & - \\
\hline - & - & $\bullet$ & - \\
\hline$\bullet$ & - & - & $\bullet$ \\
\hline - & $\bullet$ & $\bullet$ & 6.381 \\
\hline - & - & - & 3.394 \\
\hline - & - & - & 6.565 \\
\hline - & - & - & 5.924 \\
\hline - & - & - & 5.653 \\
\hline - & - & - & 5.234 \\
\hline - & - & • & 6.324 \\
\hline - & - & - & 6.953 \\
\hline - & - & - & 5.604 \\
\hline - & - & - & 5.502 \\
\hline - & - & - & 7.020 \\
\hline - & - & - & 6.171 \\
\hline$\bullet$. & - & - & 6.830 \\
\hline - & - & - & 6.343 \\
\hline - & - & - & 4.319 \\
\hline 0.172 & - & - & 5.770 \\
\hline 0.051 & - & - & 3.064 \\
\hline 0.200 & - & - & 7.666 \\
\hline 0.163 & - & - & e.599 \\
\hline 0.305 & 0.108 & 0.034 & 7.953 \\
\hline 0.034 & 0.069 & 0.028 & 6.436 \\
\hline 0.138 & $\bullet$ & • & 5.367 \\
\hline 0.075 & 0.087 & - & 5.305 \\
\hline 0.161 & 0.036 & • & 6.069 \\
\hline 0.113 & 0.030 & - & 0.165 \\
\hline 0.094 & 0.084 & - & 6.148 \\
\hline 0.063 & • & - & 5.665 \\
\hline 0.042 & • & - & 4.635 \\
\hline • & 0.012 & - & 4.481 \\
\hline - & • & - & 3.392 \\
\hline - & - & - & 2.444 \\
\hline • & - & - & 1.762 \\
\hline - & - & - & 2.015 \\
\hline - & - & - & 2.770 \\
\hline 0.018 & - & - & 2.492 \\
\hline$\bullet$ & - & - & 2.563 \\
\hline • & - & - & 2.225 \\
\hline - & - & - & 1.7 .08 \\
\hline
\end{tabular}


Basic Table 32.A. Total Tianjin Province (2): duration-specific fertility

\begin{tabular}{|c|c|c|c|c|}
\hline $\begin{array}{l}\text { Birth year } \\
\text { of child }\end{array}$ & $0-4$ & $5-9$ & $10-14$ & $15-19$ \\
\hline 1940 & 0.279 & - & - & - \\
\hline 1941 & 0.163 & • & - & - \\
\hline 1942 & 0.324 & - & - & - \\
\hline 1943 & 0.302 & - & - & - \\
\hline 1944 & 0.155 & - & • & - \\
\hline 1945 & 0.240 & 0.167 & - & • \\
\hline 1946 & 0.230 & 0.288 & - & - \\
\hline 1947 & 0.260 & 0.317 & - & • \\
\hline $\begin{array}{l}1948 \\
1949\end{array}$ & $\begin{array}{l}0.227 \\
0.239\end{array}$ & $\begin{array}{l}0.214 \\
0.315\end{array}$ & $\because$ & - \\
\hline 1950 & 0.298 & 0.262 & 0.232 & $\bullet$ \\
\hline 1951 & 0.343 & 0.246 & 0.241 & - \\
\hline 1952 & 0.315 & 0.287 & 0.317 & - \\
\hline 1953 & 0.298 & 0.275 & 0.246 & - \\
\hline 1954 & 0.372 & 0.354 & 0.258 & - \\
\hline 1955 & 0.345 & 0.335 & 0.366 & 0.269 \\
\hline 1950 & 0.343 & 0.316 & 0.246 & 0.288 \\
\hline 1957 & 0.405 & 0.371 & 0.281 & 0.277 \\
\hline 1953 & 0.386 & 0.361 & 0.286 & 0.214 \\
\hline $\begin{array}{l}1959 \\
1960\end{array}$ & $\begin{array}{l}0.317 \\
0.395\end{array}$ & $\begin{array}{l}0.235 \\
0.291\end{array}$ & $\begin{array}{l}0.109 \\
0.242\end{array}$ & $\begin{array}{l}0.155 \\
0.175\end{array}$ \\
\hline 1961 & 0.176 & 0.193 & 0.185 & 0.173 \\
\hline 1962 & 0.365 & 0.321 & 0.272 & 0.173 \\
\hline 1963 & 0.445 & 0.424 & 0.315 & 0.292 \\
\hline 1964 & 0.354 & 0.241 & 0.155 & 0.199 \\
\hline 1965 & 0.394 & 0.294 & 0.168 & 0.126 \\
\hline 1966 & 0.282 & 0.225 & 0.094 & 0.081 \\
\hline 1967 & 0.322 & 0.257 & 0.119 & 0.078 \\
\hline 1968 & 0.360 & 0.229 & 0.156 & 0.068 \\
\hline 1969 & 0.367 & 0.286 & 0.129 & 0.062 \\
\hline 1970 & 0.356 & 0.246 & 0.147 & 0.082 \\
\hline 1971 & 0.347 & 0.251 & 0.176 & 0.023 \\
\hline 1972 & 0.380 & 0.182 & 0.107 & 0.035 \\
\hline 1973 & 0.309 & 0.180 & 0.101 & 0.054 \\
\hline 1974 & 0.335 & 0.177 & 0.082 & 0.029 \\
\hline 1975 & 0.345 & 0.211 & 0.049 & 0.033 \\
\hline 1970 & 0.354 & 0.181 & 0.078 & 0.014 \\
\hline 1977 & 0.284 & 0.113 & 0.050 & 0.021 \\
\hline 1978 & 0.353 & 0.083 & 0.029 & 0.007 \\
\hline 1979 & 0.303 & 0.079 & 0.032 & 0.007 \\
\hline 1980 & 0.284 & 0.094 & • & - \\
\hline 1981 & 0.282 & 0.037 & 0.005 & - \\
\hline 1982 & 0.237 & 0.009 & 0.010 & - \\
\hline
\end{tabular}


rates, $1940-82$

\begin{tabular}{|c|c|c|c|}
\hline $20-24$ & $25-29$ & $30+$ & Total \\
\hline - & - & - & - \\
\hline$\bullet$ & - & $\bullet$ & $\bullet$ \\
\hline • & - & - & - \\
\hline • & - & $\bullet$ & $\bullet$ \\
\hline$\bullet$ & - & - & - \\
\hline$\bullet$ & - & - & 3.856 \\
\hline - & - & - & 5.673 \\
\hline - & $\bullet$ & - & 6.245 \\
\hline$\bullet$ & - & - & 4.479 \\
\hline - & - & - & 6.123 \\
\hline • & $\bullet$ & - & 5.749 \\
\hline - & $\bullet$ & - & 5.823 \\
\hline - & $\bullet$ & - & 6.634 \\
\hline • & - & - & 5.870 \\
\hline - & - & - & 5.887 \\
\hline • & - & - & 7.617 \\
\hline - & $\bullet$ & - & 6.986 \\
\hline - & - & - & $7 \cdot 695$ \\
\hline - & - & • & 7.115 \\
\hline$\bullet$ & - & - & 5.004 \\
\hline 0.121 & • & - & 6.403 \\
\hline 0.101 & $\bullet$ & • & 4.338 \\
\hline 0.142 & - & - & 6.833 \\
\hline 0.157 & - & - & 8.523 \\
\hline 0.143 & 0.021 & - & 5.570 \\
\hline 0.087 & 0.028 & 0.020 & 5.536 \\
\hline 0.058 & 0.031 & - & 3.854 \\
\hline 0.043 & 0.0 .14 & - & 4.163 \\
\hline 0.070 & 0.038 & - & 4.60 .3 \\
\hline 0.045 & 0.029 & - & 4.590 \\
\hline 0.056 & 0.016 & • & 4.514 \\
\hline 0.032 & 0.016 & 0.008 & 4.260 \\
\hline 0.030 & 0.016 & • & 3.750 \\
\hline 0.017 & - & - & 3.302 \\
\hline 0.004 & • & $\bullet$ & 3.137 \\
\hline 0.009 & - & - & 3.239 \\
\hline • & $\bullet$ & - & 3.140 \\
\hline 0.015 & - & - & 2.418 \\
\hline 0.005 & 0.004 & - & 2.402 \\
\hline 0.006 & $\bullet$ & - & $2 \cdot 130$ \\
\hline • & - & - & 1.888 \\
\hline - & - & - & 1.619 \\
\hline - & - & - & 1.282 \\
\hline
\end{tabular}


Basic Table 32.B. Urban Tianjin Province (2): duration-specific fertility

\begin{tabular}{|c|c|c|c|c|}
\hline $\begin{array}{l}\text { Birth year } \\
\text { of child }\end{array}$ & $0-4$ & $5-9$ & $10-14$ & $15-19$ \\
\hline 1940 & 0.327 & - & - & - \\
\hline 1941 & 0.131 & - & - & - \\
\hline 1942 & 0.295 & $\bullet$ & • & - \\
\hline 1943 & 0.325 & - & - & - \\
\hline 1944 & 0.207 & - & - & - \\
\hline $\begin{array}{l}1945 \\
1940\end{array}$ & $\begin{array}{r}0.203 \\
.0 .208\end{array}$ & $\begin{array}{l}0.172 \\
0.276\end{array}$ & - & . \\
\hline 1947 & 0.255 & 0.271 & - & - \\
\hline 1948 & 0.239 & 0.163 & - & - \\
\hline 1949 & 0.256 & 0.286 & - & - \\
\hline 1950 & 0.247 & 0.240 & 0.241 & - \\
\hline 1931 & 0.300 & 0.251 & 0.247 & - \\
\hline 1952 & 0.32 .3 & 0.230 & 0.283 & - \\
\hline 1953 & 0.293 & 0.282 & 0.217 & - \\
\hline 1954 & 0.389 & 0.324 & 0.168 & - \\
\hline 1955 & 0.337 & 0.313 & 0.323 & 0.224 \\
\hline 1950 & 0.341 & 0.290 & 0.251 & 0.291 \\
\hline 1957 & 0.403 & 0.364 & 0.255 & 0.246 \\
\hline 1953 & 0.395 & 0.398 & 0.273 & 0.195 \\
\hline ون 19 & 0.310 & 0.219 & 0.136 & 0.089 \\
\hline 1900 & 0.413 & 0.288 & 0.193 & 0.157 \\
\hline 1901 & 0.222 & 0.171 & 0.240 & 0.164 \\
\hline 1902 & 0.484 & 0.318 & 0.239 & 0.136 \\
\hline 1963 & 0.406 & 0.340 & 0.293 & 0.282 \\
\hline 1964 & 0.276 & 0.230 & 0.109 & 0.121 \\
\hline 1965 & 0.359 & 0.163 & 0.104 & 0.060 \\
\hline 1966 & 0.242 & 0.190 & 0.057 & 0.025 \\
\hline 1967 & 0.297 & 0.104 & 0.064 & 0.030 \\
\hline 1968 & 0.367 & 0.152 & 0.071 & 0.041 \\
\hline 1909 & 0.414 & 0.173 & 0.077 & 0.036 \\
\hline 1970 & 0.293 & 0.100 & 0.063 & 0.043 \\
\hline 1971 & 0.339 & 0.132 & 0.095 & 0.006 \\
\hline 1972 & 0.374 & 0.069 & 0.017 & 0.007 \\
\hline 1973 & 0.329 & 0.086 & 0.017 & 0.016 \\
\hline 1974 & 0.290 & 0.180 & 0.052 & • \\
\hline 1975 & 0.287 & 0.117 & 0.020 & - \\
\hline 1970 & 0.201 & 0.088 & - & - \\
\hline 1977 & 0.301 & 0.045 & - & - \\
\hline 1978 & 0.296 & 0.057 & - & - \\
\hline 1979 & 0.243 & 0.048 & - & - \\
\hline 1980 & 0.248 & 0.048 & - & - \\
\hline 1981 & 0.200 & 0.011 & 0.013 & • \\
\hline 1982 & 0.179 & - & - & - \\
\hline
\end{tabular}


rates, $1940-82$

\begin{tabular}{|c|c|c|c|}
\hline $20-24$ & $25-29$ & $30+$ & Total \\
\hline - & - & - & - \\
\hline - & - & • & • \\
\hline - & - & - & - \\
\hline - & - & - & • \\
\hline - & - & - & - \\
\hline - & - & - & 3.786 \\
\hline - & - & • & 5.787 \\
\hline - & - & - & 5.532 \\
\hline - & - & - & 3.812 \\
\hline - & - & • & 5.880 \\
\hline - & - & - & 5.373 \\
\hline - & - & - & 6.081 \\
\hline - & - & - & c.036 \\
\hline • & - & - & 5.739 \\
\hline - & - & - & 6.135 \\
\hline - & - & - & 7.010 \\
\hline - & - & - & 6.873 \\
\hline - & - & - & 7.370 \\
\hline • & • & • & 7.321 \\
\hline • & - & • & 4.306 \\
\hline 0.121 & - & $\bullet$ & 6.068 \\
\hline 0.102 & - & - & 4.082 \\
\hline 0.074 & - & - & 6.455 \\
\hline 0.087 & - & - & 7.302 \\
\hline 0.099 & - & • & 4.173 \\
\hline 0.046 & • & - & 3.660 \\
\hline - & - & • & 2.575 \\
\hline - & - & - & 2.471 \\
\hline 0.034 & - & - & 3.326 \\
\hline 0.030 & 0.020 & - & 3.749 \\
\hline 0.027 & • & - & 2.523 \\
\hline 0.006 & - & - & 2.807 \\
\hline - & - & - & 2.334 \\
\hline 0.006 & - & • & 2.271 \\
\hline - & - & - & 2.610 \\
\hline - & - & - & $2 \cdot 121$ \\
\hline - & - & • & 1.446 \\
\hline 0.007 & • & • & 1.765 \\
\hline • & 0.006 & - & 1.793 \\
\hline - & $\bullet$ & - & 1.450 \\
\hline - & - & - & $1.4 B 1$ \\
\hline - & • & - & 1.417 \\
\hline - & - & - & 0.894 \\
\hline
\end{tabular}


Basic Table 32.C. Rural Tianjin Province (2): duration-specific fertility

\begin{tabular}{|c|c|c|c|c|}
\hline $\begin{array}{l}\text { Birth year } \\
\text { of child }\end{array}$ & $0-4$ & $5-9$ & $10-14$ & $15-19$ \\
\hline 1940 & 0.222 & - & - & - \\
\hline 1941 & 0.200 & - & - & - \\
\hline 1942 & 0.359 & - & - & - \\
\hline 1943 & 0.270 & - & - & - \\
\hline 1744 & 0.178 & - & - & - \\
\hline 1945 & 0.294 & 0.161 & - & - \\
\hline 1946 & 0.187 & 0.300 & - & - \\
\hline 1947 & 0.268 & 0.374 & - & - \\
\hline 1948 & 0.206 & 0.286 & • & - \\
\hline 1949 & 0.206 & 0.356 & - & - \\
\hline 1930 & 0.416 & 0.294 & 0.222 & - \\
\hline 1951 & 0.300 & 0.240 & 0.234 & - \\
\hline 1952 & 0.295 & 0.387 & 0.359 & - \\
\hline 1953 & 0.310 & 0.264 & 0.286 & - \\
\hline 1954 & 0.326 & 0.412 & 0.384 & • \\
\hline 1455 & 0.369 & 0.385 & 0.428 & 0.323 \\
\hline 1956 & 0.347 & 0.380 & 0.240 & 0.284 \\
\hline 1957 & 0.410 & 0.388 & 0.328 & 0.314 \\
\hline 1958 & 0.367 & 0.263 & 0.308 & 0.240 \\
\hline 1959 & 0.320 & 0.277 & 0.235 & 0.247 \\
\hline 1900 & 0.374 & 0.299 & 0.354 & 0.201 \\
\hline 1961 & 0.139 & 0.256 & 0.047 & $0.1 \mathrm{et}$ \\
\hline 1902 & 0.280 & 0.328 & 0.357 & 0.238 \\
\hline 1963 & 0.470 & 0.600 & 0.372 & 0.308 \\
\hline 1964 & 0.405 & 0.259 & 0.277 & 0.353 \\
\hline 1965 & 0.413 & 0.445 & 0.352 & 0.277 \\
\hline 1960 & 0.304 & 0.252 & 0.201 & 0.221 \\
\hline 1907 & 0.337 & 0.366 & 0.246 & 0.202 \\
\hline 1968 & 0.357 & 0.280 & 0.333 & 0.139 \\
\hline 1969 & 0.342 & 0.360 & 0.213 & 0.131 \\
\hline 1970 & 0.394 & 0.326 & 0.244 & 0.193 \\
\hline 1971 & 0.352 & 0.317 & 0.239 & 0.073 \\
\hline 1972 & 0.384 & 0.246 & 0.171 & 0.098 \\
\hline 1973 & 0.289 & 0.227 & 0.157 & 0.133 \\
\hline 1974 & 0.380 & 0.176 & 0.101 & 0.076 \\
\hline 1975 & 0.409 & 0.269 & 0.065 & 0.072 \\
\hline 1970 & 0.492 & 0.247 & 0.122 & 0.025 \\
\hline 1977 & 0.273 & 0.166 & 0.078 & 0.037 \\
\hline 1978 & 0.392 & 0.107 & 0.043 & 0.011 \\
\hline 1979 & 0.351 & 0.110 & 0.049 & 0.011 \\
\hline 1980 & 0.317 & 0.145 & $\bullet$ & - \\
\hline 1981 & 0.307 & 0.060 & - & - \\
\hline 1982 & 0.329 & 0.015 & 0.018 & - \\
\hline
\end{tabular}


rates, $1940-82$

\begin{tabular}{|c|c|c|c|}
\hline $20-24$ & $25-29$ & $30+$ & Total \\
\hline - & - & - & $\bullet$ \\
\hline - & - & - & - \\
\hline - & - & - & - \\
\hline - & - & - & - \\
\hline - & - & - & - \\
\hline - & - & - & 3.941 \\
\hline - & - & - & 5.529 \\
\hline - & - & - & 7.062 \\
\hline • & - & • & $5 \cdot 395$ \\
\hline$\bullet$ & - & - & 5.476 \\
\hline - & $\bullet$ & - & 6.327 \\
\hline - & - & - & 5.411 \\
\hline - & - & - & 7.631 \\
\hline - & - & • & 6.093 \\
\hline - & - & $\bullet$ & B.192 \\
\hline$\bullet$ & - & - & 8.719 \\
\hline - & • & - & 7.193 \\
\hline - & • & - & 8.279 \\
\hline - & - & - & 6.748 \\
\hline - & - & - & 6.195 \\
\hline 0.121 & - & - & 6.965 \\
\hline 0.100 & $\bullet$ & - & 3.782 \\
\hline 0.224 & - & - & 7.424 \\
\hline 0.255 & - & - & $10 \cdot 388$ \\
\hline 0.205 & 0.050 & • & 7.748 \\
\hline 0.147 & 0.061 & 0.036 & 8.655 \\
\hline 0.147 & 0.067 & - & 5.961 \\
\hline 0.119 & 0.030 & - & 6.496 \\
\hline 0.132 & 0.090 & - & 6.657 \\
\hline 0.074 & 0.041 & - & 5.800 \\
\hline 0.123 & 0.040 & - & 6.604 \\
\hline 0.095 & 0.040 & 0.017 & 5.665 \\
\hline 0.109 & 0.045 & - & 5.264 \\
\hline 0.046 & $\bullet$ & - & 4.263 \\
\hline 0.016 & - & . & 3.746 \\
\hline 0.035 & $\bullet$ & - & 4.248 \\
\hline - & - & - & 4.426 \\
\hline 0.033 & - & - & 2.929 \\
\hline 0.017 & • & - & 2.850 \\
\hline 0.015 & - & • & 2.685 \\
\hline - & - & - & 2.308 \\
\hline - & - & - & 1.834 \\
\hline - & - & - & 1.813 \\
\hline
\end{tabular}


Basic Table 33.A. Total Hebei Province (3): duration-specific fertility

\begin{tabular}{|c|c|c|c|c|}
\hline $\begin{array}{l}\text { Birth year } \\
\text { of child }\end{array}$ & $0-4$ & $5-9$ & $10-14$ & $15-19$ \\
\hline 1940 & 0.169 & • & - & • \\
\hline 1941 & 0.162 & - & - & - \\
\hline 1942 & 0.191 & $\bullet$ & - & - \\
\hline 1943 & 0.152 & - & - & - \\
\hline 1944 & 0.149 & • & - & $\bullet$ \\
\hline 1945 & 0.198 & 0.268 & - & $\bullet$ \\
\hline 1946 & 0.187 & 0.229 & - & - \\
\hline 1947 & 0.216 & 0.262 & - & • \\
\hline 1948. & 0.195 & 0.196 & - & - \\
\hline 1949 & 0.253 & 0.291 & - & - \\
\hline 1950 & 0.240 & 0.257 & 0.236 & - \\
\hline 1951 & 0.243 & 0.232 & 0.234 & $\bullet$ \\
\hline 1952 & 0.314 & 0.321 & 0.249 & - \\
\hline 1953 & 0.294 & 0.243 & 0.247 & - \\
\hline 1954 & 0.271 & 0.288 & 0.277 & - \\
\hline 1955 & 0.318 & 0.309 & 0.255 & 0.208 \\
\hline 1956 & 0.314 & 0.295 & 0.232 & 0.202 \\
\hline 1957 & 0.312 & 0.284 & 0.276 & 0.194 \\
\hline 1950 & 0.276 & 0.279 & 0.238 & 0.228 \\
\hline 1959 & 0.236 & 0.213 & 0.178 & 0.164 \\
\hline 1960 & 0.266 & 0.242 & 0.198 & 0.153 \\
\hline 1961 & 0.150 & 0.137 & 0.109 & 0.074 \\
\hline 1962 & 0.260 & 0.305 & 0.239 & 0.157 \\
\hline 1963 & 0.359 & 0.376 & 0.346 & 0.279 \\
\hline 1964 & 0.318 & 0.295 & 0.250 & 0.202 \\
\hline 1903 & 0.331 & 0.331 & 0.277 & 0.202 \\
\hline 1966 & 0.295 & 0.319 & 0.260 & 0.189 \\
\hline 1907 & 0.307 & 0.279 & 0.230 & 0.164 \\
\hline 1908 & 0.340 & $0.30 \mathrm{e}$ & 0.260 & 0.203 \\
\hline 1969 & 0.294 & 0.287 & 0.232 & 0.154 \\
\hline 1970 & 0.365 & 0.320 & 0.242 & 0.211 \\
\hline 1971 & 0.343 & 0.274 & 0.234 & 0.192 \\
\hline 1972 & 0.318 & 0.278 & 0.198 & 0.152 \\
\hline 1973 & 0.336 & 0.247 & 0.164 & 0.111 \\
\hline 1974 & 0.320 & 0.199 & 0.127 & 0.068 \\
\hline 1975 & 0.283 & 0.213 & 0.091 & 0.049 \\
\hline 1970 & 0.338 & 0.192 & 0.084 & 0.046 \\
\hline 1977 & 0.310 & 0.163 & 0.087 & 0.042 \\
\hline 1978 & 0.336 & 0.182 & 0.075 & 0.026 \\
\hline 1979 & 0.308 & 0.186 & 0.075 & 0.025 \\
\hline 1980 & 0.305 & 0.166 & 0.064 & 0.023 \\
\hline 1981 & 0.368 & 0.176 & 0.091 & 0.036 \\
\hline 1982 & 0.274 & 0.164 & 0.093 & 0.034 \\
\hline
\end{tabular}


rates, $1940-82$

\begin{tabular}{|c|c|c|c|}
\hline $20-24$ & $25-29$ & $30+$ & Total \\
\hline - & - & - & $\bullet$ \\
\hline - & $\bullet$ & • & $\bullet$ \\
\hline - & - & $\bullet$ & - \\
\hline - & - & - & $\bullet$ \\
\hline - & - & $\bullet$ & - \\
\hline - & - & - & 5.106 \\
\hline • & - & - & 4.438 \\
\hline - & - & - & 5.110 \\
\hline - & - & $\bullet$ & 3.984 \\
\hline - & • & • & 5.740 \\
\hline - & - & - & 5.274 \\
\hline - & - & - & 5.071 \\
\hline - & - & - & 6.259 \\
\hline - & - & - & 5.532 \\
\hline - & - & - & 6.037 \\
\hline - & - & - & 6.262 \\
\hline - & • & - & 5.964 \\
\hline . & . & $\bullet$ & 6.118 \\
\hline - & - & - & 5.891 \\
\hline - & $\bullet$ & - & 4.536 \\
\hline 0.100 & . & - & 4.950 \\
\hline 0.051 & - & • & 2.697 \\
\hline 0.082 & - & - & 5.387 \\
\hline 0.185 & - & • & 8.008 \\
\hline 0.155 & 0.059 & 0.004 & 6.416 \\
\hline 0.137 & 0.057 & 0.036 & 6.707 \\
\hline 0.136 & 0.043 & 0.005 & 6.229 \\
\hline 0.101 & 0.031 & 0.007 & 5.596 \\
\hline 0.114 & 0.041 & 0.003 & 6.349 \\
\hline 0.102 & 0.037 & 0.001 & 5.533 \\
\hline 0.097 & 0.044 & 0.004 & 6.411 \\
\hline 0.094 & 0.024 & 0.001 & 5.808 \\
\hline 0.085 & 0.017 & 0.005 & 5.263 \\
\hline 0.069 & 0.013 & - & 4.702 \\
\hline 0.034 & 0.015 & • & 3.811 \\
\hline 0.029 & 0.009 & 0.001 & 3.373 \\
\hline 0.020 & 0.007 & 0.001 & 3.443 \\
\hline 0.013 & 0.005 & • & 3.097 \\
\hline 0.013 & 0.004 & - & 3.178 \\
\hline 0.008 & 0.001 & - & 3.017 \\
\hline 0.014 & 0.001 & - & 2.867 \\
\hline 0.007 & 0.003 & 0.001 & 3.406 \\
\hline 0.008 & 0.006 & - & 2.892 \\
\hline
\end{tabular}


Basic Table 33.B. Urban Hebei Province (3): duration-specific fertility

\begin{tabular}{|c|c|c|c|c|c|}
\hline $\begin{array}{l}\text { Birth year } \\
\text { of child }\end{array}$ & & $0-4$ & $5-9$ & $10-14$ & $15-19$ \\
\hline $19+0$ & & 0.147 & • & - & - \\
\hline 1941 & & 0.233 & - & - & - \\
\hline 1942 & & 0.150 & - & - & - \\
\hline 1943 & & 0.119 & - & - & - \\
\hline 1944 & & 0.181 & - & - & - \\
\hline 1945 & & 0.199 & 0.327 & - & - \\
\hline 1946 & & 0.200 & 0.124 & - & - \\
\hline 1947 & & 0.189 & 0.245 & • & • \\
\hline 1940 & & 0.185 & 0.199 & - & - \\
\hline 1949 & & 0.26 .3 & 0.194 & - & - \\
\hline 1950 & & 0.286 & 0.238 & 0.163 & - \\
\hline 1951 & & 0.346 & 0.213 & 0.280 & - \\
\hline 1952 & & 0.327 & 0.175 & 0.204 & - \\
\hline 1953 & & 0.293 & 0.259 & 0.265 & - \\
\hline 1954 & & 0.311 & 0.577 & 0.297 & $\bullet$ \\
\hline 1955 & & 0.274 & 0.265 & 0.344 & 0.114 \\
\hline 1956 & & 0.364 & 0.267 & 0.175 & 0.124 \\
\hline 1957 & & 0.326 & 0.318 & 0.310 & 0.204 \\
\hline 1958 & & 0.351 & 0.247 & 0.210 & 0.292 \\
\hline 1959 & & 0.275 & 0.188 & 0.160 & 0.181 \\
\hline 1960 & & 0.330 & 0.255 & 0.130 & 0.146 \\
\hline 1901 & & 0.142 & 0.086 & 0.089 & 0.088 \\
\hline 1902 & & 0.268 & 0.326 & 0.218 & 0.108 \\
\hline 1963 & & 0.391 & 0.408 & 0.293 & 0.210 \\
\hline 1904 & & 0.340 & 0.228 & 0.160 & 0.160 \\
\hline 1965 & 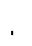 & 0.257 & 0.243 & 0.123 & 0.095 \\
\hline 1960 & & 0.285 & 0.265 & 0.115 & 0.079 \\
\hline 1907 & & 0.334 & 0.214 & 0.158 & 0.045 \\
\hline 17000 & & 0.309 & 0.326 & 0.101 & 0.119 \\
\hline 1909 & : & 0.313 & 0.189 & 0.152 & 0.057 \\
\hline 1970 & & 0.445 & 0.190 & 0.116 & 0.151 \\
\hline 1971 & & 0.343 & 0.243 & 0.123 & 0.086 \\
\hline 1972 & & 0.340 & 0.151 & 0.080 & 0.059 \\
\hline 1973 & & 0.287 & 0.168 & 0.056 & 0.009 \\
\hline 1974 & . & 0.277 & 0.115 & 0.028 & 0.019 \\
\hline 1975 & & 0.390 & 0.061 & 0.038 & - \\
\hline 1976 & & 0.258 & 0.088 & 0.011 & 0.028 \\
\hline 1977 & & 0.292 & 0.128 & 0.022 & 0.018 \\
\hline 1978 & & 0.296 & 0.096 & 0.045 & 0.009 \\
\hline 1979 & & 0.245 & 0.106 & 0.042 & - \\
\hline 1980 & & 0.233 & 0.103 & 0.010 & • \\
\hline 1981 & & 0.407 & 0.124 & 0.009 & - \\
\hline 1982 & & 0.221 & 0.039 & - & • \\
\hline
\end{tabular}


rates, $1940-82$

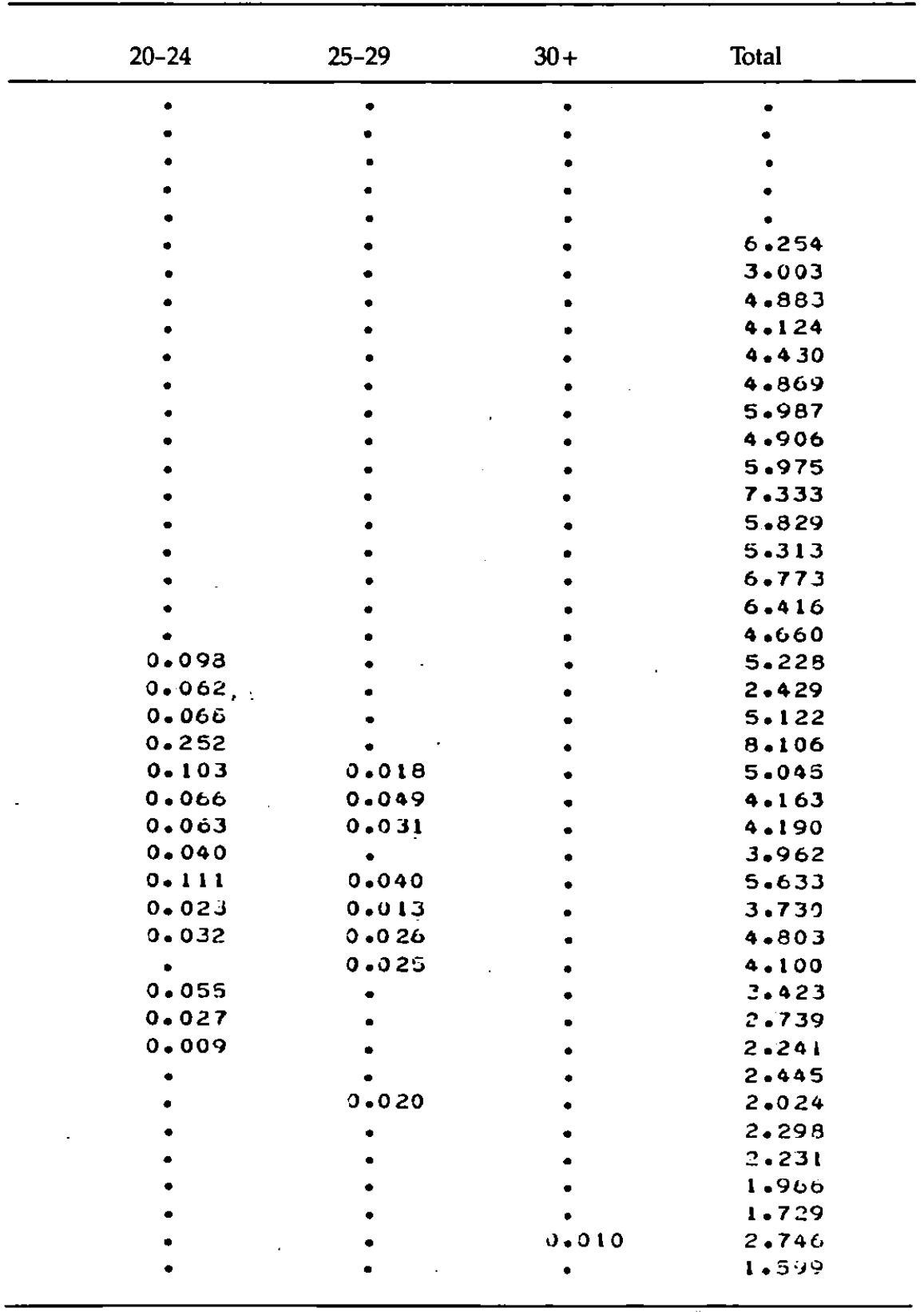


Basic Table 33.C. Rural Hebei Province (3): duration-specific fertility

\begin{tabular}{|c|c|c|c|c|}
\hline $\begin{array}{l}\text { Birth year } \\
\text { of child }\end{array}$ & $0-4$ & $5-9$ & $10-14$ & $15-19$ \\
\hline 1440 & 0.171 & - & - & - \\
\hline 1941 & 0.157 & - & - & • \\
\hline 1942 & 0.195 & - & - & - \\
\hline 1943 & 0.154 & - & - & - \\
\hline 1944 & 0.147 & - & - & - \\
\hline 1945 & 0.198 & 0.263 & • & - \\
\hline 1940 & 0.186 & 0.237 & - & - \\
\hline 1947 & 0.219 & 0.264 & - & - \\
\hline 1948 & 0.196 & 0.196 & - & - \\
\hline 1949 & 0.253 & 0.300 & - & - \\
\hline 1950 & 0.235 & 0.259 & 0.242 & - \\
\hline 1951 & 0.232 & 0.234 & 0.230 & - \\
\hline 1952 & 0.313 & 0.333 & 0.253 & • \\
\hline 1953 & 0.294 & 0.242 & 0.245 & - \\
\hline 1954 & 0.207 & 0.279 & 0.276 & - \\
\hline $\begin{array}{l}1955 \\
1950\end{array}$ & $\begin{array}{l}0.323 \\
0.308\end{array}$ & $\begin{array}{l}0.313 \\
0.298\end{array}$ & $\begin{array}{l}0.248 \\
0.237\end{array}$ & $\begin{array}{l}0.216 \\
0.208\end{array}$ \\
\hline 1957 & 0.310 & 0.280 & 0.274 & 0.193 \\
\hline 1958 & 0.267 & 0.282 & 0.241 & 0.222 \\
\hline 1959 & 0.231 & 0.216 & 0.179 & $0.1 \in 2$ \\
\hline 1960 & 0.258 & 0.240 & 0.200 & 0.154 \\
\hline 1961 & 0.151 & 0.143 & 0.111 & 0.073 \\
\hline 1962 & 0.259 & 0.303 & 0.242 & 0.161 \\
\hline 1963 & 0.356 & 0.372 & 0.352 & 0.285 \\
\hline 1964 & 0.316 & 0.303 & 0.260 & 0.206 \\
\hline 1965 & 0.338 & 0.342 & 0.294. & 0.213 \\
\hline 1960 & 0.290 & 0.326 & $0.270^{\circ}$ & 0.201 \\
\hline 1967 & 0.305 & 0.287 & 0.238 & 0.178 \\
\hline 1968 & 0.337 & 0.306 & 0.271 & 0.213 \\
\hline 1909 & 0.292 & 0.297 & 0.241 & 0.165 \\
\hline 1970 & 0.359 & 0.333 & 0.257 & 0.218 \\
\hline 1971 & 0.343 & 0.276 & 0.248 & 0.203 \\
\hline 1972 & 0.317 & 0.289 & 0.212 & 0.162 \\
\hline 1973 & 0.340 & 0.253 & 0.177 & 0.122 \\
\hline 1974 & 0.323 & 0.206 & 0.138 & 0.074 \\
\hline 1975 & 0.275 & 0.225 & 0.097 & 0.055 \\
\hline 1976 & 0.343 & 0.200 & 0.051 & 0.049 \\
\hline 1977 & 0.312 & 0.165 & 0.093 & 0.045 \\
\hline 1978 & 0.339 & 0.188 & 0.078 & 0.028 \\
\hline 1979 & 0.314 & 0.191 & 0.078 & 0.028 \\
\hline 1900 & 0.313 & 0.170 & 0.068 & 0.026 \\
\hline 1961 & 0.363 & 0.179 & 0.098 & 0.039 \\
\hline 1982 & 0.280 & 0.169 & 0.099 & 0.038 \\
\hline
\end{tabular}


rates, $1940-82$

\begin{tabular}{|c|c|c|c|}
\hline $20-24$ & $25-29$ & $30+$ & Total \\
\hline - & - & - & - \\
\hline$\bullet$ & $\bullet$ & $\bullet$ & - \\
\hline - & - & - & - \\
\hline - & - & - & - \\
\hline - & - & - & - \\
\hline - & - & - & 5.001 \\
\hline - & - & • & 4.567 \\
\hline - & - & - & 5.129 \\
\hline - & - & - & 3.972 \\
\hline - & - & - & 5.857 \\
\hline - & - & - & 5.310 \\
\hline • & • & • & 4.986 \\
\hline - & - & - & 6.386 \\
\hline - & - & - & 5.490 \\
\hline - & - & • & 5.914 \\
\hline - & • & - & 6.303 \\
\hline - & - & - & 6.020 \\
\hline - & - & - & 5.055 \\
\hline - & - & - & 5.339 \\
\hline - & - & - & 4.524 \\
\hline 0.100 & - & - & 4.923 \\
\hline 0.050 & - & - & 2.724 \\
\hline 0.084 & - & - & 5.414 \\
\hline 0.179 & - & - & 7.998 \\
\hline 0.160 & 0.063 & 0.004 & 6.557 \\
\hline 0.144 & 0.058 & 0.006 & 6.976 \\
\hline 0.142 & 0.044 & 0.005 & 0.446 \\
\hline 0.106 & 0.034 & 0.008 & 5.775 \\
\hline 0.114 & 0.041 & 0.003 & 6.435 \\
\hline 0.110 & 0.039 & 0.001 & 5.729 \\
\hline 0.103 & 0.045 & 0.004 & 6.599 \\
\hline 0.105 & 0.024 & 0.001 & 6.001 \\
\hline 0.088 & 0.019 & 0.005 & 5.463 \\
\hline 0.074 & 0.014 & • & 4.902 \\
\hline 0.036 & 0.016 & - & 3.963 \\
\hline 0.032 & 0.010 & 0.001 & 3.272 \\
\hline 0.022 & 0.005 & 0.001 & 3.561 \\
\hline 0.014 & 0.005 & $\bullet$ & 3.171 \\
\hline 0.014 & 0.004 & - & 3.257 \\
\hline 0.009 & 0.001 & - & 3.107 \\
\hline 0.015 & 0.001 & - & $2 \cdot 96,9$ \\
\hline 0.008 & 0.003 & • & 3.450 \\
\hline 0.009 & 0.007 & - & 3.009 \\
\hline
\end{tabular}


Basic Table 34.A. Total Shanxi Province (4): duration-specific fertility

\begin{tabular}{|c|c|c|c|c|}
\hline $\begin{array}{l}\text { Birth year } \\
\text { of child }\end{array}$ & $0-4$ & $5-9$ & $10-14$ & $15-19$ \\
\hline 1940 & 0.169 & - & - & - \\
\hline 1541 & 0.175 & - & - & - \\
\hline 1942 & 0.174 & - & - & - \\
\hline 1943 & 0.172 & - & - & - \\
\hline 1944 & 0.182 & - & - & - \\
\hline 1945 & 0.172 & 0.208 & • & - \\
\hline 1946 & 0.203 & 0.265 & - & - \\
\hline 1947 & 0.187 & 0.225 & - & - \\
\hline 1948 & 0.200 & 0.184 & - & - \\
\hline 1945 & 0.158 & 0.253 & - & - \\
\hline 1950 & 0.229 & 0.277 & 0.159 & - \\
\hline 1951 & 0.275 & 0.243 & 0.207 & - \\
\hline 1952 & 0.261 & 0.279 & 0.213 & - \\
\hline 1953 & 0.328 & 0.248 & 0.211 & - \\
\hline 1954 & 0.283 & 0.273 & 0.241 & - \\
\hline 1955 & 0.310 & 0.290 & 0.230 & 0.226 \\
\hline 1950 & 0.260 & 0.279 & 0.236 & 0.160 \\
\hline 1957 & 0.335 & 0.329 & 0.233 & 0.203 \\
\hline 1958 & 0.267 & 0.246 & 0.224 & 0.177 \\
\hline 1959 & 0.211 & 0.237 & 0.241 & 0.213 \\
\hline 1900 & 0.238 & 0.237 & 0.204 & 0.163 \\
\hline 1961 & 0.215 & 0.213 & $0.10 \tilde{0}$ & 0.152 \\
\hline 1962 & 0.304 & 0.343 & 0.309 & 0.203 \\
\hline 1903 & 0.352 & 0.322 & 0.320 & 0.242 \\
\hline 1964 & 0.327 & 0.282 & $0.2 \leftarrow 4$ & 0.216 \\
\hline 1905 & 0.321 & 0.292 & 0.271 & 0.180 \\
\hline 1960 & 0.305 & 0.238 & 0.254 & 0.190 \\
\hline 1967 & 0.295 & 0.308 & 0.306 & 0.189 \\
\hline 1968 & 0.318 & 0.328 & 0.289 & 0.269 \\
\hline 1969 & 0.315 & 0.296 & 0.272 & 0.180 \\
\hline 1970 & 0.347 & 0.321 & 0.240 & 0.177 \\
\hline 1971 & 0.348 & 0.303 & 0.244 & 0.226 \\
\hline 1972 & 0.341 & 0.275 & 0.235 & 0.150 \\
\hline 1973 & 0.324 & 0.266 & 0.231 & $0.130^{\circ}$ \\
\hline 1974 & 0.333 & 0.276 & 0.200 & 0.097 \\
\hline 1975 & 0.284 & 0.243 & 0.118 & 0.084 \\
\hline 1970 & 0.306 & 0.220 & 0.136 & 0.092 \\
\hline 1977 & 0.279 & 0.210 & 0.094 & 0.080 \\
\hline 1978 & 0.291 & 0.163 & 0.098 & 0.057 \\
\hline 1979 & 0.299 & 0.162 & 0.099 & 0.042 \\
\hline 1980 & 0.317 & 0.187 & 0.075 & 0.020 \\
\hline 1981 & 0.316 & 0.134 & 0.051 & 0.024 \\
\hline 1982 & 0.359 & 0.169 & 0.098 & 0.037 \\
\hline
\end{tabular}


rates, $1940-82$

\begin{tabular}{|c|c|c|c|}
\hline $20-24$ & $25-29$ & $30+$ & Total \\
\hline - & $\bullet$ & - & • \\
\hline • & - & • & - \\
\hline - & $\bullet$ & - & $\bullet$ \\
\hline - & • & - & $\bullet$ \\
\hline - & - & • & - \\
\hline - & - & - & 4.043 \\
\hline - & - & - & 5.089 \\
\hline - & - & - & 4.369 \\
\hline - & $\bullet$ & . & 3.326 \\
\hline - & - & - & 4.692 \\
\hline - & - & • & 4.710 \\
\hline - & - & - & 5.113 \\
\hline - & - & - & 5.372 \\
\hline - & - & - & 5.433 \\
\hline - & - & - & 5.701 \\
\hline - & - & - & 6.056 \\
\hline & - & - & 5.394 \\
\hline - & - & - & 6.285 \\
\hline - & - & - & 5.245 \\
\hline - & - & - & 5.248 \\
\hline 0.112 & . & - & 4.946 \\
\hline 0.087 & - & - & 4.321 \\
\hline 0.102 & - & $\bullet$ & 6.861 \\
\hline 0.152 & - & - & 7.174 \\
\hline 0.135 & 0.083 & - & 6.535 \\
\hline 0.153 & 0.057 & 0.005 & 6.454 \\
\hline 0.138 & $0 . \dot{037}$ & 0.012 & 5.870 \\
\hline 0.136 & 0.036 & 0.011 & 6.406 \\
\hline 0.142 & 0.048 & 0.007 & 7.005 \\
\hline 0.131 & 0.052 & 0.011 & 6.286 \\
\hline 0.151 & 0.050 & 0.018 & 6.550 \\
\hline 0.121 & 0.051 & 0.007 & 6.504 \\
\hline 0.097 & 0.044 & 0.002 & 5.752 \\
\hline 0.102 & 0.327 & 0.005 & 5.452 \\
\hline 0.071 & 0.021 & $\bullet$ & 4.990 \\
\hline 0.064 & 0.039 & 0.007 & 4.196 \\
\hline 0.055 & 0.010 & 0.005 & 4.122 \\
\hline 0.033 & 0.010 & 0.005 & 3.557 \\
\hline 0.014 & 0.004 & 0.004 & 3.155 \\
\hline 0.014 & 0.010 & 0.002 & 3.146 \\
\hline 0.012 & 0.002 & 0.002 & 3.076 \\
\hline 0.015 & 0.002 & $\bullet$ & 2.716 \\
\hline 0.004 & • & - & 3.330 \\
\hline
\end{tabular}


Basic Table 34.B. Urban Shanxi Province (4): duration-specific fertility

\begin{tabular}{|c|c|c|c|c|}
\hline $\begin{array}{l}\text { Birth year } \\
\text { of child }\end{array}$ & $0-4$ & $5-9$ & $10-14$ & $15-19$ \\
\hline 1940 & 0.118 & - & - & - \\
\hline 1941 & 0.116 & - & - & - \\
\hline 1942 & 0.111 & - & - & - \\
\hline 1943 & 0.192 & - & - & - \\
\hline 1944 & 0.185 & - & - & - \\
\hline 1945 & 0.306 & 0.212 & - & - \\
\hline 1940 & 0.242 & 0.325 & - & • \\
\hline 1947 & 0.275 & 0.177 & • & - \\
\hline 1940 & 0.265 & 0.234 & - &. \\
\hline 1949 & 0.119 & 0.185 & - & - \\
\hline 1950 & 0.329 & 0.402 & 0.047 & - \\
\hline 1951 & 0.310 & 0.168 & 0.302 & - \\
\hline 1952 & 0.289 & 0.344 & 0.266 & - \\
\hline 1953 & 0.399 & 0.242 & 0.152 & - \\
\hline 1954 & 0.218 & 0.356 & 0.328 & - \\
\hline 1955 & 0.367 & 0.314 & 0.208 & $0.1 \in 5$ \\
\hline 1950 & 0.178 & 0.295 & 0.317 & 0.102 \\
\hline 1957 & 0.439 & 0.289 & 0.275 & 0.155 \\
\hline 1958 & 0.204 & 0.385 & 0.282 & 0.277 \\
\hline 1959 & 0.301 & 0.218 & 0.238 & 0.144 \\
\hline 1900 & 0.278 & 0.196 & 0.251 & 0.192 \\
\hline 1901 & 0.262 & 0.191 & 0.118 & 0.149 \\
\hline 1902 & 0.316 & 0.326 & 0.289 & 0.172 \\
\hline 1963 & 0.308 & 0.292 & 0.357 & 0.232 \\
\hline 1964 & 0.303 & 0.368 & 0.167 & 0.187 \\
\hline 1965 & 0.423 & 0.156 & 0.236 & 0.094 \\
\hline 1900 & 0.313 & 0.227 & 0.191 & 0.044 \\
\hline 1967 & 0.288 & 0.334 & 0.241 & 0.115 \\
\hline 1908 & 0.305 & 0.284 & 0.219 & 0.228 \\
\hline 1969 & 0.495 & 0.112 & 0.267 & 0.141 \\
\hline 1970 & 0.317 & 0.271 & 0.087 & 0.131 \\
\hline 1971 & 0.433 & 0.221 & 0.122 & 0.096 \\
\hline 1972 & 0.507 & 0.164 & 0.130 & 0.099 \\
\hline 1973 & 0.332 & 0.279 & 0.134 & 0.044 \\
\hline 1974 & 0.292 & 0.182 & 0.080 & 0.017 \\
\hline 1975 & 0.297 & 0.170 & 0.051 & 0.052 \\
\hline 1970 & 0.331 & 0.185 & 0.074 & 0.052 \\
\hline 1977 & 0.271 & 0.210 & 0.021 & 0.056 \\
\hline 1978 & 0.338 & 0.151 & 0.025 & - \\
\hline 1979 & 0.216 & 0.125 & 0.026 & 0.048 \\
\hline 1980 & 0.333 & 0.142 & 0.049 & - \\
\hline 1981 & 0.261 & 0.071 & 0.021 & - \\
\hline 1932 & 0.424 & 0.022 & - & - \\
\hline
\end{tabular}


rates, $1940-82$

\begin{tabular}{|c|c|c|c|}
\hline $20-24$ & $25-29$ & $30+$ & Total \\
\hline - & • & - & • \\
\hline • & - & • & • \\
\hline - & - & - & - \\
\hline - & • & • & • \\
\hline - & - & - & - \\
\hline - & - & - & 4.942 \\
\hline - & - & - & 6.435 \\
\hline - & - & - & $4 \cdot 225$ \\
\hline - & - & - & 5.094 \\
\hline - & - & - & 3.560 \\
\hline - & - & - & 5.426 \\
\hline - & - & - & 5.616 \\
\hline - & - & - & 6.668 \\
\hline - & - & - & 5.709 \\
\hline - & - & - & 6.973 \\
\hline - & - & - & 6.441 \\
\hline - & • & - & 5.665 \\
\hline - & - & - & 6.632 \\
\hline - & - & - & 6.362 \\
\hline - & - & - & 5.208 \\
\hline 0.047 & - & - & 5.000 \\
\hline 0.046 & - & - & 3.967 \\
\hline 0.199 & • & - & 6.789 \\
\hline 0.064 & - & - & 6.805 \\
\hline 0.205 & 0.025 & - & 6.271 \\
\hline 0.077 & 0.024 & - & 5.047 \\
\hline 0.093 & 0.023 & - & 4.461 \\
\hline 0.103 & 0.022 & 0.035 & 5.694 \\
\hline 0.033 & 0.021 & - & 5.700 \\
\hline 0.051 & • & - & 5.327 \\
\hline 0.078 & 0.038 & - & 4.609 \\
\hline 0.059 & 0.074 & - & 5.026 \\
\hline 0.043 & • & - & 4.717 \\
\hline 0.029 & - & - & 4.087 \\
\hline 0.033 & - & - & 3.045 \\
\hline 0.013 & - & - & 2.919 \\
\hline 0.014 & - & - & 3.283 \\
\hline - & - & - & 2.786 \\
\hline - & - & - & 2.571 \\
\hline - & - & • & 2.072 \\
\hline - & - & - & 2.020 \\
\hline - & - & - & 1.763 \\
\hline - & - & - & 2.230 \\
\hline
\end{tabular}


Basic Table 34.C. Rural Shanxi Province (4): duration-specific fertility

\begin{tabular}{|c|c|c|c|c|}
\hline $\begin{array}{l}\text { Birth year } \\
\text { of child }\end{array}$ & $0-4$ & $5-9$ & $10-14$ & $15-19$ \\
\hline 1940 & 0.175 & - & - & - \\
\hline 1941 & 0.182 & • & - & - \\
\hline 1942 & 0.182 & - & - & - \\
\hline 1943 & 0.170 & - & - & - \\
\hline 1944 & 0.182 & - & - & - \\
\hline $\begin{array}{l}1945 \\
1946\end{array}$ & $\begin{array}{l}0.154 \\
0.198\end{array}$ & $\begin{array}{l}0.207 \\
0.257\end{array}$ & $\bullet$ & : \\
\hline 1947 & 0.173 & 0.230 & - & • \\
\hline 1940 & 0.189 & 0.178 & - & - \\
\hline 1944 & 0.164 & 0.202 & - & - \\
\hline 1950 & 0.214 & 0.260 & 0.172 & - \\
\hline 1951 & 0.209 & 0.254 & 0.196 & . \\
\hline 1952 & 0.257 & 0.269 & 0.200 & $\bullet$ \\
\hline 1953 & 0.316 & 0.248 & 0.214 & • \\
\hline 1954 & 0.295 & 0.261 & 0.230 & . \\
\hline 1955 & 0.299 & 0.287 & 0.225 & 0.234 \\
\hline 1956 & 0.270 & 0.276 & 0.224 & 0.159 \\
\hline 1957 & 0.317 & 0.336 & 0.226 & 0.209 \\
\hline 1958 & 0.277 & 0.221 & 0.215 & 0.165 \\
\hline 1959 & 0.198 & 0.240 & 0.242 & 0.222 \\
\hline 1900 & 0.232 & 0.245 & 0.197 & 0.159 \\
\hline 1961 & 0.209 & 0.218 & 0.173 & 0.152 \\
\hline 1962 & 0.303 & 0.346 & 0.312 & 0.208 \\
\hline 1963 & 0.350 & 0.327 & 0.313 & 0.243 \\
\hline 1904 & 0.330 & 0.270 & 0.283 & 0.221 \\
\hline 1965 & 0.309 & 0.309 & 0.278 & 0.200 \\
\hline 1906 & 0.304 & 0.240 & 0.266 & 0.213 \\
\hline 1967 & 0.296 & 0.305 & 0.317 & 0.202 \\
\hline 1908 & 0.319 & 0.334 & 0.300 & 0.276 \\
\hline 1909 & 0.302 & 0.319 & 0.272 & 0.188 \\
\hline 1970 & 0.348 & 0.323 & 0.259 & 0.186 \\
\hline 1971 & 0.342 & 0.312 & 0.259 & 0.250 \\
\hline 1972 & 0.328 & 0.286 & 0.247 & 0.166 \\
\hline 1973 & 0.324 & $0.2 \in 5$ & 0.243 & 0.151 \\
\hline 1974 & 0.337 & 0.282 & 0.215 & 0.108 \\
\hline $197 \%$ & 0.283 & 0.248 & 0.126 & 0.088 \\
\hline 1976 & 0.303 & 0.222 & 0.143 & 0.097 \\
\hline 1977 & 0.280 & 0.210 & 0.101 & 0.083 \\
\hline 1978 & 0.284 & 0.164 & 0.104 & 0.064 \\
\hline 1979 & 0.312 & 0.166 & 0.104 & 0.041 \\
\hline 1980 & 0.315 & 0.193 & 0.077 & 0.022 \\
\hline 1981 & 0.324 & 0.143 & 0.053 & 0.027 \\
\hline 1982 & 0.351 & 0.191 & 0.105 & 0.041 \\
\hline
\end{tabular}


rates, $1940-82$

\begin{tabular}{|c|c|c|c|c|c|}
\hline & $20-24$ & $25-29$ & & $30+$ & Total \\
\hline & • & - & & - & $\bullet$ \\
\hline & • & • & & - & • \\
\hline & - & - & & $\bullet$ & - \\
\hline & - & - & & - & • \\
\hline & - & • & & - & - \\
\hline & - & - & & - & 3.941 \\
\hline & - & - & & - & 4.920 \\
\hline & - & - & & $\bullet$ & 4.387 \\
\hline & - & - & & - & 3.666 \\
\hline & - & - & & - & 4.822 \\
\hline & - & - & & - & 4.617 \\
\hline & - & - & & - & $5.04 C$ \\
\hline & - & - & & • & 5.194 \\
\hline & - & & & - & 5.366 \\
\hline & - & - & & - & 5.521 \\
\hline & - & - & & - & 6.001 \\
\hline & - & - & & - & 5.355 \\
\hline & - & • & & - & 6.235 \\
\hline ' & - & - & & - & 5.014 \\
\hline & - & - & & • & 5.254 \\
\hline & 0.120 & - & . & - & 4.938 \\
\hline & 0.092 & - & & - & 4.371 \\
\hline & 0.158 & - & . & • & 6.871 \\
\hline & 0.162 & - & & - & 7.225 \\
\hline & 0.126 & 0.090 & & - & 6.597 \\
\hline & 0.170 & 0.061 & & 0.005 & 6.662 \\
\hline - & 0.144 & 0.039 & & 0.013 & 0.093 \\
\hline & 0.111 & 0.037 & & 0.008 & 6.531 \\
\hline & 0.151 & 0.052 & & 0.007 & 7.193 \\
\hline & 0.142 & 0.059 & & 0.013 & 6.474 \\
\hline & 0.162 & 0.058 & & 0.020 & 6.810 \\
\hline & 0.131 & 0.048 & . & 0.008 & 6.754 \\
\hline - & 0.106 & 0.051 & & 0.003 & 5.931 \\
\hline & 0.114 & 0.031 & & 0.005 & 5.663 \\
\hline & 0.078 & 0.024 & & - & 5.219 \\
\hline & 0.074 & 0.045 & & 0.008 & 4.356 \\
\hline & 0.063 & 0.012 & & 0.005 & 4.226 \\
\hline & 0.038 & 0.012 & & 0.005 & 3.654 \\
\hline & 0.016 & 0.005 & & 0.005 & 3.206 \\
\hline & 0.016 & 0.0 .13 &. & 0.002 & 3.272 \\
\hline & 0.013 & 0.003 & & 0.002 & 3.123 \\
\hline & 0.017 & 0.003 & & - & 2.832 \\
\hline & 0.004 & $\bullet$ & & - & 3.463 \\
\hline
\end{tabular}


Basic Table 35.A. Total Inner Mongolia Province (5): duration-specific

\begin{tabular}{|c|c|c|c|c|}
\hline $\begin{array}{l}\text { Birth year } \\
\text { of child }\end{array}$ & $0-4$ & $5-9$ & $10-14$ & $15-19$ \\
\hline 1940 & 0.252 & - & $\bullet$ & - \\
\hline 1941 & 0.271 & - & - & - \\
\hline 1942 & 0.177 & - & - & - \\
\hline 1943 & 0.231 & - & • & • \\
\hline 1944 & 0.179 & - & • & $\bullet$ \\
\hline 1945 & 0.259 & 0.262 & $\bullet$ & - \\
\hline 1940 & 0.228 & 0.262 & - & • \\
\hline 1947 & 0.242 & 0.326 & - & - \\
\hline 1940 & 0.269 & 0.255 & - & - \\
\hline 1949 & 0.243 & 0.251 & - & - \\
\hline 1950 & 0.270 & 0.293 & 0.227 & - \\
\hline 1951 & 0.292 & 0.262 & 0.247 & - \\
\hline 1952 & 0.294 & 0.312 & 0.307 & - \\
\hline 1953 & 0.313 & 0.305 & 0.294 & - \\
\hline 1954 & 0.337 & 0.271 & 0.314 & - \\
\hline 1955 & 0.336 & 0.369 & 0.288 & 0.237 \\
\hline 1950 & 0.406 & 0.322 & 0.266 & 0.242 \\
\hline 1957 & 0.296 & 0.355 & 0.364 & 0.297 \\
\hline $\begin{array}{l}1958 \\
1959\end{array}$ & $\begin{array}{l}0.340 \\
0.309\end{array}$ & $\begin{array}{l}0.308 \\
0.289\end{array}$ & $\begin{array}{l}0.272 \\
0.265\end{array}$ & $\begin{array}{l}0.275 \\
0.164\end{array}$ \\
\hline 1960 & 0.322 & 0.326 & 0.286 & 0.283 \\
\hline 1961 & 0.205 & 0.220 & 0.201 & 0.125 \\
\hline 1902 & 0.363 & 0.360 & 0.291 & 0.227 \\
\hline 1963 & 0.387 & 0.371 & 0.340 & 0.332 \\
\hline 1964 & 0.344 & 0.344 & 0.308 & 0.249 \\
\hline 1965 & 0.340 & 0.264 & 0.306 & 0.248 \\
\hline 1966 & 0.324 & 0.313 & 0.215 & 0.258 \\
\hline 1967 & 0.333 & 0.252 & 0.232 & 0.170 \\
\hline 1968 & 0.402 & 0.365 & 0.265 & 0.248 \\
\hline 1969 & 0.347 & 0.275 & 0.256 & 0.197 \\
\hline 1970 & 0.357 & 0.336 & 0.250 & 0.213 \\
\hline 1971 & 0.345 & 0.281 & 0.221 & 0.188 \\
\hline 1972 & 0.357 & 0.266 & 0.193 & 0.173 \\
\hline 1973 & 0.329 & 0.233 & 0.157 & 0.153 \\
\hline 1974 & 0.421 & 0.260 & 0.125 & 0.104 \\
\hline 1975 & 0.331 & 0.236 & 0.118 & 0.075 \\
\hline 1976 & 0.370 & 0.201 & 0.087 & 0.038 \\
\hline 1977 & 0.337 & 0.188 & 0.095 & 0.046 \\
\hline 1978 & 0.347 & 0.206 & 0.077 & 0.042 \\
\hline 1979 & 0.350 & 0.193 & 0.066 & 0.035 \\
\hline 1980 & 0.341 & 0.210 & 0.059 & 0.024 \\
\hline 1981 & 0.353 & 0.159 & 0.061 & 0.025 \\
\hline 1982 & 0.400 & 0.199 & 0.098 & 0.046 \\
\hline
\end{tabular}


fertility rates, $1940-82$

\begin{tabular}{|c|c|c|c|}
\hline $20-24$ & $25-29$ & $30+$ & Total \\
\hline$\bullet$ & • & • & - \\
\hline$\bullet$ & - & $\bullet$ & - \\
\hline - & - & $\bullet$ & $\bullet$ \\
\hline - & - & - & • \\
\hline - & - & - & $\bullet$ \\
\hline - & - & - & 5.309 \\
\hline - & - & - & 5.132 \\
\hline - & - & - & $6 \cdot 213$ \\
\hline - & - & • & 5.303 \\
\hline • & - & - & 5.094 \\
\hline - & - & - & 5.575 \\
\hline - & - & - & 5.698 \\
\hline$\bullet$ & - & - & 6.653 \\
\hline$\bullet$ & $\bullet$ & - & 6.531 \\
\hline$\bullet$ & - & - & 6.574 \\
\hline - & - & - & 7.063 \\
\hline - & - & - & 7.085 \\
\hline - & • & - & 7.641 \\
\hline - & - & - & 6.893 \\
\hline - & - & - & 5.835 \\
\hline 0.153 & - & - & 7.078 \\
\hline 0.109 & •. & - & 4.507 \\
\hline 0.225 & - & - & 7.497 \\
\hline 0.173 & $\bullet$ & • & 8.315 \\
\hline 0.145 & 0.115 & 0.022 & 7.634 \\
\hline 0.206 & 0.089 & 0.028 & 7.404 \\
\hline 0.129 & 0.059 & 0.015 & 6.56 .3 \\
\hline 0.152 & 0.043 & 0.014 & 5.98 .3 \\
\hline 0.168 & 0.039 & - & 7.437 \\
\hline 0.173 & 0.058 & 0.011 & 6.591 \\
\hline 0.146 & 0.053 & 0.005 & 6.799 \\
\hline 0.129 & 0.030 & 0.010 & 6.019 \\
\hline 0.099 & 0.037 & 0.005 & 5.643 \\
\hline 0.084 & 0.027 & - & 4.908 \\
\hline 0.076 & 0.027 & 0.005 & 5.097 \\
\hline 0.051 & 0.033 & 0.010 & 4.269 \\
\hline 0.042 & 0.016 & - & 3.764 \\
\hline 0.026 & 0.016 & - & 3.542 \\
\hline 0.009 & 0.005 & 0.003 & 3.451 \\
\hline 0.026 & 0.013 & - & 3.416 \\
\hline 0.009 & 0.003 & - & 3.226 \\
\hline 0.014 & • & 0.005 & 3.093 \\
\hline 0.004 & - & - & 3.737 \\
\hline
\end{tabular}


Basic Table 35.B. Urban Inner Mongolia Province (5): duration-specific

\begin{tabular}{|c|c|c|c|c|}
\hline $\begin{array}{l}\text { Birth year } \\
\text { of child }\end{array}$ & $0-4$ & $5-9$ & $10-14$ & . 15-19 \\
\hline 1940 & 0.199 & - & - & - \\
\hline 1941 & 0.223 & - & - & - \\
\hline 1942 & 0.211 & - & - & - \\
\hline 1943 & 0.218 & - & - & - \\
\hline 1944 & 0.140 & - & - & - \\
\hline 1945 & 0.276 & 0.199 & - & - \\
\hline 1946 & 0.173 & 0.143 & - & - \\
\hline 1947 & 0.179 & 0.211 & - & - \\
\hline 1948 & 0.255 & 0.152 & - & - \\
\hline 1949 & 0.234 & 0.260 & - & - \\
\hline 1950 & 0.326 & 0.257 & 0.057 & - \\
\hline 1951 & 0.357 & 0.191 & 0.257 & - \\
\hline 1952 & 0.307 & 0.269 & 0.342 & . \\
\hline 1953 & 0.319 & 0.282 & 0.152 & - \\
\hline 1954 & 0.310 & 0.246 & 0.360 & - \\
\hline 1955 & 0.350 & 0.348 & 0.158 & 0.170 \\
\hline 1950 & 0.382 & 0.258 & 0.225 & 0.229 \\
\hline 1957 & 0.302 & 0.327 & 0.284 & 0.263 \\
\hline 1958 & 0.342 & 0.328 & 0.228 & 0.261 \\
\hline 1959 & 0.342 & 0.263 & 0.172 & 0.080 \\
\hline 1960 & 0.357 & 0.282 & 0.293 & 0.237 \\
\hline 1901 & 0.189 & 0.174 & 0.169 & 0.069 \\
\hline 1962 & 0.339 & 0.333 & 0.228 & 0.120 \\
\hline 1963 & 0.366 & 0.335 & 0.290 & 0.269 \\
\hline 1964 & 0.295 & 0.296 & 0.178 & 0.074 \\
\hline 1965 & 0.237 & 0.172 & 0.137 & 0.141 \\
\hline $1900^{\circ}$ & 0.313 & 0.230 & 0.091 & 0.139 \\
\hline 1967 & 0.300 & 0.217 & 0.136 & 0.075 \\
\hline 1968 & 0.367 & 0.324 & 0.157 & 0.116 \\
\hline 1969 & 0.341 & 0.192 & 0.138 & 0.122 \\
\hline 1970 & 0.319 & 0.219 & 0.100 & 0.068 \\
\hline 1971 & 0.251 & 0.170 & 0.118 & 0.083 \\
\hline 1972 & 0.381 & 0.215 & 0.095 & 0.060 \\
\hline 1973 & 0.272 & $0.1+7$ & 0.103 & 0.050 \\
\hline 1974 & 0.335 & 0.091 & 0.031 & 0.013 \\
\hline 1975 & 0.377 & 0.133 & 0.028 & 0.026 \\
\hline 1976 & 0.259 & 0.090 & 0.039 & 0.006 \\
\hline 1977 & 0.243 & 0.072 & 0.016 & 0.005 \\
\hline 1978 & 0.400 & 0.124 & 0.018 & 0.009 \\
\hline 1979 & 0.194 & 0.097 & - & 0.004 \\
\hline 1980 & 0.232 & 0.113 & 0.024 & $\bullet$ \\
\hline 1931 & 0.272 & 0.049 & 0.006 & - \\
\hline 1982 & 0.423 & • & • & 0.011 \\
\hline
\end{tabular}


fertility rates, $1940-82$

\begin{tabular}{|c|c|c|c|}
\hline $20-24$ & $25-29$ & $30+$ & Total \\
\hline$\bullet$ & - & - & - \\
\hline - & - & - & - \\
\hline - & $\bullet$ & • & - \\
\hline - & - & $\bullet$ & $\bullet$ \\
\hline - & - & - & - \\
\hline - & - & - & 4.581 \\
\hline - & - & - & 3.167 \\
\hline - & - & - & 4.287 \\
\hline - & - & • & 3.729 \\
\hline$\bullet$ & - & - & 5.357 \\
\hline - & - & - & $4 \cdot 278$ \\
\hline - & - & - & 5.658 \\
\hline$\bullet$ & - & - & 6.811 \\
\hline$\bullet$ & - & - & 5.300 \\
\hline - & $\bullet$ & - & 6.794 \\
\hline - & - & - & 5.916 \\
\hline - & - & - & 6.327 \\
\hline - & - & - & 6.927 \\
\hline$\bullet$ & • & - & 6.779 \\
\hline • & - & - & 4.877 \\
\hline 0.142 & - & - & 6.820 \\
\hline 0.172 & - & • & 4.042 \\
\hline 0.079 & - & - & 5.684 \\
\hline 0.174 & - & • & 7.466 \\
\hline 0.080 & - & - & 4.620 \\
\hline 0.099 & 0.057 & - & 4.213 \\
\hline - & - & - & 3.868 \\
\hline 0.045 & 0.020 & - & 4.047 \\
\hline 0.040 & 0.022 & - & 5.129 \\
\hline 0.037 & • & - & 4.156 \\
\hline 0.022 & $\bullet$ & - & 3.681 \\
\hline 0.020 & - & - & 3.233 \\
\hline 0.020 & - & - & .3 .857 \\
\hline 0.010 & - & - & 2.909 \\
\hline - & - & 0.020 & 2.453 \\
\hline 0.017 & • & - & 2.899 \\
\hline 0.008 & - & - & 2.013 \\
\hline$\bullet$ & - & • & 1.680 \\
\hline - & - & - & 3.056 \\
\hline - & - & - & 1.479 \\
\hline - & - & - & 2.094 \\
\hline - & - & - & 1.640 \\
\hline - & - & - & 2.170 \\
\hline
\end{tabular}


Basic Table 35.C. Rural Inner Mongolia Province (5): duration-specific

\begin{tabular}{|c|c|c|c|c|}
\hline $\begin{array}{l}\text { Birth year } \\
\text { of child }\end{array}$ & $0-4$ & $5-9$ & $10-14$ & 15-19 \\
\hline 1940 & 0.263 & - & - & - \\
\hline 1941 & 0.280 & - & - & - \\
\hline 1942 & 0.170. & - & - & - \\
\hline 1943 & 0.235 & - & - & - \\
\hline 1944 & 0.191 & - & - & - \\
\hline 1945 & 0.253 & 0.275 & - & - \\
\hline 1946 & 0.246 & 0.236 & - & - \\
\hline 1947 & 0.262 & 0.352 & - & - \\
\hline 1948 & 0.273 & 0.284 & - & • \\
\hline 1949 & 0.246 & $0.24 \mathrm{e}$ & - & - \\
\hline 1950 & 0.251 & 0.304 & 0.263 & • \\
\hline 1951 & 0.268 & 0.286 & 0.245 & - \\
\hline 1952 & 0.289 & 0.327 & 0.299 & - \\
\hline 1953 & 0.311 & 0.313 & 0.334 & - \\
\hline 1954 & 0.347 & 0.279 & 0.299 & - \\
\hline 1955 & 0.331 & $0.37 \epsilon$ & 0.330 & 0.251 \\
\hline 1950 & 0.416 & 0.346 & 0.280 & 0.245 \\
\hline 1957 & 0.293 & 0.365 & 0.391 & 0.305 \\
\hline 1958 & 0.340 & 0.300 & 0.286 & 0.278 \\
\hline $195 y$ & 0.290 & 0.300 & 0.295 & 0.191 \\
\hline 1900 & 0.302 & 0.345 & 0.284 & .0 .298 \\
\hline 1961 & 0.215 & 0.240 & 0.213 & 0.143 \\
\hline 1962 & 0.380 & 0.372 & 0.316 & 0.262 \\
\hline 1963 & 0.402 & 0.389 & 0.360 & 0.353 \\
\hline 1964 & 0.381 & 0.371 & 0.358 & 0.307 \\
\hline 1965 & 0.413 & 0.317 & 0.378 & 0.284 \\
\hline 1960 & 0.332 & 0.367 & 0.268 & 0.301 \\
\hline 1907 & 0.350 & 0.278 & 0.275 & 0.206 \\
\hline 1908 & 0.422 & 0.395 & 0.319 & 0.300 \\
\hline 1909 & 0.350 & 0.337 & 0.323 & 0.226 \\
\hline 1970 & 0.373 & 0.425 & 0.332 & 0.275 \\
\hline 1971 & 0.378 & 0.357 & 0.289 & 0.233 \\
\hline 1972 & 0.350 & 0.298 & 0.262 & 0.224 \\
\hline 1973 & 0.343 & 0.281 & 0.196 & 0.203 \\
\hline 1974 & 0.438 & 0.342 & 0.195 & 0.156 \\
\hline 1973 & 0.323 & 0.279 & 0.186 & 0.103 \\
\hline 1976 & 0.389 & 0.239 & 0.121 & 0.059 \\
\hline 1977 & 0.356 & 0.222 & 0.146 & 0.076 \\
\hline 1978 & 0.321 & 0.227 & 0.110 & 0.065 \\
\hline 1979 & 0.390 & 0.212 & 0.097 & 0.057 \\
\hline 1980 & 0.359 & 0.227 & 0.073 & 0.042 \\
\hline 1981 & 0.384 & 0.179 & 0.081 & 0.043 \\
\hline 1982 & 0.390 & 0.241 & 0.126 & 0.068 \\
\hline
\end{tabular}


fertility rates, $1940-82$

\begin{tabular}{|c|c|c|c|}
\hline $20-24$ & $25-29$ & $30+$ & Total \\
\hline - & - & - & - \\
\hline - & $\bullet$ & $\bullet$ & - \\
\hline - & - & - & - \\
\hline - & - & - & - \\
\hline - & - & - & - \\
\hline - & - & • & 5.479 \\
\hline - & - & - & 5.606 \\
\hline - & - & - & 6.693 \\
\hline - & - & - & 5.715 \\
\hline - & - & - & 5.024 \\
\hline - & - & - & 5.932 \\
\hline - & - & - & 5.709 \\
\hline - & - & - & 6.607 \\
\hline - & - & - & 6.903 \\
\hline • & • & - & 6.507 \\
\hline - & - & - & $7 \cdot 428$ \\
\hline - & • & - & $7 \cdot 332$ \\
\hline - & - & - & 7.881 \\
\hline - & - & - & 6.932 \\
\hline - & - & - & 6.18 .3 \\
\hline 0.156 & • & - & 7.175 \\
\hline 0.095 & - & - & 4.689 \\
\hline 0.258 & - & - & $8 \cdot 24.3$ \\
\hline 0.173 & - & - & 8.678 \\
\hline 0.165 & 0.137 & 0.025 & 8.720 \\
\hline 0.241 & 0.096 & 0.032 & 8.830 \\
\hline 0.171 & 0.072 & 0.017 & 7.647 \\
\hline 0.138 & 0.047 & 0.016 & 6.800 \\
\hline 0.210 & 0.043 & - & 8.446 \\
\hline 0.213 & 0.076 & 0.013 & 7.721 \\
\hline 0.18$\}$ & 0.070 & 0.006 & 9.344 \\
\hline 0.169 & 0.040 & 0.012 & 7.392 \\
\hline 0.129 & 0.050 & 0.006 & 6.595 \\
\hline 0.112 & 0.036 & • & 5.859 \\
\hline 0.106 & $0.03 \epsilon$ & • & 0.300 \\
\hline 0.060 & 0.044 & 0.013 & 5.075 \\
\hline 0.056 & 0.022 & - & 4.437 \\
\hline 0.038 & 0.023 & - & 4.306 \\
\hline 0.014 & 0.007 & 0.004 & 3.747 \\
\hline 0.041 & 0.012 & • & 4.077 \\
\hline 0.015 & 0.004 & - & 3.596 \\
\hline 0.023 & - & 0.007 & $3.5 E \theta$ \\
\hline 0.008 & - & • & 4.104 \\
\hline
\end{tabular}


Basic Table 36.A. Total Liaoning Province (6): duration-specific fertility

\begin{tabular}{|c|c|c|c|c|}
\hline $\begin{array}{l}\text { Birth year } \\
\text { of child }\end{array}$ & $0-4$ & $5-9$ & $10-14$ & $15-19$ \\
\hline 1940 & 0.267 & - & - & - \\
\hline 1941 & 0.207 & - & - & - \\
\hline 1942 & 0.204 & - & - & - \\
\hline 1943 & 0.235 & - & - & - \\
\hline 1944 & 0.233 & - & - & - \\
\hline 1945 & 0.234 & 0.255 & - & - \\
\hline 1940 & 0.247 & 0.307 & - & - \\
\hline 1947 & 0.315 & 0.276 & - & • \\
\hline 1948 & 0.232 & 0.244 & - & - \\
\hline 1949 & 0.314 & 0.340 & - & - \\
\hline 1950 & 0.286 & 0.254 & 0.284 & - \\
\hline 1951 & 0.293 & 0.322 & 0.251 & - \\
\hline 1952 & 0.325 & 0.357 & 0.258 & • \\
\hline 1953 & 0.302 & 0.339 & 0.303 & - \\
\hline 1954 & 0.340 & 0.361 & 0.320 & - \\
\hline 1955 & 0.408 & 0.358 & 0.337 & 0.293 \\
\hline 1950 & 0.321 & 0.311 & 0.258 & 0.222 \\
\hline 1957 & 0.416 & 0.317 & 0.357 & 0.315 \\
\hline 1958 & 0.334 & 0.305 & 0.274 & 0.220 \\
\hline 1959 & 0.275 & 0.284 & 0.240 & 0.176 \\
\hline 1900 & 0.319 & $0.30 \mathrm{e}$ & 0.287 & 0.211 \\
\hline 1961 & 0.170 & 0.133 & 0.131 & 0.110 \\
\hline 1962 & 0.323 & 0.329 & 0.233 & 0.211 \\
\hline 1903 & 0.474 & 0.401 & 0.351 & 0.336 \\
\hline 1964 & 0.414 & 0.305 & 0.243 & 0.238 \\
\hline 1965 & 0.341 & 0.318 & 0.263 & 0.175 \\
\hline 1906 & 0.411 & 0.295 & 0.224 & 0.190 \\
\hline 1967 & 0.317 & 0.278 & 0.189 & 0.133 \\
\hline 1968 & 0.386 & 0.348 & 0.221 & 0.158 \\
\hline 1909 & 0.362 & 0.277 & 0.199 & 0.118 \\
\hline 1970 & 0.406 & 0.229 & 0.198 & 0.098 \\
\hline 1971 & 0.362 & 0.204 & 0.158 & 0.110 \\
\hline 1972 & 0.321 & 0.241 & 0.163 & 0.077 \\
\hline 1973 & 0.380 & 0.220 & 0.147 & 0.080 \\
\hline 1974 & 0.379 & 0.208 & 0.108 & 0.037 \\
\hline 1975 & 0.321 & 0.180 & 0.065 & 0.034 \\
\hline 1970 & 0.335 & $0.1+3$ & 0.056 & 0.024 \\
\hline 1977 & 0.315 & 0.134 & 0.044 & 0.031 \\
\hline 1978 & 0.404 & 0.142 & 0.047 & 0.017 \\
\hline 1979 & 0.362 & 0.146 & 0.039 & 0.014 \\
\hline 1980 & 0.297 & 0.110 & 0.017 & 0.004 \\
\hline 1981 & 0.312 & 0.065 & 0.022 & 0.003 \\
\hline 1985 & 0.327 & 0.051 & 0.016 & 0.003 \\
\hline
\end{tabular}


rates, $1940-82$

\begin{tabular}{|c|c|c|c|}
\hline $20-24$ & $25-29$ & $30+$ & Total \\
\hline - & • & • & • \\
\hline • & • & $\bullet$ & $\bullet$ \\
\hline • & • & - & • \\
\hline - & - & - & • \\
\hline$\bullet$ & - & - & - \\
\hline • & - & - & 5.156 \\
\hline - & - & - & 5.025 \\
\hline - & - & - & 5.893 \\
\hline - & - & - & 4.988 \\
\hline - & - & - & 6.872 \\
\hline • & - & - & 5.953 \\
\hline - & • & - & 6.253 \\
\hline - & - & - & 6.763 \\
\hline - & - & - & 6.379 \\
\hline - & - & - & 7.395 \\
\hline$\bullet$ & - & - & 8.067 \\
\hline - & - & - & 6.427 \\
\hline - & • & - & 8.116 \\
\hline - & - & - & 6.524 \\
\hline - & - & - & 5.636 \\
\hline 0.141 & - & - & 6.597 \\
\hline 0.076 & - & - & 3.231 \\
\hline 0.117 & - & - & 6.336 \\
\hline 0.214 & - & - & 9.236 \\
\hline 0.176 & 0.073 & - & 7.240 \\
\hline 0.146 & 0.038 & 0.004 & 6.429 \\
\hline 0.117 & 0.040 & 0.011 & $6.43 ?$ \\
\hline 0.099 & 0.038 & 0.006 & 5.301 \\
\hline 0.121 & 0.030 & 0.003 & 6.332 \\
\hline 0.100 & 0.027 & 0.003 & 5.42 .5 \\
\hline 0.055 & 0.022 & - & 5.039 \\
\hline 0.051 & 0.012 & 0.002 & $4.80 \mathrm{~J}$ \\
\hline 0.032 & 0.011 & - & 4.219 \\
\hline 0.033 & 0.016 & - & 4.380 \\
\hline 0.024 & 0.013 & 0.002 & 3.859 \\
\hline 0.012 & 0.006 & 0.003 & 3.109 \\
\hline 0.004 & 0.008 & $\bullet$ & 2.844 \\
\hline 0.005 & 0.001 & - & 2.653 \\
\hline 0.014 & • & 0.001 & 3.122 \\
\hline 0.007 & 0.001 & • & 2.943 \\
\hline$\bullet$ & • & - & 2.135 \\
\hline 0.007 & - & 0.001 & 2.056 \\
\hline 0.003 & - & $\bullet$ & 1.592 \\
\hline
\end{tabular}


Basic Table 36.B. Urban Liaoning Province (6): duration-specific

\begin{tabular}{|c|c|c|c|c|}
\hline $\begin{array}{l}\text { Birth year } \\
\text { of child }\end{array}$ & $0-4$ & $5-9$ & $10-14$ & $15-19$ \\
\hline 1940 & $0.2 \in 4$ & - & - & - \\
\hline 1941 & 0.190 & - & - & - \\
\hline $\begin{array}{l}1942 \\
1943\end{array}$ & 0.221 & $\bullet$ & $\bullet$ & $\bullet$ \\
\hline 1944 & 0.158 & . & - & . \\
\hline 1945 & 0.259 & 0.224 & - & - \\
\hline 1940 & 0.274 & 0.301 & - & - \\
\hline 1947 & 0.278 & 0.260 & - & - \\
\hline 1948 & 0.239 & 0.210 & - & - \\
\hline 1949 & 0.300 & 0.329 & - & . \\
\hline 1950 & 0.286 & 0.259 & 0.275 & - \\
\hline 1951 & 0.332 & 0.246 & 0.232 & - \\
\hline 1952 & 0.319 & 0.315 & 0.264 & . \\
\hline $\begin{array}{l}1953 \\
1954\end{array}$ & $\begin{array}{l}0.349 \\
0.329\end{array}$ & $\begin{array}{l}0.335 \\
0.330\end{array}$ & $\begin{array}{l}0.322 \\
0.299\end{array}$ & • \\
\hline 5 & 0.417 & 0.366 & 0.311 & 0.275 \\
\hline 1956 & 0.352 & 0.294 & 0.259 & 0.200 \\
\hline 1957 & 0.402 & 0.325 & 0.308 & 0.245 \\
\hline 1950 & 0.362 & 0.316 & 0.243 & 0.197 \\
\hline 1959 & 0.274 & 0.269 & 0.198 & 0.173 \\
\hline 1960 & 0.352 & 0.261 & 0.242 & 0.158 \\
\hline 1901 & 0.210 & 0.132 & 0.116 & 0.075 \\
\hline 1962 & 0.328 & 0.285 & 0.198 & 0.146 \\
\hline 1963 & 0.516 & 0.362 & 0.363 & 0.250 \\
\hline 1904 & 0.476 & 0.229 & 0.192 & 0.135 \\
\hline 1965 & 0.309 & 0.281 & 0.191 & 0.115 \\
\hline 1906 & 0.369 & 0.182 & 0.076 & 0.056 \\
\hline 1907 & 0.312 & 0.154 & 0.096 & 0.046 \\
\hline 1908 & 0.396 & 0.303 & 0.159 & 0.077 \\
\hline 1969 & 0.296 & 0.206 & 0.097 & 0.053 \\
\hline 1970 & 0.417 & 0.164 & 0.078 & 0.026 \\
\hline 1971 & 0.356 & 0.172 & 0.085 & 0.041 \\
\hline 1972 & 0.292 & 0.156 & 0.093 & 0.036 \\
\hline 1973 & 0.372 & 0.124 & 0.070 & 0.016 \\
\hline 1974 & 0.342 & 0.144 & 0.019 & 0.017 \\
\hline 1975 & 0.236 & 0.087 & 0.027 & 0.008 \\
\hline 1976 & 0.267 & 0.082 & 0.016 & 0.004 \\
\hline 1977 & 0.313 & 0.077 & 0.038 & 0.004 \\
\hline 1978 & 0.441 & 0.092 & 0.012 & 0.008 \\
\hline 1979 & 0.269 & 0.005 & 0.015 & 0.004 \\
\hline 1980 & 0.232 & 0.035 & . & - \\
\hline 1981 & 0.290 & 0.004 & 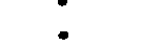 & 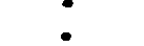 \\
\hline 1982 & 0.325 & - & 0.007 & - \\
\hline
\end{tabular}


fertility rates, $1940-82$

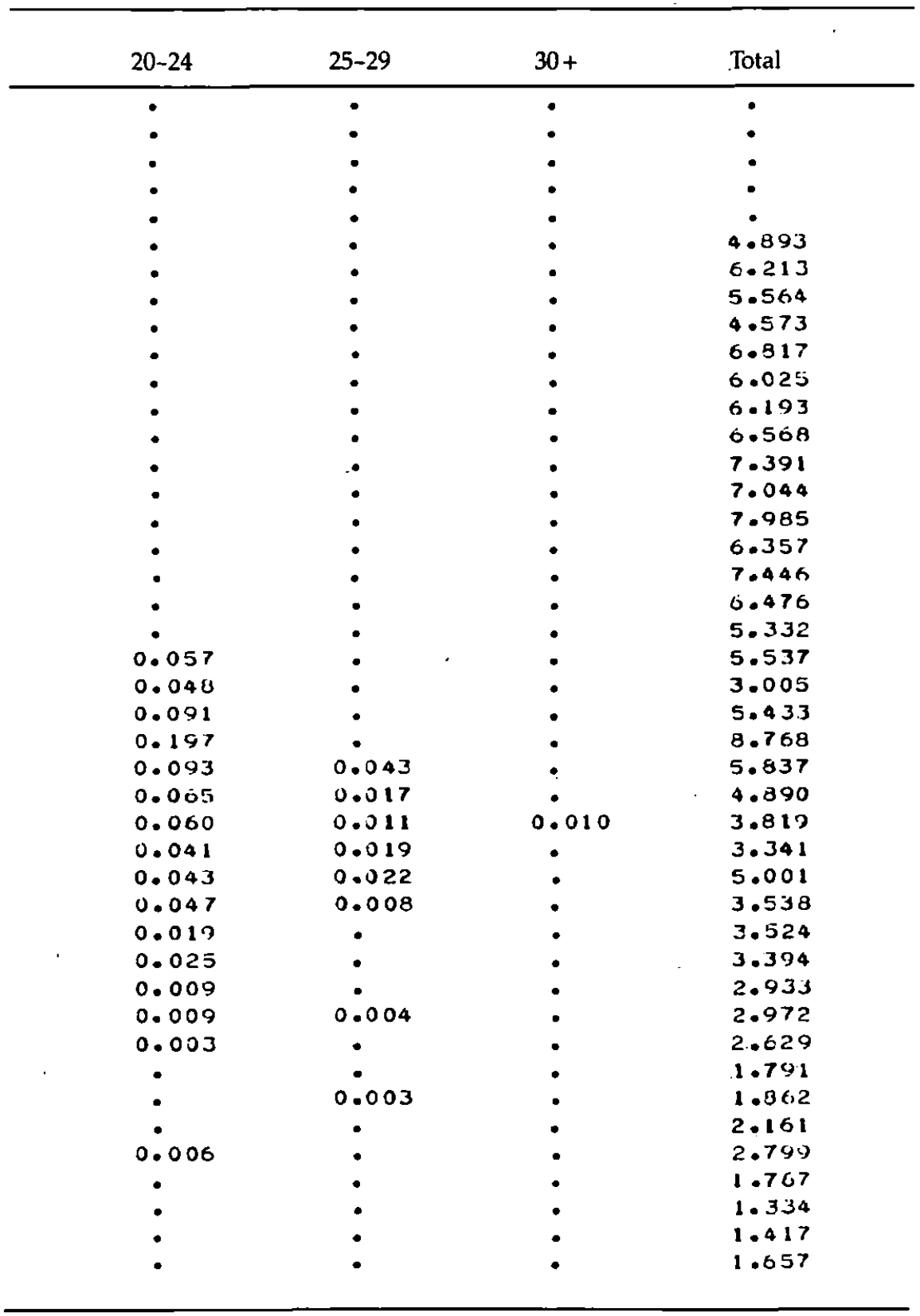


Basic Table 36.C. Rural Liaoning Province (6): duration-specific fertility

\begin{tabular}{|c|c|c|c|c|}
\hline $\begin{array}{l}\text { Birth year } \\
\text { of child }\end{array}$ & $0-4$ & $5-9$ & $10-14$ & $15-19$ \\
\hline 1940 & 0.270 & - & - & - \\
\hline 1941 & 0.219 & - & - & - \\
\hline $194 \mathrm{C}$ & 0.191 & - & - & - \\
\hline 1943 & 0.250 & - & - & - \\
\hline 1944 & 0.258 & - & - & - \\
\hline 1945 & 0.217 & 0.278 & - & - \\
\hline 1946 & 0.228 & 0.311 & - & - \\
\hline 1947 & 0.340 & 0.289 & - & - \\
\hline 1948 & 0.228 & 0.270 & - & - \\
\hline 194 & 0.319 & 0.348 & - & - \\
\hline 1950 & 0.286 & 0.251 & $0.2 \div 0$ & - \\
\hline 1951 & 0.264 & 0.339 & 0.265 & - \\
\hline 1952 & 0.329 & 0.380 & 0.254 & - \\
\hline $1>53$ & 0.262 & 0.342 & 0.290 & - \\
\hline 1554 & 0.350 & 0.381 & 0.335 & - \\
\hline 1955 & 0.398 & 0.352 & 0.355 & 0.306 \\
\hline 1950 & 0.312 & 0.323 & 0.257 & 0.234 \\
\hline 1957 & 0.428 & 0.310 & 0.391 & 0.365 \\
\hline 1956 & 0.313 & 0.295 & 0.294 & 0.237 \\
\hline 1959 & 0.270 & 0.298 & 0.267 & 0.178 \\
\hline 1900 & 0.299 & 0.353 & 0.318 & 0.248 \\
\hline 1961 & 0.147 & 0.134 & 0.141 & 0.135 \\
\hline 1902 & 0.328 & 0.365 & 0.262 & 0.255 \\
\hline 1903 & 0.453 & 0.431 & 0.335 & 0.394 \\
\hline 1904 & 0.384 & 0.359 & 0.2292 & 0.303 \\
\hline 1965 & 0.356 & 0.340 & 0.333 & 0.217 \\
\hline 1966 & 0.430 & 0.359 & 0.353 & 0.287 \\
\hline 1907 & 0.319 & 0.343 & 0.267 & 0.202 \\
\hline 1908 & 0.381 & 0.370 & 0.268 & 0.228 \\
\hline 1905 & 0.390 & 0.312 & 0.271 & 0.181 \\
\hline 1970 & 0.401 & 0.259 & 0.272 & 0.168 \\
\hline 1971 & 0.365 & 0.307 & 0.201 & 0.170 \\
\hline 1472 & 0.332 & 0.280 & 0.159 & 0.111 \\
\hline 1973 & 0.384 & 0.264 & 0.184 & 0.130 \\
\hline 1974 & 0.391 & 0.230 & 0.151 & 1 ن.0.0. \\
\hline 1975 & 0.349 & 0.221 & 0.083 & 0.051 \\
\hline 1976 & 0.360 & 0.168 & 0.074 & 0.035 \\
\hline 1977 & 0.316 & 0.157 & 0.047 & 0.045 \\
\hline 1973 & 0.385 & 0.160 & $0.0 \in 3$ & 0.021 \\
\hline 1979 & 0.416 & 0.173 & 0.049 & 0.018 \\
\hline 1980 & 0.338 & 0.135 & 0.024 & 0.000 \\
\hline 1981 & 0.336 & 0.088 & 0.032 & 0.004 \\
\hline 1982 & 0.328 & 0.075 & 0.019 & 0.004 \\
\hline
\end{tabular}


rates, $1940-82$

\begin{tabular}{|c|c|c|c|}
\hline $20-24$ & $25-29$ & $30+$ & Total \\
\hline$\bullet$ & $\bullet$ & $\bullet$ & $\bullet$ \\
\hline - & - & - & - \\
\hline$\bullet$ & - & $\bullet$ & - \\
\hline - & $\bullet$ & - & - \\
\hline - & - & - & - \\
\hline - & - & - & 5.335 \\
\hline - & - & $\bullet$ & 5.898 \\
\hline - & - & - & 6.112 \\
\hline$\bullet$ & - & - & $5 \cdot 265$ \\
\hline - & - & - & 6.908 \\
\hline - & - & - & 5.904 \\
\hline - & - & - & 6.295 \\
\hline - & - & - & 6.900 \\
\hline - & - & - & 6.509 \\
\hline - & $\bullet$ & - & 7.655 \\
\hline$\bullet$ & $\bullet$ & - & $8 \cdot 128$ \\
\hline - & - & - & 6.480 \\
\hline - & - & - & 8.610 \\
\hline - & - & - & 6.560 \\
\hline - & $\bullet$ & $\bullet$ & 5.859 \\
\hline 0.200 & $\bullet$ & $\bullet$ & 7.360 \\
\hline 0.096 & - & - & $3 \cdot 391$ \\
\hline 0.136 & - & - & 6.960 \\
\hline 0.227 & - & - & 9.553 \\
\hline 0.237 & 0.093 & • & 8.344 \\
\hline 0.203 & 0.053 & 0.007 & 7.547 \\
\hline 0.156 & 0.061 & 0.012 & 8.297 \\
\hline 0.139 & 0.052 & 0.010 & 6.658 \\
\hline 0.173 & 0.036 & 0.004 & 7.306 \\
\hline 0.133 & 0.040 & 0.004 & 6.659 \\
\hline 0.080 & 0.037 & - & 6.085 \\
\hline 0.071 & 0.021 & 0.004 & 5.691 \\
\hline 0.050 & 0.018 & - & 4.952 \\
\hline 0.054 & 0.024 & - & 5.199 \\
\hline 0.044 & 0.022 & 0.003 & 4.493 \\
\hline 0.023 & 0.011 & 0.006 & 3.714 \\
\hline 0.008 & 0.011 & • & 3.282 \\
\hline 0.010 & 0.002 & $\bullet$ & 2.882 \\
\hline 0.020 & - & 0.002 & 3.253 \\
\hline 0.012 & 0.003 & - & 3.354 \\
\hline$\bullet$ & $\bullet$ & & 2.513 \\
\hline 0.012 & $\bullet$ & 0.002 & 2.370 \\
\hline 0.004 & - & $\bullet$ & 2.151 \\
\hline
\end{tabular}


Basic Table 37.A. Total Jilin Province (7): duration-specific fertility

\begin{tabular}{|c|c|c|c|c|}
\hline $\begin{array}{l}\text { Birth year } \\
\text { of child }\end{array}$ & $0-4$ & $5-9$ & $10-14$ & . 15-19 \\
\hline 1940 & $0.24 B$ & - & - & - \\
\hline 1941 & 0.280 & - & - & - \\
\hline 1942 & 0.270 & - & - & - \\
\hline S & 0.32 .0 & - & - & - \\
\hline 1944 & 0.249 & - & - & - \\
\hline $19+3$ & 0.259 & 0.271 & - & - \\
\hline 1540 & 0.305 & 0.250 & - & - \\
\hline 1947 & 0.257 & 0.325 & - & - \\
\hline 1940 & $\left.0.2^{\prime}\right) 4$ & 0.286 & - & - \\
\hline $1 y 45$ & 0.291 & 0.300 & - & - \\
\hline 1950 & 0.310 & 0.355 & 0.2 28 & - \\
\hline $\begin{array}{l}1 \text { | } 1 \\
1952\end{array}$ & $\begin{array}{l}0.280 \\
0.38 .3\end{array}$ & $\begin{array}{l}0.281 \\
0.292\end{array}$ & $\begin{array}{l}0.300 \\
0.288\end{array}$ & : \\
\hline 1953 & 0.323 & 0.333 & 0.320 & - \\
\hline 1954 & 0.323 & 0.370 & 0.300 & - \\
\hline 1955 & 0.410 & 0.293 & 0.309 & 0.225 \\
\hline 1950 & 0.308 & 0.155 & 0.305 & 0.249 \\
\hline 1957 & 0.345 & 0.287 & 0.319 & 0.288 \\
\hline 1950 & 0.376 & 0.370 & 0.311 & 0.194 \\
\hline 1909 & 0.354 & 0.283 & 0.234 & 0.217 \\
\hline 1760 & 0.332 & 0.354 & 0.290 & 0.246 \\
\hline 1ப்1 & 0.247 & 0.250 & 0.195 & 0.106 \\
\hline 1 убठ己 & 0.337 & 0.354 & 0.314 & 0.251 \\
\hline 1363 & 0.401 & 0.393 & 0.340 & 0.368 \\
\hline 1904 & 0.370 & 0.395 & 0.296 & 0.153 \\
\hline 1905 & 0.375 & 0.340 & 0.322 & 0.251 \\
\hline 1900 & 0.379 & 0.267 & 0.205 & 0.25 \\
\hline 1507 & 0.342 & 0.312 & 0.279 & 0.208 \\
\hline 1905 & 0.448 & 0.349 & 0.28 .3 & 0.224 \\
\hline 1909 & 0.343 & 0.318 & 0.316 & 0.193 \\
\hline 1970 & 0.427 & 0.301 & 0.242 & 0.213 \\
\hline 1971 & 0.355 & 0.335 & 0.249 & 0.181 \\
\hline 1972 & $0.3 \varepsilon 1$ & 0.298 & 0.236 & 0.159 \\
\hline 197 & 0.359 & 0.278 & 0.152 & 0.103 \\
\hline 1974 & 0.362 & 0.226 & 0.121 & 0.066 \\
\hline 1975 & 0.313 & 0.158 & 0.100 & 0.044 \\
\hline 1570 & 0.367 & 0.171 & 0.066 & 0.028 \\
\hline 1977 & 0.314 & 0.140 & 0.080 & 0.034 \\
\hline 1978 & 0.396 & 0.163 & 0.056 & 0.020 \\
\hline y & 0.300 & 0.197 & 0.079 & 0.019 \\
\hline 1900 & 0.267 & 0.136 & 0.026 & 0.020 \\
\hline 1981 & 0.333 & 0.078 & 0.024 & 0.011 \\
\hline 1932 & 0.313 & 0.064 & 0.024 & • \\
\hline
\end{tabular}


rates, $1940-82$

\begin{tabular}{|c|c|c|c|c|}
\hline $20-24$ & $25-29$ & & $30+$ & Total \\
\hline - & - & & - & - \\
\hline - & - & & • & • \\
\hline - & $\bullet$ & & - & - \\
\hline - & - & & - & - \\
\hline - & • & & - & - \\
\hline - & - & & - & 5.543 \\
\hline - & - & & - & $\therefore .004$ \\
\hline - & - & & - & 5.321 \\
\hline - & - & & - & 5.375 \\
\hline - & - & & - & 0.119 \\
\hline • & - & & - & t). .557 \\
\hline - & - & & - & 6.247 \\
\hline - & - & & - & 0.561 \\
\hline - & - & & - & 7.264 \\
\hline - & - & & - & $7.24 ?$ \\
\hline - & • & & - & $7.05 E$ \\
\hline$\bullet$ & - & & - & 7.050 \\
\hline - & - & & - & 7.157 \\
\hline - & - & & - & 7.141 \\
\hline - & - & & - & 6.107 \\
\hline 0.150 & • & & - & 7.143 \\
\hline 0.117 & - & & - & $=.194$ \\
\hline 0.160 & - & & - & $7.2 \div 7$ \\
\hline 0.213 & - & & . & 0.951 \\
\hline $0.15 a$ & $0.06 \%$ & & 0.032 & 7.592 \\
\hline 0.150 & 0.052 & & 0.025 & $7.60 i$ \\
\hline 0.193 & 0.051 & & - & 7.1131 \\
\hline 0.152 & 0.037 & & - & 0.647 \\
\hline 0.162 & 0.050 & & - & 7.587 \\
\hline 0.147 & 0.043 & & - & 0.772 \\
\hline 0.130 & 0.046 & & - & 7.098 \\
\hline 0.038 & 0.028 & & - & $6.18 ?$ \\
\hline 0.107 & 0.040 & & • & 6.100 \\
\hline 0.030 & 0.0 .31 & & 0.004 & 5.037 \\
\hline 0.041 & 0.015 & & • & 4.258 \\
\hline 0.010 & 0.010 & & - & 3.213 \\
\hline 0.020 & 0.018 & & 0.004 & $3.3 e .7$ \\
\hline 0.022 & .0 .003 & & 0.003 & 2.981 \\
\hline 0.012 & • & . & $0.0 \cap 3$ & $3.24 \pi$ \\
\hline 0.000 & ذ'0.0.0 & & 0.003 & 3.436 \\
\hline 0.003 & 0.005 & & - & 2.287 \\
\hline 0.003 & $\bullet$ & & • & 2.244 \\
\hline • & 0.005 & & - & 2.033 \\
\hline
\end{tabular}


Basic Table 37.B. Urban Jilin Province (7): duration-specific fertility

\begin{tabular}{|c|c|c|c|c|}
\hline $\begin{array}{l}\text { Birth year } \\
\text { of child }\end{array}$ & $0-4$ & $5-9$ & $10-14$ & $15-19$ \\
\hline 1940 & 0.264 & - & - & - \\
\hline 1941 & 0.268 & - & - & - \\
\hline 1942 & 0.342 & - & - & - \\
\hline 1943 & 0.248 & - & - & - \\
\hline 1944 & 0.291 & - & - & - \\
\hline 1945 & 0.246 & 0.363 & - & - \\
\hline 1940 & 0.310 & 0.365 & - & $\bullet$ \\
\hline 1947 & 0.222 & 0.282 & - & - \\
\hline 1948 & 0.256 & 0.301 & - & - \\
\hline 1945 & 0.341 & 0.257 & - & • \\
\hline 1950 & 0.294 & 0.193 & 0.330 & $\bullet$ \\
\hline 1951 & 0.300 & 0.345 & 0.195 & - \\
\hline 1952 & 0.344 & 0.273 & 0.282 & - \\
\hline 1953 & 0.261 & 0.400 & 0.230 & - \\
\hline 1954 & 0.364 & 0.463 & 0.377 & - \\
\hline 1955 & 0.401 & 0.192 & 0.211 & 0.231 \\
\hline 1950 & 0.324 & $0.3 y 2$ & 0.310 & 0.170 \\
\hline 1957 & 0.326 & 0.289 & 0.256 & 0.222 \\
\hline 1958 & 0.422 & 0.380 & 0.288 & 0.248 \\
\hline 1959 & 0.291 & 0.273 & $0.1 \ddot{3} 3$ & 0.171 \\
\hline 1900 & 0.338 & 0.299 & 0.201 & 0.140 \\
\hline 1901 & 0.138 & 0.216 & 0.232 & 0.086 \\
\hline 1962 & 0.374 & 0.425 & 0.331 & 0.205 \\
\hline 1963 & 0.398 & 0.324 & 0.368 & 0.352 \\
\hline 1904 & 0.365 & 0.432 & 0.202 & 0.218 \\
\hline د190 & 0.374 & 0.293 & 0.289 & 0.115 \\
\hline 1960 & 0.326 & 0.173 & 0.141 & 0.123 \\
\hline 1967 & 0.298 & 0.272 & 0.121 & 0.096 \\
\hline 1968 & 0.406 & 0.317 & 0.170 & 0.071 \\
\hline 1 ริด & 0.356 & 0.311 & 0.160 & 0.101 \\
\hline 1970 & 0.432 & 0.314 & 0.151 & 0.075 \\
\hline 1971 & 0.332 & 0.300 & 0.181 & 0.083 \\
\hline 1972 & 0.345 & 0.255 & 0.161 & 0.068 \\
\hline 1973 & 0.397 & 0.171 & 0.081 & 0.032 \\
\hline 1974 & 0.289 & 0.155 & 0.074 & 0.019 \\
\hline 1975 & 0.312 & 0.121 & 0.047 & 0.036 \\
\hline 1976 & 0.333 & 0.144 & 0.065 & 0.009 \\
\hline 1977 & 0.384 & 0.114 & 0.043 & 0.034 \\
\hline 1978 & 0.428 & 0.074 & 0.012 & $\bullet$ \\
\hline 1979 & 0.269 & 0.065 & 0.021 & 0.007 \\
\hline 1980 & 0.184 & 0.052 & 0.009 & • \\
\hline 1981 & 0.300 & 0.030 & - & - \\
\hline 1982 & 0.334 & - & - & - \\
\hline
\end{tabular}


rates, $1940-82$

\begin{tabular}{|c|c|c|c|}
\hline $20-24$ & $25-29$ & $30+$ & Total \\
\hline - & - & - & - \\
\hline • & • & - & - \\
\hline - & • & - & - \\
\hline$\bullet$ & - & - & - \\
\hline - & - & $\bullet$ & - \\
\hline - & - & - & 7.071 \\
\hline - & - & - & 7.428 \\
\hline - & - & - & 5.047 \\
\hline - & - & - & 6.119 \\
\hline - & - & - & 5.838 \\
\hline - & - & - & 0.005 \\
\hline - & - & - & 6.104 \\
\hline - & - & - & 6.501 \\
\hline - & - & - & 6.681 \\
\hline - & - & - & 9.027 \\
\hline$\bullet$ & - & - & 5.949 \\
\hline - & - & - & 6.932 \\
\hline - & - & - & 6.383 \\
\hline - & - & - & 7.768 \\
\hline - & - & - & 4.961 \\
\hline 0.132 & - & - & 0.186 \\
\hline 0.122 & - & - & 4.151 \\
\hline 0.060 & - & - & 7.231 \\
\hline 0.177 & - & - & 8.434 \\
\hline 0.086 & 0.122 & - & 7.127 \\
\hline 0.105 & 0.033 & - & 6.052 \\
\hline 0.031 & 0.024 & - & 4.107 \\
\hline 0.085 & 0.020 & • & 4.466 \\
\hline 0.090 & 0.018 & - & 5.3 .98 \\
\hline 0.082 & - & - & 5.049 \\
\hline 0.026 & • & - & 4.986 \\
\hline 0.014 & 0.034 & - & 4.724 \\
\hline 0.041 & • & - & 4.355 \\
\hline 0.024 & - & - & 3.524 \\
\hline - & - & - & 2.687 \\
\hline - & - & - & 2.575 \\
\hline - & 0.041 & - & 2.959 \\
\hline - & - & • & 2.875 \\
\hline - & - & - & 2.507 \\
\hline - & - & - & 1.307 \\
\hline - & - & - & 1.225 \\
\hline - & - & - & 1.650 \\
\hline - & - & - & 1.603 \\
\hline
\end{tabular}


Basic Table 37.C. Rural Jilin Province (7): duration-specific fertility

\begin{tabular}{|c|c|c|c|c|}
\hline $\begin{array}{l}\text { Birth year } \\
\text { of child }\end{array}$ & $0-4$ & $5-9$ & $10-14$ & $15-19$ \\
\hline 1940 & 0.245 & - & $\bullet$ & $\bullet$ \\
\hline 1941 & 0.283 & - & • & - \\
\hline 1942 & 0.248 & - & - & - \\
\hline 1943 & 0.353 & - & - & - \\
\hline 1944 & 0.236 & - & - & - \\
\hline 1945 & 0.263 & 0.252 & - & • \\
\hline 1946 & 0.304 & 0.270 & - & • \\
\hline 1947 & 0.265 & 0.337 & - & - \\
\hline 1948 & 0.302 & 0.282 & - & - \\
\hline 1949 & 0.279 & 0.313 & - & - \\
\hline 1930 & 0.321 & 0.400 & 0.280 & - \\
\hline 1951 & 0.275 & 0.265 & 0.328 & - \\
\hline 1952 & 0.392 & 0.284 & 0.290 & - \\
\hline 1953 & 0.342 & 0.343 & 0.348 & - \\
\hline 1954 & 0.308 & 0.356 & 0.277 & - \\
\hline 1953 & 0.414 & 0.318 & 0.336 & 0.224 \\
\hline 1956 & 0.301 & 0.349 & 0.304 & 0.270 \\
\hline 1957 & 0.353 & 0.287 & $0.3 \geq 3$ & 0.308 \\
\hline 1958 & 0.357 & 0.367 & 0.316 & 0.177 \\
\hline 1959 & 0.351 & 0.286 & $0.2 \in 0$ & 0.231 \\
\hline 1960 & 0.330 & 0.377 & 0.300 & 0.278 \\
\hline 1961 & 0.292 & 0.264 & 0.186 & 0.212 \\
\hline 1962 & 0.322 & 0.321 & 0.310 & $0.2 \in 1$ \\
\hline 1903 & 0.403 & 0.423 & 0.331 & 0.371 \\
\hline 1964 & 0.382 & 0.380 & 0.331 & 0.187 \\
\hline 1965 & 0.375 & 0.359 & 0.336 & 0.284 \\
\hline 1960 & 0.405 & 0.306 & 0.320 & 0.282 \\
\hline 1907 & 0.365 & 0.329 & 0.353 & 0.235 \\
\hline 1908 & 0.470 & 0.363 & 0.332 & 0.270 \\
\hline $196 y$ & 0.336 & 0.321 & 0.377 & 0.227 \\
\hline 1970 & 0.425 & 0.385 & 0.279 & 0.268 \\
\hline 1971 & 0.365 & 0.353 & 0.277 & 0.224 \\
\hline 1972 & 0.397 & 0.320 & 0.267 & 0.202 \\
\hline 1573 & 0.343 & 0.334 & 0.183 & 0.133 \\
\hline 1974 & 0.420 & 0.262 & 0.143 & 0.084 \\
\hline 1975 & 0.313 & 0.175 & 0.127 & 0.047 \\
\hline 1976 & 0.383 & 0.183 & 0.066 & 0.036 \\
\hline 1977 & 0.284 & 0.152 & 0.098 & 0.035 \\
\hline $\begin{array}{l}1978 \\
1979\end{array}$ & $\begin{array}{l}0.382 \\
0.435\end{array}$ & $\begin{array}{l}0.200 \\
0.251\end{array}$ & $\begin{array}{l}0.079 \\
0.108\end{array}$ & $\begin{array}{l}0.029 \\
0.025\end{array}$ \\
\hline 1980 & 0.301 & 0.172 & 0.033 & 0.030 \\
\hline 1981 & 0.345 & 0.100 & 0.035 & 0.016 \\
\hline 1982 & 0.305 & 0.092 & 0.035 & - \\
\hline
\end{tabular}


rates, $1940-82$

\begin{tabular}{|c|c|c|c|c|}
\hline $20-24$ & & $25-29$ & $30+$ & Total \\
\hline - & & $\bullet$ & - & - \\
\hline - & & - & - & - \\
\hline - & & - & - & - \\
\hline - & & - & - & $\bullet$ \\
\hline - & & - & - & - \\
\hline - & & - & - & 5.160 \\
\hline - & & - & - & 5.650 \\
\hline - & & - & - & 6.483 \\
\hline - & & - & - & 5.816 \\
\hline - & & - & - & 0.186 \\
\hline - & & - & - & 7.190 \\
\hline - & & - & - & 6.281 \\
\hline - & & - & - & 6.700 \\
\hline - & & - & - & 7.417 \\
\hline - & & - & - & 6.750 \\
\hline - & & - & - & 7.372 \\
\hline - & & - & - & 7.085 \\
\hline - & & - & - & 7.390 \\
\hline - & & - & - & 0.952 \\
\hline - & & - & - & 6.455 \\
\hline 0.154 & & - & - & 7.441 \\
\hline 0.116 & & - & - & 5.527 \\
\hline 0.189 & & . & - & 7.279 \\
\hline 0.232 & & - & - & 9.122 \\
\hline 0.180 & & 0.057 & 0.038 & 7.775 \\
\hline 0.171 & & 0.056 & 0.031 & 8.052 \\
\hline 0.234 & & 0.058 & - & 0.021 \\
\hline 0.167 & & 0.041 & - & 7.451 \\
\hline 0.177 & . & 0.061 & - & 8.367 \\
\hline 0.156 & & $c .056$ & - & 7.362 \\
\hline 0.150 & & 0.058 & - & 7.859 \\
\hline 0.106 & & 0.026 & - & 6.757 \\
\hline 0.123 & & 0.049 & - & 6.789 \\
\hline 0.097 & & 0.033 & 0.000 & 5.671 \\
\hline 0.050 & & 0.019 & - & 4.919 \\
\hline 0.026 & $\theta^{\circ}$ & 0.012 & - & 3.504 \\
\hline 0.029 & . & 0.013 & 0.004 & 3.367 \\
\hline 0.032 & & 0.003 & 0.004 & 3.040 \\
\hline $\begin{array}{l}0.017 \\
0.011\end{array}$ & & 0.007 & $\begin{array}{l}0.003 \\
0.003\end{array}$ & $\begin{array}{r}3.552 \\
0.202\end{array}$ \\
\hline 0.004 & & 0.007 & . & 2.735 \\
\hline 0.004 & & • & - & 2.502 \\
\hline - & & 0.007 & - & 2.200 \\
\hline
\end{tabular}


Basic Table 38.A. Total Heilongjiang Province (8): duration-specific

\begin{tabular}{|c|c|c|c|c|}
\hline $\begin{array}{l}\text { Birth year } \\
\text { of child }\end{array}$ & $0-4$ & $5-9$ & $10-14$ & 15-19 \\
\hline 1940 & 0.246 & - & - & - \\
\hline 1941 & 0.265 & - & - & $\bullet$ \\
\hline 1942 & 0.292 & - & - & - \\
\hline 1943 & 0.264 & - & - & - \\
\hline 1944 & 0.260 & - & - & - \\
\hline 1945 & 0.298 & 0.246 & - & - \\
\hline 1946 & 0.254 & 0.265 & - & - \\
\hline 1947 & 0.300 & 0.305 & - & - \\
\hline 1948 & 0.274 & 0.275 & - & - \\
\hline 1949 & 0.313 & 0.330 & - & - \\
\hline 1950 & 0.328 & 0.310 & 0.297 & - \\
\hline 1951 & 0.279 & 0.301 & 0.275 & - \\
\hline 1952 & 0.321 & 0.337 & 0.327 & - \\
\hline 1953 & $0 \cdot 329$ & $0.2<2$ & 0.258 & - \\
\hline 1954 & 0.314 & 0.313 & 0.341 & - \\
\hline 1955 & 0.340 & 0.358 & 0.266 & 0.199 \\
\hline 1950 & 0.325 & 0.334 & 0.265 & 0.268 \\
\hline 1957 & 0.354 & 0.359 & 0.314 & 0.264 \\
\hline 1958 & 0.365 & 0.333 & 0.285 & 0.183 \\
\hline $195 y$ & 0.338 & 0.292 & 0.222 & 0.228 \\
\hline 1960 & 0.320 & 0.305 & 0.341 & 0.212 \\
\hline 1961 & 0.269 & 0.247 & 0.203 & 0.171 \\
\hline 1962 & 0.317 & 0.327 & 0.300 & 0.246 \\
\hline 1963 & 0.413 & 0.379 & 0.282 & 0.285 \\
\hline 1904 & 0.329 & 0.330 & 0.299 & 0.204 \\
\hline 1965 & 0.349 & 0.294 & 0.281 & 0.223 \\
\hline 1906 & 0.356 & 0.291 & 0.253 & 0.255 \\
\hline 1967 & 0.359 & 0.317 & 0.235 & 0.167 \\
\hline 1968 & 0.383 & 0.389 & 0.294 & 0.202 \\
\hline 1969 & 0.337 & 0.281 & 0.268 & 0.178 \\
\hline 1970 & 0.423 & 0.280 & 0.207 & 0.182 \\
\hline 1971 & 0.350 & 0.313 & 0.215 & 0.151 \\
\hline 1972 & 0.375 & 0.326 & 0.214 & 0.185 \\
\hline 1973 & 0.376 & 0.293 & 0.236 & 0.134 \\
\hline 1974 & 0.383 & 0.272 & 0.149 & 0.113 \\
\hline 1975 & 0.377 & 0.227 & 0.113 & 0.063 \\
\hline 1970 & 0.339 & 0.178 & 0.087 & 0.037 \\
\hline 1977 & 0.332 & 0.166 & 0.077 & 0.028 \\
\hline 1978 & 0.375 & 0.165 & 0.080 & 0.027 \\
\hline 1979 & 0.385 & 0.222 & 0.082 & 0.017 \\
\hline $1 \div 80$ & 0.340 & 0.130 & 0.052 & 0.008 \\
\hline 1981 & 0.348 & 0.120 & 0.029 & 0.014 \\
\hline 1982 & 0.394 & 0.133 & 0.040 & 0.009 \\
\hline
\end{tabular}


fertility rates, $1940-82$

\begin{tabular}{|c|c|c|c|}
\hline $20-24$ & $25-29$ & $30+$ & Total \\
\hline - & . & • & - \\
\hline - & - & - & • \\
\hline • & - & - & - \\
\hline - & - & - & - \\
\hline • & - & - & • \\
\hline • & - & - & 5.322 \\
\hline$\bullet$ & - & - & 5.377 \\
\hline - & - & - & 6.202 \\
\hline • & - & - & 5.665 \\
\hline$\bullet$ & $\bullet$ & - & 6.673 \\
\hline • & - & - & 6.811 \\
\hline - & • & - & 6.202 \\
\hline - & - & • & 7.162 \\
\hline$\bullet$ & • & - & 6.024 \\
\hline$\bullet$ & - & - & 7.095 \\
\hline - & - & - & 6.702 \\
\hline - & - & - & 6.999 \\
\hline - & - & - & 7.469 \\
\hline - & • & - & 6.724 \\
\hline - & - & - & 6.216 \\
\hline 0.221 & $\bullet$ & • & 7.290 \\
\hline 0.094 & - & - & 5.089 \\
\hline 0.184 & - & - & 7.146 \\
\hline 0.177 & - & - & 7.888 \\
\hline 0.152 & 0.100 & 0.010 & 7.118 \\
\hline 0.154 & 0.036 & • & 6.688 \\
\hline 0.137 & 0.057 & 0.006 & 6.773 \\
\hline 0.123 & 0.060 & - & 6.300 \\
\hline 0.161 & 0.032 & 0.004 & 7.329 \\
\hline 0.095 & 0.047 & 0.008 & 6.074 \\
\hline 0.123 & 0.029 & 0.007 & $0.25 a$ \\
\hline 0.102 & 0.037 & - & 5.842 \\
\hline 0.094 & 0.031 & 0.006 & 6.155 \\
\hline 0.072 & 0.029 & - & 5.695 \\
\hline 0.056 & 0.016 & - & 4.940 \\
\hline 0.034 & 0.011 & - & 4.125 \\
\hline 0.025 & $0.01 \mathrm{j}$ & - & 3.403 \\
\hline 0.020 & 0.010 & - & 3.159 \\
\hline 0.024 & 0.008 & - & 3.395 \\
\hline 0.017 & 0.006 & 0.007 & 3.673 \\
\hline 0.010 & 0.002 & - & 2.739 \\
\hline 0.006 & - & - & 2.597 \\
\hline 0.013 & - & - & 2.746 \\
\hline
\end{tabular}


Basic Table 38.B. Urban Heilongjiang Province (8): duration-specific

\begin{tabular}{|c|c|c|c|c|}
\hline $\begin{array}{l}\text { Birth year } \\
\text { of child }\end{array}$ & $0-4$ & $5-9$ & $10-14$ & $15-19$ \\
\hline 1940 & 0.311 & - & - & - \\
\hline 1941 & 0.212 & - & - & - \\
\hline 1942 & 0.220 & - & - & - \\
\hline 1943 & 0.245 & - & - & $\bullet$ \\
\hline 1944 & 0.251 & - & - & - \\
\hline 1945 & 0.282 & 0.269 & - & - \\
\hline 1940 & 0.203 & 0.252 & - & - \\
\hline 1947 & 0.296 & 0.137 & - & - \\
\hline 1948 & 0.279 & 0.352 & - & - \\
\hline 1449 & 0.268 & 0.242 & - & - \\
\hline 1950 & 0.300 & 0.315 & 0.425 & - \\
\hline 1951 & 0.267 & 0.261 & 0.265 & - \\
\hline $195 \ddot{~}$ & 0.284 &.$\quad 0.277$ & 0.319 & - \\
\hline 1953 & 0.331 & 0.250 & 0.313 & - \\
\hline 1954 & 0.267 & 0.326 & 0.385 & • \\
\hline $1 \cup 55$ & 0.324 & 0.388 & 0.139 & 0.241 \\
\hline 1956 & $0: 307$ & 0.324 & 0.276 & 0.371 \\
\hline 1957 & 0.342 & 0.332 & 0.347 & 0.209 \\
\hline 1958 & 0.353 & 0.362 & 0.290 & 0.225 \\
\hline 1959 & 0.305 & 0.256 & 0.189 & 0.179 \\
\hline 1960 & 0.308 & 0.263 & 0.321 & 0.166 \\
\hline 1901 & 0.256 & 0.253 & 0.211 & 0.116 \\
\hline 1962 & 0.298 & 0.288 & 0.342 & 0.200 \\
\hline 1903 & 0.502 & 0.393 & 0.330 & 0.221 \\
\hline 1964 & 0.309 & 0.297 & 0.294 & 0.137 \\
\hline 1905 & 0.340 & 0.290 & 0.197 & 0.140 \\
\hline 1900 & 0.323 & 0.206 & 0.166 & 0.113 \\
\hline 1907 & 0.304 & 0.272 & 0.139 & 0.142 \\
\hline 1968 & 0.361 & 0.309 & 0.212 & 0.116 \\
\hline 1969 & 0.305 & 0.205 & 0.180 & 0.114 \\
\hline 1970 & 0.423 & 0.259 & 0.156 & 0.091 \\
\hline 1971 & 0.314 & 0.202 & 0.144 & 0.078 \\
\hline 1972 & 0.343 & 0.233 & 0.145 & 0.072 \\
\hline 1573 & 0.415 & 0.188 & 0.193 & 0.065 \\
\hline 1974 & 0.323 & 0.232 & 0.083 & 0.059 \\
\hline 1975 & 0.308 & 0.131 & 0.062 & 0.029 \\
\hline 1970 & 0.318 & 0.118 & 0.035 & 0.017 \\
\hline 1977 & $0.3+1$ & 0.146 & 0.051 & 0.021 \\
\hline 1970 & 0.376 & 0.120 & 0.041 & 0.004 \\
\hline 1979 & 0.344 & 0.124 & 0.077 & • \\
\hline 1980 & 0.326 & 0.073 & 0.009 & - \\
\hline 1981 & 0.296 & 0.042 & - & 0.005 \\
\hline 1982 & 0.352 & 0.034 & $0.0 \geq 1$ & $\bullet$ \\
\hline
\end{tabular}


fertility rates, $1940-82$

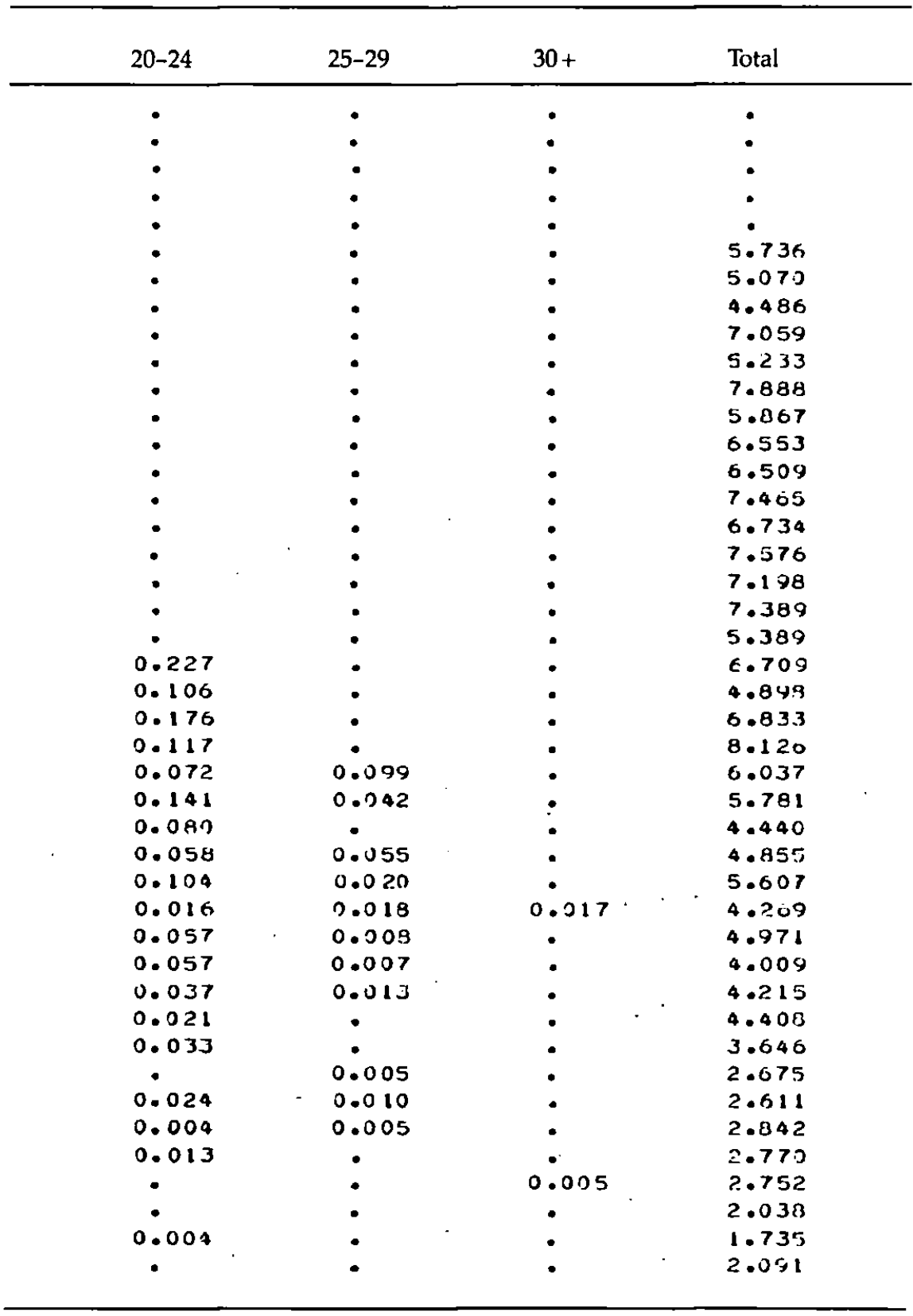


Basic Table 38.C. Rural Heilongjiang Province (8): duration-specific

\begin{tabular}{|c|c|c|c|c|}
\hline $\begin{array}{l}\text { Birth year } \\
\text { of child }\end{array}$ & $0-4$ & $5-9$ & $10-14$ & 15-19 \\
\hline 1940 & 0.224 & - & • & • \\
\hline 1941 & 0.283 & - & • & - \\
\hline 1942 & 0.322 & - & • & $\bullet$ \\
\hline 1943 & 0.272 & - & - & - \\
\hline 1944 & 0.263 & - & - & - \\
\hline 1945 & 0.304 & 0.238 & • & $\bullet$ \\
\hline 1940 & 0.277 & 0.269 & - & - \\
\hline 1947 & 0.301 & 0.353 & - & - \\
\hline 1948 & 0.271 & 0.243 & $\bullet$ & - \\
\hline 1949 & 0.336 & 0.367 & - & • \\
\hline 1950 & 0.344 & 0.308 & 0.253 & - \\
\hline 1951 & 0.285 & 0.320 & 0.278 & - \\
\hline 1952 & 0.342 & 0.365 & 0.330 & • \\
\hline 1953 & $0.3 \angle B$ & 0.268 & 0.235 & - \\
\hline 1954 & 0.344 & 0.306 & 0.322 & - \\
\hline 1955 & 0.350 & 0.341 & 0.294 & 0.185 \\
\hline 1956 & $0: 336$ & 0.340 & 0.260 & 0.233 \\
\hline 1957 & 0.362 & 0.375 & 0.298 & 0.286 \\
\hline 1950 & 0.347 & 0.315 & 0.283 & 0.173 \\
\hline 1 บ59 & 0.359 & 0.316 & 0.239 & 0.248 \\
\hline 1960 & 0.328 & 0.333 & 0.352 & 0.232 \\
\hline 1961 & 0.277 & 0.244 & 0.198 & 0.197 \\
\hline 1902 & 0.329 & 0.353 & 0.275 & 0.265 \\
\hline 1903 & 0.361 & 0.370 & $0.24 B$ & 0.317 \\
\hline 1964 & 0.340 & 0.351 & 0.302 & 0.239 \\
\hline 1965 & 0.354 & 0.293 & 0.337 & 0.268 \\
\hline 1906 & 0.371 & 0.346 & 0.308 & 0.335 \\
\hline 1907 & 0.381 & 0.346 & 0.297 & 0.181 \\
\hline 1963 & 0.390 & 0.436 & 0.347 & 0.258 \\
\hline $\begin{array}{l}1969 \\
1970\end{array}$ & $\begin{array}{l}0.348 \\
0.423\end{array}$ & $\begin{array}{l}0.323 \\
0.290\end{array}$ & $\begin{array}{l}0.326 \\
0.239\end{array}$ & $\begin{array}{l}0.220 \\
0.242\end{array}$ \\
\hline 1971 & 0.361 & 0.364 & 0.261 & 0.197 \\
\hline 1972 & 0.385 & 0.364 & 0.257 & 0.259 \\
\hline 1973 & 0.363 & 0.330 & 0.261 & 0.178 \\
\hline 1974 & 0.402 & 0.285 & 0.185 & 0.148 \\
\hline 1975 & 0.398 & 0.257 & 0.138 & 0.086 \\
\hline 1976 & 0.345 & 0.198 & 0.110 & 0.051 \\
\hline 1977 & 0.329 & 0.173 & 0.088 & 0.032 \\
\hline 1970 & 0.375 & 0.179 & 0.094 & 0.040 \\
\hline 1979 & 0.397 & 0.254 & 0.083 & 0.026 \\
\hline 1980 & 0.353 & 0.148 & 0.066 & 0.012 \\
\hline 1981 & 0.371 & 0.144 & 0.038 & 0.018 \\
\hline 1982 & 0.415 & 0.162 & 0.043 & 0.012 \\
\hline
\end{tabular}


fertility rates, $1940-82$

\begin{tabular}{|c|c|c|c|}
\hline $20-24$ & $25-29$ & $30+$ & Total \\
\hline • & - & - & • \\
\hline • & • & • & • \\
\hline$\bullet$ & - & - & • \\
\hline • & - & - & • \\
\hline - & - & - & - \\
\hline - & • & - & 5.165 \\
\hline - & • & - & 5.499 \\
\hline • & - & - & 6.903 \\
\hline - & - & - & 5.076 \\
\hline - & - & - & 7.294 \\
\hline - & - & • & 6.336 \\
\hline • & - & - & $6 \cdot 354$ \\
\hline • & • & • & 7.442 \\
\hline - & - & - & 5.791 \\
\hline - & - & • & 6.916 \\
\hline - & - & - & 6.687 \\
\hline - & - & - & 6.712 \\
\hline - & - & • & 7.607 \\
\hline • & - & - & 6.379 \\
\hline • & - & - & 6.649 \\
\hline 0.219 & - & • & 7.59 \\
\hline 0.090 & - & • & 5.190 \\
\hline 0.188 & - & - & 7.312 \\
\hline 0.202 & • & • & 7.761 \\
\hline 0.185 & 0.101 & 0.014 & 7.654 \\
\hline 0.159 & 0.034 & $\bullet$ & 7.226 \\
\hline 0.163 & 0.076 & 0.008 & 8.037 \\
\hline 0.154 & 0.063 & $\bullet$ & 7.108 \\
\hline 0.189 & 0.037 & 0.006 & 8.316 \\
\hline 0.136 & 0.059 & 0.005 & 7.085 \\
\hline 0.159 & 0.038 & 0.010 & 7.013 \\
\hline 0.128 & 0.050 & $\bullet$ & 6.809 \\
\hline 0.127 & 0.039 & 0.009 & 7.204 \\
\hline 0.104 & 0.043 & $\bullet$ & 6.393 \\
\hline 0.071 & 0.024 & - & 5.579 \\
\hline 0.057 & 0.014 & • & 4.753 \\
\hline 0.025 & 0.017 & • & 3.729 \\
\hline 0.029 & 0.012 & • & 3.315 \\
\hline 0.031 & 0.013 & • & 3.662 \\
\hline 0.027 & 0.011 & 0.003 & 4.037 \\
\hline 0.016 & 0.003 & • & 2.990 \\
\hline 0.008 & $\bullet$ & - & 2.894 \\
\hline 0.022 & - & - & 3.269 \\
\hline
\end{tabular}


Basic Table 39.A. Total Shanghai Province (9): duration-specific fertility

\begin{tabular}{|c|c|c|c|c|}
\hline $\begin{array}{l}\text { Birth year } \\
\text { of child }\end{array}$ & $0-4$ & $5-9$ & $10-14$ & $15-19$ \\
\hline 1940 & 0.254 & • & • & - \\
\hline $\begin{array}{l}1941 \\
1942\end{array}$ & $\begin{array}{l}0.270 \\
0.273\end{array}$ & • & • & • \\
\hline 1943 & 0.230 & - & - & - \\
\hline 1944 & 0.308 & - & - & - \\
\hline 1945 & 0.288 & 0.244 & - & - \\
\hline 1946 & 0.360 & 0.256 & - & - \\
\hline 1947 & 0.326 & 0.310 & $\bullet$ & • \\
\hline 1948 & 0.339 & 0.284 & - & - \\
\hline 1949 & 0.349 & 0.293 & - & - \\
\hline 1950 & 0.353 & 0.300 & 0.215 & - \\
\hline 1951 & 0.275 & 0.281 & 0.224 & - \\
\hline 1952 & 0.348 & 0.348 & 0.227 & - \\
\hline 1953 & 0.331 & 0.289 & 0.226 & - \\
\hline 1954 & 0.407 & 0.349 & 0.252 & •. \\
\hline 1455 & 0.361 & 0.298 & 0.243 & 0.191 \\
\hline 1956 & 0.359 & 0.216 & 0.169 & 0.142 \\
\hline 1957 & 0.367 & 0.360 & 0.283 & 0.219 \\
\hline 1958 & 0.356 & 0.218 & 0.225 & 0.092 \\
\hline 1959 & 0.290 & 0.245 & 0.098 & 0.078 \\
\hline 1900 & 0.255 & 0.186 & 0.116 & 0.053 \\
\hline 1961 & 0.303 & 0.184 & 0.114 & 0.060 \\
\hline 1902 & 0.311 & 0.223 & 0.098 & 0.110 \\
\hline 1903 & 0.374 & 0.274 & 0.126 & 0.108 \\
\hline 1964 & 0.354 & 0.138 & 0.077 & 0.031 \\
\hline 1465 & 0.295 & 0.100 & 0.089 & 0.043 \\
\hline $1960^{\circ}$ & 0.316 & 0.192 & 0.103 & 0.064 \\
\hline 1967 & 0.321 & 0.118 & 0.032 & 0.024 \\
\hline 1968 & 0.437 & 0.187 & 0.108 & 0.035 \\
\hline 1969 & 0.347 & 0.142 & 0.072 & 0.024 \\
\hline 1970 & 0.366 & 0.137 & 0.034 & 0.017 \\
\hline 1971 & 0.345 & 0.080 & 0.037 & 0.010 \\
\hline 1972 & 0.219 & 0.113 & 0.017 & 0.013 \\
\hline 1973 & 0.280 & 0.115 & 0.004 & 0.020 \\
\hline 1974 & 0.257 & 0.082 & 0.021 & - \\
\hline 1975 & 0.266 & 0.062 & 0.013 & - \\
\hline 1976 & 0.312 & 0.071 & 0.019 & - \\
\hline 1977 & 0.283 & 0.063 & 0.024 & 0.004 \\
\hline 1970 & 0.300 & 0.066 & 0.022 & $\bullet$ \\
\hline 1979 & 0.297 & 0.074 & 0.014 & - \\
\hline 1980 & 0.209 & 0.043 & 0.003 & - \\
\hline 1981 & 0.268 & 0.019 & 0.003 & - \\
\hline 1982 & 0.246 & 0.006 & 0.006 & - \\
\hline
\end{tabular}


rates, $1940-82$

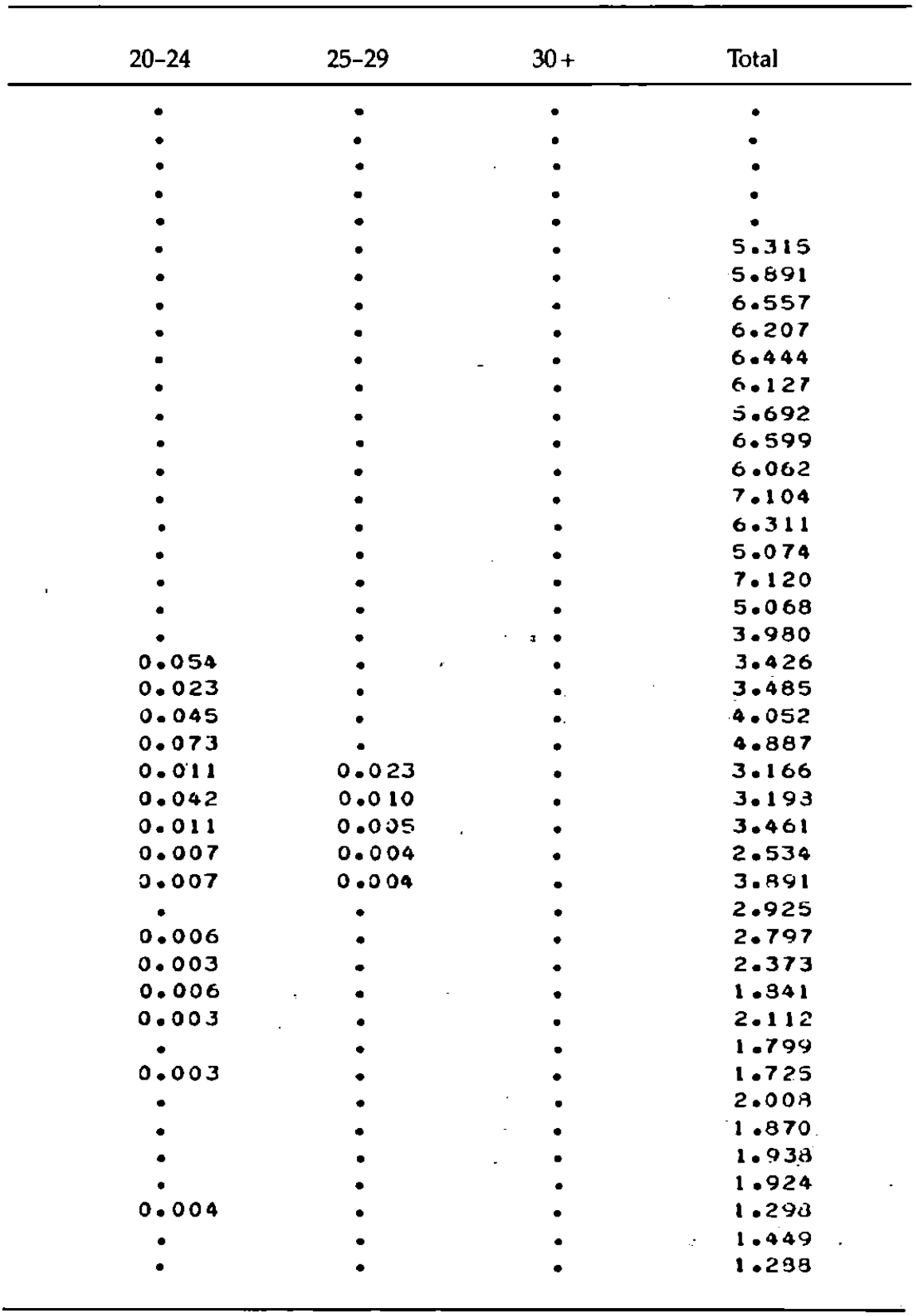


Basic Table 39.B. Urban Shanghai Province (9): duration-specific

\begin{tabular}{|c|c|c|c|c|}
\hline $\begin{array}{l}\text { Birth year } \\
\text { of child }\end{array}$ & $0-4$ & $5-9$ & $10-14$ & $15-19$ \\
\hline 1940 & 0.289 & - & - & - \\
\hline 1941 & 0.244 & - & - & - \\
\hline 1942 & 0.280 & - & - & . \\
\hline 1943 & 0.255 & - & • & - \\
\hline 1944 & 0.317 & - & - & $\bullet$ \\
\hline 1945 & 0.289 & 0.229 & - & - \\
\hline 1940 & 0.370 & 0.232 & - & - \\
\hline 1947 & 0.329 & 0.319 & - & - \\
\hline 1948 & 0.371 & 0.265 & - & - \\
\hline 1949 & 0.362 & 0.317 & - & - \\
\hline 1950 & 0.362 & 0.295 & 0.195 & - \\
\hline 1951 & 0.320 & 0.298 & 0.219 & - \\
\hline 1952 & 0.356 & 0.329 & 0.224 & $\bullet$ \\
\hline 1953 & 0.359 & 0.333 & 0.223 & - \\
\hline 1954 & 0.414 & 0.331 & 0.226 & - \\
\hline 1955 & 0.393 & 0.316 & 0.213 & 0.161 \\
\hline E 19 & 0.409 & 0.211 & 0.182 & 0.128 \\
\hline 1957 & 0.348 & 0.351 & 0.250 & 0.196 \\
\hline 1958 & 0.401 & 0.198 & 0.212 & 0.080 \\
\hline $\begin{array}{l}1959 \\
1960\end{array}$ & $\begin{array}{l}0.323 \\
0.263\end{array}$ & $\begin{array}{l}0.222 \\
0.194\end{array}$ & $\begin{array}{l}0.057 \\
0.129\end{array}$ & $\begin{array}{l}0.043 \\
0.027\end{array}$ \\
\hline 1901 & 0.329 & 0.185 & 0.110 & 0.044 \\
\hline 1962 & 0.316 & 0.194 & 0.054 & 0.052 \\
\hline 1963 & 0.324 & 0.223 & 0.069 & 0.039 \\
\hline 1904 & 0.305 & 0.087 & 0.030 & 0.009 \\
\hline 1965 & 0.227 & 0.107 & 0.030 & 0.008 \\
\hline 1906 & 0.275 & 0.100 & 0.015 & 0.013 \\
\hline 1967 & 0.326 & 0.070 & 0.005 & 0.009 \\
\hline 1908 & 0.373 & 0.144 & 0.061 & 0.005 \\
\hline 1969 & 0.315 & 0.091 & 0.031 & 0.010 \\
\hline 1970 & 0.297 & 0.076 & 0.027 & 0.005 \\
\hline 1971 & 0.282 & 0.041 & 0.022 & 0.015 \\
\hline 1972 & 0.102 & 0.070 & 0.012 & 0.005 \\
\hline 1973 & 0.205 & 0.104 & 0.006 & 0.010 \\
\hline 1974 & 0.225 & 0.066 & 0.007 & - \\
\hline 1975 & 0.228 & 0.044 & 0.008 & - \\
\hline 1970 & 0.248 & 0.028 & $\bullet$ & - \\
\hline 1977 & 0.211 & 0.042 & 0.039 & 0.000 \\
\hline 1978 & 0.260 & 0.045 & 0.028 & - \\
\hline 1979 & 0.196 & 0.059 & 0.011 & - \\
\hline 1980 & 0.200 & 0.017 & 0.005 & - \\
\hline 1981 & 0.243 & - & 0.005 & - \\
\hline 1982 & 0.254 & - & 0.009 & - \\
\hline
\end{tabular}


fertility rates, $1940-82$

\begin{tabular}{|c|c|c|c|}
\hline $20-24$ & $25-29$ & $30+$ & Total \\
\hline - & - & . & . \\
\hline - & - & $\bullet$ & $\bullet$ \\
\hline - & - & - & - \\
\hline - & - & - & - \\
\hline - & - & - & • \\
\hline - & - & - & 5.125 \\
\hline - & $\bullet$ & - & 5.577 \\
\hline - & - & - & 6.777 \\
\hline - & - & $\bullet$ & 6.124 \\
\hline - & - & - & 6.914 \\
\hline - & - & - & 6.000 \\
\hline - & $\bullet$ & - & 6.036 \\
\hline - & • & • & 6.506 \\
\hline • & - & - & 6.546 \\
\hline - & - & - & 6.836 \\
\hline - & - & - & 6.227 \\
\hline - & - & • & 5.262 \\
\hline - & - & - & 6.665 \\
\hline - & $\bullet$ & - & 5.020 \\
\hline - & $\bullet$ & - & 3.577 \\
\hline 0.034 & - & - & 3.331 \\
\hline 0.024 & - & • & 3.551 \\
\hline 0.022 & - & - & 3.272 \\
\hline 0.027 & - & - & 3.495 \\
\hline - & 0.008 & $\bullet$ & 2.190 \\
\hline - & - & • & 1.865 \\
\hline - & • & - & 2.016 \\
\hline$\bullet$ & - & - & 2.051 \\
\hline - & . & - & 2.913 \\
\hline - & - & - & 2.184 \\
\hline - & - & - & 2.023 \\
\hline • & & - & 1.798 \\
\hline 0.005 & - & $\bullet$ & 1.265 \\
\hline - & - & - & 1.625 \\
\hline • & - & - & 1.489 \\
\hline - & - & - & 1.397 \\
\hline - & - & - & $1.38 n$ \\
\hline - & - & - & 1.987 \\
\hline - & - & - & 1.664 \\
\hline$\bullet$ & - & - & 1.328 \\
\hline 0.005 & - & - & 1.132 \\
\hline - & - & - & 1.233 \\
\hline • & - & - & 1.315 \\
\hline
\end{tabular}


Basic Table 39.C. Rural Shanghai Province (9): duration-specific

\begin{tabular}{|c|c|c|c|c|c|c|}
\hline $\begin{array}{l}\text { Birth year } \\
\text { of child }\end{array}$ & & $0-4$ & $5-9$ & $10-14$ & & $15-19$ \\
\hline 1940 & & 0.161 & - & - & & - \\
\hline 1941 & & 0.346 & - & - & & - \\
\hline $\begin{array}{l}1942 \\
1943\end{array}$ & & $\begin{array}{l}0.253 \\
0.166\end{array}$ & • & • & & : \\
\hline 1944 & & 0.287 & - & - & & - \\
\hline 1945 & & 0.286 & 0.287 & - & & - \\
\hline 1940 & & 0.339 & 0.328 & $\bullet$ & & - \\
\hline 1947 & · & 0.321 & 0.285 & - & & • \\
\hline 1948 & & 0.266 & $0 \cdot 333$ & - & & - \\
\hline 1949 & & 0.320 & 0.239 & - & & - \\
\hline 1950 & & 0.333 & 0.311 & 0.269 & & - \\
\hline 1951 & & 0.172 & 0.245 & 0.237 & & • \\
\hline 1952 & & 0.332 & 0.393 & 0.237 & & - \\
\hline 1953 & & 0.271 & 0.189 & 0.236 & & - \\
\hline 1954 & & 0.392 & 0.393 & 0.311 & & - \\
\hline 1955 & & 0.289 & 0.257 & 0.311 & & 0.269 \\
\hline 1956 & & 0.260 & 0.229 & 0.140 & & 0.182 \\
\hline 1957 & & 0.402 & 0.379 & 0.357 & & 0.285 \\
\hline 1958 & & 0.265 & 0.261 & 0.255 & & 0.125 \\
\hline $\begin{array}{l}1959 \\
1960\end{array}$ & & $\begin{array}{l}0.210 \\
0.236\end{array}$ & $\begin{array}{l}0.297 \\
0.167\end{array}$ & $\begin{array}{l}0.196 \\
0.086\end{array}$ & - & $\begin{array}{l}0.155 \\
0.112\end{array}$ \\
\hline 1961 & & 0.230 & 0.183 & 0.124 & · & 0.093 \\
\hline 1962 & & 0.296 & 0.260 & 0.190 & & 0.262 \\
\hline 1903 & & 0.473 & 0.377 & 0.251 & $\cdot$ & 0.266 \\
\hline 1964 & & 0.432 & 0.260 & 0.180 & & 0.083 \\
\hline 1905 & & 0.390 & 0.288 & 0.222 & & 0.124 \\
\hline 1960 & & 0.374 & 0.444 & 0.279 & & 0.181 \\
\hline 1967 & & 0.314 & 0.237 & 0.084 & & 0.057 \\
\hline 1960 & & 0.552 & 0.272 & 0.204 & & 0.100 \\
\hline 1969 & & 0.406 & 0.237 & 0.173 & & 0.053 \\
\hline 1970 & & 0.488 & 0.221 & 0.052 & & 0.044 \\
\hline 1971 & & 0.445 & 0.136 & 0.077 & & - \\
\hline 1972 & & 0.302 & 0.181 & 0.030 & & 0.028 \\
\hline 1973 & & 0.379 & 0.135 & - & & 0.041 \\
\hline $\begin{array}{l}1974 \\
1975\end{array}$ & & $\begin{array}{l}0.301 \\
0.313\end{array}$ & $\begin{array}{l}0.109 \\
0.096\end{array}$ & $\begin{array}{l}0.043 \\
0.021\end{array}$ & & : \\
\hline 1970 & & 0.390 & 0.141 & 0.045 & & : \\
\hline 1977 & $\therefore$ & 0.368 & 0.094 & • & & - \\
\hline 1978 & & 0.343 & 0.093 & 0.012 & & - \\
\hline 1979 & & 0.397 & 0.093 & 0.020 & & • \\
\hline 1980 & & 0.220 & 0.077 & • & & - \\
\hline 1901 & & 0.301 & 0.042 & - & & - \\
\hline 1982 & & 0.235 & 0.013 & - & & - \\
\hline
\end{tabular}


fertility rates, $1940-82$

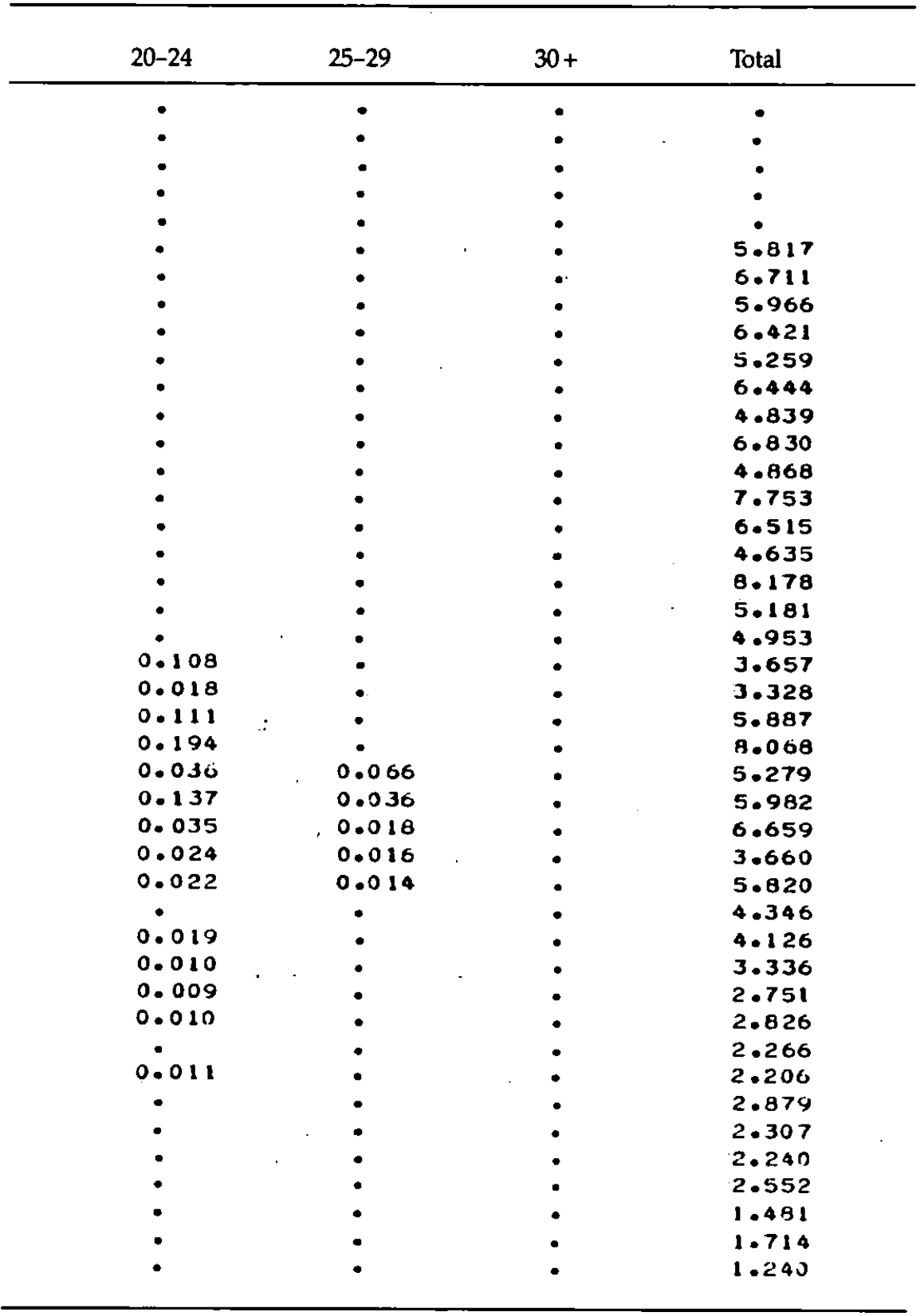


Basic Table 40.A. Total Jiangsu Province (10): duration-specific fertility

\begin{tabular}{|c|c|c|c|c|}
\hline $\begin{array}{l}\text { Birth year } \\
\text { of child }\end{array}$ & $0-4$ & $5-9$ & $10-14$ & $15-19$ \\
\hline 1940 & 0.285 & - & - & - \\
\hline 1941 & 0.293 & - & - & - \\
\hline 1942 & 0.238 & - & - & - \\
\hline 1943 & 0.308 & - & - & - \\
\hline 1944 & 0.285 & - & • & - \\
\hline 1945 & 0.277 & 0.258 & - & - \\
\hline 1946 & 0.282 & 0.257 & - & • \\
\hline 1947 & 0.292 & 0.298 & - & - \\
\hline 1948 & 0.269 & 0.258 & - & - \\
\hline 1949 & 0.308 & 0.273 & • & - \\
\hline 1950 & 0.270 & 0.256 & 0.210 & - \\
\hline 1951 & 0.280 & 0.272 & 0.223 & . \\
\hline 1952 & 0.336 & 0.277 & 0.278 & • \\
\hline 1953 & 0.309 & 0.269 & 0.239 & - \\
\hline 1954 & 0.309 & 0.345 & 0.235 & - \\
\hline 1955 & 0.335 & 0.323 & 0.233 & 0.189 \\
\hline 1956 & 0.318 & 0.266 & 0.240 & 0.176 \\
\hline 1957 & 0.342 & 0.327 & 0.252 & 0.203 \\
\hline 1958 & 0.267 & 0.267 & 0.230 & 0.164 \\
\hline 1959 & 0.236 & 0.207 & 0.170 & 0.134 \\
\hline 1960 & 0.206 & 0.217 & 0.172 & 0.122 \\
\hline 1961 & 0.195 & 0.184 & 0.150 & 0.098 \\
\hline 1902 & 0.311 & 0.303 & 0.262 & 0.189 \\
\hline 1963 & 0.381 & 0.355 & 0.310 & 0.243 \\
\hline 1904 & 0.347 & 0.286 & 0.242 & 0.157 \\
\hline 1965 & 0.368 & 0.302 & 0.230 & 0.172 \\
\hline 1966 & 0.391 & 0.306 & 0.257 & 0.179 \\
\hline 1967 & 0.325 & 0.280 & 0.186 & 0.131 \\
\hline 1968 & 0.402 & 0.346 & 0.209 & 0.148 \\
\hline 1969 & 0.363 & 0.274 & 0.193 & 0.133 \\
\hline 1970 & 0.403 & 0.256 & 0.173 & 0.135 \\
\hline 1971 & 0.384 & 0.2 .25 & 0.164 & 0.100 \\
\hline 1972 & 0.348 & 0.179 & 0.100 & 0.074 \\
\hline 1973 & 0.321 & 0.187 & 0.080 & 0.049 \\
\hline 1974 & 0.369 & 0.156 & 0.072 & 0.0 .36 \\
\hline 1975 & 0.342 & 0.154 & 0.049 & 0.038 \\
\hline 1976 & 0.364 & 0.138 & 0.047 & 0.026 \\
\hline 1977 & 0.340 & 0.114 & 0.046 & 0.024 \\
\hline 1978 & 0.321 & 0.120 & 0.040 & 0.020 \\
\hline 1979 & 0.321 & 0.108 & 0.036 & 0.012 \\
\hline 1980 & 0.250 & 0.071 & 0.016 & 0.006 \\
\hline 1981 & 0.330 & 0.077 & 0.013 & 0.004 \\
\hline 1982 & 0.272 & 0.073 & 0.012 & 0.007 \\
\hline
\end{tabular}


rates, $1940-82$

\begin{tabular}{|c|c|c|c|}
\hline $20-24$ & $25-29$ & $30+$ & Total \\
\hline - & $\bullet$ & - & - \\
\hline - & - & - & - \\
\hline - & - & - & - \\
\hline - & - & - & - \\
\hline - & - & - & - \\
\hline - & - & - & 5.355 \\
\hline - & - & - & 5.368 \\
\hline$\bullet$ & $\bullet$ & - & 6.048 \\
\hline - & - & - & 5.323 \\
\hline - & - & - & 5.762 \\
\hline$\bullet$ & - & - & 5.216 \\
\hline - & - & - & 5.497 \\
\hline - & $\bullet$ & - & 6.297 \\
\hline - & - & - & 5.747 \\
\hline - & $\bullet$ & - & 6.331 \\
\hline - & - & - & 6.185 \\
\hline - & - & - & 5.717 \\
\hline - & - & - & 6.441 \\
\hline - & - & - & $5 \cdot 336$ \\
\hline - & - & - & 4.272 \\
\hline 0.059 & - & - & 3.997 \\
\hline 0.069 & - & • & 3.592 \\
\hline 0.111 & - & - & 6.065 \\
\hline 0.122 & - & - & 7.294 \\
\hline 0.092 & 0.036 & 0.005 & 5.820 \\
\hline 0.097 & 0.026 & 0.003 & 5.994 \\
\hline 0.098 & 0.022 & 0.005 & 6.288 \\
\hline 0.059 & 0.024 & - & 5.022 \\
\hline 0.092 & 0.018 & - & 6.031 \\
\hline 0.077 & 0.014 & 0.002 & 5.290 \\
\hline 0.069 & 0.009 & - & 5.230 \\
\hline 0.056 & 0.012 & 0.002 & 4.710 \\
\hline 0.045 & 0.012 & - & 3.787 \\
\hline 0.029 & 0.007 & 0.001 & 3.363 \\
\hline 0.021 & 0.009 & - & 3.315 \\
\hline 0.013 & 0.005 & - & 3.013 \\
\hline 0.013 & 0.005 & 0.001 & 2.966 \\
\hline 0.009 & 0.002 & - & 2.672 \\
\hline 0.003 & 0.003 & - & 2.535 \\
\hline 0.003 & 0.001 & 0.001 & 2.406 \\
\hline 0.002 & 0.001 & - & 1.725 \\
\hline 0.002 & 0.001 & - & 2.129 \\
\hline 0.003 & - & 0.003 & 1.850 \\
\hline
\end{tabular}


Basic Table 40.B. Urban Jiangsu Province (10): duration-specific fertility

\begin{tabular}{|c|c|c|c|c|}
\hline $\begin{array}{l}\text { Birth year } \\
\text { of child }\end{array}$ & $0-4$ & $5-9$ & $10-14$ & $15-19$ \\
\hline 1940 & 0.286 & - & - & - \\
\hline 1941 & 0.314 & - & - & - \\
\hline 1942 & 0.235 & - & - & - \\
\hline 1943 & 0.279 & - & - & - \\
\hline 1944 & 0.321 & - & - & - \\
\hline 1945 & 0.279 & 0.222 & . & - \\
\hline 1946 & 0.270 & 0.244 & - & • \\
\hline 1947 & 0.340 & 0.285 & - & - \\
\hline 1948 & 0.271 & 0.249 & - & - \\
\hline 1949 & 0.307 & 0.338 & - & - \\
\hline 1950 & 0.328 & 0.285 & 0.186 & - \\
\hline 1951 & 0.346 & 0.208 & 0.224 & - \\
\hline 1952 & 0.346 & 0.271 & 0.260 & - \\
\hline 1953 & 0.285 & 0.241 & 0.158 & - \\
\hline 1954 & 0.364 & 0.415 & 0.212 & - \\
\hline 1955 & 0.325 & 0.323 & 0.162 & 0.150 \\
\hline 1956 & 0.364 & 0.245 & 0.191 & 0.179 \\
\hline 1957 & 0.420 & 0.326 & 0.223 & 0.149 \\
\hline 1958 & 0.301 & 0.264 & 0.216 & 0.139 \\
\hline 1959 & 0.337 & 0.303 & $0.1 \in 5$ & 0.126 \\
\hline 1960 & 0.285 & 0.266 & 0.181 & 0.106 \\
\hline 1961 & 0.275 & 0.218 & 0.190 & 0.124 \\
\hline 1962 & 0.363 & 0.276 & 0.170 & 0.128 \\
\hline 1963 & 0.421 & 0.329 & 0.310 & 0.172 \\
\hline 1904 & 0.345 & 0.245 & 0.144 & 0.061 \\
\hline 1905 & 0.356 & 0.170 & 0.118 & 0.044 \\
\hline 1960 & 0.330 & 0.174 & 0.113 & 0.045 \\
\hline 1967 & 0.260 & $0 \cdot 199$ & 0.081 & 0.030 \\
\hline 1908 & 0.407 & 0.210 & 0.056 & 0.025 \\
\hline 1969 & 0.311 & 0.177 & 0.056 & 0.021 \\
\hline 1970 & 0.380 & 0.186 & 0.074 & 0.015 \\
\hline 1971 & 0.364 & 0.117 & 0.057 & 0.028 \\
\hline 1972 & 0.292 & 0.096 & 0.039 & 0.014 \\
\hline 1973 & 0.311 & 0.140 & 0.024 & 0.004 \\
\hline 1974 & 0.291 & 0.081 & 0.044 & $\bullet$ \\
\hline 1975 & 0.385 & 0.092 & 0.028 & 0.017 \\
\hline 1970 & 0.312 & 0.084 & 0.020 & - \\
\hline 1977 & 0.310 & 0.084 & 0.004 & 0.004 \\
\hline 1970 & 0.266 & 0.068 & 0.008 & - \\
\hline 1979 & 0.247 & 0.103 & 0.019 & 0.004 \\
\hline 1980 & 0.186 & 0.040 & 0.003 & - \\
\hline 1981 & 0.365 & 0.041 & 0.003 & - \\
\hline 1982 & 0.229 & 0.014 & $\bullet$ & • \\
\hline
\end{tabular}


rates, $1940-82$

\begin{tabular}{|c|c|c|c|c|c|c|}
\hline & \multicolumn{2}{|l|}{$20-24$} & \multirow{2}{*}{$\begin{array}{l}\text { 25-29 } \\
\qquad\end{array}$} & . & \multirow{2}{*}{$\frac{30+}{.}$} & Total \\
\hline & $\bullet$ & & & & & - \\
\hline & - & & - & & - & - \\
\hline & • & & $\bullet$ & & - & - \\
\hline & $\bullet$ & & • & & $\bullet$ & • \\
\hline & • & & • & & - & - \\
\hline & - & & • & & - & 4.970 \\
\hline & - & & - & & - & $5 \cdot 268$ \\
\hline & - & & • & & - & 6.285 \\
\hline & - & & - & & - & 5.352 \\
\hline & - & & - & & - & 6.977 \\
\hline & - & & - & & - & 5.668 \\
\hline & - & & - & & - & 5.451 \\
\hline & - & & - & & - & 6.298 \\
\hline & - & & - & & - & 4.832 \\
\hline & - & & - & & - & 7.169 \\
\hline & - & & - & & - & 5.540 \\
\hline & - & & - & & - & 5.634 \\
\hline & - & & - & & - & 6.401 \\
\hline & • & . & • & & - & $5 \cdot 330$ \\
\hline & - & & - & & - & 5.342 \\
\hline & 0.064 & & - & & - & 4.675 \\
\hline & 0.083 & & - & & - & 4.615 \\
\hline & 0.037 & & - & & - & 5.023 \\
\hline & 0.067 & & - & & - & 6.716 \\
\hline & 0.052 & & 0.026 & & - & 4.365 \\
\hline & 0.039 & . & 0.021 & & - & 3.744 \\
\hline & 0.017 & & 0.006 & & - & 3.428 \\
\hline & 0.005 & & - & & - & 2.879 \\
\hline & 0.025 & & - & & - & 3.617 \\
\hline & 0.009 & . & - & & - & 2.868 \\
\hline & 0.015 & & - & & - & 3.350 \\
\hline & 0.005 & & 0.006 & & - & 2.881 \\
\hline & 0.005 & & • & & - & 2.231 \\
\hline & - & & - & & - & $2 \cdot 394$ \\
\hline & & & - & & - & 2.084 \\
\hline & - & . & - & & - & 2.606 \\
\hline & - & & - & & - & 2.082 \\
\hline . & - & & - & & - & 2.012 \\
\hline. & - & $\tau$ & - & & . & 1.714 \\
\hline & & & - & & - & 1.805 \\
\hline & - & & - & & - & 1.145 \\
\hline & - & & & & - & 2.047 \\
\hline & $\bullet$ & . & - & & - & 1.215 \\
\hline
\end{tabular}


Basic Table 40.C. Rural Jiangsu Province (10): duration-specific fertility

\begin{tabular}{|c|c|c|c|c|}
\hline $\begin{array}{l}\text { Birth year } \\
\text { of child }\end{array}$ & $0-4$ & $5-9$ & $10-14$ & $15-19$ \\
\hline 1440 & 0.285 & - & - & - \\
\hline 1941 & 0.289 & - & • & • \\
\hline 1942 & 0.239 & • & - & $\bullet$ \\
\hline 1943 & 0.314 & - & - & - \\
\hline 1944 & 0.279 & - & - & $\bullet$ \\
\hline 1945 & 0.277 & 0.264 & - & - \\
\hline 1946 & 0.284 & 0.259 & - & $\bullet$ \\
\hline 1947 & 0.284 & 0.300 & - & $\bullet$ \\
\hline 1948 & 0.268 & 0.260 & - & - \\
\hline 1949 & 0.309 & 0.261 & - & - \\
\hline 1950 & 0.259 & 0.251 & 0.215 & - \\
\hline 1951 & 0.268 & 0.283 & 0.223 & - \\
\hline 1952 & 0.334 & 0.278 & 0.282 & $\bullet$ \\
\hline 1953 & 0.313 & 0.274 & 0.253 & - \\
\hline 1954 & 0.300 & 0.330 & 0.239 & - \\
\hline 1955 & 0.337 & 0.323 & 0.246 & 0.196 \\
\hline 1956 & 0.309 & 0.270 & 0.249 & 0.176 \\
\hline 1957 & 0.327 & 0.328 & 0.257 & 0.213 \\
\hline 1958 & 0.260 & 0.268 & 0.232 & 0.169 \\
\hline 1959 & 0.213 & 0.191 & 0.171 & 0.136 \\
\hline 1960 & 0.188 & 0.208 & 0.170 & 0.124 \\
\hline 1961 & 0.177 & 0.177 & 0.143 & 0.094 \\
\hline 1962 & 0.299 & 0.308 & 0.279 & 0.200 \\
\hline 1963 & 0.373 & 0.361 & 0.310 & 0.257 \\
\hline 1904 & 0.347 & 0.295 & 0.259 & 0.176 \\
\hline 1965 & 0.370 & 0.332 & 0.250 & 0.195 \\
\hline 1966 & 0.403 & 0.337 & 0.283 & 0.203 \\
\hline 1967 & 0.337 & 0.298 & 0.206 & 0.149 \\
\hline 1968 & 0.401 & 0.374 & 0.243 & 0.170 \\
\hline 1969 & 0.374 & 0.293 & 0.224 & 0.153 \\
\hline 1970 & 0.408 & 0.269 & 0.196 & 0.156 \\
\hline 1971 & 0.388 & 0.246 & 0.188 & 0.113 \\
\hline 1972 & 0.359 & 0.195 & 0.113 & 0.085 \\
\hline 1973 & 0.323 & 0.195 & 0.091 & 0.059 \\
\hline 1974 & 0.384 & 0.170 & 0.077 & 0.044 \\
\hline 1975 & $0 \cdot 335$ & 0.167 & 0.053 & 0.043 \\
\hline 1976 & 0.373 & 0.148 & 0.052 & 0.032 \\
\hline 1977 & 0.345 & 0.119 & 0.053 & 0.028 \\
\hline 1978 & 0.330 & 0.130 & 0.046 & 0.024 \\
\hline 1979 & 0.332 & 0.109 & 0.039 & 0.013 \\
\hline 1980 & 0.261 & 0.076 & 0.018 & 0.007 \\
\hline 1981 & 0.323 & 0.083 & 0.015 & 0.005 \\
\hline 1982 & 0.281 & 0.083 & 0.014 & 0.008 \\
\hline
\end{tabular}


rates, $1940-82$

\begin{tabular}{|c|c|c|c|}
\hline $20-24$ & $25-29$ & $30+$ & Total \\
\hline - & - & - & - \\
\hline - & - & - & • \\
\hline • & - & - & - \\
\hline$\bullet$ & $\bullet$ & - & $\bullet$ \\
\hline - & - & - & - \\
\hline - & - & • & 5.42 .3 \\
\hline$\bullet$ & - & - & $5 \cdot 386$ \\
\hline - & - & - & 6.006 \\
\hline - & - & - & 5.318 \\
\hline • & - & - & 5.541 \\
\hline - & - & - & 5.134 \\
\hline - & - & - & 5.505 \\
\hline - & - & - & 6.297 \\
\hline - & - & - & 5.910 \\
\hline - & - & - & 0.181 \\
\hline - & - & - & 6.301 \\
\hline - & - & - & 5.732 \\
\hline • & - & - & 6.448 \\
\hline • & - & - & 5.337 \\
\hline$\bullet$ & - & - & 4.070 \\
\hline 0.059 & - & - & 3.869 \\
\hline 0.066 & - & - & 3.398 \\
\hline 0.124 & - & - & 6.264 \\
\hline 0.132 & - & - & 7.404 \\
\hline 0.099 & 0.037 & 0.006 & 6.096 \\
\hline 0.108 & 0.027 & 0.004 & 6.432 \\
\hline 0.112 & 0.025 & 0.005 & 6.841 \\
\hline 0.069 & 0.023 & - & 5.437 \\
\hline 0.093 & 0.021 & - & 0.511 \\
\hline 0.091 & 0.016 & 0.003 & 5.769 \\
\hline 0.079 & 0.011 & $\bullet$ & 5.597 \\
\hline 0.065 & 0.013 & 0.002 & 5.075 \\
\hline 0.052 & 0.014 & - & 4.091 \\
\hline 0.034 & 0.008 & 0.001 & 3.554 \\
\hline 0.025 & 0.010 & - & 3.553 \\
\hline 0.016 & 0.005 & - & 3.100 \\
\hline 0.015 & 0.006 & 0.001 & 3.135 \\
\hline 0.010 & 0.003 & - & 2.799 \\
\hline 0.003 & 0.004 & - & 2.684 \\
\hline 0.004 & 0.001 & 0.001 & 2.498 \\
\hline 0.003 & 0.001 & • & 1.827 \\
\hline 0.002 & 0.001 & - & 2.141 \\
\hline 0.004 & - & 0.004 & 1.969 \\
\hline
\end{tabular}


Basic Table 41.A. Total Zhejiang Province (11): duration-specific

\begin{tabular}{|c|c|c|c|c|}
\hline $\begin{array}{l}\text { Birth year } \\
\text { of child }\end{array}$ & $0-4$ & $5-9$ & $10-14$ & $15-19$ \\
\hline 1940 & 0.222 & - & $\bullet$ & - \\
\hline 1941 & 0.266 & - & • & - \\
\hline 1942 & 0.255 & - & - & - \\
\hline 1943 & 0.267 & - & - & - \\
\hline 1944 & 0.240 & - & - & - \\
\hline 1945 & 0.260 & $0.27 \epsilon$ & • & - \\
\hline 1946 & 0.276 & 0.285 & - & - \\
\hline 1947 & 0.266 & 0.310 & - & • \\
\hline 1948 & 0.293 & 0.295 & $\bullet$ & - \\
\hline 1949 & 0.293 & 0.319 & - & - \\
\hline 1950 & 0.295 & 0.294 & 0.245 & - \\
\hline 1951 & 0.293 & 0.255 & 0.275 & - \\
\hline 1952 & 0.310 & 0.324 & 0.275 & - \\
\hline 1953 & 0.289 & 0.315 & 0.269 & - \\
\hline 1954 & 0.370 & 0.312 & 0.238 & - \\
\hline 1955 & 0.332 & 0.306 & 0.255 & 0.214 \\
\hline 1956 & 0.292 & 0.310 & 0.284 & 0.216 \\
\hline 1957 & 0.385 & 0.363 & 0.294 & 0.257 \\
\hline 1958 & 0.271 & 0.290 & 0.263 & 0.185 \\
\hline 1959 & 0.243 & 0.258 & 0.208 & 0.141 \\
\hline 1960 & 0.235 & 0.266 & 0.200 & 0.183 \\
\hline 1961 & 0.171 & 0.196 & 0.168 & 0.125 \\
\hline 1962 & 0.368 & 0.348 & 0.316 & 0.290 \\
\hline 1963 & 0.422 & 0.377 & 0.360 & 0.249 \\
\hline 1964 & 0.360 & 0.328 & 0.282 & 0.241 \\
\hline 1965 & 0.377 & 0.308 & 0.262 & 0.213 \\
\hline 1966 & 0.405 & 0.339 & 0.239 & 0.193 \\
\hline 1907 & 0.340 & 0.281 & 0.214 & 0.151 \\
\hline 1960 & 0.391 & 0.314 & 0.220 & 0.176 \\
\hline 1969 & 0.386 & 0.288 & 0.219 & 0.133 \\
\hline 1970 & 0.360 & 0.265 & 0.169 & 0.122 \\
\hline 1971 & 0.397 & 0.275 & 0.121 & 0.111 \\
\hline 1972 & 0.340 & 0.214 & 0.136 & 0.083 \\
\hline 1973 & 0.357 & 0.190 & 0.115 & 0.071 \\
\hline 1974 & 0.367 & $0: 190$ & 0.080 & 0.053 \\
\hline 1975 & 0.326 & 0.169 & 0.088 & 0.055 \\
\hline 1976 & 0.366 & 0.173 & 0.085 & 0.040 \\
\hline 1977 & 0.355 & 0.157 & 0.062 & 0.038 \\
\hline 1978 & 0.341 & 0.162 & 0.062 & 0.027 \\
\hline 1979 & 0.391 & 0.160 & 0.052 & 0.014 \\
\hline 1980 & 0.293 & 0.111 & 0.046 & 0.010 \\
\hline 1981 & 0.368 & 0.131 & 0.032 & 0.012 \\
\hline 1962 & 0.312 & 0.128 & 0.038 & 0.021 \\
\hline
\end{tabular}


fertility rates, $1940-82$

\begin{tabular}{|c|c|c|c|}
\hline $20-24$ & $25-29$ & $30+$ & Total \\
\hline • & - & $\bullet$ & - \\
\hline$\bullet$ & $\bullet$ & - & - \\
\hline - & - & - & - \\
\hline - & - & • & - \\
\hline - & - & - & - \\
\hline - & - & - & 5.537 \\
\hline - & - & - & 5.732 \\
\hline - & - & - & 6.138 \\
\hline$\bullet$ & - & - & 6.022 \\
\hline - & - & • & 6.385 \\
\hline - & - & - & 5.930 \\
\hline • & - & - & 5.843 \\
\hline - & - & - & 6.496 \\
\hline - & - & - & 6.302 \\
\hline - & - & - & 6.404 \\
\hline - & - & - & $6 \cdot 348$ \\
\hline - & - & - & 6.370 \\
\hline - & - & - & 7.450 \\
\hline - & - & • & 5.826 \\
\hline - & - & - & 4.883 \\
\hline 0.101 & - & • & 5.087 \\
\hline 0.068 & - & $\bullet$ & 3.765 \\
\hline 0.110 & - & - & 7.415 \\
\hline 0.172 & - & - & 8.165 \\
\hline 0.125 & 0.040 & 0.009 & 6.925 \\
\hline 0.132 & 0.045 & 0.004 & 6.700 \\
\hline 0.140 & 0.032 & 0.003 & 6.753 \\
\hline 0.091 & 0.031 & - & 5.547 \\
\hline 0.101 & 0.026 & - & 6.137 \\
\hline 0.088 & 0.021 & 0.002 & 5.683 \\
\hline 0.057 & 0.019 & 0.002 & 4.970 \\
\hline 0.064 & 0.016 & 0.002 & 4.928 \\
\hline 0.034 & 0.014 & 0.002 & 4.114 \\
\hline 0.041 & 0.014 & 0.002 & 3.954 \\
\hline 0.032 & 0.007 & - & 3.643 \\
\hline 0.039 & 0.006 & - & 3.419 \\
\hline 0.021 & 0.006 & • & 3.455 \\
\hline 0.019 & 0.005 & 0.002 & 3.185 \\
\hline 0.008 & 0.004 & 0.003 & 3.032 \\
\hline 0.010 & 0.002 & $\bullet$ & 3.143 \\
\hline 0.007 & 0.003 & 0.004 & 2.368 \\
\hline 0.006 & 0.004 & - & 2.761 \\
\hline 0.008 & 0.005 & - & 2.567 \\
\hline
\end{tabular}


Basic Table 41.B. Urban Zhejiang Province (11): duration-specific

\begin{tabular}{|c|c|c|c|c|}
\hline $\begin{array}{l}\text { Birth year } \\
\text { of child }\end{array}$ & $0-4$ & $5-9$ & $10-14$ & $15-19$ \\
\hline 1940 & 0.091 & - & - & - \\
\hline 1941 & 0.184 & - & - & - \\
\hline 1942 & 0.282 & - & - & - \\
\hline 1943 & 0.350 & - & - & - \\
\hline 1944 & 0.285 & - & - & - \\
\hline 1945 & 0.254 & 0.238 & $\bullet$ & - \\
\hline 1946 & 0.316 & 0.220 & - & - \\
\hline 1947 & 0.402 & 0.405 & - & - \\
\hline 1948 & 0.239 & 0.333 & - & - \\
\hline 1948 & 0.428 & 0.272 & - & - \\
\hline 1950 & 0.404 & 0.406 & 0.238 & - \\
\hline 1951 & 0.334 & 0.257 & 0.165 & - \\
\hline 1952 & 0.314 & 0.380 & 0.246 & - \\
\hline 1953 & 0.295 & 0.220 & 0.266 & - \\
\hline 1954 & 0.483 & 0.300 & 0.220 & - \\
\hline 1955 & 0.288 & 0.343 & 0.152 & 0.128 \\
\hline 1940 & 0.284 & 0.283 & 0.234 & 0.220 \\
\hline 1957 & 0.411 & 0.433 & 0.326 & 0.176 \\
\hline 1958 & 0.285 & 0.284 & 0.210 & 0.200 \\
\hline 1959 & 0.285 & 0.288 & 0.257 & 0.039 \\
\hline $\begin{array}{l}1900 \\
1961\end{array}$ & $\begin{array}{l}0.337 \\
0.240\end{array}$ & $\begin{array}{l}0.355 \\
0.199\end{array}$ & $\begin{array}{l}0.172 \\
0.162\end{array}$ & $\begin{array}{l}0.266 \\
0.117\end{array}$ \\
\hline $\begin{array}{l}1962 \\
1963\end{array}$ & $\begin{array}{l}0.363 \\
0.462\end{array}$ & $\begin{array}{l}0.219 \\
0.377\end{array}$ & $\begin{array}{l}0.260 \\
0.306\end{array}$ & $\begin{array}{l}0.141 \\
0.162\end{array}$ \\
\hline 1904 & 0.370 & 0.147 & 0.134 & 0.171 \\
\hline 1965 & 0.351 & 0.202 & 0.086 & 0.051 \\
\hline 1966 & 0.262 & 0.138 & 0.076 & 0.061 \\
\hline 1967 & 0.340 & 0.098 & 0.073 & 0.065 \\
\hline 1968 & 0.338 & 0.132 & 0.046 & 0.055 \\
\hline 1909 & 0.299 & 0.172 & 0.018 & 0.041 \\
\hline 1970 & 0.337 & 0.120 & 0.106 & 0.019 \\
\hline 1971 & 0.360 & 0.081 & 0.037 & 0.009 \\
\hline 1972 & 0.306 & 0.119 & 0.049 & - \\
\hline 1973 & 0.277 & 0.101 & 0.047 & 0.009 \\
\hline 1974 & 0.323 & 0.118 & 0.018 & 0.018 \\
\hline 1975 & 0.208 & 0.078 & 0.046 & 0.019 \\
\hline 1976 & 0.279 & 0.104 & 0.030 & 0.009 \\
\hline 1977 & 0.239 & 0.083 & 0.037 & 0.010 \\
\hline 1978 & 0.261 & 0.065 & 0.018 & - \\
\hline 1979 & 0.333 & 0.087 & 0.018 & - \\
\hline 1980 & 0.191 & 0.027 & - & - \\
\hline 1981 & 0.318 & 0.010 & - & - \\
\hline 1482 & 0.194 & - & - & - \\
\hline
\end{tabular}


fertility rates, $1940-82$

\begin{tabular}{|c|c|c|c|}
\hline $20-24$ & $25-29$ & $30+$ & Total \\
\hline$\bullet$ & - & - & - \\
\hline$\bullet$ & $\bullet$ & • & • \\
\hline - & - & $\bullet$ & - \\
\hline - & - & - & - \\
\hline$\bullet$ & - & - & - \\
\hline$\bullet$ & $\bullet$ & - & 5.093 \\
\hline$\bullet$ & $\bullet$ & - & $5 \cdot 126$ \\
\hline$\bullet$ & - & - & 8.524 \\
\hline - & • & - & 6.550 \\
\hline - & - & - & 6.512 \\
\hline • & - & - & 7.517 \\
\hline - & - & - & 5.283 \\
\hline - & - & - & 6.929 \\
\hline$\bullet$ & - & - & 5.668 \\
\hline • & - & - & 6.867 \\
\hline • & • & - & 5.274 \\
\hline - & - & • & 5.991 \\
\hline - & - & - & 7.813 \\
\hline - & • & $\bullet$ & 5.719 \\
\hline - & - & - & 4.999 \\
\hline 0.091 & • & - & 6.343 \\
\hline 0.055 & - & • & 4.004 \\
\hline 0.088 & - & • & 5.547 \\
\hline 0.083 & - & - & 7.191 \\
\hline 0.065 & - & $\bullet$ & 4.435 \\
\hline - & 0.018 & - & 3.542 \\
\hline - & 0.018 & - & 2.776 \\
\hline 0.022 & $\bullet$ & - & 2.987 \\
\hline 0.019 & • & - & 2.950 \\
\hline 0.032 & 0.013 & - & 2.876 \\
\hline • & $\bullet$ & - & 2.912 \\
\hline$\bullet$ & • & - & 2.434 \\
\hline - & • & - & 2.374 \\
\hline - & - & - & 2.172 \\
\hline 0.010 & • & - & 2.438 \\
\hline$\bullet$ & - & - & I.755 \\
\hline - & - & • & 2.114 \\
\hline - & $\bullet$ & - & 1.841 \\
\hline - & $\bullet$ & • & 1.723 \\
\hline - & - & - & 2.194 \\
\hline - & - & - & 1.091 \\
\hline - & - & - & 1.037 \\
\hline - & - & • & 0.969 \\
\hline
\end{tabular}


Basic Table 41.C. Rural Zhejiang Province (11): duration-specific

\begin{tabular}{|c|c|c|c|c|}
\hline $\begin{array}{l}\text { Birth year } \\
\text { of child }\end{array}$ & $0-4$ & $5-9$ & $10-14$ & $15-19$ \\
\hline 1940 & 0.237 & - & - & - \\
\hline 1941 & 0.275 & - & - & - \\
\hline $134 z$ & 0.252 & - & - & - \\
\hline 1943 & 0.257 & - & - & - \\
\hline 1944 & 0.233 & - & - & - \\
\hline 1945 & 0.261 & 0.281 & - & - \\
\hline 1940 & 0.270 & 0.292 & - & - \\
\hline 1947 & 0.243 & 0.299 & - & - \\
\hline 1948 & 0.302 & 0.290 & - & - \\
\hline 1949 & 0.274 & 0.327 & - & - \\
\hline 1950 & 0.280 & 0.276 & 0.246 & - \\
\hline 1951 & 0.287 & 0.255 & 0.288 & - \\
\hline 1952 & 0.310 & 0.315 & 0.278 & - \\
\hline 1953 & 0.289 & 0.332 & 0.269 & - \\
\hline 1954 & 0.355 & 0.314 & 0.241 & - \\
\hline 1955 & 0.339 & 0.301 & 0.270 & 0.224 \\
\hline 1956 & 0.293 & 0.314 & 0.292 & 0.216 \\
\hline 1957 & 0.381 & 0.354 & 0.289 & 0.266 \\
\hline 1958 & 0.268 & 0.291 & 0.272 & 0.183 \\
\hline 1959 & 0.236 & 0.254 & 0.201 & 0.157 \\
\hline 1960 & 0.217 & 0.252 & 0.205 & 0.170 \\
\hline 1901 & 0.159 & 0.196 & 0.169 & 0.126 \\
\hline 1902 & 0.369 & 0.370 & 0.323 & 0.315 \\
\hline 1903 & 0.416 & 0.377 & 0.367 & 0.264 \\
\hline 1904 & 0.358 & 0.301 & 0.303 & 0.251 \\
\hline 1505 & 0.380 & 0.327 & 0.289 & 0.236 \\
\hline 1966 & 0.423 & 0.374 & 0.265 & 0.212 \\
\hline 1907 & 0.340 & 0.311 & 0.238 & 0.162 \\
\hline 1900 & 0.397 & 0.342 & 0.251 & 0.191 \\
\hline 1969 & 0.396 & 0.305 & 0.256 & 0.145 \\
\hline 1970 & 0.362 . & 0.285 & 0.181 & 0.138 \\
\hline 1971 & 0.401 & 0.279 & 0.136 & 0.127 \\
\hline 1972 & 0.344 & 0.226 & 0.150 & 0.097 \\
\hline 1973 & 0.368 & 0.201 & 0.126 & 0.082 \\
\hline 1974 & 0.373 & 0.199 & 0.089 & 0.059 \\
\hline 1975 & 0.340 & 0.180 & 0.094 & 0.062 \\
\hline 1976 & 0.375 & 0.102 & 0.091 & 0.046 \\
\hline 1977 & 0.368 & 0.166 & 0.006 & 0.043 \\
\hline 1978 & 0.349 & 0.175 & 0.067 & 0.031 \\
\hline 1979 & 0.396 & 0.169 & 0.056 & 0.016 \\
\hline 1900 & 0.303 & 0.121 & $0.05 t$ & 0.011 \\
\hline 1981 & 0.374 & 0.144 & 0.036 & 0.013 \\
\hline 1982 & 0.328 & 0.142 & 0.043 & 0.024 \\
\hline
\end{tabular}


fertility rates, $1940-82$

\begin{tabular}{|c|c|c|c|}
\hline $20-24$ & $25-29$ & $30+$ & Total \\
\hline - & - & • & - \\
\hline - & - & - & - \\
\hline - & - & - & • \\
\hline - & $\bullet$ & - & - \\
\hline - & - & - & - \\
\hline - & - & - & 5.600 \\
\hline - & - & - & 5.819 \\
\hline - & - & - & 5.785 \\
\hline - & - & - & 5.943 \\
\hline - & $\bullet$ & - & 0.367 \\
\hline - & - & - & 5.705 \\
\hline - & - & - & 5.923 \\
\hline - & - & - & 6.436 \\
\hline - & - & - & 6.391 \\
\hline - & - & - & 6.333 \\
\hline - & - & - & $\epsilon .503$ \\
\hline - & - & - & 6.425 \\
\hline - & . & - & $7 \cdot 396$ \\
\hline - & . & - & 5.842 \\
\hline - & . & - & 4.866 \\
\hline 0.102 & - & - & 4.897 \\
\hline 0.070 & - & - & 3.729 \\
\hline 0.113 & - & - & 7.094 \\
\hline 0.193 & - & - & 8.311 \\
\hline 0.135 & 0.045 & 0.010 & 7.316 \\
\hline 0.153 & 0.048 & 0.004 & 7.183 \\
\hline 0.162 & 0.034 & 0.003 & 7.301 \\
\hline 0.102 & 0.035 & - & 5.945 \\
\hline 0.115 & 0.029 & - & 6.626 \\
\hline 0.096 & 0.022 & 0.002 & 6.114 \\
\hline 0.065 & 0.022 & 0.002 & 5.274 \\
\hline 0.072 & 0.019 & 0.002 & 5.283 \\
\hline 0.038 & 0.016 & 0.002 & 4.369 \\
\hline 0.046 & 0.016 & 0.002 & 4.207 \\
\hline 0.035 & 0.008 & • & 3.810 \\
\hline 0.044 & 0.007 & - & 3.640 \\
\hline 0.024 & 0.007 & - & 3.626 \\
\hline 0.022 & 0.005 & 0.002 & $3 \cdot 352$ \\
\hline 0.010 & 0.004 & 0.003 & 3.1 .94 \\
\hline 0.012 & 0.003 & - & 3.257 \\
\hline 0.009 & 0.003 & 0.004 & 2.515 \\
\hline 0.007 & 0.004 & - & 2.390 \\
\hline 0.010 & 0.000 & - & 2.759 \\
\hline
\end{tabular}


Basic Table 42.A. Total Anhui Province (12): duration-specific fertility

\begin{tabular}{|c|c|c|c|c|}
\hline $\begin{array}{l}\text { Birth year } \\
\text { of child }\end{array}$ & $0-4$ & $5-9$ & $10-14$ & $15-19$ \\
\hline 1940 & 0.266 & - & - & - \\
\hline 1941 & 0.277 & $\bullet$ & - & - \\
\hline 1942 & 0.242 & - & - & - \\
\hline 1943 & 0.222 & - & - & - \\
\hline 1944 & 0.261 & - & - & - \\
\hline 1945 & 0.264 & 0.241 & - & - \\
\hline 1946 & 0.249 & 0.225 & - & - \\
\hline 1947 & 0.293 & 0.326 & - & - \\
\hline 1948 & 0.260 & 0.230 & - & - \\
\hline 1949 & 0.270 & 0.272 & • & - \\
\hline 1950 & 0.246 & 0.261 & 0.232 & - \\
\hline 1951 & 0.244 & 0.240 & 0.228 & - \\
\hline 1952 & 0.337 & 0.281 & 0.273 & - \\
\hline 1953 & 0.323 & 0.268 & 0.233 & - \\
\hline 1954 & 0.328 & 0.265 & 0.272 & - \\
\hline 1955 & 0.270 & 0.276 & 0.201 & 0.186 \\
\hline 1956 & 0.305 & 0.251 & 0.222 & 0.200 \\
\hline 1957 & 0.300 & 0.291 & 0.232 & 0.179 \\
\hline 1958 & 0.236 & 0.236 & 0.173 & 0.165 \\
\hline 1959 & 0.137 & 0.141 & 0.109 & 0.094 \\
\hline 1960 & 0.099 & 0.098 & 0.093 & 0.072 \\
\hline 1961 & 0.110 & 0.135 & 0.111 & 0.077 \\
\hline 1962 & 0.297 & 0.386 & 0.353 & 0.237 \\
\hline 1963 & 0.394 & 0.402 & 0.330 & 0.269 \\
\hline 1964 & 0.294 & 0.293 & 0.269 & 0.180 \\
\hline 1965 & 0.333 & 0.322 & 0.294 & 0.238 \\
\hline 1966 & 0.359 & 0.358 & 0.316 & 0.225 \\
\hline 1967 & 0.297 & 0.309 & 0.257 & 0.227 \\
\hline 1968 & 0.384 & 0.377 & 0.334 & 0.262 \\
\hline 1969 & 0.357 & 0.340 & 0.270 & 0.240 \\
\hline 1970 & 0.365 & 0.333 & 0.317 & 0.210 \\
\hline 1971 & 0.345 & 0.311 & 0.300 & 0.234 \\
\hline 1972 & 0.385 & 0.297 & 0.221 & 0.186 \\
\hline 1973 & 0.357 & 0.286 & 0.209 & 0.158 \\
\hline 1974 & 0.354 & 0.268 & 0.162 & 0.101 \\
\hline 1975 & 0.341 & 0.266 & 0.139 & 0.079 \\
\hline 1976 & 0.357 & 0.233 & 0.126 & 0.087 \\
\hline 1978 & 0.346 & 0.236 & 0.083 & 0.061 \\
\hline 1978 & 0.413 & 0.223 & 0.120 & 0.060 \\
\hline 1979 & 0.415 & 0.238 & 0.134 & 0.058 \\
\hline 1980 & 0.340 & 0.185 & 0.088 & 0.038 \\
\hline 1981 & 0.405 & 0.163 & 0.069 & 0.028 \\
\hline 1982 & 0.384 & 0.163 & 0.073 & 0.026 \\
\hline
\end{tabular}


rates, $1940-82$

\begin{tabular}{|c|c|c|c|}
\hline $20-24$ & 25-29 & $30+$ & Total \\
\hline - & - & - & - \\
\hline • & $\bullet$ & - & $\bullet$ \\
\hline - & - & - & - \\
\hline$\bullet$ & - & $\bullet$ & - \\
\hline - & $\bullet$ & $\bullet$ & - \\
\hline - & - & - & 5.042 \\
\hline - & - & - & 4.708 \\
\hline - & - & $\bullet$ & 6.475 \\
\hline - & - & - & 4.833 \\
\hline - & - & • & 5.527 \\
\hline$\bullet$ & - & - & 5.314 \\
\hline - & - & • & $5 \cdot 100$ \\
\hline - & $\bullet$ & - & 6.275 \\
\hline - & - & - & 5.740 \\
\hline - & - & - & 6.082 \\
\hline - & - & • & $5 \cdot 354$ \\
\hline - & - & • & 5.584 \\
\hline • & - & - & 5.711 \\
\hline - & $\bullet$ & - & 4.651 \\
\hline - & $\bullet$ & • & 2.746 \\
\hline 0.038 & - & - & 2.047 \\
\hline 0.040 & - & - & 2.447 \\
\hline 0.133 & - & - & 7.273 \\
\hline 0.153 & - & - & 7.993 \\
\hline 0.120 & 0.039 & - & 5.973 \\
\hline 0.126 & 0.029 & - & 6.707 \\
\hline 0.166 & 0.051 & 0.014 & 7.443 \\
\hline 0.115 & 0.022 & 0.004 & 6.152 \\
\hline 0.132 & 0.042 & 0.004 & 7.674 \\
\hline 0.127 & 0.043 & 0.003 & 6.899 \\
\hline 0.124 & 0.031 & 0.004 & 6.922 \\
\hline 0.121 & 0.026 & 0.001 & 6.688 \\
\hline 0.121 & 0.016 & 0.007 & 6.102 \\
\hline 0.087 & 0.018 & 0.004 & 5.600 \\
\hline 0.061 & 0.014 & 0.001 & 4.802 \\
\hline 0.047 & 0.019 & 0.001 & 4.455 \\
\hline 0.040 & 0.011 & 0.004 & 4.289 \\
\hline 0.040 & 0.010 & 0.001 & 3.889 \\
\hline 0.038 & 0.013 & - & 4.332 \\
\hline 0.022 & 0.010 & - & 4.388 \\
\hline 0.011 & 0.004 & 0.001 & 3.338 \\
\hline 0.012 & 0.001 & - & 3.389 \\
\hline 0.013 & 0.002 & • & 3.310 \\
\hline
\end{tabular}


Basic Table 42.B. Urban Anhui Province (12): duration-specific fertility

\begin{tabular}{|c|c|c|c|c|}
\hline $\begin{array}{l}\text { Birth year } \\
\text { of child }\end{array}$ & $0-4$ & $5-9$ & $10-14$ & $15-19$ \\
\hline 1940 & 0.155 & - & - & $\bullet$ \\
\hline 1941 & 0.426 & - & - & - \\
\hline 1942 & 0.207 & - & $\bullet$ & - \\
\hline 1943 & 0.269 & - & $\bullet$ & - \\
\hline 1944 & 0.367 & - & • & - \\
\hline 1945 & 0.269 & 0.108 & - & - \\
\hline 1946 & 0.160 & 0.142 & - & - \\
\hline 1947 & 0.176 & 0.304 & - & • \\
\hline 1948 & 0.185 & 0.209 & - & - \\
\hline 1949 & 0.274 & 0.233 & - & - \\
\hline 1950 & 0.175 & 0.202 & 0.232 & - \\
\hline 1951 & 0.266 & 0.304 & 0.199 & - \\
\hline 1952 & 0.349 & 0.264 & 0.262 & - \\
\hline 1953 & 0.337 & 0.172 & 0.150 & - \\
\hline 1954 & 0.374 & 0.320 & 0.233 & - \\
\hline 1955 & 0.328 & 0.278 & 0.236 & 0.139 \\
\hline 1956 & 0.360 & 0.266 & 0.128 & 0.255 \\
\hline 1957 & 0.400 & 0.367 & 0.205 & 0.166 \\
\hline 1958 & 0.325 & 0.238 & 0.212 & 0.239 \\
\hline 1959 & 0.238 & 0.294 & 0.217 & 0.150 \\
\hline 1960 & 0.265 & 0.164 & 0.134 & 0.118 \\
\hline 1961 & 0.123 & 0.123 & 0.106 & 0.064 \\
\hline 1962 & 0.334 & 0.376 & 0.349 & 0.161 \\
\hline 1963 & 0.445 & 0.424 & 0.296 & 0.212 \\
\hline 1964 & 0.385 & 0.279 & 0.198 & 0.137 \\
\hline 1965 & 0.354 & 0.248 & 0.239 & 0.185 \\
\hline 1966 & 0.263 & 0.221 & 0.115 & 0.097 \\
\hline 1967 & 0.323 & 0.212 & 0.172 & 0.107 \\
\hline 1968 & 0.420 & 0.215 & 0.197 & 0.090 \\
\hline 1969 & 0.351 & 0.285 & 0.180 & 0.110 \\
\hline 1970 & 0.343 & 0.217 & 0.182 & 0.060 \\
\hline 1971 & 0.340 & 0.170 & 0.131 & 0.057 \\
\hline 1972 & 0.273 & 0.219 & 0.114 & 0.031 \\
\hline 1973 & 0.283 & 0.118 & 0.067 & 0.053 \\
\hline 1974 & 0.325 & 0.182 & 0.062 & 0.016 \\
\hline 1975 & 0.413 & 0.086 & 0.032 & 0.033 \\
\hline 1976 & 0.362 & 0.164 & 0.034 & 0.033 \\
\hline 1977 & 0.302 & 0.143 & 0.021 & $\bullet$ \\
\hline 1978 & 0.417 & 0.090 & 0.053 & 0.007 \\
\hline 1979 & 0.348 & 0.163 & 0.078 & - \\
\hline 1980 & 0.318 & 0.056 & 0.012 & - \\
\hline 1981 & 0.289 & 0.020 & 0.013 & 0.008 \\
\hline 1982 & 0.233 & 0.015 & • & • \\
\hline
\end{tabular}


rates, $1940-82$

\begin{tabular}{|c|c|c|c|}
\hline $20-24$ & $25-29$ & $30+$ & Total \\
\hline - & - & • & • \\
\hline - & - & - & - \\
\hline$\bullet$ & $\bullet$ & - & $\bullet$ \\
\hline - & - & - & - \\
\hline - & • & • & $\bullet$ \\
\hline$\bullet$ & - & $\bullet$ & 3.090 \\
\hline$\bullet$ & - & - & 3.082 \\
\hline - & - & $\bullet$ & 5.765 \\
\hline$\bullet$ & - & - & 4.296 \\
\hline - & $\bullet$ & - & 5.124 \\
\hline - & $\bullet$ & - & 4.616 \\
\hline - & - & - & 5.625 \\
\hline - & - & - & 6.271 \\
\hline - & - & - & 4.448 \\
\hline - & - & - & 6.608 \\
\hline - & - & - & 5.669 \\
\hline - & • & - & 5.834 \\
\hline - & - & - & 6.543 \\
\hline • & - & - & 5.911 \\
\hline - & - & - & $5 \cdot 263$ \\
\hline 0.062 & - & - & 3.843 \\
\hline 0.028 & $\bullet$ & - & 2.304 \\
\hline 0.138 & - & - & 7.065 \\
\hline 0.105 & - & - & 7.681 \\
\hline 0.083 & 0.035 & - & 5.586 \\
\hline 0.017 & 0.031 & $\bullet$ & 5.369 \\
\hline 0.032 & $\bullet$ & - & 3.637 \\
\hline 0.044 & - & - & 4.298 \\
\hline 0.079 & - & 0.022 & 5.123 \\
\hline 0.023 & - & - & 4.743 \\
\hline 0.021 & - & - & 4.113 \\
\hline 0.018 & 0.032 & - & 3.736 \\
\hline 0.027 & - & - & 3.322 \\
\hline 0.008 & - & - & 2.645 \\
\hline 0.007 & - & - & 2.962 \\
\hline 0.007 & 0.021 & - & 2.958 \\
\hline 0.008 & - & - & 3.003 \\
\hline 0.024 & - & • & 2.443 \\
\hline - & - & - & 2.835 \\
\hline - & 0.007 & - & 2.982 \\
\hline - & - & - & 1.931 \\
\hline - & - & - & 1.646 \\
\hline - & $\bullet$ & - & 1.242 \\
\hline
\end{tabular}


Basic Table 42.C. Rural Anhui Province (12): duration-specific fertility

\begin{tabular}{|c|c|c|c|c|}
\hline $\begin{array}{l}\text { Birth year } \\
\text { of child }\end{array}$ & $0-4$ & $5-9$ & $10-14$ & $15-19$ \\
\hline 1940 & 0.277 & - & - & - \\
\hline 1941 & 0.261 & $\bullet$ & - & • \\
\hline 1942 & 0.246 & • & - & - \\
\hline 1943 & 0.218 & - & $\bullet$ & - \\
\hline 1944 & 0.252 & - & - & - \\
\hline 1945 & 0.264 & 0.255 & - & - \\
\hline 1946 & 0.257 & 0.234 & - & - \\
\hline 1947 & 0.304 & 0.328 & - & - \\
\hline 1948 & 0.267 & 0.232 & $\bullet$ & . \\
\hline 1949 & 0.269 & 0.276 & - & $\bullet$ \\
\hline 1950 & 0.254 & 0.266 & 0.232 & . \\
\hline 1951 & 0.241 & 0.234 & 0.231 & . \\
\hline 1952 & 0.336 & 0.282 & 0.275 & . \\
\hline 1953 & 0.321 & 0.278 & 0.241 & - \\
\hline 1954 & 0.321 & 0.259 & 0.276 & - \\
\hline 1955 & 0.262 & 0.276 & 0.198 & 0.191 \\
\hline 1956 & 0.297 & 0.249 & 0.230 & 0.194 \\
\hline 1957 & 0.284 & 0.281 & 0.234 & 0.181 \\
\hline 1958 & 0.221 & 0.235 & 0.169 & 0.158 \\
\hline 1959 & 0.121 & 0.118 & 0.097 & 0.089 \\
\hline 1960 & 0.073 & 0.088 & 0.088 & 0.068 \\
\hline 1961 & 0.109 & 0.137 & 0.112 & 0.078 \\
\hline 1962 & 0.292 & 0.387 & 0.354 & 0.244 \\
\hline 1963 & 0.387 & 0.399 & 0.334 & 0.275 \\
\hline 1964 & 0.283 & 0.295 & 0.279 & 0.184 \\
\hline 1965 & 0.330 & 0.333 & 0.302 & 0.244 \\
\hline 1466 & 0.369 & 0.378 & 0.345 & 0.241 \\
\hline 1967 & 0.295 & 0.323 & 0.270 & 0.242 \\
\hline 1968 & 0.381 & 0.399 & 0.357 & 0.285 \\
\hline 1969 & 0.357 & 0.347 & 0.284 & 0.259 \\
\hline 1970 & 0.366 & 0.346 & 0.339 & 0.233 \\
\hline 1971 & 0.345 & 0.325 & 0.326 & 0.259 \\
\hline 1972 & 0.392 & 0.303 & 0.236 & 0.210 \\
\hline 1973 & 0.363 & 0.297 & 0.228 & 0.176 \\
\hline 1974 & 0.355 & 0.274 & 0.173 & 0.115 \\
\hline 1975 & 0.335 & 0.278 & 0.150 & 0.086 \\
\hline 1970 & 0.356 & 0.237 & 0.136 & 0.095 \\
\hline 1977 & 0.351 & 0.242 & 0.088 & 0.070 \\
\hline 1978 & 0.413 & 0.232 & 0.125 & 0.066 \\
\hline 1979 & 0.424 & 0.244 & 0.137 & 0.065 \\
\hline 1980 & 0.343 & 0.195 & 0.093 & 0.042 \\
\hline 1981 & 0.427 & 0.175 & 0.072 & 0.030 \\
\hline 1982 & 0.416 & 0.179 & 0.078 & 0.028 \\
\hline
\end{tabular}


rates, $1940-82$

\begin{tabular}{|c|c|c|c|}
\hline $20-24$ & $25-29$ & $30+$ & Total \\
\hline - & - & - & - \\
\hline - & - & • & - \\
\hline - & $\bullet$ & - & - \\
\hline$\bullet$ & - & $\bullet$ & $\bullet$ \\
\hline - & - & - & - \\
\hline - & - & - & 5.217 \\
\hline - & - & $\bullet$ & 4.858 \\
\hline$\bullet$ & - & - & 6.542 \\
\hline - & - & - & 4.884 \\
\hline - & - & - & 5.566 \\
\hline - & - & - & 5.383 \\
\hline - & - & - & 5.045 \\
\hline • & - & - & 6.276 \\
\hline - & - & - & 5.877 \\
\hline - & • & - & 6.023 \\
\hline - & - & - & 5.318 \\
\hline - & $\bullet$ & - & 5.556 \\
\hline - & - & - & 5.615 \\
\hline - & - & • & 4.501 \\
\hline$\bullet$ & - & - & 2.441 \\
\hline 0.035 & • & - & 1.828 \\
\hline 0.041 & - & - & 2.464 \\
\hline 0.133 & - & - & 7.298 \\
\hline 0.158 & - & - & 8.031 \\
\hline 0.123 & 0.040 & - & 6.027 \\
\hline 0.135 & 0.029 & - & 6.872 \\
\hline 0.178 & 0.056 & 0.015 & 7.913 \\
\hline 0.121 & 0.024 & 0.004 & 6.398 \\
\hline 0.137 & 0.046 & 0.002 & 8.037 \\
\hline 0.139 & 0.047 & 0.003 & 7.181 \\
\hline 0.137 & 0.033 & 0.005 & 7.289 \\
\hline 0.134 & 0.025 & 0.002 & 7.079 \\
\hline 0.133 & 0.018 & 0.007 & 6.500 \\
\hline 0.098 & 0.020 & 0.004 & 5.928 \\
\hline 0.069 & 0.015 & 0.001 & 5.015 \\
\hline 0.053 & 0.019 & 0.001 & 4.609 \\
\hline 0.045 & 0.013 & 0.004 & 4.429 \\
\hline 0.043 & 0.011 & 0.001 & 4.033 \\
\hline 0.044 & 0.014 & - & 4.474 \\
\hline 0.026 & 0.010 & - & 4.533 \\
\hline 0.013 & 0.004 & 0.001 & 3.462 \\
\hline 0.013 & 0.001 & • & 3.596 \\
\hline 0.015 & 0.002 & - & 3.595 \\
\hline
\end{tabular}


Basic Table 43.A. Total Fujian Province (13): duration-specific fertility

\begin{tabular}{|c|c|c|c|c|}
\hline $\begin{array}{l}\text { Birth year } \\
\text { of child }\end{array}$ & $0-4$ & $5-9$ & $10-14$ & $15-19$ \\
\hline 1940 & 0.208 & - & - & - \\
\hline 1941 & 0.245 & - & - & - \\
\hline 1942 & 0.181 & - & - & - \\
\hline 1943 & 0.237 & - & - & - \\
\hline 1944 & 0.223 & - & - & - \\
\hline 1945 & 0.276 & 0.211 & - & $\bullet$ \\
\hline 1946 & 0.249 & 0.277 & - & - \\
\hline 1947 & 0.273 & 0.275 & - & - \\
\hline 1948 & 0.276 & 0.259 & - & . \\
\hline 1949 & 0.284 & 0.319 & - & - \\
\hline 1950 & 0.273 & 0.324 & 0.198 & . \\
\hline 1951 & 0.279 & 0.247 & 0.225 & - \\
\hline 1952 & 0.315 & 0.345 & 0.293 & - \\
\hline 1953 & 0.325 & 0.296 & 0.251 & - \\
\hline 1954 & 0.303 & 0.326 & 0.245 & - \\
\hline 1955 & 0.317 & 0.277 & 0.342 & 0.184 \\
\hline 1956 & 0.254 & 0.263 & 0.260 & 0.229 \\
\hline 1957 & 0.336 & 0.335 & 0.337 & 0.196 \\
\hline 1958 & 0.295 & 0.306 & 0.231 & 0.223 \\
\hline 1959 & 0.242 & 0.274 & 0.237 & 0.231 \\
\hline 1960 & 0.184 & 0.269 & 0.215 & 0.197 \\
\hline 1961 & 0.134 & 0.160 & 0.144 & 0.084 \\
\hline 1962 & 0.325 & 0.359 & 0.289 & 0.224 \\
\hline 1963 & 0.366 & 0.384 & 0.352 & 0.311 \\
\hline 1964 & 0.361 & 0.334 & 0.286 & 0.198 \\
\hline 1965 & 0.325 & 0.316 & 0.275 & 0.243 \\
\hline 1966 & 0.368 & 0.353 & 0.331 & 0.211 \\
\hline 1967 & 0.298 & 0.300 & 0.258 & 0.198 \\
\hline 1968 & 0.382 & 0.383 & 0.308 & 0.228 \\
\hline 1969 & 0.370 & 0.323 & 0.270 & 0.225 \\
\hline 1970 & 0.341 & 0.329 & 0.288 & 0.204 \\
\hline 1971 & 0.371 & 0.311 & 0.310 & 0.218 \\
\hline 1972 & 0.309 & 0.347 & 0.261 & 0.175 \\
\hline 1973 & 0.365 & 0.328 & 0.213 & 0.165 \\
\hline 1974 & 0.367 & 0.256 & 0.210 & 0.133 \\
\hline 1975 & 0.380 & 0.275 & 0.182 & 0.087 \\
\hline 1970 & 0.391 & 0.294 & 0.196 & 0.114 \\
\hline 1977 & 0.348 & 0.284 & 0.147 & 0.125 \\
\hline 1978 & 0.379 & 0.262 & 0.136 & 0.057 \\
\hline 1979 & 0.342 & 0.210 & 0.088 & 0.061 \\
\hline 1980 & 0.317 & 0.138 & 0.041 & 0.026 \\
\hline 1981 & 0.402 & 0.163 & 0.060 & 0.049 \\
\hline 1902 & 0.357 & 0.163 & 0.079 & 0.039 \\
\hline
\end{tabular}


rates, $1940-82$

\begin{tabular}{|c|c|c|c|}
\hline $20-24$ & $25-29$ & $30+$ & Total \\
\hline - & - & - & - \\
\hline • & • & • & • \\
\hline - & - & - & - \\
\hline • & • & • & - \\
\hline - & - & - & - \\
\hline$\bullet$ & • & - & 4.625 \\
\hline - & - & - & 5.508 \\
\hline • & - & - & 5.572 \\
\hline - & - & • & 5.366 \\
\hline • & . & • & 6.311 \\
\hline - & - & - & 5.672 \\
\hline - & - & - & 5.306 \\
\hline • & - & $\bullet$ & 6.848 \\
\hline - & - & - & 6.161 \\
\hline - & - & - & 6.230 \\
\hline • & - & - & 6.449 \\
\hline - & - & • & $5 \cdot 836$ \\
\hline • & $\bullet$ & • & 6.937 \\
\hline - & - & - & 6.096 \\
\hline - & - & - & 5.719 \\
\hline 0.128 & - & - & $5 \cdot 181$ \\
\hline 0.045 & - & - & 2.947 \\
\hline 0.105 & - & - & 6.767 \\
\hline 0.192 & . & - & 8.334 \\
\hline 0.151 & 0.042 & . & 6.858 \\
\hline 0.168 & 0.036 & 0.014 & 6.882 \\
\hline 0.165 & 0.058 & 0.005 & 7.455 \\
\hline 0.095 & 0.035 & 0.005 & 5.945 \\
\hline 0.129 & 0.039 & - & 7.345 \\
\hline 0.106 & 0.036 & - & 6.649 \\
\hline 0.111 & 0.045 & - & 6.593 \\
\hline 0.113 & 0.034 & - & 6.787 \\
\hline 0.099 & 0.018 & - & 6.040 \\
\hline 0.093 & 0.030 & - & 5.970 \\
\hline 0.066 & 0.017 & - & $5 \cdot 244$ \\
\hline 0.045 & 0.014 & 0.005 & 4.943 \\
\hline 0.050 & 0.008 & 0.003 & 5.284 \\
\hline 0.052 & 0.010 & . & 4.827 \\
\hline 0.021 & 0.014 & - & 4.347 \\
\hline 0.019 & 0.008 & 0.002 & 3.650 \\
\hline 0.004 & 0.002 & 0.002 & 2.652 \\
\hline 0.020 & 0.010 & - & 3.519 \\
\hline 0.035 & - & - & 3.372 \\
\hline
\end{tabular}


Basic Table 43.B. Urban Fujian Province (13): duration-specific fertility

\begin{tabular}{|c|c|c|c|c|}
\hline $\begin{array}{l}\text { Birth year } \\
\text { of child }\end{array}$ & $0-4$ & $5-9$ & $10-14$ & $15-19$ \\
\hline 1940 & 0.102 & - & - & - \\
\hline 1941 & 0.180 & - & - & - \\
\hline 1942 & 0.023 & - & • & - \\
\hline 1943 & 0.252 & - & - & - \\
\hline 1944 & 0.115 & - & - & - \\
\hline 1945 & 0.312 & 0.153 & - & - \\
\hline 1446 & 0.232 & 0.284 & • & - \\
\hline 1947 & 0.244 & 0.164 & $\cdot$ & • \\
\hline 1940 & 0.276 & 0.252 & • & - \\
\hline 1949 & 0.222 & 0.253 & - & - \\
\hline 1950 & 0.265 & 0.378 & 0.204 & - \\
\hline 1951 & 0.334 & 0.232 & 0.232 & - \\
\hline 1452 & 0.298 & 0.183 & 0.282 & - \\
\hline 1953 & 0.374 & 0.340 & 0.210 & - \\
\hline 1954 & 0.372 & 0.242 & 0.277 & - \\
\hline 1955 & 0.339 & 0.317 & 0.245 & 0.145 \\
\hline 1956 & 0.256 & 0.254 & 0.324 & 0.309 \\
\hline 1957 & 0.382 & 0.385 & 0.284 & 0.141 \\
\hline 1958 & 0.408 & 0.340 & 0.191 & 0.147 \\
\hline 1459 & 0.296 & 0.372 & 0.181 & 0.230 \\
\hline 1960 & 0.375 & 0.357 & 0.265 & 0.111 \\
\hline 1961 & 0.234 & 0.104 & 0.191 & 0.070 \\
\hline 1962 & 0.462 & 0.535 & 0.153 & 0.142 \\
\hline 1963 & 0.325 & 0.428 & 0.221 & 0.149 \\
\hline 1904 & 0.250 & 0.387 & 0.186 & 0.081 \\
\hline 1905 & 0.461 & 0.250 & 0.161 & 0.088 \\
\hline 1900 & 0.250 & 0.070 & 0.128 & 0.032 \\
\hline 1907 & 0.318 & 0.097 & 0.115 & 0.035 \\
\hline 1908 & 0.226 & 0.325 & 0.134 & 0.008 \\
\hline 1969 & 0.500 & 0.159 & 0.091 & 0.062 \\
\hline 1970 & 0.347 & 0.049 & 0.104 & 0.036 \\
\hline 1971 & 0.423 & 0.155 & 0.070 & 0.055 \\
\hline 1972 & 0.264 & 0.087 & 0.073 & . \\
\hline 1973 & 0.444 & 0.101 & 0.093 & - \\
\hline 1974 & 0.255 & 0.045 & 0.023 & - \\
\hline 1975 & 0.334 & 0.208 & 0.024 & . \\
\hline 1976 & 0.289 & 0.154 & 0.129 & 0.047 \\
\hline 1977 & 0.214 & 0.103 & $\cdot$ & - \\
\hline 1978 & 0.257 & 0.059 & 0.025 & - \\
\hline 1979 & 0.282 & 0.071 & 0.023 & - \\
\hline 1980 & 0.222 & 0.040 & 0.046 & - \\
\hline 1981 & 0.281 & 0.014 & - & - \\
\hline 1982 & 0.312 & - & - & - \\
\hline
\end{tabular}


rates, $1940-82$

\begin{tabular}{|c|c|c|c|}
\hline $20-24$ & $25-29$ & $30+$ & Total \\
\hline$\bullet$ & - & - & $\bullet$ \\
\hline - & $\bullet$ & - & • \\
\hline - & - & - & - \\
\hline - & - & - & - \\
\hline - & - & - & - \\
\hline$\bullet$ & - & - & 4.017 \\
\hline$\bullet$ & - & - & $5 \cdot 722$ \\
\hline • & - & - & 3.804 \\
\hline - & - & - & 5.434 \\
\hline - & - & - & 5.187 \\
\hline$\bullet$ & $\bullet$ & - & 6.288 \\
\hline - & $\bullet$ & $\bullet$ & 5.659 \\
\hline - & - & • & 5.509 \\
\hline - & - & - & 6.568 \\
\hline - & $\bullet$ & - & 6.326 \\
\hline - & - & - & 6.103 \\
\hline • & - & - & 6.804 \\
\hline - & $\bullet$ & - & 6.896 \\
\hline - & $\bullet$ & - & 6.215 \\
\hline - & - & - & 6.323 \\
\hline 0.076 & - & - & 6.128 \\
\hline$\bullet$ & - & - & 3.428 \\
\hline 0.023 & - & - & 6.806 \\
\hline 0.084 & - & - & 6.260 \\
\hline 0.069 & 0.029 & • & $5.00 B$ \\
\hline 0.022 & - & • & 4.910 \\
\hline 0.023 & $\bullet$ & - & 2.557 \\
\hline 0.020 & • & - & 2.928 \\
\hline 0.042 & 0.021 & - & 4.331 \\
\hline - & - & - & 4.063 \\
\hline 0.018 & - & • & 2.705 \\
\hline 0.016 & - & - & 3.596 \\
\hline 0.018 & • & - & 2.209 \\
\hline • & • & - & 3.186 \\
\hline • & $\bullet$ & - & 1.617 \\
\hline - & • & - & $2 \cdot 830$ \\
\hline - & • & - & 3.095 \\
\hline - & - & - & 1.584 \\
\hline - & - & - & 1.707 \\
\hline - & - & - & 1.679 \\
\hline - & - & - & 1.540 \\
\hline - & - & • & 1.271 \\
\hline - & - & - & 1.559 \\
\hline
\end{tabular}


Basic Table 43.C. Rural Fujian Province (13): duration-specific fertility

\begin{tabular}{|c|c|c|c|c|}
\hline $\begin{array}{l}\text { Birth year } \\
\text { of child }\end{array}$ & $0-4$ & $5-9$ & $10-14$ & $15-19$ \\
\hline 1940 & 0.223 & - & - & - \\
\hline 1941 & 0.254 & - & - & - \\
\hline $19+2$ & 0.204 & - & - & - \\
\hline 1943 & 0.234 & - & - & - \\
\hline 1944 & 0.237 & - & - & - \\
\hline 1945 & 0.272 & 0.219 & - & - \\
\hline 1946 & 0.252 & 0.276 & - & - \\
\hline 1947 & 0.277 & 0.291 & - & - \\
\hline 1948 & 0.276 & 0.260 & - & - \\
\hline 1945 & 0.293 & 0.328 & - & - \\
\hline 1950 & 0.274 & 0.316 & 0.197 & - \\
\hline 1951 & 0.271 & 0.249 & 0.224 & - \\
\hline 1952 & 0.317 & 0.369 & 0.294 & - \\
\hline 1953 & 0.319 & 0.290 & 0.257 & - \\
\hline 1954 & 0.293 & $0.33 \mathrm{e}$ & 0.240 & - \\
\hline 1955 & 0.315 & 0.271 & 0.355 & 0.189 \\
\hline 1956 & 0.254 & 0.264 & 0.252 & 0.217 \\
\hline 1957 & 0.331 & $0.32 \mathrm{E}$ & 0.345 & 0.204 \\
\hline 1958 & 0.282 & 0.301 & 0.237 & 0.234 \\
\hline 1959 & 0.230 & 0.261 & 0.245 & 0.231 \\
\hline 1960 & 0.102 & 0.258 & 0.207 & 0.209 \\
\hline 1901 & 0.123 & 0.160 & 0.137 & 0.086 \\
\hline 1962 & 0.311 & 0.338 & 0.306 & 0.236 \\
\hline 1903 & 0.370 & 0.379 & 0.370 & 0.332 \\
\hline 1964 & 0.372 & 0.328 & 0.299 & 0.214 \\
\hline 1965 & 0.312 & 0.323 & 0.289 & 0.266 \\
\hline 1906 & 0.377 & 0.382 & 0.355 & 0.238 \\
\hline 1907 & 0.296 & 0.321 & 0.275 & 0.219 \\
\hline 1968 & 0.394 & 0.389 & 0.322 & 0.249 \\
\hline 196y & $0.3 \in 0$ & 0.340 & 0.288 & 0.248 \\
\hline 1970 & 0.341 & 0.356 & 0.309 & 0.225 \\
\hline 1971 & 0.367 & 0.324 & 0.336 & 0.238 \\
\hline 1972 & 0.314 & 0.366 & 0.280 & 0.197 \\
\hline 1973 & 0.358 & 0.346 & 0.225 & 0.184 \\
\hline 1974 & 0.378 & 0.272 & 0.229 & 0.146 \\
\hline 1975 & 0.385 & 0.280 & 0.197 & 0.097 \\
\hline 1976 & 0.401 & 0.305 & 0.202 & 0.121 \\
\hline 1977 & 0.360 & 0.302 & 0.158 & 0.137 \\
\hline 1970 & 0.388 & 0.282 & 0.145 & 0.003 \\
\hline 1979 & 0.346 & 0.224 & 0.093 & 0.067 \\
\hline 1980 & 0.325 & 0.149 & 0.040 & 0.028 \\
\hline$|98|$ & 0.415 & 0.178 & 0.065 & 0.053 \\
\hline 1982 & 0.363 & 0.177 & 0.088 & 0.042 \\
\hline
\end{tabular}


rates, $1940-82$

\begin{tabular}{|c|c|c|c|}
\hline $20-24$ & $25-29$ & $30+$ & Total \\
\hline - & - & - & - \\
\hline • & - & - & - \\
\hline - & $\bullet$ & • & • \\
\hline$\bullet$ & - & • & $\bullet$ \\
\hline • & • & - & - \\
\hline - & - & - & 4.715 \\
\hline - & • & • & 5.477 \\
\hline - & - & - & 5.832 \\
\hline - & - & - & 5.356 \\
\hline - & - & - & 6.475 \\
\hline - & - & - & 5.581 \\
\hline - & • & - & 5.255 \\
\hline - & - & $\bullet$ & 7.041 \\
\hline - & - & - & 6.103 \\
\hline - & - & - & 6.216 \\
\hline$\bullet$ & - & - & 6.497 \\
\hline - & - & - & 5.700 \\
\hline - & - & - & 6.943 \\
\hline - & - & - & 6.079 \\
\hline$\bullet$ & - & - & 5.637 \\
\hline 0.136 & - & - & 5.053 \\
\hline 0.051 & - & - & 2.883 \\
\hline 0.117 & - & - & 6.761 \\
\hline 0.209 & - & - & 8.602 \\
\hline 0.162 & 0.044 & - & 7.103 \\
\hline 0.188 & 0.042 & 0.016 & 7.178 \\
\hline 0.184 & 0.066 & 0.006 & 3.045 \\
\hline 0.106 & 0.040 & 0.005 & 6.312 \\
\hline 0.141 & 0.042 & - & 7.680 \\
\hline 0.1 .21 & 0.041 & - & 6.987 \\
\hline 0.125 & 0.051 & - & $7 \cdot 036$ \\
\hline 0.127 & 0.033 & - & 7.155 \\
\hline 0.109 & 0.021 & - & 6.427 \\
\hline 0.106 & 0.034 & - & 6.255 \\
\hline 0.075 & 0.020 & - & 5.604 \\
\hline 0.050 & 0.016 & 0.006 & 5.157 \\
\hline 0.056 & 0.009 & 0.003 & 5.492 \\
\hline 0.058 & 0.011 & - & 5.127 \\
\hline 0.024 & 0.015 & - & 4.587 \\
\hline 0.021 & 0.009 & 0.003 & 3.814 \\
\hline 0.005 & 0.002 & 0.003 & 2.758 \\
\hline 0.022 & 0.011 & - & 3.720 \\
\hline 0.039 & $\bullet$ & - & 3.541 \\
\hline
\end{tabular}


Basic Table 44.A. Total Jiangxi Province (14): duration-specific fertility

\begin{tabular}{|c|c|c|c|c|}
\hline $\begin{array}{l}\text { Birth year } \\
\text { of child }\end{array}$ & $0-4$ & $5-9$ & $10-14$ & $15-19$ \\
\hline 1940 & 0.222 & - & - & - \\
\hline 1941 & 0.211 & - & - & - \\
\hline 1942 & 0.225 & - & - & - \\
\hline 1843 & 0.205 & - & - & - \\
\hline 1949 & 0.229 & - & - & - \\
\hline 1945 & 0.245 & 0.230 & - & - \\
\hline 1940 & 0.270 & 0.286 & - & - \\
\hline $19+7$ & 0.262 & 0.286 & - & - \\
\hline 1948 & 0.262 & 0.289 & - & - \\
\hline 1949 & 0.292 & 0.318 & - & - \\
\hline $19=0$ & 0.298 & 0.310 & 0.259 & - \\
\hline 1951 & 0.288 & 0.285 & 0.243 & - \\
\hline 1952 & 0.298 & 0.286 & 0.233 & - \\
\hline 1953 & 0.298 & 0.308 & 0.262 & - \\
\hline 1954 & 0.283 & 0.277 & 0.263 & - \\
\hline 1955 & 0.306 & 0.269 & 0.238 & 0.206 \\
\hline 1956 & $0: 275$ & 0.295 & 0.259 & 0.215 \\
\hline 1957 & 0.318 & 0.336 & 0.274 & 0.190 \\
\hline 1958 & 0.305 & 0.323 & 0.262 & 0.244 \\
\hline 1959 & 0.206 & 0.259 & 0.192 & 0.198 \\
\hline 1960 & 0.181 & 0.250 & 0.221 & 0.149 \\
\hline 1961 & 0.198 & 0.221 & 0.131 & 0.163 \\
\hline 1902 & 0.290 & 0.299 & 0.292 & 0.237 \\
\hline 1903 & 0.345 & 0.345 & 0.348 & 0.265 \\
\hline 1964 & 0.297 & 0.371 & 0.290 & 0.247 \\
\hline 1965 & 0.341 & 0.278 & 0.293 & 0.253 \\
\hline 1900 & 0.356 & 0.369 & 0.344 & 0.301 \\
\hline 1907 & 0.312 & 0.343 & 0.322 & 0.263 \\
\hline 1968 & 0.393 & 0.365 & 0.332 & 0.284 \\
\hline 1969 & 0.320 & 0.322 & 0.307 & 0.264 \\
\hline 1970 & 0.343 & 0.317 & 0.337 & 0.285 \\
\hline 1971 & 0.344 & 0.326 & 0.281 & 0.189 \\
\hline 1972 & 0.346 & 0.337 & 0.293 & 0.222 \\
\hline 1973 & 0.383 & 0.328 & 0.290 & 0.266 \\
\hline 1974 & 0.379 & 0.359 & 0.285 & 0.231 \\
\hline 1975 & 0.350 & 0.353 & 0.258 & 0.176 \\
\hline 1970 & 0.388 & 0.314 & 0.271 & 0.205 \\
\hline 1977 & 0.376 & 0.297 & 0.238 & 0.183 \\
\hline 1978 & 0.378 & 0.288 & 0.204 & 0.123 \\
\hline 1979 & 0.388 & 0.238 & 0.162 & 0.085 \\
\hline 1980 & 0.371 & 0.204 & 0.085 & 0.043 \\
\hline 1981 & 0.390 & 0.210 & 0.102 & 0.052 \\
\hline 1982 & 0.349 & 0.190 & 0.063 & 0.063 \\
\hline
\end{tabular}


rates, $1940-82$

\begin{tabular}{|c|c|c|c|}
\hline $20-24$ & $25-29$ & $30+$ & Total \\
\hline - & - & - & - \\
\hline - & - & - & - \\
\hline - & - & - & - \\
\hline - & $\bullet$ & - & - \\
\hline - & - & - & - \\
\hline - & - & - & 4.774 \\
\hline - & - & - & 5.751 \\
\hline - & - & - & 5.699 \\
\hline - & - & - & $5.75 e$ \\
\hline - & - & - & 6.330 \\
\hline - & - & $\bullet$ & 6.213 \\
\hline - & $\bullet$ & - & 5.911 \\
\hline$\bullet$ & - & - & 5.772 \\
\hline - & - & - & 6.205 \\
\hline - & - & - & 5.887 \\
\hline - & - & - & 5.856 \\
\hline - & - & - & 6.035 \\
\hline - & $\bullet$ & - & 6.425 \\
\hline • & $\bullet$ & - & 6.560 \\
\hline - & - & - & 4.966 \\
\hline 0.112 & - & - & 4.742 \\
\hline 0.091 & - & - & 4.426 \\
\hline 0.122 & - & - & 6.427 \\
\hline 0.179 & - & - & 7.707 \\
\hline 0.143 & 0.063 & 0.010 & 7.135 \\
\hline 0.164 & 0.045 & 0.008 & 6.859 \\
\hline 0.157 & 0.081 & C.009 & 8.090 \\
\hline 0.131 & 0.057 & 0.005 & 7.159 \\
\hline 0.179 & 0.045 & 0.005 & 8.014 \\
\hline 0.154 & 0.043 & • & 7.077 \\
\hline 0.156 & 0.045 & 0.002 & 7.420 \\
\hline $0.1 \epsilon 3$ & 0.039 & • & 6.711 \\
\hline 0.159 & 0.0 .31 & 0.004 & 6.963 \\
\hline 0.192 & 0.040 & 0.010 & 7.492 \\
\hline 0.149 & 0.032 & • & 7.175 \\
\hline 0.134 & 0.039 & 0.002 & 6.504 \\
\hline 0.116 & 0.035 & $\bullet$ & 6.051 \\
\hline 0.0 .32 & 0.038 & 0.003 & 6.088 \\
\hline 0.092 & 0.033 & - & j.582 \\
\hline 0.057 & 0.021 & 0.002 & 4.765 \\
\hline 0.016 & 0.003 & 0.003 & 3.622 \\
\hline 0.024 & 0.014 & • & 3.963 \\
\hline 0.037 & 0.011 & 0.003 & 3.579 \\
\hline
\end{tabular}


Basic Table 44.B. Urban Jiangxi Province (14): duration-specific fertility

\begin{tabular}{|c|c|c|c|c|}
\hline $\begin{array}{l}\text { Birth year } \\
\text { of child }\end{array}$ & $0-4$ & $5-9$ & $10-14$ & $15-19$ \\
\hline 1940 & 0.198 & - & - & - \\
\hline 1941 & 0.168 & - & - & - \\
\hline 1942 & 0.271 & - &. & - \\
\hline 1943 & 0.213 & - & - & - \\
\hline 1944 & 0.299 & - & - & - \\
\hline 1945 & 0.342 & 0.258 & - & - \\
\hline 1946 & 0.276 & 0.298 & - & - \\
\hline 1947 & 0.234 & 0.291 & - & • \\
\hline 1948 & 0.270 & 0.329 & - & $\bullet$ \\
\hline 1949 & 0.248 & 0.235 & - & - \\
\hline 1950 & 0.326 & 0.449 & 0.337 & - \\
\hline 1951 & 0.325 & 0.217 & 0.168 & - \\
\hline 1952 & 0.293 & 0.234 & 0.291 & - \\
\hline 1953 & 0.377 & 0.321 & 0.348 & - \\
\hline 1954 & 0.355 & 0.295 & 0.192 & . \\
\hline 1955 & 0.360 & 0.127 & 0.342 & 0.198 \\
\hline 1956 & 0.429 & 0.387 & 0.237 & 0.316 \\
\hline 1957 & 0.317 & 0.344 & 0.360 & 0.136 \\
\hline 1958 & 0.355 & 0.479 & 0.287 & 0.290 \\
\hline 1959 & 0.275 & 0.237 & 0.155 & 0.256 \\
\hline 1900 & 0.337 & 0.360 & 0.326 & 0.150 \\
\hline 1961 & 0.258 & 0.171 & 0.139 & 0.079 \\
\hline 1962 & 0.319 & 0.206 & 0.310 & 0.216 \\
\hline 1903 & 0.430 & 0.206 & 0.308 & 0.113 \\
\hline 1964 & 0.318 & 0.336 & 0.271 & 0.217 \\
\hline 1965 & 0.456 & 0.202 & 0.180 & 0.184 \\
\hline 1966 & 0.337 & 0.203 & 0.274 & 0.201 \\
\hline 1967 & 0.279 & 0.281 & 0.158 & 0.172 \\
\hline 1906 & 0.454 & 0.366 & 0.237 & 0.137 \\
\hline 1969 & 0.379 & 0.299 & 0.092 & 0.101 \\
\hline 1970 & 0.428 & 0.350 & 0.219 & 0.144 \\
\hline 1971 & 0.358 & 0.236 & 0.074 & 0.051 \\
\hline 1972 & 0.411 & 0.279 & 0.131 & 0.079 \\
\hline 1973 & 0.368 & 0.204 & 0.172 & 0.015 \\
\hline 1974 & 0.401 & 0.194 & 0.112 & 0.031 \\
\hline 1975 & 0.491 & 0.257 & 0.140 & 0.067 \\
\hline 1976 & 0.375 & 0.167 & 0.169 & 0.074 \\
\hline 1977 & 0.466 & 0.151 & 0.098 & - \\
\hline 1970 & 0.309 & 0.131 & 0.034 & 0.043 \\
\hline 1979 & 0.291 & 0.075 & - & 0.019 \\
\hline 1980 & 0.238 & 0.076 & - & - \\
\hline 2981 & 0.214 & 0.051 & - & • \\
\hline 1982 & 0.269 & 0.045 & 0.017 & - \\
\hline
\end{tabular}


rates, $1940-82$

\begin{tabular}{|c|c|c|c|}
\hline $20-24$ & $25-29$ & $30+$ & Total \\
\hline$\bullet$ & - & - & - \\
\hline$\bullet$ & - & - & - \\
\hline - & - & - & - \\
\hline - & - & - & - \\
\hline - & - & - & - \\
\hline - & - & - & 5.357 \\
\hline - & - & - & 6.172 \\
\hline - & - & - & 5.347 \\
\hline - & - & - & 6.641 \\
\hline - & - & - & 5.020 \\
\hline - & - & - & 3.361 \\
\hline - & - & - & 4.922 \\
\hline - & .• & • & 5.991 \\
\hline • & - & - & 7.644 \\
\hline - & - & - & 5.940 \\
\hline - & - & - & 5.955 \\
\hline - & - & $\bullet$ & 7.973 \\
\hline • & - & • & 0.755 \\
\hline - & - & - & 8.302 \\
\hline$\bullet$ & - & - & 5.407 \\
\hline 0.099 & - & - & 6.600 \\
\hline 0.037 & - & • & 3.536 \\
\hline 0.058 & - & - & 5.751 \\
\hline 0.135 & - & - & 6.518 \\
\hline 0.085 & 0.042 & - & 6.344 \\
\hline 0.171 & - & - & 5.963 \\
\hline 0.033 & 0.019 & - & 5.370 \\
\hline 0.054 & 0.039 & - & 4.917 \\
\hline 0.034 & 0.019 & • & 6.232 \\
\hline 0.093 & 0.043 & - & 5.033 \\
\hline 0.071 & - & - & 0.063 \\
\hline 0.062 & - & - & 3.905 \\
\hline 0.052 & - & - & 4.761 \\
\hline 0.017 & 0.017 & • & 3.966 \\
\hline • & 0.016 & • & 3.765 \\
\hline 0.054 & $\bullet$ & - & 5.048 \\
\hline - & 0.015 & - & 3.997 \\
\hline - & • & - & 3.573 \\
\hline - & 0.017 & - & 2.975 \\
\hline - & - & - & 1.920 \\
\hline - & - & - & 1.509 \\
\hline - & - & - & 1.328 \\
\hline$\bullet$ & - & - & 1.057 \\
\hline
\end{tabular}


Basic Table 44.C. Rural Jiangxi Province (14): duration-specific fertility

\begin{tabular}{|c|c|c|c|c|}
\hline $\begin{array}{l}\text { Birth year } \\
\text { of child }\end{array}$ & $0-4$ & $5-9$ & $10-14$ & $15-19$ \\
\hline 1940 & 0.225 & - & - & - \\
\hline 1941 & 0.216 & - & - & - \\
\hline 1942 & 0.219 & - & - & - \\
\hline 1943 & 0.204 & - & $\bullet$ & - \\
\hline 1944 & 0.222 & - & • & - \\
\hline 1945 & 0.236 & 0.227 & - & - \\
\hline 1940 & 0.270 & 0.285 & - & - \\
\hline 1947 & 0.205 & 0.285 & - & - \\
\hline 1948 & 0.261 & 0.285 & $\bullet$ & - \\
\hline 1945 & 0.297 & 0.327 & $\bullet$ & - \\
\hline 1950 & 0.295 & 0.290 & 0.250 & - \\
\hline 1951 & 0.284 & 0.292 & 0.253 & - \\
\hline 1952 & 0.298 & 0.291 & 0.226 & - \\
\hline 1953 & 0.290 & 0.307 & 0.252 & - \\
\hline 1954 & 0.276 & 0.275 & 0.270 & - \\
\hline 1955 & 0.300 & 0.285 & 0.228 & 0.207 \\
\hline 1956 & 0.258 & 0.285 & 0.262 & 0.203 \\
\hline 1957 & 0.318 & 0.335 & 0.265 & 0.197 \\
\hline 1958 & 0.299 & 0.308 & 0.259 & 0.234 \\
\hline 1959 & 0.197 & 0.262 & 0.197 & 0.193 \\
\hline 1960 & 0.163 & 0.239 & 0.208 & 0.149 \\
\hline 1901 & 0.192 & 0.227 & 0.185 & 0.172 \\
\hline 1962 & 0.287 & 0.310 & 0.290 & 0.240 \\
\hline 1903 & 0.338 & 0.356 & 0.352 & 0.282 \\
\hline 1964 & 0.295 & 0.376 & 0.292 & 0.251 \\
\hline 1965 & 0.331 & 0.287 & 0.295 & 0.261 \\
\hline 1966 & 0.358 & 0.386 & 0.352 & 0.311 \\
\hline 1967 & 0.315 & 0.349 & 0.342 & 0.271 \\
\hline 1900 & 0.380 & 0.364 & 0.346 & 0.298 \\
\hline 1969 & 0.313 & 0.325 & 0.335 & 0.281 \\
\hline 1970 & 0.332 & 0.314 & 0.350 & 0.300 \\
\hline 1971 & 0.342 & 0.334 & 0.303 & 0.205 \\
\hline 1972 & 0.338 & 0.343 & 0.309 & 0.240 \\
\hline 1973 & 0.384 & 0.343 & 0.300 & 0.301 \\
\hline 1974 & 0.370 & 0.381 & 0.300 & 0.258 \\
\hline 1975 & 0.334 & 0.366 & 0.269 & 0.189 \\
\hline 1970 & 0.390 & 0.335 & 0.280 & 0.219 \\
\hline 1977 & 0.303 & 0.316 & 0.253 & 0.201 \\
\hline 1918 & 0.374 & $0.30 \mathrm{E}$ & 0.223 & 0.129 \\
\hline 1979 & 0.403 & 0.257 & 0.184 & 0.090 \\
\hline 1980 & 0.393 & 0.218 & 0.096 & $0.0+6$ \\
\hline 1981 & 0.418 & 0.231 & 0.116 & 0.057 \\
\hline 1982 & 0.360 & 0.211 & 0.069 & 0.070 \\
\hline
\end{tabular}


rates, $1940-82$

\begin{tabular}{|c|c|c|c|}
\hline $20-24$ & $25-29$ & $30+$ & Total \\
\hline - & $\bullet$ & - & - \\
\hline - & - & • & - \\
\hline - & - & - & $\bullet$ \\
\hline - & • & - & - \\
\hline - & - & • & • \\
\hline - & - & - & 4.652 \\
\hline - & - & - & 5.703 \\
\hline - & - & - & 5.682 \\
\hline - & - & - & 5.657 \\
\hline - & - & - & 6.478 \\
\hline - & - & - & 5.970 \\
\hline - & - & - & 5.911 \\
\hline - & - & - & 5.748 \\
\hline - & - & - & 6.049 \\
\hline - & - & - & 5.881 \\
\hline - & - & - & 5.845 \\
\hline - & - & - & 5.817 \\
\hline - & - & - & 6.387 \\
\hline - & - & - & 6.360 \\
\hline - & - & - & 4.915 \\
\hline 0.114 & - & - & 4.532 \\
\hline 0.098 & - & - & 4.525 \\
\hline 0.129 & - & - & 6.501 \\
\hline 0.134 & - & - & 7.836 \\
\hline 0.149 & 0.072 & 0.011 & 7.227 \\
\hline 0.104 & 0.050 & 0.009 & 6.975 \\
\hline 0.170 & 0.089 & 0.010 & 8.381 \\
\hline 0.139 & 0.059 & 0.006 & 7.407 \\
\hline 0.195 & 0.048 & 0.005 & 8.213 \\
\hline $0.16 t$ & 0.048 & - & 7.314 \\
\hline 0.166 & 0.050 & $0.00 ?$ & 7.569 \\
\hline 0.173 & 0.043 & - & 7.000 \\
\hline 0.170 & 0.034 & 0.005 & 7.191 \\
\hline 0.197 & 0.042 & 0.011 & 7.892 \\
\hline 0.164 & 0.033 & - & 7.565 \\
\hline 0.142 & 0.044 & 0.002 & 6.730 \\
\hline 0.129 & 0.037 & - & 6.952 \\
\hline 0.092 & 0.042 & 0.004 & 6.352 \\
\hline 0.104 & 0.034 & - & 5.389 \\
\hline 0.065 & 0.023 & 0.002 & 5.121 \\
\hline 0.018 & 0.004 & 0.003 & 3.396 \\
\hline 0.027 & 0.016 & • & 4.318 \\
\hline 0.041 & 0.012 & 0.003 & 3.830 \\
\hline
\end{tabular}


Basic Table 45.A. Total Shandong Province (15): duration-specific

\begin{tabular}{|c|c|c|c|c|}
\hline $\begin{array}{l}\text { Birth year } \\
\text { of child }\end{array}$ & $0-4$ & $5-9$ & $10-14$ & $15-19$ \\
\hline 1940 & 0.199 & - & - & - \\
\hline 1941 & 0.217 & - & - & - \\
\hline 1942 & 0.194 & • & - & - \\
\hline 1943 & 0.190 & • & - & • \\
\hline 1944 & 0.201 & - & - & - \\
\hline 1945 & 0.238 & 0.270 & - & $\bullet$ \\
\hline 1946 & 0.234 & 0.255 & - & - \\
\hline 1947 & 0.223 & 0.264 & - & - \\
\hline 1940 & 0.207 & 0.235 & - & - \\
\hline 1949 & 0.239 & 0.263 & - & - \\
\hline 1950 & 0.224 & 0.246 & 0.211 & $\bullet$ \\
\hline 1951 & 0.270 & 0.267 & 0.233 & $\bullet$ \\
\hline 1952 & 0.273 & 0.300 & 0.254 & - \\
\hline 1953 & 0.283 & 0.308 & 0.248 & - \\
\hline 1954 & 0.298 & 0.279 & 0.244 & - \\
\hline 1955 & 0.354 & 0.319 & 0.276 & 0.222 \\
\hline 1956 & 0.293 & 0.268 & 0.233 & 0.193 \\
\hline 1957 & 0.364 & 0.324 & 0.302 & 0.226 \\
\hline 1958 & 0.267 & 0.227 & 0.232 & 0.182 \\
\hline 1959 & 0.198 & 0.218 & 0.169 & 0.142 \\
\hline 1960 & 0.210 & 0.204 & 0.166 & 0.140 \\
\hline 1961 & 0.168 & 0.199 & 0.150 & 0.120 \\
\hline 1962 & 0.288 & 0.345 & 0.272 & 0.209 \\
\hline 1963 & 0.394 & 0.385 & 0.332 & 0.285 \\
\hline 1904 & 0.287 & 0.279 & 0.245 & 0.189 \\
\hline 1965 & 0.309 & 0.334 & 0.268 & 0.190 \\
\hline 1960 & 0.369 & 0.334 & 0.272 & 0.199 \\
\hline 1907 & 0.311 & 0.280 & 0.211 & 0.141 \\
\hline 1968 & 0.388 & 0.343 & 0.254 & 0.174 \\
\hline 1969 & 0.344 & 0.324 & 0.239 & 0.170 \\
\hline 1970 & .0 .413 & 0.342 & 0.292 & 0.198 \\
\hline 1971 & 0.358 & 0.311 & 0.243 & 0.168 \\
\hline 1972 & 0.348 & 0.249 & 0.200 & 0.148 \\
\hline 1973 & 0.324 & 0.250 & $0.1 \in 3$ & 0.106 \\
\hline 1974 & 0.363 & 0.211 & 0.125 & 0.079 \\
\hline 1975 & 0.328 & 0.233 & 0.120 & 0.059 \\
\hline 1976 & 0.364 & 0.198 & 0.089 & 0.052 \\
\hline 1977 & 0.342 & 0.164 & 0.060 & 0.041 \\
\hline 1978 & 0.313 & 0.153 & 0.071 & 0.032 \\
\hline 1979 & 0.370 & 0.181 & 0.058 & 0.030 \\
\hline 1980 & 0.285 & 0.121 & 0.029 & 0.008 \\
\hline 1981 & 0.330 & 0.095 & 0.029 & 0.012 \\
\hline 1982 & 0.283 & 0.096 & 0.029 & 0.004 \\
\hline
\end{tabular}


fertility rates, $1940-82$

\begin{tabular}{|c|c|c|c|}
\hline $20-24$ & $25-29$ & $30+$ & Total \\
\hline - & - & • & - \\
\hline - & - & - & - \\
\hline$\bullet$ & - & - & • \\
\hline - & $\bullet$ & • & - \\
\hline - & - & - & - \\
\hline - & - & - & 5.316 \\
\hline - & • & - & 5.088 \\
\hline - & - & - & 5.169 \\
\hline - & - & - & 4.641 \\
\hline - & - & - & 5.220 \\
\hline - & - & - & 4.890 \\
\hline - & - & • & 5.475 \\
\hline - & - & - & 5.934 \\
\hline$\bullet$ & - & - & 6.016 \\
\hline - & - & - & 5.809 \\
\hline - & - & - & 6.715 \\
\hline - & - & - & $5 \cdot 663$ \\
\hline - & - & - & 6.976 \\
\hline - & - & - & $5 \cdot 221$ \\
\hline - & - & - & 4.185 \\
\hline 0.077 & - & - & 4.123 \\
\hline 0.070 & - & 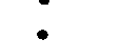 & 3.660 \\
\hline 0.103 & - & - & 6.290 \\
\hline 0.152 & - & - & 8.029 \\
\hline 0.114 & 0.034 & 0.003 & 5.753 \\
\hline 0.109 & 0.046 & 0.007 & 6.314 \\
\hline 0.120 & 0.025 & 0.005 & 6.625 \\
\hline 0.102 & 0.020 & 0.001 & 5.328 \\
\hline 0.103 & 0.027 & 0.001 & 6.453 \\
\hline 0.092 & 0.022 & 0.002 & 5.962 \\
\hline 0.103 & 0.026 & 0.003 & 6.883 \\
\hline 0.089 & 0.027 & - & 5.983 \\
\hline 0.075 & 0.024 & 0.003 & 5.229 \\
\hline 0.048 & 0.020 & 0.001 & 4.557 \\
\hline 0.030 & 0.019 & - & 4.134 \\
\hline 0.033 & 0.010 & - & 3.911 \\
\hline 0.016 & 0.003 & 0.001 & 3.617 \\
\hline 0.013 & 0.008 & - & 3.139 \\
\hline 0.010 & 0.003 & 0.001 & 2.919 \\
\hline 0.010 & 0.006 & 0.001 & 3.277 \\
\hline 0.005 & 0.002 & 0.001 & 2.253 \\
\hline 0.002 & 0.002 & - & $2 \cdot 353$ \\
\hline 0.005 & 0.001 & - & 2.093 \\
\hline
\end{tabular}


Basic Table 45.B. Urban Shandong Province (15): duration-specific

\begin{tabular}{|c|c|c|c|c|}
\hline $\begin{array}{l}\text { Birth year } \\
\text { of child }\end{array}$ & $0-4$ & $5-9$ & $10-14$ & $15-19$ \\
\hline 1940 & 0.166 & $\bullet$ & • & $\bullet$ \\
\hline 1941 & 0.219 & - & • & - \\
\hline 1942 & 0.174 & $\bullet$ & - & $\bullet$ \\
\hline 1943 & $0.290^{\circ}$ & - & - & - \\
\hline 1944 & 0.244 & - & - & - \\
\hline 1945 & 0.153 & 0.151 & - & - \\
\hline 1946 & .0 .307 & 0.219 & - & - \\
\hline 1947 & 0.296 & 0.209 & - & $\bullet$ \\
\hline 1948 & 0.188 & 0.231 & - & - \\
\hline 1949 & 0.221 & 0.305 & - & - \\
\hline 1950 & 0.228 & 0.245 & 0.151 & - \\
\hline 1951 & 0.289 & 0.296 & 0.129 & - \\
\hline 1952 & 0.358 & 0.241 & 0.290 & - \\
\hline 1953 & 0.206 & 0.292 & 0.253 & $\bullet$ \\
\hline 1954 & 0.400 & 0.317 & 0.244 & - \\
\hline 1955 & $0 \cdot 322$ & $0 \cdot 333$ & 0.235 & 0.181 \\
\hline 1956 & 0.313 & 0.249 & 0.265 & 0.193 \\
\hline 1957 & 0.451 & 0.334 & 0.339 & 0.232 \\
\hline 1958 & 0.377 & 0.256 & 0.260 & 0.154 \\
\hline 1959 & . 0.249 & 0.225 & 0.221 & 0.193 \\
\hline 1900 & 0.349 & 0.289 & 0.219 & 0.133 \\
\hline 1961 & 0.208 & 0.132 & 0.144 & 0.106 \\
\hline 1962 & 0.349 & 0.243 & 0.318 & 0.131 \\
\hline 1963 & 0.457 & $0.52 B$ & 0.214 & 0.260 \\
\hline 1904 & 0.369 & 0.249 & 0.186 & 0.115 \\
\hline 1965 & 0.453 & 0.277 & 0.122 & 0.053 \\
\hline 1966 & 0.218 & 0.169 & 0.084 & 0.024 \\
\hline 1967 & 0.337 & 0.228 & 0.104 & 0.008 \\
\hline 1968 & 0.418 & 0.180 & 0.119 & 0.033 \\
\hline 1909 & 0.339 & 0.250 & 0.054 & 0.039 \\
\hline 1970 & 0.562 & 0.233 & 0.108 & 0.033 \\
\hline 1971 & 0.378 & 0.160 & 0.039 & 0.048 \\
\hline 1972 & 0.322 & 0.152 & 0.040 & 0.023 \\
\hline 1973 & 0.295 & 0.109 & 0.083 & • \\
\hline 1974 & 0.287 & 0.113 & 0.053 & 0.011 \\
\hline 1975 & 0.376 & 0.163 & 0.052 & 0.036 \\
\hline 1976 & 0.236 & 0.108 & 0.015 & 0.013 \\
\hline 1977 & 0.277 & 0.002 & 0.017 & • \\
\hline $\begin{array}{l}1978 \\
1979\end{array}$ & $\begin{array}{l}0.294 \\
0.210\end{array}$ & $\begin{array}{l}0.035 \\
0.025\end{array}$ & 0.032 & 0.013 \\
\hline 1980 & 0.218 & 0.071 & - & 0.013 \\
\hline 1981 & 0.256 & • & • & - \\
\hline 1982 & 0.263 & 0.021 & 0.025 & - \\
\hline
\end{tabular}


fertility rates, $1940-82$

\begin{tabular}{|c|c|c|c|}
\hline $20-24$ & $25-29$ & $30+$ & Total \\
\hline - & - & - & - \\
\hline$\bullet$ & • & • & - \\
\hline - & • & $\bullet$ & - \\
\hline$\bullet$ & • & $\bullet$ & - \\
\hline - & - & • & - \\
\hline - & - & • & 3.190 \\
\hline$\bullet$ & - & - & 5.056 \\
\hline - & • & • & 4.843 \\
\hline - & - & - & 4.648 \\
\hline • & - & - & 6.019 \\
\hline - & - & • & 4.521 \\
\hline - & - & • & 5.059 \\
\hline - & • & - & 0.373 \\
\hline - & - & $\bullet$ & 5.704 \\
\hline$\bullet$ & • & $\bullet$ & 6.812 \\
\hline - & - & • & 6.231 \\
\hline - & - & - & 5.939 \\
\hline - & - & - & 7.857 \\
\hline - & - & - & 6.025 \\
\hline - & - & $\bullet$ & 5.208 \\
\hline 0.060 & $\bullet$ & $\bullet$ & 5.431 \\
\hline 0.026 & - & $\bullet$ & 3.189 \\
\hline 0.105 & - & - & 5.944 \\
\hline 0.105 & - & - & 8.439 \\
\hline 0.041 & 0.019 & - & 4.895 \\
\hline 0.041 & 0.015 & - & 4.804 \\
\hline 0.032 & 0.026 & - & 2.767 \\
\hline 0.022 & - & - & 3.496 \\
\hline 0.010 & - & - & 3.798 \\
\hline & - & - & 3.411 \\
\hline 0.035 & 0.010 & • & 4.910 \\
\hline - & - & - & 3.127 \\
\hline$\bullet$ & $\bullet$ & $\bullet$ & 2.686 \\
\hline - & - & - & 2.437 \\
\hline • & 0.019 & • & 2.411 \\
\hline - & $\bullet$ & $\bullet$ & 3.134 \\
\hline - & - & $\bullet$ & 2.106 \\
\hline • & $\bullet$ & - & 1.777 \\
\hline - & - & - & 1.649 \\
\hline - & - & $\bullet$ & 1.401 \\
\hline - & - & - & 1.510 \\
\hline • & - & - & 1.282 \\
\hline - & • & - & 1.545 \\
\hline
\end{tabular}


Basic Table 45.C. Rural Shandong Province (15): duration-specific

\begin{tabular}{|c|c|c|c|c|}
\hline $\begin{array}{l}\text { Birth year } \\
\text { of child }\end{array}$ & $0-4$ & $5-9$ & $10-14$ & 15-19 \\
\hline 1940 & 0.201 & - & - & - \\
\hline 1941 & 0.216 & - & - & - \\
\hline 1942 & 0.196 & - & • & - \\
\hline 1943 & 0.182 & - & - & - \\
\hline 1944 & 0.197 & - & • & - \\
\hline 1945 & 0.245 & 0.277 & - & - \\
\hline 1946 & $0 \cdot 228$ & 0.258 & - & $\bullet$ \\
\hline 1947 & 0.218 & 0.268 & - & • \\
\hline 1948 & 0.208 & 0.235 & - & • \\
\hline 1949 & 0.240 & 0.259 & - & • \\
\hline 1950 & 0.224 & 0.246 & 0.215 & - \\
\hline 1951 & 0.269 & 0.264 & 0.240 & • \\
\hline 1952 & 0.265 & 0.304 & 0.251 & - \\
\hline 1953 & 0.290 & 0.310 & 0.248 & - \\
\hline 1954 & 0.290 & 0.276 & 0.244 & - \\
\hline 1955 & 0.356 & $0.31 \mathrm{e}$ & 0.280 & 0.225 \\
\hline 1956 & 0.291 & 0.270 & 0.230 & 0.193 \\
\hline 1957 & 0.358 & 0.323 & 0.299 & 0.226 \\
\hline 1958 & 0.260 & 0.224 & 0.230 & 0.184 \\
\hline 1959 & 0.195 & 0.218 & 0.165 & 0.137 \\
\hline 1960 & 0.201 & 0.199 & 0.162 & 0.140 \\
\hline 1961 & 0.165 & 0.203 & 0.151 & 0.122 \\
\hline 1962 & 0.235 & 0.351 & 0.267 & 0.215 \\
\hline 1963 & 0.391 & 0.376 & 0.343 & 0.287 \\
\hline 1964 & 0.283 & 0.281 & 0.250 & 0.194 \\
\hline 1965 & 0.301 & 0.338 & 0.278 & 0.200 \\
\hline 1966 & 0.376 & 0.343 & 0.283 & 0.214 \\
\hline 1967 & 0.310 & 0.283 & 0.218 & 0.153 \\
\hline 1960 & 0.387 & 0.351 & 0.263 & 0.187 \\
\hline 1909 & 0.344 & 0.327 & 0.251 & 0.180 \\
\hline 1970 & 0.407 & 0.348 & 0.303 & 0.209 \\
\hline 1971 & 0.358 & 0.318 & 0.254 & 0.175 \\
\hline 1972 & 0.349 & 0.253 & 0.208 & 0.155 \\
\hline 1973 & 0.326 & 0.255 & 0.167 & 0.113 \\
\hline 1974 & 0.366 & 0.215 & 0.129 & 0.083 \\
\hline 1975 & 0.326 & 0.236 & 0.123 & 0.060 \\
\hline 1976 & 0.368 & 0.202 & 0.093 & 0.054 \\
\hline 1977 & 0.345 & 0.168 & 0.062 & 0.043 \\
\hline 1978 & 0.314 & 0.158 & 0.074 & 0.033 \\
\hline 1979 & 0.378 & 0.187 & 0.059 & 0.031 \\
\hline 1980 & 0.289 & 0.123 & 0.030 & 0.000 \\
\hline 1981 & 0.335 & 0.100 & 0.030 & 0.012 \\
\hline 1982 & 0.284 & 0.100 & 0.029 & 0.004 \\
\hline
\end{tabular}


fertility rates, $1940-82$

\begin{tabular}{|c|c|c|c|}
\hline $20-24$ & $25-29$ & $30+$ & Total \\
\hline • & - & - & - \\
\hline - & - & - & • \\
\hline - & • & - & - \\
\hline - & - & - & - \\
\hline - & - & - & - \\
\hline - & - & - & 5.468 \\
\hline - & • & - & 5.090 \\
\hline - & - & - & 5.191 \\
\hline - & - & - & 4.641 \\
\hline • & $\bullet$ & - & 5.163 \\
\hline - & - & - & 4.918 \\
\hline - & - & - & 5.507 \\
\hline - & - & - & 5.901 \\
\hline - & - & - & 6.039 \\
\hline - & - & - & 5.736 \\
\hline - & - & - & 6.750 \\
\hline - & • & - & 5.643 \\
\hline - & - & - & 0.912 \\
\hline - & - & - & 5.163 \\
\hline$\bullet$ &. & - & 4.113 \\
\hline 0.078 & - & - & 4.032 \\
\hline 0.073 & - & - & .3 .692 \\
\hline 0.103 & - & - & 6.313 \\
\hline 0.151 & - & - & 8.002 \\
\hline 0.120 & 0.035 & 0.003 & 5.829 \\
\hline 0.115 & 0.048 & 0.007 & 6.438 \\
\hline 0.128 & 0.025 & 0.005 & 6.876 \\
\hline 0.108 & 0.021 & 0.001 & 5.460 \\
\hline 0.110 & 0.030 & 0.001 & 6.640 \\
\hline 0.098 & 0.024 & 0.002 & 0.137 \\
\hline 0.109 & 0.027 & 0.003 & 7.027 \\
\hline 0.097 & 0.029 & - & 0.156 \\
\hline 0.082 & 0.026 & 0.003 & 5.379 \\
\hline 0.052 & 0.021 & 0.001 & 4.673 \\
\hline 0.033 & 0.019 & - & 4.221 \\
\hline 0.035 & 0.011 & . & 3.953 \\
\hline 0.017 & 0.003 & 0.001 & 3.688 \\
\hline 0.014 & 0.009 & - & 3.202 \\
\hline 0.010 & 0.004 & 0.001 & 2.975 \\
\hline 0.010 & 0.007 & 0.001 & 3.366 \\
\hline 0.005 & 0.002 & 0.001 & 2.290 \\
\hline 0.002 & 0.002 & - & 2.409 \\
\hline 0.006 & 0.001 & - & 2.123 \\
\hline
\end{tabular}


Basic Table 46.A. Total Henan Province (16): duration-specific fertility

\begin{tabular}{|c|c|c|c|c|}
\hline $\begin{array}{l}\text { Birth year } \\
\text { of child }\end{array}$ & $0-4$ & $5-9$ & $10-14$ & $15-19$ \\
\hline 1940 & 0.195 & - & - & - \\
\hline 1941 & 0.208 & - & - & - \\
\hline 1942 & 0.209 & - & - & - \\
\hline 1943 & 0.172 & - & - & - \\
\hline 1944 & 0.177 & - & - & - \\
\hline 1945 & 0.207 & 0.254 & • & - \\
\hline 1940 & 0.239 & 0.236 & - & - \\
\hline 1947 & 0.234 & 0.284 & - & - \\
\hline 1940 & 0.229 & 0.240 & - & - \\
\hline 1949 & 0.228 & 0.249 & $\bullet$ & - \\
\hline 1950 & 0.227 & 0.274 & 0.218 & - \\
\hline 1951 & 0.255 & 0.281 & 0.239 & - \\
\hline 1952 & 0.311 & 0.296 & 0.245 & - \\
\hline 1953 & 0.265 & 0.277 & 0.241 & - \\
\hline 1954 & 0.282 & 0.278 & 0.274 & - \\
\hline 1955 & 0.289 & 0.288 & 0.276 & 0.191 \\
\hline 1956 & .0 .260 & 0.242 & 0.233 & 0.192 \\
\hline 1957 & 0.297 & 0.232 & 0.268 & 0.208 \\
\hline 1958 & 0.207 & 0.237 & 0.214 & 0.181 \\
\hline 1959 & 0.160 & 0.188 & 0.163 & 0.154 \\
\hline 1960 & 0.144 & 0.190 & 0.143 & 0.140 \\
\hline 1961 & 0.144 & 0.148 & 0.128 & 0.088 \\
\hline 1962 & $0.29 d$ & 0.312 & 0.282 & 0.223 \\
\hline 1903 & 0.393 & 0.414 & 0.369 & 0.304 \\
\hline 1904 & 0.288 & 0.283 & 0.266 & 0.231 \\
\hline 1965 & 0.296 & 0.323 & 0.302 & 0.227 \\
\hline 1960 & 0.347 & 0.340 & 0.312 & 0.273 \\
\hline 1967 & 0.319 & 0.318 & 0.282 & 0.234 \\
\hline 1968 & 0.399 & 0.371 & 0.314 & 0.265 \\
\hline 1969 & 0.342 & 0.322 & 0.272 & 0.234 \\
\hline 1970 & 0.353 & 0.330 & 0.302 & 0.244 \\
\hline 1971 & 0.352 & 0.307 & 0.288 & 0.224 \\
\hline 1972 & $0 \cdot 336$ & 0.323 & 0.270 & 0.214 \\
\hline 1973 & 0.324 & 0.307 & 0.224 & 0.179 \\
\hline 1974 & 0.372 & 0.299 & 0.236 & 0.167 \\
\hline 1975 & 0.305 & 0.254 & 0.146 & 0.103 \\
\hline 1976 & 0.332 & 0.221 & 0.151 & 0.083 \\
\hline 1977 & 0.342 & 0.221 & 0.122 & 0.066 \\
\hline 1978 & 0.355 & 0.239 & 0.133 & 0.079 \\
\hline 1979 & 0.371 & 0.246 & 0.136 & 0.064 \\
\hline 1980 & 0.321 & 0.182 & 0.072 & 0.029 \\
\hline 1981 & 0.370 & 0.194 & 0.077 & 0.028 \\
\hline 1982 & 0.302 & 0.158 & 0.073 & 0.025 \\
\hline
\end{tabular}


rates, $1940-82$

\begin{tabular}{|c|c|c|c|}
\hline $20-24$ & $25-29$ & $30+$ & Total \\
\hline . & • & • & - \\
\hline - & - & - & - \\
\hline - & - & • & - \\
\hline - & - & • & - \\
\hline - & $\bullet$ & - & • \\
\hline - & - & - & 4.926 \\
\hline - & - & - & 4.807 \\
\hline - & - & - & 5.534 \\
\hline - & • & - & 4.832 \\
\hline - & - & - & 4.961 \\
\hline - & - & - & 5.201 \\
\hline - & - & - & 5.560 \\
\hline - & - & $\bullet$ & 6.029 \\
\hline - & - & - & 5.602 \\
\hline - & - & - & 5.972 \\
\hline$\bullet$ & - & - & 6.005 \\
\hline • & - & - & 5.344 \\
\hline - & - & - & 6.069 \\
\hline - & - & - & 4.856 \\
\hline$\bullet$ & $\bullet$ & • & 3.854 \\
\hline 0.082 & - & • & 3.553 \\
\hline 0.049 & - & - & 2.853 \\
\hline 0.122 & - & - & 6.389 \\
\hline 0.214 & - & - & 8.762 \\
\hline 0.141 & 0.042 & 0.009 & 6.297 \\
\hline 0.144 & 0.042 & 0.006 & 6.702 \\
\hline 0.166 & 0.048 & 0.006 & 7.456 \\
\hline 0.139 & 0.041 & 0.003 & 6.679 \\
\hline 0.170 & 0.059 & 0.001 & 7.897 \\
\hline 0.147 & 0.050 & 0.004 & 6.861 \\
\hline 0.170 & 0.047 & 0.005 & $7 \cdot 254$ \\
\hline 0.144 & 0.030 & 0.001 & 6.731 \\
\hline 0.138 & 0.033 & 0.003 & 6.582 \\
\hline 0.118 & 0.024 & 0.002 & 5.885 \\
\hline 0.089 & 0.024 & 0.004 & 5.958 \\
\hline 0.065 & 0.019 & - & 4.461 \\
\hline 0.039 & 0.014 & 0.001 & 4.200 \\
\hline 0.034 & 0.009 & 0.002 & 3.984 \\
\hline 0.023 & 0.010 & 0.001 & 4.199 \\
\hline 0.023 & 0.006 & - & 4.230 \\
\hline 0.015 & 0.002 & 0.001 & 3.100 \\
\hline 0.011 & 0.003 & 0.001 & 3.413 \\
\hline 0.004 & 0.002 & - & 2.819 \\
\hline
\end{tabular}


Basic Table 46.B. Urban Henan Province (16): duration-specific fertility

\begin{tabular}{|c|c|c|c|c|}
\hline $\begin{array}{l}\text { Birth year } \\
\text { of child }\end{array}$ & $0-4$ & $5-9$ & $10-14$ & $15-19$ \\
\hline 1940 & 0.232 & - & - & - \\
\hline 1941 & 0.166 & - & - & - \\
\hline 1942 & 0.220 & - & - & - \\
\hline 1943 & $0.180^{\circ}$ & • & - & - \\
\hline 1944 & 0.155 & - & - & - \\
\hline 1945 & 0.170 & 0.232 & - & $\bullet$ \\
\hline 1946 & 0.242 & 0.212 & - & - \\
\hline 1947 & 0.163 & 0.246 & - & - \\
\hline 1948 & 0.185 & 0.186 & - & - \\
\hline 1945 & 0.276 & 0.200 & . & - \\
\hline 1950 & 0.203 & 0.223 & 0.232 & - \\
\hline 1951 & 0.232 & 0.252 & 0.151 & - \\
\hline 1952 & 0.333 & 0.326 & 0.298 & $\bullet$ \\
\hline 1953 & 0.227 & 0.202 & 0.137 & - \\
\hline 1954 & 0.259 & 0.360 & 0.200 & . \\
\hline 1955 & 0.285 & 0.196 & 0.329 & 0.108 \\
\hline 1950 & 0.231 & 0.204 & 0.171 & 0.197 \\
\hline 1957 & 0.288 & 0.270 & 0.217 & 0.194 \\
\hline 1958 & 0.202 & 0.293 & 0.202 & 0.124 \\
\hline 19 ن 19 & 0.226 & 0.243 & 0.153 & 0.211 \\
\hline 1960 & 0.233 & 0.221 & 0.166 & 0.149 \\
\hline 1961 & 0.197 & 0.166 & 0.126 & 0.081 \\
\hline 1902 & 0.349 & 0.213 & 0.241 & 0.199 \\
\hline 1963 & 0.434 & 0.404 & 0.242 & 0.337 \\
\hline 1964 & 0.291 & 0.314 & 0.266 & 0.214 \\
\hline 1965 & 0.392 & 0.345 & 0.142 & 0.158 \\
\hline 1966 & 0.292 & 0.202 & 0.166 & 0.175 \\
\hline 1967 & 0.343 & 0.317 & 0.144 & 0.099 \\
\hline 1968 & 0.409 & 0.308 & 0.232 & 0.117 \\
\hline 1969 & 0.324 & 0.280 & 0.221 & 0.160 \\
\hline 1970 & 0.424 & 0.253 & 0.216 & 0.064 \\
\hline 1971 & 0.328 & 0.156 & 0.159 & 0.094 \\
\hline 1972 & 0.351 & 0.260 & 0.166 & 0.082 \\
\hline 1973 & 0.319 & 0.195 & 0.136 & 0.080 \\
\hline 1974 & 0.380 & 0.176 & 0.148 & 0.093 \\
\hline 1975 & 0.235 & 0.182 & 0.121 & 0.050 \\
\hline 1976 & 0.287 & 0.144 & 0.068 & 0.058 \\
\hline 1977 & 0.310 & 0.092 & 0.059 & 0.052 \\
\hline 1978 & 0.325 & 0.103 & 0.074 & 0.045 \\
\hline 1979 & 0.302 & 0.152 & 0.102 & 0.016 \\
\hline 1980 & 0.266 & 0.039 & 0.009 & $\bullet$ \\
\hline 1901 & 0.252 & 0.061 & 0.008 & 0.020 \\
\hline 1982 & 0.244 & 0.038 & - & - \\
\hline
\end{tabular}


rates, $1940-82$

\begin{tabular}{|c|c|c|c|}
\hline $20-24$ & $25-29$ & $30+$ & Total \\
\hline$\bullet$ & - & • & - \\
\hline$\bullet$ & • & - & $\bullet$ \\
\hline - & - & - & • \\
\hline - & • & • & • \\
\hline - & - & - & $\bullet$ \\
\hline - & - & - & 4.581 \\
\hline - & - & - & 4.614 \\
\hline - & - & - & 4.773 \\
\hline - & - & - & 3.926 \\
\hline - & - & - & 4.593 \\
\hline - & - & - & 4.931 \\
\hline - & • & - & 4.599 \\
\hline - & - & - & 7.025 \\
\hline - & • & - & 4.037 \\
\hline - & - & - & 6.069 \\
\hline • & - & - & $5 \cdot 333$ \\
\hline - & - & - & 4.702 \\
\hline • & - & - & 5.656 \\
\hline - & • & - & 4.827 \\
\hline - & - & - & 4.901 \\
\hline 0.093 & - & - & 4.477 \\
\hline 0.0 .30 & - & - & 3.102 \\
\hline 0.065 & - & - & 5.523 \\
\hline 0.137 & - & - & 8.074 \\
\hline 0.078 & - & 0.009 & 6.155 \\
\hline 0.053 & 0.015 & $\bullet$ & 5.552 \\
\hline 0.121 & 0.030 & - & 4.930 \\
\hline 0.072 & 0.013 & - & 4.948 \\
\hline 0.194 & 0.050 & - & 6.553 \\
\hline 0.107 & 0.011 & 0.017 & 5.596 \\
\hline 0.098 & 0.021 & - & $5 \cdot 332$ \\
\hline 0.070 & 0.020 & - & 4.138 \\
\hline 0.035 & 0.027 & - & 4.612 \\
\hline 0.029 & - & - & 3.797 \\
\hline 0.015 & 0.015 & - & $4 \cdot 133$ \\
\hline 0.007 & 0.015 & - & 3.045 \\
\hline 0.007 & 0.007 & - & 2.850 \\
\hline 0.021 & $\bullet$ & - & 2.663 \\
\hline$\bullet$ & 0.007 & - & 2.778 \\
\hline 0.012 & • & - & 2.919 \\
\hline - & - & - & 1.567 \\
\hline - & - & $\bullet$ & 1.709 \\
\hline - & - & - & $1.41 ?$ \\
\hline
\end{tabular}


Basic Table 46.C. Rural Henan Province (16): duration-specific fertility

\begin{tabular}{|c|c|c|c|c|}
\hline $\begin{array}{l}\text { Birth year } \\
\text { of child }\end{array}$ & $0-4$ & $5-9$ & $10-14$ & $15-19$ \\
\hline 1940 & 0.192 & - & - & - \\
\hline 1941 & 0.211 & - & - & - \\
\hline 1942 & 0.209 & - & - & - \\
\hline 1943 & 0.171 & $\bullet$ & : & $\bullet$ \\
\hline $\begin{array}{l}1944 \\
1945\end{array}$ & 0.210 & 0.255 & $\dot{\bullet}$ & . \\
\hline $1940^{\circ}$ & 0.238 & 0.237 & • & - \\
\hline 1947 & 0.240 & 0.287 & - & - \\
\hline 1948 & 0.234 & 0.244 & - & - \\
\hline 1949 & 0.223 & 0.254 & - & - \\
\hline 1950 & 0.229 & 0.279 & 0.217 & - \\
\hline 1951 & 0.257 & 0.284 & 0.245 & $\bullet$ \\
\hline 1952 & 0.309 & 0.293 & 0.241 & - \\
\hline 1953 & 0.269 & 0.284 & 0.249 & - \\
\hline 1954 & 0.285 & 0.269 & 0.280 & - \\
\hline 1955 & 0.290 & 0.297 & 0.272 & 0.196 \\
\hline 1956 & 0.263 & 0.246 & 0.238 & 0.192 \\
\hline 1957 & 0.298 & 0.284 & 0.272 & 0.209 \\
\hline 1958 & 0.208 & 0.231 & 0.215 & 0.185 \\
\hline 1959 & 0.150 & 0.183 & 0.164 & 0.150 \\
\hline 1900 & 0.130 & 0.175 & 0.141 & 0.139 \\
\hline 1961 & 0.135 & 0.145 & 0.128 & 0.088 \\
\hline 1902 & 0.290 & 0.324 & 0.286 & 0.225 \\
\hline 1903 & 0.387 & 0.415 & 0.383 & 0.300 \\
\hline 1964 & 0.288 & 0.278 & 0.266 & 0.232 \\
\hline 1965 & 0.285 & 0.319 & 0.320 & 0.234 \\
\hline 1966 & 0.353 & 0.363 & 0.329 & 0.284 \\
\hline 1967 & 0.317 & 0.318 & 0.299 & 0.248 \\
\hline 1968 & 0.399 & 0.381 & 0.325 & 0.281 \\
\hline 1909 & 0.344 & 0.328 & 0.280 & 0.242 \\
\hline 1970 & 0.348 & 0.339 & 0.316 & 0.265 \\
\hline 1971 & 0.353 & 0.323 & 0.310 & 0.238 \\
\hline 1972 & 0.335 & 0.328 & 0.287 & 0.230 \\
\hline 1973 & 0.324 & 0.315 & 0.238 & 0.193 \\
\hline 1974 & 0.371 & 0.308 & 0.249 & 0.278 \\
\hline 1975 & 0.309 & 0.259 & 0.150 & 0.111 \\
\hline 1976 & 0.334 & 0.226 & 0.160 & 0.087 \\
\hline 1977 & 0.343 & 0.231 & 0.127 & 0.068 \\
\hline 1978 & 0.357 & 0.247 & 0.138 & 0.084 \\
\hline 1979 & 0.377 & 0.251 & 0.138 & 0.071 \\
\hline 1980 & 0.325 & 0.190 & 0.076 & 0.033 \\
\hline 1981 & 0.380 & 0.201 & 0.082 & 0.028 \\
\hline 1982 & 0.307 & 0.165 & 0.078 & 0.027 \\
\hline
\end{tabular}


rates, $1940-82$

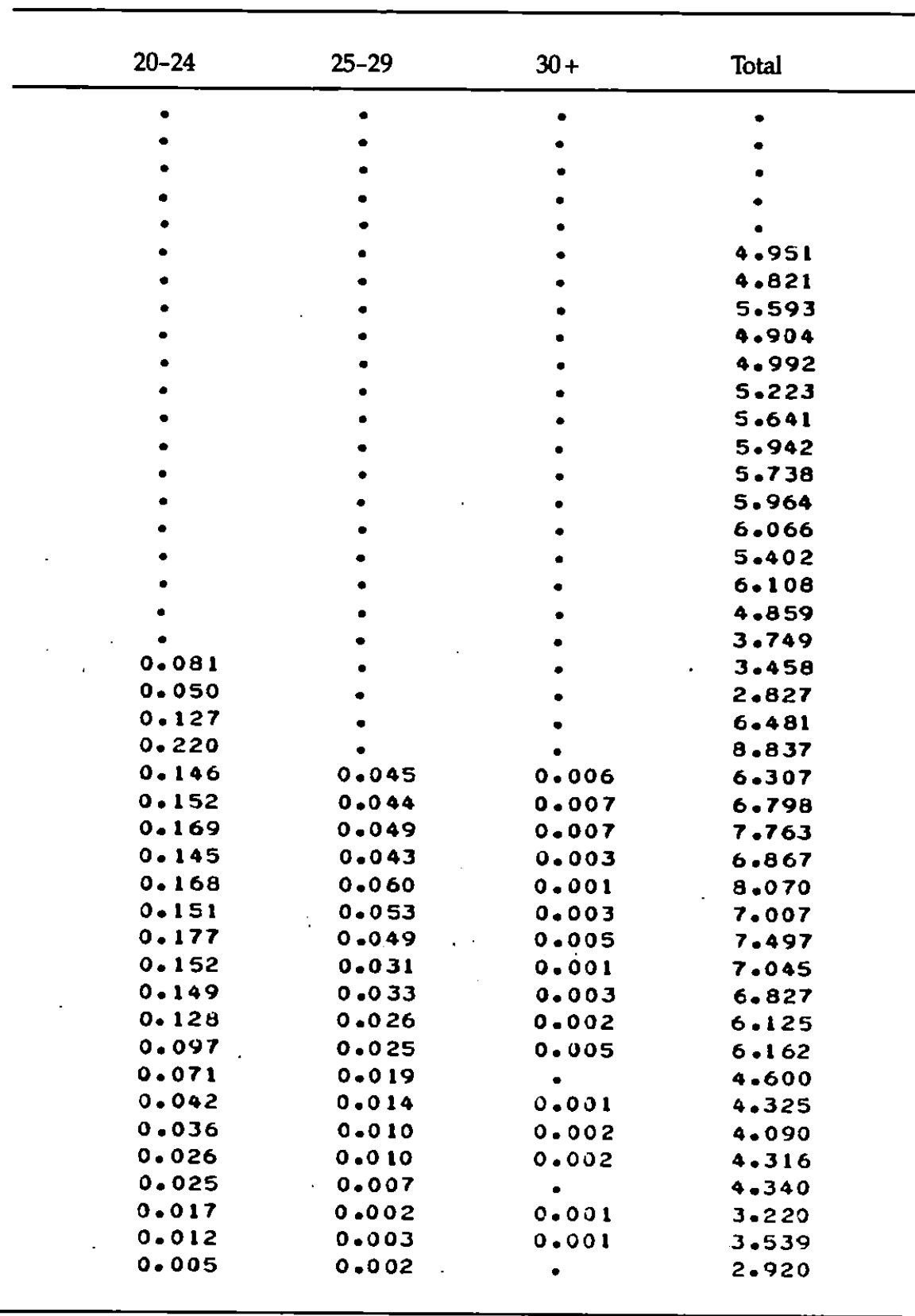


Basic Table 47.A. Total Hubei Province (17): duration-specific fertility

\begin{tabular}{|c|c|c|c|c|}
\hline $\begin{array}{l}\text { Birth year } \\
\text { of child }\end{array}$ & $0-4$ & $5-9$ & $10-14$ & $15-19$ \\
\hline 1940 & 0.222 & - & $\bullet$ & $\bullet$ \\
\hline 1941 & 0.290 & - & - & - \\
\hline 1942 & 0.202 & - & - & - \\
\hline 1943 & 0.280 & • & - & $\bullet$ \\
\hline 1944 & 0.242 & - & - & - \\
\hline 1945 & 0.249 & 0.264 & • & - \\
\hline 1946 & 0.262 & 0.296 & - & - \\
\hline 1947 & 0.280 & 0.262 & - & - \\
\hline 1948 & 0.298 & 0.301 & - & - \\
\hline 1949 & 0.265 & 0.284 & - & - \\
\hline 1950 & 0.269 & 0.258 & 0.235 & - \\
\hline 1951 & 0.275 & 0.296 & 0.273 & - \\
\hline 1952 & 0.324 & 0.323 & 0.255 & - \\
\hline 1953 & 0.314 & 0.274 & 0.251 & • \\
\hline 1954 & 0.347 & 0.305 & 0.272 & • \\
\hline 1955 & 0.324 & 0.313 & 0.287 & 0.203 \\
\hline 1956 & 0.287 & 0.296 & 0.230 & 0.180 \\
\hline 1957 & 0.362 & 0.359 & 0.309 & 0.261 \\
\hline 1958 & 0.339 & 0.312 & 0.253 & 0.211 \\
\hline 1959 & 0.227 & 0.289 & 0.228 & 0.172 \\
\hline $\begin{array}{l}1960 \\
1961\end{array}$ & $\begin{array}{l}0.156 \\
0.217\end{array}$ & $\begin{array}{l}0.166 \\
0.270\end{array}$ & $\begin{array}{l}0.150 \\
0.224\end{array}$ & $\begin{array}{l}0.103 \\
0.100\end{array}$ \\
\hline 1962 & 0.374 & 0.408 & 0.340 & 0.268 \\
\hline 1963 & 0.419 & 0.391 & 0.335 & 0.316 \\
\hline 1964 & 0.329 & 0.362 & 0.304 & 0.211 \\
\hline 1965 & 0.365 & 0.312 & 0.293 & 0.227 \\
\hline 1960 & 0.360 & 0.377 & 0.288 & 0.248 \\
\hline 1967 & 0.329 & 0.278 & 0.275 & 0.205 \\
\hline 1968 & 0.446 & 0.393 & 0.370 & 0.253 \\
\hline 1909 & 0.382 & 0.321 & 0.276 & 0.250 \\
\hline 1970 & 0.378 & 0.332 & 0.282 & 0.199 \\
\hline 1971 & 0.392 & 0.325 & 0.259 & 0.200 \\
\hline 1972 & 0.384 & 0.261 & 0.222 & 0.167 \\
\hline 1973 & 0.356 & 0.277 & 0.181 & 0.117 \\
\hline 1974 & 0.407 & 0.261 & 0.145 & 0.093 \\
\hline 1975 & 0.390 & 0.247 & 0.124 & 0.069 \\
\hline 1976 & 0.427 & 0.214 & 0.107 & 0.052 \\
\hline 1977 & 0.375 & 0.205 & 0.075 & 0.039 \\
\hline 1978 & 0.366 & 0.167 & 0.090 & 0.034 \\
\hline 1979 & 0.410 & 0.205 & 0.106 & 0.032 \\
\hline 1980 & 0.297 & 0.156 & 0.052 & 0.026 \\
\hline 1981 & 0.359 & 0.124 & 0.041 & 0.019 \\
\hline 1982 & 0.301 & 0.100 & 0.049 & 0.032 \\
\hline
\end{tabular}


rates, $1940-82$

\begin{tabular}{|c|c|c|c|}
\hline $20-24$ & $25-29$ & $30+$ & Total \\
\hline • & - & - & - \\
\hline$\bullet$ & $\bullet$ & - & - \\
\hline - & - & - & - \\
\hline$\bullet$ & • & - & - \\
\hline - & - & - & - \\
\hline - & - & - & $5 \cdot 308$ \\
\hline - & - & - & 5.863 \\
\hline$\bullet$ & - & - & 5.439 \\
\hline - & - & $\bullet$ & 6.127 \\
\hline - & - & - & 5.696 \\
\hline - & • & $\bullet$ & 5.420 \\
\hline • & - & • & 6.077 \\
\hline$\bullet$ & - & • & $6 \cdot 383$ \\
\hline - & - & - & 5.885 \\
\hline • & - & $\bullet$ & 6.492 \\
\hline • & - & - & 6.480 \\
\hline - & - & - & 5.681 \\
\hline - & - & • & 7.451 \\
\hline - & - & - & 6.343 \\
\hline - & - & - & 5.280 \\
\hline 0.063 & • & - & 3.264 \\
\hline 0.084 & • & - & 4.929 \\
\hline 0.162 & - & - & 7.985 \\
\hline 0.179 & $\bullet$ & • & 8.480 \\
\hline 0.121 & 0.040 & - & 6.838 \\
\hline 0.116 & 0.040 & - & 6.764 \\
\hline 0.173 & 0.062 & 0.002 & 7.583 \\
\hline 0.108 & 0.023 & 0.002 & 6.102 \\
\hline 0.149 & 0.044 & 0.003 & 8.290 \\
\hline 0.139 & 0.045 & - & 7.064 \\
\hline 0.138 & 0.034 & 0.001 & 6.822 \\
\hline 0.128 & 0.026 & 0.003 & 6.666 \\
\hline 0.089 & 0.027 & - & 5.749 \\
\hline 0.063 & 0.018 & 0.004 & 5.076 \\
\hline 0.061 & 0.018 & - & 4.929 \\
\hline 0.039 & 0.010 & 0.003 & 4.411 \\
\hline 0.028 & 0.009 & - & 4.186 \\
\hline 0.022 & 0.008 & 0.003 & 3.630 \\
\hline 0.014 & 0.004 & - & 3.372 \\
\hline 0.014 & 0.003 & - & 3.948 \\
\hline 0.009 & 0.002 & - & 2.715 \\
\hline 0.005 & - & - & 2.739 \\
\hline 0.005 & 0.004 & - & 2.459 \\
\hline
\end{tabular}


Basic Table 47.B. Urban Hubei Province (17): duration-specific fertility

\begin{tabular}{|c|c|c|c|c|}
\hline $\begin{array}{l}\text { Birth year } \\
\text { of child }\end{array}$ & $0-4$ & $5-9$ & $10-14$ & $15-19$ \\
\hline 1940 & 0.246 & - & - & - \\
\hline 1941 & 0.343 & - & - & - \\
\hline 1942 & 0.248 & $\bullet$ & • & - \\
\hline 1943 & 0.299 & - & - & - \\
\hline 1944 & 0.170 & $\bullet$ & - & - \\
\hline 1945 & 0.203 & 0.246 & - & $\bullet$ \\
\hline 1946 & 0.284 & 0.252 & - & - \\
\hline $\begin{array}{l}1947 \\
1948\end{array}$ & $\begin{array}{l}0.269 \\
0.324\end{array}$ & $\begin{array}{l}0.218 \\
0.224\end{array}$ & : & : \\
\hline 1949 & 0.270 & 0.250 & - & - \\
\hline 1450 & 0.413 & 0.215 & 0.246 & - \\
\hline 1951 & 0.298 & 0.247 & 0.242 & - \\
\hline 1952 & 0.254 & 0.202 & 0.208 & - \\
\hline 1953 & 0.453 & 0.205 & 0.235 & - \\
\hline 1954 & 0.363 & 0.260 & 0.204 & - \\
\hline 1955 & 0.328 & 0.292 & 0.305 & 0.190 \\
\hline 1956 & 0.333 & 0.275 & 0.185 & 0.141 \\
\hline 1957 & 0.398 & 0.367 & 0.376 & 0.268 \\
\hline 1958 & 0.322 & 0.327 & 0.097 & 0.096 \\
\hline 1959 & 0.337 & 0.322 & 0.260 & 0.204 \\
\hline 1960 & 0.275 & 0.279 & 0.215 & 0.136 \\
\hline 1961 & 0.252 & 0.235 & 0.207 & 0.123 \\
\hline 1902 & 0.473 & 0.300 & 0.281 & 0.148 \\
\hline 1963 & 0.420 & 0.370 & 0.358 & 0.302 \\
\hline 1964 & 0.333 & 0.260 & 0.208 & 0.190 \\
\hline 1965 & 0.307 & 0.138 & 0.194 & 0.112 \\
\hline 1906 & 0.217 & 0.221 & 0.098 & 0.138 \\
\hline 1907 & 0.268 & 0.177 & 0.164 & 0.047 \\
\hline 1968 & 0.397 & 0.345 & 0.185 & 0.094 \\
\hline $196 y$ & 0.371 & 0.201 & 0.143 & 0.149 \\
\hline 1970 & 0.385 & 0.157 & 0.148 & 0.049 \\
\hline 1971 & 0.348 & 0.237 & 0.118 & 0.069 \\
\hline 1972 & 0.344 & 0.141 & 0.083 & 0.082 \\
\hline 1973 & 0.310 & 0.199 & 0.059 & 0.032 \\
\hline 1974 & 0.297 & 0.135 & 0.075 & 0.015 \\
\hline 1975 & 0.434 & 0.121 & 0.054 & 0.015 \\
\hline 1976 & 0.306 & 0.121 & 0.026 & 0.010 \\
\hline 1977 & 0.390 & $0 \cdot 128$ & 0.007 & 0.010 \\
\hline 1978 & 0.372 & 0.081 & 0.064 & • \\
\hline 1979 & 0.303 & 0.092 & 0.024 & 0.006 \\
\hline 1980 & 0.213 & 0.048 & 0.011 & 0.006 \\
\hline 1981 & 0.270 & 0.052 & 0.011 & $\bullet$ \\
\hline 1982 & 0.198 & 0.015 & $\bullet$ & - \\
\hline
\end{tabular}


rates, $1940-82$

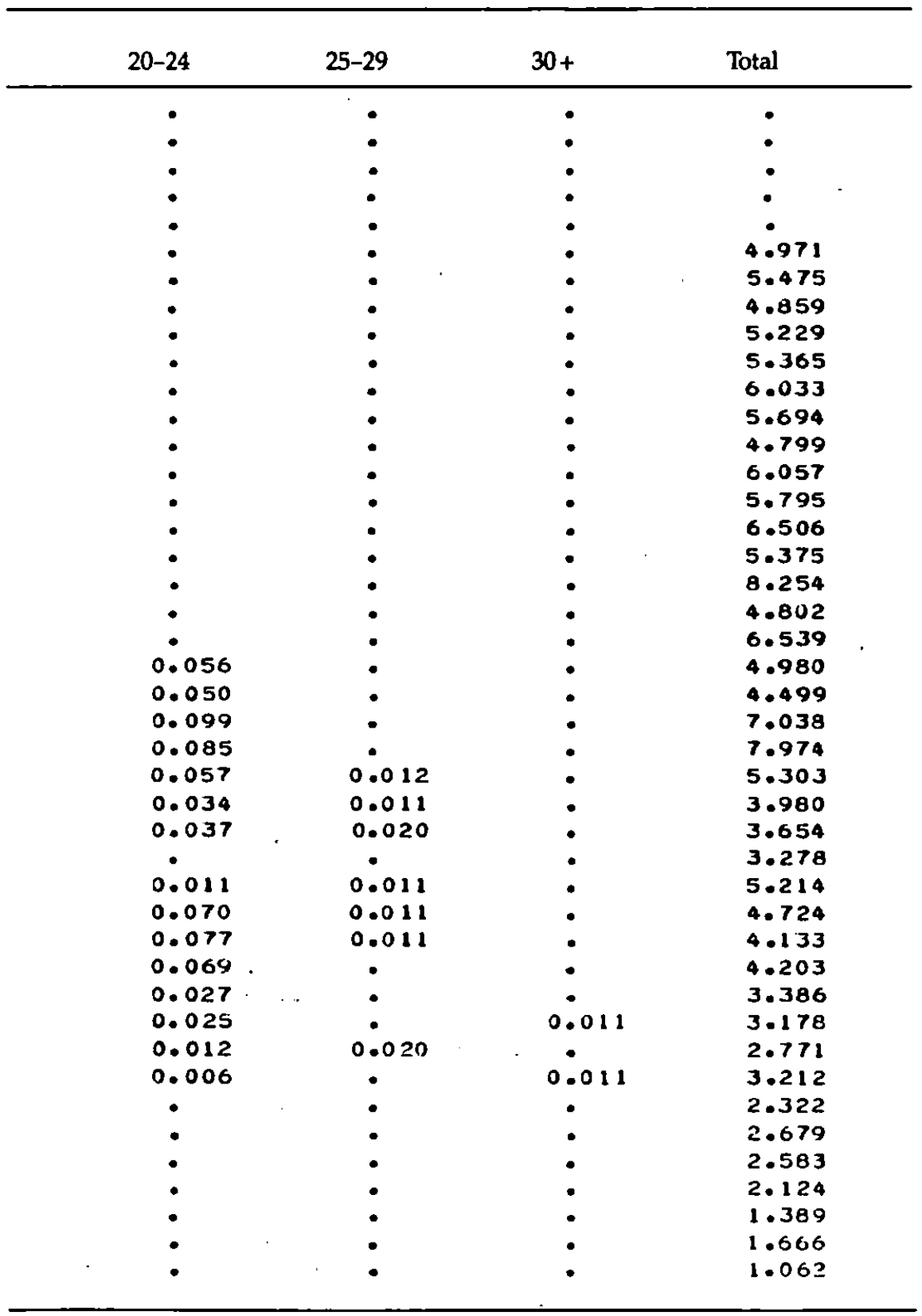


Basic Table 47.C. Rural Hubei Province (17): duration-specific fertility

\begin{tabular}{|c|c|c|c|c|}
\hline $\begin{array}{l}\text { Birth year } \\
\text { of child }\end{array}$ & $0-4$ & $5-9$ & $10-14$ & 15-19 \\
\hline 1940 & 0.219 & $\bullet$ & - & • \\
\hline 1941 & 0.283 & - & - & - \\
\hline 1942 & 0.196 & - & • & - \\
\hline 1943 & 0.277 & - & - & - \\
\hline 1944 & 0.252 & • & - & - \\
\hline 1945 & 0.255 & 0.266 & - & $\bullet$ \\
\hline 1946 & 0.259 & 0.302 & - & - \\
\hline 1947 & 0.281 & 0.269 & • & $\bullet$ \\
\hline 1948 & 0.295 & 0.312 & - & $\bullet$ \\
\hline 1949 & 0.264 & 0.289 & - & - \\
\hline 1950 & 0.248 & 0.264 & 0.234 & - \\
\hline 1951 & 0.271 & 0.302 & 0.277 & $\bullet$ \\
\hline 1952 & 0.337 & 0.336 & 0.262 & $\bullet$ \\
\hline $\begin{array}{l}1953 \\
1954\end{array}$ & $\begin{array}{l}0.289 \\
0.344\end{array}$ & $\begin{array}{l}0.283 \\
0.311\end{array}$ & $\begin{array}{l}0.253 \\
0.280\end{array}$ & - \\
\hline 1955 & 0.323 & 0.315 & 0.285 & 0.205 \\
\hline 1950 & 0.278 & 0.300 & 0.235 & 0.185 \\
\hline $\begin{array}{l}1957 \\
1950\end{array}$ & $\begin{array}{l}0.354 \\
0.343\end{array}$ & $\begin{array}{l}0.357 \\
0.309\end{array}$ & $\begin{array}{l}0.302 \\
0.273\end{array}$ & $\begin{array}{l}0.260 \\
0.226\end{array}$ \\
\hline 1959 & 0.200 & 0.283 & 0.224 & 0.168 \\
\hline $\begin{array}{l}1960 \\
1961\end{array}$ & $\begin{array}{l}0.127 \\
0.210\end{array}$ & $\begin{array}{l}0.145 \\
0.277\end{array}$ & $\begin{array}{l}0.140 \\
0.226\end{array}$ & $\begin{array}{l}0.055 \\
0.165\end{array}$ \\
\hline 1962 & 0.353 & 0.418 & 0.350 & 0.281 \\
\hline 1963 & 0.419 & 0.396 & 0.331 & 0.318 \\
\hline 1964 & 0.328 & 0.387 & 0.323 & 0.214 \\
\hline 1965 & 0.375 & 0.355 & 0.312 & 0.244 \\
\hline 1966 & 0.390 & 0.412 & 0.328 & 0.265 \\
\hline 1967 & 0.338 & 0.300 & 0.300 & 0.232 \\
\hline 1968 & 0.453 & 0.402 & 0.413 & 0.282 \\
\hline 1969 & 0.384 & 0.341 & 0.309 & 0.270 \\
\hline 1970 & 0.377 & 0.362 & 0.315 & 0.228 \\
\hline 1971 & 0.399 & 0.340 & 0.292 & 0.227 \\
\hline 1972 & 0.390 & 0.279 & 0.251 & 0.186 \\
\hline 1973 & 0.363 & 0.288 & 0.205 & 0.137 \\
\hline 1974 & 0.424 & 0.281 & 0.157 & 0.112 \\
\hline 1975 & 0.383 & 0.267 & 0.136 & 0.082 \\
\hline 1970 & 0.451 & 0.228 & 0.121 & 0.061 \\
\hline 1977 & 0.371 & 0.216 & 0.086 & 0.045 \\
\hline 1978 & $0 \cdot 364$ & 0.179 & 0.094 & 0.040 \\
\hline 1979 & 0.436 & 0.222 & 0.119 & 0.036 \\
\hline 1980 & 0.319 & 0.174 & 0.059 & 0.029 \\
\hline 1981 & 0.381 & 0.138 & 0.045 & 0.023 \\
\hline 1982 & 0.325 & 0.120 & 0.057 & 0.037 \\
\hline
\end{tabular}


rates, $1940-82$

\begin{tabular}{|c|c|c|c|}
\hline $20-24$ & $25-29$ & $30+$ & Total \\
\hline - & - & - & - \\
\hline • & • & - & • \\
\hline - & - & - & - \\
\hline - & $\bullet$ & $\bullet$ & $\bullet$ \\
\hline - & - & - & - \\
\hline - & - & - & 5.351 \\
\hline - & - & - & 5.912 \\
\hline - & - & - & 5.511 \\
\hline - & - & - & 6.243 \\
\hline$\bullet$ & - & - & 5.738 \\
\hline - & - & - & $5 \cdot 338$ \\
\hline - & - & - & 6.129 \\
\hline - & - & - & 6.603 \\
\hline - & - & - & 5.861 \\
\hline - & - & - & 6.594 \\
\hline - & - & - & 6.476 \\
\hline - & $\bullet$ & $\bullet$ & 5.727 \\
\hline - & - & - & $7 \cdot 325$ \\
\hline$\bullet$ & - & - & 6.594 \\
\hline - & - & - & 5.070 \\
\hline $0.0 \in 4$ & $\bullet$ & • & 2.978 \\
\hline 0.089 & - & • & 5.001 \\
\hline 0.171 & - & $\bullet$ & $8 \cdot 145$ \\
\hline 0.192 & - & - & 8.565 \\
\hline 0.129 & 0.044 & - & 7.129 \\
\hline 0.126 & 0.043 & - & $7 \cdot 275$ \\
\hline 0.189 & 0.069 & 0.003 & 8.276 \\
\hline 0.120 & 0.027 & 0.002 & 6.591 \\
\hline 0.167 & 0.049 & 0.004 & 8.843 \\
\hline 0.148 & 0.049 & • & 7.500 \\
\hline 0.146 & 0.037 & 0.001 & $7 \cdot 332$ \\
\hline 0.138 & 0.029 & 0.003 & 7.132 \\
\hline 0.099 & 0.030 & - & $\epsilon .180$ \\
\hline 0.064 & 0.020 & 0.003 & 5.425 \\
\hline 0.071 & 0.018 & $\bullet$ & 5.317 \\
\hline 0.046 & 0.011 & 0.001 & 4.633 \\
\hline 0.033 & 0.011 & - & 4.526 \\
\hline 0.027 & 0.009 & 0.003 & 3.785 \\
\hline 0.017 & 0.005 & - & 3.499 \\
\hline 0.018 & 0.003 & - & 4.173 \\
\hline 0.011 & 0.002 & • & 2.975 \\
\hline 0.006 & $\bullet$ & - & 2.967 \\
\hline 0.007 & 0.005 & - & 2.753 \\
\hline
\end{tabular}


Basic Table 48.A. Total Hunan Province (18): duration-specific fertility

\begin{tabular}{|c|c|c|c|c|}
\hline $\begin{array}{l}\text { Birth year } \\
\text { of child }\end{array}$ & $0-4$ & $5-9$ & $10-14$ & $15-19$ \\
\hline 1940 & 0.246 & - & - & - \\
\hline 1941 & 0.268 & $\bullet$ & • & - \\
\hline 1942 & 0.258 & - & - & $\bullet$ \\
\hline 1943 & 0.243 & - & - & - \\
\hline 1944 & 0.272 & - & - & $\bullet$ \\
\hline 1945 & 0.242 & 0.273 & - & - \\
\hline 1946 & 0.240 & 0.315 & - & - \\
\hline 1947 & 0.295 & 0.304 & - & • \\
\hline 1948 & 0.298 & 0.316 & - & - \\
\hline 1949 & 0.284 & 0.337 & - & • \\
\hline 1950 & 0.269 & 0.319 & 0.264 & - \\
\hline 1951 & 0.291 & 0.292 & 0.283 & - \\
\hline 1952 & 0.303 & 0.321 & 0.300 & $\bullet$ \\
\hline 1953 & 0.334 & 0.322 & 0.223 & $\bullet$ \\
\hline 1954 & 0.310 & 0.302 & 0.300 & - \\
\hline 1955 & 0.271 & 0.313 & 0.254 & 0.223 \\
\hline 1956 & 0.295 & 0.325 & 0.300 & 0.225 \\
\hline 1957 & 0.321 & 0.329 & 0.272 & 0.239 \\
\hline 1958 & 0.276 & 0.269 & 0.261 & 0.220 \\
\hline 1959 & 0.189 & 0.207 & 0.203 & 0.156 \\
\hline 1960 & 0.174 & 0.197 & $0.1 \in 0$ & 0.150 \\
\hline 1961 & 0.122 & 0.174 & 0.132 & 0.084 \\
\hline 1962 & 0.313 & 0.346 & 0.339 & 0.262 \\
\hline 1963 & 0.387 & 0.417 & 0.362 & 0.302 \\
\hline 1904 & 0.356 & 0.356 & 0.303 & 0.237 \\
\hline 1965 & 0.320 & 0.369 & 0.290 & 0.246 \\
\hline 1960 & 0.372 & 0.336 & 0.300 & 0.263 \\
\hline 1967 & 0.290 & 0.286 & 0.243 & 0.216 \\
\hline 1908 & 0.422 & 0.414 & 0.355 & 0.266 \\
\hline 1969 & 0.354 & 0.316 & 0.275 & 0.247 \\
\hline 1970 & 0.349 & 0.324 & 0.224 & 0.220 \\
\hline 1971 & 0.369 & 0.344 & 0.248 & 0.174 \\
\hline 1972 & 0.387 & 0.290 & 0.227 & 0.157 \\
\hline 1973 & 0.368 & 0.289 & 0.193 & 0.133 \\
\hline 1974 & 0.417 & 0.285 & 0.192 & 0.129 \\
\hline 1975 & 0.405 & 0.283 & 0.149 & 0.095 \\
\hline 1970 & 0.383 & 0.215 & 0.124 & 0.061 \\
\hline 1977 & 0.383 & 0.191 & 0.091 & 0.042 \\
\hline 1970 & 0.362 & 0.183 & 0.069 & 0.028 \\
\hline 1979 & 0.390 & 0.181 & 0.066 & 0.024 \\
\hline 1980 & $0 \cdot 352$ & 0.152 & 0.050 & 0.014 \\
\hline 1981 & 0.402 & 0.160 & 0.054 & 0.022 \\
\hline 1982 & 0.403 & 0.206 & 0.068 & 0.025 \\
\hline
\end{tabular}


rates, $1940-82$

\begin{tabular}{|c|c|c|c|}
\hline $20-24$ & $25-29$ & $30+$ & Total \\
\hline - & - & - & - \\
\hline - & - & - & - \\
\hline - & - & - & - \\
\hline - & - & - & - \\
\hline - & - & - & - \\
\hline • & - & - & 5.452 \\
\hline - & - & - & 6.105 \\
\hline - & - & - & 0.175 \\
\hline - & - & - & 6.360 \\
\hline - & - & - & 6.621 \\
\hline - & - & - & 6.211 \\
\hline - & - & - & 6.263 \\
\hline - & - & - & 6.684 \\
\hline - & - & • & 6.725 \\
\hline - & - & - & 6.553 \\
\hline - & - & - & 6.156 \\
\hline & - & - & 6.660 \\
\hline - & - & - & 6.742 \\
\hline - & - & - & 5.953 \\
\hline - & - & - & 4.402 \\
\hline 0.100 & - & - & 4.082 \\
\hline 0.055 & - & - & 2.949 \\
\hline 0.115 & - & - & 7.153 \\
\hline 0.190 & - & - & 0.608 \\
\hline 0.175 & 0.073 & - & 7.532 \\
\hline 0.160 & 0.060 & 0.015 & 7.304 \\
\hline 0.180 & 0.043 & 0.008 & 7.505 \\
\hline 0.129 & 0.034 & 0.011 & 6.045 \\
\hline 0.159 & 0.052 & 0.003 & 8.358 \\
\hline 0.139 & 0.054 & 0.004 & 6.944 \\
\hline 0.138 & 0.038 & 0.006 & 6.800 \\
\hline 0.121 & 0.030 & 0.006 & 6.457 \\
\hline 0.112 & 0.031 & 0.002 & 6.039 \\
\hline 0.086 & 0.019 & 0.006 & 5.466 \\
\hline 0.076 & 0.026 & 0.005 & 5.652 \\
\hline 0.055 & 0.023 & 0.003 & 5.069 \\
\hline 0.027 & 0.018 & 0.002 & 4.152 \\
\hline 0.023 & 0.011 & 0.003 & 3.721 \\
\hline 0.018 & 0.010 & $\bullet$ & 3.348 \\
\hline 0.017 & 0.006 & 0.001 & 3.424 \\
\hline 0.007 & 0.001 & 0.002 & 2.837 \\
\hline 0.007 & 0.006 & 0.001 & 3.250 \\
\hline 0.014 & 0.003 & 0.002 & 3.623 \\
\hline
\end{tabular}


Basic Table 48.B. Urban Hunan Province (18): duration-specific fertility

\begin{tabular}{|c|c|c|c|c|}
\hline $\begin{array}{l}\text { Birth year } \\
\text { of child }\end{array}$ & $0-4$ & $5-9$ & $10-14$ & $15-19$ \\
\hline 1940 & 0.268 & • & - & - \\
\hline 1941 & 0.272 & - & - & - \\
\hline 1942 & 0.283 & - & - & - \\
\hline 1943 & 0.246 & • & $\bullet$ & - \\
\hline 1944 & 0.309 & - & - & . \\
\hline 1945 & 0.301 & 0.230 & - & • \\
\hline 1946 & 0.236 & 0.198 & - & - \\
\hline $\begin{array}{l}1947 \\
1948\end{array}$ & $\begin{array}{l}0.354 \\
0.298\end{array}$ & $\begin{array}{l}0.271 \\
0.213\end{array}$ & $\bullet$ & 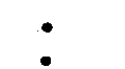 \\
\hline 1949 & 0.376 & 0.237 & - & - \\
\hline 1950 & 0.267 & 0.294 & 0.185 & • \\
\hline 1951 & 0.296 & 0.282 & 0.191 & - \\
\hline 1952 & 0.271 & 0.264 & 0.245 & • \\
\hline 1953 & 0.412 & 0.228 & 0.199 & . \\
\hline 1954 & 0.318 & 0.259 & 0.178 & - \\
\hline 1955 & 0.326 & 0.287 & 0.224 & 0.204 \\
\hline 1956 & 0.290 & 0.272 & 0.249 & 0.099 \\
\hline 1957 & 0.462 & 0.336 & 0.204 & 0.167 \\
\hline 1958 & 0.320 & 0.300 & 0.240 & 0.140 \\
\hline 1959 & 0.311 & 0.228 & 0.141 & 0.105 \\
\hline 1960 & 0.328 & 0.184 & 0.141 & 0.133 \\
\hline 1961 & 0.202 & 0.180 & 0.124 & 0.085 \\
\hline 1962 & 0.421 & 0.246 & 0.213 & 0.186 \\
\hline 1903 & 0.357 & 0.343 & 0.209 & 0.146 \\
\hline 1964 & 0.444 & 0.287 & 0.166 & 0.135 \\
\hline 1965 & 0.381 & 0.268 & 0.113 & 0.070 \\
\hline 1966 & 0.272 & 0.147 & 0.141 & 0.071 \\
\hline 1967 & 0.343 & 0.211 & 0.069 & 0.065 \\
\hline 1968 & 0.366 & 0.203 & 0.152 & 0.028 \\
\hline 1969 & 0.353 & 0.162 & 0.160 & 0.055 \\
\hline 1970 & 0.333 & 0.165 & 0.112 & 0.064 \\
\hline 1971 & 0.285 & 0.142 & 0.055 & 0.031 \\
\hline 1972 & 0.329 & 0.100 & 0.096 & 0.038 \\
\hline 1973 & 0.203 & 0.080 & 0.058 & 0.008 \\
\hline 1974 & 0.341 & 0.128 & 0.061 & 0.008 \\
\hline 1975 & 0.388 & 0.073 & 0.052 & 0.026 \\
\hline 1976 & 0.294 & 0.123 & - & $\bullet$ \\
\hline 1977 & 0.339 & 0.090 & 0.011 & - \\
\hline 1978 & 0.272 & 0.054 & $\bullet$ & - \\
\hline 1979 & 0.212 & 0.085 & 0.011 & - \\
\hline $\begin{array}{l}1980 \\
1981\end{array}$ & $\begin{array}{l}0.265 \\
0.239\end{array}$ & $\begin{array}{l}0.048 \\
0.034\end{array}$ & 0.010 & 0.022 \\
\hline 1982 & 0.205 & 0.082 & - & - \\
\hline
\end{tabular}


rates, $1940-82$

\begin{tabular}{|c|c|c|c|}
\hline $20-24$ & $25-29$ & $30+$ & Total \\
\hline - & - & - & - \\
\hline • & • & $\bullet$ & - \\
\hline - & - & - & - \\
\hline - & $\bullet$ & - & - \\
\hline - & - & - & - \\
\hline - & - & - & 5.197 \\
\hline - & - & $\bullet$ & 4.359 \\
\hline$\bullet$ & - & - & 6.122 \\
\hline • & $\bullet$ & - & 4.912 \\
\hline • & - & - & 5.695 \\
\hline - & • & - & 5.526 \\
\hline - & - & - & 5.530 \\
\hline$\bullet$ & - & - & 5.725 \\
\hline - & - & - & 5.728 \\
\hline • & - & - & $5 \cdot 326$ \\
\hline - & - & - & 6.055 \\
\hline • & - & • & 5.265 \\
\hline - & - & - & 6.668 \\
\hline - & $\bullet$ & - & 5.787 \\
\hline$\bullet$ & - & - & 4.481 \\
\hline 0.077 & - & - & 4.459 \\
\hline 0.049 & - & - & 3.319 \\
\hline 0.045 & - & - & 5.736 \\
\hline 0.106 & $\bullet$ & - & 6.025 \\
\hline 0.059 & 0.044 & - & 5.678 \\
\hline 0.049 & 0.032 & $\because$ & 4.567 \\
\hline 0.039 & $\bullet$ & $\bullet$ & 3.352 \\
\hline 0.018 & 0.006 & - & 3.559 \\
\hline 0.041 & - & - & 3.949 \\
\hline 0.029 & 0.026 & - & 3.925 \\
\hline & 0.007 & - & 3.406 \\
\hline 0.012 & - & 0.006 & 2.655 \\
\hline 0.013 & - & $\bullet$ & $2 . A 78$ \\
\hline 0.007 & - & - & 1.780 \\
\hline$\bullet$ & - & - & 2.688 \\
\hline - & - & - & 2.690 \\
\hline - & - & $\bullet$ & 2.086 \\
\hline$\bullet$ & - & 0.006 & 2.230 \\
\hline$\bullet$ & - & - & $1.62 A$ \\
\hline$\bullet$ & - & - & 1.538 \\
\hline - & & 0.006 & 1.647 \\
\hline$\bullet$ & $\bullet$ & $\bullet$ & 1.472 \\
\hline$\bullet$ & - & - & 1.435 \\
\hline
\end{tabular}


Basic Table 48.C. Rural Hunan Province (18): duration-specific fertility

\begin{tabular}{|c|c|c|c|c|}
\hline $\begin{array}{l}\text { Birth year } \\
\text { of child }\end{array}$ & $0-4$ & $5-9$ & $10-14$ & 15-19 \\
\hline 1940 & 0.241 & - & - & $\bullet$ \\
\hline 1941 & 0.268 & - & - & - \\
\hline 1942 & 0.252 & - & - & - \\
\hline 1943 & 0.242 & • & - & - \\
\hline 1944 & 0.265 & - & - & • \\
\hline 1945 & 0.231 & 0.284 & - & . \\
\hline 1946 & 0.240 & 0.344 & - & - \\
\hline 1947 & 0.283 & 0.312 & - & - \\
\hline 1948 & 0.298 & 0.337 & - & - \\
\hline 1949 & 0.268 & 0.357 & $\bullet$ & - \\
\hline 1950 & 0.266 & 0.323 & 0.284 & - \\
\hline 1951 & 0.290 & 0.293 & 0.306 & - \\
\hline 1952 & 0.308 & 0.333 & 0.312 & - \\
\hline 1933 & 0.324 & 0.340 & 0.301 & - \\
\hline 1954 & 0.308 & 0.309 & 0.324 & - \\
\hline 1955 & 0.263 & 0.317 & 0.260 & 0.228 \\
\hline 1956 & 0.296 & 0.333 & 0.309 & 0.256 \\
\hline 1957 & 0.302 & 0.328 & 0.286 & 0.255 \\
\hline 1958 & 0.269 & 0.265 & 0.265 & 0.237 \\
\hline 1959 & 0.172 & 0.204 & 0.214 & 0.166 \\
\hline 1960 & 0.153 & 0.198 & 0.163 & 0.154 \\
\hline 1961 & 0.112 & 0.173 & 0.133 & 0.084 \\
\hline 1962 & 0.302 & 0.359 & 0.356 & 0.277 \\
\hline 1963 & 0.389 & 0.428 & 0.382 & 0.332 \\
\hline 1904 & 0.349 & 0.366 & 0.329 & 0.255 \\
\hline 1965 & 0.315 & 0.383 & 0.316 & 0.274 \\
\hline 1960 & 0.380 & 0.358 & 0.322 & 0.291 \\
\hline 1907 & 0.286 & 0.293 & 0.267 & 0.237 \\
\hline 1968 & 0.427 & 0.433 & 0.384 & 0.297 \\
\hline 1909 & 0.354 & 0.328 & 0.292 & 0.274 \\
\hline 1970 & 0.350 & 0.336 & $0.30 \mathrm{e}$ & 0.243 \\
\hline 1971 & 0.376 & 0.358 & 0.271 & 0.194 \\
\hline $1 \rightarrow 72$ & 0.391 & 0.304 & 0.241 & 0.174 \\
\hline 1973 & 0.380 & 0.304 & 0.204 & 0.152 \\
\hline 1974 & 0.423 & 0.297 & 0.202 & 0.146 \\
\hline 1975 & 0.406 & 0.298 & 0.157 & 0.105 \\
\hline 1970 & 0.391 & 0.222 & 0.133 & 0.068 \\
\hline 1977 & 0.388 & 0.198 & 0.097 & 0.047 \\
\hline 1978 & 0.373 & 0.192 & 0.074 & 0.031 \\
\hline 1979 & 0.413 & 0.188 & 0.070 & 0.026 \\
\hline 1980 & 0.363 & 0.161 & 0.052 & 0.016 \\
\hline 1981 & 0.424 & 0.172 & 0.058 & 0.022 \\
\hline 1982 & 0.430 & 0.221 & 0.073 & 0.027 \\
\hline
\end{tabular}


rates, $1940-82$

\begin{tabular}{|c|c|c|c|}
\hline $20-24$ & $25-29$ & $30+$ & Total \\
\hline - & - & • & - \\
\hline$\bullet$ & - & - & • \\
\hline - & - & - & - \\
\hline$\bullet$ & - & - & - \\
\hline - & - & - & • \\
\hline - & - & - & 5.504 \\
\hline - & - & - & 6.460 \\
\hline - & - & - & 6.186 \\
\hline - & - & - & 6.651 \\
\hline - & - & • & 6.799 \\
\hline - & - & - & 6.339 \\
\hline - & - & - & $6 \cdot 398$ \\
\hline - & - & - & 6.959 \\
\hline - & . & - & 6.902 \\
\hline - & - & - & 6.771 \\
\hline - & - & - & 6.173 \\
\hline$\bullet$ & - & - & 6.902 \\
\hline - & - & - & 6.755 \\
\hline - & - & - & 5.981 \\
\hline - & - & - & 4.388 \\
\hline 0.106 & - & - & 4.019 \\
\hline 0.056 & - & - & 2.889 \\
\hline 0.130 & . & - & 7.377 \\
\hline 0.207 & . & - & 9.006 \\
\hline 0.198 & 0.080 & . & 7.882 \\
\hline 0.180 & 0.067 & 0.018 & 7.769 \\
\hline 0.206 & 0.053 & 0.009 & 8.093 \\
\hline 0.151 & 0.040 & 0.014 & 6.442 \\
\hline 0.181 & 0.063 & 0.004 & 9.944 \\
\hline 0.158 & 0.060 & 0.005 & 7.349 \\
\hline 0.160 & 0.044 & 0.008 & 7.244 \\
\hline 0.137 & 0.036 & 0.006 & 6.885 \\
\hline 0.126 & 0.038 & 0.003 & 6.383 \\
\hline 0.096 & 0.023 & 0.007 & 5.827 \\
\hline 0.086 & 0.031 & 0.006 & 5.960 \\
\hline 0.063 & 0.027 & 0.004 & 5.297 \\
\hline 0.031 & 0.021 & 0.002 & 4.347 \\
\hline 0.026 & 0.012 & 0.002 & 3.855 \\
\hline 0.021 & 0.011 & - & 3.509 \\
\hline 0.020 & 0.007 & 0.001 & 3.622 \\
\hline 0.008 & 0.001 & 0.001 & 3.003 \\
\hline 0.007 & 0.007 & 0.001 & 3.454 \\
\hline 0.015 & 0.009 & 0.002 & 3.879 \\
\hline
\end{tabular}


Basic Table 49.A. Total Guangdong Province (19): duration-specific

\begin{tabular}{|c|c|c|c|c|}
\hline $\begin{array}{l}\text { Birth year } \\
\text { of child }\end{array}$ & $0-4$ & $5-9$ & $10-14$ & 15-19 \\
\hline 1940 & 0.151 & - & - & - \\
\hline 1941 & 0.168 & - & - & - \\
\hline 1942 & 0.184 & - & • & - \\
\hline 1943 & 0.181 & - & - & - \\
\hline 1944 & 0.184 & - & - & - \\
\hline 1945 & 0.217 & 0.235 & • & - \\
\hline 1946 & 0.193 & 0.204 & - & - \\
\hline 1947 & 0.192 & 0.247 & - & $\bullet$ \\
\hline 1948 & 0.216 & 0.239 & - & • \\
\hline 1949 & 0.205 & 0.274 & $\bullet$ & - \\
\hline 1950 & 0.232 & 0.270 & 0.249 & $\bullet$ \\
\hline 1951 & 0.220 & 0.283 & 0.224 & - \\
\hline 1952 & 0.264 & 0.254 & 0.232 & - \\
\hline 1953 & 0.277 & 0.276 & 0.251 & - \\
\hline 1954 & 0.271 & 0.254 & 0.277 & - \\
\hline 1955 & 0.286 & 0.277 & 0.225 & 0.208 \\
\hline 1956 & 0.273 & 0.260 & 0.257 & 0.197 \\
\hline 1957 & 0.328 & 0.309 & 0.207 & 0.197 \\
\hline 1958 & 0.295 & 0.287 & 0.233 & 0.207 \\
\hline 1959 & 0.258 & 0.239 & 0.229 & 0.189 \\
\hline 1960 & 0.189 & 0.205 & 0.157 & 0.150 \\
\hline 1961 & 0.224 & 0.215 & 0.179 & 0.128 \\
\hline 1962 & 0.311 & 0.357 & 0.311 & 0.222 \\
\hline 1963 & 0.332 & 0.329 & 0.283 & 0.220 \\
\hline 1964 & 0.327 & 0.321 & 0.278 & 0.177 \\
\hline 1965 & 0.326 & 0.274 & 0.249 & 0.222 \\
\hline 1966 & 0.345 & 0.326 & 0.245 & 0.211 \\
\hline 1907 & 0.343 & 0.256 & 0.230 & 0.184 \\
\hline 1968 & 0.350 & 0.343 & 0.260 & 0.221 \\
\hline 1969 & 0.336 & 0.312 & 0.238 & 0.200 \\
\hline 1970 & 0.363 & 0.287 & 0.252 & 0.203 \\
\hline 1971 & 0.337 & 0.330 & 0.232 & 0.187 \\
\hline 1972 & 0.362 & 0.282 & 0.215 & 0.187 \\
\hline 1973 & 0.357 & 0.299 & 0.210 & 0.153 \\
\hline 1974 & 0.372 & 0.294 & 0.181 & 0.108 \\
\hline 1975 & 0.334 & 0.276 & 0.168 & 0.078 \\
\hline 1970 & 0.342 & 0.264 & 0.151 & 0.103 \\
\hline 1977 & 0.355 & 0.220 & 0.110 & 0.076 \\
\hline 1978 & 0.350 & 0.268 & 0.137 & 0.085 \\
\hline 1979 & 0.408 & 0.254 & 0.123 & 0.086 \\
\hline 1980 & 0.374 & 0.230 & 0.103 & 0.070 \\
\hline 1981 & 0.407 & 0.235 & 0.111 & 0.070 \\
\hline 1982 & 0.376 & 0.251 & 0.099 & 0.037 \\
\hline
\end{tabular}


fertility rates, $1940-82$

\begin{tabular}{|c|c|c|c|}
\hline $20-24$ & $25-29$ & $30+$ & Total \\
\hline - & - & $\bullet$ & - \\
\hline - & $\bullet$ & - & - \\
\hline - & - & - & - \\
\hline - & $\bullet$ & - & - \\
\hline - & $\bullet$ & - & - \\
\hline - & • & - & 4.702 \\
\hline - & - & - & 4.126 \\
\hline - & • & - & 4.761 \\
\hline • & - & - & 4.780 \\
\hline - & $\bullet$ & - & $5 \cdot 251$ \\
\hline$\bullet$ & • & - & 5.468 \\
\hline - & & - & $5 \cdot 297$ \\
\hline - & - & - & 5.359 \\
\hline - & • & - & 5.753 \\
\hline - & - & - & 5.769 \\
\hline - & • & - & 5.720 \\
\hline - & $\bullet$ & - & 5.683 \\
\hline - & - & - & 6.274 \\
\hline - & • & - & 5.852 \\
\hline - & - & - & 5.250 \\
\hline 0.101 & - & - & 4.118 \\
\hline $0.08 B$ & - & - & 4.296 \\
\hline 0.105 & - & - & 6.741 \\
\hline 0.167 & $\bullet$ & : & 6.910 \\
\hline 0.121 & 0.037 & 0.010 & 6.361 \\
\hline 0.107 & 0.037 & 0.029 & 6.220 \\
\hline 0.133 & 0.046 & 0.002 & 6.544 \\
\hline 0.098 & 0.034 & 0.011 & 5.786 \\
\hline 0.117 & 0.040 & 0.011 & 6.747 \\
\hline 0.104 & 0.039 & 0.006 & 6.169 \\
\hline 0.096 & 0.040 & 0.007 & 6.235 \\
\hline 0.101 & 0.020 & 0.004 & 6.055 \\
\hline 0.116 & 0.028 & 0.002 & 5.961 \\
\hline 0.072 & 0.013 & 0.009 & 5.565 \\
\hline 0.065 & 0.020 & 0.003 & 5.214 \\
\hline 0.048 & 0.018 & $0.00 s$ & 4.619 \\
\hline 0.041 & 0.017 & 0.001 & 4.537 \\
\hline 0.041 & 0.012 & - & 4.072 \\
\hline 0.033 & 0.012 & 0.002 & 4.439 \\
\hline 0.029 & 0.014 & 0.003 & 4.582 \\
\hline 0.035 & 0.010 & 0.001 & 4.121 \\
\hline 0.0 .36 & 0.013 & 0.003 & 4.374 \\
\hline 0.023 & 0.003 & 0.002 & 3.953 \\
\hline
\end{tabular}


Basic Table 49.B. Urban Guangdong Province (19): duration-specific

\begin{tabular}{|c|c|c|c|c|}
\hline $\begin{array}{l}\text { Birth year } \\
\text { of child }\end{array}$ & $0-4$ & $5-9$ & $10-14$ & $15-19$ \\
\hline 1940 & 0.147 & - & - & - \\
\hline 1941 & 0.223 & - & - & $\bullet$ \\
\hline 1942 & 0.295 & - & - & - \\
\hline 1943 & 0.218 & - & - & - \\
\hline 1944 & 0.208 & $\cdot$ & - & - \\
\hline 1945 & 0.270 & $0.25 \mathrm{e}$ & - & - \\
\hline 1946 & 0.320 & 0.283 & - & - \\
\hline 1947 & 0.275 & 0.278 & - & - \\
\hline 1948 & 0.366 & 0.318 & • & - \\
\hline 1949 & 0.261 & 0.331 & - & - \\
\hline 1950 & 0.328 & 0.375 & 0.203 & - \\
\hline 1951 & 0.319 & 0.341 & 0.206 & • \\
\hline 1952 & 0.302 & 0.301 & 0.253 & - \\
\hline 1953 & 0.333 & 0.335 & 0.251 & - \\
\hline 1954 & 0.318 & 0.278 & 0.293 & - \\
\hline 1955 & 0.373 & 0.328 & 0.195 & 0.194 \\
\hline 1956 & 0.362 & 0.209 & 0.284 & 0.223 \\
\hline 1957 & 0.443 & 0.346 & 0.308 & 0.126 \\
\hline 1958 & 0.375 & 0.247 & 0.171 & 0.176 \\
\hline 1959 & 0.334 & 0.242 & 0.130 & 0.185 \\
\hline 1900 & 0.248 & 0.224 & 0.153 & 0.105 \\
\hline 1961 & 0.250 & 0.232 & 0.120 & 0.107 \\
\hline 1962 & 0.309 & 0.381 & 0.239 & 0.167 \\
\hline 1963 & 0.422 & 0.341 & 0.276 & 0.202 \\
\hline 1964 & 0.362 & 0.248 & 0.195 & 0.107 \\
\hline 1961 & 0.345 & 0.194 & 0.103 & 0.077 \\
\hline 1960 & 0.303 & 0.162 & 0.076 & 0.073 \\
\hline 1967 & 0.383 & 0.204 & 0.050 & 0.063 \\
\hline 1968 & 0.356 & 0.192 & 0.088 & 0.043 \\
\hline 1969 & 0.333 & 0.187 & 0.086 & 0.047 \\
\hline 1970 & 0.386 & 0.162 & 0.068 & 0.042 \\
\hline 1971 & 0.323 & 0.227 & 0.068 & 0.021 \\
\hline 1972 & 0.405 & 0.156 & 0.055 & 0.017 \\
\hline 1973 & 0.364 & 0.192 & 0.053 & 0.017 \\
\hline 1974 & 0.340 & 0.137 & 0.033 & - \\
\hline 1975 & 0.290 & 0.176 & 0.032 & • \\
\hline 1976 & 0.318 & 0.106 & 0.011 & - \\
\hline 1977 & 0.286 & 0.092 & 0.022 & - \\
\hline 1978 & 0.280 & 0.088 & 0.011 & - \\
\hline 1979 & 0.305 & 0.085 & - & - \\
\hline 1980 & 0.311 & 0.070 & 0.005 & - \\
\hline 1981 & 0.283 & 0.033 & 0.005 & - \\
\hline 1982 & 0.212 & 0.046 & 0.009 & • \\
\hline
\end{tabular}


fertility rates, $1940-82$

\begin{tabular}{|c|c|c|c|c|c|}
\hline & $20-24$ & $25-29$ & & $30+$ & Total \\
\hline & - & - & & - & - \\
\hline & $\bullet$ & • & & - & • \\
\hline & - & - & & - & - \\
\hline & - & $\bullet$ & & - & - \\
\hline & - & - & & - & - \\
\hline & - & - & & - & 5.504 \\
\hline & - & - & & - & 6.158 \\
\hline & - & - & & - & 5.847 \\
\hline & - & - & & - & 6.952 \\
\hline & - & - & & - & 6.632 \\
\hline & - & - & & - & 6.573 \\
\hline & - & - & & - & 6.269 \\
\hline & - & - & & - & 6.267 \\
\hline & - & - & & - & 6.686 \\
\hline & - & - & & - & 6.505 \\
\hline & • & - & & - & 6.294 \\
\hline & - & - & & - & 6.264 \\
\hline & - & - & & - & 7.018 \\
\hline & - & - & & - & 5.548 \\
\hline & - & - & & - & 5.121 \\
\hline & 0.028 & • & & - & 3.915 \\
\hline & 0.034 & - & & - & 3.842 \\
\hline & 0.067 & - & & - & 6.335 \\
\hline . & 0.059 & - & & - & 6.722 \\
\hline & 0.062 & - & & - & 4.867 \\
\hline . & 0.030 & $\bullet$ & & - & 3.738 \\
\hline & 0.014 & - & . & - & 3.139 \\
\hline & 0.013 & - & & - & 3.504 \\
\hline & 0.019 & 0.017 & & 0.011 & 3.654 \\
\hline & 0.012 & - & & - & 3.321 \\
\hline & 0.011 & - & & - & 3.342 \\
\hline & 0.005 & - & & - & 3.219 \\
\hline & 0.015 & - & & - & 3.238 \\
\hline & 0.005 & - & & - & 3.158 \\
\hline & - & - & & - & 2.551 \\
\hline & 0.005 & - & & - & 2.514 \\
\hline & • & - & & - & 2.172 \\
\hline & - & - & & - & 2.000 \\
\hline & - & & & - & 1.895 \\
\hline & - & - & & - & 1.950 \\
\hline & - & - & & - & 1.931 \\
\hline & - & 0.004 & & - & 1.621 \\
\hline & - & - & & - & 1.338 \\
\hline
\end{tabular}


Basic Table 49.C. Rural Guangdong Province (19): duration-specific

\begin{tabular}{|c|c|c|c|c|}
\hline $\begin{array}{l}\text { Birth year } \\
\text { of child }\end{array}$ & $0-4$ & $5-9$ & $10-14$ & 15-19 \\
\hline 1940 & 0.151 & - & - & - \\
\hline 1941 & 0.159 & $\bullet$ & - & - \\
\hline 1942 & 0.167 & - & - & - \\
\hline 1943 & 0.175 & - & - & - \\
\hline 1944 & 0.180 & - & - & - \\
\hline 1945 & 0.209 & 0.231 & - & - \\
\hline 1940 & 0.172 & 0.191 & - & - \\
\hline 1947 & 0.177 & 0.242 & - & - \\
\hline 1948 & 0.189 & 0.227 & - & - \\
\hline 1949 & 0.194 & 0.264 & - & • \\
\hline 1950 & 0.213 & 0.254 & 0.257 & - \\
\hline 1951 & 0.201 & 0.274 & 0.227 & - \\
\hline 1952 & 0.257 & 0.240 & 0.228 & - \\
\hline 1953 & 0.260 & 0.265 & 0.251 & - \\
\hline 1954 & 0.261 & 0.250 & 0.274 & • \\
\hline 1955 & 0.268 & 0.268 & 0.229 & 0.211 \\
\hline 1956 & 0.252 & 0.269 & 0.252 & 0.193 \\
\hline 1957 & 0.299 & 0.301 & 0.259 & 0.207 \\
\hline 1958 & 0.275 & 0.294 & 0.245 & 0.212 \\
\hline 1959 & 0.238 & 0.238 & 0.247 & 0.190 \\
\hline 1960 & 0.173 & 0.201 & 0.157 & 0.157 \\
\hline 1901 & 0.218 & 0.212 & 0.190 & 0.132 \\
\hline 1902 & 0.300 & 0.351 & 0.325 & 0.232 \\
\hline 1903 & 0.315 & 0.326 & 0.285 & 0.231 \\
\hline 1964 & 0.321 & 0.340 & 0.295 & 0.191 \\
\hline 1905 & 0.323 & 0.294 & 0.279 & 0.250 \\
\hline 1966 & 0.353 & 0.362 & 0.285 & 0.237 \\
\hline 1967 & 0.335 & 0.266 & 0.276 & 0.208 \\
\hline 1968 & 0.349 & 0.371 & 0.312 & 0.254 \\
\hline 1969 & 0.330 & 0.332 & 0.278 & 0.230 \\
\hline 1970 & 0.359 & 0.308 & 0.300 & 0.236 \\
\hline 1971 & 0.340 & 0.348 & 0.268 & 0.226 \\
\hline 1972 & 0.354 & 0.306 & 0.245 & 0.231 \\
\hline 1973 & 0.356 & 0.318 & 0.239 & 0.188 \\
\hline 1974 & 0.378 & 0.323 & 0.205 & 0.137 \\
\hline 1975 & 0.341 & 0.295 & 0.191 & 0.098 \\
\hline 1976 & 0.345 & 0.292 & 0.175 & 0.126 \\
\hline 1977 & 0.365 & 0.242 & 0.127 & 0.091 \\
\hline 1978 & 0.361 & 0.300 & 0.159 & 0.101 \\
\hline 1979 & 0.425 & 0.282 & 0.146 & 0.101 \\
\hline 1980 & 0.386 & 0.256 & 0.121 & 0.082 \\
\hline 1981 & 0.433 & 0.266 & 0.130 & 0.082 \\
\hline 1982 & 0.414 & 0.281 & 0.115 & 0.043 \\
\hline
\end{tabular}


fertility rates, $1940-82$

\begin{tabular}{|c|c|c|c|}
\hline $20-24$ & $25-29$ & $30+$ & Total \\
\hline - & - & • & - \\
\hline • & - & - & - \\
\hline - & - & $\bullet$ & - \\
\hline$\bullet$ & - & - & - \\
\hline$\bullet$ & $\bullet$ & - & - \\
\hline$\bullet$ & $\bullet$ & - & 4.572 \\
\hline$\bullet$ & - & - & 3.788 \\
\hline$\bullet$ & $\bullet$ & - & 4.579 \\
\hline - & $\bullet$ & - & 4.413 \\
\hline - & - & - & 5.013 \\
\hline$\bullet$ & - & - & 5.277 \\
\hline - & - & - & 5.127 \\
\hline$\bullet$ & - & - & 5.199 \\
\hline$\bullet$ & $\bullet$ & - & 5.588 \\
\hline - & - & - & $5 \cdot 637$ \\
\hline$\bullet$ & $\bullet$ & - & 5.615 \\
\hline - & - & - & 5.573 \\
\hline$\bullet$ & - & - & 6.130 \\
\hline$\bullet$ & $\bullet$ & - & 5.911 \\
\hline • & $\bullet$ & - & 5.275 \\
\hline 0.113 & - & - & 4.158 \\
\hline 0.096 & - & - & 4.385 \\
\hline 0.110 & - & $\bullet$ & 6.820 \\
\hline 0.183 & • & - & 6.946 \\
\hline 0.131 & 0.043 & 0.012 & 6.665 \\
\hline 0.120 & 0.043 & 0.034 & 6.714 \\
\hline 0.153 & 0.054 & 0.003 & $7 \cdot 230$ \\
\hline 0.113 & 0.039 & 0.013 & 6.255 \\
\hline 0.135 & 0.044 & 0.011 & 7.386 \\
\hline 0.121 & 0.046 & 0.00 .7 & 6.752 \\
\hline 0.112 & 0.046 & 0.008 & 6.344 \\
\hline 0.119 & 0.024 & 0.004 & 6.644 \\
\hline 0.135 & 0.033 & 0.003 & 0.533 \\
\hline 0.085 & 0.015 & 0.010 & 6.056 \\
\hline 0.077 & 0.024 & 0.004 & 5.739 \\
\hline 0.056 & 0.021 & 0.004 & 5.026 \\
\hline 0.050 & 0.020 & 0.001 & 5.048 \\
\hline 0.051 & 0.014 & - & 4.452 \\
\hline 0.041 & 0.015 & 0.002 & 4.897 \\
\hline 0.037 & 0.017 & 0.003 & 5.052 \\
\hline 0.044 & 0.012 & 0.001 & 4.513 \\
\hline 0.043 & 0.015 & 0.004 & 4.866 \\
\hline 0.027 & 0.004 & 0.002 & 4.431 \\
\hline
\end{tabular}


Basic Table 50.A. Total Guangxi Province (20): duration-specific

\begin{tabular}{|c|c|c|c|c|}
\hline $\begin{array}{l}\text { Birth year } \\
\text { of child }\end{array}$ & $0-4$ & $5-9$ & $10-14$ & $15-19$ \\
\hline 1940 & 0.244 & • & - & - \\
\hline 1941 & 0.166 & - & - & • \\
\hline 1942 & 0.229 & - & $\bullet$ & - \\
\hline 1943 & 0.215 & - & $\bullet$ & - \\
\hline 1944 & 0.239 & - & - & - \\
\hline 1945 & 0.226 & 0.298 & - & - \\
\hline 1940 & 0.209 & 0.260 & - & - \\
\hline 1947 & 0.177 & 0.245 & - & • \\
\hline 1948 & 0.203 & 0.258 & . & $\bullet$ \\
\hline 1949 & 0.251 & 0.302 & - & • \\
\hline 1950 & 0.248 & 0.287 & 0.227 & - \\
\hline 1951 & 0.228 & 0.192 & 0.220 & - \\
\hline 1952 & 0.289 & 0.307 & 0.271 & - \\
\hline 1953 & 0.279 & $0.2 \in 0$ & 0.226 & $\bullet$ \\
\hline 1954 & 0.306 & 0.288 & 0.245 & - \\
\hline 1955 & 0.287 & 0.279 & 0.228 & 0.251 \\
\hline 1956 & 0.274 & 0.261 & 0.254 & 0.199 \\
\hline 1957 & 0.336 & 0.279 & 0.257 & 0.249 \\
\hline 1958 & 0.324 & 0.329 & 0.277 & 0.221 \\
\hline 1959 & 0.191 & 0.249 & 0.196 & 0.174 \\
\hline 1960 & 0.240 & 0.217 & 0.219 & 0.212 \\
\hline 1961 & 0.236 & 0.216 & 0.153 & 0.151 \\
\hline 1962 & 0.332 & 0.381 & 0.282 & 0.193 \\
\hline 1963 & 0.381 & 0.376 & 0.345 & 0.273 \\
\hline 1964 & 0.379 & 0.326 & 0.343 & 0.218 \\
\hline 1965 & 0.331 & 0.360 & 0.282 & 0.225 \\
\hline 1906 & 0.403 & 0.370 & 0.285 & 0.247 \\
\hline 1967 & 0.278 & 0.285 & 0.243 & 0.191 \\
\hline 1968 & 0.435 & 0.342 & 0.289 & 0.257 \\
\hline 1969 & 0.330 & 0.341 & 0.270 & 0.194 \\
\hline 1970 & 0.324 & 0.295 & 0.274 & 0.220 \\
\hline 1971 & 0.377 & 0.336 & 0.249 & 0.199 \\
\hline 1972 & 0.321 & 0.331 & 0.232 & 0.155 \\
\hline 1973 & 0.390 & 0.315 & 0.266 & 0.173 \\
\hline 1974 & 0.414 & 0.305 & 0.245 & 0.197 \\
\hline 1975 & 0.395 & 0.317 & 0.252 & 0.150 \\
\hline 1970 & 0.367 & 0.296 & 0.175 & 0.125 \\
\hline 1977 & 0.415 & 0.266 & 0.155 & 0.092 \\
\hline 1978 & 0.391 & 0.250 & 0.151 & 0.074 \\
\hline 1979 & 0.389 & 0.291 & 0.164 & 0.078 \\
\hline 1980 & 0.434 & 0.267 & 0.138 & 0.072 \\
\hline 1981 & 0.380 & 0.264 & 0.131 & 0.075 \\
\hline 1982 & 0.485 & 0.252 & 0.159 & 0.082 \\
\hline
\end{tabular}


fertility rates, $1940-82$

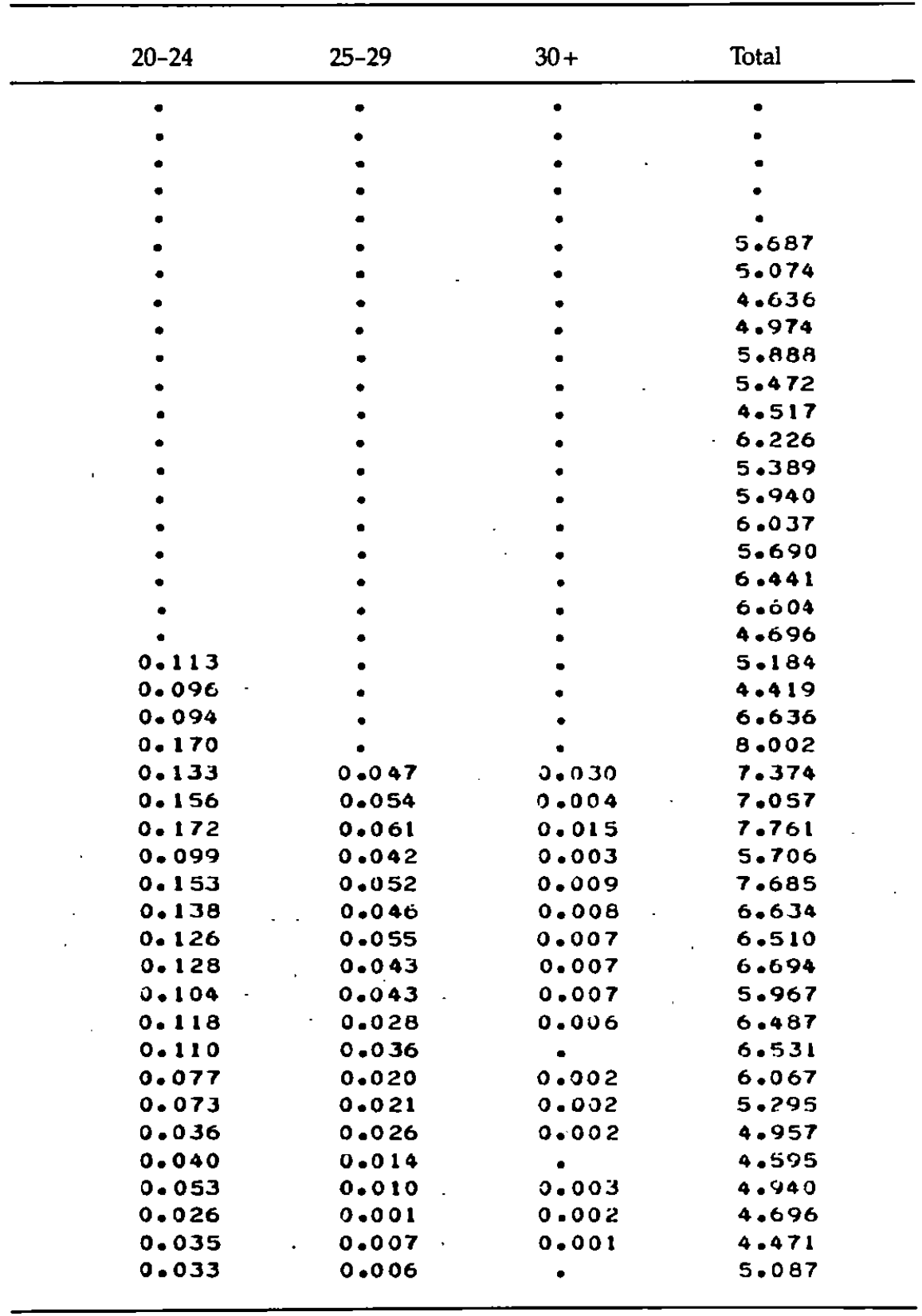


Basic Table 50.B. Urban Guangxi Province (20): duration-specific

\begin{tabular}{|c|c|c|c|c|}
\hline $\begin{array}{l}\text { Birth year } \\
\text { of child }\end{array}$ & $0-4$ & $5-9$ & $10-14$ & 15-19 \\
\hline 1940 & 0.181 & - & • & • \\
\hline 1941 & 0.178 & $\bullet$ & - & $\bullet$ \\
\hline 1942 & 0.234 & - & - & - \\
\hline 1943 & 0.242 & - & - & - \\
\hline 1944 & 0.401 & - & - & • \\
\hline 1945 & 0.204 & 0.181 & $\bullet$ & - \\
\hline 1946 & 0.311 & 0.445 & - & - \\
\hline 1947 & 0.255 & 0.201 & - & • \\
\hline 1948 & 0.168 & 0.181 & - & - \\
\hline 1949 & 0.351 & 0.368 & $\bullet$ & - \\
\hline 1950 & 0.254 & 0.272 & 0.181 & $\bullet$ \\
\hline IYSI & 0.437 & 0.259 & 0.133 & • \\
\hline 1952 & 0.255 & 0.368 & 0.234 & $\bullet$ \\
\hline 1953 & 0.496 & 0.337 & 0.242 & - \\
\hline 1954 & 0.319 & 0.332 & 0.268 & - \\
\hline 1955 & 0.351 & 0.220 & 0.272 & 0.226 \\
\hline 1956 & 0.349 & 0.245 & 0.311 & 0.222 \\
\hline 1957 & 0.389 & 0.300 & 0.510 & 0.134 \\
\hline 1958 & 0.303 & 0.375 & 0.288 & 0.060 \\
\hline 1959 & 0.345 & 0.212 & 0.176 & 0.067 \\
\hline 1960 & 0.206 & 0.244 & 0.237 & 0.204 \\
\hline 1961 & 0.424 & 0.152 & 0.087 & 0.052 \\
\hline 1902 & 0.419 & 0.440 & 0.195 & 0.198 \\
\hline 1963 & 0.417 & 0.285 & 0.315 & 0.168 \\
\hline 1964 & 0.393 & 0.287 & 0.288 & 0.078 \\
\hline 1905 & 0.379 & 0.299 & 0.122 & 0.051 \\
\hline 1960 & 0.320 & 0.221 & 0.106 & 0.070 \\
\hline 1967 & 0.256 & 0.227 & 0.085 & 0.030 \\
\hline 1968 & 0.417 & 0.225 & 0.125 & 0.090 \\
\hline 1969 & 0.339 & 0.227 & 0.057 & 0.091 \\
\hline 1970 & 0.325 & 0.126 & 0.094 & 0.046 \\
\hline 1971 & 0.442 & 0.219 & 0.037 & • \\
\hline 1972 & 0.313 & 0.328 & 0.105 & 0.068 \\
\hline 1973 & 0.316 & 0.155 & 0.112 & 0.018 \\
\hline 1974 & 0.323 & 0.221 & 0.076 & 0.038 \\
\hline 1975 & $0 \cdot 355$ & 0.113 & 0.063 & 0.019 \\
\hline 1976 & 0.275 & 0.174 & 0.067 & $\bullet$ \\
\hline 1977 & 0.465 & 0.136 & 0.033 & $\bullet$ \\
\hline 1978 & $0 \cdot 368$ & 0.111 & 0.031 & - \\
\hline 1979 & 0.381 & 0.097 & 0.029 & 0.015 \\
\hline 1980 & 0.221 & 0.016 & • & • \\
\hline 1981 & 0.237 & 0.061 & 0.027 & - \\
\hline 1982 & 0.265 & 0.082 & - & • \\
\hline
\end{tabular}


fertility rates, $1940-82$

\begin{tabular}{|c|c|c|c|}
\hline $20-24$ & $25-29$ & $30+$ & Total \\
\hline - & - & - & $\bullet$ \\
\hline - & - & $\bullet$ & - \\
\hline - & - & - & - \\
\hline - & - & - & • \\
\hline - & - & - & $\bullet$ \\
\hline • & - & - & 3.928 \\
\hline - & - & • & 8.714 \\
\hline - & - & - & 4.505 \\
\hline - & - & - & 3.756 \\
\hline - & - & - & 7.678 \\
\hline - & - & - & 5.141 \\
\hline$\bullet$ & - & $\bullet$ & 5.532 \\
\hline - & - & - & 6.425 \\
\hline - & - & - & 7.427 \\
\hline - & • & - & 6.736 \\
\hline - & - & - & 6.215 \\
\hline - & • & • & 6.570 \\
\hline - & - & - & 7.766 \\
\hline - & - & - & 5.949 \\
\hline - & - & - & 4.520 \\
\hline 0.090 & - & - & 5.118 \\
\hline 0.044 & - & - & 3.884 \\
\hline 0.134 & - & - & 7.192 \\
\hline 0.060 & - & - & 6.443 \\
\hline 0.067 & 0.099 & - & 6.065 \\
\hline 0.068 & $\bullet$ & - & 4.594 \\
\hline • & $\bullet$ & - & 3.585 \\
\hline - & - & - & 3.187 \\
\hline 0.024 & - & - & 4.404 \\
\hline 0.039 & - & - & 3.767 \\
\hline 0.051 & 0.034 & - & 3.376 \\
\hline 0.052 & - & - & 3.751 \\
\hline 0.060 & $\bullet$ & - & 4.368 \\
\hline 0.015 & - & - & 3.078 \\
\hline 0.030 & - & - & 3.444 \\
\hline - & - & - & 2.750 \\
\hline 0.015 & - & - & 2.660 \\
\hline$\bullet$ & - & - & 3.169 \\
\hline - & • & - & 2.550 \\
\hline 0.038 & - & - & 2.804 \\
\hline$\bullet$ & - & - & 1.187 \\
\hline - & - & - & 1.625 \\
\hline - & - & - & 1.733 \\
\hline
\end{tabular}


Basic Table 50.C. Rural Guangxi Province (20): duration-specific

\begin{tabular}{|c|c|c|c|c|}
\hline $\begin{array}{l}\text { Birth year } \\
\text { of child }\end{array}$ & $0-4$ & $5-9$ & $10-14$ & $15-19$ \\
\hline 1940 & 0.248 & - & - & - \\
\hline 1941 & 0.166 & - & - & - \\
\hline 1942 & 0.229 & - & - & - \\
\hline 1943 & 0.213 & $\bullet$ & - & - \\
\hline 1944 & 0.228 & - & - & - \\
\hline 1945 & 0.227 & 0.305 & - & - \\
\hline 1946 & 0.200 & 0.250 & $\bullet$ & - \\
\hline 1947 & 0.171 & 0.248 & - & - \\
\hline 1948 & 0.206 & 0.264 & • & - \\
\hline 1949 & 0.241 & 0.297 & - & - \\
\hline 1950 & 0.248 & 0.288 & 0.229 & - \\
\hline 1951 & 0.208 & 0.185 & 0.225 & - \\
\hline 1952 & 0.293 & 0.302 & 0.274 & • \\
\hline 1953 & 0.257 & 0.253 & 0.224 & • \\
\hline 1954 & 0.305 & 0.284 & 0.244 & - \\
\hline 1955 & 0.281 & 0.285 & 0.225 & 0.253 \\
\hline 1956 & 0.267 & 0.262 & 0.249 & 0.198 \\
\hline 1957 & 0.331 & 0.277 & 0.237 & 0.257 \\
\hline 1958 & 0.326 & 0.324 & 0.276 & 0.234 \\
\hline 1959 & 0.179 & 0.252 & 0.198 & 0.181 \\
\hline 1960 & 0.243 & 0.215 & 0.217 & 0.213 \\
\hline 1961 & 0.222 & 0.223 & 0.159 & 0.160 \\
\hline 1962 & 0.325 & 0.376 & 0.291 & 0.193 \\
\hline 1903 & 0.378 & 0.384 & 0.349 & 0.282 \\
\hline 1964 & 0.378 & 0.329 & 0.348 & 0.232 \\
\hline 1965 & 0.327 & 0.365 & 0.298 & 0.242 \\
\hline 1966 & 0.410 & 0.381 & 0.303 & 0.264 \\
\hline 1967 & 0.277 & 0.290 & 0.256 & 0.208 \\
\hline 1968 & 0.437 & 0.351 & 0.303 & 0.274 \\
\hline 1969 & 0.329 & 0.350 & 0.287 & 0.204 \\
\hline 1970 & 0.324 & 0.309 & 0.288 & 0.238 \\
\hline 1971 & 0.370 & 0.340 & 0.264 & 0.218 \\
\hline 1972 & 0.322 & 0.331 & 0.241 & 0.163 \\
\hline 1973 & 0.396 & 0.332 & 0.278 & 0.186 \\
\hline 1974 & 0.421 & 0.314 & 0.258 & 0.209 \\
\hline 1975 & 0.398 & 0.340 & 0.267 & 0.161 \\
\hline 1970 & 0.374 & 0.310 & 0.184 & 0.134 \\
\hline 1977 & 0.411 & 0.279 & 0.166 & 0.099 \\
\hline 1978 & 0.393 & 0.261 & 0.164 & 0.079 \\
\hline 1979 & 0.390 & 0.306 & 0.179 & 0.083 \\
\hline 1980 & 0.401 & 0.285 & 0.154 & 0.078 \\
\hline 1981 & 0.400 & 0.279 & 0.143 & 0.082 \\
\hline 1982 & 0.522 & 0.267 & 0.175 & 0.090 \\
\hline
\end{tabular}


fertility rates, $1940-82$

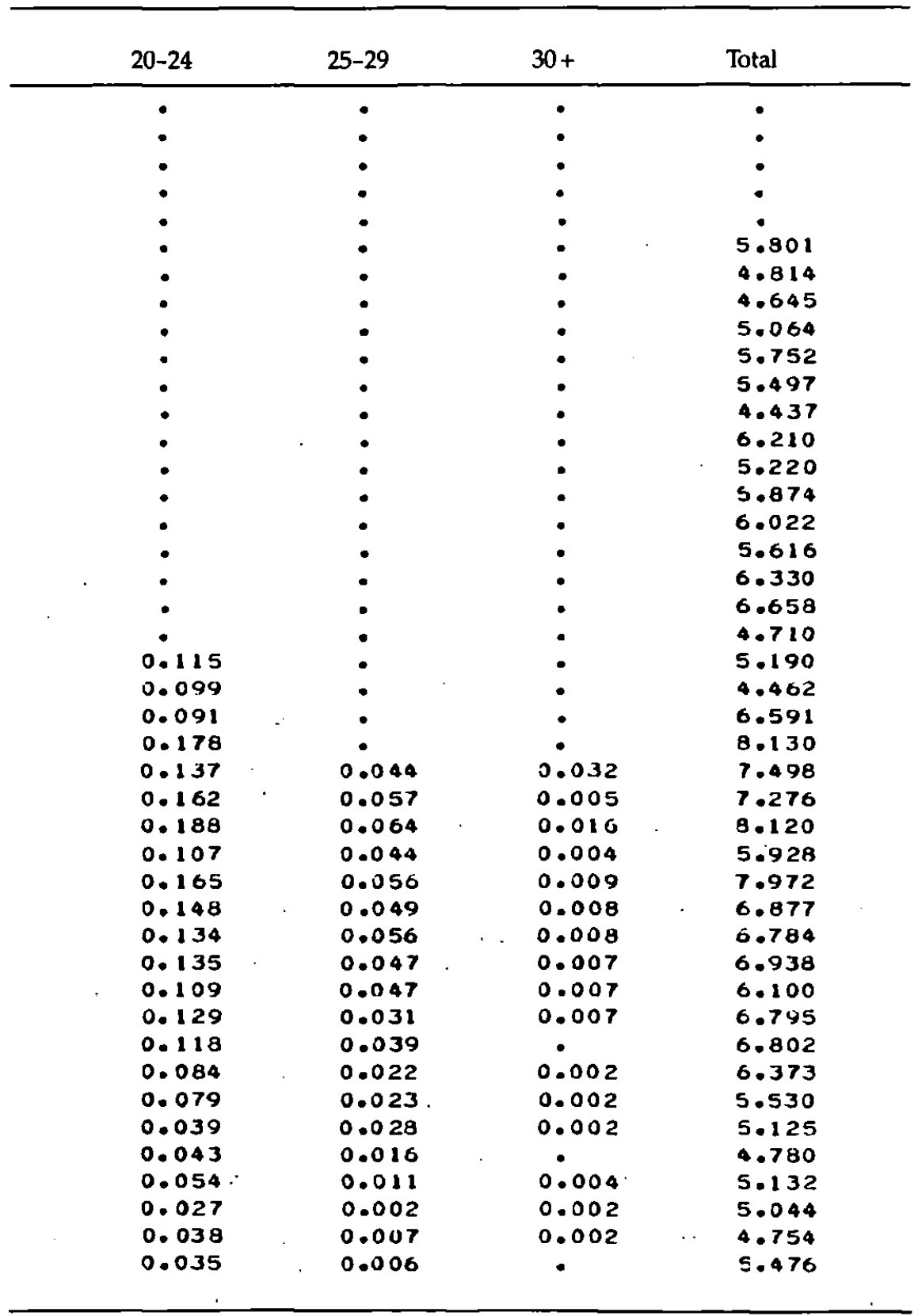


Basic Table 51.A. Total Sichuan Province (21): duration-specific fertility

\begin{tabular}{|c|c|c|c|c|}
\hline $\begin{array}{l}\text { Birth year } \\
\text { of child }\end{array}$ & $0-4$ & $5-9$ & $10-14$ & $15-19$ \\
\hline 1940 & 0.257 & - & - & - \\
\hline 1941 & 0.276 & • & • & - \\
\hline 1942 & 0.275 & • & - & $\bullet$ \\
\hline 1943 & 0.261 & $\bullet$ & $\bullet$ & $\bullet$ \\
\hline 1944 & 0.271 & $\bullet$ & - & $\bullet$ \\
\hline 1945 & 0.260 & 0.278 & - & - \\
\hline 1946 & 0.283 & 0.316 & $\bullet$ & $\bullet$ \\
\hline 1947 & 0.288 & 0.279 & - & - \\
\hline 1948 & 0.289 & 0.299 & - & • \\
\hline 1949 & 0.304 & 0.335 & - & . \\
\hline 1950 & 0.269 & 0.300 & 0.257 & - \\
\hline 1951 & 0.307 & 0.261 & 0.241 & - \\
\hline 1952 & 0.355 & 0.329 & 0.300 & - \\
\hline 1953 & 0.345 & 0.323 & 0.263 & $\bullet$ \\
\hline 1954 & 0.361 & 0.289 & 0.271 & - \\
\hline 1955 & 0.337 & 0.325 & 0.272 & 0.243 \\
\hline 1956 & 0.314 & 0.305 & 0.263 & 0.218 \\
\hline 1957 & 0.316 & 0.317 & 0.285 & 0.228 \\
\hline 1958 & 0.317 & 0.324 & 0.275 & 0.219 \\
\hline 1959 & 0.187 & 0.202 & 0.166 & 0.147 \\
\hline 1960 & 0.143 & 0.136 & 0.134 & 0.112 \\
\hline 1961 & 0.131 & 0.138 & 0.114 & 0.084 \\
\hline 1962 & 0.215 & 0.258 & 0.219 & 0.154 \\
\hline 1963 & 0.392 & 0.422 & 0.380 & 0.274 \\
\hline 1964 & 0.364 & 0.323 & 0.294 & 0.208 \\
\hline 1905 & 0.322 & 0.333 & 0.263 & 0.234 \\
\hline 1966 & 0.369 & 0.333 & 0.277 & 0.257 \\
\hline 1967 & 0.303 & 0.306 & 0.251 & 0.203 \\
\hline 1908 & 0.385 & 0.372 & 0.278 & 0.251 \\
\hline 1909 & 0.343 & 0.342 & 0.289 & 0.235 \\
\hline 1970 & 0.359 & 0.333 & 0.270 & 0.222 \\
\hline 1971 & 0.358 & 0.347 & 0.291 & 0.200 \\
\hline 1972 & 0.347 & 0.302 & 0.264 & 0.195 \\
\hline 1973 & 0.373 & 0.307 & 0.247 & 0.165 \\
\hline 1974 & 0.382 & 0.292 & 0.228 & 0.150 \\
\hline 1975 & 0.393 & 0.266 & 0.189 & 0.128 \\
\hline 1976 & $0 \cdot 333$ & $0.224^{\circ}$ & 0.124 & 0.087 \\
\hline 1977 & 0.316 & 0.161 & 0.088 & 0.063 \\
\hline 1978 & 0.292 & 0.131 & 0.062 & 0.025 \\
\hline 1979 & 0.319 & 0.129 & 0.053 & 0.025 \\
\hline 1980 & 0.271 & 0.081 & 0.025 & 0.010 \\
\hline 1981 & 0.374 & 0.117 & 0.045 & 0.017 \\
\hline 1982 & 0.330 & 0.117 & 0.037 & 0.018 \\
\hline
\end{tabular}


rates, $1940-82$

\begin{tabular}{|c|c|c|c|}
\hline $20-24$ & 25-29 & $30+$ & Total \\
\hline - & - & - & $\bullet$ \\
\hline - & - & $\bullet$ & • \\
\hline - & - & - & - \\
\hline - & $\bullet$ & $\bullet$ & $\bullet$ \\
\hline - & - & - & $\bullet$ \\
\hline - & - & - & 5.568 \\
\hline - & - & $\bullet$ & 6.264 \\
\hline$\bullet$ & - & - & 5.732 \\
\hline - & - & $\bullet$ & 6.049 \\
\hline - & • & - & 6.678 \\
\hline - & $\bullet$ & - & 5.965 \\
\hline - & - & - & 5.684 \\
\hline - & • & - & 6.980 \\
\hline - & $\bullet$ & • & 6.574 \\
\hline$\bullet$ & • & - & 6.448 \\
\hline - & - & $\bullet$ & 6.746 \\
\hline - & $\bullet$ & - & 6.316 \\
\hline$\bullet$ & $\bullet$ & - & 6.590 \\
\hline - & $\bullet$ & • & 6.524 \\
\hline$\bullet$ & - & - & 4.038 \\
\hline 0.085 & - & - & 3.162 \\
\hline 0.048 & - & - & 2.678 \\
\hline 0.077 & - & - & 4.809 \\
\hline 0.174 & - & - & 8.521 \\
\hline 0.144 & 0.071 & 0.006 & 7.054 \\
\hline 0.137 & 0.045 & 0.008 & 6.708 \\
\hline 0.135 & 0.047 & 0.002 & 7.106 \\
\hline 0.137 & 0.039 & 0.006 & 6.232 \\
\hline 0.135 & 0.043 & 0.015 & $7 \cdot 395$ \\
\hline 0.135 & 0.046 & 0.007 & 6.983 \\
\hline 0.147 & 0.034 & 0.010 & 6.875 \\
\hline 0.134 & $0.036^{\circ}$ & 0.004 & 6.843 \\
\hline 0.110 & 0.036 & 0.004 & 6.286 \\
\hline 0.101 & 0.023 & 0.003 & 6.091 \\
\hline 0.099 & 0.025 & 0.002 & 5.896 \\
\hline 0.064 & 0.026 & 0.001 & $5 \cdot 335$ \\
\hline 0.051 & 0.021 & 0.001 & 4.205 \\
\hline 0.024 & 0.007 & 0.001 & 3.299 \\
\hline 0.013 & 0.004 & 0.001 & 2.632 \\
\hline 0.013 & 0.001 & • & 2.725 \\
\hline 0.008 & 0.001 & 0.000 & 1.980 \\
\hline 0.014 & 0.004 & 0.000 & 2.856 \\
\hline 0.011 & 0.002 & - & 2.575 \\
\hline
\end{tabular}


Basic Table 51.B. Urban Sichuan Province (21): duration-specific

\begin{tabular}{|c|c|c|c|c|}
\hline $\begin{array}{l}\text { Birth year } \\
\text { of child }\end{array}$ & $0-4$ & $5-9$ & $10-14$ & $15-19$ \\
\hline 1940 & 0.262 & • & - & - \\
\hline 1941 & 0.272 & - & - & - \\
\hline 1942 & 0.312 & - & - & - \\
\hline 1943 & 0.245 & - & - & - \\
\hline 1944 & 0.236 & - & - & - \\
\hline $\begin{array}{l}1945 \\
1946\end{array}$ & $\begin{array}{l}0.306 \\
0.288\end{array}$ & $\begin{array}{l}0.200 \\
0.279\end{array}$ & - & : \\
\hline 1947 & 0.267 & 0.260 & - & - \\
\hline 1948 & 0.320 & 0.282 & $\bullet$ & - \\
\hline 1949 & 0.306 & 0.318 & - & - \\
\hline 1950 & 0.340 & 0.226 & 0.262 & • \\
\hline 1951 & 0.346 & 0.221 & 0.199 & - \\
\hline 1952 & 0.382 & 0.310 & 0.254 & - \\
\hline 1953 & 0.345 & 0.341 & 0.234 & - \\
\hline 1954 & 0.390 & 0.264 & 0.241 & - \\
\hline 1955 & 0.372 & 0.311 & 0.260 & 0.158 \\
\hline 1950 & 0.325 & 0.333 & 0.221 & 0.159 \\
\hline 1957 & 0.375 & 0.355 & 0.281 & 0.197 \\
\hline 1958 & 0.345 & 0.286 & 0.233 & 0.154 \\
\hline 1959 & 0.281 & 0.217 & 0.113 & 0.120 \\
\hline 1900 & 0.237 & 0.153 & 0.134 & 0.084 \\
\hline 1961 & 0.217 & 0.130 & 0.090 & 0.049 \\
\hline 1962 & 0.311 & 0.212 & 0.152 & 0.110 \\
\hline 1903 & 0.425 & 0.421 & 0.324 & 0.184 \\
\hline 1964 & 0.373 & 0.224 & 0.220 & 0.140 \\
\hline 1965 & 0.317 & 0.252 & 0.132 & 0.126 \\
\hline 1966 & 0.292 & 0.169 & 0.115 & 0.135 \\
\hline 1967 & 0.321 & 0.204 & 0.112 & 0.061 \\
\hline 1968 & 0.488 & 0.279 & 0.122 & 0.114 \\
\hline 1969 & 0.421 & 0.200 & 0.158 & 0.076 \\
\hline 1970 & 0.364 & 0.210 & 0.088 & 0.064 \\
\hline 1971 & 0.335 & 0.135 & 0.064 & 0.039 \\
\hline 1972 & 0.284 & 0.127 & $0: 066$ & 0.042 \\
\hline 1973 & 0.255 & 0.195 & 0.051 & 0.015 \\
\hline 1974 & 0.349 & 0.115 & 0.056 & 0.025 \\
\hline 1975 & 0.346 & 0.104 & 0.046 & 0.015 \\
\hline 1976 & 0.309 & 0.079 & 0.030 & 0.013 \\
\hline 1977 & 0.226 & 0.034 & 0.004 & 0.007 \\
\hline 1978 & 0.200 & 0.046 & 0.018 & • \\
\hline 1979 & 0.205 & 0.050 & 0.006 & 0.004 \\
\hline 1980 & 0.224 & 0.020 & 0.003 & - \\
\hline 1981 & 0.338 & 0.003 & - & - \\
\hline 1982 & 0.267 & - & - & - \\
\hline
\end{tabular}


fertility rates, $1940-82$

\begin{tabular}{|c|c|c|c|}
\hline $20-24$ & $25-29$ & $30+$ & Total \\
\hline - & - & $\bullet$ & - \\
\hline • & - & • & - \\
\hline - & • & - & - \\
\hline • & • & • & - \\
\hline - & - & • & - \\
\hline • & - & - & 4.739 \\
\hline - & - & - & 5.928 \\
\hline - & - & - & 5.521 \\
\hline - & - & - & 6.143 \\
\hline - & - & $\bullet$ & 6.646 \\
\hline - & $\bullet$ & - & 5.903 \\
\hline - & - & - & $5 \cdot 338$ \\
\hline - & - & - & 6.748 \\
\hline - & - & - & 6.649 \\
\hline - & • & - & 6.294 \\
\hline - & - & $\bullet$ & 6.351 \\
\hline - & $\bullet$ & - & 6.026 \\
\hline - & - & - & 7.014 \\
\hline - & - & - & 5.881 \\
\hline - & - & - & 4.188 \\
\hline 0.110 & - & - & 3.730 \\
\hline 0.060 & - & $\bullet$ & 2.820 \\
\hline 0.029 & - & - & 4.201 \\
\hline 0.144 & • & - & 7.778 \\
\hline 0.053 & 0.041 & - & 5.250 \\
\hline 0.092 & 0.021 & - & 4.700 \\
\hline 0.064 & 0.020 & - & 3.975 \\
\hline 0.028 & 0.017 & 0.009 & 3.768 \\
\hline 0.052 & 0.011 & - & 5.326 \\
\hline 0.039 & 0.010 & - & 4.516 \\
\hline 0.043 & 0.004 & - & 3.862 \\
\hline 0.038 & . & - & 3.054 \\
\hline 0.022 & 0.004 & • & 2.722 \\
\hline 0.021 & - & - & 2.688 \\
\hline 0.007 & 0.003 & - & 2.776 \\
\hline 0.008 & • & - & 2.597 \\
\hline 0.005 & 0.005 & - & 2.205 \\
\hline • & • & - & 1.355 \\
\hline 0.002 & $\bullet$ & $\bullet$ & 1.332 \\
\hline 0.003 & - & - & $1 \cdot .337$ \\
\hline - & - & - & 1.236 \\
\hline - & - & - & 1.706 \\
\hline$\bullet$ & • & - & 1.333 \\
\hline
\end{tabular}


Basic Table 51.C. Rural Sichuan Province (21): duration-specific

\begin{tabular}{|c|c|c|c|c|}
\hline $\begin{array}{l}\text { Birth year } \\
\text { of child }\end{array}$ & $0-4$ & $5-9$ & $10-14$ & $15-19$ \\
\hline 1940 & 0.257 & - & - & - \\
\hline 1941 & 0.276 & • & • & - \\
\hline 1942 & 0.269 & $\bullet$ & - & - \\
\hline 1943 & 0.264 & - & - & - \\
\hline 1944 & 0.277 & - & - & - \\
\hline 1945 & 0.258 & 0.288 & $\bullet$ & - \\
\hline 1940 & 0.282 & 0.321 & • & - \\
\hline 1947 & 0.292 & 0.282 & - & - \\
\hline 1948 & 0.283 & 0.302 & - & - \\
\hline 1949 & 0.304 & 0.338 & - & • \\
\hline 1950 & 0.255 & 0.315 & 0.256 & - \\
\hline 1951 & 0.299 & 0.269 & 0.246 & - \\
\hline 1952 & 0.349 & 0.333 & 0.307 & - \\
\hline 1953 & 0.345 & 0.319 & 0.268 & $\bullet$ \\
\hline 1954 & 0.355 & 0.293 & 0.277 & - \\
\hline 1955 & 0.329 & 0.328 & 0.274 & 0.254 \\
\hline 1956 & 0.311 & 0.299 & 0.272 & 0.226 \\
\hline 1957 & 0.300 & 0.309 & 0.286 & 0.233 \\
\hline 1958 & 0.310 & 0.333 & 0.283 & 0.230 \\
\hline 1959 & 0.164 & 0.199 & 0.177 & 0.152 \\
\hline 1960 & 0.122 & 0.132 & 0.134 & 0.117 \\
\hline 1961 & 0.115 & 0.140 & 0.119 & 0.091 \\
\hline 1962 & 0.201 & 0.270 & 0.232 & 0.163 \\
\hline 1463 & 0.388 & 0.423 & ופ9.3 & 0.292 \\
\hline 1964 & 0.363 & 0.346 & 0.311 & 0.222 \\
\hline 1965 & 0.322 & 0.350 & 0.294 & 0.256 \\
\hline 1906 & 0.378 & 0.363 & 0.317 & 0.282 \\
\hline 1967 & 0.301 & 0.321 & 0.288 & 0.232 \\
\hline 1968 & 0.372 & 0.383 & 0.320 & 0.281 \\
\hline 1969 & 0.332 & 0.358 & 0.320 & 0.271 \\
\hline 1970 & 0.358 & 0.347 & 0.309 & 0.260 \\
\hline 1971 & 0.361 & 0.370 & 0.332 & 0.240 \\
\hline 1972 & 0.355 & 0.322 & 0.292 & 0.235 \\
\hline 1973 & 0.387 & 0.321 & 0.271 & 0.205 \\
\hline 1974 & 0.385 & $0.31 \mathrm{e}$ & 0.248 & 0.180 \\
\hline 1975 & 0.399 & 0.289 & 0.206 & 0.152 \\
\hline 1976 & 0.336 & 0.245 & 0.135 & 0.100 \\
\hline 1977 & 0.328 & 0.177 & 0.098 & 0.071 \\
\hline 1978 & 0.304 & 0.141 & 0.067 & 0.028 \\
\hline 1979 & 0.333 & 0.138 & 0.060 & 0.028 \\
\hline 1480 & 0.277 & 0.088 & 0.028 & 0.011 \\
\hline 1981 & 0.380 & 0.131 & 0.051 & 0.019 \\
\hline 1982 & 0.343 & 0.132 & 0.042 & 0.021 \\
\hline
\end{tabular}


fertility rates, $1940-82$

\begin{tabular}{|c|c|c|c|}
\hline $20-24$ & $25-29$ & $30+$ & Total \\
\hline - & - & - & - \\
\hline - & - & - & - \\
\hline - & $\bullet$ & - & - \\
\hline - & - & - & - \\
\hline - & - & - & - \\
\hline - & - & - & 5.698 \\
\hline - & - & - & 6.319 \\
\hline - & • & - & 5.767 \\
\hline - & • & - & 6.033 \\
\hline - & - & $\bullet$ & 6.683 \\
\hline - & - & - & 5.975 \\
\hline - & - & - & 5.746 \\
\hline - & - & - & 7.021 \\
\hline - & - & - & 6.500 \\
\hline • & $\bullet$ & - & 6.476 \\
\hline - & - & - & 6.820 \\
\hline - & - & - & 6.371 \\
\hline - & - & - & 6.506 \\
\hline - & - & - & 6.652 \\
\hline - & - & - & 4.009 \\
\hline 0.082 & - & - & 3.052 \\
\hline 0.046 & - & - & 2.650 \\
\hline 0.084 & - & - & 4.922 \\
\hline 0.179 & - & - & 8.655 \\
\hline 0.161 & 0.075 & 0.007 & 7.423 \\
\hline 0.146 & 0.048 & 0.009 & 7.126 \\
\hline 0.150 & 0.051 & 0.003 & 7.714 \\
\hline 0.160 & 0.043 & 0.006 & 6.751 \\
\hline 0.151 & 0.048 & 0.017 & 7.857 \\
\hline 0.153 & 0.052 & 0.008 & 7.475 \\
\hline 0.167 & 0.041 & 0.011 & 7.464 \\
\hline 0.154 & 0.043 & 0.005 & 7.516 \\
\hline 0.128 & 0.043 & 0.004 & 6.895 \\
\hline 0.117 & 0.027 & 0.003 & 6.660 \\
\hline 0.120 & 0.030 & 0.003 & 6.421 \\
\hline 0.077 & 0.031 & 0.002 & 5.776 \\
\hline 0.062 & 0.024 & 0.002 & 4.515 \\
\hline 0.030 & 0.005 & 0.002 & 3.568 \\
\hline 0.016 & 0.005 & 0.001 & 2.807 \\
\hline 0.021 & 0.002 & - & 2.909 \\
\hline 0.010 & 0.001 & 0.001 & 2.079 \\
\hline 0.017 & 0.004 & 0.001 & 3.014 \\
\hline 0.012 & 0.003 & - & 2.766 \\
\hline
\end{tabular}


Basic Table 52.A. Total Guizhou Province (22): duration-specific

\begin{tabular}{|c|c|c|c|c|}
\hline $\begin{array}{l}\text { Birth year } \\
\text { of child }\end{array}$ & $0-4$ & $5-9$ & $10-14$ & $15-19$ \\
\hline 1940 & 0.238 & - & - & • \\
\hline 1941 & 0.274 & - & $\bullet$ & - \\
\hline 1942 & 0.245 & - & - & $\bullet$ \\
\hline 1943 & 0.281 & - & - & - \\
\hline 1944 & 0.280 & - & - & - \\
\hline 1945 & 0.213 & 0.276 & - & • \\
\hline $\begin{array}{l}1946 \\
1947\end{array}$ & $\begin{array}{l}0.277 \\
0.286\end{array}$ & $\begin{array}{l}0.317 \\
0.245\end{array}$ & : & : \\
\hline $\begin{array}{l}1948 \\
1949\end{array}$ & $\begin{array}{l}0.286 \\
0.318\end{array}$ & $\begin{array}{l}0.290 \\
0.316\end{array}$ & : & - \\
\hline 1950 & 0.264 & 0.274 & 0.255 & • \\
\hline 1951 & 0.227 & 0.259 & 0.228 & - \\
\hline 1952 & 0.289 & 0.342 & 0.270 & - \\
\hline 1953 & 0.311 & 0.277 & 0.240 & - \\
\hline 1954 & 0.352 & 0.295 & 0.340 & - \\
\hline 1955 & 0.314 & 0.355 & 0.246 & 0.248 \\
\hline $1950^{\circ}$ & $0: 315$ & 0.296 & 0.275 & 0.231 \\
\hline 1957 & 0.319 & 0.321 & 0.302 & 0.185 \\
\hline 1958 & 0.289 & 0.343 & 0.256 & 0.222 \\
\hline 1959 & 0.233 & 0.265 & 0.190 & 0.168 \\
\hline 1960 & 0.164 & 0.204 & 0.161 & 0.140 \\
\hline 1961 & 0.141 & 0.171 & 0.143 & 0.077 \\
\hline 1902 & 0.317 & 0.317 & 0.294 & 0.195 \\
\hline 1403 & 0.334 & 0.388 & 0.364 & 0.299 \\
\hline 1964 & 0.328 & 0.296 & 0.308 & 0.211 \\
\hline 1905 & 0.339 & 0.359 & 0.318 & 0.231 \\
\hline 1960 & 0.343 & $0.33 \epsilon$ & 0.310 & 0.271 \\
\hline 1967 & 0.274 & 0.311 & 0.297 & 0.222 \\
\hline 1968 & 0.374 & 0.413 & 0.344 & $0 \cdot 291$ \\
\hline 1969 & 0.318 & 0.346 & 0.293 & 0.232 \\
\hline 1970 & 0.328 & 0.365 & 0.317 & 0.264 \\
\hline 1971 & 0.356 & 0.350 & 0.284 & 0.240 \\
\hline 1972 & 0.300 & 0.314 & 0.309 & 0.207 \\
\hline 1973 & 0.334 & 0.362 & 0.301 & 0.265 \\
\hline 1974 & 0.378 & 0.360 & 0.298 & 0.238 \\
\hline 1975 & 0.381 & 0.313 & 0.268 & 0.231 \\
\hline 1976 & 0.368 & 0.285 & 0.214 & 0.162 \\
\hline 1977 & 0.365 & 0.268 & 0.212 & 0.142 \\
\hline 1978 & 0.322 & 0.289 & 0.164 & 0.088 \\
\hline 1979 & 0.404 & 0.252 & 0.157 & 0.118 \\
\hline 1900 & 0.309 & 0.283 & 0.151 & 0.091 \\
\hline 1981 & 0.400 & 0.262 & 0.175 & 0.096 \\
\hline 1982 & 0.375 & 0.259 & 0.219 & 0.164 \\
\hline
\end{tabular}


fertility rates, $1940-82$

\begin{tabular}{|c|c|c|c|}
\hline $20-24$ & $25-29$ & $30+$ & Total \\
\hline$\bullet$ & - & - & - \\
\hline - & • & - & • \\
\hline$\bullet$ & - & - & $\bullet$ \\
\hline - & - & - & - \\
\hline - & - & $\bullet$ & - \\
\hline - & - & $\bullet$ & 5.324 \\
\hline - & - & - & 0.294 \\
\hline$\bullet$ & • & - & 5.199 \\
\hline - & - & - & 5.930 \\
\hline - & - & - & 6.449 \\
\hline - & - & - & 5.726 \\
\hline - & - & • & 5.191 \\
\hline - & - & - & 6.523 \\
\hline - & - & - & 5.857 \\
\hline - & - & - & 7.064 \\
\hline - & $\bullet$ & $\bullet$ & 6.714 \\
\hline - & $\bullet$ & - & 6.452 \\
\hline$\bullet$ & - & - & 6.503 \\
\hline - & - & - & 6.42 .3 \\
\hline - & - & - & 4.950 \\
\hline 0.073 & - & - & 3.865 \\
\hline 0.073 & - & - & 3.140 \\
\hline 0.115 & - & - & 6.425 \\
\hline 0.166 & - & - & 8.090 \\
\hline 0.111 & 0.037 & 0.024 & 6.578 \\
\hline 0.148 & 0.056 & 0.003 & 7.295 \\
\hline 0.134 & 0.036 & 0.012 & 7.210 \\
\hline 0.150 & 0.039 & 0.010 & 6.513 \\
\hline 0.194 & 0.053 & 0.004 & 8.364 \\
\hline 0.151 & 0.036 & 0.007 & 6.920 \\
\hline 0.161 & 0.036 & 0.003 & 7.374 \\
\hline 0.152 & 0.044 & 0.010 & 7.176 \\
\hline 0.163 & 0.023 & 0.003 & 6.62' \\
\hline 0.138 & 0.030 & 0.015 & 7.219 \\
\hline 0.154 & 0.065 & 0.003 & 7.481 \\
\hline 0.153 & 0.048 & 0.011 & 7.02 .9 \\
\hline 0.100 & 0.035 & $\bullet$ & 5.822 \\
\hline 0.076 & 0.034 & 0.005 & 5.510 \\
\hline 0.059 & 0.020 & • & 4.713 \\
\hline 0.061 & 0.016 & 0.004 & 5.066 \\
\hline 0.046 & 0.011 & 0.002 & 4.465 \\
\hline 0.068 & 0.019 & 0.022 & 5.119 \\
\hline 0.042 & 0.034 & 0.003 & 5.481 \\
\hline
\end{tabular}


Basic Table 52.B. Urban Guizhou Province (22): duration-specific

\begin{tabular}{|c|c|c|c|c|}
\hline $\begin{array}{l}\text { Birth year } \\
\text { of child }\end{array}$ & $0-4$ & $5-9$ & $10-14$ & $15-19$ \\
\hline 1940 & 0.255 & - & - & - \\
\hline 1941 & 0.247 & - & - & - \\
\hline 1942 & 0.368 & - & - & - \\
\hline 1943 & 0.176 & - & - & - \\
\hline 1944 & 0.280 & - & - & - \\
\hline 1945 & 0.270 & 0.332 & - & - \\
\hline 1946 & 0.243 & 0.164 & - & - \\
\hline 1947 & 0.388 & 0.221 & - & - \\
\hline 1948 & 0.222 & 0.193 & - & - \\
\hline 1949 & 0.375 & 0.315 & - & - \\
\hline 1950 & 0.283 & 0.222 & 0.230 & - \\
\hline 1951 & 0.169 & 0.214 & 0.185 & - \\
\hline 1952 & 0.303 & 0.347 & $0.16 \epsilon$ & - \\
\hline 1953 & 0.335 & 0.245 & $0.17 \epsilon$ & - \\
\hline 1954 & 0.332 & 0.257 & 0.280 & - \\
\hline 1950 & 0.306 & 0.260 & 0.191 & 0.128 \\
\hline 1956 & 0.249 & 0.221 & 0.214 & 0.185 \\
\hline 1957 & 0.335 & 0.268 & 0.130 & 0.166 \\
\hline 1958 & 0.318 & $0.21 \mathrm{~s}$ & 0.257 & 0.176 \\
\hline $195 \%$ & 0.328 & 0.214 & 0.246 & 0.070 \\
\hline 1960 & 0.174 & 0.136 & 0.125 & 0.095 \\
\hline 1901 & 0.173 & 0.141 & 0.078 & 0.071 \\
\hline 1902 & 0.313 & 0.212 & 0.103 & 0.125 \\
\hline 1903 & 0.322 & 0.294 & 0.242 & 0.175 \\
\hline 1904 & 0.417 & 0.231 & 0.154 & 0.128 \\
\hline 1965 & 0.349 & 0.212 & 0.193 & 0.170 \\
\hline 1900 & 0.345 & 0.196 & 0.205 & 0.052 \\
\hline 1907 & 0.243 & 0.235 & 0.089 & 0.070 \\
\hline 1966 & 0.393 & $0.26 e$ & 0.129 & 0.115 \\
\hline 1969 & 0.419 & 0.239 & 0.109 & 0.095 \\
\hline 1970 & 0.357 & 0.201 & 0.125 & 0.113 \\
\hline 1971 & 0.310 & 0.211 & 0.104 & 0.054 \\
\hline 1972 & 0.216 & 0.207 & 0.089 & 0.011 \\
\hline 1973 & 0.331 & 0.210 & 0.075 & 0.024 \\
\hline 1974 & 0.355 & 0.183 & 0.096 & 0.036 \\
\hline 1975 & 0.347 & 0.132 & - & 0.025 \\
\hline 1970 & 0.258 & 0.113 & 0.011 & $\bullet$ \\
\hline 1977 & 0.370 & 0.037 & 0.030 & 0.022 \\
\hline 1978 & 0.260 & 0.187 & - & . \\
\hline 1479 & 0.311 & 0.0 .26 & 0.039 & - \\
\hline 1400 & 0.241 & 0.045 & - & - \\
\hline 1901 & 0.271 & 0.100 & - & - \\
\hline 1932 & 0.211 & - & - & - \\
\hline
\end{tabular}


fertility rates, $1940-82$

\begin{tabular}{|c|c|c|c|}
\hline $20-24$ & $25-29$ & $30+$ & Total \\
\hline - & • & • & . \\
\hline • & • & - & • \\
\hline - & - & - & - \\
\hline - & - & - & - \\
\hline$\bullet$ & - & • & - \\
\hline - & - & $\bullet$ & $0.69 ?$ \\
\hline - & - & - & 3.361 \\
\hline - & - & - & 5.495 \\
\hline - & - & - & $4 \cdot 220$ \\
\hline - & - & - & 0.940 \\
\hline - & - & - & 5.306 \\
\hline - & - & - & 4.272 \\
\hline • & $\bullet$ & - & 5.977 \\
\hline - & $\bullet$ & - & 5.276 \\
\hline • & - & - & 5.285 \\
\hline - & - & • & ة.0 \\
\hline - & • & - & 5.087 \\
\hline - & - & - & 5.470 \\
\hline - & - & - & 5.620 \\
\hline - & • & - & 4.399 \\
\hline 0.051 & • & - & 3.016 \\
\hline 0.021 & - & - & 2.497 \\
\hline 0.092 & • & - & $4.68 y$ \\
\hline 0.088 & • & - & 5.318 \\
\hline 0.052 & - & - & 4.917 \\
\hline 0.016 & 0.051 & - & 4.952 \\
\hline 0.043 & 0.021 & - & 4.309 \\
\hline $0.0 ? t ;$ & O.JIE & - & 3.414 \\
\hline 0.047 & - & - & 4.702 \\
\hline 0.011 & 0.0 .35 & - & $4.78: 3$ \\
\hline 0.034 & $\bullet$ & 0.026 & 4.273 \\
\hline • & - & • & 3.396 \\
\hline - & 0.014 & - & 2.982 \\
\hline 0.023 & $\bullet$ & 0.018 & 2.703 \\
\hline - & 0.021 & • & $3 \cdot 400$ \\
\hline 0.011 & - & $0.01 \mathrm{C}$ & 2.650 \\
\hline - & - & • & 1.307 \\
\hline 0.011 & - & - & 2.631 \\
\hline - & - & - & 2.235 \\
\hline 0.012 & - & - & 2.044 \\
\hline • & - & - & 1. 1. 12: \\
\hline • & 0.011 & - & 1.907 \\
\hline - & - & - & 1.057 \\
\hline
\end{tabular}


Basic Table 52.C. Rural Guizhou Province (22): duration-specific

\begin{tabular}{|c|c|c|c|c|}
\hline $\begin{array}{l}\text { Birth year } \\
\text { of child }\end{array}$ & $0-4$ & $5-9$ & $10-14$ & $15-19$ \\
\hline 1940 & 0.235 & - & • & - \\
\hline 1941 & 0.280 & - & - & • \\
\hline 1942 & 0.221 & - & - & - \\
\hline 1943 & 0.303 & - & - & - \\
\hline 1944 & 0.280 & - & - & - \\
\hline 1945 & 0.201 & 0.268 & - & - \\
\hline 1946 & 0.285 & 0.347 & - & - \\
\hline 1947 & 0.264 & 0.250 & - & - \\
\hline 1948 & 0.301 & 0.310 & - & - \\
\hline 1949 & 0.306 & 0.316 & • & - \\
\hline 1950 & 0.261 & 0.286 & 0.259 & - \\
\hline 1951 & 0.236 & 0.269 & 0.236 & - \\
\hline 1952 & 0.287 & 0.341 & 0.290 & - \\
\hline 1953 & 0.307 & 0.284 & 0.253 & - \\
\hline 1954 & 0.356 & 0.304 & 0.352 & - \\
\hline 1955 & 0.316 & 0.372 & 0.258 & 0.268 \\
\hline 1956 & 0.328 & 0.307 & 0.288 & 0.240 \\
\hline 1957 & 0.316 & 0.330 & 0.329 & 0.189 \\
\hline 1958 & 0.285 & 0.365 & 0.256 & 0.231 \\
\hline 1959 & 0.218 & 0.275 & 0.178 & 0.189 \\
\hline 1960 & 0.163 & 0.217 & 0.168 & 0.150 \\
\hline 1961 & 0.136 & 0.176 & 0.154 & 0.078 \\
\hline 1962 & 0.317 & $0.330^{\circ}$ & 0.310 & 0.211 \\
\hline 1963 & 0.336 & 0.403 & 0.385 & 0.330 \\
\hline 1964 & 0.315 & 0.306 & 0.335 & 0.229 \\
\hline 1905 & 0.338 & 0.381 & 0.341 & 0.242 \\
\hline 1966 & 0.343 & 0.358 & 0.330 & 0.305 \\
\hline 1967 & 0.279 & 0.323 & 0.334 & 0.247 \\
\hline 1968 & 0.372 & 0.435 & 0.377 & 0.321 \\
\hline 1969 & 0.306 & 0.355 & 0.321 & 0.256 \\
\hline 1970 & 0.325 & 0.390 & 0.346 & 0.292 \\
\hline 1971 & 0.360 & 0.371 & 0.312 & 0.275 \\
\hline 1972 & 0.308 & 0.321 & 0.344 & 0.242 \\
\hline 1973 & 0.334 & 0.380 & 0.336 & 0.303 \\
\hline 1974 & 0.380 & 0.382 & 0.328 & 0.269 \\
\hline 1975 & 0.385 & 0.333 & 0.308 & 0.262 \\
\hline 1976 & 0.379 & 0.303 & 0.244 & 0.187 \\
\hline 1977 & 0.365 & 0.286 & 0.237 & 0.160 \\
\hline 1978 & 0.331 & 0.299 & 0.183 & 0.101 \\
\hline 1979 & 0.419 & 0.271 & 0.171 & 0.136 \\
\hline 1980 & 0.320 & 0.307 & 0.168 & 0.104 \\
\hline 1981 & 0.421 & 0.279 & 0.193 & 0.111 \\
\hline 1982 & 0.400 & 0.291 & 0.240 & 0.186 \\
\hline
\end{tabular}


fertility rates, $1940-82$

\begin{tabular}{|c|c|c|c|}
\hline $20-24$ & 25-29 & $30+$ & Total \\
\hline - & - & - & • \\
\hline$\bullet$ & • & - & - \\
\hline$\bullet$ & - & - & $\bullet$ \\
\hline - & - & - & - \\
\hline - & - & - & $\bullet$ \\
\hline - & - & - & 5.096 \\
\hline - & - & - & 6.729 \\
\hline - & - & - & 5.146 \\
\hline - & - & . & 6.243 \\
\hline - & - & - & 6.361 \\
\hline • & - & - & 5.799 \\
\hline - & - & - & 5.347 \\
\hline - & - & - & 6.634 \\
\hline - & - & - & 5.961 \\
\hline - & - & - & 7.203 \\
\hline - & - & - & 7.003 \\
\hline - & - & - & 6.691 \\
\hline - & - & - & 6.083 \\
\hline - & - & - & 6.561 \\
\hline$\cdot$ & - & - & 4.959 \\
\hline 0.077 & - & - & 4.011 \\
\hline 0.083 & - & - & $3 \cdot 251$ \\
\hline 0.120 & - & - & 6.721 \\
\hline 0.182 & - & - & 8.474 \\
\hline 0.123 & 0.042 & 0.025 & 6.880 \\
\hline 0.177 & 0.057 & 0.008 & 7.722 \\
\hline 0.153 & 0.039 & 0.013 & 7.712 \\
\hline 0.177 & 0.044 & 0.011 & 7.068 \\
\hline 0.230 & 0.064 & 0.005 & 9.020 \\
\hline 0.182 & 0.036 & 0.008 & 7.322 \\
\hline 0.185 & 0.044 & $\bullet$ & 7.907 \\
\hline 0.176 & 0.053 & 0.012 & 7.797 \\
\hline 0.190 & 0.031 & 0.004 & $7 \cdot 200$ \\
\hline 0.157 & 0.037 & 0.014 & 7.807 \\
\hline 0.181 & 0.075 & 0.004 & 8.087 \\
\hline 0.180 & 0.057 & 0.010 & 7.675 \\
\hline 0.119 & 0.041 & $\bullet$ & 6.367 \\
\hline 0.087 & 0.039 & 0.000 & 5.902 \\
\hline 0.068 & 0.024 & $\bullet$ & 5.037 \\
\hline 0.069 & 0.019 & 0.005 & 5.446 \\
\hline 0.052 & 0.013 & 0.002 & 4.032 \\
\hline 0.079 & 0.020 & 0.002 & $5.52 ?$ \\
\hline 0.049 & 0.040 & 0.004 & 0.050 \\
\hline
\end{tabular}


Basic Table 53.A. Total Yunnan Province (23): duration-specific fertility

\begin{tabular}{|c|c|c|c|c|}
\hline $\begin{array}{l}\text { Birth year } \\
\text { of child }\end{array}$ & $0-4$ & $5-9$ & $10-14$ & $15-19$ \\
\hline 1940 & 0.218 & - & - & - \\
\hline 1941 & 0.233 & - & - & - \\
\hline 1942 & 0.264 & - & - & - \\
\hline 1943 & 0.274 & - & - & - \\
\hline 1944 & 0.259 & - & - & - \\
\hline 1945 & 0.251 & 0.253 & - & - \\
\hline 1946 & 0.303 & 0.307 & - & - \\
\hline 1947 & 0.300 & 0.300 & - & - \\
\hline 1948 & 0.307 & 0.288 & - & - \\
\hline 1949 & 0.331 & 0.361 & - & - \\
\hline 1950 & 0.301 & 0.297 & 0.253 & - \\
\hline 1951 & 0.290 & 0.283 & 0.248 & - \\
\hline 1952 & 0.361 & 0.288 & 0.302 & - \\
\hline 1953 & 0.291 & 0.350 & 0.251 & . \\
\hline 1954 & 0.366 & 0.336 & 0.316 & - \\
\hline 1955 & 0.347 & 0.361 & 0.284 & 0.223 \\
\hline 1956 & 0.283 & 0.344 & 0.251 & 0.218 \\
\hline 1957 & 0.370 & 0.300 & 0.271 & 0.222 \\
\hline 1958 & 0.301 & 0.307 & 0.274 & 0.255 \\
\hline 1959 & 0.194 & 0.241 & 0.222 & 0.154 \\
\hline 1960 & 0.223 & 0.215 & 0.235 & 0.158 \\
\hline 1961 & 0.215 & 0.217 & 0.168 & 0.117 \\
\hline 1962 & 0.360 & 0.394 & 0.321 & 0.225 \\
\hline 1963 & 0.366 & 0.375 & 0.337 & 0.302 \\
\hline 1964 & 0.378 & 0.349 & 0.306 & 0.211 \\
\hline 1965 & 0.363 & 0.331 & 0.304 & 0.218 \\
\hline 1966 & 0.354 & 0.341 & 0.294 & 0.221 \\
\hline 1967 & 0.307 & 0.326 & 0.236 & 0.187 \\
\hline 1908 & 0.372 & 0.337 & 0.328 & 0.242 \\
\hline 1969 & 0.348 & 0.360 & 0.268 & 0.222 \\
\hline 1970 & 0.345 & 0.336 & 0.263 & 0.196 \\
\hline 1971 & 0.368 & 0.307 & 0.288 & 0.196 \\
\hline 1972 & 0.378 & 0.303 & 0.238 & 0.184 \\
\hline 1973 & 0.336 & 0.280 & 0.271 & 0.177 \\
\hline 1974 & 0.380 & 0.311 & 0.211 & 0.156 \\
\hline 1975 & 0.394 & 0.297 & 0.232 & 0.179 \\
\hline 1976 & 0.380 & 0.302 & 0.216 & 0.194 \\
\hline 1977 & 0.388 & 0.297 & 0.211 & 0.168 \\
\hline 1978 & 0.334 & 0.267 & 0.205 & 0.147 \\
\hline 1975 & 0.362 & 0.232 & 0.166 & 0.118 \\
\hline 1980 & 0.309 & 0.188 & 0.128 & 0.075 \\
\hline 1981 & 0.364 & 0.176 & 0.162 & 0.105 \\
\hline 1982 & 0.363 & 0.196 & 0.137 & 0.111 \\
\hline
\end{tabular}


rates, $1940-82$

\begin{tabular}{|c|c|c|c|}
\hline $20-24$ & $25-29$ & $30+$ & Total \\
\hline - & $\bullet$ & - & - \\
\hline - & $\bullet$ & • & - \\
\hline - & • & - & - \\
\hline - & - & - & - \\
\hline$\bullet$ & - & - & - \\
\hline - & - & - & 5.166 \\
\hline - & - & - & 6.246 \\
\hline - & - & - & 6.112 \\
\hline - & • & - & 5.975 \\
\hline - & - & • & $7 \cdot 247$ \\
\hline - & - & • & 6.052 \\
\hline - & - & - & 5.844 \\
\hline - & - & - & 6.685 \\
\hline - & - & - & 6.435 \\
\hline - & - & - & 7.273 \\
\hline - & - & - & 6.962 \\
\hline - & - & - & 6.314 \\
\hline - & - & - & 6.660 \\
\hline - & • & • & 6.573 \\
\hline - & - & - & 4.708 \\
\hline 0.105 & - & - & 4.860 \\
\hline 0.072 & - & - & 4.081 \\
\hline 0.118 & - & - & 7.375 \\
\hline 0.207 & - & - & 8.256 \\
\hline 0.140 & 0.045 & 0.019 & 7.244 \\
\hline 0.156 & 0.062 & 0.005 & $7 \cdot 192$ \\
\hline 0.133 & 0.041 & 0.008 & 6.952 \\
\hline 0.101 & 0.050 & 0.003 & 6.050 \\
\hline 0.122 & 0.058 & 0.003 & 7.312 \\
\hline 0.151 & 0.051 & 0.006 & 7.034 \\
\hline 0.110 & 0.044 & 0.003 & 6.484 \\
\hline 0.109 & 0.030 & 0.003 & 6.503 \\
\hline 0.096 & 0.029 & - & 6.142 \\
\hline 0.109 & 0.038 & 0.005 & 6.076 \\
\hline 0.112 & 0.024 & 0.009 & 6.016 \\
\hline 0.083 & 0.019 & - & 6.020 \\
\hline 0.077 & 0.022 & - & 5.952 \\
\hline 0.074 & 0.019 & 0.002 & 5.795 \\
\hline 0.089 & 0.025 & 0.003 & 5.351 \\
\hline 0.059 & 0.016 & • & 4.764 \\
\hline 0.050 & 0.011 & 0.001 & 3.809 \\
\hline 0.052 & 0.011 & - & 4.350 \\
\hline 0.063 & 0.014 & - & 4.419 \\
\hline
\end{tabular}


Basic Table 53.B. Urban Yunnan Province (23): duration-specific

\begin{tabular}{|c|c|c|c|c|}
\hline $\begin{array}{l}\text { Birth year } \\
\text { of child }\end{array}$ & $0-4$ & $5-9$ & $10-14$ & $15-19$ \\
\hline 1940 & 0.256 & $\bullet$ & - & - \\
\hline 1941 & 0.200 & - & - & - \\
\hline 1942 & 0.287 & - & - & - \\
\hline 1943 & 0.200 & - & - & - \\
\hline 1944 & 0.310 & • & - & - \\
\hline 1945 & 0.202 & 0.277 & - & - \\
\hline 1946 & 0.302 & 0.301 & - & - \\
\hline 1947 & 0.320 & 0.287 & - & - \\
\hline 1948 & 0.240 & 0.273 & - & - \\
\hline 1949 & 0.303 & 0.418 & - & - \\
\hline 1950 & 0.293 & 0.277 & 0.213 & - \\
\hline 1951 & 0.359 & 0.257 & 0.221 & - \\
\hline 1952 & 0.305 & 0.289 & 0.215 & - \\
\hline 1953 & 0.376 & 0.269 & 0.273 & - \\
\hline 1954 & 0.304 & 0.295 & 0.341 & - \\
\hline 1955 & 0.420 & 0.310 & 0.227 & 0.128 \\
\hline 1956 & 0.220 & 0.367 & 0.224 & 0.140 \\
\hline 1957 & 0.513 & 0.267 & 0.247 & 0.197 \\
\hline 1958 & 0.366 & 0.342 & 0.231 & 0.219 \\
\hline 1959 & 0.236 & 0.290 & 0.134 & 0.124 \\
\hline 1960 & 0.245 & 0.199 & 0.204 & 0.101 \\
\hline 1961 & 0.253 & 0.220 & 0.152 & 0.101 \\
\hline 1962 & 0.409 & 0.303 & 0.201 & 0.134 \\
\hline 1963 & 0.449 & 0.402 & 0.301 & 0.269 \\
\hline 1964 & 0.434 & 0.227 & 0.184 & 0.214 \\
\hline 1965 & 0.401 & 0.304 & 0.183 & 0.090 \\
\hline 1960 & 0.303 & 0.253 & 0.126 & 0.128 \\
\hline 1967 & 0.247 & 0.097 & 0.050 & 0.045 \\
\hline 1968 & 0.430 & 0.327 & 0.192 & 0.075 \\
\hline 1969 & 0.385 & 0.231 & 0.138 & 0.092 \\
\hline 1970 & 0.292 & 0.222 & 0.137 & 0.046 \\
\hline 1971 & 0.368 & 0.218 & 0.107 & 0.055 \\
\hline 1972 & 0.287 & 0.137 & 0.136 & 0.084 \\
\hline 1973 & 0.431 & 0.267 & 0.094 & 0.046 \\
\hline 1974 & 0.388 & 0.120 & 0.058 & 0.020 \\
\hline 1975 & 0.315 & 0.125 & 0.032 & 0.039 \\
\hline 1976 & 0.437 & 0.156 & 0.061 & 0.029 \\
\hline 1977 & 0.372 & 0.121 & 0.082 & 0.010 \\
\hline 1978 & 0.309 & 0.133 & 0.059 & 0.009 \\
\hline 1979 & 0.203 & 0.053 & 0.040 & 0.010 \\
\hline 1980 & 0.215 & 0.057 & - & . \\
\hline 1981 & 0.206 & 0.012 & - & 0.012 \\
\hline 1982 & 0.299 & 0.060 & - & - \\
\hline
\end{tabular}


fertility rates, $1940-82$

\begin{tabular}{|c|c|c|c|}
\hline $20-24$ & $25-29$ & $30+$ & Total \\
\hline • & - & - & - \\
\hline - & - & - & $\bullet$ \\
\hline - & - & - & - \\
\hline - & - & - & $\bullet$ \\
\hline - & - & - & - \\
\hline - & - & - & 5.471 \\
\hline - & - & - & 6.349 \\
\hline - & - & - & 6.208 \\
\hline - & - & - & 5.600 \\
\hline • & - & - & 8.242 \\
\hline - & - & - & 5.671 \\
\hline - & - & - & 5.899 \\
\hline - & - & - & 5.837 \\
\hline • & - & - & 6.546 \\
\hline - & - & - & 6.995 \\
\hline - & - & - & 6.194 \\
\hline - & - & - & 5.599 \\
\hline - & - & - & 6.970 \\
\hline - & - & - & 6.718 \\
\hline - & . & - & 4.554 \\
\hline 0.107 & - & - & 4.447 \\
\hline 0.060 & - & - & 4.066 \\
\hline 0.072 & - & - & 5.775 \\
\hline 0.182 & - & - & $8 \cdot 336$ \\
\hline 0.124 & - & 0.050 & 6.160 \\
\hline 0.113 & 0.085 & $\bullet$ & 5.887 \\
\hline 0.067 & 0.020 & - & 4.482 \\
\hline 0.041 & $\bullet$ & - & 2.405 \\
\hline 0.038 & 0.036 & - & 5.496 \\
\hline 0.036 & 0.015 & • & 4.488 \\
\hline 0.026 & 0.025 & - & 3.691 \\
\hline 0.048 & 0.022 & - & 4.092 \\
\hline 0.007 & • & - & $3 \cdot 262$ \\
\hline 0.020 & - & - & 4.287 \\
\hline 0.014 & - & - & 2.996 \\
\hline 0.008 & - & - & 2.594 \\
\hline 0.008 & - & - & 3.450 \\
\hline 0.008 & - & - & 2.965 \\
\hline • & - & - & 2.550 \\
\hline 0.020 & - & - & 1.629 \\
\hline$\bullet$ & - & - & 1.362 \\
\hline - & - & - & 1.151 \\
\hline - & • & - & 1.793 \\
\hline
\end{tabular}


Basic Table 53.C. Rural Yunnan Province (23): duration-specific fertility

\begin{tabular}{|c|c|c|c|c|}
\hline $\begin{array}{l}\text { Birth year } \\
\text { of child }\end{array}$ & $0-4$ & $5-9$ & $10-14$ & $15-19$ \\
\hline 1940 & 0.212 & - & - & - \\
\hline 1941 & 0.238 & - & - & - \\
\hline 1942 & 0.261 & - & - & - \\
\hline 1943 & 0.285 & - & - & - \\
\hline 1944 & 0.250 & - & - & - \\
\hline 1945 & 0.260 & 0.249 & - & - \\
\hline 1946 & 0.303 & 0.308 & - & - \\
\hline 1947 & 0.296 & 0.302 & - & - \\
\hline 1948 & 0.322 & 0.290 & - & - \\
\hline 1949 & 0.338 & 0.351 & - & - \\
\hline 1950 & 0.303 & 0.301 & 0.258 & - \\
\hline 1951 & 0.275 & 0.288 & 0.252 & - \\
\hline 1952 & 0.374 & 0.287 & 0.315 & $\bullet$ \\
\hline 1953 & 0.271 & 0.369 & 0.247 & - \\
\hline 1954 & 0.381 & 0.345 & 0.312 & - \\
\hline 1955 & 0.331 & 0.372 & 0.296 & 0.237 \\
\hline 1956 & 0.296 & 0.338 & 0.257 & 0.229 \\
\hline 1957 & 0.342 & 0.308 & 0.276 & 0.226 \\
\hline 1958 & 0.289 & 0.299 & 0.283 & 0.261 \\
\hline 1959 & 0.187 & 0.230 & 0.241 & 0.159 \\
\hline 1960 & 0.220 & 0.219 & 0.241 & 0.169 \\
\hline 1961 & 0.209 & 0.216 & 0.172 & 0.120 \\
\hline 1962 & 0.353 & 0.412 & 0.349 & 0.245 \\
\hline 1963 & 0.355 & 0.370 & 0.346 & 0.309 \\
\hline 1964 & 0.370 & 0.369 & 0.334 & 0.210 \\
\hline 1965 & 0.358 & 0.336 & 0.330 & 0.247 \\
\hline 1966 & 0.359 & 0.355 & 0.329 & 0.242 \\
\hline 1967 & 0.313 & 0.360 & 0.273 & 0.220 \\
\hline 1968 & 0.367 & 0.338 & 0.353 & 0.282 \\
\hline 1969 & 0.344 & 0.378 & 0.290 & 0.253 \\
\hline 1970 & 0.350 & 0.350 & 0.284 & 0.229 \\
\hline 1971 & 0.368 & 0.317 & 0.317 & 0.226 \\
\hline 1972 & 0.384 & 0.320 & 0.253 & 0.204 \\
\hline 1973 & 0.330 & 0.281 & 0.297 & 0.201 \\
\hline 1974 & 0.379 & 0.330 & 0.232 & 0.179 \\
\hline 1975 & 0.400 & 0.313 & 0.257 & 0.202 \\
\hline 1976 & 0.375 & 0.314 & 0.233 & 0.220 \\
\hline 1977 & 0.390 & 0.309 & 0.224 & 0.192 \\
\hline 1978 & 0.337 & 0.276 & 0.218 & 0.167 \\
\hline 1979 & 0.381 & 0.243 & 0.179 & 0.133 \\
\hline 1980 & 0.320 & 0.198 & 0.139 & 0.084 \\
\hline 1981 & 0.383 & 0.191 & 0.175 & 0.115 \\
\hline 1982 & 0.370 & 0.212 & 0.146 & 0.122 \\
\hline
\end{tabular}


rates, $1940-82$

\begin{tabular}{|c|c|c|c|}
\hline $20-24$ & $25-29$ & $30+$ & Total \\
\hline - & - & - & - \\
\hline$\bullet$ & • & • & • \\
\hline - & - & $\bullet$ & - \\
\hline - & - & - & - \\
\hline • & - & - & - \\
\hline - & - & - & 5.114 \\
\hline - & $\bullet$ & - & 6.228 \\
\hline • & - & - & 6.095 \\
\hline - & - & - & 6.042 \\
\hline - & - & - & 7.064 \\
\hline$\bullet$ & - & - & 6.125 \\
\hline - & - & - & 5.833 \\
\hline$\bullet$ & - & - & 6.850 \\
\hline - & • & - & 6.413 \\
\hline - & - & - & 7.329 \\
\hline - & - & - & 7.114 \\
\hline - & - & - & 6.455 \\
\hline - & - & - & 6.599 \\
\hline - & - & - & 6.545 \\
\hline - & - & - & 4.737 \\
\hline 0.105 & - & - & 4.939 \\
\hline 0.073 & - & - & 4.084 \\
\hline 0.125 & - & - & 7.672 \\
\hline 0.210 & - & - & 8.242 \\
\hline 0.143 & 0.053 & 0.015 & 7.474 \\
\hline 0.164 & 0.058 & 0.006 & 7.493 \\
\hline 0.147 & 0.044 & 0.009 & 7.419 \\
\hline 0.114 & 0.057 & 0.004 & 6.704 \\
\hline 0.141 & 0.061 & 0.003 & 7.728 \\
\hline 0.177 & 0.057 & 0.007 & 7.527 \\
\hline 0.131 & 0.048 & 0.003 & 6.970 \\
\hline 0.123 & 0.031 & 0.003 & 6.922 \\
\hline 0.117 & 0.036 & $\bullet$ & 6.567 \\
\hline 0.130 & 0.047 & 0.005 & 6.451 \\
\hline 0.135 & 0.029 & 0.010 & 6.474 \\
\hline 0.099 & 0.024 & - & 6.469 \\
\hline 0.092 & 0.026 & - & 6.300 \\
\hline 0.088 & 0.024 & 0.002 & 6.141 \\
\hline 0.105 & 0.031 & 0.004 & 5.692 \\
\hline 0.065 & 0.020 & • & 5.103 \\
\hline 0.058 & 0.013 & 0.002 & 4.073 \\
\hline 0.061 & 0.013 & • & 4.690 \\
\hline 0.072 & 0.017 & - & 4.696 \\
\hline
\end{tabular}


Basic Table 54.A. Total Shaanxi Province (25): duration-specific fertility

\begin{tabular}{|c|c|c|c|c|}
\hline $\begin{array}{l}\text { Birth year } \\
\text { of child }\end{array}$ & $0-4$ & $5-9$ & $10-14$ & $15-19$ \\
\hline 1940 & 0.204 & • & - & $\bullet$ \\
\hline 1941 & 0.232 & - & - & - \\
\hline 1942 & 0.237 & - & - & - \\
\hline 1943 & 0.236 & - & - & - \\
\hline 1944 & 0.271 & $\bullet$ & - & - \\
\hline 1945 & 0.215 & 0.239 & - & - \\
\hline 1946 & 0.263 & 0.297 & - & - \\
\hline 1947 & 0.311 & 0.260 & • & - \\
\hline 1948 & 0.201 & 0.275 & - & - \\
\hline 1949 & 0.249 & 0.289 & - & - \\
\hline 1950 & 0.250 & 0.248 & 0.236 & $\bullet$ \\
\hline $\begin{array}{l}1951 \\
1952\end{array}$ & $\begin{array}{l}0.278 \\
0.308\end{array}$ & $\begin{array}{l}0.263 \\
0.268\end{array}$ & $\begin{array}{l}0.240 \\
0.283\end{array}$ & : \\
\hline $\begin{array}{l}1953 \\
1954\end{array}$ & $\begin{array}{l}0.288 \\
0.314\end{array}$ & $\begin{array}{l}0.270 \\
0.314\end{array}$ & $\begin{array}{l}0.267 \\
0.273\end{array}$ & : \\
\hline 1955 & 0.293 & 0.309 & 0.286 & 0.204 \\
\hline 1956 & 0.292 & 0.273 & 0.261 & 0.195 \\
\hline 1957 & 0.348 & $0 \cdot 333$ & 0.295 & 0.260 \\
\hline 1958 & 0.321 & 0.288 & 0.249 & 0.217 \\
\hline 1959 & 0.249 & 0.248 & 0.222 & $\begin{array}{l}0.135 \\
0.181\end{array}$ \\
\hline $\begin{array}{l}1900 \\
1961\end{array}$ & 0.216 & 0.183 & 0.218 & 0.170 \\
\hline $\begin{array}{l}1962 \\
1963\end{array}$ & $\begin{array}{l}0.316 \\
0.416\end{array}$ & $\begin{array}{l}0.385 \\
0.349\end{array}$ & $\begin{array}{l}0.301 \\
0.294\end{array}$ & $\begin{array}{l}0.207 \\
0.268\end{array}$ \\
\hline 1904 & 0.319 & 0.315 & 0.263 & 0.213 \\
\hline 1965 & 0.284 & 0.261 & 0.267 & 0.198 \\
\hline $\begin{array}{l}1966 \\
1967\end{array}$ & $\begin{array}{l}0.347 \\
0.314\end{array}$ & $\begin{array}{l}0.351 \\
0.269\end{array}$ & $\begin{array}{l}0.264 \\
0.260\end{array}$ & $\begin{array}{l}0.205 \\
0.188\end{array}$ \\
\hline 1968 & 0.361 & 0.374 & 0.279 & 0.225 \\
\hline 1969 & 0.347 & 0.298 & 0.237 & 0.197 \\
\hline 1970 & 0.339 & 0.289 & 0.263 & 0.187 \\
\hline 1971 & 0.345 & 0.292 & 0.247 & 0.147 \\
\hline 1972 & 0.323 & 0.308 & 0.225 & 0.192 \\
\hline 1973 & 0.319 & 0.250 & 0.229 & 0.132 \\
\hline 1974 & 0.357 & 0.292 & 0.180 & 0.111 \\
\hline 1975 & 0.284 & 0.237 & 0.138 & 0.093 \\
\hline 1976 & 0.364 & 0.220 & 0.123 & 0.075 \\
\hline $\begin{array}{l}1977 \\
1978\end{array}$ & $\begin{array}{l}0.322 \\
0.336\end{array}$ & $\begin{array}{l}0.190 \\
0.202\end{array}$ & $\begin{array}{l}0.108 \\
0.128\end{array}$ & $\begin{array}{l}0.054 \\
0.043\end{array}$ \\
\hline 1979 & 0.384 & 0.223 & 0.108 & 0.038 \\
\hline 1980 & 0.304 & $0.16 i$ & 0.079 & 0.033 \\
\hline 1981 & 0.354 & 0.148 & 0.071 & 0.018 \\
\hline 1982 & 0.354 & 0.165 & 0.071 & 0.016 \\
\hline
\end{tabular}


rates, $1940-82$

\begin{tabular}{|c|c|c|c|}
\hline $20-24$ & $25-29$ & $30+$ & Total \\
\hline • & - & - & - \\
\hline$\bullet$ & $\bullet$ & $\bullet$ & - \\
\hline$\bullet$ & $\bullet$ & - & - \\
\hline$\bullet$ & - & • & $\bullet$ \\
\hline - & • & - & 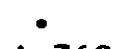 \\
\hline - & • & - & 4.762 \\
\hline - & - & - & 5.885 \\
\hline - & $\bullet$ & • & 5.545 \\
\hline - & - & - & 5.246 \\
\hline - & - & • & 5.700 \\
\hline • & • & - & 5.255 \\
\hline - & - & - & 5.550 \\
\hline - & • & • & 6.142 \\
\hline - & - & - & 5.925 \\
\hline - & - & - & 6.437 \\
\hline$\bullet$ & - & - & 6.315 \\
\hline - & - & - & 5.898 \\
\hline • & - & - & 7.070 \\
\hline - & - & - & $6 \cdot 218$ \\
\hline - & - & - & 4.901 \\
\hline 0.135 & - & $\bullet$ & 5.486 \\
\hline 0.075 & - & - & 4.467 \\
\hline 0.121 & - & - & 6.890 \\
\hline 0.196 & - & • & 7.850 \\
\hline 0.156 & 0.061 & 0.006 & 6.667 \\
\hline 0.124 & 0.037 & 0.013 & 5.921 \\
\hline 0.120 & 0.047 & 0.017 & 6.757 \\
\hline 0.119 & 0.039 & - & 5.947 \\
\hline 0.127 & 0.056 & 0.003 & 7.128 \\
\hline 0.109 & 0.044 & 0.008 & 6.195 \\
\hline 0.132 & 0.030 & 0.012 & 6.265 \\
\hline 0.122 & 0.027 & 0.002 & 5.915 \\
\hline 0.121 & 0.029 & 0.002 & 6.004 \\
\hline 0.079 & 0.022 & 0.011 & 5.208 \\
\hline 0.057 & 0.028 & - & 5.126 \\
\hline 0.058 & 0.014 & 0.002 & 4.128 \\
\hline 0.045 & 0.018 & - & 4.222 \\
\hline 0.023 & 0.007 & 0.004 & 3.539 \\
\hline 0.031 & 0.009 & 0.004 & 3.764 \\
\hline 0.027 & 0.009 & 0.002 & 3.957 \\
\hline 0.006 & 0.002 & 0.002 & 2.934 \\
\hline 0.014 & 0.004 & 0.002 & 3.053 \\
\hline 0.009 & 0.007 & $\bullet$ & 3.114 \\
\hline
\end{tabular}


Basic Table 54.B. Urban Shaanxi Province (25): duration-specific

\begin{tabular}{|c|c|c|c|c|}
\hline $\begin{array}{l}\text { Birth year } \\
\text { of child }\end{array}$ & $0-4$ & $5-9$ & $10-14$ & $15-19$ \\
\hline 1940 & 0.154 & - & - & - \\
\hline 1941 & 0.237 & - & - & - \\
\hline 1942 & 0.207 & - & - & - \\
\hline 1943 & 0.148 & - & - & - \\
\hline 1944 & 0.307 & - & - & - \\
\hline 1945 & 0.217 & 0.273 & - & - \\
\hline 1946 & 0.285 & 0.288 & - & - \\
\hline 1947 & 0.326 & 0.177 & - & - \\
\hline 1948 & 0.207 & 0.282 & - & . \\
\hline 1949 & 0.270 & 0.246 & - & - \\
\hline 1950 & 0.208 & 0.152 & 0.120 & - \\
\hline 1951 & 0.265 & 0.275 & 0.187 & - \\
\hline 1952 & 0.284 & 0.240 & 0.310 & - \\
\hline 1953 & 0.244 & 0.216 & 0.242 & - \\
\hline 1954 & 0.322 & 0.337 & 0.282 & - \\
\hline 1955 & 0.219 & 0.241 & 0.260 & 0.102 \\
\hline 1956 & 0.286 & 0.240 & 0.304 & 0.220 \\
\hline 1957 & 0.415 & 0.363 & 0.268 & 0.163 \\
\hline 1958 & 0.271 & 0.244 & 0.207 & 0.202 \\
\hline 1959 & 0.257 & 0.251 & 0.194 & 0.123 \\
\hline 1960 & 0.224 & 0.274 & 0.191 & 0.119 \\
\hline 1961 & 0.204 & 0.136 & 0.166 & 0.157 \\
\hline 1962 & 0.324 & 0.321 & 0.315 & 0.182 \\
\hline 1963 & 0.362 & 0.390 & 0.312 & 0.207 \\
\hline 1964 & 0.294 & 0.193 & 0.228 & 0.152 \\
\hline 1905 & 0.337 & 0.243 & 0.149 & 0.083 \\
\hline 1966 & 0.226 & 0.224 & 0.193 & 0.058 \\
\hline 1967 & 0.273 & 0.151 & 0.181 & 0.095 \\
\hline 1968 & 0.293 & 0.354 & 0.227 & 0.107 \\
\hline 1969 & 0.317 & 0.244 & 0.173 & 0.133 \\
\hline 1970 & 0.353 & 0.195 & 0.178 & 0.055 \\
\hline 1971 & 0.351 & 0.172 & 0.145 & 0.064 \\
\hline 1972 & 0.336 & 0.211 & 0.079 & 0.074 \\
\hline 1973 & 0.278 & 0.140 & 0.102 & 0.038 \\
\hline 1974 & 0.363 & 0.083 & 0.017 & 0.019 \\
\hline $\begin{array}{l}1975 \\
1976\end{array}$ & $\begin{array}{l}0.295 \\
0.359\end{array}$ & $\begin{array}{l}0.149 \\
0.085\end{array}$ & $\begin{array}{l}0.080 \\
0.032\end{array}$ & $\begin{array}{l}0.013 \\
0.033\end{array}$ \\
\hline 1977 & 0.402 & 0.128 & 0.037 & 0.007 \\
\hline 1978 & 0.309 & 0.139 & 0.070 & 0.016 \\
\hline 1979 & 0.224 & 0.117 & 0.028 & 0.008 \\
\hline 1980 & 0.268 & 0.062 & 0.014 & $\bullet$ \\
\hline 1981 & 0.215 & 0.093 & 0.012 & 0.011 \\
\hline 1982 & 0.271 & 0.040 & 0.024 & - \\
\hline
\end{tabular}


fertility rates, $1940-82$

\begin{tabular}{|c|c|c|c|}
\hline $20-24$ & $25-29$ & $30+$ & Total \\
\hline$\bullet$ & $\bullet$ & - & - \\
\hline$\bullet$ & - & - & $\bullet$ \\
\hline • & $\bullet$ & • & • \\
\hline - & - & $\bullet$ & - \\
\hline$\bullet$ & - & $\bullet$ & $\bullet$ \\
\hline - & • & $\bullet$ & 5.480 \\
\hline - & - & - & 6.063 \\
\hline - & - & - & 4.483 \\
\hline - & - & - & 5.578 \\
\hline - & $\bullet$ & - & 5.300 \\
\hline • & - & - & $3 \cdot 368$ \\
\hline • & • & - & 5.276 \\
\hline - & $\bullet$ & - & 6.167 \\
\hline - & $\bullet$ & • & 5.166 \\
\hline - & - & - & 6.925 \\
\hline - & - & - & 4.819 \\
\hline - & • & - & 6.177 \\
\hline • & - & - & 6.965 \\
\hline - & - & - & 5.399 \\
\hline - & $\bullet$ & • & 4.785 \\
\hline 0.068 & • & - & 4.554 \\
\hline 0.034 & - & • & 3.615 \\
\hline 0.044 & - & - & 6.153 \\
\hline 0.134 & - & $\bullet$ & 7.314 \\
\hline 0.086 & 0.020 & • & 4.859 \\
\hline 0.076 & $\bullet$ & - & 4.441 \\
\hline 0.029 & 0.017 & 0.031 & 3.897 \\
\hline 0.038 & 0.015 & - & $3: 764$ \\
\hline 0.054 & 0.013 & - & 5.244 \\
\hline 0.017 & 0.012 & 0.020 & 4.583 \\
\hline 0.083 & - & - & 4.319 \\
\hline 0.050 & - & - & 3.914 \\
\hline 0.047 & - & - & 3.738 \\
\hline 0.030 & - & - & 2.941 \\
\hline 0.008 & - & - & 2.446 \\
\hline - & • & - & 2.688 \\
\hline 0.021 & - & - & 2.651 \\
\hline$\bullet$ & - & • & 2.871 \\
\hline 0.013 & - & - & 2.730 \\
\hline - & - & 0.003 & 1.923 \\
\hline - & - & • & 1.716 \\
\hline - & - & - & 1.653 \\
\hline - & - & - & 1.674 \\
\hline
\end{tabular}


Basic Table 54.C. Rural Shaanxi Province (25): duration-specific fertility

\begin{tabular}{|c|c|c|c|c|}
\hline $\begin{array}{l}\text { Birth year } \\
\text { of child }\end{array}$ & $0-4$ & $5-9$ & $10-14$ & $15-19$ \\
\hline 1940 & 0.213 & - & $\bullet$ & - \\
\hline 1941 & 0.231 & • & - & - \\
\hline 1942 & 0.243 & - & - & - \\
\hline 1943 & 0.253 & - & - & - \\
\hline 1944 & 0.263 & - & - & • \\
\hline 1945 & 0.215 & 0.233 & - & - \\
\hline 1946 & 0.258 & 0.298 & - & - \\
\hline 1947 & 0.307 & 0.275 & - & - \\
\hline 1948 & 0.199 & 0.274 & - & - \\
\hline 1949 & 0.243 & 0.298 & • & - \\
\hline 1950 & 0.261 & 0.270 & 0.256 & - \\
\hline 1951 & 0.282 & 0.260 & 0.249 & - \\
\hline 1952 & 0.314 & 0.275 & 0.278 & - \\
\hline 1953 & 0.302 & 0.284 & 0.271 & - \\
\hline 1954 & 0.312 & 0.308 & 0.271 & - \\
\hline 1955 & 0.318 & 0.327 & 0.292 & 0.221 \\
\hline 1956 & 0.295 & 0.282 & 0.250 & 0.190 \\
\hline 1957 & 0.324 & 0.325 & 0.302 & 0.278 \\
\hline $\begin{array}{l}1958 \\
1959\end{array}$ & $\begin{array}{l}0.339 \\
0.246\end{array}$ & $\begin{array}{l}0.302 \\
0.247\end{array}$ & $\begin{array}{l}0.260 \\
0.230\end{array}$ & 0.220 \\
\hline 1960 & 0.220 & 0.294 & 0.246 & 0.195 \\
\hline 1961 & 0.219 & 0.199 & 0.231 & 0.173 \\
\hline 1962 & 0.314 & 0.408 & 0.297 & 0.213 \\
\hline 1963 & 0.428 & 0.335 & 0.288 & 0.284 \\
\hline 1964 & 0.324 & 0.356 & 0.274 & 0.230 \\
\hline 1965 & 0.274 & 0.267 & 0.308 & 0.227 \\
\hline 1966 & 0.366 & 0.390 & 0.290 & 0.242 \\
\hline 1967 & 0.321 & 0.299 & 0.288 & 0.214 \\
\hline 1968 & 0.369 & 0.379 & 0.298 & 0.261 \\
\hline 1969 & $0 \cdot 350$ & 0.309 & 0.259 & 0.217 \\
\hline 1970 & 0.338 & 0.307 & 0.289 & 0.233 \\
\hline 1971 & 0.345 & 0.311 & 0.278 & 0.176 \\
\hline 1972 & 0.322 & 0.322 & 0.262 & 0.234 \\
\hline 1973 & 0.324 & 0.264 & 0.258 & 0.164 \\
\hline 1974 & 0.357 & 0.316 & 0.213 & 0.142 \\
\hline 1975 & 0.283 & 0.246 & 0.149 & 0.118 \\
\hline 1976 & 0.365 & 0.235 & 0.137 & 0.087 \\
\hline 1977 & 0.309 & 0.197 & 0.119 & 0.066 \\
\hline 1978 & 0.341 & 0.209 & 0.135 & 0.049 \\
\hline 1979 & 0.413 & 0.234 & 0.117 & 0.045 \\
\hline 1980 & 0.310 & 0.172 & 0.086 & 0.040 \\
\hline 1981 & 0.375 & 0.155 & 0.078 & 0.019 \\
\hline 1982 & 0.364 & 0.185 & 0.076 & 0.019 \\
\hline
\end{tabular}


rates, $1940-82$

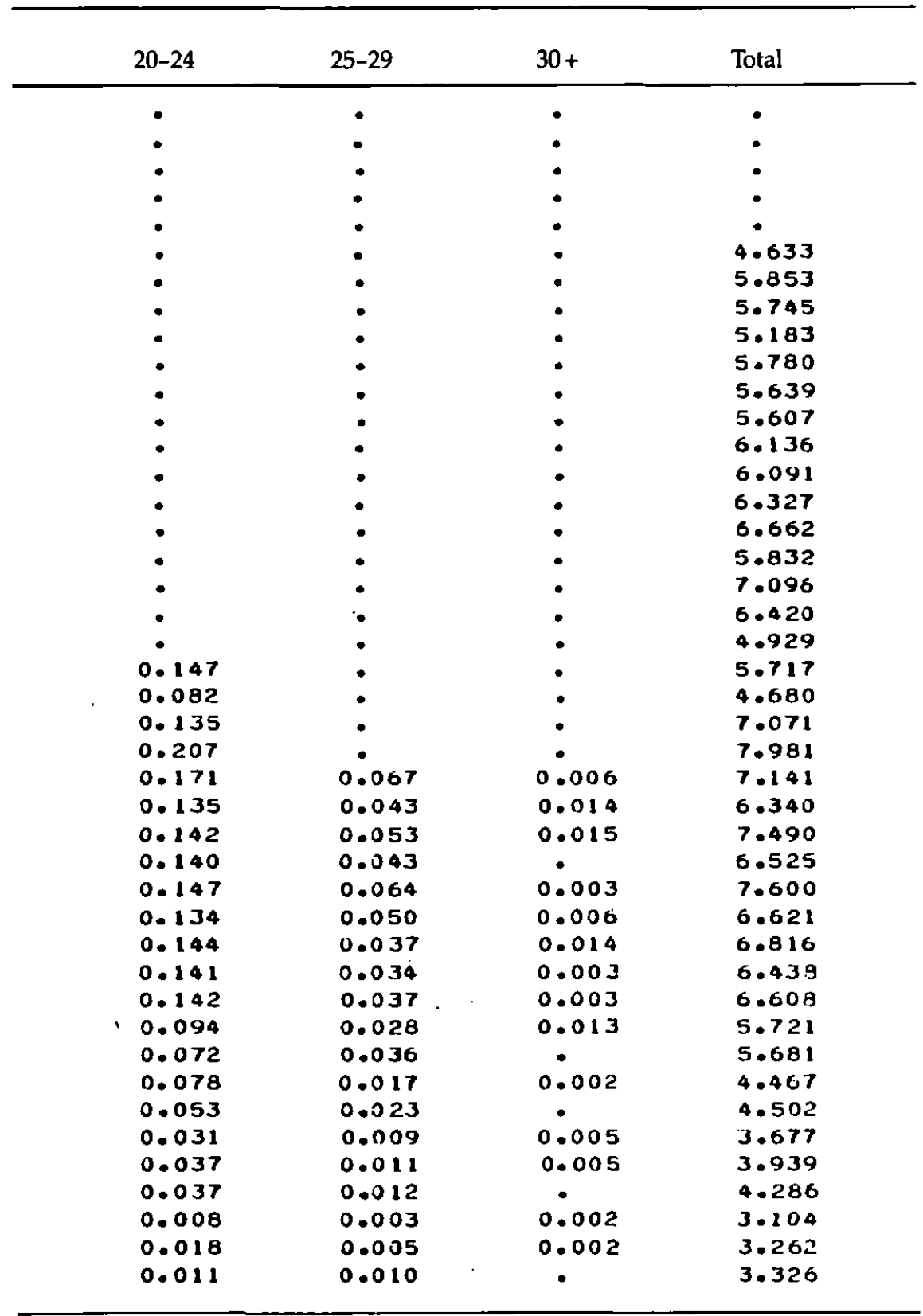


Basic Table 55.A. Total Gansu Province (26): duration-specific fertility

\begin{tabular}{|c|c|c|c|c|}
\hline $\begin{array}{l}\text { Birth year } \\
\text { of child }\end{array}$ & $0-4$ & $5-9$ & $10-14$ & $15-19$ \\
\hline 1940 & 0.204 & - & - & • \\
\hline 1941 & 0.239 & - & - & - \\
\hline 1942 & 0.210 & - & - & - \\
\hline 1943 & 0.206 & - & - & - \\
\hline 1944 & 0.238 & - & - & - \\
\hline 1945 & 0.238 & 0.308 & - & - \\
\hline 1940 & 0.275 & 0.291 & - & - \\
\hline 1947 & 0.237 & 0.284 & - & - \\
\hline 1948 & 0.203 & 0.285 & - & - \\
\hline 1949 & 0.293 & 0.330 & - & • \\
\hline 1950 & 0.253 & 0.315 & 0.283 & - \\
\hline 1951 & 0.238 & 0.258 & 0.213 & • \\
\hline 1952 & 0.313 & 0.342 & 0.273 & $\bullet$ \\
\hline 1953 & 0.281 & 0.297 & 0.267 & - \\
\hline 1954 & 0.268 & 0.321 & 0.253 & - \\
\hline 1455 & 0.283 & 0.355 & 0.295 & 0.233 \\
\hline 1950 & 0.315 & 0.275 & 0.323 & 0.191 \\
\hline 1957 & 0.287 & 0.280 & 0.267 & 0.214 \\
\hline 1958 & 0.262 & 0.274 & 0.250 & 0.235 \\
\hline 1959 & 0.161 & 0.169 & 0.173 & 0.147 \\
\hline 1960 & 0.205 & 0.175 & 0.191 & 0.111 \\
\hline 1961 & 0.173 & 0.187 & 0.157 & 0.127 \\
\hline 1962 & 0.283 & 0.360 & 0.359 & 0.287 \\
\hline 1903 & 0.325 & 0.394 & 0.313 & 0.229 \\
\hline 1964 & 0.316 & 0.355 & 0.330 & 0.296 \\
\hline 1965 & 0.316 & 0.310 & 0.278 & 0.242 \\
\hline 1900 & 0.330 & 0.379 & 0.345 & 0.270 \\
\hline 1967 & 0.293 & 0.294 & 0.291 & 0.241 \\
\hline 1968 & 0.344 & 0.374 & 0.306 & 0.291 \\
\hline 1909 & 0.318 & 0.303 & 0.271 & 0.241 \\
\hline 1970 & 0.303 & 0.345 & 0.277 & 0.256 \\
\hline 1971 & 0.317 & 0.326 & 0.280 & 0.220 \\
\hline 1972 & 0.318 & 0.309 & 0.272 & 0.230 \\
\hline 1973 & 0.298 & 0.331 & 0.264 & 0.187 \\
\hline 1974 & 0.359 & 0.277 & 0.211 & 0.144 \\
\hline 1975 & 0.322 & 0.208 & 0.132 & 0.080 \\
\hline 1976 & 0.312 & 0.212 & 0.101 & 0.087 \\
\hline 1977 & 0.310 & 0.197 & 0.109 & 0.058 \\
\hline 1978 & 0.383 & 0.231 & 0.116 & 0.065 \\
\hline 1979 & 0.374 & 0.277 & 0.162 & 0.056 \\
\hline 1980 & 0.324 & 0.213 & 0.080 & 0.028 \\
\hline 1981 & 0.339 & 0.205 & 0.078 & 0.039 \\
\hline 1982 & 0.307 & 0.181 & 0.084 & 0.043 \\
\hline
\end{tabular}


rates, $1940-82$

\begin{tabular}{|c|c|c|c|}
\hline $20-24$ & $25-29$ & $30+$ & Total \\
\hline - & - & - & - \\
\hline - & - & - & - \\
\hline - & - & - & - \\
\hline - & - & - & - \\
\hline - & - & • & - \\
\hline - & - & - & 5.925 \\
\hline - & - & - & 5.343 \\
\hline - & • & - & 5.519 \\
\hline •. & - & - & 5.416 \\
\hline - & - & - & 6.546 \\
\hline - & - & - & 6.220 \\
\hline - & - & - & 5.108 \\
\hline - & - & - & 6.626 \\
\hline - & - & - & 6.094 \\
\hline - & - & - & 6.088 \\
\hline - & - & - & 6.773 \\
\hline - & - & - & 6.345 \\
\hline - & - & • & 6.029 \\
\hline - & - & - & 5.921 \\
\hline - & - & - & 3.765 \\
\hline 0.079 & • & . & 3.904 \\
\hline 0.071 & - & - & 3.691 \\
\hline 0.181 & - & - & 7.612 \\
\hline 0.159 & - & - & 7.338 \\
\hline 0.172 & 0.063 & 0.009 & 7.708 \\
\hline 0.170 & 0.124 & 0.007 & 7.167 \\
\hline 0.170 & 0.093 & 0.024 & 8.059 \\
\hline $0.1<7$ & 0.063 & 0.005 & 6.769 \\
\hline 0.167 & 0.097 & 0.019 & 7.989 \\
\hline 0.161 & 0.051 & - & 6.727 \\
\hline 0.210 & 0.045 & 0.00 .5 & 7.259 \\
\hline 0.172 & 0.048 & 0.004 & 6.834 \\
\hline 0.181 & 0.062 & 0.011 & 6.919 \\
\hline 0.141 & 0.069 & 0.007 & 6.784 \\
\hline 0.099 & 0.060 & 0.004 & 5.765 \\
\hline 0.053 & 0.046 & 0.004 & 4.253 \\
\hline 0.040 & 0.020 & • & 3.853 \\
\hline 0.031 & 0.024 & 0.004 & 3.666 \\
\hline 0.022 & 0.015 & 0.004 & 4.181 \\
\hline 0.049 & 0.010 & - & 4.645 \\
\hline 0.002 & 0.018 & $0.00 ?$ & 3.337 \\
\hline 0.016 & 0.005 & 0.007 & $3.44 \pi$ \\
\hline 0.022 & 0.014 & 0.005 & 3.284 \\
\hline
\end{tabular}


Basic Table 55.B. Urban Gansu Province (26): duration-specific fertility

\begin{tabular}{|c|c|c|c|c|}
\hline $\begin{array}{l}\text { Birth year } \\
\text { of child }\end{array}$ & $0-4$ & $5-9$ & $10-14$ & $15-19$ \\
\hline 1940 & 0.248 & - & - & - \\
\hline 1941 & 0.286 & - & - & - \\
\hline 1942 & 0.151 & - & - & - \\
\hline 1943 & 0.138 & - & - & - \\
\hline 1944 & 0.248 & - & - & - \\
\hline 1945 & 0.352 & 0.273 & - & - \\
\hline 1940 & 0.318 & 0.260 & - & - \\
\hline 1947 & 0.205 & 0.151 & - & - \\
\hline 1948 & 0.253 & 0.322 & - & - \\
\hline 1949 & 0.420 & 0.338 & - & - \\
\hline 1950 & 0.299 & 0.117 & 0.248 & - \\
\hline 1951 & 0.136 & 0.295 & 0.260 & - \\
\hline 1452 & 0.349 & 0.365 & 0.176 & - \\
\hline 1953 & 0.361 & 0.232 & 0.322 & - \\
\hline 1954 & 0.191 & 0.385 & 0.180 & - \\
\hline 1955 & 0.288 & 0.285 & 0.399 & 0.248 \\
\hline 1950 & 0.369 & 0.314 & 0.295 & 0.156 \\
\hline 1957 & 0.250 & 0.323 & 0.160 & 0.126 \\
\hline 1958 & 0.334 & 0.321 & 0.274 & 0.299 \\
\hline 1959 & 0.215 & 0.235 & 0.175 & 0.135 \\
\hline 1960 & 0.282 & 0.176 & 0.257 & 0.094 \\
\hline 1961 & 0.240 & 0.150 & 0.150 & 0.068 \\
\hline 1962 & 0.335 & 0.408 & 0.298 & 0.274 \\
\hline $1 \overline{0} 3$ & 0.394 & 0.429 & 0.387 & 0.034 \\
\hline 1964 & 0.304 & 0.403 & 0.264 & 0.263 \\
\hline 1965 & 0.349 & 0.317 & $0 \cdot 192$ & 0.143 \\
\hline 1900 & 0.333 & 0.330 & 0.273 & 0.164 \\
\hline 1967 & 0.316 & 0.168 & 0.193 & 0.129 \\
\hline 1968 & 0.434 & 0.321 & 0.277 & 0.174 \\
\hline 1909 & 0.350 & 0.157 & 0.152 & 0.073 \\
\hline 1970 & 0.424 & 0.287 & 0.150 & 0.112 \\
\hline 1971 & 0.359 & 0.222 & 0.134 & 0.137 \\
\hline 1972 & 0.449 & 0.260 & 0.186 & 0.091 \\
\hline 1973 & 0.303 & 0.316 & 0.114 & 0.057 \\
\hline 1974 & 0.339 & 0.136 & 0.079 & 0.045 \\
\hline 1975 & 0.279 & 0.173 & 0.012 & 0.035 \\
\hline 1976 & 0.343 & 0.125 & 0.032 & 0.009 \\
\hline 1977 & 0.339 & 0.139 & 0.056 & 0.009 \\
\hline 1978 & 0.394 & 0.084 & 0.039 & 0.010 \\
\hline 1979 & 0.385 & 0.054 & 0.058 & - \\
\hline 1980 & 0.211 & 0.035 & • & - \\
\hline 1981 & 0.286 & 0.034 & 0.016 & - \\
\hline 1982 & 0.246 & $\bullet$ & $\bullet$ & - \\
\hline
\end{tabular}


rates, 1940-82

\begin{tabular}{|c|c|c|c|}
\hline $20-24$ & $25-29$ & $30+$ & Total \\
\hline - & - & - & - \\
\hline - & • & - & - \\
\hline - & $\bullet$ & - & - \\
\hline$\bullet$ & - & $\bullet$ & - \\
\hline$\bullet$ & - & - & $\bullet$ \\
\hline - & - & - & 6.146 \\
\hline - & $\bullet$ & - & 5.776 \\
\hline - & - & • & 3.452 \\
\hline$\bullet$ & - & - & 6.452 \\
\hline - & - & - & 7.541 \\
\hline - & - & • & 4.669 \\
\hline - & - & - & 5.452 \\
\hline - & - & • & 6.354 \\
\hline - & - & - & 6.593 \\
\hline - & - & - & 5.767 \\
\hline - & - & - & 7.215 \\
\hline - & - & - & 6.565 \\
\hline$\bullet$ & - & - & 4.993 \\
\hline • & - & - & 7.218 \\
\hline - & - & - & 4.439 \\
\hline 0.050 & - & - & 4.440 \\
\hline 0.078 & - & - & $3 \cdot 560$ \\
\hline 0.126 & • & - & 7.500 \\
\hline 0.138 & - & - & 7.437 \\
\hline 0.045 & 0.049 & - & 6.634 \\
\hline 0.164 & 0.025 & • & 5.951 \\
\hline 0.023 & 0.078 & - & 6.001 \\
\hline 0.114 & $\bullet$ & - & 4.599 \\
\hline 0.084 & - & - & 6.450 \\
\hline 0.070 & 0.023 & - & 4.128 \\
\hline 0.057 & - & 0.025 & 5.272 \\
\hline 0.041 & - & - & 4.461 \\
\hline 0.013 & - & - & 4.996 \\
\hline 0.067 & - & - & 4.282 \\
\hline 0.015 & - & - & 3.066 \\
\hline • & $\bullet$ & - & 2.499 \\
\hline • & 0.014 & - & 2.610 \\
\hline 0.011 & $\bullet$ & - & 2.772 \\
\hline 0.010 & - & - & 2.689 \\
\hline 0.009 & - & - & 2.531 \\
\hline$\bullet$ & - & - & 1.230 \\
\hline - & - & • & 1.678 \\
\hline - & - & - & 1.228 \\
\hline
\end{tabular}


Basic Table 55.C. Rural Gansu Province (26): duration-specific fertility

\begin{tabular}{|c|c|c|c|c|}
\hline $\begin{array}{l}\text { Birth year } \\
\text { of child }\end{array}$ & $0-4$ & $5-9$ & $10-14$ & $15-19$ \\
\hline 1940 & 0.195 & • & - & - \\
\hline 1941 & 0.231 & $\bullet$ & - & - \\
\hline 1942 & 0.220 & - & - & - \\
\hline 1943 & 0.218 & • & - & - \\
\hline 2944 & 0.236 & $\cdot$ & - & - \\
\hline 1945 & 0.213 & 0.315 & • & - \\
\hline 1946 & 0.265 & 0.297 & - & - \\
\hline 1947 & 0.245 & 0.307 & - & - \\
\hline 1948 & 0.193 & 0.278 & $\bullet$ & - \\
\hline 1949 & 0.265 & 0.328 & - & - \\
\hline 1950 & 0.242 & 0.357 & 0.290 & - \\
\hline 1951 & 0.260 & 0.249 & 0.205 & - \\
\hline 1952 & 0.305 & 0.336 & 0.289 & - \\
\hline 1953 & 0.263 & 0.311 & 0.257 & - \\
\hline 1954 & 0.284 & 0.307 & 0.267 & • \\
\hline 1955 & 0.283 & 0.372 & 0.273 & 0.230 \\
\hline 1956 & 0.303 & 0.266 & 0.330 & 0.196 \\
\hline 1957 & 0.296 & 0.270 & 0.291 & 0.229 \\
\hline 1958 & 0.241 & 0.263 & 0.245 & 0.223 \\
\hline 1959 & 0.144 & 0.156 & 0.173 & 0.149 \\
\hline 1960 & 0.181 & 0.174 & 0.176 & 0.114 \\
\hline 1961 & 0.154 & 0.196 & 0.159 & 0.141 \\
\hline 1962 & 0.270 & 0.347 & 0.373 & 0.291 \\
\hline 1963 & 0.311 & 0.384 & 0.297 & 0.259 \\
\hline 1964 & 0.318 & 0.339 & 0.344 & 0.303 \\
\hline 1965 & 0.310 & 0.308 & 0.295 & 0.265 \\
\hline 1960 & 0.329 & 0.394 & 0.361 & 0.293 \\
\hline 1967 & 0.290 & 0.326 & 0.317 & 0.267 \\
\hline 1968 & 0.333 & 0.385 & 0.315 & 0.318 \\
\hline 1969 & 0.315 & 0.331 & 0.308 & 0.275 \\
\hline 1970 & 0.291 & 0.356 & 0.316 & 0.283 \\
\hline 1971 & 0.312 & 0.341 & 0.323 & 0.239 \\
\hline 1972 & 0.302 & 0.316 & 0.293 & 0.267 \\
\hline 1973 & 0.298 & 0.333 & 0.295 & 0.226 \\
\hline 1974 & 0.361 & 0.293 & 0.236 & 0.175 \\
\hline 1975 & 0.328 & 0.212 & 0.153 & 0.102 \\
\hline 1976 & 0.307 & 0.222 & 0.111 & 0.110 \\
\hline 1977 & 0.305 & 0.204 & 0.116 & 0.070 \\
\hline 1978 & 0.381 & 0.248 & 0.125 & 0.077 \\
\hline 1979 & 0.371 & 0.304 & 0.174 & 0.067 \\
\hline 1980 & 0.353 & 0.238 & 0.088 & 0.033 \\
\hline 1981 & 0.353 & 0.233 & 0.086 & 0.044 \\
\hline 1982 & 0.322 & 0.213 & 0.094 & 0.049 \\
\hline
\end{tabular}


rates, $1940-82$

\begin{tabular}{|c|c|c|c|}
\hline $20-24$ & $25-29$ & $30+$ & Total \\
\hline - & - & - & - \\
\hline - & - & - & $\bullet$ \\
\hline - & • & - & - \\
\hline$\bullet$ & - & - & - \\
\hline - & - & - & - \\
\hline - & - & - & 5.983 \\
\hline - & - & - & 5.861 \\
\hline - & - & - & 5.912 \\
\hline - & - & - & 5.218 \\
\hline - & - & - & 6.347 \\
\hline - & - & - & 6.540 \\
\hline$\bullet$ & - & - & 5.038 \\
\hline - & - & - & $6.68 ?$ \\
\hline - & - & - & 5.993 \\
\hline - & - & - & 6.153 \\
\hline - & - & - & 6.685 \\
\hline - & - & - & 6.300 \\
\hline - & - & - & 6.255 \\
\hline$\bullet$ & - & - & 5.626 \\
\hline - & - & - & 3.611 \\
\hline 0.085 & - & - & 3.782 \\
\hline 0.070 & - & - & 3.720 \\
\hline 0.190 & - & - & 7.638 \\
\hline 0.163 & - & • & 7.316 \\
\hline 0.197 & 0.056 & 0.010 & 7.893 \\
\hline 0.179 & 0.120 & 0.008 & 7.421 \\
\hline 0.206 & 0.0 .96 & 0.026 & 8.534 \\
\hline 0.178 & 0.073 & 0.006 & 7.287 \\
\hline 0.194 & 0.116 & 0.023 & 8.364 \\
\hline 0.180 & 0.057 & - & $7 \cdot 334$ \\
\hline 0.256 & 0.055 & 0.005 & 7.799 \\
\hline 0.200 & 0.059 & 0.004 & $7.39 ?$ \\
\hline 0.219 & 0.076 & 0.013 & 7.432 \\
\hline 0.157 & 0.083 & 0.009 & 7.006 \\
\hline 0.117 & 0.073 & 0.004 & 6.295 \\
\hline 0.063 & 0.056 & 0.005 & 4.597 \\
\hline 0.049 & 0.021 & - & 4.095 \\
\hline 0.036 & 0.030 & 0.005 & 3.830 \\
\hline 0.02 .6 & 0.018 & 0.004 & 4.398 \\
\hline 0.002 & 0.012 & • & 4.951 \\
\hline 0.003 & 0.021 & 0.003 & 3.697 \\
\hline 0.021 & 0.006 & 0.009 & 3.755 \\
\hline 0.028 & 0.018 & 0.006 & 3.645 \\
\hline
\end{tabular}


Basic Table 56. Total Qinghai Province (27): duration-specific fertility

\begin{tabular}{|c|c|c|c|c|}
\hline $\begin{array}{l}\text { Birth year } \\
\text { of child }\end{array}$ & $0-4$ & $5-9$ & $10-14$ & $15-19$ \\
\hline 1940 & 0.174 & $\bullet$ & - & - \\
\hline 1941 & 0.105 & - & • & - \\
\hline 1942 & 0.192 & - & - & - \\
\hline 1943 & 0.188 & - & - & - \\
\hline 1944 & 0.176 & - & - & - \\
\hline 1945 & 0.078 & 0.240 & - & - \\
\hline 1946 & 0.239 & 0.281 & - & - \\
\hline 1947 & 0.207 & 0.221 & - & - \\
\hline 1948 & 0.093 & 0.126 & - & - \\
\hline 1949 & 0.270 & 0.188 & $\bullet$ & - \\
\hline 1950 & 0.156 & 0.285 & 0.218 & - \\
\hline 1951 & 0.140 & 0.189 & 0.176 & - \\
\hline 1952 & 0.229 & 0.233 & 0.162 & - \\
\hline 1953 & 0.200 & 0.213 & 0.214 & - \\
\hline 1954 & 0.173 & 0.307 & 0.263 & - \\
\hline 1955 & 0.255 & 0.292 & 0.272 & 0.262 \\
\hline 1950 & 0.185 & 0.210 & 0.189 & 0.176 \\
\hline 1957 & 0.239 & 0.257 & 0.181 & 0.133 \\
\hline 1958 & 0.167 & 0.255 & 0.147 & 0.214 \\
\hline 1959 & 0.157 & 0.155 & 0.208 & 0.075 \\
\hline 1960 & 0.070 & 0.082 & 0.094 & 0.091 \\
\hline 1961 & 0.095 & 0.102 & 0.110 & 0.063 \\
\hline 1962 & 0.277 & 0.334 & 0.172 & 0.207 \\
\hline $196 \mathrm{~J}$ & 0.248 & 0.241 & 0.228 & 0.173 \\
\hline 1904 & 0.301 & 0.407 & 0.327 & 0.270 \\
\hline 1905 & 0.212 & 0.303 & 0.274 & 0.219 \\
\hline 1966 & 0.269 & 0.307 & 0.268 & 0.240 \\
\hline 1907 & 0.247 & 0.284 & 0.239 & 0.210 \\
\hline 1968 & 0.337 & 0.322 & 0.241 & 0.282 \\
\hline 1909 & 0.301 & 0.243 & 0.277 & 0.182 \\
\hline 1970 & 0.320 & 0.307 & 0.229 & 0.228 \\
\hline 1971 & 0.215 & 0.269 & 0.229 & 0.176 \\
\hline 1972 & 0.326 & 0.326 & 0.234 & 0.210 \\
\hline 1973 & 0.256 & 0.277 & 0.208 & 0.223 \\
\hline 1974 & 0.324 & 0.341 & 0.237 & 0.111 \\
\hline 1975 & 0.231 & 0.291 & 0.178 & 0.132 \\
\hline 1970 & 0.374 & 0.260 & 0.166 & 0.095 \\
\hline 1977 & 0.323 & 0.227 & 0.213 & 0.113 \\
\hline 1978 & 0.282 & 0.243 & 0.196 & 0.148 \\
\hline 1979 & 0.297 & 0.310 & 0.239 & 0.081 \\
\hline 1980 & 0.152 & 0.210 & 0.184 & 0.100 \\
\hline 1981 & 0.285 & 0.252 & 0.221 & 0.143 \\
\hline 1982 & 0.206 & 0.196 & 0.263 & 0.124 \\
\hline
\end{tabular}


rates, $1940-82$

\begin{tabular}{|c|c|c|c|}
\hline $20-24$ & $25-29$ & $30+$ & Total \\
\hline - & - & - & - \\
\hline - & - & • & - \\
\hline - & - & - & - \\
\hline - & $\bullet$ & $\bullet$ & - \\
\hline - & • & - & $\bullet$ \\
\hline - & • & - & 3.954 \\
\hline - & - & - & 5.423 \\
\hline - & $\bullet$ & - & 4.404 \\
\hline - & - & - & 2.367 \\
\hline - & - & - & 4.221 \\
\hline - & - & - & 4.774 \\
\hline - & - & - & 3.621 \\
\hline - & - & - & 4.488 \\
\hline - & • & - & 4.508 \\
\hline - & - & - & 5.571 \\
\hline$\bullet$ & - & - & 6.233 \\
\hline - & - & - & 4.385 \\
\hline - & - & - & 4.741 \\
\hline - & - & - & 4.822 \\
\hline - & - & - & $3 \cdot 385$ \\
\hline 0.065 & - & - & 1.951 \\
\hline 0.070 & - & - & 2.246 \\
\hline 0.103 & - & • & 5.484 \\
\hline 0.100 & - & - & 5.194 \\
\hline 0.100 & 0.079 & 0.062 & 7.720 \\
\hline 0.169 & 0.065 & 0.054 & 6.505 \\
\hline 0.139 & 0.053 & 0.054 & 6.652 \\
\hline 0.103 & 0.029 & 0.042 & 5.768 \\
\hline 0.187 & 0.100 & 0.031 & 7.508 \\
\hline 0.172 & 0.050 & 0.026 & 6.253 \\
\hline 0.125 & 0.039 & - & 6.239 \\
\hline 0.170 & 0.038 & - & 5.483 \\
\hline 0.172 & 0.039 & 0.015 & 6.605 \\
\hline 0.155 & 0.040 & 0.013 & 5.859 \\
\hline 0.173 & 0.074 & • & 6.297 \\
\hline 0.100 & 0.073 & 0.013 & 5.093 \\
\hline 0.074 & 0.050 & 0.013 & 5.154 \\
\hline 0.105 & 0.038 & 0.013 & 5.165 \\
\hline 0.065 & 0.046 & 0.053 & $5 \cdot 1 \in 2$ \\
\hline 0.065 & 0.036 & 0.012 & $5 \cdot 204$ \\
\hline 0.114 & 0.092 & 0.010 & 4.266 \\
\hline 0.079 & 0.028 & 0.010 & 5.088 \\
\hline 0.085 & 0.039 & • & 4.504 \\
\hline
\end{tabular}


Basic Table 57. Total Ningxia Province (28): duration-specific fertility

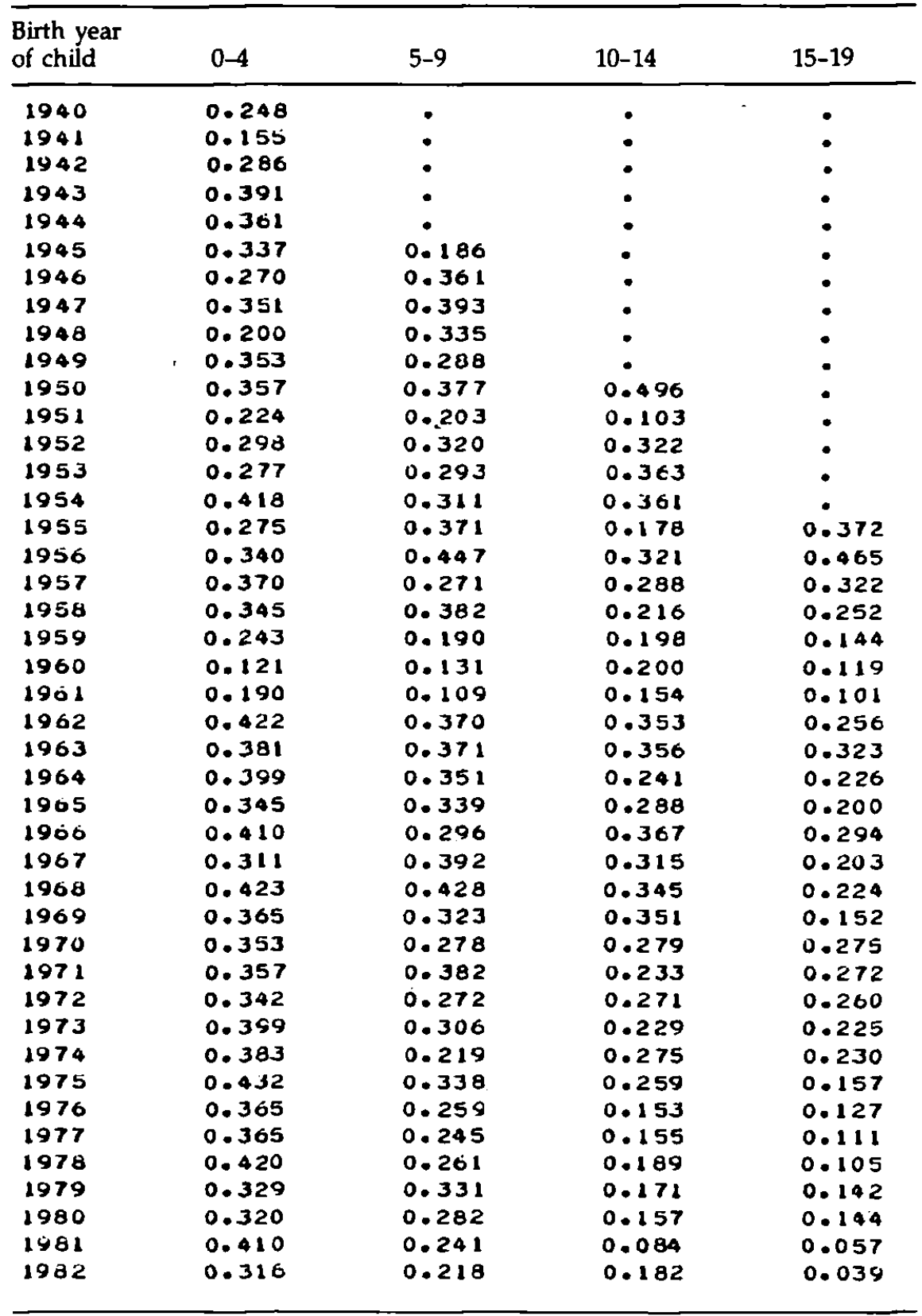


rates, $1940-82$

\begin{tabular}{|c|c|c|c|}
\hline $20-24$ & $25-29$ & $30+$ & Total \\
\hline$\bullet$ & - & - & - \\
\hline • & $\bullet$ & • & - \\
\hline - & - & - & - \\
\hline - & - & - & - \\
\hline - & - & - & - \\
\hline - & - & - & 4.838 \\
\hline - & - & - & 7.115 \\
\hline - & - & - & 7.798 \\
\hline - & - & - & 6.050 \\
\hline - & - & - & 5.942 \\
\hline$\bullet$ & - & - & 9.008 \\
\hline - & - & - & 3.791 \\
\hline - & - & • & 6.825 \\
\hline - & - & •. & 6.933 \\
\hline - & - & - & 7.612 \\
\hline - & - & - & 6.910 \\
\hline - & - & - & $9.1 \in 2$ \\
\hline - & - & - & 7.200 \\
\hline - & - & - & 6.907 \\
\hline - & - & - & 4.419 \\
\hline 0.124 & - & - & 3.621 \\
\hline 0.103 & $\bullet$ & - & 3.437 \\
\hline 0.286 & - & - & 8.743 \\
\hline 0.084 & - & - & 7.770 \\
\hline 0.168 & 0.108 & 0.097 & 7.953 \\
\hline 0.139 & 0.124 & - & $7 \cdot 1.69$ \\
\hline 0.152 & 0.206 & - & 8.630 \\
\hline 0.144 & 0.071 & - & 7.180 \\
\hline 0.154 & 0.112 & 0.050 & 0.678 \\
\hline 0.184 & 0.096 & - & 7.359 \\
\hline 0.214 & 0.040 & - & $7 \cdot 188$ \\
\hline 0.126 & 0.034 & - & 7.018 \\
\hline 0.149 & 0.048 & - & 0.711 \\
\hline 0.224 & 0.031 & - & 7.065 \\
\hline 0.152 & 0.071 & - & 6.053 \\
\hline 0.157 & 0.171 & - & 7.569 \\
\hline 0.136 & 0.056 & 0.034 & 5.647 \\
\hline 0.055 & 0.108 & • & $5 \cdot 196$ \\
\hline 0.053 & 0.053 & 0.031 & 5.556 \\
\hline 0.135 & 0.087 & • & 5.981 \\
\hline 0.061 & 0.052 & 0.029 & 5.21 .3 \\
\hline 0.042 & 0.014 & - & 4.240 \\
\hline 0.059 & 0.027 & 0.027 & 4.347 \\
\hline
\end{tabular}


Basic Table 58.A. Total Xinjiang Province (29): duration-specific fertility

\begin{tabular}{|c|c|c|c|c|}
\hline $\begin{array}{l}\text { Birth year } \\
\text { of child }\end{array}$ & $0-4$ & $5-9$ & $10-14$ & $15-19$ \\
\hline 1940 & 0.188 & • & - & - \\
\hline 1941 & 0.145 & - & - & - \\
\hline 1942 & 0.217 & - & - & - \\
\hline 1943 & 0.180 & - & - & - \\
\hline 1944 & 0.180 & - & - & - \\
\hline 1945 & 0.171 & 0.207 & - & • \\
\hline 1946 & 0.182 & 0.264 & - & $\bullet$ \\
\hline 1947 & 0.172 & 0.189 & - & - \\
\hline 1948 & 0.155 & 0.216 & - & - \\
\hline 1949 & 0.168 & 0.263 & - & - \\
\hline 1950 & 0.187 & 0.232 & 0.213 & • \\
\hline 1951 & 0.126 & 0.263 & 0.172 & - \\
\hline 1952 & 0.276 & 0.242 & 0.343 & - \\
\hline 1953 & 0.203 & 0.203 & 0.180 & - \\
\hline 1954 & 0.252 & 0.263 & 0.366 & - \\
\hline 1955 & 0.280 & 0.179 & 0.184 & 0.170 \\
\hline 1956 & 0.255 & 0.299 & 0.344 & 0.238 \\
\hline 1957 & 0.271 & 0.224 & 0.226 & 0.217 \\
\hline 1958 & 0.268 & 0.340 & 0.280 & 0.266 \\
\hline 1959 & 0.223 & 0.210 & 0.202 & 0.180 \\
\hline 1960 & 0.200 & 0.248 & 0.212 & 0.232 \\
\hline 1961 & 0.208 & 0.218 & 0.192 & 0.200 \\
\hline 1962 & 0.278 & 0.320 & 0.298 & 0.226 \\
\hline 1903 & $0 \cdot 336$ & 0.287 & 0.245 & 0.218 \\
\hline 1904 & 0.282 & 0.327 & 0.274 & 0.263 \\
\hline 1965 & 0.322 & 0.314 & 0.248 & 0.212 \\
\hline 1960 & 0.335 & $0.32 \mathrm{e}$ & 0.284 & 0.203 \\
\hline 1907 & 0.334 & 0.255 & 0.261 & 0.164 \\
\hline 1968 & 0.376 & 0.329 & 0.235 & 0.279 \\
\hline 1969 & 0.356 & 0.327 & 0.253 & 0.199 \\
\hline 1970 & 0.327 & 0.299 & 0.266 & 0.216 \\
\hline 1971 & 0.305 & 0.321 & 0.203 & 0.169 \\
\hline 1972 & 0.315 & 0.269 & 0.225 & 0.178 \\
\hline 1973 & 0.328 & 0.293 & 0.251 & 0.160 \\
\hline 1974 & 0.345 & 0.292 & 0.248 & 0.199 \\
\hline 1975 & 0.321 & 0.257 & 0.183 & 0.152 \\
\hline 1976 & 0.333 & 0.257 & 0.224 & 0.133 \\
\hline 1977 & 0.310 & 0.232 & 0.166 & 0.117 \\
\hline 1973 & 0.345 & 0.216 & 0.152 & 0.135 \\
\hline 1979 & 0.296 & 0.225 & 0.156 & 0.102 \\
\hline 1900 & 0.287 & 0.183 & 0.137 & 0.091 \\
\hline 1981 & 0.309 & 0.227 & 0.124 & 0.112 \\
\hline 1982 & 0.350 & 0.210 & 0.212 & 0.166 \\
\hline
\end{tabular}


rates, $1940-82$

\begin{tabular}{|c|c|c|c|}
\hline $20-24$ & $25-29$ & $30+$ & Total \\
\hline - & - & - & - \\
\hline - & - & - & - \\
\hline - & - & - & • \\
\hline - & - & - & - \\
\hline - & - & - & - \\
\hline - & - & - & 4.012 \\
\hline - & - & - & 4.919 \\
\hline - & - & - & 3.789 \\
\hline - & - & - & 4.053 \\
\hline - & - & - & 4.905 \\
\hline - & - & - & 4.625 \\
\hline - & - & - & 4.158 \\
\hline - & - & - & 6.200 \\
\hline - & - & - & 4.202 \\
\hline - & - & - & 6.418 \\
\hline - & . & - & 4.663 \\
\hline - & - & - & 0.519 \\
\hline • & - & - & 5.353 \\
\hline - & - & - & 6.676 \\
\hline - & - & - & 4.632 \\
\hline 0.163 & - & - & 5.363 \\
\hline 0.059 & - & - & 4.600 \\
\hline 0.161 & - & - & 6.641 \\
\hline 0.130 & - & - & 6.214 \\
\hline 0.228 & 0.128 & 0.067 & 7.843 \\
\hline 0.219 & 0.138 & 0.080 & 7.666 \\
\hline 0.188 & 0.073 & 0.063 & 7.369 \\
\hline 0.135 & 0.112 & 0.061 & 6.012 \\
\hline 0.131 & 0.079 & 0.063 & 7.454 \\
\hline 0.133 & 0.076 & 0.038 & 6.913 \\
\hline 0.151 & 0.082 & 0.050 & 6.956 \\
\hline 0.134 & 0.038 & 0.033 & 6.009 \\
\hline 0.157 & 0.059 & 0.028 & 6.154 \\
\hline 0.084 & 0.029 & 0.022 & 5.835 \\
\hline 0.146 & 0.034 & 0.028 & 6.467 \\
\hline 0.103 & 0.049 & 0.007 & $5 \cdot 356$ \\
\hline $0 .-119$ & 0.061 & 0.031 & 5.795 \\
\hline 0.071 & 0.045 & 0.005 & 4.731 \\
\hline 0.047 & 0.031 & 0.005 & 4.655 \\
\hline 0.064 & 0.026 & 0.013 & 4.404 \\
\hline 0.051 & 0.025 & 0.012 & 3.927 \\
\hline 0.058 & 0.026 & 0.015 & 4.356 \\
\hline 0.056 & 0.024 & 0.008 & 5.128 \\
\hline
\end{tabular}


Basic Table 58.B. Urban Xinjiang Province (29): duration-specific

\begin{tabular}{|c|c|c|c|c|}
\hline $\begin{array}{l}\text { Birth year } \\
\text { of child }\end{array}$ & $0-4$ & $5-9$ & $10-14$ & $15-19$ \\
\hline 1940 & 0.341 & - & - & - \\
\hline 1941 & 0.200 & - & - & - \\
\hline 1942 & 0.135 & - & - & - \\
\hline 1943 & 0.212 & - & - & - \\
\hline 1944 & 0.243 & - & - & - \\
\hline 1945 & 0.368 & 0.272 & - & • \\
\hline 1946 & 0.312 & 0.200 & $\bullet$ & $\bullet$ \\
\hline 1947 & 0.180 & 0.271 & - & - \\
\hline 1948 & 0.171 & 0.141 & - & - \\
\hline 1945 & 0.215 & 0.364 & - & - \\
\hline 1950 & 0.299 & 0.105 & 0.341 & - \\
\hline 1 & 0.201 & 0.389 & 0.133 & - \\
\hline 1452 & 0.356 & 0.209 & 0.271 & - \\
\hline 1953 & 0.198 & 0.220 & 0.212 & - \\
\hline 1954 & 0.309 & 0.344 & 0.303 & - \\
\hline 1955 & 0.259 & 0.187 & 0.158 & 0.204 \\
\hline $\begin{array}{l}1956 \\
1957\end{array}$ & $\begin{array}{l}0.357 \\
0.270\end{array}$ & $\begin{array}{l}0.335 \\
0.204\end{array}$ & $\begin{array}{l}0.312 \\
0.209\end{array}$ & $\begin{array}{l}0.200 \\
0.135\end{array}$ \\
\hline 1958 & 0.327 & 0.270 & 0.293 & 0.282 \\
\hline 1959 & 0.278 & 0.250 & 0.301 & 0.121 \\
\hline 1960 & 0.252 & 0.218 & 0.149 & 0.105 \\
\hline 1961 & 0.209 & 0.250 & 0.201 & 0.234 \\
\hline 1962 & 0.341 & 0.307 & 0.288 & 0.299 \\
\hline 1963 & 0.406 & 0.280 & 0.252 & 0.220 \\
\hline 1964 & 0.309 & 0.347 & 0.132 & 0.280 \\
\hline 1965 & 0.358 & 0.290 & 0.191 & 0.131 \\
\hline $1960^{\circ}$ & 0.371 & 0.271 & 0.167 & 0.100 \\
\hline 1967 & 0.376 & 0.219 & 0.149 & 0.102 \\
\hline 1968 & 0.380 & 0.310 & 0.179 & 0.090 \\
\hline 1969 & 0.401 & 0.302 & 0.193 & 0.132 \\
\hline 1970 & 0.326 & 0.307 & 0.114 & 0.055 \\
\hline 1971 & 0.405 & 0.265 & 0.139 & 0.143 \\
\hline 1972 & 0.366 & 0.194 & 0.122 & 0.084 \\
\hline 1973 & 0.419 & 0.235 & 0.239 & 0.093 \\
\hline 1974 & 0.449 & 0.191 & 0.173 & 0.062 \\
\hline 1975 & 0.373 & 0.142 & 0.089 & 0.061 \\
\hline 1976 & 0.407 & 0.165 & 0.029 & 0.023 \\
\hline 1977 & 0.333 & 0.161 & 0.040 & 0.016 \\
\hline 1978 & 0.307 & 0.134 & 0.024 & 0.016 \\
\hline 1979 & 0.282 & 0.068 & 0.013 & - \\
\hline 1980 & 0.213 & 0.049 & 0.007 & - \\
\hline 1981 & 0.226 & 0.010 & - & • \\
\hline 1982 & 0.300 & 0.044 & 0.018 & - \\
\hline
\end{tabular}


fertility rates, $1940-82$

\begin{tabular}{|c|c|c|c|}
\hline $20-24$ & $25-29$ & $30+$ & Total \\
\hline - & - & - & $\bullet$ \\
\hline - & • & - & - \\
\hline - & $\bullet$ & - & - \\
\hline - & - & $\bullet$ & - \\
\hline - & - & - & • \\
\hline - & - & • & 6.222 \\
\hline - & - & - & 4.776 \\
\hline - & - & - & 5.258 \\
\hline - & - & - & 3.125 \\
\hline - & - & - & 6.934 \\
\hline : & - & - & 5.385 \\
\hline - & - & - & 5.439 \\
\hline - & - & - & 5.929 \\
\hline - & - & - & 4.700 \\
\hline . & - & - & .7 .105 \\
\hline - & - & - & 4.707 \\
\hline - & - & - & 7.013 \\
\hline - & - & - & 4.743 \\
\hline - & $\bullet$ & - & 6.886 \\
\hline - & - & - & .5 .537 \\
\hline 0.136 & • & - & 4.479 \\
\hline 0.133 & - & - & 5.363 \\
\hline 0.135 & - & . & 7.142 \\
\hline 0.071 & - & - & 6.358 \\
\hline 0.121 & - & - & 5.948 \\
\hline 0.158 & 0.204 & - & 6.656 \\
\hline 0.078 & - & . & 4.931 \\
\hline 0.060 & 0.068 & . & 4.866 \\
\hline 0.073 & - & - & 5.164 \\
\hline 0.086 & - & - & 5.573 \\
\hline 0.056 & - & - & 4.288 \\
\hline 0.050 & - & - & 5.011 \\
\hline 0.051 & 0.090 & 0.063 & 4.869 \\
\hline 0.018 & - & . & 5.024 \\
\hline 0.015 & - & - & 4.444 \\
\hline . & . & - & 3.324 \\
\hline 0.012 & - & . & 3.182 \\
\hline 0.009 & - & . & 2.795 \\
\hline 0.008 & - & - & 2.445 \\
\hline$\bullet$ & - & - & 1.813 \\
\hline 0.008 & - & - & 1.382 \\
\hline • & • & - & 1.173 \\
\hline - & - & - & 1.812 \\
\hline
\end{tabular}


Basic Table 58.C. Rural Xinjiang Province (29): duration-specific

\begin{tabular}{|c|c|c|c|c|}
\hline $\begin{array}{l}\text { Birth year } \\
\text { of child }\end{array}$ & $0-4$ & $5-9$ & $10-14$ & $15-19$ \\
\hline 1940 & 0.173 & - & - & - \\
\hline 1941 & 0.139 & - & - & - \\
\hline $\begin{array}{l}1942 \\
1943\end{array}$ & $\begin{array}{l}0.226 \\
0.176\end{array}$ & • & : & : \\
\hline 1944 & 0.172 & - & - & - \\
\hline 1945 & 0.141 & 0.201 & - & - \\
\hline 1946 & 0.157 & 0.272 & - & - \\
\hline 1947 & 0.171 & 0.180 & - & - \\
\hline 1948 & 0.151 & 0.224 & - & - \\
\hline 1949 & 0.156 & 0.250 & - & - \\
\hline 1950 & 0.156 & 0.251 & 0.201 & - \\
\hline 1951 & 0.104 & 0.239 & 0.176 & - \\
\hline 1952 & 0.253 & 0.249 & 0.351 & - \\
\hline 1953 & 0.204 & 0.199 & 0.176 & - \\
\hline 1954 & 0.232 & 0.242 & 0.374 & - \\
\hline 1955 & 0.287 & 0.177 & 0.188 & 0.106 \\
\hline 1956 & 0.215 & 0.288 & 0.351 & 0.242 \\
\hline 1957 & 0.271 & 0.229 & 0.230 & 0.226 \\
\hline 1958 & 0.236 & 0.359 & 0.277 & 0.265 \\
\hline 1959 & 0.195 & 0.197 & 0.178 & 0.187 \\
\hline 1960 & 0.175 & 0.258 & 0.229 & 0.251 \\
\hline 1961 & 0.208 & 0.206 & 0.189 & 0.194 \\
\hline 1962 & 0.250 & 0.326 & 0.301 & 0.210 \\
\hline 1963 & 0.305 & 0.291 & 0.243 & 0.217 \\
\hline 1964 & 0.270 & 0.316 & 0.323 & 0.258 \\
\hline 1965 & 0.305 & 0.327 & 0.268 & 0.234 \\
\hline 1966 & 0.316 & 0.356 & 0.330 & 0.234 \\
\hline 1967 & 0.314 & 0.272 & 0.317 & 0.182 \\
\hline 1908 & 0.374 & 0.336 & 0.266 & 0.330 \\
\hline 1969 & 0.337 & 0.339 & 0.284 & 0.222 \\
\hline 1970 & 0.328 & 0.295 & 0.342 & 0.273 \\
\hline 1971 & 0.275 & 0.349 & 0.234 & 0.179 \\
\hline 1972 & 0.301 & 0.306 & 0.272 & 0.225 \\
\hline 1973 & 0.303 & 0.319 & 0.257 & 0.197 \\
\hline 1974 & 0.318 & 0.335 & 0.283 & 0.272 \\
\hline 1975 & 0.307 & 0.299 & 0.229 & 0.197 \\
\hline 1976 & 0.312 & 0.285 & 0.325 & 0.185 \\
\hline 1977 & 0.303 & 0.252 & 0.228 & 0.164 \\
\hline 1978 & 0.355 & 0.239 & 0.211 & 0.187 \\
\hline 1979 & 0.300 & 0.265 & 0.215 & 0.148 \\
\hline 1980 & 0.306 & 0.219 & 0.185 & 0.135 \\
\hline 1981 & 0.332 & 0.287 & 0.161 & 0.170 \\
\hline 1982 & 0.365 & 0.256 & 0.204 & 0.246 \\
\hline
\end{tabular}


fertility rates, $1940-82$

\begin{tabular}{|c|c|c|c|}
\hline $20-24$ & $25-29$ & $30+$ & Total \\
\hline - & - & - & • \\
\hline - & $\bullet$ & $\bullet$ & - \\
\hline - & $\bullet$ & - & - \\
\hline - & - & $\bullet$ & - \\
\hline - & $\bullet$ & - & - \\
\hline - & - & - & 3.774 \\
\hline - & - & - & 4.936 \\
\hline - & - & - & 3.600 \\
\hline - & - & - & $4 \cdot 186$ \\
\hline - & - & - & 4.598 \\
\hline - & - & - & 4.500 \\
\hline - & - & - & 3.933 \\
\hline - & $\bullet$ & - & 6.248 \\
\hline - & $\bullet$ & - & 4.113 \\
\hline • & - & - & 6.278 \\
\hline - & - & - & 4.653 \\
\hline • & - & • & 6.405 \\
\hline - & - & - & 5.508 \\
\hline - & - & - & 6.619 \\
\hline - & - & - & 4.374 \\
\hline 0.166 & $\bullet$ & - & 5.616 \\
\hline 0.051 & $\bullet$ & - & 4.378 \\
\hline 0.164 & - & - & 6.489 \\
\hline 0.136 & - & - & 6.170 \\
\hline 0.242 & 0.142 & 0.070 & 8.101 \\
\hline 0.228 & 0.131 & 0.087 & 7.895 \\
\hline 0.209 & 0.081 & 0.066 & 7.950 \\
\hline 0.151 & 0.117 & 0.065 & 7.085 \\
\hline 0.145 & 0.088 & 0.069 & 8.038 \\
\hline 0.145 & 0.086 & 0.043 & 7.276 \\
\hline 0.177 & 0.094 & 0.055 & 7.819 \\
\hline 0.159 & 0.045 & .0 .037 & 6.385 \\
\hline 0.186 & 0.053 & 0.023 & 6.829 \\
\hline 0.102 & 0.036 & 0.024 & 6.189 \\
\hline 0.192 & 0.043 & $0.03 i$ & 7.365 \\
\hline 0.139 & 0.062 & 0.008 & 6.209 \\
\hline 0.160 & 0.080 & 0.037 & 6.924 \\
\hline 0.101 & 0.057 & 0.007 & 5.559 \\
\hline 0.069 & 0.039 & 0.006 & 5.528 \\
\hline 0.097 & 0.035 & 0.016 & 5.384 \\
\hline 0.072 & 0.034 & 0.016 & 4.829 \\
\hline 0.085 & 0.037 & 0.020 & 5.460 \\
\hline 0.081 & 0.0 .37 & 0.010 & 6.294 \\
\hline
\end{tabular}




\section{APPENDIX A. COMMENTS ON THE TOTAL AGE-SPECIFIC FERTILITY RATE, TOTAL AGE-SPECIFIC MARRIED FERTILITY RATE, AND TOTAL DURATION-SPECIFIC FERTILITY RATE}

To summarize the time pattern of changing fertility in China from 1940 to 1982 , it is convenient to use an index of overall fertility. The measure of overall fertility for a specified period that is generally accepted as most useful is the total age-specific fertility rate, the sum of age-specific rates at which women from 15 to 50 years of age bear children. The TAFR is the mean number of children who would have been born to women reaching age 50 , had the women been subject at each age to the rates in question. It has the advantage over alternative measures of being independent of the age distribution of the population in question, and it is readily understood, since it answers the simple question: How many children, on average, would women bear according to this year's rates of childbearing?

An analogous aggregate measure of marital fertility is the total agespecific marital fertility rate, defined as the sum of age-specific marital fertility rates for ages 15 to 50 . It is the mean number of children who would have been born to women reaching age 50 , had all the women married at age 15, remained married, and experienced the marital fertility rates in question. If all women from 15 to 50 are currently married, the total fertility rate and the total marital fertility rate are equal. Otherwise, when all births occur to currently married women, the total fertility rate equals the total marital fertility rate multiplied by an index of proportion married $\left(C_{m}\right) . C_{m}$ is the weighted average of the proportion married at each age from 15 to 50; the weights are age-specific marital fertility rates. (See John Bongaarts, "A framework for analyzing proximate determinants of fertility," Population and Development Review, vol. 4, no. 1, March 1978.)

The TAMFR must be interpreted with caution. A higher value of this index for one population than for a second does not imply that married women in the first would have a larger number of children if subject to the observed marital fertility rates. A complicating factor is the effect on TAMFR of high marital fertility rates at young ages that prevail in populations where most marriages occur in the mid-20s or later. When the mean age at first marriage is over 25 , the few women who marry before age 20 typically have very high marital fertility at these ages. Many marry because they are pregnant, rather than become pregnant because they have married; the fertility rate at ages less than 20 can be as high as 0.70 in such populations. Marital fertility is usually higher early in marriage than in later years (especially when contraception leads to low fertility at greater durations); marital fertility at all ages up to and beyond the mean age at first 
marriage is experienced mostly by women at early durations. In Ireland in 1961 (where marriage was very late), the total marital fertility rate was 10.34 , even though the total age-specific fertility rate was only 3.78 , and married women reaching age 50 had borne an average of fewer than five children. Because of these ambiguities we have not calculated TAMFR for China.

An alternative cumulative measure of marital fertility is the total duration-specific fertility rate, or TDFR. It is the sum of fertility rates at each duration of marriage up to the highest duration at which nonzero fertility occurs, and it equals the average number of children that married women would bear if subject to the given rates at each duration from the first year after marriage until the highest duration with nonzero fertility.

TDFR for a birth cohort multiplied by the proportion ever married at age 50 in the cohort equals TAFR for the cohort. This equality can be understood intuitively by imagining a retrospective fertility survey conducted when a cohort reaches age 50. For the ever-married women in the cohort, the average number of children they have borne by age 50 is at once the sum of the age-specific fertility rates they have experienced and the sum of the duration-specific rates. But since TMR (the total first-marriage rate) equals the proportion ever married at age 50 (on the assumption of no marriages beyond age 50 and a negligible effect of differential mortality between the single and ever married), the total fertility rate of the whole cohort is the total fertility rate of the ever-married at 50 times TMR, or TDFR.TMR.

As will be demonstrated later, the relation of TAFR in a period to TDFR for the same period depends not on the total first-marriage rate for the period, but on the series of total first-marriage rates in the preceding years.

In a population with an approximately equal number at each age at which marital fertility rates are high, the total duration fertility rate can be converted into the total age fertility rate by multiplying the duration-specific rate at each duration $d$ by the total first-marriage rate at $t-d$ and summing the duration-specific rates so modified. As noted already, TAFR for a cohort is equal to TDFR.TMR because the ever-married women in the cohort have the same cumulative fertility by age (TAFR) as by duration since first marriage (TDFR); each total is equal to the average final parity of the cohort of ever-married women. If nuptiality is constant in a population with the same number of women at each childbearing age, if the proportion ever married at each age and the duration composition of ever-married women at each age are fixed, the cohort relation (TAFR=TDFR TMR) also applies to period total fertility rates and total nuptiality rates.

When first-marriage rates have been changing, causing variation in TMR, duration-specific fertility rates are not much affected. Women who married at age $\mathbf{2 4}$ have about the same fertility at early durations as women who married at age 23 . If mean age at first marriage changes from 23 to 
24, however, the number of first marriages is reduced, even though the total marriage rate in each cohort remains fixed.

The effect on the total fertility rate and the total first-marriage rate of a change in the age schedule of entry into first marriage is illustrated by a deliberately simplified hypothetical example.

\section{Hypothetical example of duration-specific and age-specific fertility when age at marriage changes}

Consider a regime of nuptiality and marital fertility in which 80 percent of all women marry, 16 percent of each cohort marrying at the exact ages of 20,21, 22, 23, and 24. Each married woman bears just one child, always within the first year of marriage. Total duration-specific fertility is 1.0 as a result of a rate of 1.0 at duration $0-1$ and 0.0 at other durations. Total agespecific fertility is 0.80 as a result of a fertility rate of 0.16 at five consecutive ages, from 20-21 through 24-25.

Now suppose that after the long prevalence of this regime, there is a change, beginning at time $t_{0}$, such that all cohorts arriving at age 20 postpone marriage for one year. In the new regime 16 percent marry at each exact age from 21 to 25 and each married woman bears one child in the first year of marriage. Duration-specific fertility remains 1.0 at duration $0-1$ and 0.0 at other durations, but age-specific fertility is altered. In the first year after $t_{0}, f_{20}$ is 0.0 and $f_{21}, f_{22}, f_{23}$, and $f_{24}$ are each 0.16 . After five years the first cohort subject to the new regime of marriage reaches age 25; the last 16 percent of the cohort who will ever marry marry at that age, leaving 20 percent single. At $t_{0}+5$, then, the age-specific fertility schedule is 0.16 at 21-22 through 25-26. During the five years following $t_{0}$, when no marriages occur at age 20 or 25 , but only at $21,22,23$, and 24 , the total agespecific fertility is only 0.64 ; at $t_{0}+5$ and subsequent times, age-specific TFR is again 0.80 . At all times duration-specific TFR remains at 1.0. A oneyear increase in age at marriage causes TAFR to fall from 0.80 to 0.64 for five years; it also causes the total first-marriage rate to fall from 0.80 to 0.64 for five years.

In this hypothetical example and also in real situations (at least when marital fertility is strongly controlled), a change in age at marriage has no necessary effect on duration-specific fertility rates, but it does affect both age-specific fertility rates and the total first-marriage rate. To reconcile total duration-specific fertility with total age-specific fertility when age at marriage changes, one must multiply duration-specific fertility at duration $y$ at time $t$ by total first-marriage rate at $t-y$. After this adjustment, the modified duration-specific rates sum to the same total as the sum of the agespecific fertility rates for the same year. 
In our example the reconciliation of the total duration-specific fertility rate with the total age-specific fertility rate is simple, since duration-specific fertility consists entirely of a rate of 1.0 at duration $0-1$. Because fertility is restricted to the first year of marriage, to convert the total duration-specific fertility rate for each year into the total age-specific fertility rate, TDFR is multiplied by the total first-marriage rate at the beginning of that year. The total duration-specific rates for years before $t_{0}$ are thus multiplied by 0.8 , for the next five years by 0.64 , and thereafter by 0.8 again, precisely converting the TDFRs into the TAFRs for these years.

Suppose that $f(d, t)$ is the fertility at time $t$ of ever-married women at duration $d$ since first marriage, and $\operatorname{TMR}(t)$ is the total first-marriage rate at time $t$. It can be proved (see the next section) that the total fertility rate (by age) at time $t$ is the sum of corrected duration-specific rates at time $t$, where the correction factor at each duration $d$ is simply the total firstmarriage rate $d$ years earlier. To be precise,

$$
\text { TAFR }=\int_{0}^{35} f(d, t) \operatorname{TMR}(t-d) d d ;
$$

but to be more precise, Equation (1) is exact only if the number of women at each age from 15 to 50 is the same. If, instead, the number of women at each age declines by an average fraction of $g$ per year of age, durationspecific fertility differs from its value with a flat age distribution by a factor of $\left(1+\left[\bar{a}_{d}-\bar{a}_{b}\right] 8\right)$, where $\bar{a}_{d}$ is the mean age of women at marriage duration $d$, and $\bar{a}_{b}$ is the mean age of childbearing of women at duration $d$. These two mean ages would be the same were it not for changing rates of childbearing with age for women at duration $d$. Suppose the average proportional decline in duration-specific fertility over the age range where women at duration $d$ are found is $h$ per year of age. Then the difference between $\bar{a}_{d}$ and $\bar{a}_{b}$ is $h \sigma_{d}^{2}$, where $\sigma_{d}^{2}$ is the variance of the age distribution of women at duration $d$, or the variance of age at first marriage $d$ years earlier. The overall effect of an age distribution that diminishes rather than is constant (over the range in which women at duration $d$ are found) is to increase duration-specific fertility at duration $d$ by a factor of $\left(1+g h \sigma_{d}^{2}\right)$. In a growing population, the age distribution may diminish by an average of 3 percent per year, and fertility at duration $d$ may decline with age by some 2 to 4 percent. The average decline in "natural" fertility from age 20 to age 35 is 1.9 percent per year; at higher ages the decline is greater. Allowance for a declining number of women as age increases thus multiplies the estimate of TAFR from TDFR by a factor of about $\left(1+.0009 \sigma_{d}^{2}\right)$. The variance of first-marriage frequencies is as little as 7 in China and was as much as 43 in nineteenth-century Sweden; the effect of a nonconstant age distribution is thus to make the sum of the duration-specific rates adjusted according to Equation (1) exceed the actual TFR by 1.0 to 4.0 percent. 


\section{Conversion of TDFR into TAFR in China}

In any closed population in which only married women bear children, a change in the age schedule of entry into marriage has no necessary effect on duration-specific fertility. Women who marry at age 24 instead of age 23 can be expected to bear children after marriage at about the same rate. The change in age at marriage does affect the number of women marrying each year, however, even when the proportion ever marrying within each cohort is constant. In the hypothetical example presented earlier, an increase of one year in the mean age at marriage depressed the total first-marriage rate by 20 percent of its original value for an interval of five years. This effect is an example of a general one: A permanent increase of one year in the mean age at first marriage causes the loss of one year's normal quota of first marriages, a loss that is usually spread over several years. Conversely, a reduction of one year in the mean age at first marriage adds altogether an extra one year's normal quota to the number of first marriages, again spread over a period. In a stationary regime of first-marriage rates, the total first-marriage rate is constant. The proportion ever married at each age does not change, remaining equal to the unchanging cohort proportion ever married at each age. In China, the proportion ultimately ever marrying in each cohort is very near 1.0. For example, the proportion ever married was above 0.99 at all ages greater than 31 in 1982 . However, the total first-marriage rate was below 1.0 in many years for which it can be calculated, as reported in the State Family Planning Commission's publication of its survey. Consequently, a total first-marriage rate of less than 1-for example, 0.88indicates that either a temporary avoidance of marriage or a rising age at marriage has depressed the number of marriages in the year in question (in this case by 12 percent). Suppose the total first-marriage rate in the year from 1 July 1978 to 1 July 1979 is 0.88 . This means that the number of women experiencing duration-specific fertility at duration $0-1$ is only about 88 percent of what the number would be if the TMR were 1.0 in that period. The smaller number of women in duration 0-1 in 1979 does not reduce the duration-specific rate, but it does reduce the contribution of women at duration $0-1$ (at the different ages at which they are found) to the overall agespecific fertility rate. Therefore, in converting the total duration-specific fertility rate into a number equal to the total age-specific fertility rate, one should multiply the contribution of births at duration $0-1$ by 0.88 . The general procedure for converting the total duration-specific rate into a number approximately equal to the total age-specific rate is to multiply the durationspecific rate at each duration $y$ by the total first-marriage rate in the year $t-y$ (Equation 1). Such a modification of the total duration-specific rates year by year in the rural population of Liaoning Province has been calculated. The adjusted total duration-specific fertility rates are compared with 
Table A.1. Total duration-specific fertility, total age-specific fertility, and adjusted total duration-specific fertility, for rural Liaoning Province, 1960-82, and for China and selected provinces, 1979

\begin{tabular}{|c|c|c|c|}
\hline Year & TDFR & TAFR & $\begin{array}{l}\text { Adjusted } \\
\text { TDFR }\end{array}$ \\
\hline 1960 & 7.51 & 6.78 & 6.96 \\
\hline 1961 & 3.36 & 3.03 & 3.08 \\
\hline 1962 & 6.88 & 6.17 & 6.36 \\
\hline 1963 & 9.77 & 8.91 & 9.22 \\
\hline 1964 & 8.28 & 7.59 & 7.81 \\
\hline 1965 & 7.55 & 6.67 & 6.91 \\
\hline 1966 & 8.27 & 7.30 & 7.56 \\
\hline 1967 & 6.66 & 5.76 & 5.92 \\
\hline 1968 & 7.35 & 6.38 & 6.51 \\
\hline 1969 & 6.62 & 5.84 & 5.88 \\
\hline 1970 & 6.08 & 5.18 & 5.31 \\
\hline 1971 & 5.77 & 4.86 & 4.99 \\
\hline 1972 & 4.94 & 4.16 & 4.18 \\
\hline 1973 & 5.22 & 4.22 & 4.32 \\
\hline 1974 & 4.47 & 3.55 & 3.55 \\
\hline 1975 & 3.70 & 2.75 & 2.85 \\
\hline 1976 & 3.31 & 2.43 & 2.47 \\
\hline 1977 & 2.89 & 2.17 & 2.20 \\
\hline 1978 & 3.19 & 2.46 & 2.55 \\
\hline 1979 & 3.29 & 2.57 & 2.67 \\
\hline 1980 & 2.45 & 2.08 & 2.13 \\
\hline 1981 & 2.32 & 2.15 & 2.21 \\
\hline 1982 & 2.08 & 2.13 & 2.22 \\
\hline \multicolumn{4}{|l|}{ China, 1979} \\
\hline Urban & 1.87 & 1.38 & 1.43 \\
\hline Rural & 3.91 & 3.06 & 3.12 \\
\hline City of Shanghai & 1.23 & .78 & .80 \\
\hline Rural Qinghai & 6.12 & 5.76 & 5.89 \\
\hline
\end{tabular}

the total age-specific rates from 1960 to 1982 in Table A.1. The agreement is quite good. From 1960 to 1981, the adjusted TDFRs exceed the TAFRs by less than 4 percent (an average of 2.7 percent), about the difference predicted as the effect of nonconstant age distribution and fertility rates. In selected areas of China in 1979, the adjusted TDFR exceeds the TAFR by 2 .or 3 percent. 


\section{TAFR and TDFR: Summary}

In a cohort, the total age-specific fertility rate equals the total durationspecific rate of ever-married women multiplied by the proportion who ever marry before age 50 . When all women marry at least once, when duration is measured from the date of first marriage, and when all births occur to married women, the total cohort age-specific fertility rate and the total duration-specific fertility rate are equal.

When age at marriage changes but all cohorts attain 100 percent ever married, the annual number of marriages and the total first-marriage rate are affected. They are reduced if age at marriage is increasing. Although duration-specific fertility rates are not affected by such a reduction in the size of marriage cohorts, the number of births and the age-specific fertility rates are reduced by the reduction in the number of married women at the relevant durations. For given duration-specific rates, the modified total duration-specific rate occasioned by past deviations in the number of marriages caused by changing age at marriage is $\mathrm{TDFR}^{*}=\sum_{j=0}^{30} \mathrm{TMR}_{t-j} d_{j}(t)$. The modified total exceeds (by only 1 to 3 percent) the total age-specific fertility rate, which is affected by the deficits (or excesses) of women at different durations produced by past fluctuations in the age schedule of first marriage. The conclusion is simple: In China, TAFR and TDFR would be very nearly equal if age at marriage were fixed. The time sequence of TDFR shows how marital fertility has changed, and the time sequence of TAFR shows how overall fertility has changed. Each year the difference between TDFR and TAFR shows how total fertility has been reduced or increased because of changes in age at marriage. 


\section{APPENDIX B. PROOFS OF TWO PROPOSITIONS ABOUT THE RELATION OF TDFR AND TAFR \\ by German Rodriguez ${ }^{\star}$ and Ansley J. Coale}

\section{Proposition 1}

When the period duration-specific fertility rate at each duration $d$ is multiplied by the total first-marriage rate $d$ years earlier, the sum of the durationspecific fertility rates so modified equals the total age-specific fertility rate, provided that the number of women at each childbearing age is the same.

\section{Proposition 2}

Total duration-specific fertility, when adjusted as in Proposition 1, exceeds rather than equals total age-specific fertility provided that, first, the number of women in the childbearing ages and, second, the rate of childbearing by women at duration $d$ both decline with age. The proportionate excess of adjusted TDFR over TAFR is $\left(1+g h \sigma_{d}^{2}\right)$, where $g$ is the average annual proportionate rate of decline with age in the number of women at duration $d, h$ is the average annual proportionate rate of decline with age in the fertility of women at duration $d$, and $\sigma_{d}^{2}$ is the variance of the age distribution of women at duration $d$ or the variance in age at first marriage $d$ years earlier.

\section{Notation}

$(\alpha, \beta)=$ reproductive span. No marriages or births occur outside this span. $g(a, t)=$ first-marriage density at age $a$ at time $t$.

$r(a, d, t)=$ ever-married fertility rate at $a, d, t$. $w(a, d, t)=$ number of ever-married women at $a, d, t$.

$W(a, t)=$ number of ever-married women (all durations) at $a, t$.

$W(d, t)=$ number of women (all ages) at $d, t$.

$N(a, t)=$ number of women at $a, t$.

$\operatorname{TMR}(t)=$ total first-marriage rate at $t$.

$B(a, d, t)=$ number of births to women at $a, d, t$.

$f(a, t)=$ age-specific fertility rate at $a, t$.

$f(d, t)=$ duration-specific fertility rate at $d, t$.

\section{Assumptions}

No premarital fertility exists.

Mortality for the single and for the ever-married is the same, so that

$$
w(a, t, d)=N(a, t) g(a-d, t-d)
$$

\footnotetext{
* German Rodriguez is Director, Department of Statistics, Catholic University of Chile.
} 
Proof of proposition 1

The age-specific fertility rate is

$$
\begin{aligned}
& f(a, t)=\int_{0}^{a-\alpha} B(a, d, t) d d / N(a, t), \text { or } \\
& f(a, t)=\int_{0}^{a-\alpha} N(a, t) g(a-d, t-d) r(a, t) d d / N(a, t), \text { or } \\
& f(a, t)=\int_{0}^{a-\alpha} g(a-d, t-d) r(a, t, d) d d .
\end{aligned}
$$

Thus age-specific fertility is independent of the age distribution. The total age-specific fertility rate is

$$
\operatorname{TAFR}(t)=\int_{\alpha}^{\beta} \int_{a}^{a-\alpha} g(a-d, t-d) r(a, t, d) d d d a .
$$

The duration-specific fertility rate is

$$
f(d, t)=\int_{\alpha+d}^{\beta} B(a, d, t) d a / W(d, t) .
$$

But $W(d, t)=\int_{\alpha+d}^{\beta+d} N(a) g(a-d, t-d) d a$ and

$$
\begin{aligned}
& B(a, d, t)=N(a) g(a-d, t-d) r(a, d, t), \text { so that } \\
& f(d, t)=\int_{\alpha+d}^{\beta} N(a) g(a-d, t-d) r(a, d, t) d a / \int_{\alpha+d}^{\beta+d} N(a) g(a-d, t-d) d a .
\end{aligned}
$$

At this point, the assumption of the same number of women at each childbearing age is invoked. If $N(a)$ is a constant, it cancels from $f(d, t)$. With $N(a)$ canceled, the denominator of $f(d, t)$ in equation $\mathrm{A} 2$ becomes

$$
\int_{\alpha+d}^{\beta+d} g(a-d, t-d) d a .
$$

If we let $x=a+d$, the denominator is

$$
\int_{\alpha}^{\beta} g(x, t-d) d x \text {, or the total first-marriage rate at time } t-d, \operatorname{TMR}(t-d) .
$$

If $f(d, t)$ is converted to $f^{\star}(d, t)$ by being multiplied by $\operatorname{TMR}(t-d)$,

$$
\begin{aligned}
& f^{*}(t, d)=\int_{\alpha+d}^{\beta} g(a-d, t-d) r(a, d, t) d a ; \text { adjusted TDFR is } \\
& \text { TDFR }^{\star}=\int_{0}^{\beta-\alpha} \int_{\alpha+d}^{\beta} g(a-d, t-d) r(a, d, t) d a d d .
\end{aligned}
$$

If one changes the order of integration, 
$\mathrm{TDFR}^{\star}=\int_{\alpha}^{\beta} \int_{0}^{a-\alpha} g(a-d, t-d) r(a, t, d) d d d a$ or $\mathrm{TDFR}^{\star}=\mathrm{TAFR}$ (see Equation $\mathrm{Al}$ ). QED.

\section{Proof of proposition 2}

In the proof of Proposition 1, an assumption that $N(a)$ is constant was introduced to make $f(d, t)$ independent of the age distribution. Suppose now that variation of $N(a)$ within the span of ages where there are women at duration $d$ is approximated by an exponential, such that $N(a) \doteq \mathrm{Ke}^{-g^{a}}$. In a population with an average growth rate of $r$ over this range and an average death rate of $\mu, g$ would equal $r+\mu$. With this approximation,

$$
f(d, t)=\int_{\alpha+d}^{\beta} e^{-g^{a}} g(a-d, t-d) r(a, d, t) d a / \int_{\alpha+d}^{\beta+d} e^{-g^{a}} g(a-d, t-d) d a .
$$

When the age distribution is constant, $g$ is zero. What is the effect of a nonzero $g$ on $f(d, t)$ ?

Let $f_{0}(d, t)$ be duration-specific fertility that would result from given $g(a-d, t-d)$ and $r(a, d, t)$ and a flat age distribution (so that $g=0$ ), and consider the rate of change in $f(d, t), \frac{d f(d, t)}{d g}$, as the steepness of the age distri-
bution increases from zero.

$$
\begin{aligned}
\frac{d f(d, t)}{d g}= & \frac{\int_{\alpha+d^{3}}^{\beta} a e^{-8 a} g(a-d, t-d) r(a, d, t) d a \int_{\alpha+d}^{\beta+d} e^{-g a} g(a-d, t-d) d a}{\left[\int_{\alpha+d}^{\beta+d} e^{-g^{a}} g(a-d, t-d) d a\right]^{2}} \\
& +\frac{\int_{\alpha+d}^{\beta+d} a e^{-g a} g(a-d, t-d) d a \int_{\alpha+d^{2}}^{\beta} e^{-g a} g(a-d, t-d) r(a, d, t) d a}{\left[\int_{\alpha+d}^{\beta+d} e^{-g a} g(a-d, t-d) d a\right]^{2}},
\end{aligned}
$$

or $\frac{d f(d, t)}{d g}=f(d, t)\left(\bar{a}_{d}-\bar{a}_{b}\right)$, where $\bar{a}_{d}$ is the mean age of women at duration $d$ (equal to the mean age at marriage at $t-d$ plus $d$ ) and $\bar{a}_{b}$ is the mean age at childbirth of ever-married women at duration $d$. Thus, for a small value of $g, f(d, t)=f_{0}(d, t)\left[1+g\left(\bar{a}_{d}-\bar{a}_{b}\right)\right]$.

The mean age at childbirth of women at duration $d$ is the mean age of $w(a, d, t) r(a, d, t)$. If $r(a, d, t)$ were constant over the relevant ages, $\bar{a}_{b}$ would equal $\bar{a}_{d}$. But marital fertility at duration $d$ often declines with age. Suppose the average proportionate decline over the central ages of women at duration $d$ is $h$. Then 


$$
\begin{aligned}
& \bar{a}_{b}=\int_{\alpha+d}^{\beta} a w(a, d, t) r(a, d, t) d a / \int_{\alpha+d}^{\beta} w(a, d, t) r(a, d, t) a d \text { becomes } \\
& \bar{a}_{b}=\int_{\alpha+d}^{\beta+d} a e^{-h a} g(a-d, t-d) d a / \int_{\alpha+d}^{\beta+d} e^{-h a} g(a-d, t-d) d a .
\end{aligned}
$$

If $h$ is zero, $\bar{a}_{b}$ is simply the mean age of women at duration $d$, or $\bar{a}_{d}$. To approximate the value of $\bar{a}_{b}$ for a small value of $h$, take the derivative of $\bar{a}_{b}$ with respect to $h$, evaluated at $h=0$.

$$
\frac{d \bar{a}_{b}}{d h}=\frac{-\int_{\alpha+d}^{\beta+d} a^{2} e^{-h a} g(a-d, t-d) d a}{\int_{\alpha+d}^{\beta+d} e^{-h a} g(a-d, t-d) d a}+\frac{\left[\int_{\alpha+d}^{\beta+d} a e^{-h a} g(a-d, t-d) d a\right]^{2}}{\left[\int_{\alpha+d}^{\beta+d} e^{-h a} g(a-d, t-d) d a\right]^{2}}, \text { or }
$$

$\left[\frac{d \bar{a}_{b}}{d h}\right]_{h=0}=\sigma_{d}^{2}$, the variance of the age distribution of women at duration $d$. Thus, for small values of $h, \bar{a}_{b}=\bar{a}_{d}-h \sigma_{d}^{2}$, or $\bar{a}_{d}-\bar{a}_{b}$ equals $h \sigma_{d}^{2}$. Since $f(d, t)$ with an age distribution that falls off at a rate $e^{-g^{a}}$ is $f_{0}(d, t)\left[1+g\left(\bar{a}_{d}-\bar{a}_{g}\right)\right]$, if the fertility schedule for women at duration $d$ falls off at a rate of $e^{-h a}, f(d, t)=f_{0}(d, t)\left(1+g h \sigma_{d}^{2}\right)$. QED. 


\section{RECENT AVAILABLE PAPERS OF THE EAST-WEST POPULATION INSTITUTE}

No.

85 Development perspectives and population change, by Ozzie G. Simmons, April 1983, $\mathrm{vi}+41 \mathrm{pp}$.

86 The effects of induced abortion on subsequent reproductive function and pregnancy outcome: Hawaii, by Chin Sik Chung and Patricia G. Steinhoff in collaboration with Roy G. Smith and Ming-Pi Mi, June 1983, xii + 144 pp.

60-F Influences on childbearing intentions across the fertility career: demographic and socioeconomic factors and the value of children, by Rodolfo $\mathrm{A}$. Bulatao and James $\mathrm{T}$. Fawcett, June 1983, x +152 pp.

87 Population mobility and wealth transfers in Indonesia and other Third World societies, by Graeme J. Hugo, July 1983, vi +50 pp.

88 Structural change and prospects for urbanization in Asian countries, by Gavin W. Jones, August 1983, vi + 46 pp.

89 Urban growth and local taxes in less developed countries, by Roy Bahl, Daniel Holland, and Johannes Linn, September 1983, vi +33 pp.

90 A false fertility transition: the case of American blacks, by Paul Wright and Peter Pirie, February 1984, viii +81 pp.

60-G The old-age economic security value of children in the Philippines and Taiwan, by Susan De Vos, March 1984, viii + 72 pp.

91 A profile of Hawaii's elderly population, by Eleanor C. Nordyke, Richard K.C. Lee, and Robert W. Gardner, August 1984, viii + 39 pp.

92 City characteristics, migration, and urban development policies in India, by Mahendra K. Premi with Judith Ann L. Tom, June 1985, viii + 127 pp.

93 Urbanization in China: new insights from the 1982 Census, by Sidney Goldstein, July 1985, vi +73 pp.

94 Comparison of fertility trends estimated alternatively from birth histories and own children, by Robert D. Retherford and Iqbal Alam, July 1985, viii + 39 pp.

95 Population mobility in the People's Republic of China, by Sidney Goldstein and Alice Goldstein, October 1985, vi + 46 pp.

96 Factors in the achievement of below-replacement fertility in Chiang Mai, Thailand, by Tieng Pardthaisong, March 1986, viii +46 pp.

97 The Population of Burma: An analysis of the 1973 Census, by M. Ismael Khin Maung, April 1986, viii + 32 pp.

98 Population aging in Australia: Implications for social and economic policy, by Graeme Hugo, April 1986, viii +47 pp.

99 Marriage and fertility in Tianjin, China: Fifty years of transition, by Burton Pasternak, July 1986, viii + 76 pp.

100 Migration in Thailand: A twenty-five-year review, by Sidney Goldstein and Alice Goldstein, July 1986, viii + 54 pp.

101 Recent fertility trends in the Pacific Islands, by Michael J. Levin and Robert D. Retherford, August 1986, viii $+72 \mathrm{pp}$.

102 Malnourished children: An economic approach to the causes and consequences in rural Thailand, by Sirilaksana Chutikul, December 1986, viii + 64 pp.

103 Consistent correction of census and vital registration data for Thailand, 1960-80, by Norman Y. Luther, Neramit Dhanasakdi, and Fred Arnold, December 1986, viii +40 pp. 
THE EAST-WEST CENTER is a public, nonprofit educational institution with an international board of governors. Some 2,000 research fellows, graduate students, and professionals in business and government each year work with the Center's international staff in cooperative study, training, and research. They examine major issues related to population, resources and development, the environment, culture, and communication in Asia, the Pacific, and the United States. The Center was established in 1960 by the United States Congress, which provides principal funding. Support also comes from more than 20 Asian and Pacific governments, as well as private agencies and corporations.

Situated on 21 acres adjacent to the University of Hawaii's Manoa Campus, the Center's facilities include a 300-room office building housing research and administrative offices for an international staff of 250 , three residence halls for participants, and a conference center with meeting rooms equipped to provide simultaneous translation and a complete range of audiovisual services.

THE EAST-WEST POPULATION INSTITUTE, established as a unit of the EastWest Center in 1969, carries out multidisciplinary research, training, and related activities in the field of population, placing emphasis on economic, social, psychological, and environmental aspects of population problems in Asia, the Pacific, and the United States. 